\section{Magnetic Fusion Eñergy (UC-20) \\ MFE--Reactor Materials (UC-20c) \\ MFE--Fusion Systems (UC-20d) \\ MFE--Environment and Safety \\ Ânalyses (UC-20e)}

ANL-84-55

ARGONNE NATIONAL LABORATORY

ANL $--84-55$

9700 South Cass Avenue

DE85 008345

\title{
THE TRIO EXPERIMENT
}

by

R. G. Clemmer, P. A. Finn, R. F. Malecha, B. Misra, M. C. Billone, D. L. Bowers, A. K. Fischer, L. R. Greenwood, R. F. Mattas, S. W. Tam, R. B. Poeppel, and G. T. Reedy

Argonne National Laboratory

I. T. Dudley, F. F. Dyer, E. D. Clemmer, J. S. Watson, P. W. Fisher, J. R. Conlin, R. L. Childs, and J. L. Scott

Oak Ridge Nationai Laboratory

R. M. Arons

Celanese Research Company

Summit, N. J.

A. E. Scandora

Science Applications, Inc.

Schaumberg, I11. 
TABLE OF CONBNTS

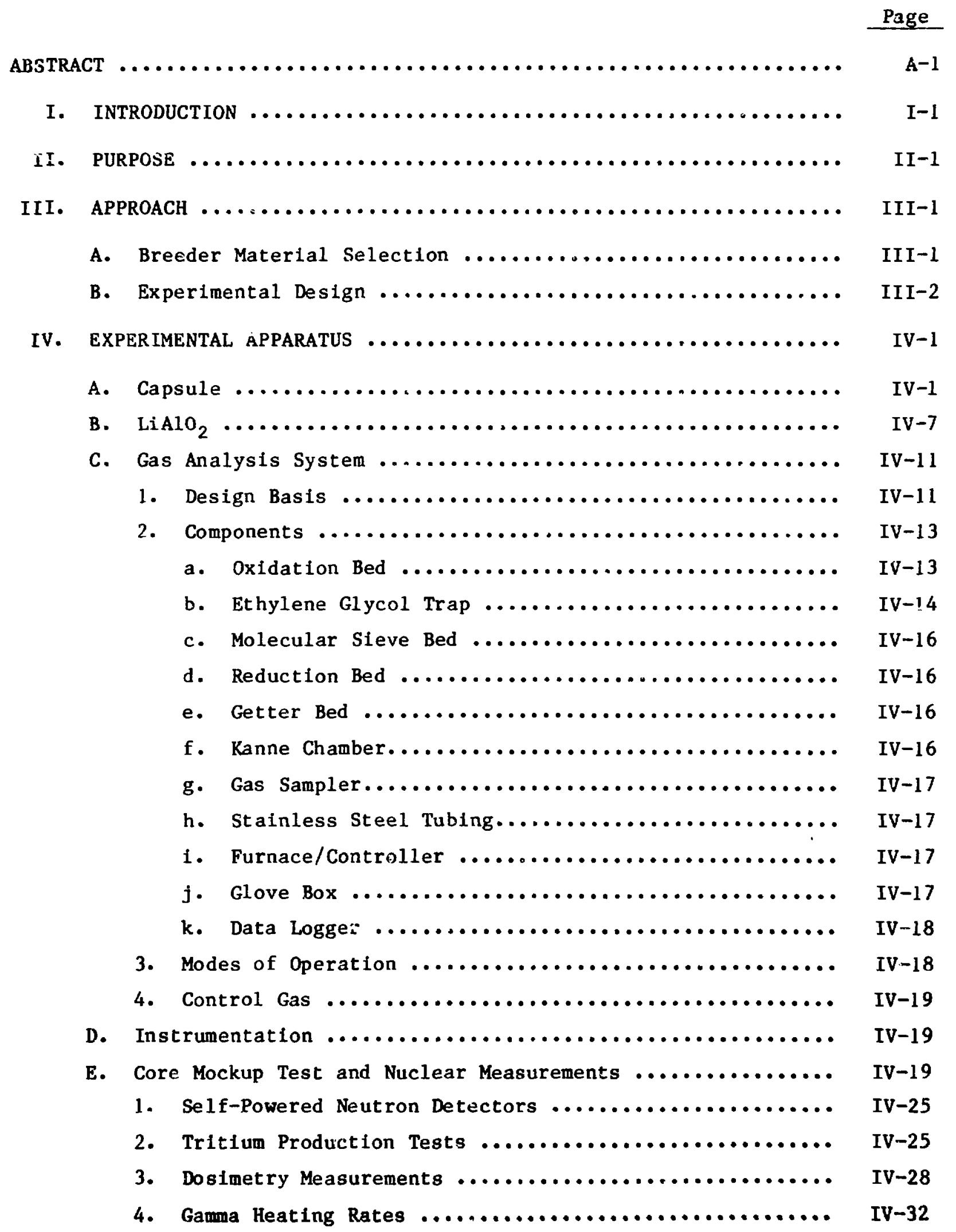




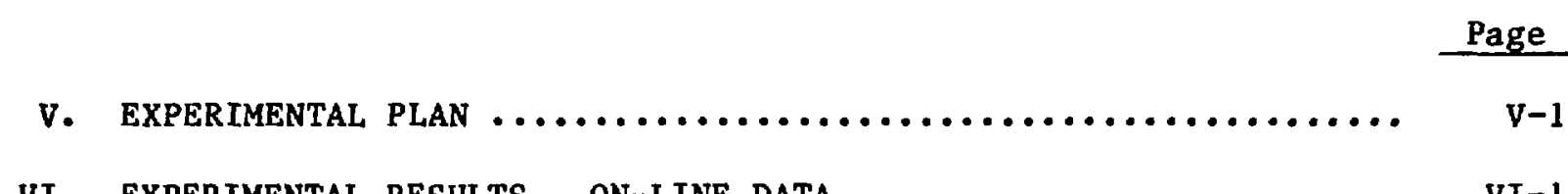

vi EXPERIMENTAL RESULTS - ON-LINE DATA $\ldots \ldots \ldots \ldots \ldots \ldots \ldots \ldots \ldots$ vI-1

A. Time and Recorded Experimental History $\ldots \ldots \ldots \ldots \ldots \ldots \ldots$ VI-1

B. Neutron Flu. and Dosimetry.$\ldots \ldots \ldots \ldots \ldots \ldots \ldots \ldots \ldots \ldots$ vI-1

C. Temperature Profiles ........................... vI-12

D. Sweep Gas Analyses $\ldots \ldots \ldots \ldots \ldots \ldots \ldots \ldots \ldots \ldots \ldots \ldots \ldots$ vI-12

E. Tritium Collected $\ldots \ldots \ldots \ldots \ldots \ldots \ldots \ldots \ldots \ldots \ldots \ldots \ldots \ldots$ VI-1 2

1. HT Collected $\ldots \ldots \ldots \ldots \ldots \ldots \ldots \ldots \ldots \ldots \ldots \ldots \ldots \ldots$ VI-14

2. Tritium Collected as HTO $\ldots \ldots \ldots \ldots \ldots \ldots \ldots \ldots \ldots \ldots$ VI-22

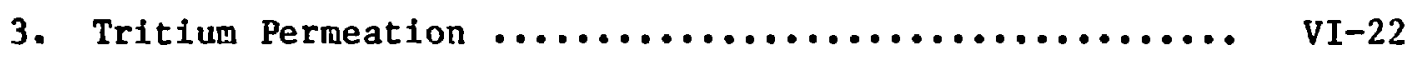

4. Total Tritium Collected $\ldots \ldots \ldots \ldots \ldots \ldots \ldots \ldots \ldots \ldots$ vI 33

F. Tritium (HT) Release Rates ..................... vI-33

G. Release Rates of HTO, Condensable Form .............. VI-81

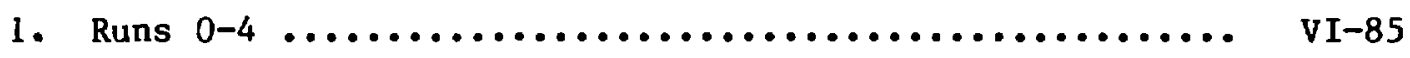

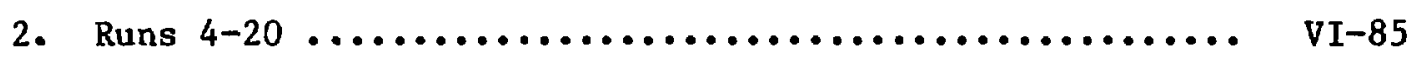

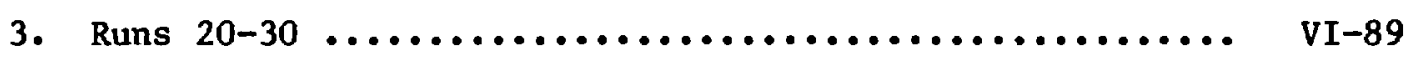

H. Radionuclides $\ldots \ldots \ldots \ldots \ldots \ldots \ldots \ldots \ldots \ldots \ldots \ldots \ldots \ldots \ldots \ldots$ VI-92

vit. POST-IRRADIATION EXAMINATIONS (PIE) $\ldots \ldots \ldots \ldots \ldots \ldots \ldots \ldots \ldots$ viI-1

A. Capsule Disassembly $\ldots \ldots \ldots \ldots \ldots \ldots \ldots \ldots \ldots \ldots \ldots \ldots$ vII -1

B. Tritium Retention in the Lithium Aluminate Pellets ....... VII-5

C. Lithium Isotopy $\ldots \ldots \ldots \ldots \ldots \ldots \ldots \ldots \ldots \ldots \ldots \ldots \ldots$ vII-8

D. Dosimetry $\ldots \ldots \ldots \ldots \ldots \ldots \ldots \ldots \ldots \ldots \ldots \ldots \ldots \ldots \ldots \ldots$ VII 8

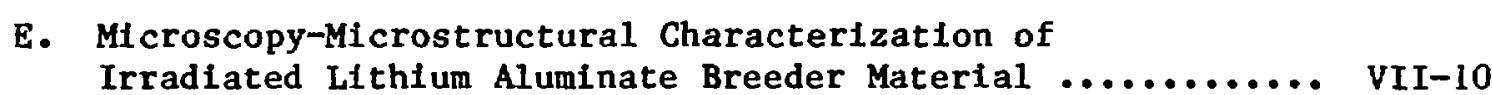

F. Radioactivity .............................. vII-11

G. X-Ray Diffraction $\ldots \ldots \ldots \ldots \ldots \ldots \ldots \ldots \ldots \ldots \ldots \ldots \ldots$ vII-15

viII. Data ANALYSIS $\ldots \ldots \ldots \ldots \ldots \ldots \ldots \ldots \ldots \ldots \ldots \ldots \ldots \ldots \ldots \ldots$. vili-

A. Dosimetry and Damage Analysis $\ldots \ldots \ldots \ldots \ldots \ldots \ldots \ldots \ldots \ldots$ vIII-1

1. Tritium Production Rates ....................... viII-1

2. Danage Analysis.$\ldots \ldots \ldots \ldots \ldots \ldots \ldots \ldots \ldots \ldots \ldots \ldots$ vIII -2

3. Nuclear Heating Rates...$\ldots \ldots \ldots \ldots \ldots \ldots \ldots \ldots \ldots$ vIII-2 


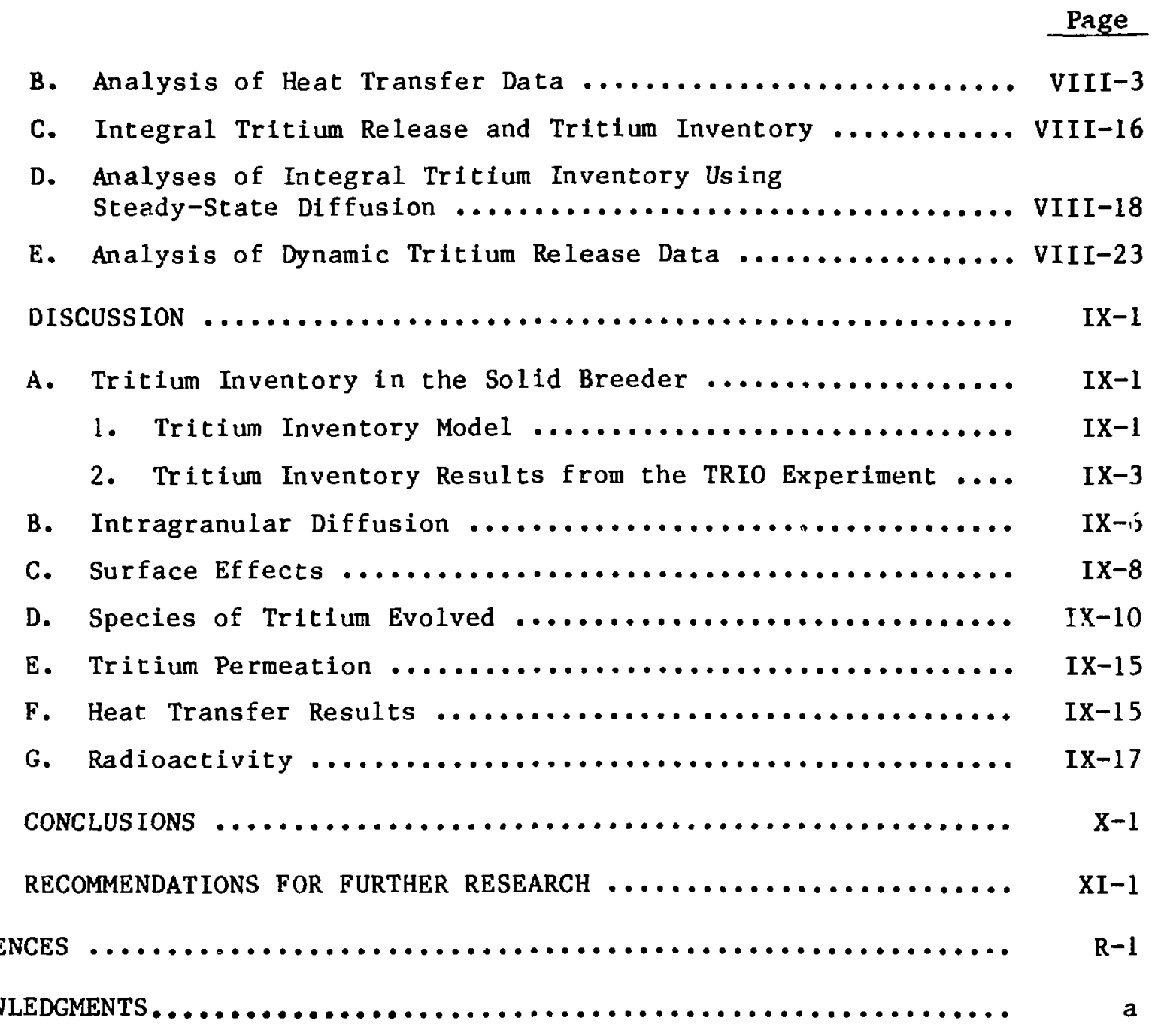




\section{LIST OP F LGURBS}

\begin{tabular}{|c|c|c|}
\hline No. & Title & Page \\
\hline IV -1 & TRIO sweep gas flow path. ...................... & IV-2 \\
\hline IV -2 & rRIo design concept. $\ldots \ldots \ldots \ldots \ldots \ldots \ldots \ldots \ldots \ldots \ldots \ldots \ldots \ldots$ & IV -2 \\
\hline IV -3 & Detailed design of TRIO capsule. ................... & IV-5 \\
\hline IV -4 & Scanning electron micrographs of $\gamma-\mathrm{LiAlO}_{2}$ pellet. $\ldots \ldots \ldots \ldots$ & IV -10 \\
\hline IV -5 & Flow diagram for the gas analysis system. ............. & IV-12 \\
\hline IV -6 & Modes of operation for the sweep gas analysis system. ..... & IV -18 \\
\hline IV -7 & Core mockup test assembly. $\ldots \ldots \ldots \ldots \ldots \ldots \ldots \ldots \ldots \ldots \ldots \ldots$ & IV -23 \\
\hline IV -8 & 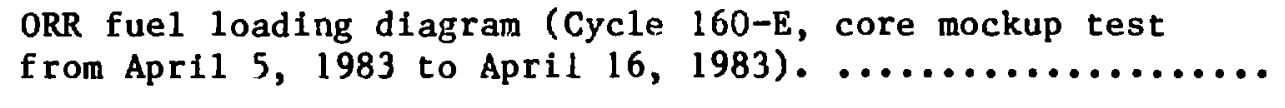 & IV -24 \\
\hline IV-9 & Cross section of TRIO core mockup. ................. & IV -25 \\
\hline IV -10 & $\begin{array}{l}\text { Flux spectrum measured in the A2 core position of } \text { ORR for } \\
\text { the TRIO experiment. } \ldots \ldots \ldots \ldots \ldots \ldots \ldots \ldots \ldots \ldots \ldots \ldots \ldots \ldots \ldots \ldots \ldots \ldots \ldots \ldots \ldots\end{array}$ & IV -31 \\
\hline IV-1I & Vertical fuel gradients measured in position A2 of ORR. .... & IV -32 \\
\hline IV-12 & $\begin{array}{l}\text { Fuel loading diagram for the gamma heating measurements } \\
\text { (July } 12,1981) . \ldots \ldots \ldots \ldots \ldots \ldots \ldots \ldots \ldots \ldots \ldots \ldots \ldots \ldots \ldots \ldots \ldots\end{array}$ & IV -33 \\
\hline$V I-1$ & 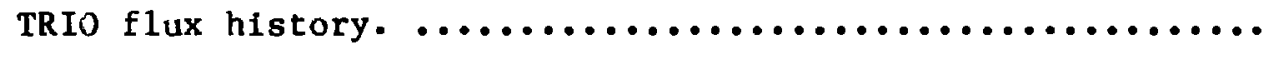 & $V I-4$ \\
\hline$V I-2$ & ORR reactor power history $\ldots \ldots \ldots \ldots \ldots \ldots \ldots \ldots \ldots \ldots \ldots$ & VI-4 \\
\hline$V I-3 a$ & 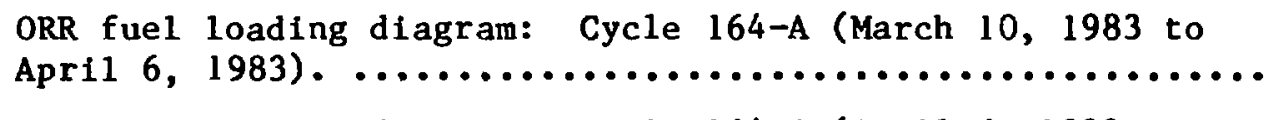 & VI-5 \\
\hline$V I-3 b$ & 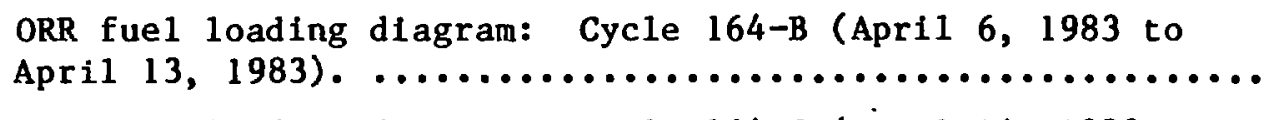 & $V I-6$ \\
\hline$V I-3 c$ & 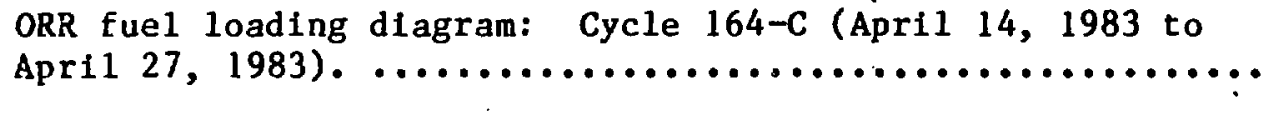 & $\mathrm{VI}-7$ \\
\hline$V I-3 d$ & $\begin{array}{l}\text { ORR fuel loading diagram: Cycle 164-D (April 28, } 1983 \text { to } \\
\text { May } 6,1983) . \ldots \ldots \ldots \ldots \ldots \ldots \ldots \ldots \ldots \ldots \ldots \ldots \ldots \ldots \ldots \ldots \ldots \ldots \ldots\end{array}$ & VI-8 \\
\hline$V I-3 e$ & $\begin{array}{l}\text { ORR fuel loading diagram: Cycle } 164-E \text { (May } 6,1983 \text { to } \\
\text { May } 16,1983) . \ldots \ldots \ldots \ldots \ldots \ldots \ldots \ldots \ldots \ldots \ldots \ldots \ldots \ldots \ldots \ldots \ldots \ldots \ldots \ldots\end{array}$ & VI-9 \\
\hline$V I-3 f$ & $\begin{array}{l}\text { ORR fuel loading diagram: Cycle } 164-F \text { (May } 16,1983 \text { to } \\
\text { May } 31,1983) \ldots \ldots \ldots \ldots \ldots \ldots \ldots \ldots \ldots \ldots \ldots \ldots \ldots \ldots \ldots \ldots \ldots \ldots \ldots \ldots \ldots \ldots \ldots\end{array}$ & $V I-10$ \\
\hline$V I-3 g$ & $\begin{array}{l}\text { ORR fuel loading diagram: Cycle } 164-\text { G (May } 31,1983 \text { to } \\
\text { June } 13,1983) . \ldots \ldots \ldots \ldots \ldots \ldots \ldots \ldots \ldots \ldots \ldots \ldots \ldots \ldots \ldots \ldots \ldots \ldots \ldots \ldots \ldots \ldots\end{array}$ & VI-11 \\
\hline$V I-4$ & TRIO capsule temperatures. .. & VI-13 \\
\hline$V I-5$ & TRIO ca & VI-13 \\
\hline $\begin{array}{l}V I-6 \\
V I-7\end{array}$ & $\begin{array}{l}\text { Recorded temperatures for } \operatorname{Run} 0 . \ldots \ldots \\
\text { Trit } 1 \text { m release rate for } \operatorname{Run} 0, \ldots \ldots\end{array}$ & $\begin{array}{l}V I-40 \\
V I-40\end{array}$ \\
\hline
\end{tabular}




\section{LIST OF FIGURES (Contd.)}

No.

VI -8

VI-9

VI-10

$V I-11$

VI-12

VI -13

$V I-14$

VI -15

VI-16

VI -17

VI-18

$\mathrm{VI}-13$

VI -20

VI-2

VI -22

VI -23

VI -24

$V I-25$

VI-26

$\mathrm{VI}-27$

VI-28

VI-29

$V I-30$

VI-3

VI-32

VI-33

VI -34

VI-35

VI-36

VI -37

$V I-38$

VI -39

$V I-40$
Title

Recorded temperature for Run 1.

Tritium release rate and generation rate for Run 1.......

Recorded temperature for Run 2.

Tritium release rate for Run 2 .

Recorded temperatures for Run 3.

Tritium release rate for Run 3.

Recorded temperatures for Run 4.

Tritium release rate for Run 4

Recorded temperatures for Run 5.

Tritium release rate for Run 5 .

Recorded temperatures for Run 6

Tritium release rate for Run 6 .

Recorded temperatures for Run 7.

Tritium release rate for Run 7

Recorded temperatures for Run 8

Tritium release rate for Run 8 .

Recorded temperatures for Run 9.

Tritium release rate for Run 9

Recorded temperatures for Run 10 .

Recorded temperatures for Run 11

Tritium release rate for Runs 10 and 11 .

Recorded temperatures for Run 12

Tritium release rate for Run 12

Recorded temperatures for Run 13.

Tritium release rate for Run 13.

Recorded temperatures for Run 14.

Tritium release rate for Run 14

Recorded temperatures for Run 15

Tritium release rate for Run 15 .

Recorded temperatures for Run 16.

Tritium release rate for Run 16.

Sweep gas pressure (psig) for Run 16.

Hellum flow to seep gas for Run 16 .
Page

$\mathrm{VI}-42$

VI-42

VI-44

$\mathrm{VI}-44$

$V I-45$

$V I-45$

$V I-46$

VI-46

$V I-48$

$V I-48$

VI-49

$V I-49$

VI-50

$V I-50$

VI-5I

VI-5I

VI-52

VI-52

VI-54

VI -54

VI-55

VI-55

VI -56

$V I-56$

VI -57

VI-57

VI -58

$V I-58$

VI-59

VI-60

VI-60

VI-6I

$V I-61$ 


\section{LIST OF FIGURES (Contd.)}

\begin{tabular}{|c|c|c|}
\hline o. & itle & Page \\
\hline VI-41 & 1low of helium $+4 \% \mathrm{H}_{2}$ to sweep gas for Run $16 . \ldots \ldots \ldots \ldots$ & VI-62 \\
\hline VI -42 & Sweep gas exhaust $\left(\mathrm{cm}^{3} / \mathrm{min}\right)$ for Run $16 . \ldots \ldots \ldots \ldots \ldots \ldots$ & $I-62$ \\
\hline $1-43$ & ded temperatures for Run $17, \ldots \ldots \ldots$ & {$[-64$} \\
\hline $1-44$ & Iritium release rate for Run $17, \ldots \ldots \ldots$ & $I-64$ \\
\hline $1 \mathrm{I}-45$ & peratures for Run $18 . \ldots \ldots \ldots \ldots$ & VI -65 \\
\hline$I-46$ & ate for $\operatorname{Run} 18, \ldots \ldots \ldots$ & $V I-6$ \\
\hline$I-47$ & res for Run $19 . . . . .$. & VI-66 \\
\hline$I-48$ & e for Run $19 . \ldots \ldots \ldots$ & VI-6e \\
\hline$I-49$ & res for Run $20 . . . .$. . & VI -67 \\
\hline$I-50$ & for Run $20 . \ldots$ & VI-67 \\
\hline $\mid I-51$ & es for Run $21 . \ldots \ldots \ldots \ldots$. & VI-68 \\
\hline$I-52$ & or $\operatorname{Run} 21 . \ldots \ldots \ldots \ldots \ldots \ldots \ldots \ldots \ldots \ldots$ & VI-68 \\
\hline$I-5$ & r Run $22 . \ldots$ & VI-70 \\
\hline$I-5$ & for Run $22 . \ldots$ & VI-70 \\
\hline$I-5$ & es for Run 23 . & VI-71 \\
\hline$I-5$ & Run 23. & $V I-71$ \\
\hline$I-57$ & es for Run $24 . \ldots$ & VI-72 \\
\hline$I-58$ & for Run $24 . \ldots \ldots \ldots$ & vI -72 \\
\hline $\mathrm{I}-5$ & or Run $25, \ldots \ldots \ldots$. & vI-73 \\
\hline$I-6$ & for Run $25 . \ldots \ldots \ldots$ & VI-73 \\
\hline$I-6$ & $r \operatorname{Run} 26 . \ldots \ldots \ldots \ldots \ldots \ldots \ldots \ldots$ & VI-75 \\
\hline $1-6$ & Run $26 . \ldots \ldots \ldots$ & -75 \\
\hline$I-6$ & Run $27 . \ldots \ldots \ldots$ & 76 \\
\hline$I-$ & $\cdots \cdots \cdots$ & 76 \\
\hline$I-6$ & May 20 & 77 \\
\hline I- & (....... & .77 \\
\hline 1 & $\cdots$ & 78 \\
\hline$-c$ & r Ru & \\
\hline $\mathrm{I}-\mathrm{C}$ & 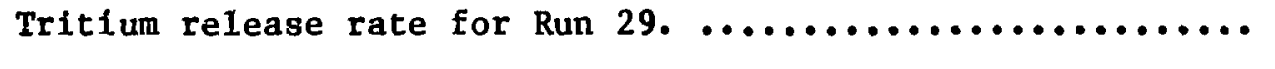 & 79 \\
\hline VI-7C & $\cdots \cdots$ & VI-80 \\
\hline $1 \mathrm{I}-71$ & $\bullet \bullet \bullet$ & $V I-80$ \\
\hline VI-7 & ( & VI-82 \\
\hline-73 & ease & VI-82 \\
\hline
\end{tabular}




\section{LIST OF PIGURES (Contd.)}

VI-74 Recorded temperatures for Run 32. ..................... VI-83

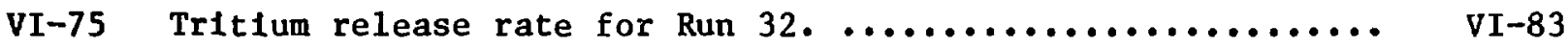

VI-76 Recorded temperatures for Run 33. ..................... VI-84

VI-77 Tritium release rate for Run 33. ..................... VI-84

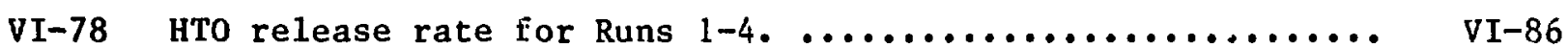

VI-79 HTO release rate for Run 1. ........................ VI-86

VI-80 HTO release rate for Runs $4-7 . \ldots \ldots \ldots \ldots \ldots \ldots \ldots \ldots \ldots \ldots \ldots \ldots \ldots$ VI-87

VI-81 HTO release rate for Run $8 . \ldots \ldots \ldots \ldots \ldots \ldots \ldots \ldots \ldots \ldots \ldots \ldots \ldots \ldots$ VI-87

VI-82 HTO release rate for Runs 9-13. ...................... VI-88

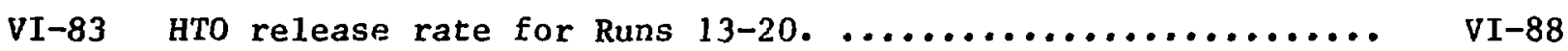

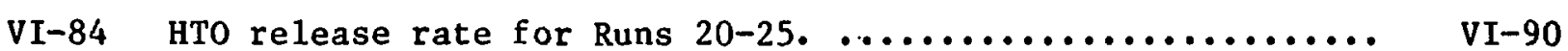

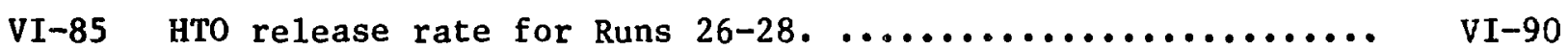

VI-86 HTO release rate for Run $28 . \ldots \ldots \ldots \ldots \ldots \ldots \ldots \ldots \ldots \ldots \ldots \ldots \ldots \ldots$ VI-91

VI-87 HTO release rate for Runs $29-30 . \ldots \ldots \ldots \ldots \ldots \ldots \ldots \ldots \ldots \ldots \ldots \ldots . . . \ldots$ VI-91

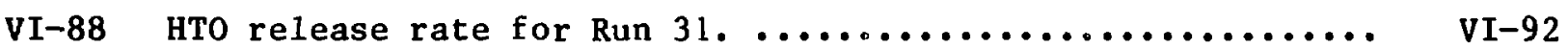

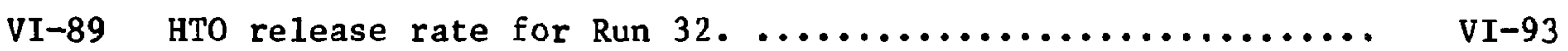

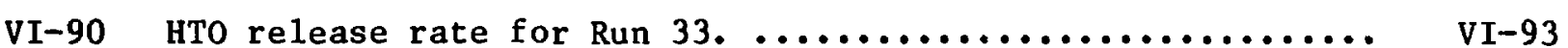

VII-1 Drawing of TRIO capsule showing location of first cut for the disassembly procedure. ...................... VII-3

.I-2 Photograph of TRIO capsule after first cut made. ......... VII-4

VII-3 Photograph of irradiated LIAlO pellet. ................ VII-6

VII-4 Scanning electron micrographs of j.rradiated $\mathrm{LIAlO}_{2}$

from TRIO. ................................. VII-12

VII-5 Scanning electron micrographs of irradiated $\mathrm{LiAlO}_{2}$

from TRIO.

VIII-13

VIII-1 Diffusion coefficient for tritium in $\mathrm{LiAlO}_{2}$ determined froin selected TRIo runs in which intragranular diffusion appeared to be the rate-limiting phenomenon in tritium transport. .................................... vIII-22

VIII-2 Fractional release rate and inventory for step increase In temperature $\left(\tau_{1} / \tau_{2}=19.4\right) \ldots \ldots \ldots \ldots \ldots \ldots \ldots \ldots \ldots \ldots \ldots$ vIII-25

VIII-3 Comparison between analytical predictions ( $k_{\text {nom }}$ ) for Run 8 and TRIO tritium rate $\left(\mathbf{R}_{e}\right)$ for a mean temperature Increase from $478^{\circ} \mathrm{C}$ to $627^{\circ} \mathrm{C}$. 


\section{LIST OF PIGURES (Contd.)}

No.

Title

Page

VIII-4 Comparison between analytical predictions ( $\dot{R}_{\mathrm{min}}, \dot{R}_{\mathrm{nom}}$, $\left.\dot{R}_{\max }\right)$ for Run 31 and TRIO tritium release rate $\left(k_{\mathrm{e}}\right)$ for a mean temperature increase from $474^{\circ} \mathrm{C}$ to $757^{\circ} \mathrm{C} . \ldots \ldots \ldots \ldots$.... VIII-27

VIII-5 Comparison between analytical predictions ( $\left.\dot{R}_{\text {nom }}\right)$ for Run 31 and TRIO rritium release rate $\left(\hat{R}_{e}\right)$ for a mean temperature increase from $747^{\circ} \mathrm{C}$ to $757^{\circ} \mathrm{C}$..................... vIII-28

IX-1 Comparison of selected data for tritium diffusion in $\mathrm{\gamma}^{-\mathrm{LiAlO}_{2}}$ with TRIO corelation (includes uncertainties in grain size, Inventory, and generation rate. ..................... IX-1

IX-2 Tritium permeation results for TRI0. .................. IX-16

IX-3 Thermal conductivcity of $\gamma-\mathrm{LiAlO}_{2}$ measured in pile....... IX-16 


\section{LIST OF TABLES}

III-1 Design and Operating Conditions of STARFIRE and TRIO

Breeder Blankets.

IV-1 Characteristics of $\mathrm{\gamma LiAlO}_{2}$ Breeder Material for TRIO

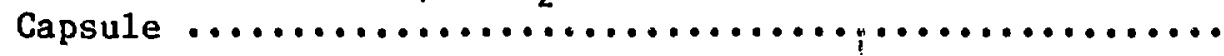

IV-2 Characteristics of TRIO Capsule and Capsule

Instrumentation $\ldots \ldots \ldots \ldots \ldots \ldots \ldots \ldots \ldots \ldots \ldots \ldots \ldots$ IV -4

IV-3 Location of Capsule Thermocouples ................. IV -6

IV-4 Chemical Analysis of $\mathrm{LiAlO}_{2}$ Powder $\ldots \ldots \ldots \ldots \ldots \ldots \ldots \ldots$ IV -8

IV-5 Density Data for the Firing of the First Test Pe1let .... IV-11

IV-6 Parameter List of TRIO ........................ IV-20

IV-7 Comparison of Predicted and Measured Activities of

Tritium in Irradiated Samples of Lithium Carbonate and

Lithium Aluminate ............................... IV -27

IV-8 Tritium Production in the Core Mockup Test

(Apri1 27, 1982) ........................... IV -28

IV-9 Act1vation Rates for TRIo ...................... IV-29

IV-10 Neutron F1ux Values for TRIo (Apri1 26, 1982) ......... IV-30

IV-11 Tritium Production Rates in the TRIO Core Mockup Test .... IV-30

V-1 TRIo Test Matrix $\ldots \ldots \ldots \ldots \ldots \ldots \ldots \ldots \ldots \ldots \ldots \ldots \ldots \ldots \ldots, \quad v-2$

VI-1 TRIO Operating History $\ldots \ldots \ldots \ldots \ldots \ldots \ldots \ldots \ldots \ldots \ldots \ldots . \ldots \ldots$ VI-2

VI-2 Sweep Gas Analysis .......................... vI-14

VI-3 Tritium Collected, Noncondensable or HT Form

VI-4 Tritium Collected, Condensable, or HTO Trorm

(January 26, 1983) ............................. VI-23

VI-5 TRIO Tritium Permeation Data ..................... VI-34

VI-6 Tritium Permeation Data ....................... vi-36

vi-7 Summary of Tritium Collected .................... vI-38 
LIST OF TABLBS (Contd.)

\begin{tabular}{|c|c|c|}
\hline No. & Title & Page \\
\hline VII-1 & Measured Capsule Asymmetry ..................... & VII-4 \\
\hline VII-2 & $\begin{array}{l}\text { Measured Tritium Activities Retained in Lithium } \\
\text { Aluminate Breeder } \ldots \ldots \ldots \ldots \ldots \ldots \ldots \ldots \ldots \ldots \ldots \ldots \ldots \ldots \ldots\end{array}$ & VII-7 \\
\hline VII-3 & ${ }^{6} \mathrm{L1}$ Isotope Content of Irradiated TRIo Pellets .......... & VII-9 \\
\hline VII-4 & Measured Activation Rates for TRIo ................ & VII-10 \\
\hline VII -5 & Adjusted Neutron Fluences in TRIO $\ldots \ldots \ldots \ldots \ldots \ldots \ldots \ldots$ & VII -10 \\
\hline VII-6 & $\begin{array}{l}\text { Gamma Analysts of Irradiated TRIo Lithium Aluminate } \\
\text { Samples } \ldots \ldots \ldots \ldots \ldots \ldots \ldots \ldots \ldots \ldots \ldots \ldots \ldots \ldots \ldots \ldots \ldots \ldots \ldots \ldots \ldots \ldots\end{array}$ & VII \\
\hline VII -7 & $\begin{array}{l}\text { Possible Fission Products }(\mu / \text { Pu }) \text { of Irradiation } \\
\text { Aluminate Samples } \ldots \ldots \ldots \ldots \ldots \ldots \ldots \ldots \ldots \ldots \ldots \ldots \ldots \ldots \ldots \ldots \ldots \ldots \ldots\end{array}$ & VII-15 \\
\hline VIII-1 & $\begin{array}{l}\text { Spectral Averaged Kerma (MACKLIB) Factors and Dawage } \\
\text { per Component Wuclide in the } \mathrm{LiAlO}_{2} \ldots \ldots \ldots \ldots \ldots \ldots \ldots \ldots \ldots\end{array}$ & VIII-3 \\
\hline VII I -2 & Spectral Averaged Gas Production File (ENDF 533) ........ & VIII-5 \\
\hline VIII-3 & Damage from Various Sources of TRIO ................. & VIII-6 \\
\hline VIII -4 & Nuclear Heating Rates & VIII-7 \\
\hline VIII-5 & Heat Transfer Data from TRIO: Temperatures ........... & VIII-9 \\
\hline VIII-6 & Heat Transfer Data irom TRIO: Temperature Gradients ..... & VIII -12 \\
\hline VIII-7 & Heat Transfer Data from TRIO: Thermal Conductivity ..... & VIII-14 \\
\hline VIII-8 & 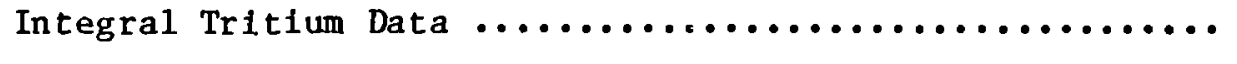 & VIII-16 \\
\hline PIII-9 & Total Tritium $\ldots \ldots \ldots$ & VIII-17 \\
\hline I I-10 & Calculated Tritium Inventory for each TRIO Run ......... & VIII-19 \\
\hline II -11 & Calculated Diffusivities from TRIO Data ............. & VIII-21 \\
\hline VIII-12 & Diffusive Inventories for Runs $9-27 \ldots \ldots \ldots$ & VIII-23 \\
\hline$I-13$ & $\begin{array}{l}\text { Comparison Between Predictions and TRIo Results for } \\
\text { Time to Reach } 67 \% \text { of Steady-State Release Rate After } \\
\text { a Temperature Change } \ldots \ldots \ldots \ldots \ldots \ldots \ldots \ldots \ldots \ldots \ldots \ldots \ldots \ldots \ldots \ldots \ldots \ldots \ldots\end{array}$ & VIII-28 \\
\hline $\mathbf{I X}-1$ & $\begin{array}{l}\text { Tritium Inventories for Runs Which Were Not Diffusion } \\
\text { Controlled } \ldots \ldots \ldots \ldots \ldots \ldots \ldots \ldots \ldots \ldots \ldots \ldots \ldots \ldots \ldots \ldots\end{array}$ & IX -5 \\
\hline $\mathbf{I X}-2$ & $\begin{array}{l}\text { Calculated Diffusive Inventories for a } 4000-\text { MW Fusion } \\
\text { Reactor Blanket } \ldots \ldots \ldots \ldots \ldots \ldots \ldots \ldots \ldots \ldots \ldots \ldots \ldots \ldots \ldots \ldots \ldots \ldots \ldots \ldots\end{array}$ & $\mathbf{I X}-9$ \\
\hline$I X-3$ & Lithium Aluminate Equilibria: Fixed Oxygen Content $\ldots .$. & $\mathrm{IX}-12$ \\
\hline $1 \mathrm{X}-4$ & Lithium Aluminate Equilibria: Low Oxygen Activity ....... & $\mathrm{IX}-13$ \\
\hline IX -5 & Lithium Aluminate Equilibria: High Oxygen Activity ..... & $1 X-14$ \\
\hline
\end{tabular}




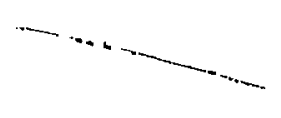

\begin{abstract}
The TRIO experiment is a cest of in-situ tritium recovery and heat transfer performance of a miniaturized solid breeder blanket assembly. The assembly (capsule) was monitored for temperature and neutron flux profiles during irradiation and a sweep gas flowed through the capsule to an analytical train wherein the amounts of tritium in its various chemical forms were determined. The capsule was designed to operate at different temperatures and sweep gas conditions. At the end of the experiment the amount of tritium retained in the solid was at a concentration of less than 0.1 wppt. More than $99.9 \%$ of tritium generated during the exper1ment was successfully recovered. The results of the experiment showed that the tritium inventories at the beginning and at the end of the experiment follow a relationship which appears to be characteristic of intragranular diffusion. The derived diffusivity for tritfum in lithfum aluminate is expressed by $D=1.1$ $\times 10^{-6} \mathrm{e}^{(-35.8 \mathrm{Kcal} / \mathrm{RT})}$, where $\mathrm{D}$ in $\mathrm{cm}^{2} / \mathrm{s}$ is the absolute temperature in $\mathrm{K}$, and $R$ is the gas constant, $0.001987 \mathrm{Kcal} / \mathrm{mol}-\mathrm{K}$. It was found that the addition of hydrogen to the sweep gas appeared to enhance the release of tritium - this enhancement is attributed to a surface effect. The predominant form of tritium observed was the noncondensible, or HT, form. Thermodynamic calculations are consistent with this observation. The conditions of irradiation, equivalent to about two months in a fusion reactor, did not appreciably change the morphology of the breeder material and there was no significant change in thermal conductivity of the 1ithium aluminate.
\end{abstract}


namas I 


\section{INTRODOCTION}

Lithium aluminate, $\mathrm{LiAlO}_{2}$, is representative of a group of ceramics, called the "Solid Breeders," that are candidate materials for use as the tritium breeding medium in fusion reactors. In the past several years, a number of conceptual blanket designs for fusion reactors have used solid breeders, ${ }^{1-16}$ and the data base has been reviewed.17-24 Although the data base is limited, experimental programs are in progress and more results are becoming available. 25-39 The solid breeders include $\mathrm{Li}_{2} \mathrm{O}, \mathrm{LiAlO}_{2}, \mathrm{LI}_{2} \mathrm{ZrO}_{3}$, $\mathrm{Li}_{4} \mathrm{SiO}_{4}$, and $\mathrm{Li}_{2} \mathrm{SiO}_{3}$. The ternary oxides, in general, have much better chemical and thermophysical stability than $\mathrm{LI}_{2} \mathrm{O}$, but owing to its high lithium atom density, $\mathrm{Li}_{2} \mathrm{O}$ has better tritium breeding potential.

The TRIO experiment involved the irradiation of $\mathrm{LHAlO}_{2}$ in the Oak Ridge Research Reactor (ORR) under well-defined conditions of temperature, neutron flux, sweep gas flow, and sample configuration. Lithium aluminate was selected for the experiment because of its attractive properties (discussed later). Tritium, in its various chemical forms (and other gases evolved from the $\mathrm{LiAlO}_{2}$ during irradiation), was moved by a sweep gas to an analytical train in which measurements on the composition of the effluent were performed. 
SBCTION II

PURPOSE 


\section{PURPOSE}

The primary purpose of the TRIO experiment was to test in-situ tritium recovery from a candidate solid breeder material under conditions representing those expected in a fusion reactor blanket. The tritium recovery results tested the feasibllity of in-situ tritium recovery from solid breeders. The results also provided tritium transport data from which models can be developed to predict tritium inventories in fusion reactor blankets. Heat transfer performance of the $\mathrm{LAAlO}_{2}$ was a key part of the experiment. The heat transfer data (heat transfer coefficients, thermal conductivity, etc.) derived from the experimental results will be of considerable value for the design of fusion reactor blankets.

Seven objectives were proposed for this experiment:

(1) Demonstrate tritium release and recovery for a miniaturized solid breeder blanket assembly. This objective is the most important one of the experiment. The capsule is a small-scale but operational solid breeder blanket which will be tested under limited conditions. The experiment will demonstrate for these limited conditions whether in-situ tritium recovery is feasible.

(2) Determine the effects of key operational factors upon tritium release. These factors include temperature, time, tritium partial pressure, sweep gas flow rate, $\mathrm{H}_{2}$ partial pressure, neutron fluence (to a limited extent), and impurities. It is necessary to know how tritium release is affected by operational factors so that an understanding of tritium transport can be acquired. This should enable predictions to be made of blanket performance under conditions that have not been or cannot be determined experimentally.

(3) Demonstrate heat transfer performance in a miniaturized solid breeder blanket assembly. The control of temperatures and temperature profiles to maintain solid breeder blankets within operating linits is regarded as a significant design issue. Thus, experimental denonstration of the ability to control temperature profiles is important. In addition, data from the experiment will be used to 
determine values of heat transfer coefficients and thermal conductivity of the $\mathrm{LAAlO}_{2}$ and to access the effects of radiation damage upon these values.

(4) Measure tritium permeation through primary cladding. A measurable quantity of tritium may permeate through the primary cladding into the gas gap of the breeder capsule. This tritium which permeates will be collected and assayed.

(5) Investigate the form of the tritium species (e.g., $\mathrm{I}_{2} \cdot: \mathrm{T}_{2}$ ratio) in the sweep gas stream. The amount of tritium released in each chemical form is to be quantitatively determined.

(6) Establish the methodology for in-pile testing. This experiment is the first truly comprehensive test of in-situ tritium recovery from solid breeders. Thus, a methodology for this kind of test will be developed.

(7) Measure radioactive species in the sweep gas stream. Certain impurities in the $\mathrm{LiAlO}_{2}$ (e.g., uranium, potassium, sodium, chlorine, etc.) produce volatile radionuclides during irradiation. These will be quantified to ensure that tritium monitoring is not affected. In addition, transport data on other specles (mostly nobel gases), which may provide some insight into transport of helium, will be obtained. Helium transport is potentially important for solid breeders because it can cause bubbles, which may act as traps for tritium and may also cause swelling. 
SECTION III

APPROACH 


\section{APPROACH}

The experimental approach included provision for safe operation of the experiment and acceptable uncertainty in tritium $r$ ease behavior. Conditions were chosen to be favorable for tritium release, and the monitoring and control systems were provided with appropriate redundancy and flexibility. The experimental plan included a test matrix in which certain parameters were systematically varied; these parameters were time, temperature, and sweep gas conditions. Otiner factors, notably sample configuration and microstructure, were fixed. The grain size and the microstructure were kept essentially unchanged so that they would be well defined throughout the course of the experiment. Quality assurance was judiciously applied in the planning, design, Installation, and operation of the experiment. The quality control thus ensured the validity of results from the experiment.

\section{A. Breeder ifaterial Selection}

The first consideration was selection of the appropriate breeder material for the experiment. The selection criteria were:

(1) It must be a candidate solid breeder. The choice was limited to $\mathrm{Li}_{2} \mathrm{O}$ and certain ternary oxides, viz., $\mathrm{LiAlO}_{2}, \mathrm{Li}_{4} \mathrm{SiO}_{4}$, and $\mathrm{Li}_{2} \mathrm{ZrO}_{3}$. other materials for testing can be considered as data on them is developed; an example of such a material is $\mathrm{Li}_{8} \mathrm{ZrO}_{6} \cdot 37$

(2) It must maintain structural and microstructural integrity during the experiment, so that temperacure profiles and microstructural properties are we11 defined throughout.

(3) It must be readily fabricated in appropriate form, and there must be a sufficient data base such that extensive property measurements are not required to be able to design the experiment.

(4) Chemical inertness is desirabie.

Based upon these criteria, $\mathrm{LiAlO}_{2}$ was selected as the breeder material for TRIO. 


\section{B. Eperinental Design}

A test capsule was designed to function as a small-scale blanket assembly with temperature control and with continuous, in-situ tritium recovery. Parameters such as tritium production rates, temperatures, temperature profiles, damage rates, and sample configuration were, in general, selected to correspond to STARFIRE. 1 Selected features for the STARFIRE and TRIO blankets are compared in Table III-1.

\section{TABLE III-1}

Design and Operating Conditions of STARFIRE and TRIO Breeder Blankets

\begin{tabular}{|c|c|c|}
\hline & TRIO & STARFIRE \\
\hline \multicolumn{3}{|l|}{ Breeder Material } \\
\hline $\mathrm{LIAlO}_{2}$ allotrope & $\gamma$ & $\alpha$ \\
\hline Pore size distribution & Bimodal & Bimodal \\
\hline Grain radius, $\mu \mathrm{m}$ & $0.1-0.2$ & $0.1-0.2$ \\
\hline Agglomerate diameter, $\mu^{m}$ & $50-100$ & 100 \\
\hline \multicolumn{3}{|l|}{ Temperatures/Heat Transfer } \\
\hline Minimum temperature, ${ }^{\circ} \mathrm{C}$ & 400 & 500 \\
\hline Maximum temperature, ${ }^{\circ} \mathrm{C}$ & 900 & 900 \\
\hline Temperature range, ${ }^{\circ} \mathrm{C}$ & 150 & 400 \\
\hline \multicolumn{3}{|l|}{ Tritium Generation } \\
\hline Production rate, $\mathrm{T} / \mathrm{cm}^{3} . \mathrm{s}$ & $8 \times 10^{12}$ & $8 \times 10^{12}$ \\
\hline Lithium burnup, $\% / m o$ & 0.08 & 0.08 \\
\hline $\begin{array}{l}\text { Atomic displacements } \\
\text { in } \mathrm{LiAlO}_{2}, \mathrm{dpa} / \mathrm{mo}\end{array}$ & 0.2 & 0.2 \\
\hline Dose, rads/mo & $3 \times 10^{12}$ & $3 \times 10^{12}$ \\
\hline${ }^{6}$ L, isotopy, \% & 0.5 & 30 \\
\hline \multicolumn{3}{|l|}{ Configuration } \\
\hline Sweep gas composition & $\mathrm{He} / \mathrm{H}_{2}$ or $\mathrm{O}_{2}$ & $\mathrm{He}$ \\
\hline Tritium partial pressure, $\mathrm{Pa}$ & $0.1-10$ & 1 \\
\hline Purge channel spacing, $\mathrm{cm}$ & 1 & 1 \\
\hline Purge channel location & $\begin{array}{l}\text { High-temp. } \\
\text { zone }\end{array}$ & $\begin{array}{l}\text { High-temp. } \\
\text { zone }\end{array}$ \\
\hline
\end{tabular}


The TRIO and STARFIRE features are similar with respect to configuration, microstructure, tritium production, damage rates, and temperature range. However, some features of TRIO are different. The gamma phase of $\mathrm{LiAlO}_{2}$ was chosen for TRIO because it is the stable form at high temperature and does not revert to the alpha form. The ${ }^{6} \mathrm{Li}$ content was reduced to compensate for differences in the neutron spectrum; ORR has a mixed spectrum, with roughly equal fluxes of thermal, epithermal, and fast neutrons -- each about 1.4 $10^{14} \mathrm{n} / \mathrm{cm}^{2} . \mathrm{s}$. Because the ${ }^{6} \mathrm{Li}$ cross section is very much larger at low neutron energies, the isotope is diluted to have the same tritium production rate as that in a fusion reactor, which has a much "harder" neutron spectrum (more high-energy neutrons). The "softer" spectrum of the TRIO experiment (more low-energy neutrons) has another effect; namely, the ${ }^{6} \mathrm{LI}$ burns up in a rather short time, and the tritium production rate decreases by about $12 \%$ every month. The temperature and sweep gas conditions are fixed in STARFIRE but the TRIO-01 experimental design allows for variation of both teraperature and sweep-gas flow rate. In addition, the temperature range at a given point in time is minimized so that the effects of temperature upon tritium transport can be determined. As later discussed, the experiment includes a matrix of 33 tests involving different combinations of temperature and flow conditions. 
SECTION IV

EXPERIMENTAL APPARATUS 


\section{BXPERIMRNTAL APPARATUS}

The TRIO experimental configuration is schematically illustrated in Fig. IV-1. The sweep gas flows through the capsule and then passes to the gas analysis system. The essential components of the experimental apparatus are the capsule containing the breeder material, the gas analysis system, the core mockup test, and the instrumentation system. Each of these components is discussed below, as is the core mockup test.

\section{A. Capsule}

The design for the capsule is illustrated schematically in Fig. IV-2. The $\mathrm{LiAlO}_{2}$ breeder pellets are in the form of hollow cylinders, designed to limit the radial temperature gradients to about $100^{\circ} \mathrm{C}$. The pellets are enclosed in the inner capsule, which is separated from the outer capsule by a gas gap. The heat generated in the inner capsule flows radially outward to the reactor coolant, which is water at $60^{\circ} \mathrm{C}$. The gap acts as a resistance to heat flow, and temperature control is achieved by varying the helium/argon ratio in the flowing gap gas. The sweep gas flows down through the center of the qapsule, back up through the annulus between the inner tube and the inside surface of the breeder pellets, and out to the gas analysis system. Temperature is monttored by ten thermocouples located on the inside and outside surfaces of the breeder pellets. Neutron flux is monitored by three self-powered neutron detectors wrapped around the outside of the capsule. In addition, dosimetry wires are placed in the center of the capsule and on the outside of the capsule assembly.

Table IV-1 presents various characteristics of the $\mathrm{YLiAlO}_{2}$ pellets used In the TRIO capsule, and Table IV-2 gives various characteristics of the capsule itself and capsule instrumentation. A detalled capsule design 1s illustrated in Fig. IV-3. In general, the TRIO design conforms with the criteria selected for the design concept (see Table III-1). The major exception is the maximum temperature, which was limited by stress considerations. The essential elements of the experimental design are discussed below.

The pellet dimensions were selected so that the radial temperature gradients were 1 imited to $100^{\circ} \mathrm{C}, 1 . e$. , the inside surface of the pellets was $100^{\circ} \mathrm{C}$ hotter than the outside surface. The sweep gas flows past the inside surface 


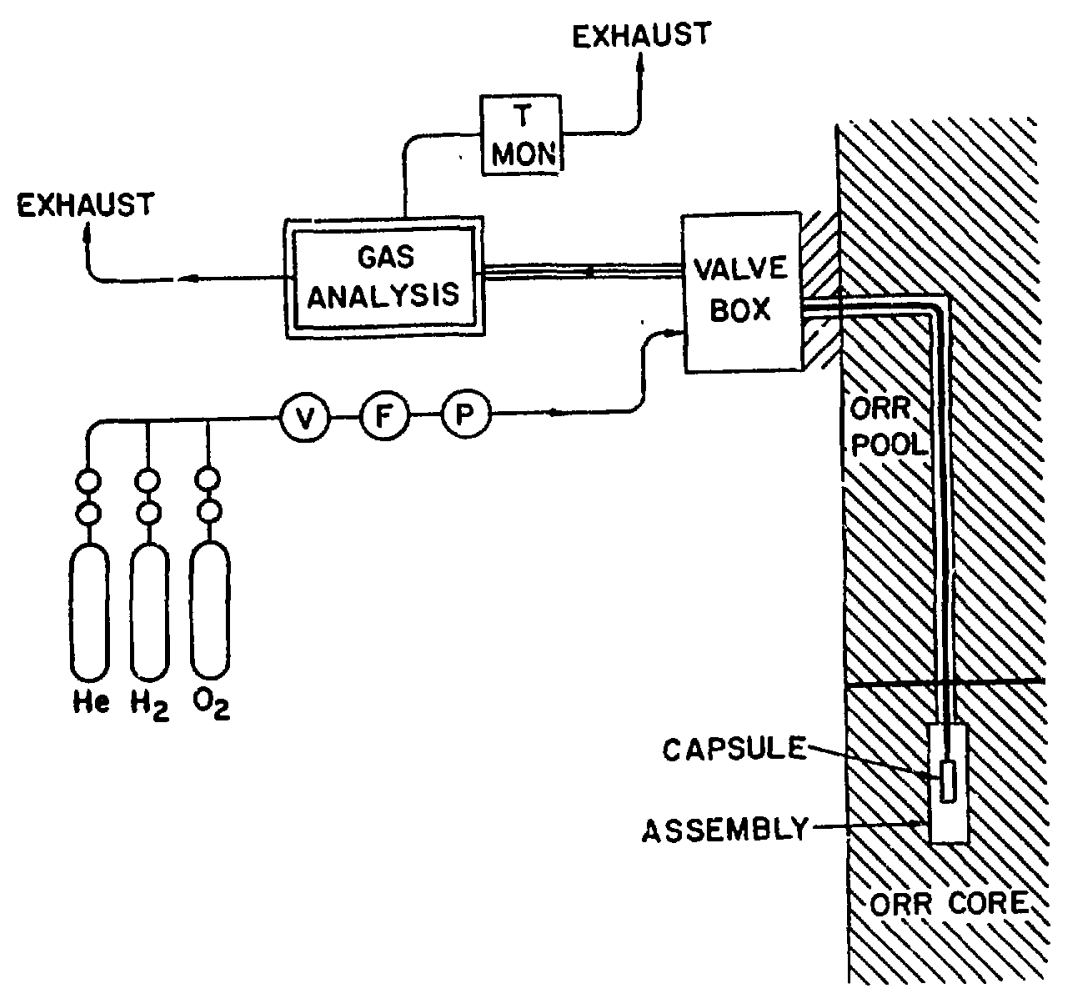

Fig. IV-1. TRIO sweep gas flow path.

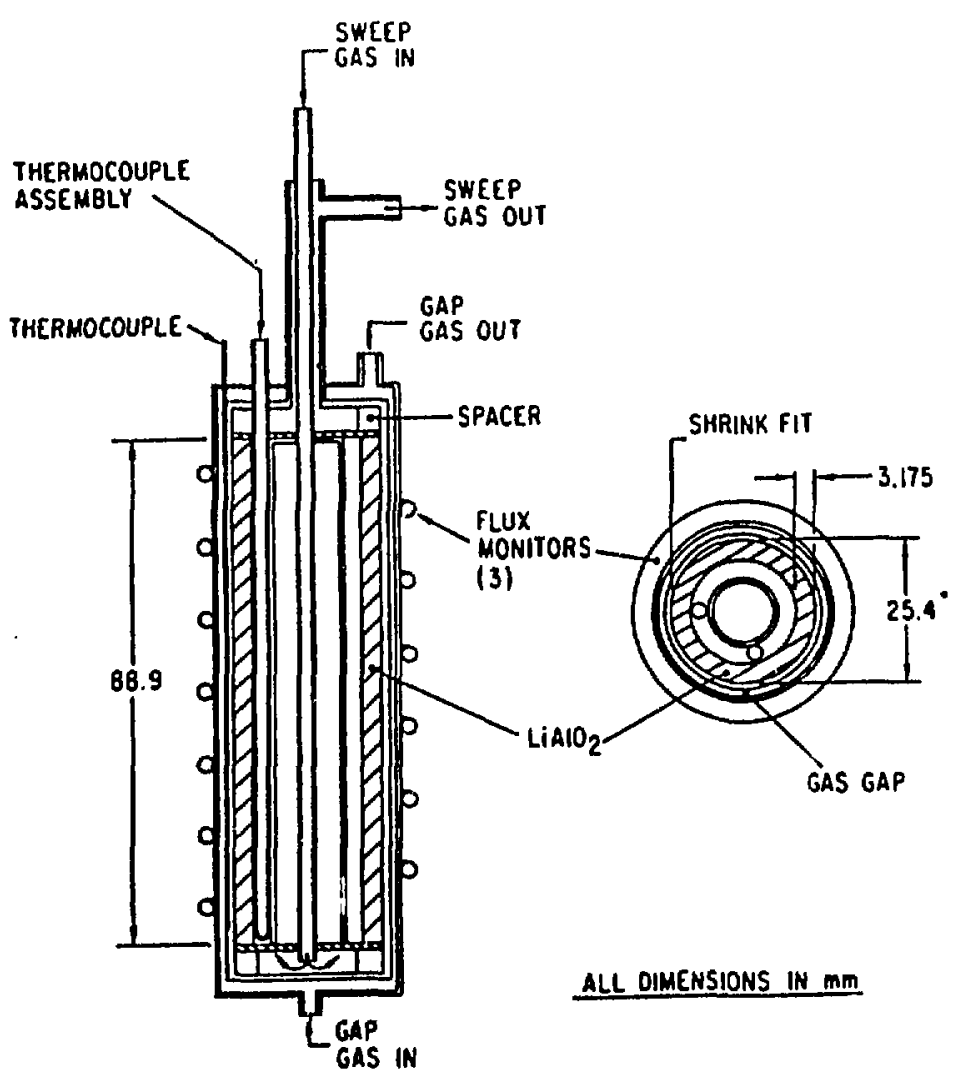

Fig. IV-2. TRIO design concept. 
TABLE IV-1

Characteristics of $\gamma-\mathrm{LiAlO}_{2}$ Breeder Material for TRIO Capsule

\begin{tabular}{|c|c|c|}
\hline Characteristics & \multicolumn{2}{|c|}{ Values } \\
\hline Number of pellets & 7 & \\
\hline Mass, ${ }^{a} \mathrm{~g}$ & 42.9 & \\
\hline \multicolumn{3}{|l|}{ Pellet dimensions, mm (1n.) } \\
\hline Inside diameter & 15.87 & $(5 / 8)$ \\
\hline Outside diameter & 25.4 & $(0.999)$ \\
\hline Height & $\sim 13$ & $(1 / 2)$ \\
\hline Smear density, \% of theoretical & 65 & \\
\hline Elemental impurities, wppm & $<100$ & \\
\hline Carbonate impurity, \% & $<0.5$ & \\
\hline Moisture content, $\%$ & $<0.5$ & \\
\hline${ }^{6} \mathrm{L1}$ isotopy, \% & 0.55 & \\
\hline Grain radius, $\mu$ & 0.1 & \\
\hline Nominal diameter agglomerates, $\mu \mathrm{m}$ & $\sim 50$ & \\
\hline Maximum temperature, $b{ }^{\circ} \mathrm{C}$ & 800 & \\
\hline Minimum temperature, ${ }^{\circ} \mathrm{C}$ & 400 & \\
\hline Radial temperature gradient, ${ }^{\circ} \mathrm{C}$ & 100 & \\
\hline Total temperature range, ${ }^{\circ} \mathrm{C}$ & 150 & \\
\hline
\end{tabular}

${ }^{a}$ Total of the seven pellets.

bimited by capsule stresses. 
TABLE IV-2

Characteristics of TRIO Capsule and Capsule Instrumentation

\begin{tabular}{|c|c|}
\hline Capsule Characteristics & Values \\
\hline Breeder material & $\gamma-\mathrm{LiAlO}_{2}$ \\
\hline Structural material & 304 SS \\
\hline Maximum temperature, ${ }^{a}{ }^{\circ} \mathrm{C}\left({ }^{\circ} \mathrm{F}\right)$ & $815(1500)$ \\
\hline \multicolumn{2}{|l|}{ Inner capsule dimensons, mm (in.) } \\
\hline Minimum wall thickness & $2.59(0.0625)$ \\
\hline Inside diameter & $25.40(1.000)$ \\
\hline Outside diameter & $31.75(1.250)$ \\
\hline \multicolumn{2}{|l|}{ Outer capsule dimensions, mm (in.) } \\
\hline Minimum wall thickness & $1.59(0.0625)$ \\
\hline Inside diameter & $32.77(1.290)$ \\
\hline Outside diameter & $39.12(1.540)$ \\
\hline \multicolumn{2}{|l|}{ Pressure, kPa (psig) } \\
\hline Sweep gas & $340(35)$ \\
\hline Gap gas & $450(50)$ \\
\hline Coolant water & $340(35)$ \\
\hline Coolant water temperature, ${ }^{\circ} \mathrm{C}$ & 60 \\
\hline Average (nominal) breeder temperature, ${ }^{\circ} \mathrm{C}$ & $400-700$ \\
\hline Sweep gas flow, $\mathrm{cm}^{3} / \mathrm{min}$ & $30-300$ \\
\hline Sweep gas composition & $\mathrm{He}+0-1 \% \mathrm{H}_{2}$ or $0-0.2 \% \mathrm{O}_{2}$ \\
\hline \multicolumn{2}{|l|}{ Capsule instrumentation } \\
\hline Temperature & 10 thermocouples \\
\hline Thermal flux & 3 self-powered neutron detectors \\
\hline Dose & $\begin{array}{l}\text { Dosimetry wire (inside and } \\
\text { outside capsule) }\end{array}$ \\
\hline Sweep gas prese 'e and flow & Gas instrumentation \\
\hline Tritium release, sweep gas & SGAS $^{b}$ \\
\hline Sweep gas chemistry & SGAS \\
\hline Radionuclides in sweep gas & SGAS \\
\hline Tritium permeation & Gap gas monitor \\
\hline
\end{tabular}

${ }^{a}$ Limited by stress, ASME code.

b $_{\text {SGAS }}=$ sweep gas analysis system. 


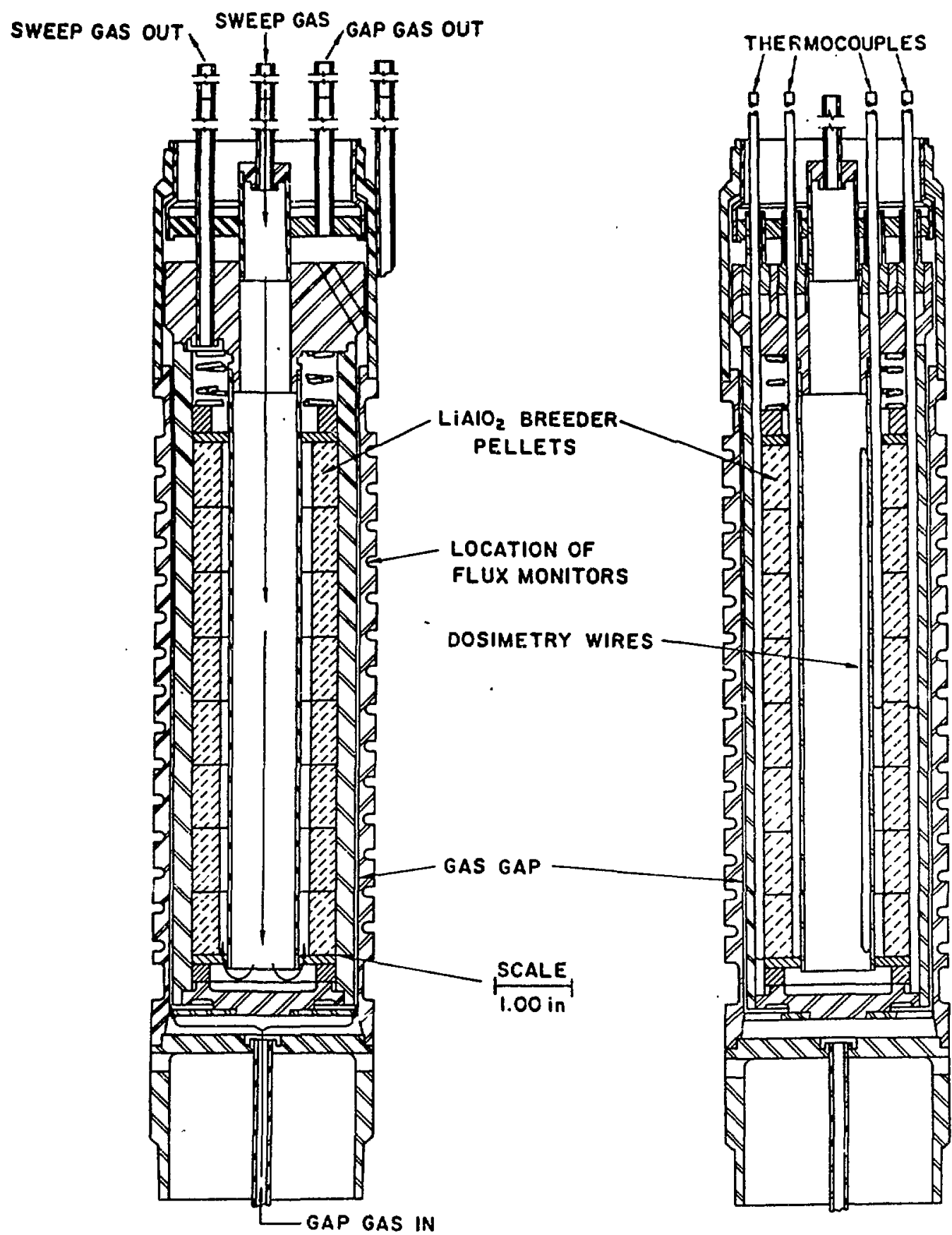

Fig. IV-3. Detailed design of TRIO capsule.

of the breeder pellets, which is the hot zone. In STARFIRE, the purge channels are also located in the hot zone. The breeder thickness for TRIO is about $5 \mathrm{~mm}$, compared to a nominal purge channel spacing of $1 \mathrm{~cm}$ in STARFIRE.

The capsule assembly (F1g. IV-3) includes an Inner capsule, which is separated from an outer capsule by the gas gap. The gas gap has a nominal 
thickness of $0.50 \mathrm{~mm}(0.020 \mathrm{in.})$ at room temperature. This gap decreas $\epsilon$ s by up to $50 \%$ during operation of the experiment owing to the greater thermal expansion of the inner capsule, which is at significantly higher temperatures than the outer capsule. Attached to the bottom end of the inner capsule is a guide plate that contacts the bevelled inside surface of the outer cladding. The purpose of this cladding is to center the inner capsule and maintain the gap. The inner cladding has two grooves machined into the inside surface to hold the thermocouples in position. The outer cladding has spiral grooves on the outside surface for holding the three self-powered neutron detectors in place.

There are a number of monitors for the capsule. Thermocouples (Type $\mathrm{K}$ Chromel-Alume1) measure temperature at 10 locations. Two of them are singleelement thermocouples located on the east side of the capsule. Two are fourelement thermocouple assemblies, located on the west side of the capsule. The thermocouple locations are given in Table IV-3. The thermocouples were suijected to extensive inspection by $X$-radiography to ensure that failures would

TABLE IV-3

Location of Capsule Thermocouples

\begin{tabular}{l|c|c|c|c}
\hline Thermocouple Type & $\begin{array}{c}\text { Element } \\
\text { No. }\end{array}$ & $\begin{array}{c}\text { Geographic } \\
\text { Direction }\end{array}$ & $\begin{array}{c}\text { Distance } \\
\text { from Bottom } \\
(\mathrm{cm})\end{array}$ & $\begin{array}{c}\text { Location } \\
\text { with Respect } \\
\text { to Breeder }\end{array}$ \\
\hline Single element & TE-1 & East & 4.32 & Inside \\
Single element & TE-2 & East & 4.32 & Outside \\
Multi-element & TE-3 & West & 6.22 & Inside \\
Multi-element & TE-4 & West & 4.32 & Inside \\
Multi-element & TE-5 & West & 2.54 & Inside \\
Multi-element & TE-6 & West & 0.73 & Inside \\
Multi-element & TE-7 & West & 6.22 & Outside \\
Multi-element & TE-8 & West & 4.32 & Outside \\
Multi-element & TE-9 & West & 2.54 & Outside \\
Multi-element & TE-10 & West & 0.73 & Outside \\
\hline
\end{tabular}


not occur. The accuracy (tolerance) of the thermocouples as per specifications is $\pm 3 / 8 \%$ for the single-element ones and $\pm 3 / 4 \%$ for the four-element ones. Further, these thermocouples normally read, at $400^{\circ} \mathrm{C}$, high by about half of the tolerance. 36 Radiation effects, which are primarily important with respect to transmutation, are negligible for the experimental conditions. At $500^{\circ} \mathrm{C}$, the absolute error is thus estimated to be $2{ }^{\circ} \mathrm{C}$ for the singleelement units and $4^{\circ} \mathrm{C}$ for the multi-element units.

Neutron flux is measured by active and passive methods. The active measurement consists of three self-powered neutron detectors wound around the outside of the capsule. The passive dosimetry consists of sets of dosimetry wires encapsulaied in Type 316 stainless steel tubing with an o.d. of $1.6 \mathrm{~mm}$ $(1 / 16 \mathrm{in.})$, having a length of $8.9 \mathrm{~cm}(31 / 2 \mathrm{in})$. There are three such dosimetry packets, one located near the center of the capsule, and two located outside the capsule on the east and west sides. As discussed later in this section, a core mockup test was performed to characterize the neutronic environment of the A2 core position, wherein the TRIO experiment was subsequently performed. The precision of the self-powered neutron detectors appears to be better than $5 \%$, on the basis of the observed agreement of the three detectors. The accuracy of the self-powered neutron detectors is approximately $10 \%$. The accuracy in total fluence as measured by the dosimetry wires is $10-15 \%$.

\section{B. $\mathrm{LAAlO}_{2}$}

The $\mathrm{LiAlO}_{2}$ powder (alpha form) was prepared by mixing stoichiometric quantities of $\mathrm{Al}_{2} \mathrm{O}_{3}$ and $\mathrm{Li}_{2} \mathrm{CO}_{3}$, which were then ball-milled in a water slurry, spray dried, and calcined in air at $800^{\circ} \mathrm{C}$ for $8 \mathrm{~h}$. Chemical analyses (Table IV-4) showed that the powder was reasonably pure (impurities <100 wppm) except for chloride, which was 2000 wppm.

The chloride level was a matter of considerable concern because of the corrosiveness of that ion. After exploration of several methods, it was found that vacuum heat treatment of the lithium aluminate powder for $20 \mathrm{~h}$ at $830^{\circ} \mathrm{C}$ reduced the chloride levels to less than 500 wppm. This heat treatment apparently reduced the sinterability of the final product, because the final pellet firing required more stringent conditions in comparison to procedures developed earlier to fabricate "bimodal" lithium aluminate from powder that had not 
TABLE IV-4

Chemical Analysis of $\mathrm{LiAlO}_{2}$ Powder

\begin{tabular}{|c|c|c|c|}
\hline \multirow[b]{2}{*}{ Impurity } & \multicolumn{3}{|c|}{ Amount of Impurity (wppm) } \\
\hline & $\begin{array}{c}\text { Emission } \\
\text { Spectroscopy }\end{array}$ & $\begin{array}{l}\text { Neutron } \\
\text { Activation }\end{array}$ & Other \\
\hline $\begin{array}{l}\mathrm{Ag} \\
\mathrm{As} \\
\mathrm{Au} \\
\mathrm{B} \\
\mathrm{Ba} \\
\mathrm{Be} \\
\mathrm{BI} \\
\mathrm{Br} \\
\mathrm{Ca} \\
\mathrm{Cd} \\
\mathrm{Cl} \\
\mathrm{Co} \\
\mathrm{Cr} \\
\mathrm{Cu} \\
\mathrm{Fe} \\
\mathrm{Ga} \\
\mathrm{In} \\
\mathrm{K} \\
\mathrm{La} \\
\mathrm{Mg} \\
\mathrm{Mn} \\
\mathrm{Mo} \\
\mathrm{Na} \\
\mathrm{Ni} \\
\mathrm{P} \\
\mathrm{Pb} \\
\mathrm{Rb} \\
\mathrm{Sb} \\
\mathrm{Sc} \\
\mathrm{Si} \\
\mathrm{Sn} \\
\mathrm{Sr} \\
\mathrm{TI} \\
\mathrm{U} \\
\mathrm{Zn} \\
\mathrm{Zr}\end{array}$ & $\begin{array}{l}<10,<10,<1 \\
<500,<500,<300 \\
\mathrm{~b} \\
<10,<10, \quad 5) \\
<100,<100,<20 \\
<10,<10,<5 \\
<10,<10,<10 \\
\mathrm{~b} \\
30,50 ?,<50 \\
<1000,<1000,<100 \\
\mathrm{~b} \\
<30,<30,<5 \\
<30,<30,50 \\
\mathrm{FT}, \mathrm{FT}, 2 \\
30,70,100 \\
\mathrm{~b}, 20,15 \\
\mathrm{~b} \\
<500, \quad(<1000),<100 \\
\mathrm{~b} \\
(10), 20,40 \\
<10, \mathrm{FT}, 2 \\
<30,<30,<30 \\
50,100,200 \\
20 ?, 30,40 \\
<500,<500,<500 \\
<10,<10,<2 \\
\mathrm{~b},<1000,<50 \\
<100,<1000,<10 \\
\mathrm{~b} \\
(50),(70),(150) \\
<30,<30,<5 \\
<1000,<1000,<100 \\
<30,<100,<30 \\
\mathrm{~b} \\
<500,<300,<100 \\
<100,<300,<300 \\
\end{array}$ & $\begin{array}{l}0.10,0.065 \\
1960 \\
0.22 \\
25.5,27 \\
99.0 \\
17.1,15.0 \\
0.03 \\
<18,7.8 \\
0.14,0.086 \\
1.55 \\
83.5 \\
(400.0) \\
\\
1.2 \\
0.08,0.015 \\
4.90 \\
0.19 \\
48.6,52.0\end{array}$ & $2300 \pm 100$ \\
\hline
\end{tabular}

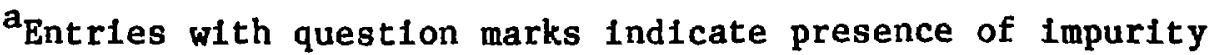
uncertain; those values in parentheses have uncertain accuracies; $F T=$ falnt trace, $1-10$ wppm.

bot reported.

$c_{\text {The }} \mathrm{Cl}$ level of the final product was $15 \pm 5$ wppm. 
been heat treated. In particular, somewhat higher temperatures $\left(1050^{\circ} \mathrm{C}\right.$ instead of $1000^{\circ} \mathrm{C}$ ) and longer firing times ( $17 \mathrm{~h}$ instead of $1 \mathrm{~h}$ ) were necessary to achieve the target density of $65 \%$. The final firing procedures reduced the chloride level to 15 wppm.

The pellets were fabricated by preparing agglomerates of appropriate size and then cold pressing and firing them. The agglomerates were prepared by first mixing $12 \mathrm{~g}$ of polyvinyl alcohol (PVA) in $300 \mathrm{~cm}^{3}$ of water at $60^{\circ} \mathrm{C}, \mathrm{mix}-$ ing in $120 \mathrm{~g}$ of $\mathrm{LiAlO}_{2}$ powder, and then heating the mixture until a thick paste was obtained. The paste was then vacuum dried at $80^{\circ} \mathrm{C}$ for $8 \mathrm{~h}$ with periodic stirring. The resultant agglomerates were weakly bound; ball-milling produced an excessively high fraction of fine particles. The lithium aluminate was then mixed with water $\left(200 \mathrm{~cm}^{3}\right)$, dried into a cake, and manually ground with a mortar and pestle; and once again there were too many fines. The material was then added to $300 \mathrm{~cm}^{3}$ of warm water with an additional $3.6 \mathrm{~g}$ PVA, dried as before, manually ground with a mortar and pestle, and sieved to retain the fraction passing a 200-mesh screen but not a 325-mesh one. The fines were again added to water and reworked to produce 200- to 325- mesh agglomerates. These agglomerates from the second batch were added to those of the first batch to use for pellet fabrication.

The prepared material was then cold-pressed at $62 \times 10^{3} \mathrm{~N}(14,0001 \mathrm{~b})$, producing a green pellet having dimensions of $3.00-\mathrm{cm} 0 . \mathrm{d} . \times 1.76-\mathrm{cm} 1 . \mathrm{d} . \times$ 1.30-cm high (1.18-in. o.d. x 0.695-in. i.d. x 0.512-in. high). The pe1let firing data for the first test pellet are shown in Table IV-5. After the firing conditions were verifled, the subsequent pellets, i.e., those used in the TRIO experiment, were fired for $17 \mathrm{~h}$ at $1050^{\circ} \mathrm{C}$. Scanning electron microscopy showed that the microstructure had the desired bimodal pore-size distribution, with a grain diameter of about $0.2 \mu \mathrm{m}$ (see FIg. IV-4).

The preparation procedures involved exposure of the pellets to humid ambient air. Chemical analysts of a pellet showed that the water content was 0.4 wt\%. Nearly all (>99\%) of this moisture was removed by heating the pellet to $300^{\circ} \mathrm{C}$ for $20 \mathrm{~min}$. Before final assembly of the capsule, the finished pellets (which had been ground to the desired dimension) were dried by heating to $400^{\circ} \mathrm{C}$ for $1 \mathrm{~h}$ and kept in a desiccator until they were loaded into the capsule in an inert atmosphere box. These procedures Involved a very brief exposure 

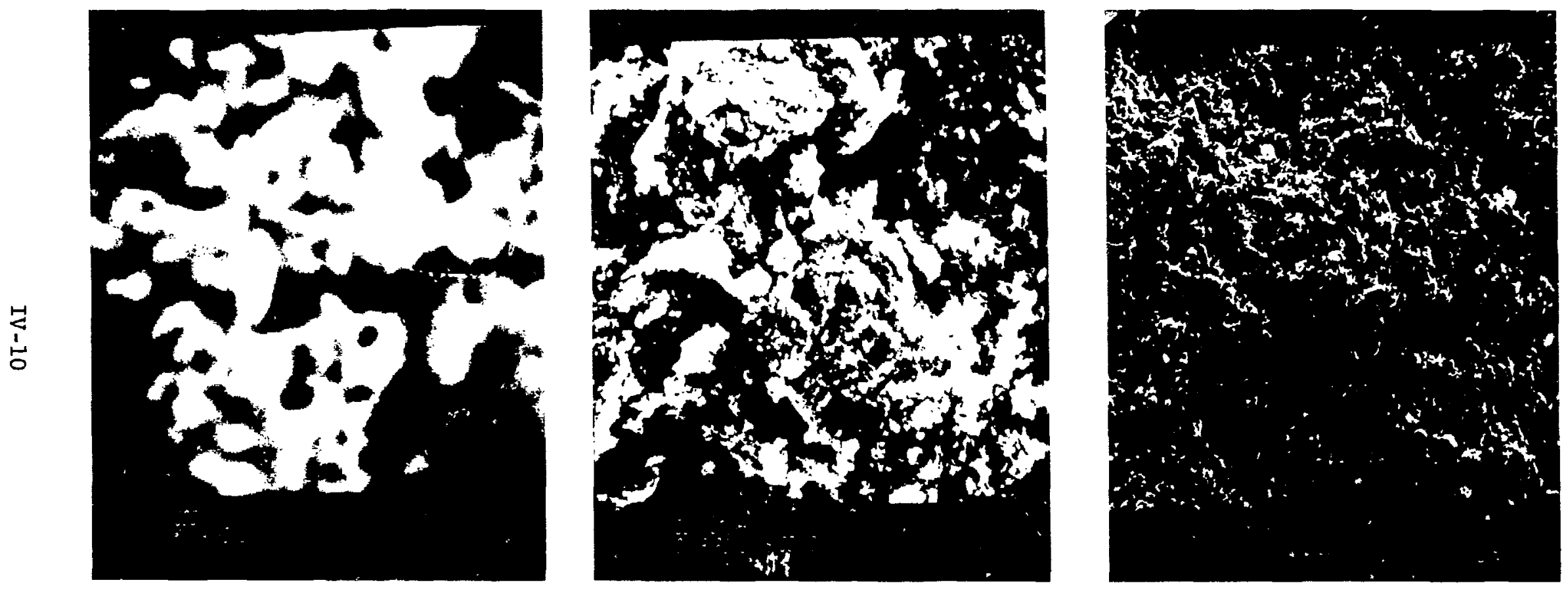

Fig. IV-4. Scanning electron micrographs of $\gamma-\mathrm{LiAlO}_{2}$ pellet. Note the bimodal pore distribution. 
TABLE IV-5

Density Data for the Firing of the First Test Pellet

\begin{tabular}{l|l|l|l|l|l|l}
\hline & $\begin{array}{c}\text { Weight } \\
(\mathrm{g})\end{array}$ & $\begin{array}{l}\text { o.d. } \\
(\text { In.) }\end{array}$ & $\begin{array}{l}\text { i.d. } \\
(\text { in.) }\end{array}$ & $\begin{array}{c}\text { Height } \\
(\text { in.) }\end{array}$ & $\begin{array}{c}\text { Density } \\
\left(\mathrm{g} / \mathrm{cm}^{3}\right)\end{array}$ & \% TD \\
\hline Green & 9.1413 & 1.180 & 0.695 & 0.512 & 1.524 & 59.5 \\
First firing & 7.2318 & 1.137 & 0.670 & 0.495 & 1.345 & 52.5 \\
Second firing & 7.2050 & 1.095 & 0.645 & 0.476 & 1.502 & 58.7 \\
Third firingc & 7.2074 & 1.078 & 0.635 & 0.467 & 1.580 & 61.7 \\
\hline TRLO & 42.9 & 1.000 & 0.625 & 3.20 & 1.709 & 65.4 \\
\hline
\end{tabular}

AFiring schedule: $15 \mathrm{~h}$ to $500^{\circ} \mathrm{C}, 8 \mathrm{~h}$ at $1000^{\circ} \mathrm{C}, 1 \mathrm{~h}$ to $1000^{\circ} \mathrm{C}$, $4 \mathrm{~h}$ of cooling.

biring schedule: $10 \mathrm{~h}$ to $1050^{\circ} \mathrm{C}, 6.5 \mathrm{~h}$ at $1050^{\circ} \mathrm{C}, 4 \mathrm{~h}$ of cooling.

CFiring schedule: $10 \mathrm{~h}$ to $1050^{\circ} \mathrm{C}, 10 \mathrm{~h}$ at $1050^{\circ} \mathrm{C}, 4 \mathrm{~h}$ of cooling.

to the ambient air. The moisture content of the pellets in the experiment was estimated to be less than 0.1 wt.\%.

A sample of a pellet exposed to air for one week had a $\mathrm{CO}_{2}$ content of 0.4 wt.\%. Another sample, exposed to air for four weeks, had a carbonate $\left(\mathrm{CO}_{2}\right)$ content of $1 \mathrm{wt. \%}$. The heat-treatment procedure was estimated to reduce the carbonate content of the TRIO pellets to about 0.1 wt.\%.

\section{Gas Analysis Systen}

The gas analysis system was designed to quantitatively measure both the amounts of tritium released in its various chemical forms and the real-time tritium release rates. The components of the system were designed as modular units that could be easily replaced and could be grouped as needed to meet the needs of the experiment. The performance of each component was verified in a mockup rig prior to assembly of the gas analysis system.

\section{Design Basis}

The TRIO experiment required a gas analysjs systen with capabilities for quantitatively determining amounts of tritium released in its various chemical 
forms and for continuously measuring tritium release rates. Also, measurement of chemical impurities, radionuclides, and tritium permeation through the inner cladding into the gas gap were required. In view of considerable uncertainties regarding tritium release, the experimental system had to handle many contingencies. Proper and safe operation was necessary for the duration of the experiment, including provision for unattended operation over weekends.

The flow paths for the sweep gas and the control gas (gap gas) are shown schematically in Fig. IV-5. The sweep gas is routed sequentially to (1) a cleanup system which purifies the gas; (2) the capsule, where tritium released from the lithium aluminate is removed by the flowing sweep gas; (3) the sweep gas analysis train, where the tritium is collected and analyzed; and (4) the exhaust. The sweep gas analysis train is enclosed in a glove box, which has an atmosphere of dry ("instrument") alr. The atmosphere of the glove box is routed to the ORR cell vent and exhaust. The sweep gas line leading from the capsule to the glove box is enclosed within a purged jacket to provide secondary containment of tritium. The control gas flows sequentially from its supply station through (1) a cleanup system, (2) the gas gap between the inner

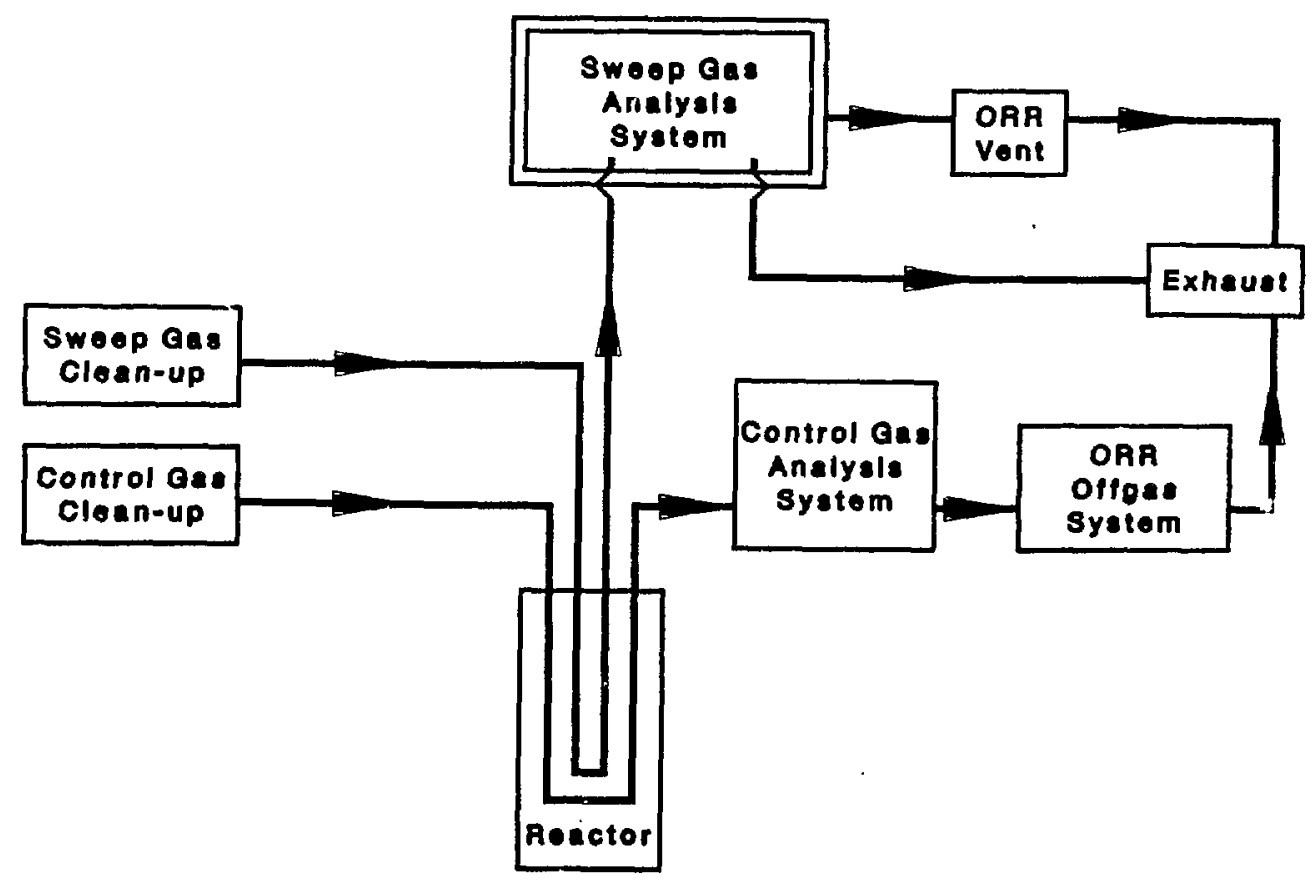

Fig. IV-5. Flow diagram for the gas analysis system. 
and outer cladding of the capsule, (3) the control gas analysis system, a smal1 scrubber which collects permeated trjtium, (4) the ORR off-gas system, which consists of a charcoal bed, and (5) the exhaust. The cleanup systems ccnsist of $4 \mathrm{~A}$ molecular sleve beds operating at room temperature and, for a11 gases except oxygen and hydrogen, titanium getter beds operated at $400^{\circ} \mathrm{C}$.

\section{Components}

The components for the gas analysis system were designed to be replaceable units that could be grouped as needed for the experiment. The performance of selected units was tested in a mockup of the gas analysis train prior to final design of the gas analysis system. The purpose of each component is described below.

$\underline{\text { Unit }}$

Oxidation bed

Ethylene glycol trap

Mole seive bed

Reduction bed

Getter bed

Kanne chamber

Gas sampler

Stainless steel tubing

Furnace/controller

Glove box

Data logger

\section{Purpose}

Converting HT to HTO

Collecting HTO

Collecting HTO

Converting HTO to HT

Collecting HT

Measuring HT concentration in gas stream Analyzing of composition and radionuclides Proving gas lines

Controlling bed temperature

Providing secondary containment of tritium

Collecting data collection

\section{a. Oxidation Bed}

The oxidation beds, as well as the mole sieve beds, reduction beds, getter beds and the gas sampler, were constructed from Whitey sample cylinders, Part No. 304-HDF4-300. These cylinders have an internal volume of $300 \mathrm{~cm}^{3}$ and are constructed of Type 304 stainless steel. The cylinders were loaded with appropriate material, and pipe thread adapter fittings were attached to each end. For the beds which operated at high temperature (oxidation beds, reduction beds, and getter beds), the pipe thread seals were welded to ensure that leakage did not occur. The ends of the cylinder were then connected to stain- 
less steel tubing ( $1 / 4$ in. o.d.) with Swagelock fittings. The stainless steel tubing was then fitted with quick-connects to the rest of the system so that components could be readily replaced.

The oxidation beds were used in the experiment for converting tritium in the noncondensable form (HT) to the condensable form (HTO). Reagent-grade copper oxide wire was used as the oxidant. In the sweep gas system, $0.1 \%$ oxygen in helium was added upstream of the oxidation bed to ensure that the oxidant was not consumed. In the control gas system, oxygen was not added; however, the bed was designed to have a 11fetime of one year for that system. Tests of a prototype bed showed that the conversion efficiency (HT to HTO) was $99.5 \%$ at a temperature of $500^{\circ} \mathrm{C}$, for a sweep gas with nominal conditions (helium- $0.1 \% \mathrm{H}_{2}$ flowing at $100 \mathrm{~cm}^{3} / \mathrm{min}$ ). The mean residence time of tritium in the bed was approximately $30 \mathrm{~min}$.

\section{b. Ethylene Glycol Trap}

Ethylene glycol is used as a liquid sorbent for HTO at room temperature. The use of ethylene glycol for this purpose has been demonstrated. 40 For the TRIO experiment, the traps are so arranged that the sweep gas flows through a coarse glass frit and bubbles up through $20 \mathrm{~mL}$ of ethylene glycol in the traps. The traps are operated as two in series so that the HTO which is not collected by the first trap is collected in the second one. Each set of such traps is backed by a bed of $4 \mathrm{~A}$ molecular sieves to ensure removal of HTO. The traps are constructed of Pyrex glass and attached to the metal system with glass-to-metal seals having flexible metal tubing to reduce strain and shock. The outer portion of each trap is removable, having an 0-ring seal joint. This outer portion serves as a container for the ethylene glycol. Samples were changed by bypassing flow, opening the seal, and removing and replacing the container. The removed container was then sealed, placed in a special carrying container, bagged and taken to the analytical laboratory for performing dilutions and preparing solutions for scintillation counting. Extreme care was taken not to drop or spill the solutions of ethlylene glycol, which, in some instances, contained upwards of one curie of HTO.

Estimates based on existing data ${ }^{41}$ indicated that the traps removed more than $98 \%$ of the HTO when operated at nominal sweep gas conditions for three 
days. It is estimated that increasing the $f$ low rate to 11 iter/min would reduce the efficlency to about $92 \%$. The vapor pressure of ethylene glycol at $25^{\circ} \mathrm{C}$ is $0.9 \mathrm{MPa}(0.12$ torr). With nominal sweep gas flow, about $60 \mathrm{mg}$ is transported from the bubblers each day. The ethylent glycol vapor is expected to be removed by the molecular sieve bed. The carryover of ethylene glycol from one sample to the next was measured to be $0.30 \%$ for the first bubbler (which was minimized by blowing out the grit with a momentary pulse of sweep gas) and $0.6 \%$ for the second bubbler. The effect of ethylene glycol upon quenching the scintillation signal was a decrease of about $4 \%$ for an addition of $1 \%$ ethylene glycol to the liquid scintillator solution. ${ }^{42}$ since the lowest dilution used has a factor of 100 less ethylene glycol and the quenching curve is almost linear, the error introduced from this factor is less than $0.05 \%$.

The following analytical procedure was used to determine the tritium content of a trap sample. Using an Eppindorf pippete, a 0.5-mL sample of the ethylene glycol solution was diluted to $25 \mathrm{~mL}$. If necessary, a second dilution was made by diluting $0.5 \mathrm{~mL}$ of the first solution to $25 \mathrm{~mL}$. In some cases, a third dilution was prepared from $0.5 \mathrm{~mL}$ of the second dilution. After appropriate dilution, a $0.10-\mathrm{mL}$ sample of the diluted solution was added to $15.0 \mathrm{~mL}$ of Beckman GP scintillator solution, and the mixture was counted for tritium. After background corrections, the counts were ratioed to NBS standards so that the tritium activity in the sample could be determined. The accuracy of all pipettes was ensured by weighing the amounts of liquid delivered in not less than ten tests. It was found that the tesi pipettes delivered to within $1 \%$ of the nominal value. The absolute accuracy of a single determination is estimated to be $5 \%$, including random errors. from the NBS standard $(<2 \%)$, the dilution $(3 \%)$, and the counting statistics $(<1 \%)$.

A technique was developed to remove some samples from a trap without removing the trap. For this procedure, the trap had a side arm with a rubber

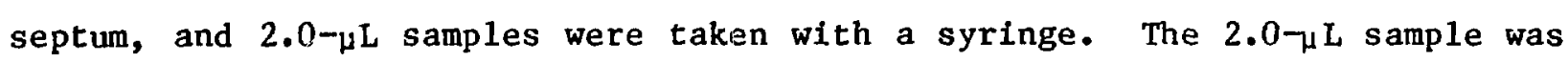
then added directly to the scintillator solution and counted. This procedure is not as accurate as the above one, owing to sampling errors of approximately $5 \%$. (overall absolute error, $<10 \%$ ), but it did permit frequent samples to be taken. Such a procedure was used for many Hro determinations. 


\section{c. Molecular Sieve Bed}

Beds containir:- Type 4A molecular sieves were used to back up the scintillation traps and to collect permeated tritium from the gap gas. The molecular sieve beds were operated at ambient temperature. Tritium was removed from the molecular sieves by refluxing in water for a period of not less than four hours. The water was then analyzed for tritium by liquid scintillation counting.

\section{d. Reduction Bed}

Although not used in the experiment, the gas analysis system was provided with reduction beds designed to convert HTO to HT. The beds contained reagent grade magnesium turnings and were to be operated at $400^{\circ} \mathrm{C}$. Mockup tests of a prototype bed showed that the conversion efficiency was $99.5 \%$, with negligible holdup of tritium. The residence time was approximately one hour.

\section{e. Getter Bed}

A getter bed, consisting of titanium sponge, was provided for the purpose of removing HT from the sweep gas stream. The bed was to be operated at $230^{\circ} \mathrm{C}$ and regenerated at $600^{\circ} \mathrm{C}$. The bed was not used for the experiment.

\section{f. Kanne Chamber}

Kanne chambers were used to monitor tritium concentration of the sweep gas and to measure tritium levels in the glove box and in the room. These instruments are particularly useful because a continuous measure of tritium level is provided. When calibrated, the instruments have an absolute accuracy of about $10 \%$. The instruments operate properly over a range of about two decades. However, exposure of a meter to very high levels of tritium causes a significant Increase in background levels for an extended period of time -this is called a "memory" effect. Tritium monitors used for the room and for the glove box were calibrated with a commercially avallable unit that supplies known amounts of tritlated methane. The sweep-gas line monitors were tested and calibrated at levels of up to $1000 \mu \mathrm{C} / \mathrm{m}^{3}$ with this unit. However, the tritium levels in the sweep gas during the experiment were three orders of magnftude higher, and the calibration unit could not operate at this level. 
Therefore, the Kanne chambers used as sweep gas monitors were calibrated using the integral data obtained from scintillation counting during operation of the experiment.

\section{g. Gas Sampler}

The purpose of the gas sampler was to provide samples of the sweep gas for chemical analysis. The unit consisted of a $300-\mathrm{cm}^{3}$ cylinder in a flowthrough configuration fictad with quick-disconnects. As later discussed, the unit was modified for measuring radionuclides.

\section{h. Stainless Steel Tubing}

The sweep gas, upon leaving the capsule, must traverse a $15-\mathrm{m}$ (50-ft) length of stainless steel tubing ( $1 / 8 \mathrm{in.} \mathrm{o.d.)} \mathrm{leading} \mathrm{to} \mathrm{the} \mathrm{analytical}$ train. To determine the tritium holdup of such a line, a 15-m long coll of stainless steel tubing was tested in the mockup rig. It was found that the holdup time was less than 30 min for the reference gas flow conditions.

\section{i. Furnace/Controller}

Replaceable furnace units, constructed of semi-cylindrical heating elements, were provided for the purpose of operating beds at elevated temperature. The heating elements were coated with Sauerisen paste, dried, provided with insulation, and installed. Temperature was controlled with a Variac rheostat and a Leeds on-off type controller.

\section{j. Glove Box}

The above units were located in a glove box. The purpose of the glove box was to provide secondary containment of the tritium for protection of personne1. The atmosphere of the glove box was dry instrument air which is available at the ORR site as a utility. The glove box is maintained at a negative pressure of one inch of water, and the air is flushed through the box at a rate of 100-200 1iters/min. Pressure control is provided to ensure against pressurization of the box. 


\section{k. Data Logger}

Data from tritium monitors, flow rates, pressures, bed temperatures, etc., from the above units were collected at timed intervals on a Fluke data logger and recorded both on paper and magnetic tape. Alarm levels were specified where appropriate. Alarms that could affect safety were wired directly to the control room. Alarms that could affect only the operation of the experiment were combined into a single alarm for the reactor control room. In the event the latter alarm was tripped, the cause was investigated and proper actions were taken to clear the alarm $\cap r$ the experimenter was contacted for advice.

\section{Modes of Operation}

The sweep gas analysis system was designed to operate in four different modes, as illustrated in Fig. IV-6. The different modes were selected by appropriate manipulation of valves in the glovebox. Mode 1 was found to be most appropriate for the experiment, and it was the only mode used. In this mode, the sweep gas flows in the following sequence: (1) the tritiumcontaining gas is sent through the gas sampler, (2) HTO is removed by the
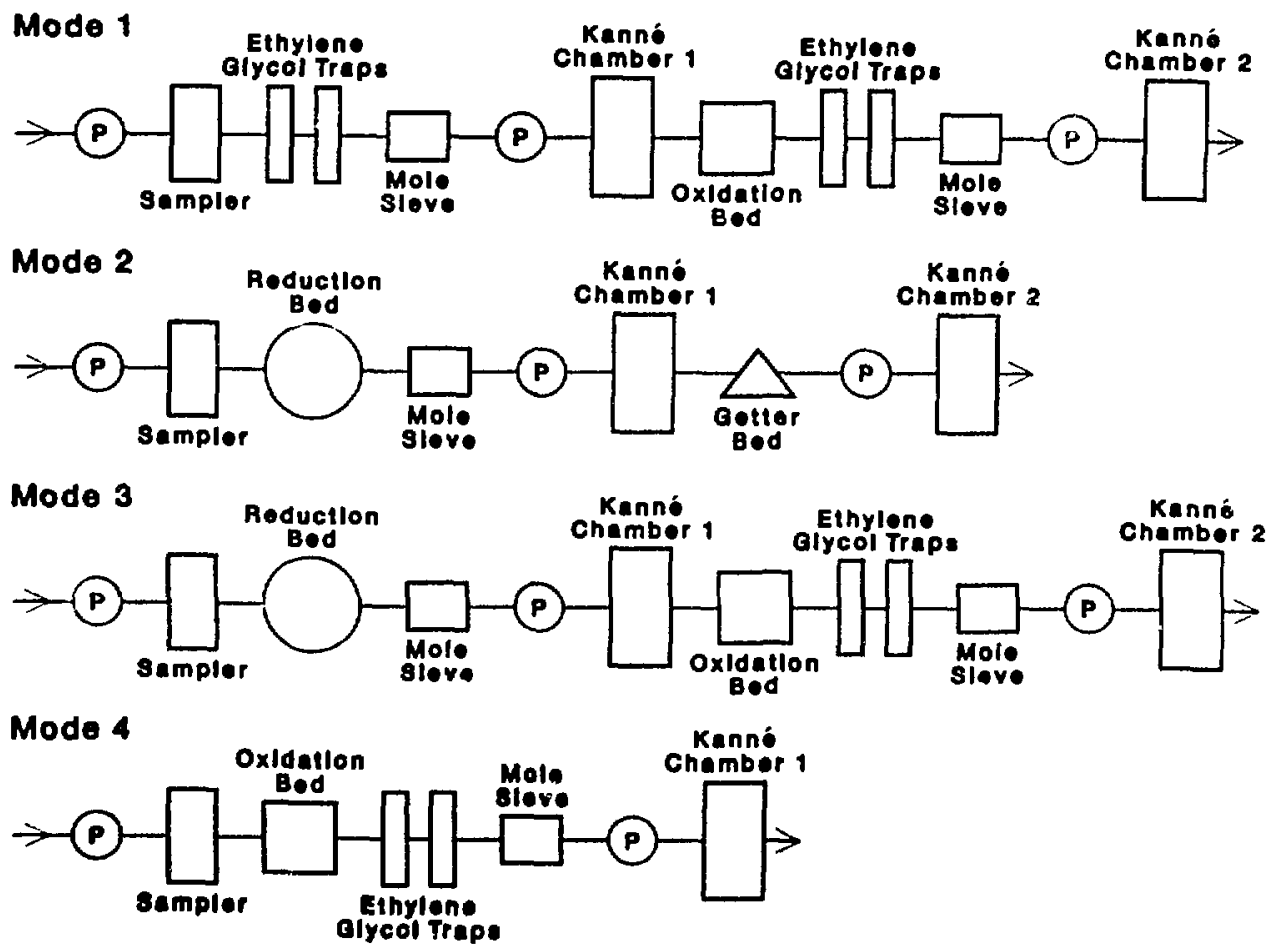

Fig. IV-6. Modes of operation for the sweep gas analysis system. 
ethylene glycol traps and the mole sleve bed, (3) the HT level is monitored with Kanne Chamber 1, (4) the HT is oxidized to HTO, (5) the converted HTO is trappei, and (6) the exhaust stream is monitored by Kanne chamber 2 . Owing to instrumental problems, the exhaust monitor did not function for most of the experiment.

\section{Control Gas}

The analysis system was provided with an arrangement designed to collect the tritium that permeated into the control gas. Such tritium was oxidized, then collected on molecular sieves. Owing to radioactivity, the system was accessible only when the reactor was down (every two weeks). The molecular sieve bed was then removed and replaced every two weeks. The tritium content was determined as previously discussed.

\section{Instrumentation}

The experimental system includes a number of on-line monitors which record data from the components described above. The data from the gas analysis system include tritfum production, temperatures, and flow conditions in the train. These data were recorded on a Fluke data logger and magnetic tape. Monitors record the ORR power parameters, which were manually recorded in $O R R$ logbooks. Capsule monitors and some gas supply system data were recorded on the ORRDACS (ORR data acquisition system) and on ORRDACS magnetic tapes. From these monitors, data were transferred to computer lisc storage at ANL. The parameters so recorded are defined in Table IV-6.

\section{E. Core Mockup Test and Nuclear Measurements}

Before irradiation of the capsule, the core mockup test was performed to characterize the nuclear environment and to calibrate the flux monitors. A mockup of the TRIO capsule assembly (Fig. IV-7) was fabricated and installed in the A2 core position of ORR. The configuration of the ORR core, with the grams of fuel in each position during the mockup test, is schematically shown in Fig. IV-8. The mockup included three of the self-powered neutron detectors and provision for inserting tubes in eight different locations, as 11lustrated in Fig. IV-9. The test included a comprehensive dosimetry experiment used to measure the neutron spectrum in a number of appropriate geometric locations. 
TABLE IV-6

Parameter List for TRIO

\begin{tabular}{|c|c|c|}
\hline $\begin{array}{l}\text { Parameter } \\
\text { Name }\end{array}$ & Description & Units \\
\hline \multicolumn{3}{|c|}{ Gas Analysis System } \\
\hline $\mathrm{KCl}$ & Line monitor Kanne chamber $\mathrm{KCl}$ & $\mu \mathrm{Ci} / \mathrm{min}$ \\
\hline $\mathrm{KClC}$ & Corrected line monitor $\mathrm{KCl}$ & ${ }_{\mu} \mathrm{Ci} / \mathrm{min}$ \\
\hline $\mathrm{KClR}$ & Line monitor Kanne chamber KC: & $\mathrm{Ci} / \mathrm{m}^{3}$ \\
\hline PI-3 & $\mathrm{PI}-3$ line monitor pressure for $\mathrm{KCl}$ & psi \\
\hline PI-3R & $\mathrm{PI}-3$ pressure for $\mathrm{KCl}$ (raw) & psi \\
\hline FI-3 & FI-3 line monitor flow for $\mathrm{KCl}$ & $\mathrm{cm}^{3} / \mathrm{min}$ \\
\hline $\mathrm{KC2}$ & Exhaust monitor Kanne chamber $\mathrm{KC} 2$ & $\mu \mathrm{Ci} / \mathrm{min}$ \\
\hline $\mathrm{KC} 2 \mathrm{R}$ & Exhaust monitor Kanne chamber $\mathrm{KC2}$ & $\mathrm{Ci} / \mathrm{m}^{3}$ \\
\hline PI-2 & PI-2 exhaust monitor pressure for $\mathrm{KC} 2$ & psi \\
\hline$P I-2 R$ & PI-2 pressure for KC2 (raw) & psi \\
\hline FI-2 & FI-2 exhaust monitor flow for $\mathrm{KC} 2$ & $\mathrm{~cm}^{3} / \mathrm{min}$ \\
\hline $\mathrm{KC} 3$ & Box monitor Kanne chamber KC3 & $\mu \mathrm{Ci} / \mathrm{min}$ \\
\hline KC3R & Box monitor Kanne chamber KC3 & $\mu \mathrm{Ci} / \mathrm{m}^{3}$ \\
\hline PI-4 & PI-4 box monitor pressure for KC3 & psi \\
\hline$P I-4 R$ & PI -4 box pressure for KC3 (raw) & psi \\
\hline FI-4 & FI-4 box monitor flow for $\mathrm{KC} 3$ & $\mathrm{~cm}^{3} / \mathrm{min}$ \\
\hline $\mathrm{TC2}$ & TC2 temperature from Box $O B-1$ & ${ }^{\circ} \mathrm{C}$ \\
\hline TC4 & TC4 temperature from Box RB-1 & ${ }^{\circ} \mathrm{C}$ \\
\hline TC6 & TC6 temperature from Box RB-2 & ${ }^{\circ} \mathrm{C}$ \\
\hline TC8 & TC8 temperature from Box $\mathrm{OB}-2$ & ${ }^{\circ} \mathrm{C}$ \\
\hline $\mathrm{TC10}$ & TC10 temperature from Box GB-1 & ${ }^{\circ} \mathrm{C}$ \\
\hline $\mathrm{TC} 12$ & TC12 temperature from Box GB-2 & ${ }^{\circ} \mathrm{C}$ \\
\hline $\mathrm{PI}-1$ & PI-1 inlet sweep gas pressure & psi \\
\hline PI-1R & PI-1 inlet sweep gas pressure (raw) & psi \\
\hline TE-1 & TE-1 capsule temperature & ${ }^{\circ} \mathrm{C}$ \\
\hline TE-2 & TE-2 capsule temperature & ${ }^{\circ} \mathrm{C}$ \\
\hline $\mathrm{TE}-3$ & TE-3 capsule temperature & ${ }^{\circ} \mathrm{C}$ \\
\hline TE-4 & TE -4 capsule temperature & ${ }^{\circ} \mathrm{C}$ \\
\hline TE -5 & TE-5 capsule temperature & ${ }^{\circ} \mathrm{C}$ \\
\hline
\end{tabular}


TABLE IV-6 (Contd.)

\begin{tabular}{|c|c|c|}
\hline $\begin{array}{c}\text { Paramet } \\
\text { Name }\end{array}$ & Parameter & Units \\
\hline \multicolumn{3}{|c|}{ Gas Analysis System (Contd.) } \\
\hline $\mathrm{TE}-6$ & $\mathrm{TE}-6$ capsule temperature & ${ }^{\circ} \mathrm{C}$ \\
\hline $\mathrm{TE}-7$ & TE-7 capsule temperature & ${ }^{\circ} \mathrm{C}$ \\
\hline $\mathrm{TE}-8$ & TE-8 capsule temperature & ${ }^{\circ} \mathrm{C}$ \\
\hline \multicolumn{3}{|c|}{ Power History } \\
\hline POWER & Daily power (energy) & MWh \\
\hline CPOWER & Cumulative power (energy) & MWh \\
\hline FPD & Daily FPD (Full-power days) & \\
\hline CFPD & Cumulative FPD & \\
\hline $\mathrm{T}$ & Daily tritium & $\mathrm{Ci}$ \\
\hline $\mathrm{CT}$ & Cumulative tritium & $\mathrm{Ci}$ \\
\hline DFLUX & Daily average $\mathrm{flux}$ & $10^{14} \mathrm{nv}$ \\
\hline SHIELD & Self-shielding & \\
\hline $6 \mathrm{LIBU}$ & ${ }^{6} \mathrm{Li}$ burnup & $\%$ \\
\hline 6LIBUT & ${ }^{6} \mathrm{Li}$ total burnup & $\%$ \\
\hline BUDAY & $\%$ burnup/day & \\
\hline BUTOT & Total burnup & \\
\hline LILEF' & $\%$ lithium left & \\
\hline TDAY & Tritium production & Ci/day \\
\hline TNET & Total tritium rroduction & $\mathrm{Ci}$ \\
\hline \multicolumn{3}{|c|}{ ORRDACS } \\
\hline T901 & TE-1 capsule temperature & ${ }^{\circ} \mathrm{C}$ \\
\hline $\mathrm{T} 902$ & $\mathrm{TE}-2$ capsule temperature & ${ }^{\circ} \mathrm{C}$ \\
\hline T903 & TE-3 capsule temperature & ${ }^{\circ} \mathrm{C}$ \\
\hline T904 & $\mathrm{TE}-4$ capsule temperature & ${ }^{\circ} \mathrm{C}$ \\
\hline T905 & $\mathrm{TE}-5$ capsule temperature & ${ }^{\circ} \mathrm{C}$ \\
\hline T906 & TE- 6 capsule temperature & ${ }^{\circ} \mathrm{C}$ \\
\hline
\end{tabular}


TABLE IV 6 (Contd.)

\begin{tabular}{|c|c|c|}
\hline $\begin{array}{l}\text { Parameter } \\
\text { Name }\end{array}$ & Description & Units \\
\hline \multicolumn{3}{|c|}{ ORRDACS (Contd.) } \\
\hline T907 & $\mathrm{TE}-7$ capsule temperature & ${ }^{\circ} \mathrm{C}$ \\
\hline T908 & TE-8 capsule temperature & ${ }^{\circ} \mathrm{C}$ \\
\hline TR801 & TE-9 capsule temperature & ${ }^{\circ} \mathrm{C}$ \\
\hline TR901 & TE-10 capsule temperature & ${ }^{\circ} \mathrm{C}$ \\
\hline F12 & Helium inlet flow-gap gas & $\mathrm{cm}^{3} / \mathrm{min}$ \\
\hline F18 & Argon inlet flow-gap gas & $\mathrm{cm}^{3} / \mathrm{min}$ \\
\hline F25 & Flow-gap gas exhaust & $\mathrm{cm}^{3} / \mathrm{min}$ \\
\hline F26 & Flow-gap gas exhaust & $\mathrm{cm}^{3} / \mathrm{min}$ \\
\hline F52 & Helium flow-sweep gas & $\mathrm{cm}^{3} / \mathrm{min}$ \\
\hline F53 & Helium/4\% $\mathrm{H}_{2}$ flow-sweep gas & $\mathrm{cm}^{3} / \mathrm{min}$ \\
\hline F64 & Sweep gas exhaust & $\mathrm{cm}^{3} / \mathrm{min}$ \\
\hline REAPWR & ORR reactor power & W \\
\hline TREF6 & Reference thermocouple & \\
\hline $\mathrm{R} 100$ & R100 neutron f1ux & $10^{14} \mathrm{nv}$ \\
\hline $\mathrm{R} 100 \mathrm{R}$ & R100 neutron flux (raw) & $10^{14} \mathrm{nv}$ \\
\hline $\mathrm{R} 200$ & R200 neutron f1ux & $10^{14} \mathrm{nv}$ \\
\hline R200R & R200 neutron f1ux (raw) & $10^{14} \mathrm{nv}$ \\
\hline R300 & R300 neutron flux & $10^{14} \mathrm{nv}$ \\
\hline $\mathrm{R} 300 \mathrm{R}$ & R300 neutron flux (raw) & $10^{14} \mathrm{nv}$ \\
\hline $\mathrm{R} 400$ & Average neutron flux & $10^{14} \mathrm{nv}$ \\
\hline
\end{tabular}




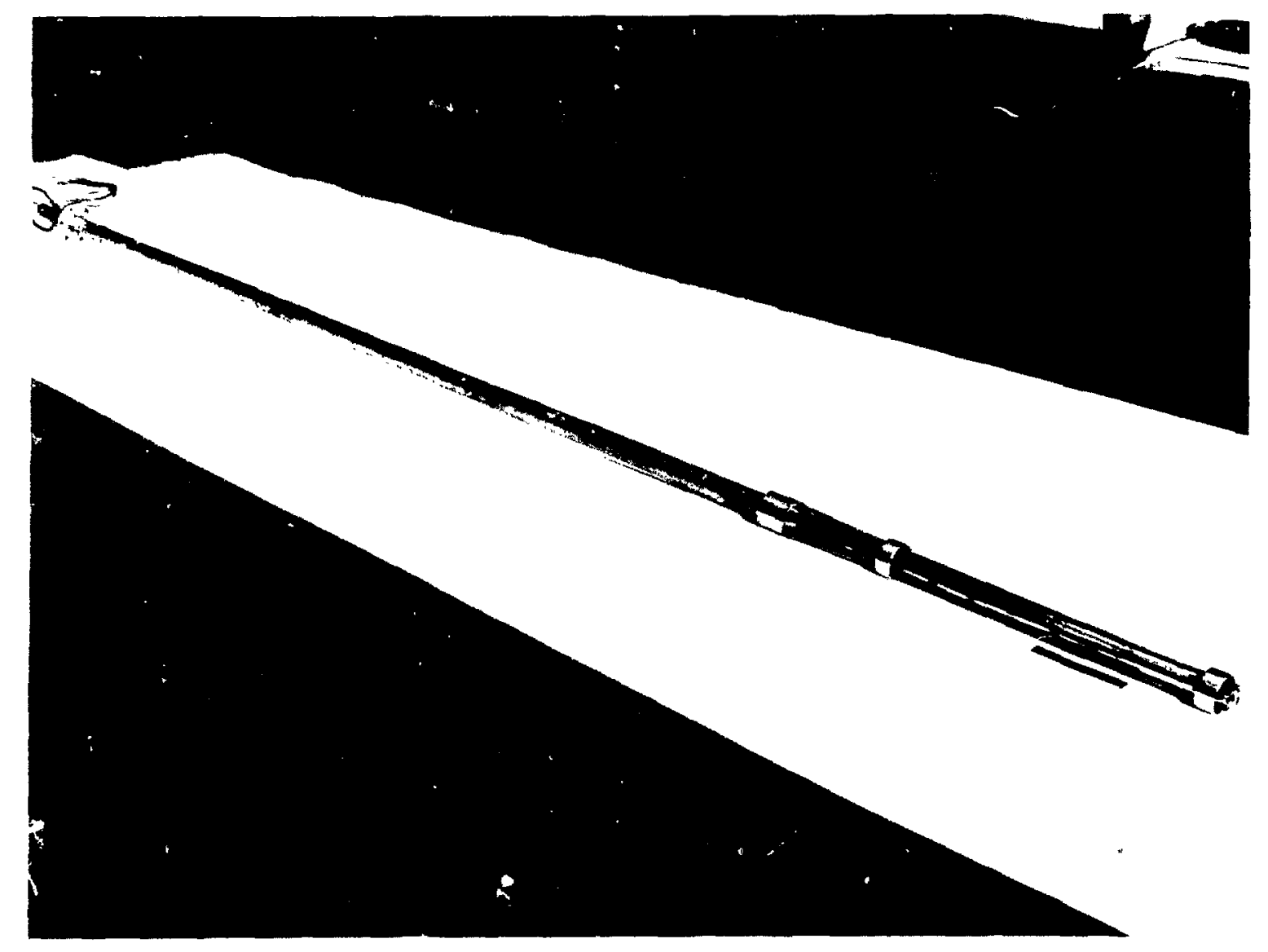

Fig. IV-7. Core mockup test assembly. 


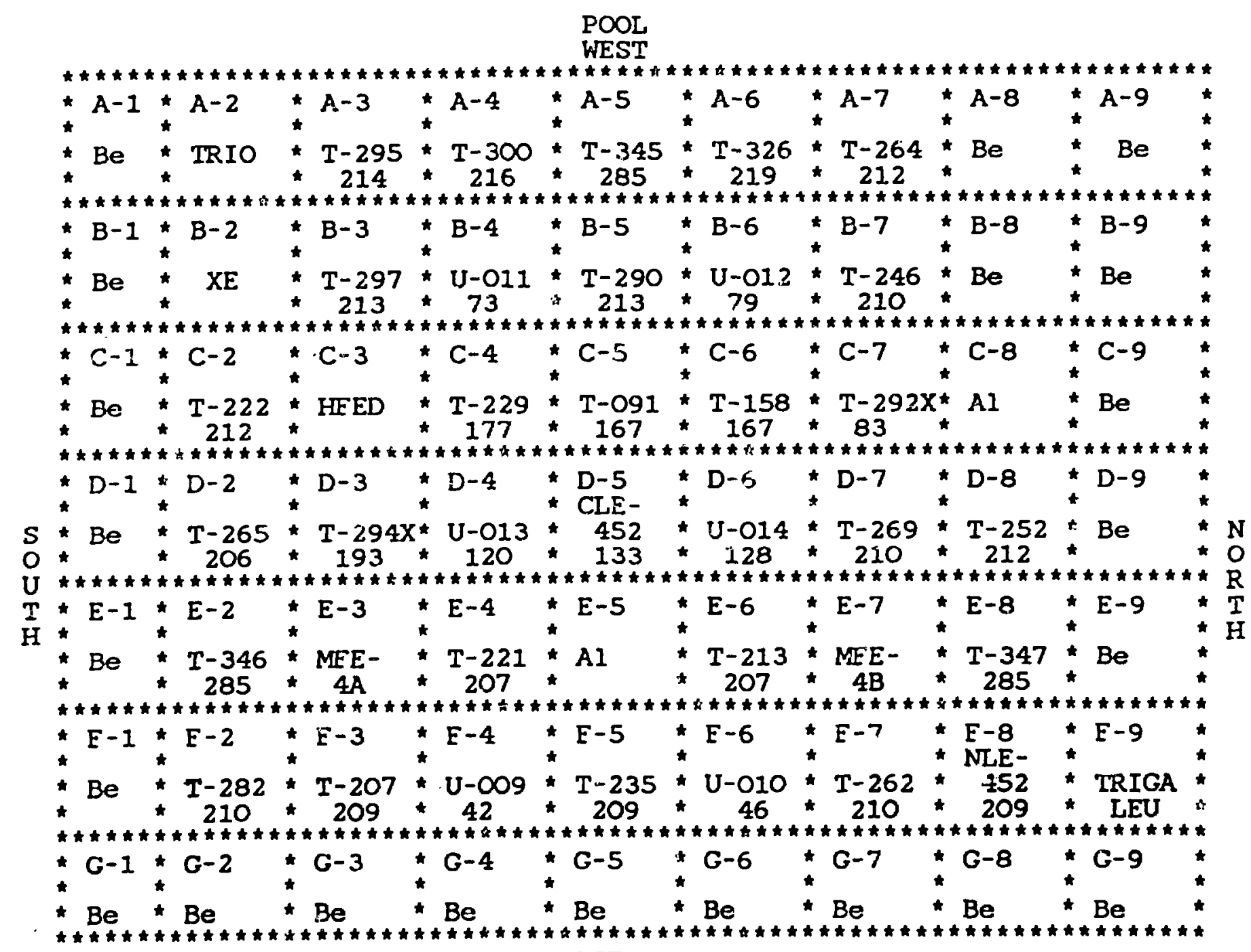

EAST

Note: $T$ - is fuel element.

$\mathrm{V}$ - is control element.

Grams of fuel shown, other designations are other experiments.

Fig. IV-8. ORR fuel loading diagram (Cycle 160-E, core mockup test, from Apri1 5, 1983, to Apri1 16, 1983). 


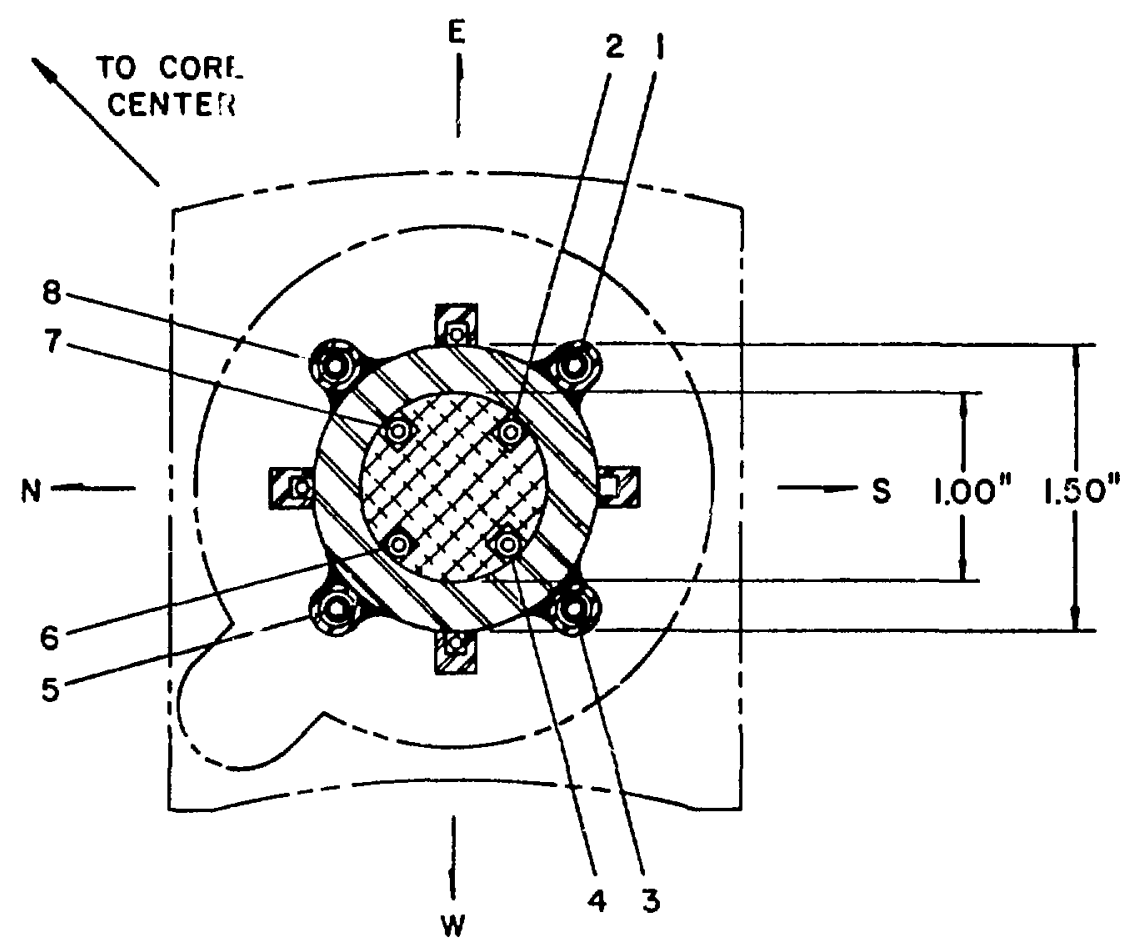

Fig. IV-9. Cross section of TRIO core mockup.

Tritium production in samples of the isotopically tailored breeder material used for the TRIO experiment was also measured. In related but separate tests, neutron flux profiles and gamma heating rates were measured in the $A 2$ core position of ORR. Uescribed herein are the calibration of the selfpowered neutron detectors, and measurements of essential nuclear parameters, including gamma heating rates, neutron spectrum, and tritium production.

\section{Se1f-Powered Neutron Detectors}

The three self-powerea neutron detectors were found to consistently give thermal flux readings that agreed within one percent. Typical values for the three detectors were $1.33,1.32$, and $1.3410^{14} \times \mathrm{n} \cdot \mathrm{cm}^{-2} \cdot \mathrm{s}^{-1}\left(\mathrm{n} \cdot \mathrm{cm}^{-2} \cdot \mathrm{s}^{-1}=\mathrm{nv}\right)$, or an average of $1.33 \times 10^{-14} \mathrm{nv}$. The precision of these detectors $1 \mathrm{~s}$ thus very good. Since the three detectors used in the TRIO experiment are from the same batch, their precision is expected to also be very good.

\section{Tritium Production Tests}

Before the tritium production was measured in the core mockup test, experiments were conducted to verify the tritium assay techniques. Specimens 
of lithium carbonate (natural abundance, ${ }^{6} \mathrm{Li}=7.5 \%$ ) and the $\mathrm{LiAlO}_{2}$ powder used to fabricate the TRIO pellets (isotopically tailored, ${ }^{6} \mathrm{Li}=0.55 \%$ ) were sealed in quartz capillaries and irradiated in the pneumatic tube of the Oak Ridge High Flux Isotope Reactor (HFIR) at a nominal thermal neutron flux of $4.6 \times 10^{-14} \mathrm{nv}$. The neutron fluence was monitored using wires of aluminum alloys containing known amounts of manganese and gold. After irradiation, each quartz capillary containing a sample of lithium aluminate or lithium carbonate was dissolved in concentrated $\mathrm{HF}$ in a sealed polyethylene vial. The solutions were diluted with water, made basic with $\mathrm{NaOH}$, diluted to a known volume, and distilled to recover the HTO. The tritium was then measured by beta counting in a Packard liquid scintillation counter. The counter was calibrated using known aliquots of an NBS standard.

Comparisons of predicted and measured amounts of tritium al presented in Table IV-7. Predicted activities of tritium (A) were based on the relation:

$$
A=N \times P_{t h}\left[S_{0}+\left(P_{r} / P_{t h}\right) I\right](1-\exp \{-1 t\})
$$

where

$$
\begin{aligned}
\mathrm{N} & =\text { number of }{ }^{6} \mathrm{Li} \text { atoms irradiated } \\
\mathrm{P}_{\mathrm{th}} & =\text { thermal neutron flux } \\
\mathrm{S}_{0} & =2200 \mathrm{~m} / \mathrm{s}^{*} \text { neutron cross section of }{ }^{6} \mathrm{Li}(940 \mathrm{~b}) \\
P_{\mathrm{r}} & =\text { resonance neutron flux } \\
\mathrm{I} & =\text { resonance integral for the reaction of }{ }^{6} \mathrm{Li}(425 \mathrm{~b}) \\
\mathrm{I} & =\text { decay constant for tritium (half } 1 \text { ife }=12.33 \mathrm{y}) \\
\mathrm{t} & =\text { the irradiation time }
\end{aligned}
$$

As can be seen from Table IV-7, the agreement between predicted and measured tritium activities is quite good. Uncertainties in predicted activities are thought to be mainly due to uncertainties in the measured neutron flux, estimated to be $5 \%$. The error in the NBS standard is about $2 \%$. The results

\footnotetext{
*This value is the nominal velocity of a thermal neutron.
} 
TABLE IV -7

Comparison of Predicted and Measured Activities of Tritium in Irradiated Samples of Lithium Carbonate and Lithium Aluminate

\begin{tabular}{l|c|c|c}
\hline \multirow{2}{*}{$\begin{array}{l}\text { Sample } \\
\text { No. }\end{array}$} & \multicolumn{3}{|c|}{ Tritium Activity, Bq/mg } \\
\cline { 2 - 4 } & Predicted & Measured & $\%$ Diff. \\
\hline
\end{tabular}
Lithium Carbonate

\begin{tabular}{l|l|ll|l}
1 & $5.98 \times 10^{4}$ & $5.84 \times 10^{4}$ & -2.3 \\
3 & $5.98 \times 10^{4}$ & $6.10 \times 10^{4}$ & +2.0 \\
$5.98 \times 10^{4}$ & $6.05 \times 10^{4}$ & +1.2 \\
& & & &
\end{tabular}

Lithium Aluminate (TRIO) $^{\text {b }}$

\begin{tabular}{l|l|l|l|l}
1 & $7.07 \times 10^{4}$ & $6.87 \times 10^{4}$ & -2.8 \\
2 & $7.07 \times 10^{4}$ & $6.93 \times 10^{4}$ & -2.0 \\
3 & $7.07 \times 10^{4}$ & $7.24 \times 10^{4}$ & +2.4 \\
4 & $7.07 \times 10^{4}$ & $6.97 \times 10^{4}$ & -1.4 \\
6 & $7.07 \times 10^{4}$ & $7.44 \times 10^{4}$ & +5.2 \\
$7.07 \times 10^{4}$ & $6.94 \times 10^{4}$ & -1.8 \\
\hline
\end{tabular}

$a_{\text {Thermal flux }}{ }^{*}=4.68 \times 10^{14} \mathrm{nv}$;

irradiation time $=61.6 \mathrm{~s}$.

$\mathrm{b}_{\text {Thermal flux }}{ }^{*}=4.61 \times 10^{14} \mathrm{nv}$;

irradiation time $=1800 \mathrm{~s}$.

* The thermal-tc-resonance $\mathrm{flux}$ ratio was measured to be 36 .

demonstrate that the analytical techniques developed for the core mockup test yield quantitative recovery of tritium. 
Tritium production in the core mockup test was determined by inserting quartz ampoules contalning the isotopically tallored $\mathrm{LiAlO}_{2}$ into various locations in the mockup assembly (Fig. IV-9) and assaying for tritium. The measured tritium activities for the core mockup test are given in Table IV-8.

Table IV-8

Tritium Production in the Core Mockup Test

(Apri1 27, 1982)

\begin{tabular}{l|l|l|l}
\hline $\begin{array}{c}\text { Sample } \\
\text { No. }\end{array}$ & $\begin{array}{c}\text { Irradiation } \\
\text { Location }\end{array}$ & $\begin{array}{c}\text { Sample } \\
\text { Weight }^{\mathrm{a}} \\
\left(\mathrm{mg} \mathrm{LiAl0}{ }_{2}\right)\end{array}$ & $\begin{array}{c}\text { Measured } \\
\text { Tritium } \\
\text { Activity } \\
(\mu \mathrm{Ci} / \mathrm{mg})\end{array}$ \\
\hline 1 & 2 top & 2.415 & 6.58 \\
2 & 2 bottom & 1.173 & 7.04 \\
3 & 4 top & 1.396 & 6.72 \\
4 & 4 bottom & 1.509 & 6.23 \\
5 & 6 top & 1.358 & 6.24 \\
6 & 6 bottom & 1.569 & 6.41 \\
7 & 8 top & 1.858 & 6.55 \\
8 & 3 bottom & 1.920 & 6.53 \\
\hline
\end{tabular}

${ }^{a}$ The lithium aluminate used is isotopically tailored, having a ${ }^{6} \mathrm{~L}$ i content of $0.55 \%$.

\section{Dosimetry Measurements}

A comprehensive dosimetry experiment was conducted as a part of the core mockup test to characterize the nuclear environment. 43 Flux monitor wires were irradiated in the A2 core position of ORR (see Fig. IV-8) for $8.93 \mathrm{~h}$, which amounted to a total exposure of $253.35 \mathrm{MWh}$. Eight aluminum tubes were positioned in the core mockup assembly (Fig. VI-9) to measure flux and spectral gradients. Seven tubes contained titanium, iron, nickel, and cobaltaluminum gradient wires, $15 \mathrm{~cm}$ ( $6 \mathrm{in.}$ ) in length. These wires were positioned to span the flux peak, which is located about $15 \mathrm{~cm}$ (6 in.) below the reactor midplane. The elghth tube measured $89 \mathrm{~cm}$ (35 in.) in length and contained 17 different materials, including gadolinium covers and fission monitors for a 
spectral analysis. Gradient wires were also included to span the entire length of the core mockup assembly.

The activities of the samples were counted with $\mathrm{Ge}(\mathrm{Li})$ spectrometers, and the resultant saturated activities are 1isted in Table IV-9. These results were then used to adjust a calculated neutron spectrum using the STAYSL computer code. 54 The resultant neutron flux values are 1isted in Table IV-10.

TABLE IV -9

Activation Rates for $\mathrm{TRIO}^{\mathrm{a}}$

\begin{tabular}{|c|c|c|}
\hline Reaction & $\begin{array}{l}\text { Activation } \\
\text { Rate } \\
\text { (atom/atom·s) }\end{array}$ & $\begin{array}{c}\text { Gd } \\
\text { Cover }\end{array}$ \\
\hline${ }^{58} \mathrm{Fe}\left(\mathrm{n}, \gamma^{59} \mathrm{Fe}\right.$ & $1.08 \times 10^{-10}$ & No \\
\hline${ }^{59} \mathrm{Co}(n, \gamma)^{60} \mathrm{Co}$ & $2.70 \times 10^{-9}$ & No \\
\hline${ }^{176} \mathrm{Lu}(n, \gamma) 177 \mathrm{~m} \mathrm{Lu}$ & $3.92 \times 10^{-9}$ & No \\
\hline${ }^{45} \mathrm{Sc}(n, \gamma)^{46} \mathrm{Sc}$ & $2.53 \times 10^{-10}$ & Yes \\
\hline${ }^{197} \mathrm{Au}(\mathrm{n}, \gamma){ }^{198} \mathrm{Au}$ & $2.15 \times 10^{-8}$ & Yes \\
\hline${ }^{237} \mathrm{~Np}(\mathrm{n}, \gamma)^{238 \mathrm{~Np}}$ & $7.22 \times 10^{-9}$ & Yes \\
\hline $238 \mathrm{U}(\mathrm{n}, \gamma)^{239} \mathrm{~Np}$ & $1.02 \times 10^{-10}$ & Yes \\
\hline${ }^{238} \mathrm{U}(\mathrm{n}$, fission $)$ & $2.26 \times 10^{-11}$ & Yes \\
\hline${ }^{54} \mathrm{Fe}(n, p){ }^{54} \mathrm{Mn}$ & $5.38 \times 10^{-12}$ & No \\
\hline${ }^{58} \mathrm{Ni}(\mathrm{n}, \mathrm{p}){ }^{58} \mathrm{Co}$ & $6.86 \times 10^{-12}$ & No \\
\hline${ }^{46} \mathrm{Ti}(\mathrm{n}, \mathrm{p}){ }^{46} \mathrm{Sc}$ & $7.30 \times 10^{-13}$ & No \\
\hline${ }^{47} \mathrm{Ti}(\mathrm{n}, \mathrm{p})^{47} \mathrm{Sc}$ & $1.26 \times 10^{-12}$ & No \\
\hline${ }^{48} \mathrm{Ti}(\mathrm{n}, \mathrm{p})^{48} \mathrm{Sc}$ & $1.99 \times 10^{-1} t_{r}$ & No \\
\hline${ }^{27} \mathrm{Al}\left(\mathrm{n}, \mathrm{N}^{24} \mathrm{va}\right.$ & $4.59 \times 10^{-14}$ & No \\
\hline $55_{M n}(n, 2 n)^{54} M n$ & $1.69 \times 10^{-14}$ & No \\
\hline${ }^{9}{ }^{3} \mathrm{Nb}(\mathrm{n}, 2 \mathrm{n}){ }^{92 \mathrm{~m}_{\mathrm{Nb}}}$ & $3.05 \times 10^{-14}$ & No \\
\hline
\end{tabular}

a Determined on Apri1 26, 1982, at rRR, A2 position. Values renormalized to $30-\mathrm{MW}$ reactor power. Maximum flux height is in pusition 8 . 
Table IV-10

Neutron Flux Values for TRIO

(Apr11 26, 1982)

\begin{tabular}{l|c|c}
\hline \multicolumn{1}{c|}{ Energy Range } & $\begin{array}{c}\text { F1ux } \\
\left(10^{14}-\mathrm{v}\right)\end{array}$ & $\begin{array}{c}\text { Uncertainty } \\
(\%)\end{array}$ \\
\hline Total & 3.79 & 5.0 \\
Thermal $(<0.5 \mathrm{eV})$ & 1.31 & 6.0 \\
Thermal $(2200 \mathrm{~m} / \mathrm{s})^{\mathrm{a}}$ & 1.16 & 6.0 \\
Epithermal $(0.5 \mathrm{eV}-0.11 \mathrm{MeV})$ & 1.20 & 9.0 \\
Fast $(>0.11 \mathrm{MeV})$ & 1.27 & 7.0 \\
Fast $(>1 \mathrm{Mev})$ & 0.58 & 6.0 \\
\hline
\end{tabular}

${ }^{a_{N o m i n a l}}$ velocity of a thermal neutron.

The flux spectrum is shown in Fig. IV-10 for the A2 position. The dotted and dashed lines represent errors for each flux group; however, the group; are highly correlated. A covariance error matrix must then be used to calculate errors in integral quantities. Integral flux errors are typically 5-10\%, as 1isted in Table IV-10.

The measured spectrum was also used to calculate the tritium production rate from ${ }^{6} \mathrm{LI}$. The result agrees quite well with the measured tritium production rate (Table IV-7) and related helium dosimetry results, 44 as shown in Table IV-11. The measured thermal neutron flux also agrees well with the self-powered neutron detector readings $\left(1.31 \mathrm{vs} .1 .33 \times 10^{14}\right)$.

TABLE IV-11

Tritium Production Rates in the TRIO Core Mockup Test

\begin{tabular}{l|c}
\hline \multicolumn{1}{c|}{ Method Used } & $\begin{array}{c}\text { Production Rate } \\
\left(10^{-8} \mathrm{~T} / 6 \mathrm{Li} \cdot \mathrm{s}\right)\end{array}$ \\
\hline Dosimetry (calculated) & 9.22 \\
Measured (Table IV-8) & 8.91 \\
Helium dosimetry (Ref. 44) & $\mathbf{8 . 4 5}$ \\
\hline
\end{tabular}




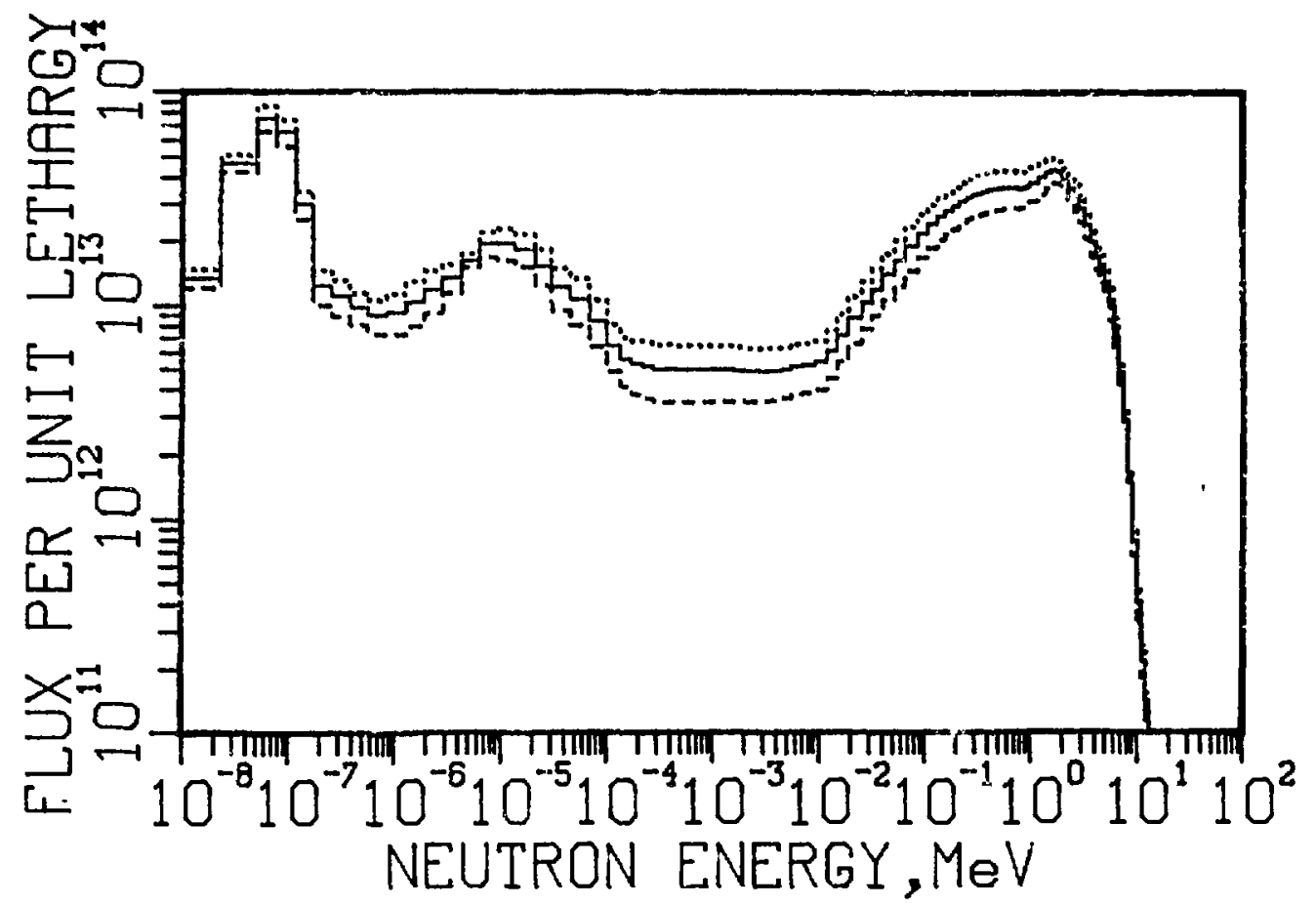

Fig. IV-10. F1ux spectrum measured in the A2 core position of ORR for the TRIO experiment. (Dotted and dashed lines represent one standard deviation; however, the flux groups are highly correlated.)

Vertical flux gradients are shown in Figure IV-11. The maximum flux occurs about $13 \mathrm{~cm}$ ( 5 in.) below the reactor midplane, as expected at the start of a fuel cycle in ORR. Over the course of a fuel cycle, the flux peak muves upward about $10 \mathrm{~cm}$ ( $4 \mathrm{in.)}$. Thus, the TRIO capsule, centered at $8 \mathrm{~cm}$ ( 3 in.) below reactor midplane, is near or on the flux peak throughout the fuel cycle.

Horizontal flux gradients are summarized as follows: (1) the thermal flux decreases about $21 \%$ across the assembly $(4.5 \mathrm{~cm})$ from east to west [away from the center of the core (Fig. IV-7)]; (2) the thermal flux is about 23\% lower inside the mockup assembly, owing to absorption by the stainless steel cladding; (3) the fast flux clecreases sharply (about 60\%) from northeast to southwest on a line roughly pointing away from the center of the core; (4) the thermal flux varies less than $10 \%$ inside the mockup assembly, representing the region where the lithium aluminate breeder material will be located during operation of the TRIO experiment. 


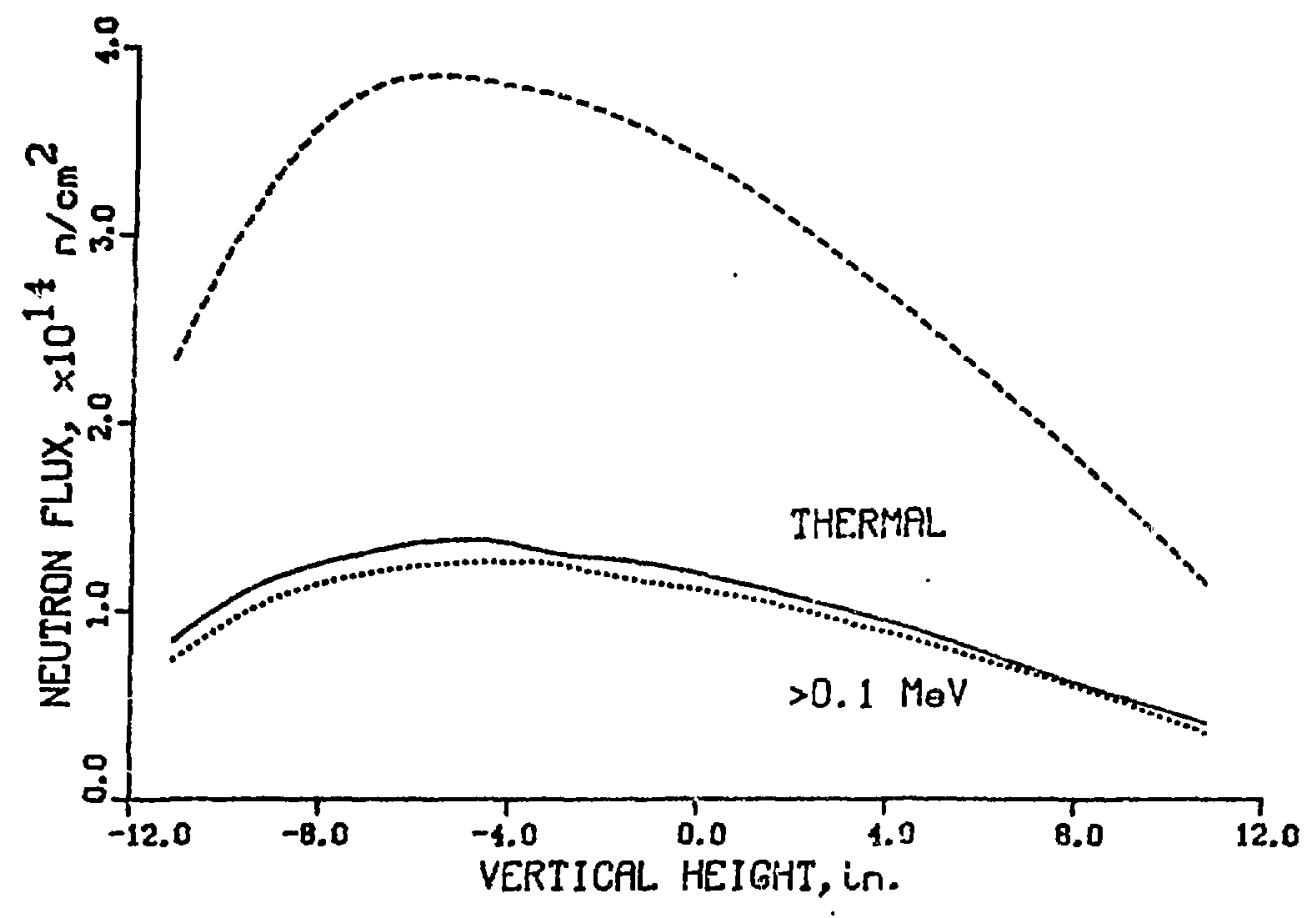

Fig. IV-11. Vertical flux gradients measured in position A2 of ORR. The maximum flux is $5 \mathrm{in}$. below midplane at the start of the fuel cycle, but will rise to 3 in. below midplane where TRIo is centered.

\section{Gamma Heating Rates}

Gamma flux profiles in the A2 core position were measured with an ionization chamber. The fuel loading diagram for the measurement is given in Figure IV-12. The values of ionization current were correlated to a prior experiment with known gamma heating rates, and the heating rates derived as a function of axial position. It was found that the gamma heating rate in stainless steel in the region of the TRIO capsule is $4.8 \mathrm{~W} / \mathrm{g}$. The estimated accuracy of this value is 5-10\% (42) for a given core loading in ORR. However, changes in core loading in ORR cause significant variations in the local nuclear environment. The uncertainty in gamma flux owing to fuel changes is estimated to be $20 \%$. The overall accuracy of the values is thus estimated to be about $25 \%$. The value of $4.8 \mathrm{~W} / \mathrm{g}$ was used for the capsule. Because gamma heating in units of watts per gram is roughly the same for most materials, the measured value was used for both the stainless steel and the $\mathrm{LiAlO}_{2}$ in the capsule. 


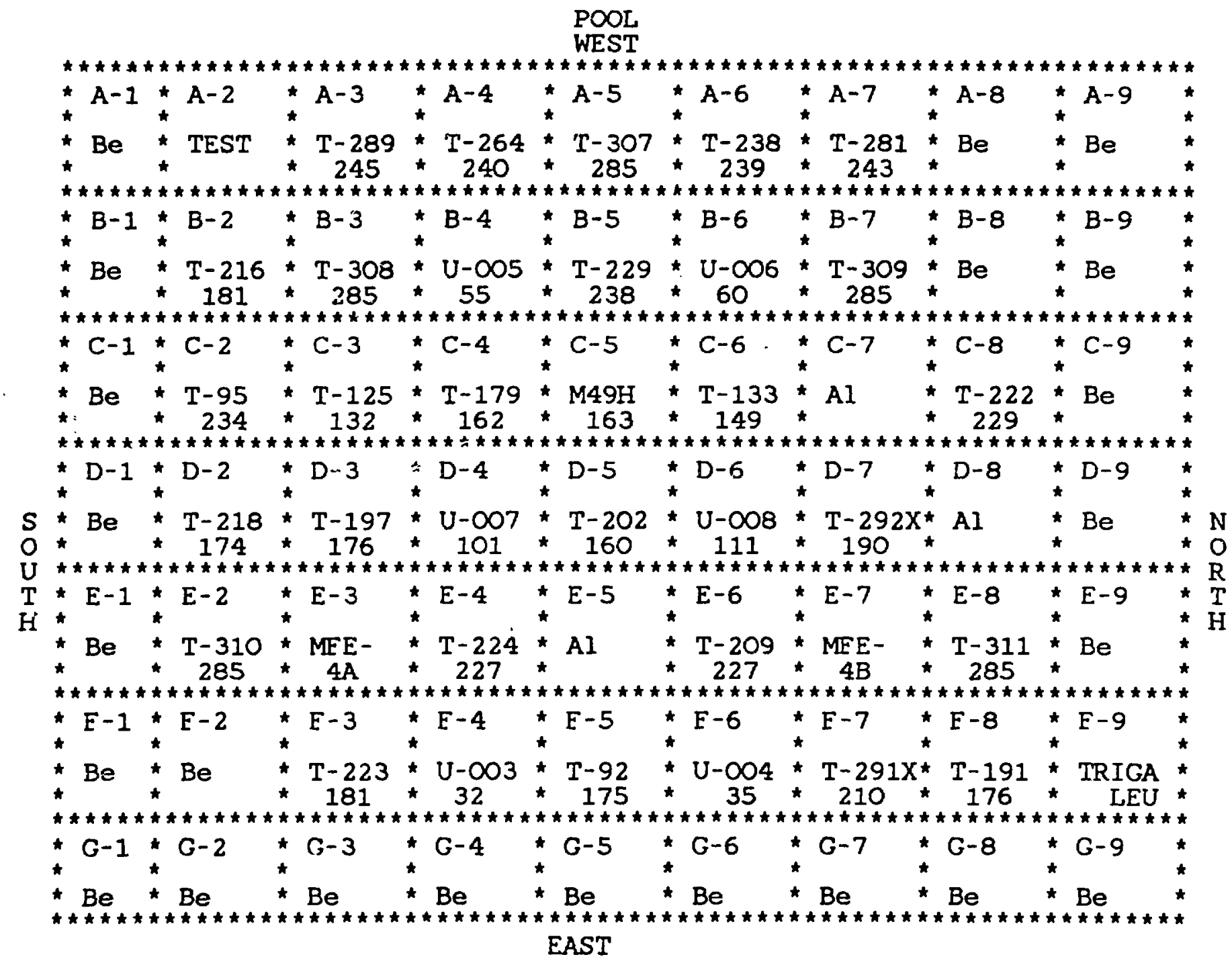

Fig. IV-12. Fuel loading diagram for the gamma heating measurements (July 12, 1981). 
$=$ 


\section{v. EXPER IMENTAL PLAN}

Tritium release is a function of operational paramters such as temperature, purge gas conditions, radiation damage, etc. In the TRIo experiment, three parameters were independently controlled: temperature, sweep gas flow rate, and sweep gas composition. Average (nominal) temperature was varied from 400 to $700^{\circ} \mathrm{C}$ by changing che composition of the gap gas; the flow rate was operated at 30 to $300 \mathrm{cc} / \mathrm{min}$; and up to $1 \% \mathrm{H}_{2}$ or $\mathrm{O}_{2}$ was added to the helium sweep gas. The TRIO test matrix (Table V-1) is composed of combinations of the three parameters. The mairix was designed to systematically investigate the parameter space. Temperature is the most importanic variable, and for most runs the sweep gas was maintained at standard conditions, namely, a flow rate of $100 \mathrm{~cm}^{3} / \mathrm{min}$ and $0.1 \% \mathrm{H}_{2}$ added to the helium swee, gas.

The basic strategy was to operate under preset conditions and to monitor the tritium release rate until steady state was achieved. Sceady state was observable when the release rate was invariant in time, and also when the release rate was equal to the generation rate. Upon completion of such a test run, the conditions were changed and another run was performed. It was expected that, when the second run was at steady state, the release rate would be the same as that of the first run.

The conditions selected for the first run were predicted to achieve steady state within about one day. Thus, the remperature was chosen to he moderately high so that there would be a good chance of achieving steady state. The second run was set at maximum temperature so that it could be checked that the first test had actually reached steady-state conditions.

The next several runs (up to Run 8) tested the effects of the temperature range, with the exception of Run 3, which tested the effect of increased hydrogen in the sweep gas. Runs 9 through 14 tested the effect of temperature when no hydrogen was added to the sweep gas. Runs 10 and 11 were used to measure low-temperature release rates of radionuclides. The next series, Runs 16-20, tested the effects of sweep gas composition and flow rate. The later series of runs tested low temperatures in an effort to obtain steady state at as low a temperature as possible. Finally, the temperature was increased to standard conditions so that the amount of tritium retained in the solid breeder could be minimized. 
TABLE VI-1

TRIO Test Matrix

\begin{tabular}{|c|c|c|c|c|c|}
\hline \multirow[b]{2}{*}{$\begin{array}{l}\text { Run } \\
\text { No. }\end{array}$} & \multirow[b]{2}{*}{$\begin{array}{l}\text { Time } \\
\text { (d) }\end{array}$} & \multirow{2}{*}{$\begin{array}{l}\text { Nominal } \\
\text { Temp. } \\
\left({ }^{\circ} \mathrm{C}\right)\end{array}$} & \multicolumn{2}{|c|}{ Sweep Gas } & \multirow[b]{2}{*}{ Test Purpose } \\
\hline & & & Composition & $\begin{array}{l}\text { Flow Rate } \\
\left(\mathrm{cm}^{3} / \text { min }\right)\end{array}$ & \\
\hline 1 & 2 & 600 & $\mathrm{He} / 0.1 \% \mathrm{H}_{2}$ & 100 & \\
\hline 2 & 2 & 700 & $\mathrm{He} / 0.1 \% \mathrm{H}_{2}$ & 100 & Temperature \\
\hline 3 & 1 & 700 & $\mathrm{He} / 1 \% \mathrm{H}_{2}$ & 100 & $\mathrm{H}_{2}$ level \\
\hline 4 & 5 & 550 & $\mathrm{He} / 0.1 \% \mathrm{H}_{2}$ & 100 & Temperature \\
\hline 5 & 2 & 500 & $\mathrm{He} / 0.1 \% \mathrm{H}_{2}^{2}$ & 100 & Temperature \\
\hline 6 & 1 & 550 & $\mathrm{He} / 0.1 \% \mathrm{H}_{2}$ & 100 & Repeat Run 4 \\
\hline 7 & 3 & 600 & $\mathrm{He} / 0.1 \% \mathrm{H}_{2}^{2}$ & 100 & Repeat Run 1 \\
\hline 8 & 2 & 650 & $\mathrm{He} / 0.1 \% \mathrm{H}_{2}^{2}$ & 100 & Standard run \\
\hline 9 & 6 & 650 & $100 \% \mathrm{He}$ & 100 & $\mathrm{H}_{2}$ level \\
\hline 10 & 1 & 400 & $100 \% \mathrm{He}$ & 100 & Rádionuclides \\
\hline 11 & 1 & 500 & $100 \% \mathrm{He}$ & 100 & Radionuclides \\
\hline 12 & 4 & 550 & $100 \% \mathrm{He}$ & 100 & $\mathrm{H}_{2}$ level \\
\hline 13 & 5 & 600 & $100 \% \mathrm{He}$ & 100 & $\mathrm{H}_{2}$ level \\
\hline 14 & 2 & 700 & $100 \% \mathrm{He}$ & 100 & $\mathrm{H}_{2}$ level \\
\hline 15 & 1 & 700 & $\mathrm{He} / 0.1 \% \mathrm{H}_{2}$ & 100 & $\mathrm{H}_{2}$ level, temperature \\
\hline 16 & 1 & 700 & $\mathrm{He} / 0.1 \% \mathrm{H}_{2}^{2}$ & 100 & $\mathrm{H}_{2}$ leve1, temperature \\
\hline 17 & 3 & 700 & $\mathrm{He} / 0.1 \% \mathrm{H}_{2}$ & 30 & Flow rate \\
\hline 18 & 1 & 700 & $\mathrm{He} / 0.1 \% \mathrm{H}_{2}$ & 100 & Flow rate \\
\hline 19 & 1 & 650 & $\mathrm{He} / 0.1 \% \mathrm{H}_{2}$ & 100 & Standard run \\
\hline 20 & 4 & 650 & $\mathrm{He} / 0.2 \% \mathrm{O}_{2}$ & 100 & $\mathrm{O}_{2}$ \\
\hline 21 & 2 & 650 & $\mathrm{He} / 0.1 \% \mathrm{H}_{2}$ & 100 & Standard run \\
\hline 22 & 1 & 000 & $\mathrm{He} / 0.1 \% \mathrm{H}_{2}$ & 100 & Repeat Runs 1,7 \\
\hline 23 & 3 & 550 & $\mathrm{He} / 0.1 \% \mathrm{H}_{2}$ & 100 & Repeat Runs 4,6 \\
\hline 24 & 1 & 600 & $\mathrm{He} / 0.1 \% \mathrm{H}_{2}^{2}$ & 100 & Repeat Runs $1,7,22$ \\
\hline 25 & 2 & 560 & $\mathrm{He} / 0.1 \% \mathrm{H}_{2}^{2}$ & 100 & New temperature \\
\hline 26 & 3 & 550 & $\mathrm{He} / 0.1 \% \mathrm{H}_{2}^{2}$ & 100 & Repeat Runs 4,6,23 \\
\hline 27 & 7 & 525 & $\mathrm{He} / 0.1 \% \mathrm{H}_{2}^{2}$ & 100 & Low temperature \\
\hline 28 & 7 & 500 & $\mathrm{He} / 0.1 \% \mathrm{H}_{2}^{2}$ & 100 & Low temperature \\
\hline 29 & 3 & 480 & $\mathrm{He} / 0.1 \% \mathrm{H}_{2}^{2}$ & 300 & Flow rate \\
\hline 30 & 4 & 480 & $\mathrm{He} / 0.1 \% \mathrm{H}_{2}$ & 100 & Low temperature \\
\hline 31 & 2 & 500 & $\mathrm{He} / 0.1 \% \mathrm{H}_{2}^{2}$ & 100 & Repeat Run 28 \\
\hline 32 & 2 & 550 & $\mathrm{He} / 0.1 \% \mathrm{H}_{2}^{2}$ & 100 & Repeat Run 26 \\
\hline 33 & 3 & 650 & $\mathrm{He} / 0.1 \% \mathrm{H}_{2}$ & 100 & St andard run \\
\hline
\end{tabular}


SECTION VI

EXPERIMENTAL RESULTS - ON LINE DATA 


\section{EXPERINENTAL RESULTS - ON-LINE DATA}

The measurement of in-situ tritium release and the correlation of relationships between tritiun release and operational parameters constitute the essential part of the TRIO experiment. The most important operational parameters in the experiment are time, neutron flux, temperature and temperature profiles, and sweep gas chemistry. Presented herein are the data on operational parameters and the observed release behavior of tritium in its various chemical forms.

\section{A. Time and Recorded Experimental History}

The TRIO experiment was installed in CRR on January 14, 1983. Installation of the experiment occurred during a time when the reactor was down for a three-month period for coolant system repairs. The full-power irradiation began on March 12, 1983, and the 33 tests performed in subsequent months. The operating history is given in Table VI-1. In addition to the 33 runs, there are a large number of (about 50) transient runs. The transients provide useful dynamic data.

\section{B. Neutron Flux and Dosimetry}

Thermal neutron flux incident on the capsule was continuously monitored with the three self-powered neutron detectors wound around the outside of the capsule. Calibration of the self-powered neutron detectors was done in the core mockup test, as previously discussed. The recorded fluxes for the entire experiment are shown in Fig. VI-1. The variation in local neutron flux was considerably more than that of the reactor power (Fig. VI-2). The reason for the rather large variations in local flux was that the entire fuel for the reactor is changed every two weeks. The core loading diagrams, showing the grams of fuel in each core position for the six different fuel loadings used in the experiment, are given in Figs. VI-3a through $-3 g$. In addition to these effects, the flux levels are approximately $30 \%$ lower than those in the core mockup test so that tritium production is corrospondingly reduced. The flux data, in conjunction with dosimetry results, are used to calculate instantaneous tritium production rates. 
TABLE VI-1

TRIO Operating History

\begin{tabular}{|c|c|c|c|c|c|c|}
\hline \multirow[b]{2}{*}{$\begin{array}{l}\text { Run } \\
\text { No. }\end{array}$} & \multirow[b]{2}{*}{$\begin{array}{l}1983 \\
\text { Dete }\end{array}$} & \multirow[b]{2}{*}{ Time } & \multirow{2}{*}{$\begin{array}{l}\text { Noninal } \\
\text { Teap. } \\
\text { ( } \mathrm{\circ} \text { C) }\end{array}$} & \multicolumn{2}{|c|}{ Sweep Cas } & \multirow[b]{2}{*}{ Coment $:^{b}$} \\
\hline & & & & Composition & $\begin{array}{l}\text { Flow Rate } \\
\left(\mathrm{cm}^{3} / \mathrm{m} 1 \mathrm{n}\right)\end{array}$ & \\
\hline 0 & $\begin{array}{l}1 / 14 \\
1 / 27 \\
2 / 28 \\
3 / 2 \\
3 / 9 \\
3 / 10 \\
3 / 10 \\
3 / 11\end{array}$ & $\begin{array}{l}1500 \\
1200 \\
0900 \\
0930 \\
1400 \\
1000 \\
1900 \\
0357 \\
1013 \\
1051 \\
1625 \\
1701 \\
1720 \\
1746 \\
1826 \\
1846 \\
2025 \\
2125\end{array}$ & $\begin{array}{r}30 \\
30 \\
30 \\
30 \\
30 \\
30 \\
35 \\
200 \\
35 \\
35 \\
35 \\
35 \\
100 \\
35 \\
35 \\
100 \\
200 \\
35\end{array}$ & 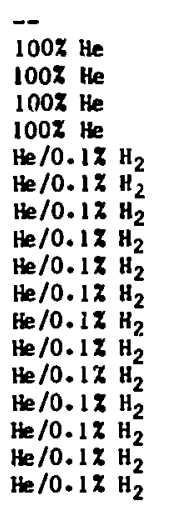 & $\begin{array}{r}0 \\
20 \\
0 \\
100 \\
100 \\
100 \\
100 \\
100 \\
100 \\
100 \\
100 \\
100 \\
100 \\
100 \\
100 \\
100 \\
100 \\
100\end{array}$ & 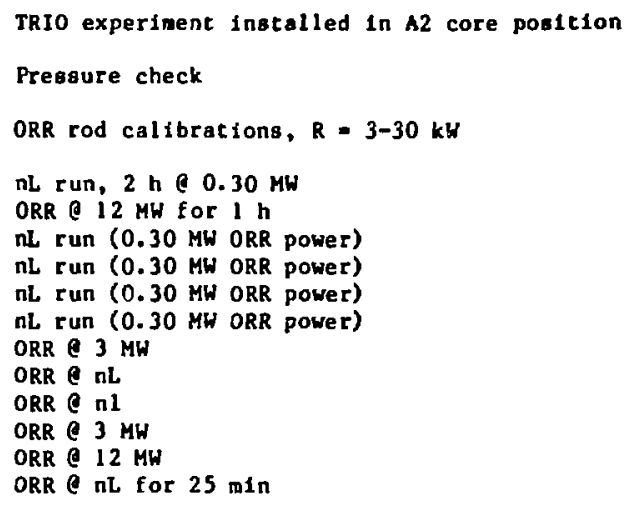 \\
\hline 1 & $3 / 12$ & $\begin{array}{l}0754 \\
0838 \\
0841 \\
0848 \\
0849 \\
0850 \\
0851 \\
0852 \\
0853 \\
0900 \\
0924 \\
0937 \\
1050 \\
1100 \\
1200 \\
1016 \\
1226\end{array}$ & $\begin{array}{r}35 \\
50 \\
87 \\
150 \\
180 \\
214 \\
242 \\
269 \\
300 \\
350 \\
380 \\
450 \\
450 \\
450 \\
600 \\
600 \\
600\end{array}$ & 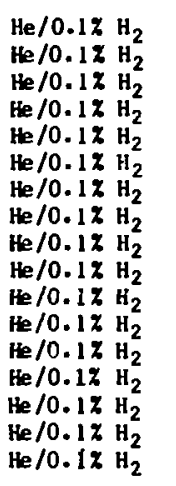 & $\begin{array}{l}100 \\
100 \\
100 \\
100 \\
100 \\
100 \\
100 \\
100 \\
100 \\
100 \\
100 \\
100 \\
100 \\
100 \\
100 \\
100 \\
100\end{array}$ & 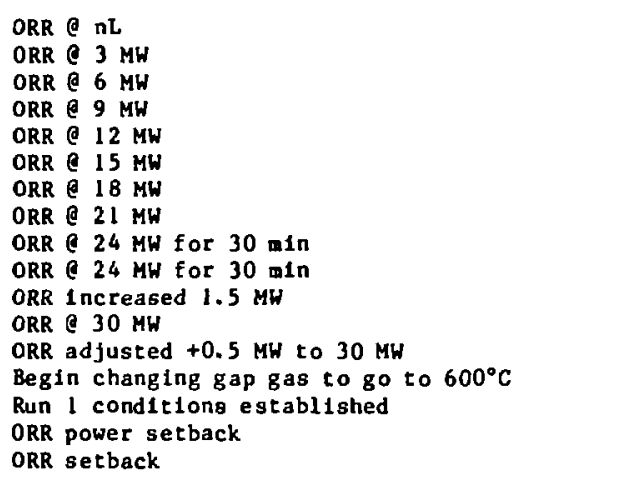 \\
\hline 2 & $\begin{array}{l}3 / 14 \\
3 / 15\end{array}$ & $\begin{array}{l}1300 \\
0930\end{array}$ & $\begin{array}{l}700 \\
700\end{array}$ & $\begin{array}{lll}\mathrm{He} / 0.1 \% & \mathrm{H}_{2} \\
\mathrm{He} / 0.1 \times \mathrm{H}_{2}\end{array}$ & $\begin{array}{l}100 \\
100\end{array}$ & $\begin{array}{l}\text { Beg 1n Run } 2 \\
\text { ORR setback }\end{array}$ \\
\hline 3 & $3 / 16$ & $\begin{array}{l}1124 \\
1525\end{array}$ & $\begin{array}{l}700 \\
700\end{array}$ & $\begin{array}{l}\mathrm{He} / 0.17 \mathrm{H}_{2} \\
\mathrm{He} / 0.1 \approx \mathrm{H}_{2}\end{array}$ & $\begin{array}{l}100 \\
100\end{array}$ & $\begin{array}{l}\text { Begin Run } 3 \\
\text { Power reduced } 0.12 \mathrm{MW} \mathrm{to} 30 \mathrm{MH}\end{array}$ \\
\hline 4 & $\begin{array}{l}3 / 17 \\
3 / 19 \\
3 / 21\end{array}$ & $\begin{array}{l}1405 \\
1206 \\
0856 \\
0956\end{array}$ & $\begin{array}{r}550 \\
550 \\
30 \\
550\end{array}$ & $\begin{array}{l}\mathrm{He} / 0.1 \% \mathrm{H}_{2} \\
\mathrm{He} / 0.1 \% \mathrm{H}_{2} \\
\mathrm{He} / 0.1 \% \mathrm{H}_{2} \\
\mathrm{He} / 0.1 \% \mathrm{H}_{2}\end{array}$ & $\begin{array}{l}100 \\
100 \\
100 \\
100\end{array}$ & $\begin{array}{l}\text { Begin Run } 4 \\
\text { Temperature of breeder reduced } 8 \mathrm{deg} \\
\text { ORR sliut down for } 1 \mathrm{~h} \\
\text { Resume Run } 4\end{array}$ \\
\hline$s$ & $3 / 22$ & 1127 & 500 & $\mathrm{He} / 0.1 \mathrm{HH}_{2}$ & 100 & Begin Run 5 \\
\hline 6 & $3 / 24$ & 1052 & 550 & $\mathrm{He} / 0.1 \times \mathrm{H}_{2}$ & 100 & Begin Run 6 \\
\hline 7 & $3 / 25$ & 1050 & 600 & $\mathrm{He} / 0.1 \mathrm{x} \mathrm{H}_{2}$ & 100 & BegIn Run 7 \\
\hline 8 & $3 / 28$ & 1300 & 650 & $\mathrm{He} / 0.1 \% \mathrm{H}_{2}$ & 100 & Begin Run $8 ;$ flux up $3 z$ \\
\hline 9 & $\begin{array}{l}3 / 30 \\
4 / 4 \\
4 / 5 \\
4 / 6\end{array}$ & $\begin{array}{l}1015 \\
1424 \\
0400 \\
0800 \\
0915\end{array}$ & $\begin{array}{r}650 \\
650 \\
30 \\
30 \\
150\end{array}$ & $\begin{array}{l}100 \% \text { He } \\
100 \% \text { He } \\
0.1 \% \mathrm{H}_{2} \\
0.1 \% \mathrm{H}_{2} \\
100 \% \mathrm{He}^{2}\end{array}$ & $\begin{array}{l}100 \\
100 \\
100 \\
100 \\
100\end{array}$ & $\begin{array}{l}\text { Begin Run } 9 \\
\text { Temperature of breeder increased } 8 \mathrm{deg} \\
\text { ORR shutdown } \\
\text { Duration } 45 \mathrm{~min} \text { during shutdown } \\
\text { Orr up to } 9 \mathrm{MW} \text { and down }\end{array}$ \\
\hline $\begin{array}{l}10 \\
11\end{array}$ & $\begin{array}{l}4 / 6 \\
4 / 7\end{array}$ & $\begin{array}{l}1230 \\
0922\end{array}$ & $\begin{array}{l}400 \\
500\end{array}$ & $\begin{array}{l}1002 \mathrm{He} \\
1002 \mathrm{He}\end{array}$ & $\begin{array}{l}100 \\
100\end{array}$ & $\begin{array}{l}\text { Regin Run } 10 \\
\text { Begin Run } 11\end{array}$ \\
\hline 12 & $\begin{array}{l}4 / 8 \\
4 / 11\end{array}$ & $\begin{array}{l}0915 \\
0900 \\
1011\end{array}$ & $\begin{array}{r}550 \\
30 \\
550\end{array}$ & $\begin{array}{l}1002 \text { the } \\
100 \% \text { the } \\
1002 \text { the }\end{array}$ & $\begin{array}{l}100 \\
100 \\
100\end{array}$ & $\begin{array}{l}\text { Begin Run } 12 \\
\text { ORR shutdown } \\
\text { Resume Run } 12\end{array}$ \\
\hline 13 & $\begin{array}{l}4 / 12 \\
4 / 13\end{array}$ & $\begin{array}{l}0920 \\
1345 \\
1350 \\
1408\end{array}$ & $\begin{array}{r}600 \\
35 \\
600 \\
30\end{array}$ & $\begin{array}{l}100 \pi \text { the } \\
100 \pi \text { the } \\
100 \pi \text { th } \\
100 \pi \text { tit }\end{array}$ & $\begin{array}{l}100 \\
100 \\
100 \\
100\end{array}$ & $\begin{array}{l}\text { Regin Run } 13 \\
\text { Set back to } \mathrm{nL} \\
\text { Re sues Run } 13 \\
\text { ORk ohutdurn }\end{array}$ \\
\hline
\end{tabular}


TABLE VI-1 (Contd.)

\begin{tabular}{|c|c|c|c|c|c|c|}
\hline \multirow[b]{2}{*}{$\begin{array}{l}\text { Run } \\
\text { No. }\end{array}$} & \multirow[b]{2}{*}{$\begin{array}{l}1983 \\
\text { Date }\end{array}$} & \multirow[b]{2}{*}{ T1me } & \multirow{2}{*}{$\begin{array}{l}\text { Nominal } \\
\text { Teap. } \\
\left({ }^{\circ} \mathrm{C}\right)\end{array}$} & \multicolumn{2}{|c|}{ Sweep Cas } & \multirow[b]{2}{*}{ Coment $s^{b}$} \\
\hline & & & & Composition & $\begin{array}{l}\text { Plow Rate } \\
\left(\mathrm{cm}^{3} / \mathrm{aln}\right)\end{array}$ & \\
\hline & $\begin{array}{l}4 / 14 \\
4 / 15\end{array}$ & $\begin{array}{l}1420 \\
1440 \\
1614 \\
0840\end{array}$ & $\begin{array}{r}35 \\
30 \\
600 \\
600\end{array}$ & $\begin{array}{l}100 \% \text { He } \\
100 \% \text { He } \\
100 \% \text { He } \\
100 \% \text { He }\end{array}$ & $\begin{array}{l}100 \\
100 \\
100 \\
100\end{array}$ & $\begin{array}{l}\text { ORR e nL } \\
\text { ORR shutdown } \\
\text { ReBume Run } 13 \text {, ORR \& } 29 \mathrm{MH} \\
\text { ORR to } 30 \mathrm{MH} \text {, readjuat tenp. }\end{array}$ \\
\hline 14 & $4 / 18$ & 0951 & 700 & 1007 He & 100 & Begin Run 14 \\
\hline 15 & $4 / 20$ & 1400 & 700 & $0.17 \mathrm{H}_{2}$ & 100 & Begin Run 15 \\
\hline 16 & $4 / 21$ & 0946 & 700 & $0.17 \mathrm{H}_{2}$ & 300 & Beg1n Run 16 \\
\hline 17 & $4 / 22$ & 1230 & 700 & $0.17 \mathrm{H}_{2}$ & 30 & Begin Run 17 \\
\hline 18 & $4 / 25$ & 1300 & 700 & $0.17 \mathrm{H}_{2}$ & 100 & Beg1n Run 18 \\
\hline 19 & $\begin{array}{l}4 / 26 \\
4 / 27\end{array}$ & $\begin{array}{l}1000 \\
0400\end{array}$ & $\begin{array}{r}650 \\
30\end{array}$ & $\begin{array}{ll}0.1 \% & \mathrm{H}_{2} \\
0.1 \% & \mathrm{H}_{2}\end{array}$ & $\begin{array}{l}100 \\
100\end{array}$ & $\begin{array}{l}\text { Beg1n Run } 19 \\
\text { ORR s hutdown }\end{array}$ \\
\hline 20 & $\begin{array}{l}4 / 28 \\
4 / 29 \\
5 / 2\end{array}$ & $\begin{array}{l}1130 \\
0810 \\
1110 \\
0915\end{array}$ & $\begin{array}{l}400 \\
400 \\
650 \\
650\end{array}$ & $\begin{array}{l}100 \% \text { He } \\
\text { He } / 0.27 \mathrm{O}_{2} \\
\mathrm{He} / 0.2 \% \mathrm{O}_{2} \\
100 \% \text { He }\end{array}$ & $\begin{array}{l}100 \\
100 \\
100 \\
100-200\end{array}$ & Begin Run 20 \\
\hline 21 & $\begin{array}{l}5 / 2 \\
5 / 3\end{array}$ & $\begin{array}{l}1425 \\
0837\end{array}$ & $\begin{array}{l}650 \\
650\end{array}$ & $\begin{array}{l}\mathrm{He} / 0.1 \% \mathrm{H}_{2} \\
\mathrm{He} / 0.1 \% \mathrm{H}_{2}\end{array}$ & $\begin{array}{l}100 \\
100\end{array}$ & $\begin{array}{l}\text { Begin Run } 21 \\
\text { Temperature adjusted - Increased } 9 \mathrm{deg}\end{array}$ \\
\hline 22 & $5 / 4$ & 0900 & 600 & $\mathrm{He} / 0.1 \% \mathrm{H}_{2}$ & 100 & Begin Run 22 \\
\hline 23 & $\begin{array}{l}5 / 5 \\
5 / 6 \\
5 / 6 \\
5 / 7\end{array}$ & $\begin{array}{l}0900 \\
0001 \\
1400 \\
1710 \\
1720 \\
1730 \\
1743 \\
2051 \\
2106 \\
0305 \\
0351\end{array}$ & $\begin{array}{r}550 \\
30 \\
550 \\
300 \\
550 \\
300 \\
550 \\
550 \\
550 \\
30 \\
550\end{array}$ & 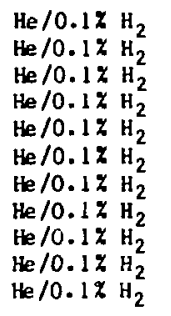 & $\begin{array}{l}100 \\
100 \\
100 \\
100 \\
100 \\
100 \\
100 \\
100 \\
100 \\
100 \\
100\end{array}$ & $\begin{array}{l}\text { Begin Run } 23 \\
\text { ORR shutdown } \\
\text { Re sume Run } 23 \text { e } 28.5 \mathrm{MW} \\
\text { ORR set back to } 15 \mathrm{MW} \\
\text { Resume Run } 23 \text { e } 28.5 \mathrm{MW} \\
\text { ORR set back to } 15 \mathrm{MW} \\
\text { Resume Run } 23 \text { \& } 28.5 \mathrm{MW} \\
\text { ORR \& } 29 \mathrm{MW} \\
\text { ORR E } 29.5 \mathrm{MW} \\
\text { ORR shutdown } \\
\text { Resume Run } 23 \text { \& } 29.5 \mathrm{MW}\end{array}$ \\
\hline 24 & $5 / 9$ & 1100 & 600 & $\mathrm{He} / 0.1 \% \mathrm{H}_{2}$ & 100 & Begin Run 24 巳 $29.5 \mathrm{MW}$ \\
\hline 25 & $\begin{array}{l}5 / 10 \\
5 / 11\end{array}$ & $\begin{array}{l}1351 \\
10: 45 \\
1129\end{array}$ & $\begin{array}{r}560 \\
35 \\
560\end{array}$ & $\begin{array}{l}\mathrm{He} / 0.1 \text { ₹ } \mathrm{H}_{2} \\
\mathrm{He} / 0.1 \% \mathrm{H}_{2} \\
\mathrm{He} / 0.1 \% \mathrm{H}_{2}\end{array}$ & $\begin{array}{l}100 \\
100 \\
100\end{array}$ & $\begin{array}{l}\text { Begin Run } 25 \text { @ } 29.5 \mathrm{MH} \\
\text { ORR set back to nL }(0.30 \mathrm{MW}) \\
\text { ORR \& } 30 \mathrm{MW} \text {; resura Run } 25\end{array}$ \\
\hline 26 & $\begin{array}{l}5 / 12 \\
5 / 16\end{array}$ & $\begin{array}{l}0815 \\
0400\end{array}$ & $\begin{array}{r}550 \\
30\end{array}$ & $\begin{array}{l}\mathrm{He} / 0.1 \% \mathrm{H}_{2} \\
\mathrm{He} / 0.17 \mathrm{H}_{2}\end{array}$ & $\begin{array}{l}100 \\
100\end{array}$ & $\begin{array}{l}\text { Begin Run } 26 \text { \& } 30 \mathrm{MW} \\
\text { ORR shutdown }\end{array}$ \\
\hline 27 & $\begin{array}{l}5 / 16 \\
5 / 17\end{array}$ & $\begin{array}{l}1600 \\
1400\end{array}$ & $\begin{array}{l}525 \\
525\end{array}$ & $\begin{array}{l}\mathrm{He} / 0.1 \% \mathrm{H}_{2} \\
\mathrm{He} / 0.1 \% \mathrm{H}_{2}\end{array}$ & $\begin{array}{l}100 \\
100\end{array}$ & $\begin{array}{l}\text { Begin Run } 27 @ 28 \mathrm{MW} \\
\text { ORR } 28.5 \mathrm{MW}\end{array}$ \\
\hline 28 & $\begin{array}{l}5 / 23 \\
5 / 31 \\
6 / 1\end{array}$ & $\begin{array}{l}1050 \\
0400 \\
0125\end{array}$ & $\begin{array}{r}500 \\
30 \\
500\end{array}$ & $\begin{array}{l}\mathrm{He} / 0.1 \% \mathrm{H}_{2} \\
\mathrm{He} / 0.1 \% \mathrm{H}_{2} \\
\mathrm{He} / 0.1 \% \mathrm{H}_{2}\end{array}$ & $\begin{array}{l}100 \\
100 \\
100\end{array}$ & $\begin{array}{l}\text { Begin Run } 28 \text { e } 28.5 \mathrm{mw} \\
\text { ORR shutdown } \\
\text { ORR up } 2 \mathrm{~h} \text {, then down }\end{array}$ \\
\hline 29 & $6 / 1$ & 1357 & 480 & $\mathrm{He} / 0.17 \quad \mathrm{H}_{2}$ & 300 & Begin Run 29 C $29 \mathrm{MW}$ \\
\hline 30 & $6 / 3$ & 1500 & 480 & $\mathrm{He} / 0.1 \% \mathrm{H}_{2}$ & 100 & Begin Run $30 @ 29 \mathrm{MW}$ \\
\hline 31 & $6 / 6$ & 0900 & 500 & $\mathrm{He} / 0.12 \mathrm{H}_{2}$ & 100 & Begin Run 31$] 30 \mathrm{mw}$ \\
\hline 32 & $6 / 10$ & 0830 & 650 & $\mathrm{He} / 0.1 \% \mathrm{H}_{2}$ & 100 & Beg1n Run 32 \\
\hline 33 & $\begin{array}{l}6 / 10 \\
6 / 13\end{array}$ & $\begin{array}{l}0830 \\
0400\end{array}$ & $\begin{array}{r}650 \\
30\end{array}$ & $\begin{array}{l}\text { He } / 0.17 \mathrm{H}_{2} \\
\mathrm{He} / 0.17 \mathrm{H}_{2}\end{array}$ & $\begin{array}{l}100 \\
100\end{array}$ & $\begin{array}{l}\text { Begin Run } 33 \\
\text { ORR shut down; end of experiment }\end{array}$ \\
\hline
\end{tabular}

ulocal tine at Oak Ridge, EST or EDT.

blote: nL 1t the lowest power at which the autonstic reactor control system will function - it correapond to resctor power of $\sim 0.30 \mathrm{mw}$. R refers to reactor power. 


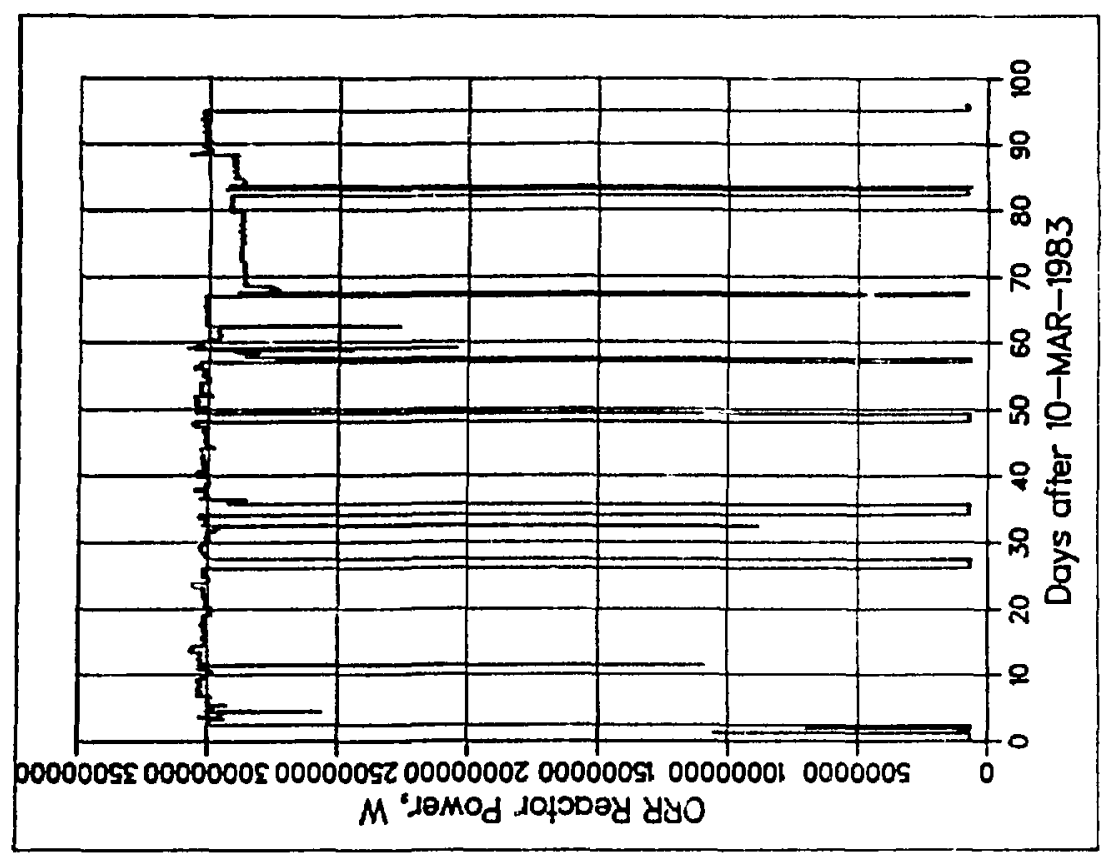

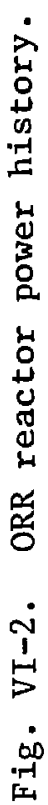

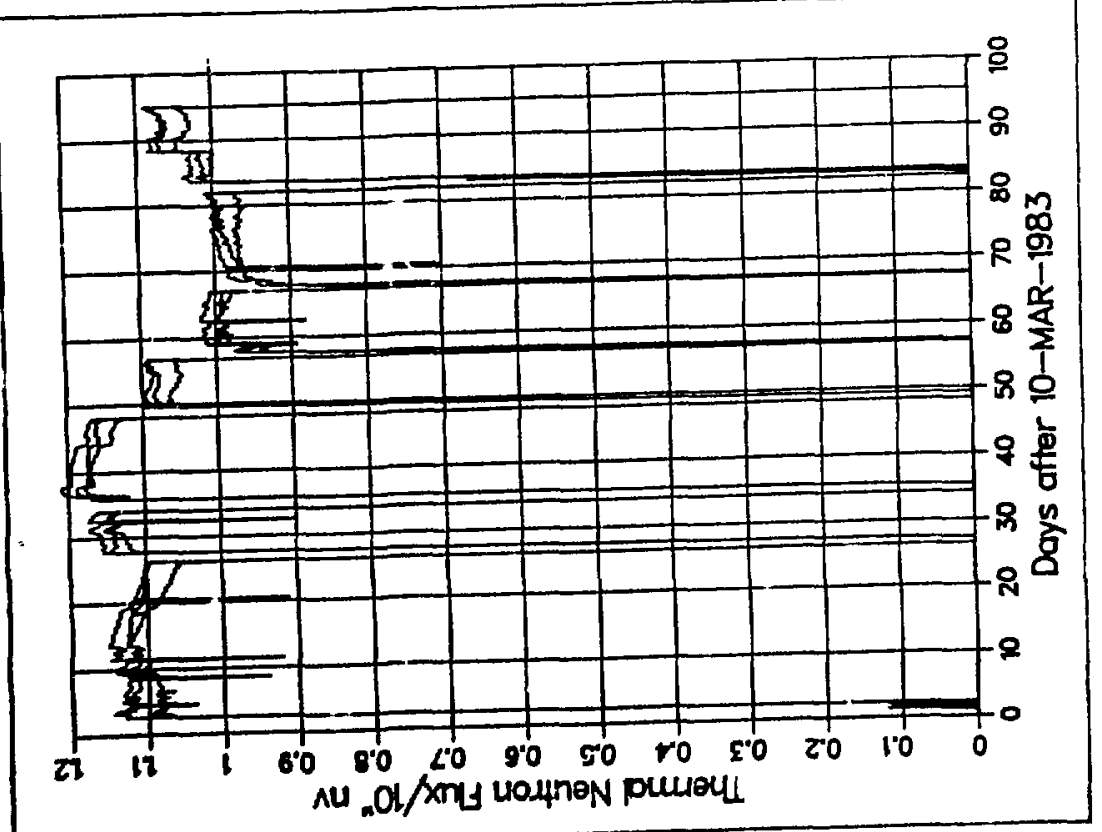

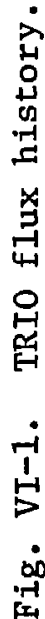




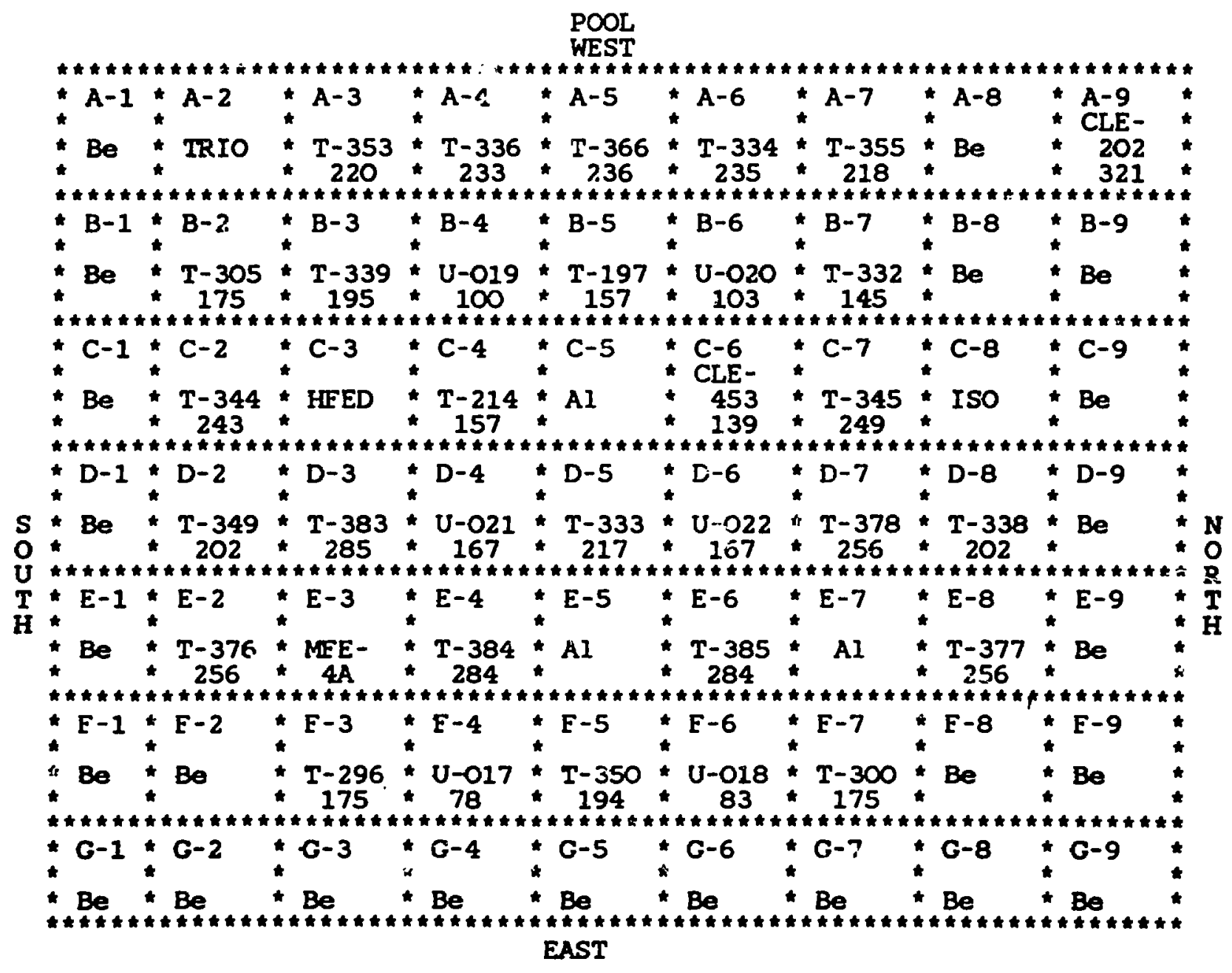

Note: - T-xxx refers to a fuel element

- U-xxx is a control rod.

- other alphanumeric designations refer to experiments.

Fig. VI-3a. ORR fuel loading diagram: Cycle 164-A. (March 10, 1983 to April 6, 1983). 


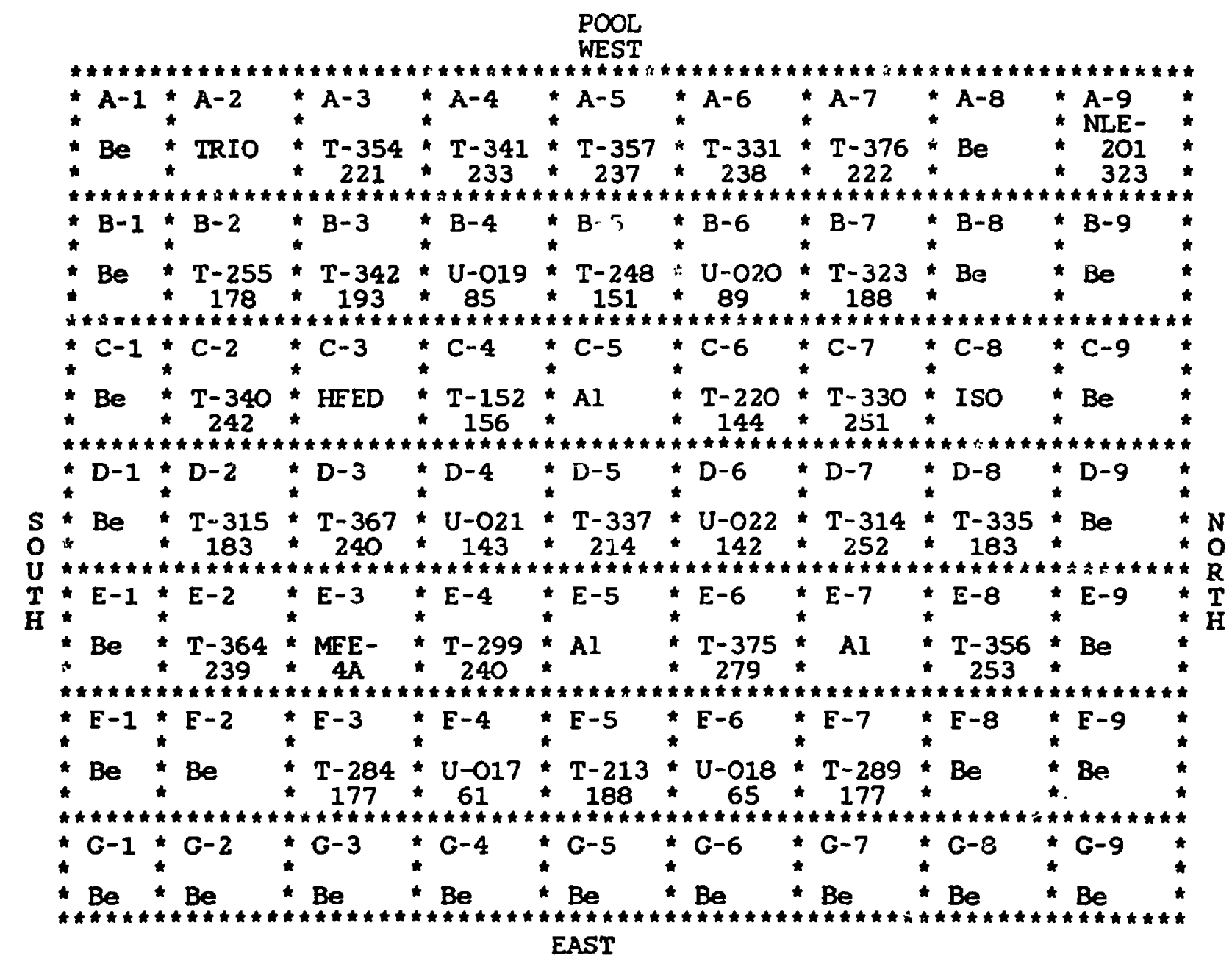

Fig. VI-3b. ORR fuel loading diagram: Cycle 164-B.

(April 6, 1983 to April 13, 1983). 


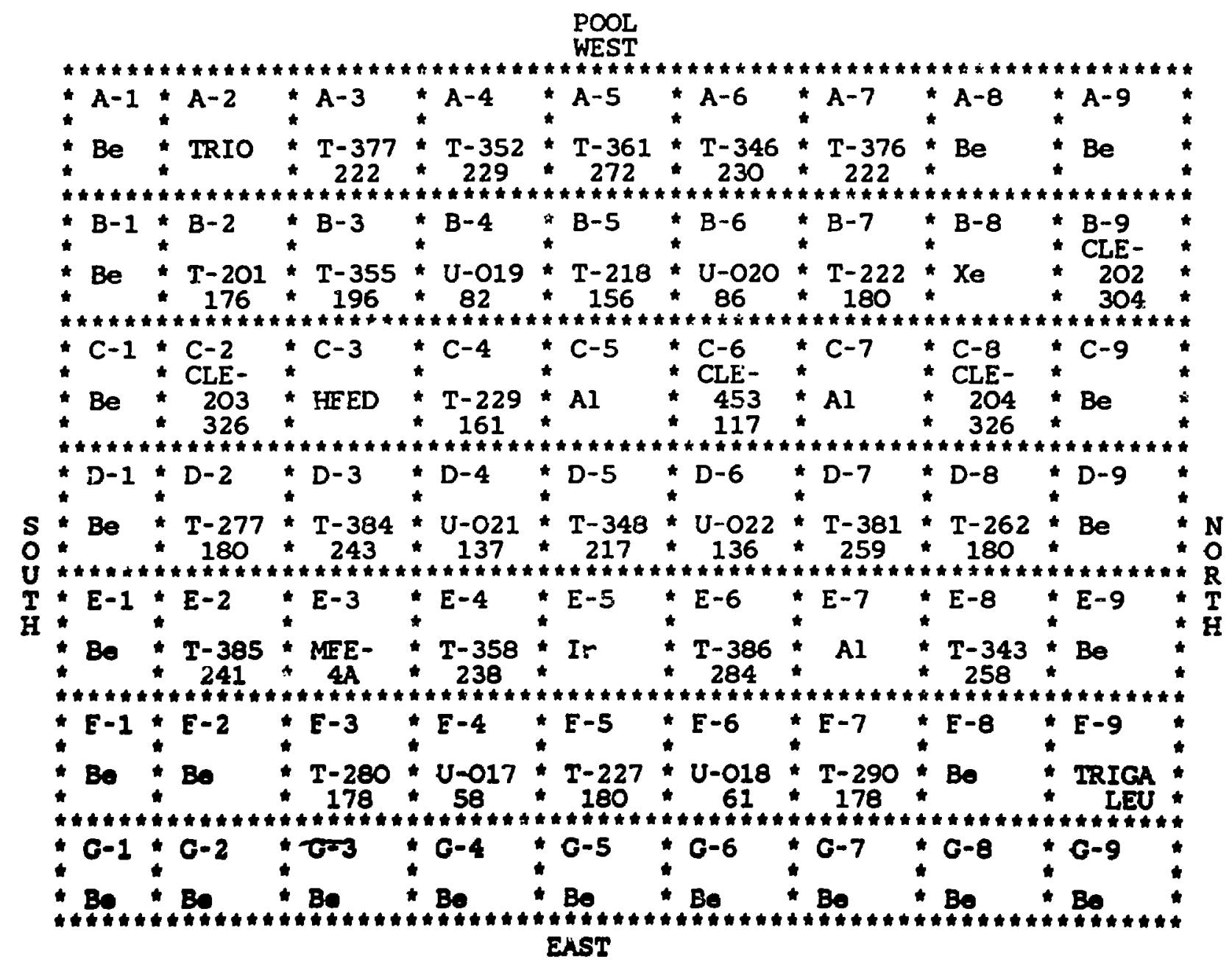

Fig. VI-3c. ORR fuel loading diagram: Cycle 164-C (April 14, 1983 to Aprii 27, 1983). 


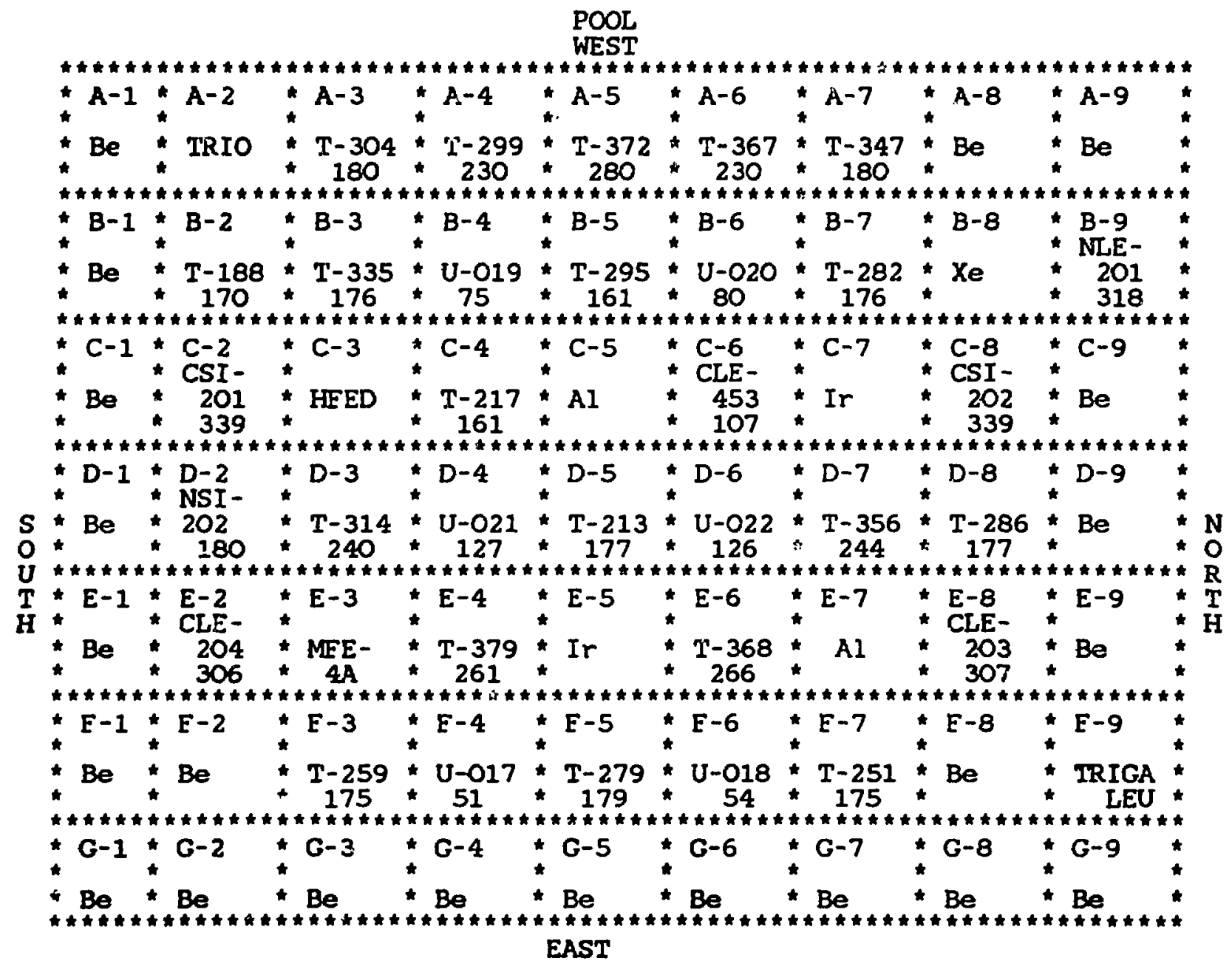

Fig. VI-3d. ORR fuel loading diagram: Cycle 164-D.

(Apri1 28, 1983 to May 6, 1983). 


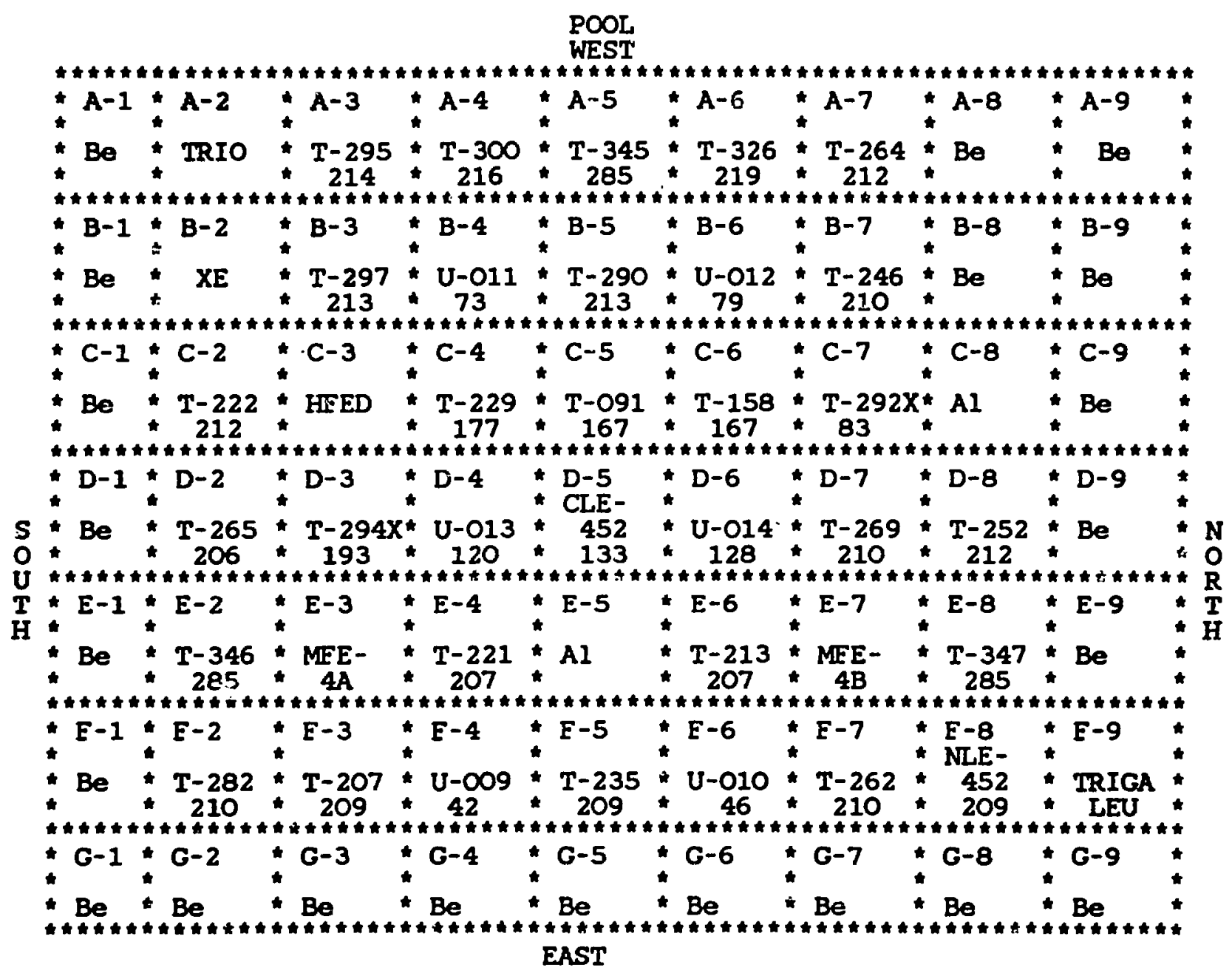

Fig. VI-3e. ORR fuel loading diagram: Cycle 164-E.

(May 6, 1983 to May 16, 1983). 


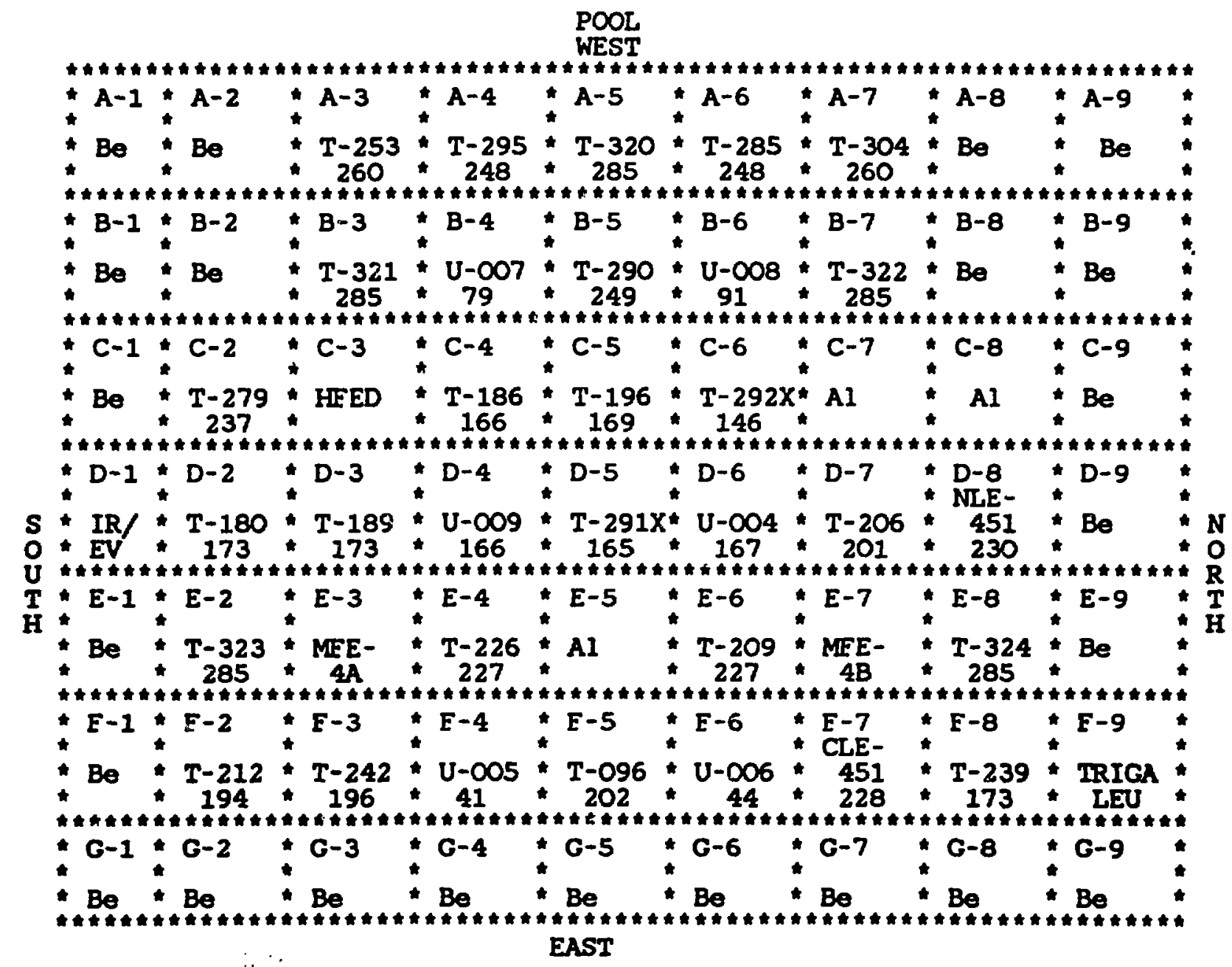

Fig. VI-3f, ORR fuel loading diagram: Cycle 164-F. (May 16, 1983 to May 31,1983 ). 


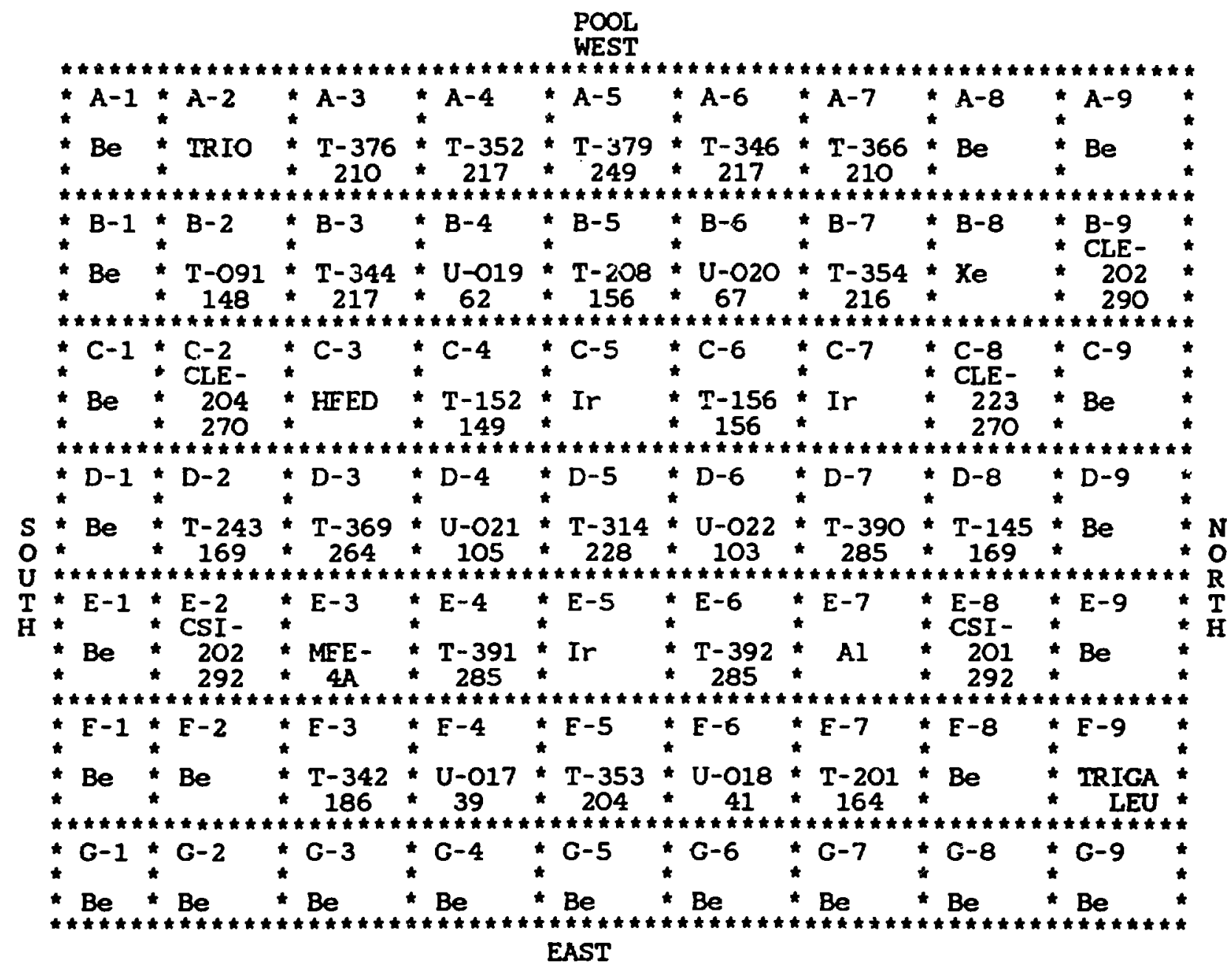

Fig. VI-3g. ORR fuel loading diagram: Cycle 164-G.

(May 31, 1983 to June 13, 1983). 


\section{Tenperature Profiles}

The recorded temperatures for the entire experiment are given in Fig. VI-4. The location of the thermocouples is shown in a cross-sectional diagram of the capsule (Fig. VI-5). Besides defining the temperature profiles for the tritium release, a number of observations were made regarding temperature. First, the readings from the thermocouples have a distinct pattern; the highest temperatures are. always on the west side and the inside surface. These are from thermocouples T903, T904, T905, and T906. Of these four, T906, the one on the capsule bottom, consistently shows the lowest temperature by about $10^{\circ} \mathrm{C}$. The next highest temperature is from $\mathrm{T} 902$, located on the east side and the inside surface of the breeder. The four thermocouples on the west outside (T907, T908, TR801, and TR901) read within $20^{\circ} \mathrm{C}$ of one another. The lowest temperature is always from $\mathrm{T} 902$, located on the east outside surface of the breeder. The fact that this pattern remained the same throughout the course of the experiment provides evidence that the heat transfer pathways did not change as a result of effects of temperature and radiation. Therefore, the integrity of the capsule was maintained throughout the experiment. The lower temperature on the east side of the capsule is attributed to asymmetry in the gap, i.e., the gap is smaller on the east side. The temperature profiles for each run are presented later.

\section{Sweep Gas Analyses}

The total level of contaminants in the supply cylinders was less than 30 vppm. Significant impurities were neon $(<20 \mathrm{vppm})$ and $\mathrm{N}_{2}+\mathrm{Co}(<6 \mathrm{ppm})$. Oxygen was less than $2 \mathrm{vppm}$, and the gas purifier ensured that levels $<1 \mathrm{ppm}$ were maintained. Samples of the effluent sweep gas were taken during the time when no hydrogen was added to the system. The results (shown in Table VI-2) show that no measurable amounts of impurities were added to the sweep gas stream by the capsule. The concentrations of the tritium species noted in the effluent were not high enough to provide truly quantitative data.

\section{E. Tritiun Collected}

The tritium released to the sweep gas was collected and quantitatively analyzed by liquid scintillation counting. The tritium collected is in three 


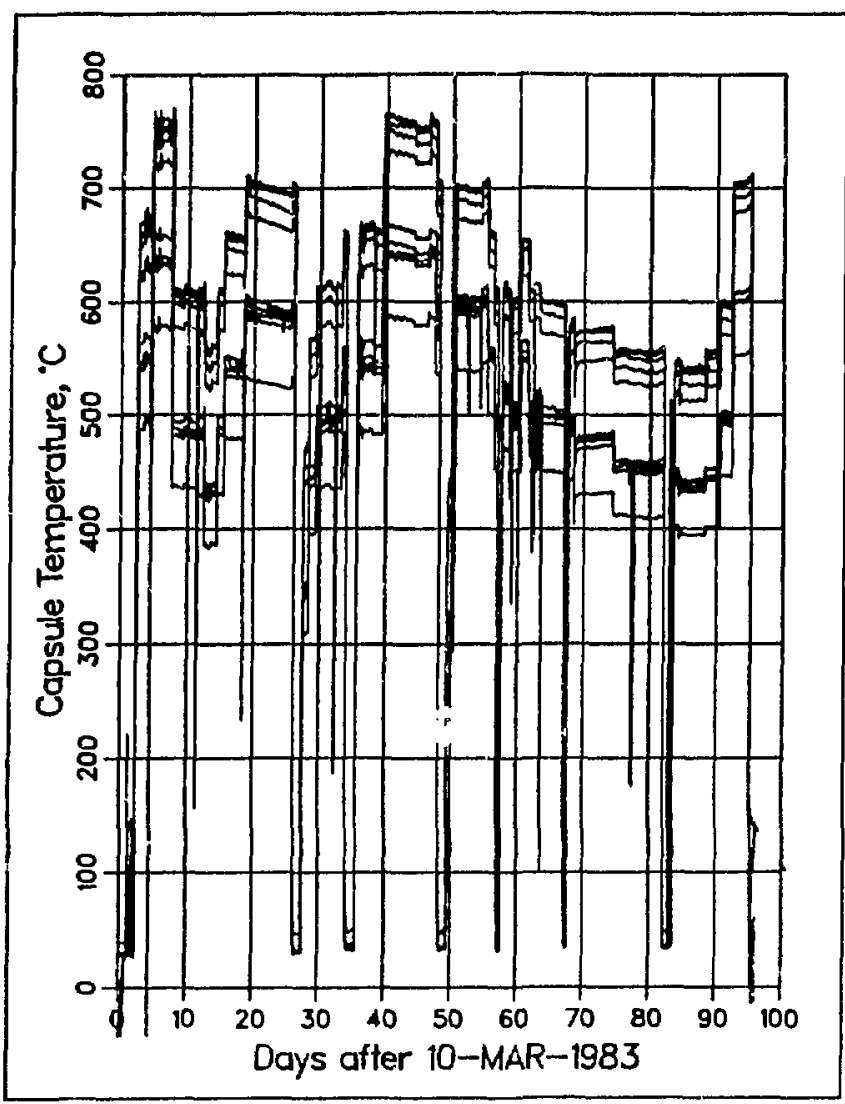

Fig. VI-4. TRIO capsule temperature.

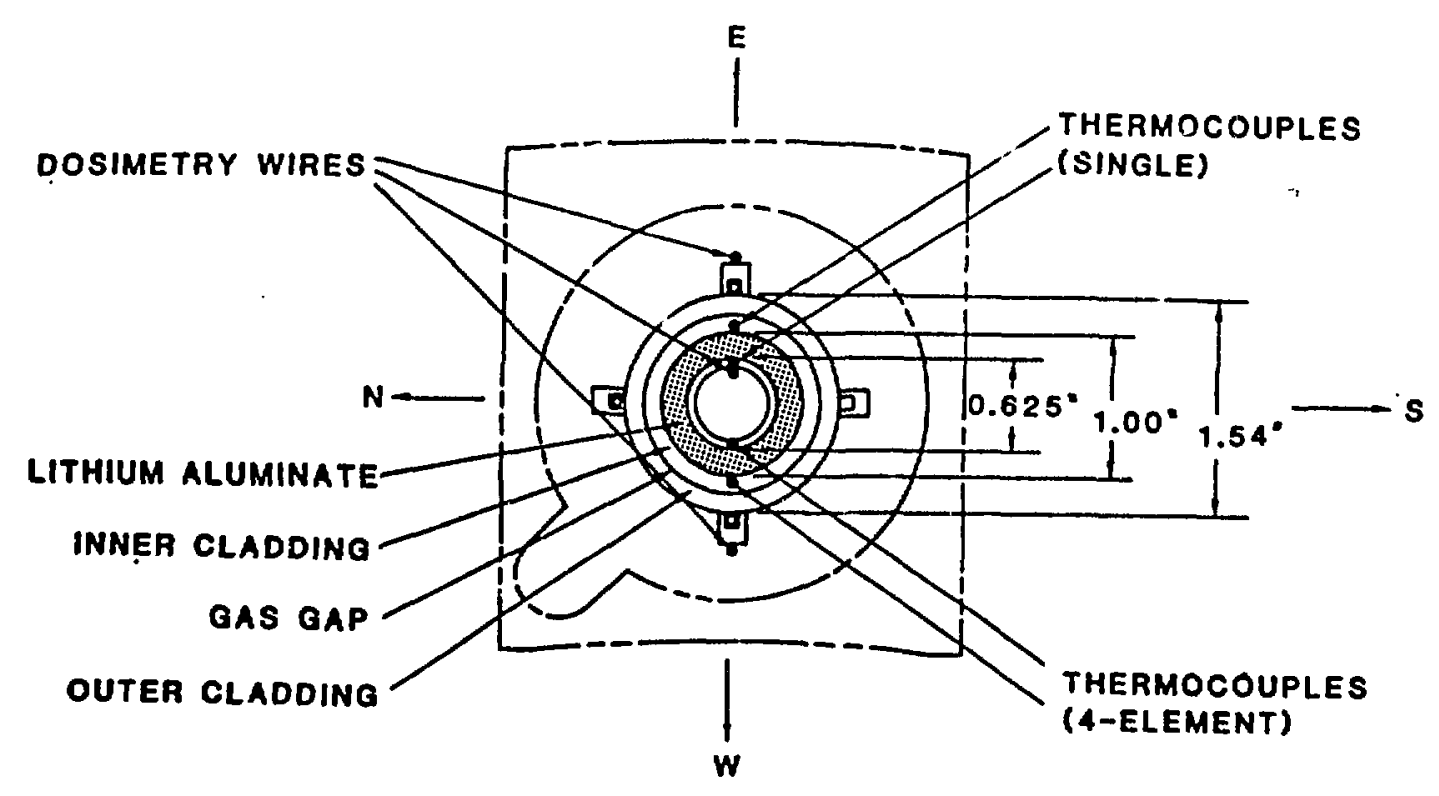

Fig. VI-5. TRIO capsule assembly. 
TABLE VI-2

Sweep Gas Analysis

\begin{tabular}{l|c|c|c}
\hline \multirow{2}{*}{ Species } & \multicolumn{3}{|c}{ Concentration (\%) } \\
\cline { 2 - 4 } & Supply & Exhaust (1) & Exhaust (2) \\
\hline $\mathrm{H}_{2}$ & 0.0003 & 0.0003 & 0.0003 \\
$\mathrm{He}$ & 99.997 & 99.998 & 99.997 \\
$\mathrm{CH}_{4}$ & $<0.0002$ & $<0.0002$ & $<0.0002$ \\
$\mathrm{H}_{2} \mathrm{O}$ & $<0.0002$ & $<0.0002$ & $<0.0002$ \\
$\mathrm{~N}_{2}+\mathrm{CO}$ & 0.0005 & $<0.0005$ & $<0.0005$ \\
$\mathrm{O}_{2}$ & $<0.0002$ & $<0.0001$ & 0.0001 \\
$\mathrm{Ar}$ & 0.0001 & $<0.0001$ & $<0.0001$ \\
$\mathrm{CO}_{2}$ & $<0.0001$ & $<0.0001$ & $<0.0001$ \\
$\mathrm{Ne}$ & 0.0020 & 0.0018 & 0.0020 \\
$\mathrm{~T}_{2}$ & - & $<0.0001$ & $<0.0001$ \\
$\mathrm{HT}$ & - & - & $<0.0002$ \\
\hline
\end{tabular}

forms: (1) tritium as HT or noncondensable form, (2) tritium as HTO or condensable form, and (3) tritium that permeated from the sweep gas through the inner capsule to the gap gas stream. Data for tritium collected in these three forms for the 33 experimental runs are presented below.

\section{HT Collected}

Tritium in the sweep gas which passed the first set of traps was monitored by the line monitor ( $\mathrm{KCl}$ ), oxidized, and then trapped and analyzed as described in Sec. IV-C. The samples represent noncondensable tritium collected a specified time interval. The data for HT thus collected are given in Table VI-3.

The predominant form of tritium collected was HT, representing more than 95\% of all the tritium. The data in Table VI-3 show the amount of HT collected during the amount of 33 experimental runs. In those instances when a sample overlapped two runs, the amount collected was apportioned between the two runs. 
TABLE VI-3

Tritium Coilected, Noncondensable or HT Form (November 25, 1983)

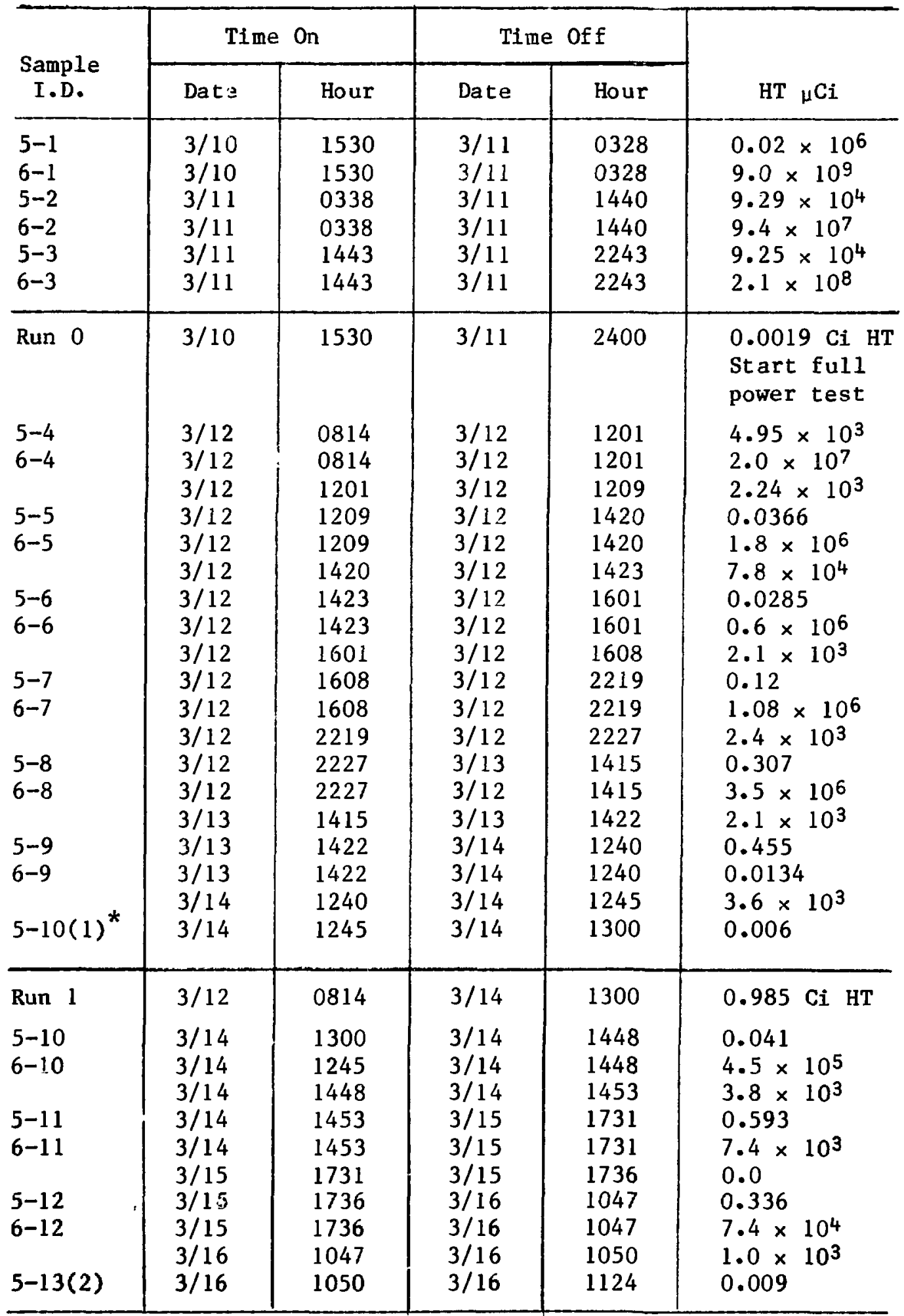


TABLE VI-3 (Contd.)

\begin{tabular}{|c|c|c|c|c|c|}
\hline \multirow{2}{*}{$\begin{array}{c}\text { Sample } \\
\text { I.D. }\end{array}$} & \multicolumn{2}{|c|}{ Time On } & \multicolumn{2}{|c|}{ Time of $f$} & \multirow[b]{2}{*}{$\mathrm{HT} \mu \mathrm{Ci}$} \\
\hline & Date & Hour & Date & Hour & \\
\hline Run 2 & $3 / 14$ & 1300 & $3 / 16$ & 1124 & $0.985 \mathrm{Ci} \mathrm{HT}$ \\
\hline $\begin{array}{l}5-13 \\
6-13 \\
5-14 \\
6-14 \\
5-14 \\
5-15 \\
6-15 \\
5-16(3)\end{array}$ & $\begin{array}{l}3 / 16 \\
3 / 16 \\
3 / 16 \\
3 / 16 \\
3 / 16 \\
3 / 17 \\
3 / 16 \\
3 / 17 \\
3 / 17 \\
3 / 17 \\
3 / 17\end{array}$ & $\begin{array}{l}1124 \\
1050 \\
1437 \\
1441 \\
1441 \\
1125 \\
1441 \\
1129 \\
1129 \\
1344 \\
1347\end{array}$ & $\begin{array}{l}3 / 16 \\
3 / 16 \\
3 / 16 \\
3 / 17 \\
3 / 17 \\
3 / 17 \\
3 / 17 \\
3 / 17 \\
3 / 20 \\
3 / 17 \\
3 / 17\end{array}$ & $\begin{array}{l}1437 \\
1437 \\
1441 \\
1125 \\
1125 \\
1129 \\
1125 \\
1344 \\
1747 \\
1347 \\
1405\end{array}$ & $\begin{array}{l}0.0504 \\
1.74 \times 10^{3} \\
1.2 \times 10^{3} \\
0.07245 \\
8.5 \times 10^{3} \\
1.2 \times 10^{3} \\
0.336^{a} \\
0.0444 \\
6.0 \times 10^{4} \\
9.0 \times 10^{3} \\
0.005\end{array}$ \\
\hline Run 3 & $3 / 16$ & 1124 & $3 / 17$ & 1405 & $0.513 \mathrm{Ci} \mathrm{HT}$ \\
\hline $\begin{array}{l}5-16 \\
5-17 \\
5-18 \\
5-19 \\
5-16 \\
5-20\end{array}$ & $\begin{array}{l}3 / 18 \\
3 / 18 \\
3 / 18 \\
3 / 19 \\
3 / 19 \\
3 / 20 \\
3 / 20 \\
3 / 20 \\
3 / 21 \\
3 / 21\end{array}$ & $\begin{array}{l}1405 \\
1600 \\
1603 \\
2132 \\
2135 \\
1747 \\
1753 \\
1753 \\
0815 \\
0820\end{array}$ & $\begin{array}{l}3 / 18 \\
3 / 18 \\
3 / 19 \\
3 / 19 \\
3 / 20 \\
3 / 20 \\
3 / 21 \\
3 / 21 \\
3 / 21 \\
3 / 22\end{array}$ & $\begin{array}{l}1600 \\
1603 \\
2132 \\
2135 \\
1747 \\
1753 \\
0815 \\
0815 \\
0820 \\
1127\end{array}$ & $\begin{array}{l}0.0428 \\
0.0009 \\
0.541 \\
0.0009 \\
0.36 \\
0.0018 \\
0.291 \\
0.00381 \\
0.0015 \\
0.47\end{array}$ \\
\hline $\begin{array}{l}\text { Run } 4 \\
5-20(5) \\
5-21 \\
5-22\end{array}$ & $\begin{array}{l}3 / 18 \\
3 / 22 \\
3 / 22 \\
3 / 22 \\
3 / 23 \\
3 / 23\end{array}$ & $\begin{array}{l}1405 \\
1127 \\
1606 \\
1611 \\
0834 \\
0841\end{array}$ & $\begin{array}{l}3 / 22 \\
3 / 22 \\
3 / 22 \\
3 / 23 \\
3 / 23 \\
3 / 24\end{array}$ & $\begin{array}{l}1127 \\
1606 \\
1611 \\
0834 \\
0841 \\
1053\end{array}$ & $\begin{array}{l}2.099 \mathrm{Ci} \mathrm{HT} \\
0.081 \\
0.001 \\
0.179 \\
0.0014 \\
0.355\end{array}$ \\
\hline Run 5 & $3 / 22$ & 1127 & $3 / 24$ & 1052 & $0.617 \mathrm{Ci} \mathrm{HT}$ \\
\hline $\begin{array}{l}5-23 \\
6-17 \\
5-24 \\
5-25(6)\end{array}$ & $\begin{array}{l}3 / 24 \\
3 / 24 \\
3 / 21 \\
3 / 24 \\
3 / 24 \\
3 / 25 \\
3 / 25\end{array}$ & $\begin{array}{l}1053 \\
1058 \\
0820 \\
1538 \\
1543 \\
1018 \\
1022\end{array}$ & $\begin{array}{l}3 / 24 \\
3 / 24 \\
3 / 28 \\
3 / 24 \\
3 / 25 \\
3 / 25 \\
3 / 25\end{array}$ & $\begin{array}{l}1058 \\
1538 \\
0855 \\
1543 \\
1018 \\
1022 \\
1050\end{array}$ & $\begin{array}{l}0.001 \\
0.18 \\
0.0098 \\
0.0015 \\
0.505 \\
0.0024 \\
0.025\end{array}$ \\
\hline
\end{tabular}


TABLE VI-3 (Contd.)

\begin{tabular}{|c|c|c|c|c|c|}
\hline \multirow{2}{*}{$\begin{array}{c}\text { Sample } \\
\text { I.D. }\end{array}$} & \multicolumn{2}{|c|}{ Time On } & \multicolumn{2}{|c|}{ Time of $\mathrm{f}$} & \multirow[b]{2}{*}{$\mathrm{HT} \mu \mathrm{Ci}$} \\
\hline & Date & Hour & Date & Hour & \\
\hline Run 6 & $3 / 24$ & 1053 & $3 / 25$ & 1050 & $0.725 \mathrm{Cl} \mathrm{HT}$ \\
\hline $\begin{array}{l}5-25 \\
5-26 \\
5-27 \\
5-28(7)\end{array}$ & $\begin{array}{l}3 / 25 \\
3 / 25 \\
3 / 25 \\
3 / 27 \\
3 / 27 \\
3 / 28 \\
3 / 28\end{array}$ & $\begin{array}{l}1050 \\
1423 \\
1428 \\
1751 \\
1756 \\
0855 \\
0858\end{array}$ & $\begin{array}{l}3 / 25 \\
3 / 25 \\
3 / 27 \\
3 / 27 \\
3 / 28 \\
3 / 28 \\
3 / 28\end{array}$ & $\begin{array}{l}1423 \\
1428 \\
1751 \\
1756 \\
0855 \\
0858 \\
1300\end{array}$ & $\begin{array}{l}0.194 \\
0.004 \\
1.21 \\
0.002 \\
0.321 \\
0.0012 \\
0.079\end{array}$ \\
\hline Run 7 & $3 / 25$ & 1050 & $3 / 28$ & 1300 & $1.811 \mathrm{Ci} \mathrm{HT}$ \\
\hline $\begin{array}{l}5-28 \\
5-29 \\
5-30(8)\end{array}$ & $\begin{array}{l}3 / 28 \\
3 / 29 \\
3 / 29 \\
3 / 30 \\
3 / 30\end{array}$ & $\begin{array}{l}1300 \\
0906 \\
0907 \\
0920 \\
0925\end{array}$ & $\begin{array}{l}3 / 29 \\
3 / 29 \\
3 / 30 \\
3 / 30 \\
3 / 30\end{array}$ & $\begin{array}{l}0906 \\
0907 \\
0920 \\
0925 \\
1015\end{array}$ & $\begin{array}{l}0.539 \\
0.0004 \\
0.452 \\
0.0015 \\
0.015\end{array}$ \\
\hline Run 8 & $3 / 28$ & 1300 & $3 / 30$ & 1015 & 1.008 \\
\hline $\begin{array}{l}5-30 \\
5-31 \\
6-18\end{array}$ & $\begin{array}{l}3 / 30 \\
4 / 04 \\
4 / 04 \\
3 / 28\end{array}$ & $\begin{array}{l}1015 \\
1355 \\
1402 \\
0858\end{array}$ & $\begin{array}{l}4 / 04 \\
4 / 04 \\
4 / 05 \\
4 / 04\end{array}$ & $\begin{array}{l}1355 \\
1402 \\
0754 \\
1355\end{array}$ & $\begin{array}{l}1.525 \\
0.0014 \\
0.285 \\
0.012\end{array}$ \\
\hline Run 9 & $3 / 30$ & 1015 & $4 / 0$ & 0400 & $1.823 \mathrm{Cl} \mathrm{HT}$ \\
\hline $\begin{array}{l}5-32 \\
5-33(10)\end{array}$ & $\begin{array}{l}4 / 06 \\
4 / 07 \\
4 / 07\end{array}$ & $\begin{array}{l}0855 \\
0811 \\
0825\end{array}$ & $\begin{array}{l}4 / 07 \\
4 / 07 \\
4 / 07\end{array}$ & $\begin{array}{l}0811 \\
0825 \\
0922\end{array}$ & $\begin{array}{l}0.033 \\
0.0014 \\
0.006\end{array}$ \\
\hline $\begin{array}{l}\text { Run } 10 \\
5-33\end{array}$ & $\begin{array}{l}4 / 06 \\
4 / 07\end{array}$ & $\begin{array}{l}0855 \\
0922\end{array}$ & $\begin{array}{l}4 / 07 \\
4 / 08\end{array}$ & $\begin{array}{l}0922 \\
0915\end{array}$ & $\begin{array}{l}0.0404 \mathrm{Ci} \text { HT } \\
0.3\end{array}$ \\
\hline Run 11 & $4 / 07$ & 0922 & $4 / 08$ & 0915 & $0.3 \mathrm{Cl} \mathrm{HT}$ \\
\hline $\begin{array}{l}5-33(12) \\
5-34 \\
6-19 \\
6-20 \\
5-35(12)\end{array}$ & $\begin{array}{l}4 / 08 \\
4 / 11 \\
4 / 11 \\
4 / 04 \\
4 / 11 \\
4 / 12 \\
4 / 12\end{array}$ & $\begin{array}{l}0915 \\
1430 \\
1432 \\
1402 \\
1432 \\
0847 \\
0903\end{array}$ & $\begin{array}{l}4 / 11 \\
4 / 11 \\
4 / 12 \\
4 / 11 \\
4 / 13 \\
4 / 12 \\
4 / 12\end{array}$ & $\begin{array}{l}1430 \\
1432 \\
0847 \\
1430 \\
0913 \\
0903 \\
0920\end{array}$ & $\begin{array}{l}0.828 \text { Ci HT } \\
0.0004 \\
0.246 \\
0.0805 \\
0.0807 \\
0.0048 \\
0.005\end{array}$ \\
\hline
\end{tabular}


TABLE VI-3 (Contd.)

\begin{tabular}{|c|c|c|c|c|c|}
\hline \multirow{2}{*}{$\begin{array}{c}\text { Sample } \\
\text { I.D. }\end{array}$} & \multicolumn{2}{|c|}{ Time On } & \multicolumn{2}{|c|}{ Time of $\mathrm{f}$} & \multirow[b]{2}{*}{$\mathrm{HT}{ }_{\mu} \mathrm{Ci}$} \\
\hline & Date & Hour & Date & Hour & \\
\hline Run 12 & $4 / 08$ & 0915 & $4 / 12$ & 0920 & $1.245 \mathrm{Ci} \mathrm{HT}$ \\
\hline $\begin{array}{l}5-35 \\
5-36 \\
5-37 \\
5-38(13)\end{array}$ & $\begin{array}{l}4 / 12 \\
4 / 13 \\
4 / 13 \\
4 / 15 \\
4 / 15 \\
4 / 18\end{array}$ & $\begin{array}{l}0920 \\
1109 \\
1113 \\
0908 \\
0910 \\
0913\end{array}$ & $\begin{array}{l}4 / 13 \\
4 / 13 \\
4 / 15 \\
4 / 15 \\
4 / 18 \\
4 / 18\end{array}$ & $\begin{array}{l}1109 \\
1113 \\
0908 \\
0910 \\
0913 \\
0951\end{array}$ & $\begin{array}{l}0.77 \\
0.0012 \\
0.0858 \\
0.0 \\
1.324 \\
0.016\end{array}$ \\
\hline Run 13 & $4 / 12$ & 0920 & $4 / 18$ & 0951 & $2.197 \mathrm{CI} \mathrm{HT}$ \\
\hline $\begin{array}{l}6-21 \\
5-38 \\
5-39 \\
5-40 \\
5-41(13)\end{array}$ & $\begin{array}{l}4 / 18 \\
4 / 18 \\
4 / 19 \\
4 / 19 \\
4 / 19 \\
4 / 19 \\
4 / 20 \\
4 / 20\end{array}$ & $\begin{array}{l}0913 \\
0951 \\
0853 \\
0857 \\
1604 \\
1607 \\
1245 \\
1248\end{array}$ & $\begin{array}{l}4 / 21 \\
4 / 19 \\
4 / 19 \\
4 / 19 \\
4 / 19 \\
4 / 20 \\
4 / 20 \\
4 / 20\end{array}$ & $\begin{array}{l}0917 \\
0853 \\
0857 \\
1604 \\
1607 \\
1245 \\
1248 \\
1400\end{array}$ & $\begin{array}{l}0.00687 \\
0.976 \\
0.002 \\
0.177 \\
0.0009 \\
0.366 \\
0.0012 \\
0.03\end{array}$ \\
\hline Run 14 & $4 / 18$ & 0951 & $4 / 20$ & 1400 & $1.560 \mathrm{Ci} \mathrm{HT}$ \\
\hline $\begin{array}{l}5-41 \\
5-42(14)\end{array}$ & $\begin{array}{l}4 / 20 \\
4 / 21 \\
4 / 21\end{array}$ & $\begin{array}{l}1400 \\
0917 \\
0921\end{array}$ & $\begin{array}{l}4 / 21 \\
4 / 21 \\
4 / 21\end{array}$ & $\begin{array}{l}0917 \\
0921 \\
0946\end{array}$ & $\begin{array}{l}0.705 \\
0.002 \\
0.008\end{array}$ \\
\hline Run 15 & $4 / 20$ & 1400 & $4 / 21$ & 0946 & $0.715 \mathrm{Ci} \mathrm{HT}$ \\
\hline $\begin{array}{l}5-42 \\
6-22 \\
5-43(16)\end{array}$ & $\begin{array}{l}4 / 21 \\
4 / 21 \\
4 / 22 \\
4 / 22\end{array}$ & $\begin{array}{l}0946 \\
0921 \\
0852 \\
0856\end{array}$ & $\begin{array}{l}4 / 22 \\
5 / 02 \\
4 / 22 \\
4 / 22\end{array}$ & $\begin{array}{l}0852 \\
0856 \\
0856 \\
1230\end{array}$ & $\begin{array}{l}0.434 \\
0.0203 \\
0.001 \\
0.048\end{array}$ \\
\hline Run 16 & $4 / 21$ & 0946 & $4 / 22$ & 1230 & $0.503 \mathrm{Ci} \mathrm{HT}$ \\
\hline $\begin{array}{l}5-43 \\
5-44\end{array}$ & $\begin{array}{l}4 / 22 \\
4 / 22 \\
4 / 22\end{array}$ & $\begin{array}{l}1230 \\
1543 \\
1546\end{array}$ & $\begin{array}{l}4 / 22 \\
4 / 22 \\
4 / 25\end{array}$ & $\begin{array}{l}1543 \\
1546 \\
1300\end{array}$ & $\begin{array}{l}0.0434 \\
0.0006 \\
1.065\end{array}$ \\
\hline Run 17 & $4 / 22$ & 1230 & $4 / 25$ & 1300 & $1.109 \mathrm{CI} \mathrm{HT}$ \\
\hline $\begin{array}{l}5-44(18) \\
5-45\end{array}$ & $\begin{array}{l}4 / 25^{b} \\
4 / 25 \\
4 / 25\end{array}$ & $\begin{array}{l}1300 \\
1319 \\
1322\end{array}$ & $\begin{array}{l}4 / 25 \\
4 / 25 \\
4 / 26\end{array}$ & $\begin{array}{l}1319 \\
1322 \\
1000\end{array}$ & $\begin{array}{l}0.005 \\
0.0005 \\
0.374\end{array}$ \\
\hline Run 18 & $4 / 25$ & 1300 & $4 / 26$ & 1000 & $0.380 \mathrm{CI} \mathrm{HT}$ \\
\hline $\begin{array}{l}5-45(19) \\
5-46\end{array}$ & $\begin{array}{l}4 / 26 \\
4 / 26 \\
4 / 26 \\
4 / 27\end{array}$ & $\begin{array}{l}1000 \\
1047 \\
1052 \\
0920\end{array}$ & $\begin{array}{l}4 / 26 \\
4 / 26 \\
4 / 27 \\
4 / 27\end{array}$ & $\begin{array}{l}1047 \\
1052 \\
0920 \\
0923\end{array}$ & $\begin{array}{l}0.015 \\
0.0012 \\
0.277 \\
0.0006\end{array}$ \\
\hline
\end{tabular}


TABLE VI-3 (Contd.)

\begin{tabular}{|c|c|c|c|c|c|}
\hline \multirow{2}{*}{$\begin{array}{c}\text { Sample } \\
\text { I.D. }\end{array}$} & \multicolumn{2}{|c|}{ Time On } & \multicolumn{2}{|c|}{ Time of $f$} & \multirow[b]{2}{*}{$\mathrm{HT}{ }_{\mu} \mathrm{Cl}$} \\
\hline & Date & Hour & Date & Hour & \\
\hline Run 19 & $4 / 26$ & 1000 & $4 / 27$ & 0923 & $0.294 \mathrm{Ci} \mathrm{HT}$ \\
\hline $5-47$ & $\begin{array}{l}4 / 27 \\
5 / 02\end{array}$ & $\begin{array}{l}0923 \\
0856\end{array}$ & $\begin{array}{l}5 / 02 \\
5 / 02\end{array}$ & $\begin{array}{l}0856 \\
0902\end{array}$ & $\begin{array}{l}0.0278 \\
0.0\end{array}$ \\
\hline Run 20 & $4 / 29$ & 1110 & $5 / 02$ & 0915 & $0.0278 \mathrm{CI} \mathrm{HT}$ \\
\hline $\begin{array}{l}5-48 \\
6-23 \\
5-49 \\
5-50(21)\end{array}$ & $\begin{array}{l}5 / 02 \\
5 / 02 \\
5 / 03 \\
5 / 03 \\
5 / 04 \\
5 / 04\end{array}$ & $\begin{array}{l}0902 \\
0902 \\
0915 \\
0920 \\
0835 \\
0840\end{array}$ & $\begin{array}{l}5 / 03 \\
5 / 06 \\
5 / 03 \\
5 / 04 \\
5 / 04 \\
5 / 04\end{array}$ & $\begin{array}{l}0915 \\
0810 \\
0920 \\
0835 \\
0840 \\
0900\end{array}$ & $\begin{array}{l}0.671 \\
0.00311 \\
0.002 \\
0.385 \\
0.0063 \\
0.025\end{array}$ \\
\hline Run 21 & $5 / 02$ & 1425 & $5 / 04$ & 0900 & $1.092 \mathrm{Ci} \mathrm{HT}$ \\
\hline $\begin{array}{l}5-50 \\
-51(22) \\
\end{array}$ & $\begin{array}{l}5 / 04 \\
5 / 05 \\
5 / 05 \\
\end{array}$ & $\begin{array}{l}0900 \\
0830 \\
0837 \\
\end{array}$ & $\begin{array}{l}5 / 05 \\
5 / 05 \\
5 / 05\end{array}$ & $\begin{array}{l}0830 \\
0837 \\
0900\end{array}$ & $\begin{array}{l}0.347 \\
0.0014 \\
0.003\end{array}$ \\
\hline $\begin{array}{l}\text { Run } 22 \\
5-51 \\
5-52 \\
6-24 \\
5-53(23)\end{array}$ & $\begin{array}{l}5 / 04 \\
5 / 05 \\
5 / 06 \\
5 / 06 \\
5 / 06 \\
5 / 09 \\
5 / 09\end{array}$ & $\begin{array}{l}0900 \\
0900 \\
0840 \\
1440 \\
0850 \\
0858 \\
0903\end{array}$ & $\begin{array}{l}5 / 05 \\
5 / 06 \\
5 / 06 \\
5 / 09 \\
5 / 09 \\
5 / 09 \\
5 / 09\end{array}$ & $\begin{array}{l}0900 \\
0840 \\
0850 \\
0858 \\
0858 \\
0903 \\
1100\end{array}$ & $\begin{array}{l}0.351 \text { Ci HT } \\
0.191 \\
0.0 \\
0.855 \\
0.00712 \\
0.001 \\
0.023\end{array}$ \\
\hline Run 23 & $5 / 05$ & 0900 & $5 / 09$ & 1100 & $1.077 \mathrm{Ci}$ HT \\
\hline $\begin{array}{l}5-53 \\
6-25 \\
5-54(24)\end{array}$ & $\begin{array}{l}5 / 09 \\
5 / 09 \\
5 / 10 \\
5 / 10\end{array}$ & $\begin{array}{l}1100 \\
0903 \\
0920 \\
0926\end{array}$ & $\begin{array}{l}5 / 10 \\
5 / 19 \\
5 / 10 \\
5 / 10\end{array}$ & $\begin{array}{l}0920 \\
0816 \\
0926 \\
1351\end{array}$ & $\begin{array}{l}0.476 \\
0.0308 \\
0.0018 \\
0.067\end{array}$ \\
\hline Run 24 & $5 / 09$ & 1100 & $5 / 10$ & 1351 & $0.576 \mathrm{Ci} \mathrm{HT}$ \\
\hline $\begin{array}{l}5-54 \\
5-55\end{array}$ & $\begin{array}{l}5 / 10 \\
5 / 11 \\
5 / 11\end{array}$ & $\begin{array}{l}1351 \\
0835 \\
0840\end{array}$ & $\begin{array}{l}5 / 11 \\
5 / 11 \\
5 / 12\end{array}$ & $\begin{array}{l}0835 \\
0840 \\
0815\end{array}$ & $\begin{array}{l}0.218 \\
0.001 \\
0.319\end{array}$ \\
\hline Run 25 & $5 / 10$ & 1351 & $5 / 12$ & 0815 & $0.537 \mathrm{Ci} \mathrm{HT}$ \\
\hline $\begin{array}{l}5-55(26) \\
5-56 \\
5-57\end{array}$ & $\begin{array}{l}5 / 12 \\
5 / 12 \\
5 / 12 \\
5 / 13 \\
5 / 13 \\
5 / 16\end{array}$ & $\begin{array}{l}0815 \\
0829 \\
0833 \\
0815 \\
0819 \\
0944\end{array}$ & $\begin{array}{l}5 / 12 \\
5 / 12 \\
5 / 13 \\
5 / 13 \\
5 / 16 \\
5 / 16\end{array}$ & $\begin{array}{l}0829 \\
0833 \\
0815 \\
0819 \\
0944 \\
0948\end{array}$ & $\begin{array}{l}0.003 \\
0.0008 \\
0.294 \\
0.0008 \\
0.914 \\
0.0008\end{array}$ \\
\hline
\end{tabular}


TABLE VI-3 (Contd.)

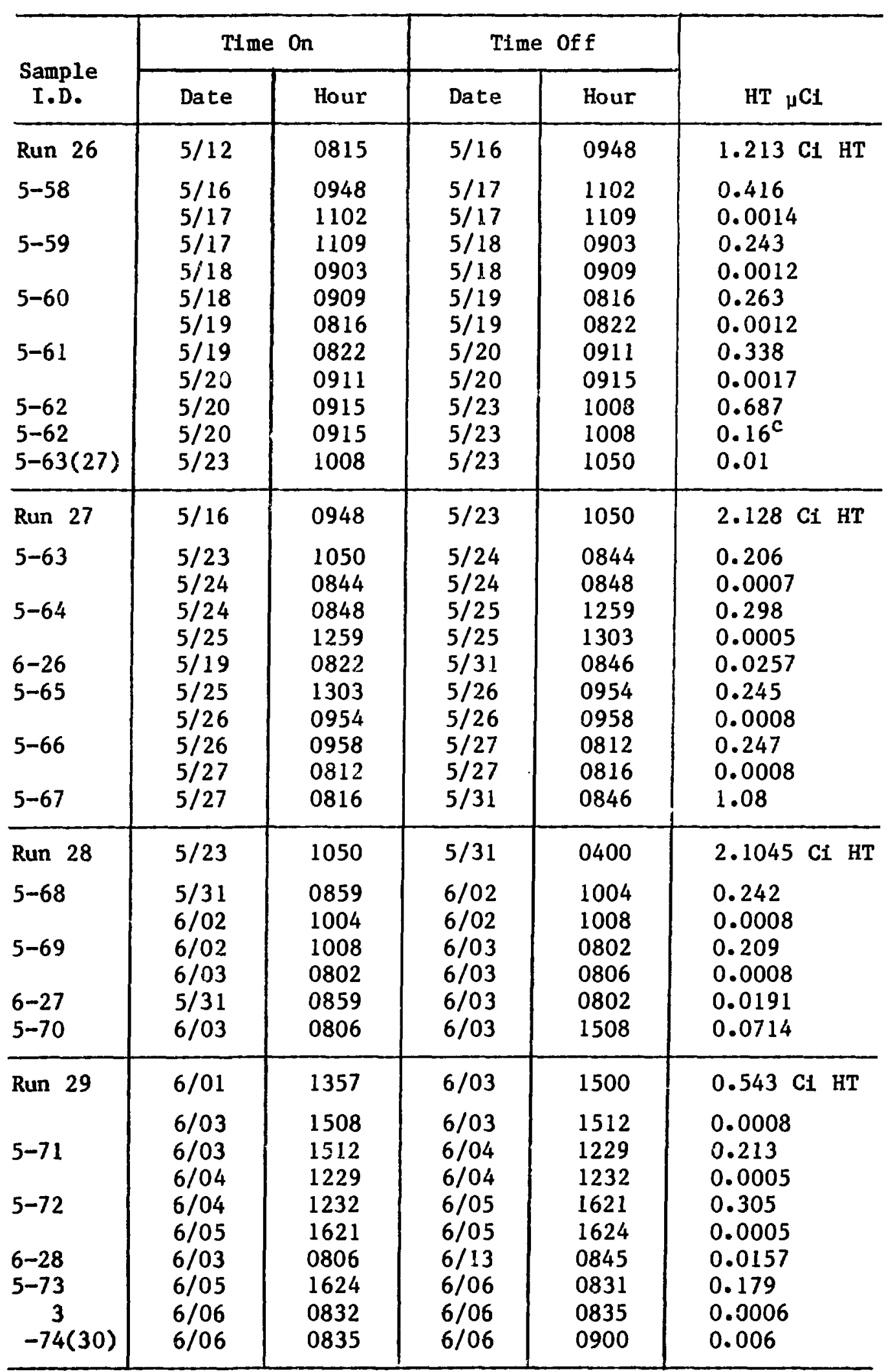


TABLE VI-3 (Contd.)

\begin{tabular}{|c|c|c|c|c|c|}
\hline \multirow{2}{*}{$\begin{array}{c}\text { Sample } \\
\text { I.D. }\end{array}$} & \multicolumn{2}{|c|}{ Time On } & \multicolumn{2}{|c|}{ Time off } & \multirow[b]{2}{*}{$\mathrm{HT} \mu \mathrm{CI}$} \\
\hline & Date & Hour & Date & Hour & \\
\hline Run 30 & $6 / 03$ & 1500 & $6 / 06$ & 0900 & $0.721 \mathrm{Ci} \mathrm{HT}$ \\
\hline $\begin{array}{r}5-74 \\
4 \\
5-75 \\
4 \\
5-76 \\
4 \\
-77(31)\end{array}$ & $\begin{array}{l}6 / 06 \\
6 / 07 \\
6 / 07 \\
6 / 08 \\
6 / 08 \\
6 / 08 \\
6 / 08\end{array}$ & $\begin{array}{l}0900 \\
0836 \\
0840 \\
0846 \\
0850 \\
1228 \\
1232\end{array}$ & $\begin{array}{l}6 / 07 \\
6 / 07 \\
6 / 08 \\
6 / 08 \\
6 / 08 \\
6 / 08 \\
6 / 08\end{array}$ & $\begin{array}{l}0836 \\
0840 \\
0846 \\
0850 \\
1228 \\
1232 \\
1240\end{array}$ & $\begin{array}{l}0.345 \\
0.0008 \\
0.342 \\
0.0008 \\
0.049 \\
0.0008 \\
0.002\end{array}$ \\
\hline Run 31 & $6 / 06$ & 0900 & $6 / 08$ & 1240 & $0.74 \mathrm{Ci} \mathrm{HT}$ \\
\hline $\begin{array}{r}5-77 \\
4 \\
5-78 \\
\\
5-79\end{array}$ & $\begin{array}{l}6 / 08 \\
6 / 09 \\
6 / 09 \\
6 / 10 \\
6 / 10\end{array}$ & $\begin{array}{l}1240 \\
0831 \\
0835 \\
0808 \\
0812\end{array}$ & $\begin{array}{l}6 / 09 \\
6 / 09 \\
6 / 10 \\
6 / 10 \\
6 / 10\end{array}$ & $\begin{array}{l}0831 \\
0835 \\
0808 \\
0812 \\
0830\end{array}$ & $\begin{array}{l}0.767 \\
0.0008 \\
0.498 \\
0.0008 \\
0.004\end{array}$ \\
\hline Run 32 & $6 / 08$ & 1240 & $6 / 10$ & 0830 & $1.271 \mathrm{Ci} \mathrm{HT}$ \\
\hline $\begin{array}{l}5-79 \\
5-80 \\
5-81\end{array}$ & $\begin{array}{l}6 / 10 \\
6 / 11 \\
6 / 11 \\
6 / 13\end{array}$ & $\begin{array}{l}0830 \\
0725 \\
0728 \\
0819\end{array}$ & $\begin{array}{l}6 / 11 \\
6 / 11 \\
6 / 13 \\
6 / 13\end{array}$ & $\begin{array}{l}0725 \\
0728 \\
0819 \\
0845\end{array}$ & $\begin{array}{l}0.987 \\
0.0006 \\
0.675 \\
3.85 \times 10^{3}\end{array}$ \\
\hline Run 33 & $6 / 10$ & 0830 & $6 / 13$ & 0845 & $1.666 \mathrm{Ci} \mathrm{HT}$ \\
\hline
\end{tabular}

*Note: Parentheses refer to run proapportioned samples which overlapped two runs.

${ }^{a}$ Calculated loss, copper oxide bed of fluorine.

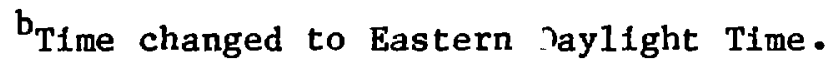

$\mathrm{c}_{\text {Leak }}$ into glovebox, calculated to $\mathrm{g}$. 
The data show tritlum collected in the first and the second HT trap (Nos. 5 and 6). The sample identification numbers indicate which trap was used. The blanks represent HT which was calculated to pass during the short time intervals when the traps were not on-1ine. The absolute accuracy of a single determination is $5 \%$. The tritium collected in the second trap (No. 6) is consistently less than $1 \%$ of the tritium collected in the first trap. The exhaust monitor recorded tritium levels less than one per cent of the levels of the line monitor. Thus, a single trap removes about $99 \%$ of the tritium.

\section{Tritium Collected as HTO}

The HTO was scrubbed from the sweep gas stream as previously described (Sec. IV.C). The HTO data are presented in Table VI-4. The first trap is either No. 1 or 3 and the second trap is either No. 2 or 4. Designations $A$, $B, C$, etc., as the last character of the sample identification number refer to $2 \mu \mathrm{L}$, samples taken from the ethylene glycol during operation by means of inserting a calibrated syringe through a septum. These samples were then added directly to the scintillator solution without dilution. The designations $D$ as the first character of the sample identification number refers to HTO collected over intervals between different syringe samples. The absolute accuracy for the syringe samples is estimated to be $10 \%$. The absolute accuracy of the regular samples is again 5\%. The amounts of HTO were much less (generally by a factor of 100 to 1000) than those of HT.

\section{Tritium Permeation}

The tritium which permeated through the wall of the inner capsule into the Jap gas was collected by passing the gap gas through an oxidizer and then trapping the tritium on Type $4 \mathrm{~A}$ molecular sieves. The molecular sieves were removed at times when the ORR was down and analyzed for tritium by refluxing in wacer for not less than four hours and then analyzing an aliquot by liquid scintillation. Seven such samples were collected and analyzed. Each sample thus represents several runs. For each sample, the amounts were apportioned to each run using the temperature, the amount of HT released (Table VI-2) and the duration of each run by a method described below. Temperatures used were the average of the recorded thermocouple readings on the two sides (east and west) of the capsule for the thermocouple elements in contact with the inner cladding. The permeation data are given in Table VI-5. 
TABLE VI-4

Tritium Collected, Condensable, or HTO FORM (January 26, 1983)

\begin{tabular}{|c|c|c|c|c|c|}
\hline \multirow{2}{*}{$\begin{array}{c}\text { Sample } \\
\text { I.D. }\end{array}$} & \multicolumn{2}{|c|}{ Time on } & \multicolumn{2}{|c|}{ Time of $f$} & \multirow[b]{2}{*}{ HTO, $\mu \mathrm{Ci}$} \\
\hline & Date & Hour & Date & Hour & \\
\hline $\begin{array}{l}1-1 \\
2-1 \\
1-2 \\
2-2\end{array}$ & $\begin{array}{l}3 / 11 \\
3 / 11 \\
3 / 11 \\
3 / 11\end{array}$ & $\begin{array}{l}0340 \\
0340 \\
1443 \\
1443\end{array}$ & $\begin{array}{l}3 / 11 \\
3 / 11 \\
3 \\
3\end{array}$ & $\begin{array}{l}0540 \\
0540 \\
2230 \\
2230\end{array}$ & $\begin{array}{c}56.9 \\
0.28 \\
2.4 \\
0.0\end{array}$ \\
\hline Run 0 & $3 / 11$ & 0340 & $3 / 11$ & 2230 & 59.6 \\
\hline $\begin{array}{l}1-3 \\
2-3 \\
1-4 \\
2-4 \\
\\
1-5 \\
2-5 \\
\\
1-6 \\
2-6 \\
1-7 \\
2-7 \\
\\
j-1 \\
1-8 \\
2-8 \\
3-2\end{array}$ & $\begin{array}{l}3 / 12 \\
3 / 12 \\
3 / 12 \\
3 / 12 \\
3 / 12 \\
3 / 12 \\
3 / 12 \\
3 / 12 \\
3 / 12 \\
3 / 12 \\
3 / 12 \\
3 / 12 \\
3 / 12 \\
3 / 12 \\
3 / 12 \\
3 / 12 \\
3 / 12 \\
3 / 12 \\
3 / 13\end{array}$ & $\begin{array}{l}0812 \\
0812 \\
1033 \\
1043 \\
1043 \\
1243 \\
1255 \\
1255 \\
1411 \\
1413 \\
1413 \\
1531 \\
1544 \\
1544 \\
1714 \\
1725 \\
2216 \\
2216 \\
0458\end{array}$ & $\begin{array}{l}3 / 12 \\
3 / 12 \\
3 / 12 \\
3 / 12 \\
3 / 12 \\
3 / 12 \\
3 / 12 \\
3 / 12 \\
3 / 12 \\
3 / 12 \\
3 / 12 \\
3 / 12 \\
3 / 12 \\
3 / 12 \\
3 / 12 \\
3 / 12 \\
3 / 13 \\
3 / 13 \\
3 / 13\end{array}$ & $\begin{array}{l}1033 \\
1033 \\
1043 \\
1243 \\
1243 \\
1255 \\
1411 \\
1411 \\
1413 \\
1531 \\
1531 \\
1544 \\
1714 \\
1714 \\
1725 \\
2211 \\
0458 \\
0458 \\
1407\end{array}$ & $\begin{array}{c}153.0 \\
0.77 \\
500.0 \\
6218.0 \\
4.7 \\
121.0 \\
849.0 \\
1.7 \\
60.0 \\
3718.0 \\
0.91 \\
260.0 \\
1218.0 \\
0.73 \\
110.0 \\
2910.0^{\mathrm{a}} \\
4045.0 \\
0.0 \\
5490.0^{\mathrm{a}}\end{array}$ \\
\hline Run 1 & $3 / 12$ & 0812 & $3 / 13$ & 1407 & $2.566 \times 10^{4}$ \\
\hline $\begin{array}{l}1-9 \\
2-9 \\
3-3 \\
1-10 \\
2-10 \\
3-4 \\
1-11 A \\
2-11 A \\
3-5 / 6\end{array}$ & $\begin{array}{l}3 / 13 \\
3 / 13 \\
3 / 14 \\
3 / 14 \\
3 / 14 \\
3 / 14 \\
3 / 14 \\
3 / 14 \\
3 / 14\end{array}$ & $\begin{array}{l}1407 \\
1407 \\
1313 \\
1439 \\
1439 \\
1548 \\
1745 \\
1745 \\
2304\end{array}$ & $\begin{array}{l}3 / 14 \\
3 / 14 \\
3 / 14 \\
3 / 14 \\
3 / 14 \\
3 / 14 \\
3 / 14 \\
3 / 14 \\
3 / 16\end{array}$ & $\begin{array}{l}1313 \\
1313 \\
1439 \\
1548 \\
1548 \\
1745 \\
2374 \\
2304 \\
1410\end{array}$ & $\begin{array}{c}3490.0 \\
0.4 \\
172.0^{\mathrm{a}} \\
133.0 \\
0.043 \\
234.0^{\mathrm{a}} \\
565.0 \\
0.0 \\
4000.0\end{array}$ \\
\hline Run 2 & $3 / 13$ & 1407 & $3 / 16$ & 1410 & $8.594 \times 10^{3}$ \\
\hline $\begin{array}{l}3-7 \\
1-11 B\end{array}$ & $\begin{array}{l}3 / 16 \\
3 / 17 \\
3 / 17\end{array}$ & $\begin{array}{l}1410 \\
0857 \\
1344\end{array}$ & $\begin{array}{l}3 / 17 \\
3 / 17 \\
3 / 17\end{array}$ & $\begin{array}{l}0850 \\
1344 \\
1359\end{array}$ & $\begin{array}{r}11200.0 \\
259.0 \\
10.0\end{array}$ \\
\hline
\end{tabular}


TABLE VI-4 (Contd.)

\begin{tabular}{|c|c|c|c|c|c|}
\hline \multirow{2}{*}{$\begin{array}{c}\text { Sample } \\
\text { T. D. }\end{array}$} & \multicolumn{2}{|c|}{ Time on } & \multicolumn{2}{|c|}{ Time of $f$} & \multirow[b]{2}{*}{ HTO, $\mu \mathrm{CI}$} \\
\hline & Date & Hour & Date & Hour & \\
\hline Run 3 & $3 / 16$ & 1410 & $3 / 17$ & 1359 & $1.147 \times 10^{4}$ \\
\hline $\begin{array}{l}1-12 A \\
3-8 / 11 \\
1-13 A\end{array}$ & $\begin{array}{l}3 / 17 \\
3 / 18 \\
3 / 21 \\
3 / 22\end{array}$ & $\begin{array}{l}1359 \\
0827 \\
0943 \\
1008\end{array}$ & $\begin{array}{l}3 / 18 \\
3 / 21 \\
3 / 22 \\
3 / 22\end{array}$ & $\begin{array}{l}0827 \\
0943 \\
1008 \\
1012\end{array}$ & $\begin{array}{r}747.0 \\
4243.0 \\
768.0 \\
2.0\end{array}$ \\
\hline Run 4 & $3 / 17$ & 1359 & $3 / 22$ & 1012 & $5.76 \times 10^{3}$ \\
\hline $\begin{array}{l}2-11 B \\
1-12 B A \\
1-12 B B \\
1-12 B \\
D 1-12 B B \\
D-112 B\end{array}$ & $\begin{array}{l}3 / 17 \\
3 / 22 \\
3 / 22 \\
3 / 22 \\
3 / 22 \\
3 / 23 \\
3 / 24\end{array}$ & $\begin{array}{l}0950 \\
1012 \\
1012 \\
1012 \\
1445 \\
0851 \\
1104\end{array}$ & $\begin{array}{l}3 / 24 \\
3 / 22 \\
3 / 23 \\
3 / 24 \\
3 / 23 \\
3 / 24 \\
3 / 24\end{array}$ & $\begin{array}{l}1104 \\
1445 \\
0851 \\
1104 \\
0851 \\
1104 \\
1110\end{array}$ & $\begin{array}{c}9.4 \\
(109.0)^{b} \\
(683.0) \\
1383.0 \mathrm{c} \\
(574.0) \\
(500.0) \\
3.0\end{array}$ \\
\hline Run 5 & $3 / 17$ & 0950 & $3 / 24$ & 1110 & $1.395 \times 10^{3}$ \\
\hline $\begin{array}{l}1-13 B A \\
1-13 B B \\
1-13 B C\end{array}$ & $\begin{array}{l}3 / 24 \\
3 / 24 \\
3 / 24\end{array}$ & $\begin{array}{l}1110 \\
1110 \\
1110\end{array}$ & $\begin{array}{l}3 / 24 \\
3 / 24 \\
3 / 25\end{array}$ & $\begin{array}{l}1355 \\
1546 \\
1025\end{array}$ & $\begin{array}{l}(62.0) \\
(172.0) \\
(718.0)\end{array}$ \\
\hline Run 6 & $3 / 24$ & 1110 & $3 / 25$ & 1025 & $7.18 \times 10^{2}$ \\
\hline $\begin{array}{l}1-13 B D \\
1-13 B E \\
1-13 B F \\
1-13 B G \\
1-13 B \\
D 1-13 B B \\
D 1-13 B C \\
D 1-13 B D \\
D 1-13 B E \\
D 1-13 B F \\
D 1-13 B G \\
D 1-13 B \\
2-12\end{array}$ & $\begin{array}{l}3 / 24 \\
3 / 24 \\
3 / 24 \\
3 / 24 \\
3 / 24 \\
3 / 24 \\
3 / 24 \\
3 / 25 \\
3 / 25 \\
3 / 27 \\
3 / 28 \\
3 / 28 \\
3 / 24 \\
3 / 28\end{array}$ & $\begin{array}{l}1110 \\
1110 \\
1110 \\
1110 \\
1110 \\
1355 \\
1546 \\
1025 \\
1428 \\
1748 \\
0906 \\
1906 \\
1110 \\
1<30\end{array}$ & $\begin{array}{l}3 / 25 \\
3 / 27 \\
3 / 28 \\
3 / 25 \\
3 / 25 \\
3 / 24 \\
3 / 25 \\
3 / 25 \\
3 / 27 \\
3 / 28 \\
3 / 28 \\
3 / 28 \\
4 / 05 \\
3 / 28\end{array}$ & $\begin{array}{l}1428 \\
1748 \\
0906 \\
1200 \\
1200 \\
1546 \\
1025 \\
1428 \\
1748 \\
0906 \\
1200 \\
1200 \\
0759 \\
1205\end{array}$ & $\begin{array}{c}(906.0) \\
(1910.0) \\
(2320.0) \\
(2110.0) \\
2420.0 \\
(110.0) \\
(546.0) \\
(188.0) \\
(1004.0) \\
(410.0) \\
(-210.0) \\
(100.0) \\
5.2 \\
2.0\end{array}$ \\
\hline Run 7 & $3 / 25$ & 1025 & $3 / 28$ & 1205 & $1.71 \times 10^{3}$ \\
\hline $\begin{array}{l}1-14 \mathrm{~A} \\
1-14 \mathrm{~B} \\
1-14 \mathrm{C} \\
1-14 \mathrm{D}\end{array}$ & $\begin{array}{l}3 / 28 \\
3 / 28 \\
3 / 28 \\
3 / 28\end{array}$ & $\begin{array}{l}1205 \\
1205 \\
1205 \\
1205\end{array}$ & $\begin{array}{l}3 / 28 \\
3 / 29 \\
3 / 30 \\
3 / 30\end{array}$ & $\begin{array}{l}1537 \\
0901 \\
0915 \\
1100\end{array}$ & $\begin{array}{r}(169.0) \\
(604.0) \\
(1350.0) \\
(1400.0)\end{array}$ \\
\hline
\end{tabular}


TABLE VI-4 (Contd.)

\begin{tabular}{|c|c|c|c|c|c|}
\hline \multirow{2}{*}{$\begin{array}{c}\text { Sample } \\
\text { I.D. }\end{array}$} & \multicolumn{2}{|c|}{ Time On } & \multicolumn{2}{|c|}{ Tyme of $f$} & \multirow[b]{2}{*}{ HTO, $\mu \mathrm{Ci}$} \\
\hline & Date & Hour & Date & Hour & \\
\hline Run 8 & $3 / 28$ & 1205 & $3 / 30$ & 1100 & $1.40 \times 10^{3}$ \\
\hline $\begin{array}{l}1-14 \mathrm{E} \\
1-14 \mathrm{~F} \\
1-14 \mathrm{G} \\
1-14 \mathrm{H} \\
1-14 \mathrm{~F} \\
1-14 \\
\mathrm{D} 1-14 \mathrm{~B} \\
\mathrm{D} 1-14 \mathrm{C} \\
\mathrm{D} 1-14 \mathrm{D} \\
\mathrm{D} 1-14 \mathrm{E} \\
\mathrm{D} 1-14 \mathrm{~F} \\
\mathrm{D} 1-14 \mathrm{G} \\
\mathrm{D} 1-14 \mathrm{H} \\
\mathrm{D} 1-14 \mathrm{I} \\
\mathrm{D} 1-14\end{array}$ & $\begin{array}{l}3 / 28 \\
3 / 28 \\
3 / 28 \\
3 / 28 \\
3 / 28 \\
3 / 28 \\
3 / 28 \\
3 / 29 \\
3 / 30 \\
3 / 30 \\
3 / 30 \\
3 / 30 \\
3 / 30 \\
3 / 31 \\
4 / 04\end{array}$ & $\begin{array}{l}1205 \\
1205 \\
1205 \\
1205 \\
1205 \\
1205 \\
1537 \\
0901 \\
0915 \\
1100 \\
1300 \\
1500 \\
1624 \\
0918 \\
1353\end{array}$ & $\begin{array}{l}3 / 30 \\
3 / 30 \\
3 / 30 \\
3 / 31 \\
4 / 04 \\
4 / 05 \\
3 / 29 \\
3 / 30 \\
3 / 30 \\
3 / 30 \\
3 / 30 \\
3 / 30 \\
3 / 31 \\
4 / 04 \\
4 / 05\end{array}$ & $\begin{array}{l}1300 \\
1500 \\
1624 \\
0918 \\
1353 \\
0754 \\
0901 \\
0915 \\
1100 \\
1300 \\
1500 \\
1624 \\
0918 \\
1353 \\
0754\end{array}$ & $\begin{array}{c}(1460.0) \\
(1500.0) \\
(1430.0) \\
(1600.0) \\
(2466.0) \\
2950.0 \\
(435.0) \\
(746.0) \\
(50.0) \\
(60.0) \\
(40.0) \\
(-70.0) \\
(170.0) \\
(866.0) \\
(481.0)\end{array}$ \\
\hline Run 9 & $3 / 30$ & 1100 & $4 / 05$ & 0754 & $1.55 \times 10^{3}$ \\
\hline $1-15 A$ & $\begin{array}{l}4 / 05 \\
4 / 06\end{array}$ & $\begin{array}{l}0754 \\
0842\end{array}$ & $\begin{array}{l}4 / 06 \\
4 / 07\end{array}$ & $\begin{array}{l}0842 \\
0825\end{array}$ & $\begin{array}{c}0.0 \\
(90.8)\end{array}$ \\
\hline Run 10 & $4 / 05$ & 0754 & $4 / 07$ & 0825 & 91.0 \\
\hline $\begin{array}{l}1-15 B \\
1-15 C \\
1-15 D\end{array}$ & $\begin{array}{l}4 / 06 \\
4 / 06 \\
4 / 06\end{array}$ & $\begin{array}{l}0842 \\
0842 \\
0842\end{array}$ & $\begin{array}{l}4 / 07 \\
4 / 08 \\
4 / 08\end{array}$ & $\begin{array}{l}1558 \\
0824 \\
1530\end{array}$ & $\begin{array}{l}(1360.0) \\
(2445.0)^{c} \\
(2511.0)\end{array}$ \\
\hline Run 11 & $4 / 07$ & 0825 & $4 / 08$ & 1530 & $2.51 \times 10^{3}$ \\
\hline $\begin{array}{l}1-15 \\
2-13 \\
D 1-15 B \\
D 1-15 C \\
D 1-15 D \\
D 1-15 \\
1-16 A \\
\end{array}$ & $\begin{array}{l}4 / 06 \\
4 / 06 \\
4 / 07 \\
4 / 07 \\
4 / 08 \\
4 / 08 \\
4 / 11 \\
4 / 11\end{array}$ & $\begin{array}{l}0842 \\
0842 \\
0829 \\
1558 \\
0824 \\
1530 \\
1415 \\
1420\end{array}$ & $\begin{array}{l}4 / 11 \\
4 / 11 \\
4 / 07 \\
4 / 08 \\
4 / 08 \\
4 / 11 \\
4 / 11 \\
4 / 12\end{array}$ & $\begin{array}{l}1415 \\
1415 \\
1558 \\
0824 \\
1530 \\
1415 \\
1420 \\
0829\end{array}$ & $\begin{array}{c}2988.0 \\
15.0 \\
(1269.1) \\
(1085.0) \\
(66.0) \\
(477.0) \\
2.0 \\
(67.2)\end{array}$ \\
\hline Run 12 & $4 / 08$ & 1530 & $4 / 12$ & 0829 & 494.0 \\
\hline $\begin{array}{l}1-16 B \\
1-16 C \\
1-16 D \\
1-16 \\
2-14 \\
D 1-16 B \\
D 1-16 C \\
D 1-16 D\end{array}$ & $\begin{array}{l}4 / 11 \\
4 / 11 \\
4 / 11 \\
4 / 11 \\
4 / 11 \\
4 / 12 \\
4 / 12 \\
4 / 12\end{array}$ & $\begin{array}{l}1420 \\
1420 \\
1420 \\
1420 \\
1420 \\
0829 \\
1315 \\
1700\end{array}$ & $\begin{array}{l}4 / 12 \\
4 / 12 \\
4 / 13 \\
4 / 14 \\
4 / 10 \\
4 / 12 \\
4 / 12 \\
4 / 13\end{array}$ & $\begin{array}{l}1315 \\
1700 \\
1514 \\
1445 \\
0859 \\
1315 \\
1700 \\
1514\end{array}$ & $\begin{array}{c}(74.3) \\
(714.0) \\
(1168.0) \\
881.0 \\
8.21 \\
(7.1) \\
(639.7) \\
(454.0)\end{array}$ \\
\hline
\end{tabular}


TABLE VI-4 (Contd.)

\begin{tabular}{|c|c|c|c|c|c|}
\hline \multirow{2}{*}{$\begin{array}{c}\text { Sample } \\
\text { I.D. }\end{array}$} & \multicolumn{2}{|c|}{ Time On } & \multicolumn{2}{|c|}{ Time of $\mathrm{f}$} & \multirow[b]{2}{*}{ нТо, $\mu \mathrm{Cl}$} \\
\hline & Date & Hour & Date & Hour & \\
\hline \multicolumn{2}{|c|}{ Run 12 (Contd.) } & & & & \\
\hline $\begin{array}{l}D 1-16 \\
1-17 A \\
1-17\end{array}$ & $\begin{array}{l}4 / 13 \\
4 / 14 \\
4 / 14 \\
4 / 14 \\
4 / 18\end{array}$ & $\begin{array}{l}1514 \\
1445 \\
1444 \\
1444 \\
0859\end{array}$ & $\begin{array}{l}4 / 14 \\
4 / 14 \\
4 / 15 \\
4 / 18 \\
4 / 18\end{array}$ & $\begin{array}{l}1445 \\
1444 \\
0904 \\
0859 \\
0903\end{array}$ & $\begin{array}{c}(-287.0) \\
0.0 \\
(370.0) \\
108.0 \\
1.2\end{array}$ \\
\hline $\begin{array}{l}\text { Run } 13 \\
1-18 A \\
1-18 B \\
1-18 C \\
1-18 D \\
1-18\end{array}$ & $\begin{array}{l}4 / 12 \\
4 / 18 \\
4 / 18 \\
4 / 18 \\
4 / 18 \\
4 / 18\end{array}$ & $\begin{array}{l}0829 \\
0903 \\
0903 \\
0903 \\
0903 \\
0903\end{array}$ & $\begin{array}{l}4 / 18 \\
4 / 18 \\
4 / 19 \\
4 / 19 \\
4 / 20 \\
4 / 20\end{array}$ & $\begin{array}{l}0903 \\
1513 \\
0850 \\
1602 \\
0907 \\
1239\end{array}$ & $\begin{array}{c}496.0 \\
(36.9) \\
(667.0) \\
(678.0) \\
(681.0) \\
670.0\end{array}$ \\
\hline Run 14 & $4 / 18$ & 0930 & $4 / 20$ & 1239 & 670.0 \\
\hline $\begin{array}{l}2-15 \\
D 1-18 B \\
D 1-18 C \\
D 1-18 D \\
D 1-18\end{array}$ & $\begin{array}{l}4 / 18 \\
4 / 19 \\
4 / 19 \\
4 / 19 \\
4 / 20 \\
4 / 20\end{array}$ & $\begin{array}{l}0903 \\
1513 \\
0850 \\
1602 \\
0907 \\
1239\end{array}$ & $\begin{array}{l}5 / 02 \\
4 / 19 \\
4 / 19 \\
4 / 20 \\
4 / 20 \\
4 / 20\end{array}$ & $\begin{array}{l}0830 \\
0850 \\
1602 \\
0907 \\
1239 \\
1242\end{array}$ & $\begin{array}{c}84.8 \\
(630.1) \\
(11.0) \\
(3.0) \\
(-11.0) \\
0.9\end{array}$ \\
\hline Run 15 & $4 / 20$ & 1239 & $4 / 20$ & 1242 & 86.0 \\
\hline $\begin{array}{l}1-19 A \\
1-19 B \\
1-19 C \\
1-19 D \\
1-19 \\
D 1-19 B \\
D 1-19 C \\
D 1-19 D \\
D 1-19\end{array}$ & $\begin{array}{l}4 / 20 \\
4 / 20 \\
4 / 20 \\
4 / 20 \\
4 / 20 \\
4 / 20 \\
4 / 21 \\
4 / 21 \\
4 / 22\end{array}$ & $\begin{array}{l}1242 \\
1242 \\
1242 \\
1242 \\
1242 \\
1559 \\
0914 \\
1551 \\
0905\end{array}$ & $\begin{array}{l}4 / 20 \\
4 / 21 \\
4 / 21 \\
4 / 22 \\
4 / 22 \\
4 / 20 \\
4 / 21 \\
4 / 22 \\
4 / 22\end{array}$ & $\begin{array}{l}1559 \\
0914 \\
1551 \\
0905 \\
1527 \\
0914 \\
1551 \\
0905 \\
1527\end{array}$ & $\begin{array}{c}(59.3) \\
(36.6) \\
(320.0) \\
(418.0) \\
508.0 \\
(-22.7) \\
(283.4) \\
(98.0) \\
(90.0)\end{array}$ \\
\hline Run 16 & $4 / 20$ & 1242 & $4 / 22$ & 1527 & 508.0 \\
\hline $1-20$ & $\begin{array}{l}4 / 22 \\
4 / 22 \\
4 / 25\end{array}$ & $\begin{array}{l}1527 \\
1532 \\
1249\end{array}$ & $\begin{array}{l}4 / 22 \\
4 / 25 \\
4 / 25\end{array}$ & $\begin{array}{l}1532 \\
1249 \\
1253\end{array}$ & $\begin{array}{r}2.0 \\
267.3 \\
1.6\end{array}$ \\
\hline Run 17 & $4 / 22$ & 1527 & $4 / 25$ & 1253 & 271.0 \\
\hline $\begin{array}{l}1-21 A \\
1-21 B \\
1-21 C \\
1-21 D \\
1-21\end{array}$ & $\begin{array}{l}4 / 25 \\
4 / 25 \\
4 / 25 \\
4 / 25 \\
4 / 25\end{array}$ & $\begin{array}{l}1233 \\
1233 \\
1233 \\
1233 \\
1233\end{array}$ & $\begin{array}{l}4 / 26 \\
4 / 26 \\
4 / 27 \\
4 / 29 \\
4 / 29\end{array}$ & $\begin{array}{l}0942 \\
1559 \\
0906 \\
0856 \\
1014\end{array}$ & $\begin{array}{c}(18.5) \\
(16.6) \\
(15.6) \\
(32.0) \\
32.8\end{array}$ \\
\hline
\end{tabular}


TABLE VI-4 (Contd.)

\begin{tabular}{|c|c|c|c|c|c|}
\hline \multirow{2}{*}{$\begin{array}{c}\text { Sample } \\
\text { I.D. }\end{array}$} & \multicolumn{2}{|c|}{ Tima On } & \multicolumn{2}{|c|}{ Time of $\mathrm{f}$} & \multirow[b]{2}{*}{$\mathrm{HTO}, \mu \mathrm{CI}$} \\
\hline & Date & Hour & Date & Hour & \\
\hline Runs 18,19 & $4 / 25$ & 1233 & $4 / 29$ & 1014 & 32.8 \\
\hline $\begin{array}{l}1-22 \\
1-23 \\
2-16\end{array}$ & $\begin{array}{l}4 / 29 \\
4 / 29 \\
5 / 02 \\
5 / 02 \\
5 / 02\end{array}$ & $\begin{array}{l}1014 \\
1018 \\
0830 \\
0846 \\
0846\end{array}$ & $\begin{array}{l}4 / 29 \\
5 / 02 \\
5 / 02 \\
5 / 06 \\
5 / 06\end{array}$ & $\begin{array}{l}1018 \\
0830 \\
0846 \\
0827 \\
0827\end{array}$ & $\begin{array}{r}0.2 \\
33.4 \\
1.6 \\
69.4 \\
27.6\end{array}$ \\
\hline Runs 20-22 & $4 / 29$ & 1014 & $5 / 06$ & 0827 & 132.0 \\
\hline $\begin{array}{l}1-24 A \\
1-24 B \\
1-24 C \\
1-24 D \\
1-24 E\end{array}$ & $\begin{array}{l}5 / 06 \\
5 / 06 \\
5 / 06 \\
5 / 06 \\
5 / 06 \\
5 / 06\end{array}$ & $\begin{array}{l}0827 \\
1300 \\
1300 \\
1300 \\
1300 \\
1300\end{array}$ & $\begin{array}{l}5 / 06 \\
5 / 06 \\
5 / 06 \\
5 / 07 \\
5 / 09 \\
5 / 09\end{array}$ & $\begin{array}{l}1300 \\
1318 \\
1620 \\
1152 \\
0855 \\
1156\end{array}$ & $\begin{array}{r}0.00 \\
(36.6) \\
(46.1) \\
(172.0) \\
(1230.0) \\
(1370.0)\end{array}$ \\
\hline Run 23 & $5 / 06$ & 0827 & $5 / 09$ & 1156 & $1.37 \times 10^{3}$ \\
\hline $\begin{array}{l}1-24 F \\
1-24 G \\
1-24 \\
2-17 A \\
2-17 B \\
2-17 C \\
2-17 D \\
2-17 E \\
2-17 F \\
2-17 G \\
2-17 \\
D 1-24 B \\
D 1-24 C \\
D 1-24 D \\
D 1-24 E \\
D 1-24 F \\
D 1-24 G \\
D 1-24\end{array}$ & $\begin{array}{l}5 / 06 \\
5 / 06 \\
5 / 06 \\
5 / 06 \\
5 / 06 \\
5 / 06 \\
5 / 06 \\
5 / 06 \\
5 / 06 \\
5 / 06 \\
5 / 06 \\
5 / 06 \\
5 / 06 \\
5 / 07 \\
5 / 09 \\
5 / 09 \\
5 / 09 \\
5 / 10 \\
5 / 10\end{array}$ & $\begin{array}{l}1300 \\
1300 \\
1300 \\
1300 \\
1300 \\
1300 \\
1300 \\
1300 \\
1300 \\
1300 \\
1300 \\
1318 \\
1620 \\
1152 \\
0855 \\
1156 \\
1555 \\
0919 \\
1251\end{array}$ & $\begin{array}{l}5 / 09 \\
5 / 10 \\
5 / 10 \\
5 / 06 \\
5 / 06 \\
5 / 07 \\
5 / 09 \\
5 / 09 \\
5 / 09 \\
5 / 10 \\
5 / 10 \\
5 / 06 \\
5 / 07 \\
5 / 09 \\
5 / 09 \\
5 / 09 \\
5 / 10 \\
5 / 10 \\
5 / 10\end{array}$ & $\begin{array}{l}1555 \\
0919 \\
1251 \\
1322 \\
1618 \\
1149 \\
0853 \\
1153 \\
1553 \\
0916 \\
1251 \\
1620 \\
1152 \\
0855 \\
1156 \\
1555 \\
0919 \\
1251 \\
1257\end{array}$ & $\begin{array}{c}(1540.0) \\
(2600.0) \\
3290.0 \\
(7.88) \\
(7.07) \\
(16.7) \\
(17.6) \\
(25.8) \\
(22.8) \\
(22.0) \\
17.2 \\
(9.5) \\
(125.9) \\
(1058.0) \\
(140.0) \\
(170.0) \\
(1060.0) \\
(690.0) \\
10.0\end{array}$ \\
\hline Run 24 & $5 / 06$ & 1300 & $5 / 10$ & 1257 & $1.947 \times 10^{3}$ \\
\hline $\begin{array}{l}1-25 A \\
1-25 B \\
1-25 C \\
1-25 D\end{array}$ & $\begin{array}{l}5 / 10 \\
5 / 10 \\
5 / 10 \\
5 / 10\end{array}$ & $\begin{array}{l}1257 \\
1257 \\
1257 \\
1257\end{array}$ & $\begin{array}{l}5 / 10 \\
5 / 11 \\
5 / 11 \\
5 / 12\end{array}$ & $\begin{array}{l}1553 \\
0830 \\
1546 \\
0827\end{array}$ & $\begin{array}{r}(263.0) \\
(2160.0) \\
(3890.0) \\
(6950.0)\end{array}$ \\
\hline
\end{tabular}


TABLE VI-4 (Contd.)

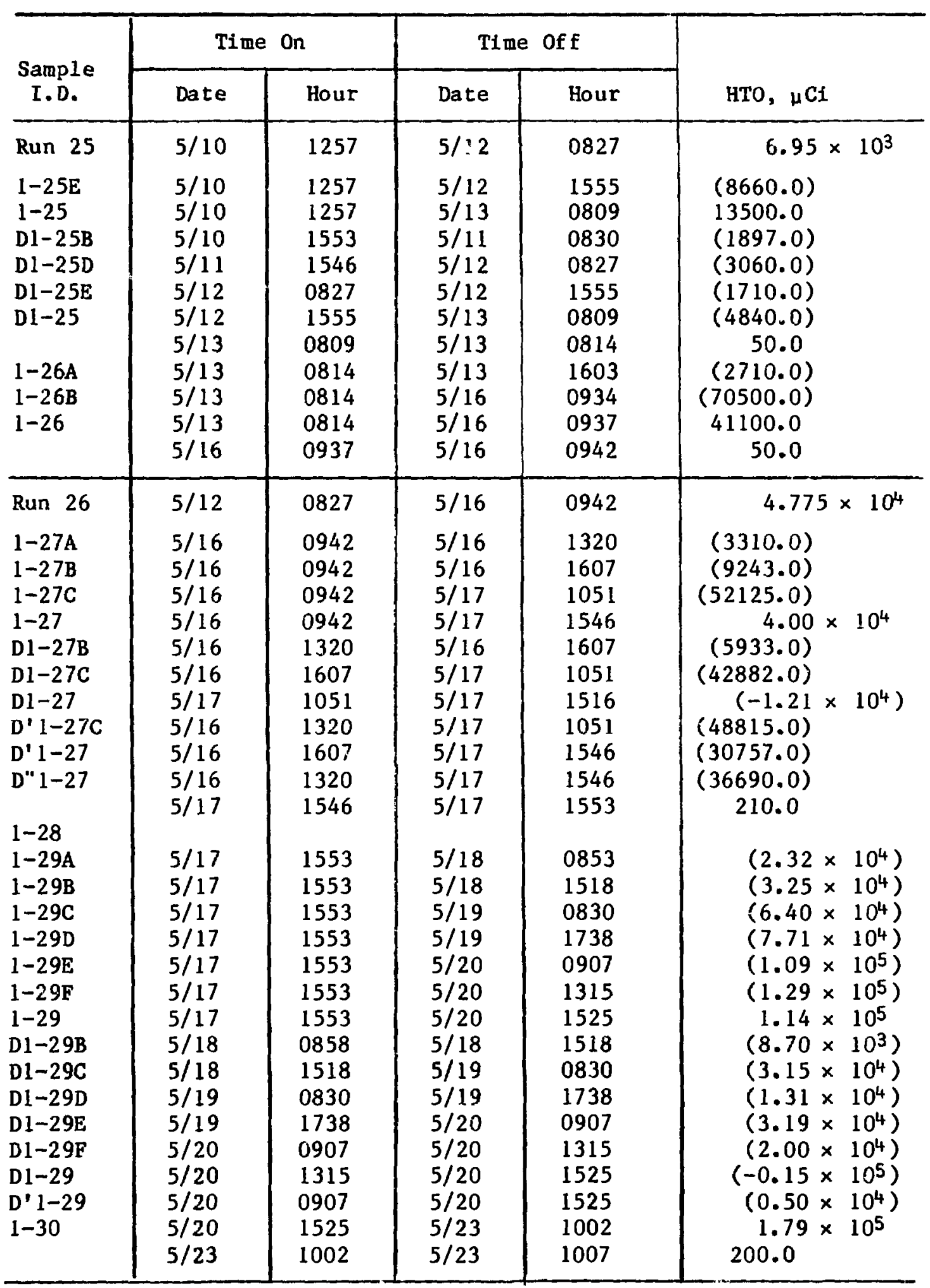


TABLE VI-4 (Contd.)

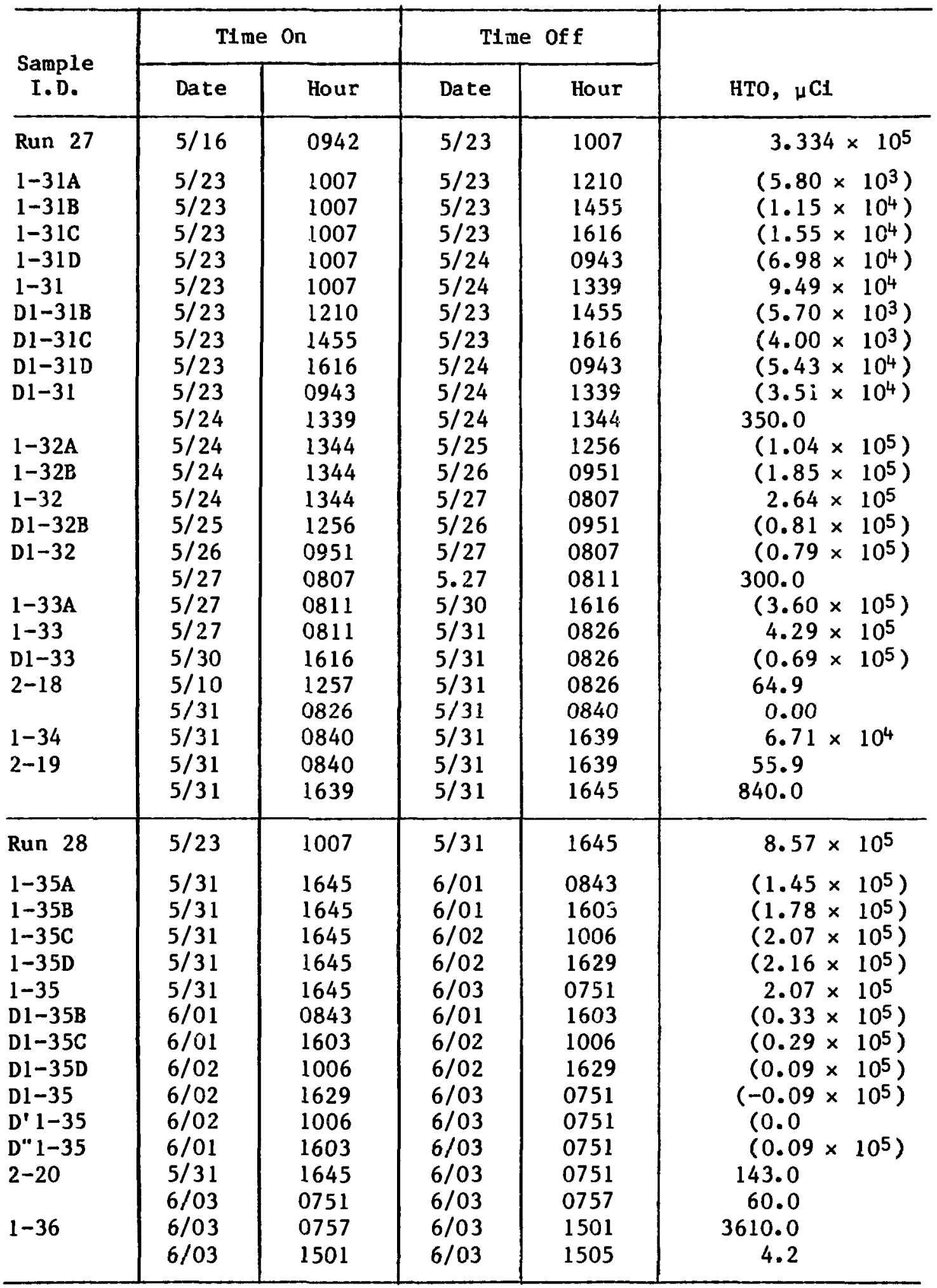


TABLE VI-4 (Contd.)

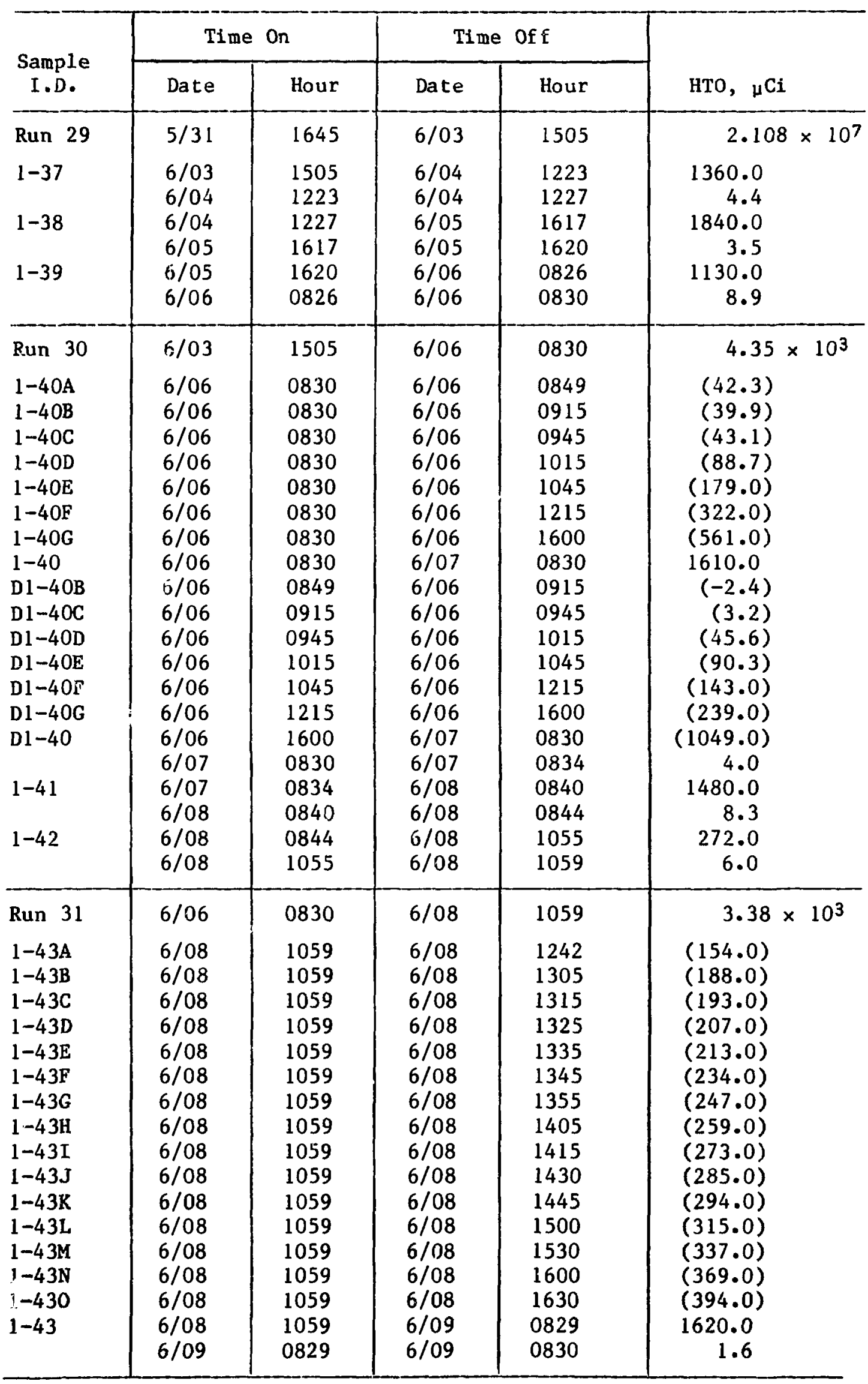


TABLE VI-4 (Contd.)

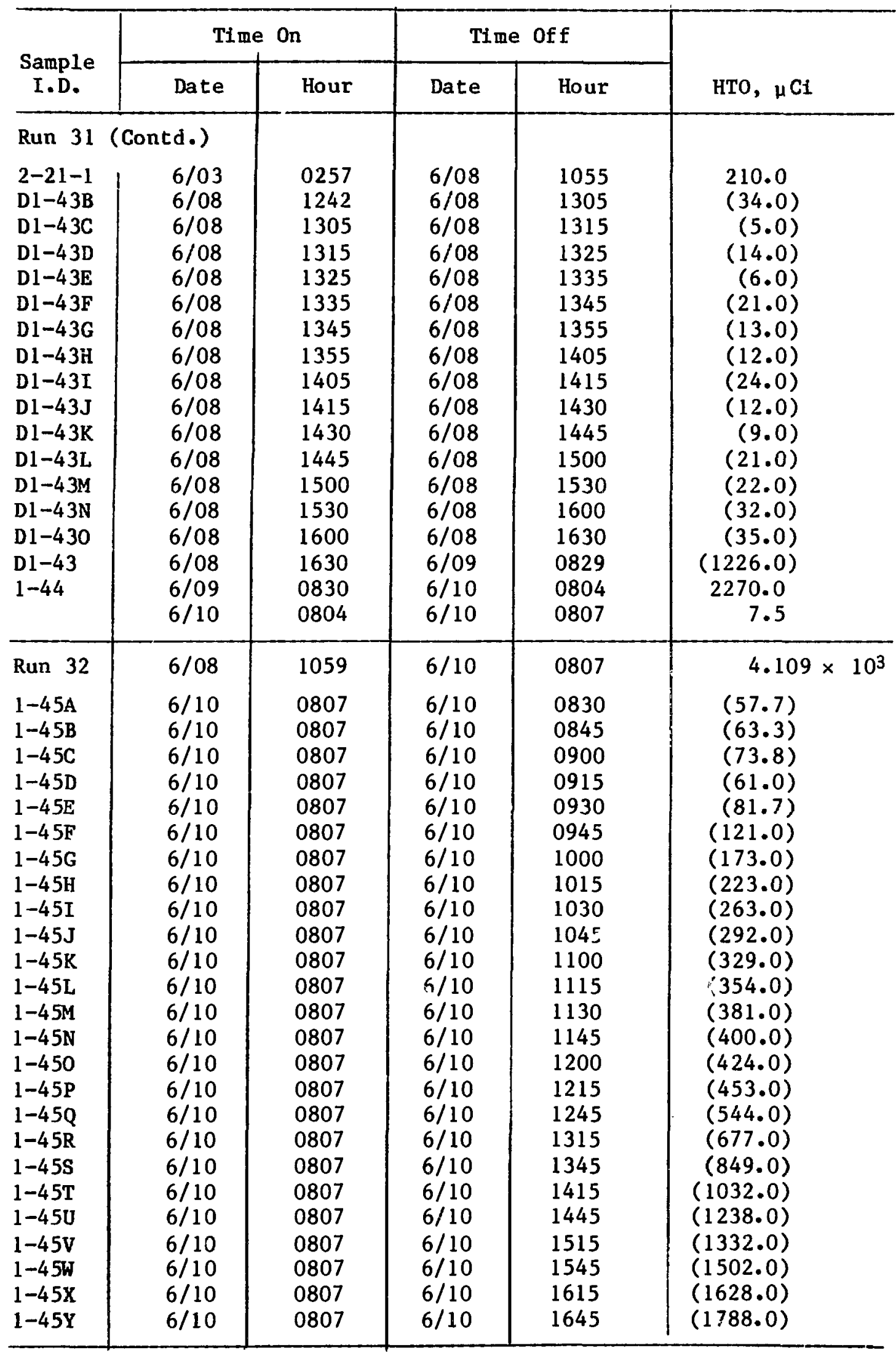


TABLE VI -4 (Contd.)

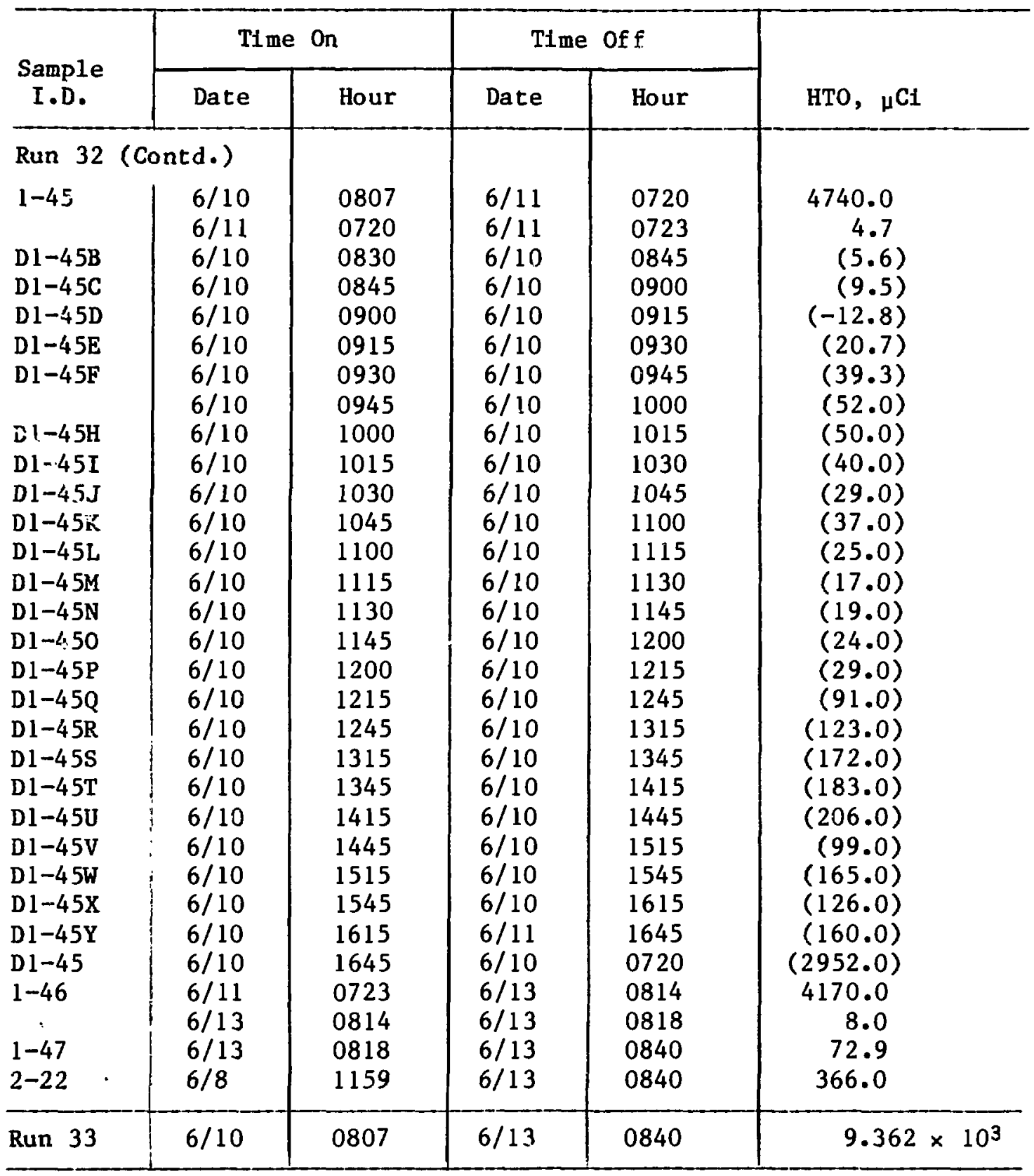

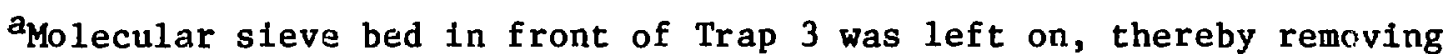
the HTO. Numbers are estimated.

b Numbers in parentheses are for syringe samples taken at various time intervals. These samples are not included in the sum of the total amount collected per rua.

${ }^{c}$ Counting time less than 1 min for scintillation sample - accuracy may be reduced about $5 \%$. 
The procedure used to apportion the total amount of tritium collected in a sample into the amounts for each run was as follows. The contribution from each run in a sample was assumed to be proportional to three factors: (1) the permeability of the inner cladding at the temperature measured for the run, (2) the square root of the average tritium (HT) pressure, and (3) the time for each run. The tritium permeability for Type 316 stainless stee $1^{46}$ was used for Type 304 stainless steel cladding in TRIO. [Note: In order to convert the permeability into units of Curies/(m•day•pa), it is necessary to multiply the data in reference 46 by a factor of 677.] The average HT pressure is proportional to the total amount of HT released per unit time. The data for cladding temperature, tritium permeability, calculated amounts of tritium permeation, reciprocal temperatire, and the logarithm of the permeation rates are given in Table VI-6.

On observing the permeation data (Table VI-6) some permeation rates appear to be much higher than the rest, particularly those for Samples 2 and 3. These samples include Runs 10-14 which had no hydrogen added to the sweep gas. It appears that the added hydrogen may have reduced the permeation rates for the other runs. It is possible that the addition of hydrogen reduced the permeation rates of tritium by isotopic swamping. This possibility is discussed further in Sec. IX.5.

\section{Total Tritium Collected}

The data presented in Tables VI-4 and -5 are summarized in Table VI-7. The tritlum collected in the three forms, the total (TCOLL), and the cumulative total (CTCOLL) are presented for the 33 runs.

\section{F. Tritiun (HT) Release Rates}

One of the key features of the TRIO experiment is the on-line measurement of tritium release rate. In the experiment, the level of tritium released in the HT (noncondensable) form was continuously measured with $\mathrm{KCl}$, the line monitor. The HT form accounted for about $94 \%$ of the released tritium. Also, for the majority of the experimental runs, the HT form was about $99 \%$ of the released tritium. Thus, the dynamic data on HT release rate provide a reasonably good measure of the overall rates of tritium release from the solid. 
TABLE VI-5

TRIO Tritium Permeation Data

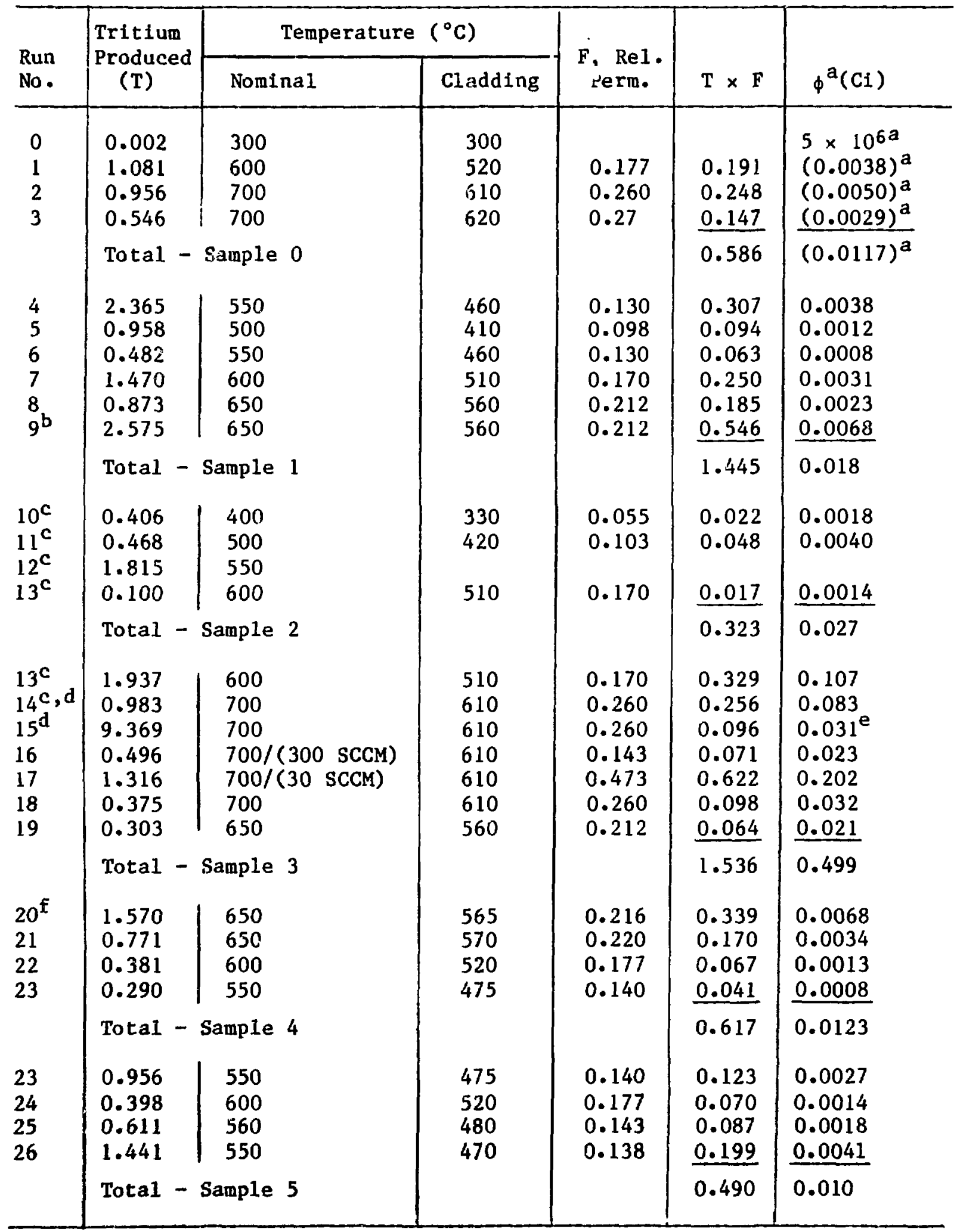


TABLE VI-5 (Contd.)

\begin{tabular}{|c|c|c|c|c|c|c|}
\hline \multirow{2}{*}{$\begin{array}{l}\text { Run } \\
\text { No. }\end{array}$} & \multirow{2}{*}{$\begin{array}{l}\text { Tritium } \\
\text { Produced } \\
\quad \text { (T) }\end{array}$} & \multicolumn{2}{|c|}{ Temperature $\left({ }^{\circ} \mathrm{C}\right)$} & \multirow{2}{*}{$\begin{array}{c}\text { F, } \operatorname{Re} 1 . \\
\text { Perm. }\end{array}$} & \multirow[b]{2}{*}{$\mathrm{T} \times \mathrm{F}$} & \multirow[b]{2}{*}{$\phi^{a}(C 1)$} \\
\hline & & Nominal & Cladding & & & \\
\hline \multirow[t]{2}{*}{$\begin{array}{l}27 \\
28\end{array}$} & $\begin{array}{l}2.107 \\
2.488\end{array}$ & $\begin{array}{l}525 \\
500\end{array}$ & $\begin{array}{l}450 \\
430\end{array}$ & $\begin{array}{l}0.124 \\
0.100\end{array}$ & $\begin{array}{l}0.261 \\
0.269 \\
\end{array}$ & $\begin{array}{l}0.0103 \\
0.0107 \\
\end{array}$ \\
\hline & \multicolumn{2}{|c|}{ Total - Sample 6} & & & 0.530 & 0.021 \\
\hline $\begin{array}{l}29 \\
30 \\
31 \\
32 \\
33\end{array}$ & $\begin{array}{l}0.726 \\
0.892 \\
0.718 \\
0.601 \\
0.906\end{array}$ & $\begin{array}{l}480 /(300 \text { SCCM }) \\
480 \\
500 \\
550 \\
650\end{array}$ & $\begin{array}{l}410 \\
410 \\
425 \\
470 \\
580\end{array}$ & $\begin{array}{l}0.054 \\
0.098 \\
0.107 \\
0.139 \\
0.230\end{array}$ & $\begin{array}{l}0.039 \\
0.087 \\
0.077 \\
0.084 \\
0.208 \\
\end{array}$ & $\begin{array}{l}0.0007 \\
0.0016 \\
0.0014 \\
0.0015 \\
0.0038 \\
\end{array}$ \\
\hline $\begin{array}{l}32 \\
33\end{array}$ & \multicolumn{2}{|c|}{ Total - Sample 7} & & & 0.495 & 0.009 \\
\hline
\end{tabular}

$a_{\phi}=T \times F \times F F$, where $F F$ is assumed to be 0.02 for Sample 0 , 0.0125 for Sample 1, 0.0836 for Sample 2, 0.325 for Sample 3, 0.020 for Sample 4, 0.204 for Sample 5, 0.0396 for Sample 6, and 0.018 for Sample 7.

$b_{\text {Run }} 9$ had no $\mathrm{H}_{2}$; tritium produced may be overestimated.

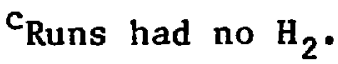

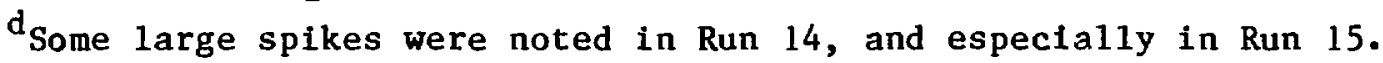

e Large spike.

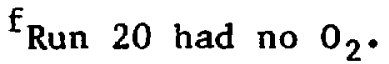


TABLE VI-6

Tritium Permeation Data

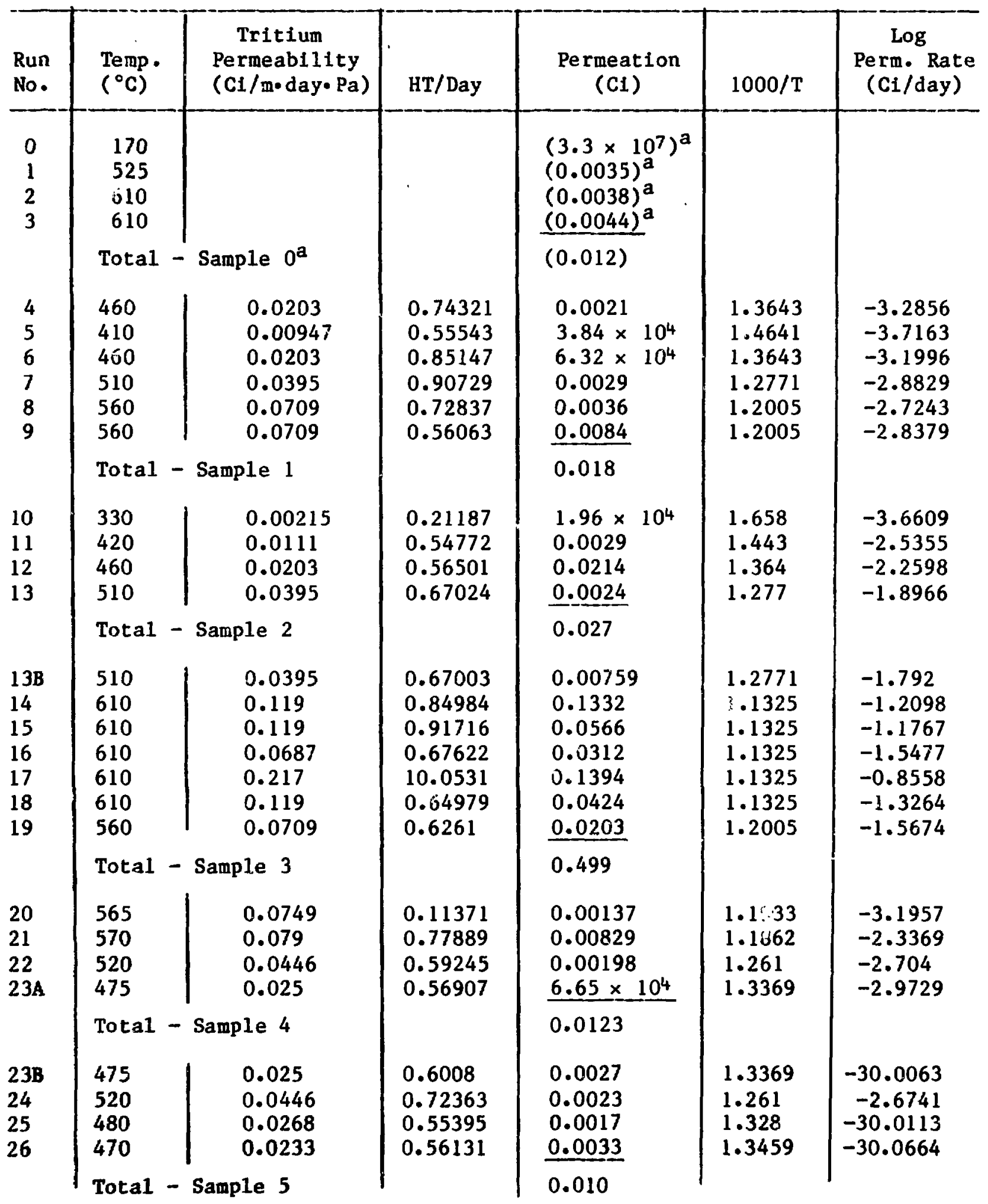


TABLE VI.-6 (Contd.)

\begin{tabular}{|c|c|c|c|c|c|c|}
\hline $\begin{array}{l}\text { Run } \\
\text { No. }\end{array}$ & $\begin{array}{l}\text { Temp. } \\
\left({ }^{\circ} \mathrm{C}\right)\end{array}$ & $\begin{array}{l}\text { Tritium } \\
\text { Permeability } \\
(\mathrm{C} i / \mathrm{m} \bullet \text { day•Pa) }\end{array}$ & $\mathrm{niT} / \mathrm{Day}$ & $\begin{array}{c}\text { Permeation } \\
\text { (Ci) }\end{array}$ & $1000 / T$ & $\begin{array}{c}\text { Log } \\
\text { Perm. Rate } \\
\text { (Ci/day) }\end{array}$ \\
\hline \multirow[t]{2}{*}{$\begin{array}{l}27 \\
28\end{array}$} & $\begin{array}{l}450 \\
430\end{array}$ & $\begin{array}{l}0.0176 \\
0.013\end{array}$ & $\begin{array}{l}0.56065 \\
0.52279\end{array}$ & $\begin{array}{l}0.0118 \\
0.0092 \\
\end{array}$ & $\begin{array}{l}1.3831 \\
1.4225\end{array}$ & $\begin{array}{l}-2.7596 \\
-2.9215\end{array}$ \\
\hline & \multicolumn{2}{|c|}{ Total - Sample 6} & & 0.021 & & \\
\hline $\begin{array}{l}29 \\
30 \\
31 \\
32 \\
33\end{array}$ & $\begin{array}{l}410 \\
410 \\
425 \\
470 \\
580\end{array}$ & $\begin{array}{l}0.00947 \\
0.00547 \\
0.012 \\
0.0233 \\
0.0879\end{array}$ & $\begin{array}{l}0.51466 \\
0.51204 \\
0.58262 \\
0.83339 \\
0.76999\end{array}$ & $\begin{array}{l}3.48 \times 10^{4} \\
2.68 \times 10^{4} \\
5.30 \times 10^{6} \\
0.0012 \\
0.0066 \\
\end{array}$ & $\begin{array}{l}1.4641 \\
1.4641 \\
1.4327 \\
1.3459 \\
1.1723\end{array}$ & $\begin{array}{l}-3.7706 \\
-40.0112 \\
-3.6139 \\
-3.1703 \\
-2.628\end{array}$ \\
\hline & \multicolumn{2}{|c|}{ Total - Sample 7} & & 0.090 & & \\
\hline
\end{tabular}

${ }^{a}$ Estimated. 
TABLE VI -7

Summary of $\operatorname{Tr} i t i u m$ Collected

\begin{tabular}{|c|c|c|c|c|c|c|}
\hline Run & Conditions $^{a}$ & $\mathrm{HT}$ & HTO & $\phi$ & TCOLL & CTCOLL \\
\hline 0 & Tests to 300 & 0.00186 & 0.00006 & $3.3 \times 10^{7}$ & 0.002 & 0.002 \\
\hline 1 & 600/STD & 0.985 & 0.026 & $(0.004)$ & 1.015 & 1.017 \\
\hline 2 & 700/STD & 0.985 & 0.009 & $(0.004)$ & 0.998 & 2.015 \\
\hline 3 & $700 / 1 \% \mathrm{H}_{2}$ & 0.513 & 0.011 & $(0.004)$ & 0.528 & 2.543 \\
\hline 4 & 550/STD & 2.099 & 0.006 & 0.0021 & 2.107 & 4.650 \\
\hline 5 & 500/STD & 0.617 & 0.001 & 0.0004 & 0.618 & 5.268 \\
\hline 6 & $550 / \mathrm{STD}$ & 0.725 & 0.001 & 0.0006 & 0.727 & 5.995 \\
\hline 7 & $600 /$ STD & 1.811 & 0.002 & 0.0029 & 1.816 & 7.811 \\
\hline 8 & 650/STD & 1.008 & 0.001 & 0.0036 & 1.013 & 8.824 \\
\hline 9 & $650 / \mathrm{No} \mathrm{H}_{2}$ & 1.823 & 0.002 & $0.0084^{\mathrm{b}}$ & 1.833 & 10.657 \\
\hline 10 & $400 / \mathrm{No} \mathrm{H}_{2}^{2}$ & 0.040 & 0.000 & 0.0004 & 0.041 & 10.698 \\
\hline 11 & $500 /$ No $\mathrm{H}_{2}$ & 0.300 & 0.003 & 0.0029 & 0.306 & 11.004 \\
\hline 12 & $550 / \mathrm{No} \mathrm{H}_{2}^{2}$ & 1.245 & 0.000 & 0.0214 & 1.267 & 12.271 \\
\hline 13 & $600 / \mathrm{No} \mathrm{H}_{2}^{2}$ & 2.197 & 0.000 & $0.0783^{b}$ & 2.275 & 14.546 \\
\hline 14 & $700 /$ No $\mathrm{H}_{2}$ & 1.560 & 0.001 & 0.1332 & 1.694 & 16.240 \\
\hline 15 & $700 / \mathrm{STD}^{2}$ & 0.715 & 0.000 & 0.0566 & 0.772 & 17.012 \\
\hline 16 & $700 /(300 \mathrm{SCCM})$ & 0.503 & 0.001 & 0.0312 & 0.535 & 17.547 \\
\hline 17 & $700 /(30 \mathrm{scCM})$ & 1.109 & 0.000 & 0.1394 & 1.248 & 18.795 \\
\hline 18 & $700 /$ STD & 0.380 & 0.000 & 0.0424 & 0.422 & 19.217 \\
\hline 19 & 650/STD & 0.294 & 0.000 & $0.0203^{b}$ & 0.314 & 19.531 \\
\hline 20 & $650 / 0.2 \% \quad \mathrm{O}_{2}$ & 0.028 & 0.000 & 0.0014 & 0.029 & 19.560 \\
\hline 21 & 650/STD & 1.092 & 0.000 & 0.0083 & 1.100 & 20.660 \\
\hline 22 & $600 / \mathrm{STD}$ & 0.351 & 0.000 & 0.0020 & 0.353 & 21.013 \\
\hline 23 & $550 / \mathrm{STD}$ & 1.077 & $\simeq .001$ & $0.0033^{b}$ & 1.082 & 22.095 \\
\hline 24 & $600 / \mathrm{STD}$ & 0.576 & 0.002 & 0.0023 & 0.580 & 22.675 \\
\hline 25 & 560/STD & 0.537 & 0.007 & 0.0017 & 0.546 & 23.221 \\
\hline 26 & $550 / \mathrm{STD}$ & 1.213 & 0.048 & $0.0033^{b}$ & 1.264 & 24.485 \\
\hline 27 & 525/STD & 2.128 & 0.333 & 0.0118 & 2.473 & 26.958 \\
\hline 28 & $500 /$ STD & 2.104 & 0.857 & $0.0093^{b}$ & 2.970 & 29.928 \\
\hline 29 & $480 /(300$ SCCM $)$ & 0.543 & 0.211 & 0.0003 & 0.754 & 30.682 \\
\hline 30 & 480/STD & 0.721 & 0.004 & 0.0003 & 0.725 & 31.408 \\
\hline 31 & 500/STD & 0.740 & 0.003 & 0.0005 & 0.744 & 32.152 \\
\hline 32 & $550 / \mathrm{STD}$ & 1.271 & 0.004 & 0.0012 & 1.276 & 33.428 \\
\hline \multirow[t]{2}{*}{33} & 650/STD & 1.666 & $\underline{0.009}$ & $0.0066^{b}$ & 1.682 & 35.110 \\
\hline & Total: & 32.958 & 1.543 & 0.608 & 35.110 & \\
\hline
\end{tabular}

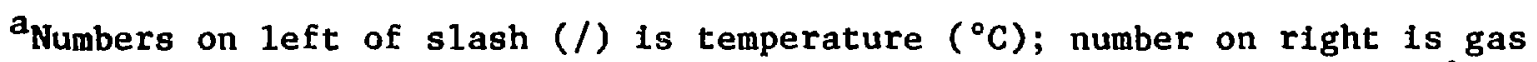
composition; STP means standard conditions, $1 . e$. flow rate of $100 \mathrm{~cm}^{3} / \mathrm{min}$ and $0.1 \% \mathrm{H}_{3}$ added.

bermeation samples. 
The output of the Xanne chamber records the HT signal in volts, which is equal to the logarithm of the tritium concentration in microcuries per cubic meter. From the outputs of the calibrated transducers which monttor pressire and flow, the HT release rate, in units of microcuries per minute, is then calculated. The absolute accuracy of this release rate was about $30 \%$, owing to the many scale changes (three to four orders of magnitude) which occurred after calibrating the Kanne chamber at low levels. However, the observed data were recalibrated to the data for the HT, which was collected and analyzed by scintillation counting. The latter data had an absolute accuracy of $5 \%$. This callbration procedure involved multiplying the release rates for HT by a factor of 0.65 and subtracting the baseline correction, which increased from zero at the beginning of the experiment to a value of $75 \mu \mathrm{Cl} / \mathrm{min}$ at the end. The dynamic data for tritium release, after calibration, agreed with the integral data from the scintillation counting within $10 \%$; thus the absolute accuracy of the HT release rate was about $10 \%$.

For a given run, the experimental parameters (e.g., temperature) are fixed and the tritium (HT) release rate was monitored. At steady state, the release rate is invariant in time and equal to the generation rate. The tricium generation rate was calculated from the recorded neutron flux levels, as later discussed in Sec. VII.D. The absolute accuracy of the tritium generation rate was $10 \%$.

Presented below are the dynamic data for temperature, tritium (HT) release rate, and tritium generation rate for the 33 experimental runs. The time given for all graphical data are in Central Standard Time.

Before the full-power operation of the TRIO irradiation experiment, tests of the ORR were carried out at low power, $3 \mathrm{~kW}$ to $12 \mathrm{MW}$ (full power, $30 \mathrm{MW}$ ); they were done subsequent to the perfod of about three months when the reactor was down for repalrs to the reactor coolant system. A reactor power of 300 $\mathrm{kW}$, or $1 \%$ of full power, is given the name " $\mathrm{N}_{\mathrm{L}}$ " because it is considered to be the lowest power that can be quantitavely measured. The data for temperature and tritium release rate data from Run 0 are presented in Figs. VI-6 and -7 , respectively.

The recording of temperature began at 2100 on March 10, 1983. One thermocouple element, TR-901, read $\sim 15 \mathrm{deg}$ high owing to an electronic (reference 


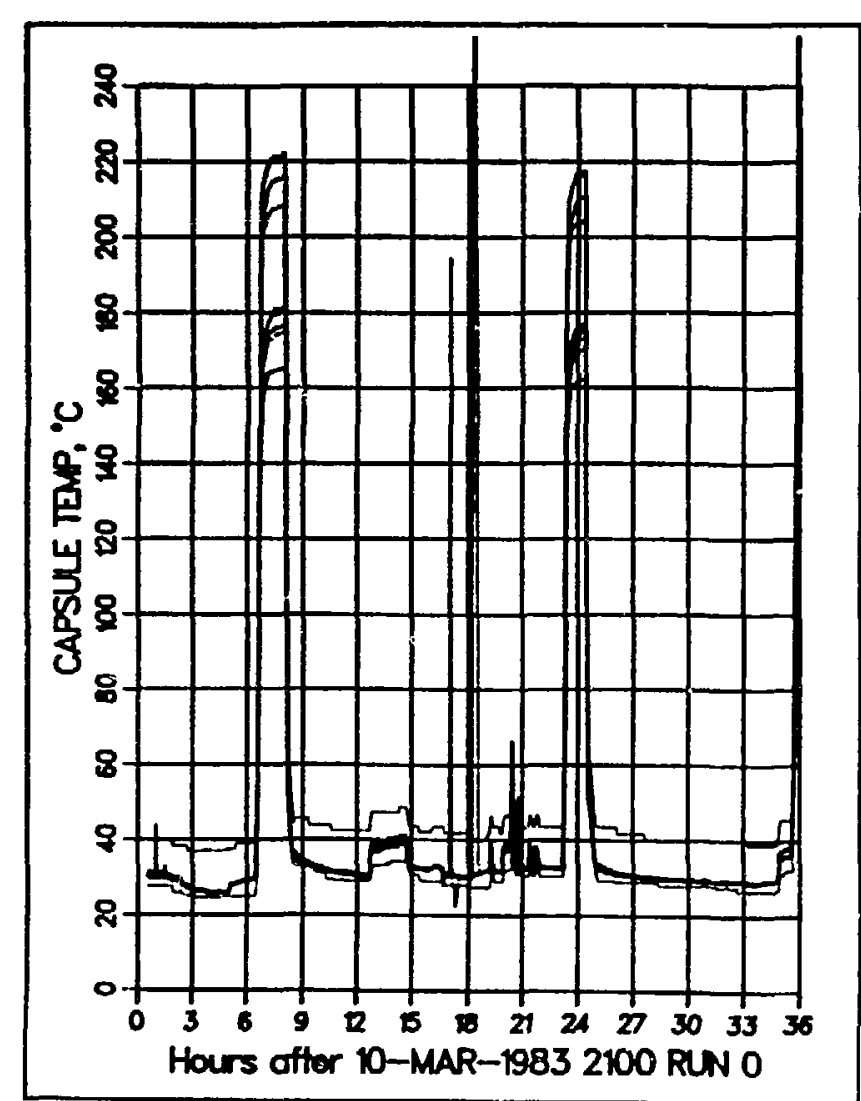

Fig. VI-6. Recorded temperatures for Run 0.

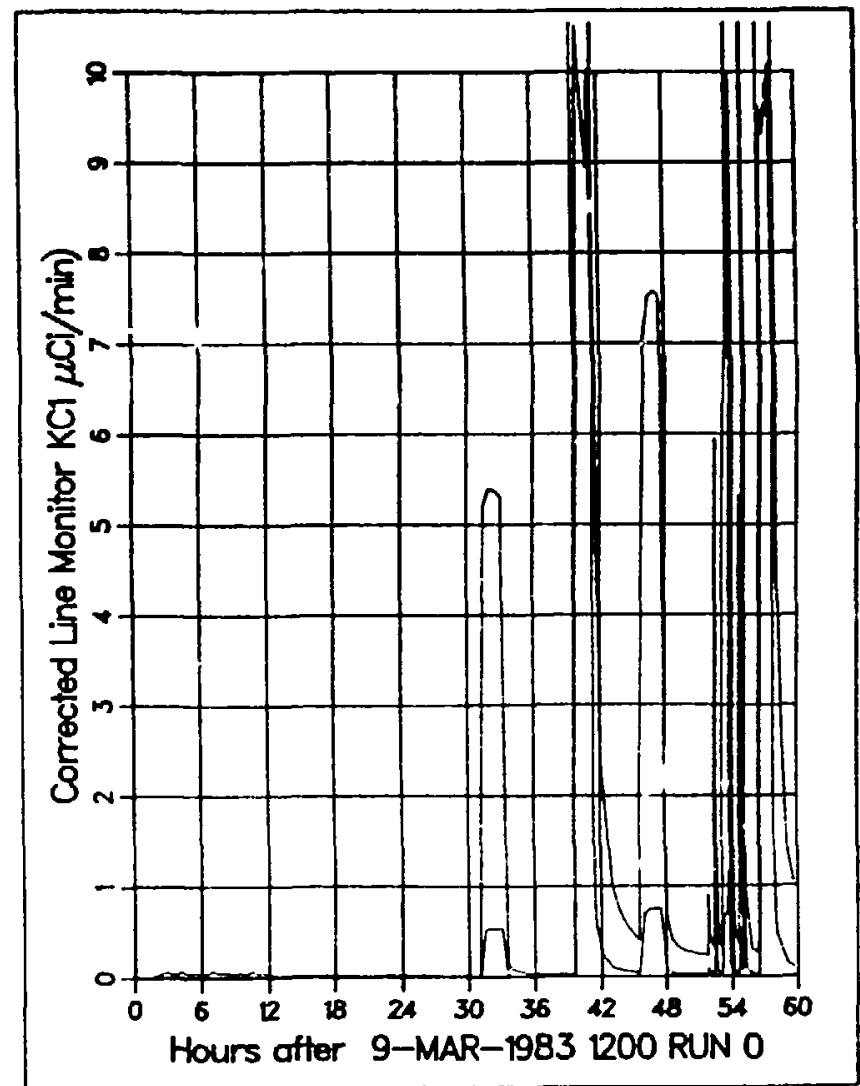

Fig. VI-7. Tritium release rate for Run 0 (shaded). (The unshaded line represents scale expansion by a factor of 100.) 
point) problem. As shown, the greatest responses were at 0400 and at 2030 on March 11; these peaks correspond to 1-h tests when the reactor was at $12 \mathrm{MW}$. With pure helium in the gas gap, the temperature rose to $\sim 200^{\circ} \mathrm{C}$ when $\mathrm{ORK}$ was at $40 \%$ full power. Other tests at $1 \%$ full power showed a measurable temperature rise of $\sim 4^{\circ} \mathrm{C}$. As will be discussed later, the data provide a good deal of information on heat transfer. For example, thermal conductivity of lithium aluminate from 30 to $200^{\circ} \mathrm{C}$ can be derived from the radial temperature gradient .

The recording of tritium release began on March 9, 1983. Generally, the peak heights are proportional to the tritium generation rate, with approximately $10 \%$ of the tritlum released. This release is attributed to a recoil mechanism, which is discussed is Sec. IX.A. Another feature is that measurable tritium release occurred on the afternoon of March 9, when the reactor power was at only 3 to $30 \mathrm{~kW}$. These levels are barely detectable by the reactor instruments. It appears that the tritium monitor is the most sensitive detector for neutrons at the ORR facility.

Run 1 had a nominal temperature (the average of $\mathrm{T} 804$ and T808) of $600^{\circ} \mathrm{C}$ with a helium $+0.1 \% \mathrm{H}_{2}$ sweep gas flowing at $100 \mathrm{~cm}^{3} / \mathrm{min}$. The data temperature and tritium release data are given in Figs. VI-8 and VI-9, respectively. The nominal temperature was held at $400^{\circ} \mathrm{C}$ for $2 \mathrm{~h}$ at the start to reduce the chances for thermal shock. At 1200 on March 12, the nominal conditions were established. The thermal data show that the total temperature range is about $180^{\circ} \mathrm{C}$, that the radial gradients are about $120^{\circ} \mathrm{C}$, and that longitudinal gradients are $\sim 20^{\circ} \mathrm{C}$. One thermocouple, TR801 $\left(\sim 630^{\circ} \mathrm{C}\right)$ showed a large number of spikes which were caused by the electronic recording system. Also, oscillations in temperature are shown at hours 21 and 31.

The tritium release rate and the generation rate are shown in Fig. VI-8. The ORR reached full power at hour 4, when the generation rate thus reached the level of $350 \mu \mathrm{Cl} / \mathrm{min}$. Because the temperature was 1 ow $\left(400^{\circ} \mathrm{C}\right)$ from hour 4 until hour 6, trittum accumulated in the solid. When the temperature was increased to nominal conditions, which were chosen such that steady state could be achleved in a few hours, the release curve briefly rose above the generation curve; and some of the excess tritium that had built up over the preceeding two hours was released. Within approximately two hours, the release rate 


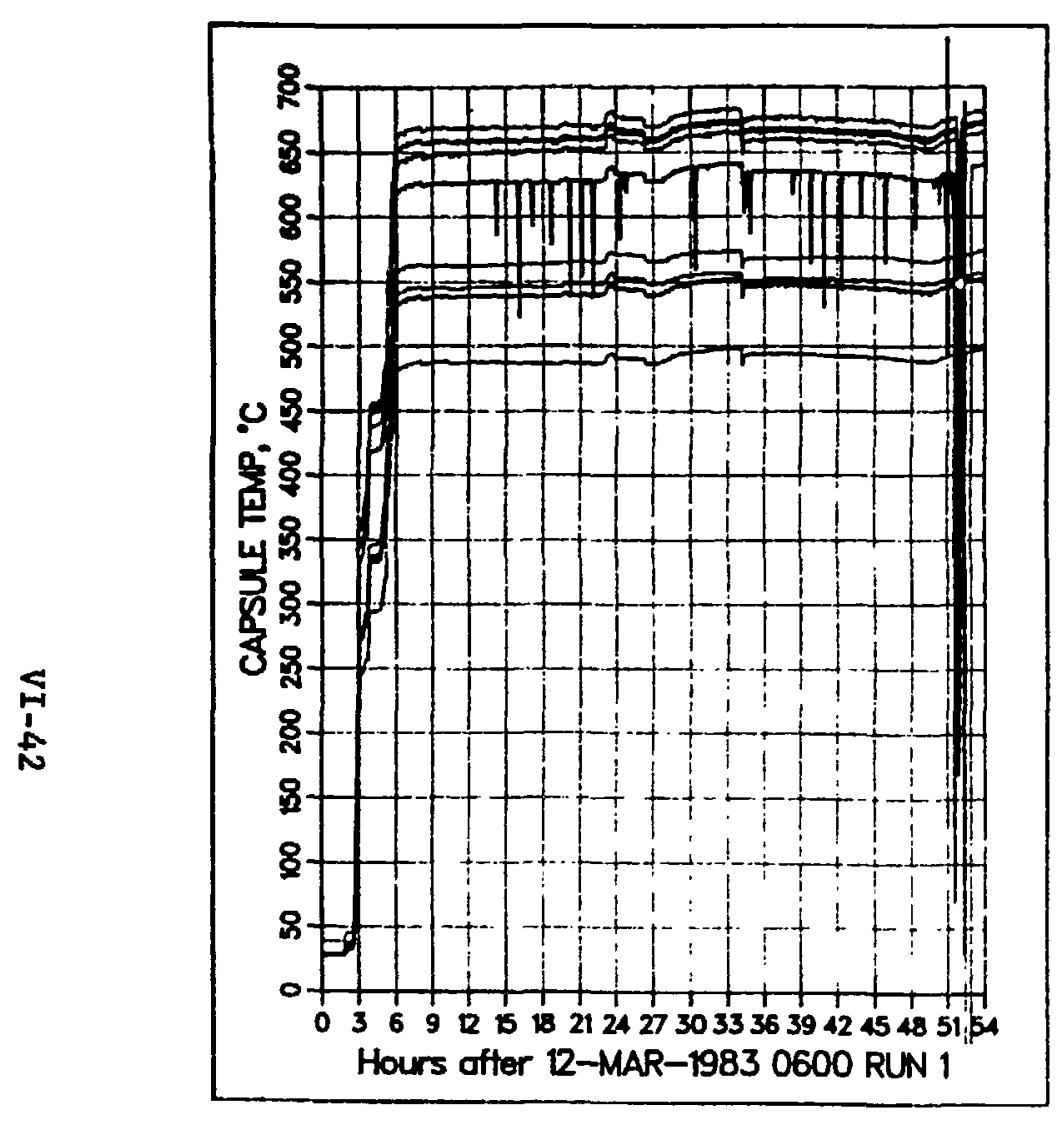

Fig. VI-8. Recorded temperature for Run 1.

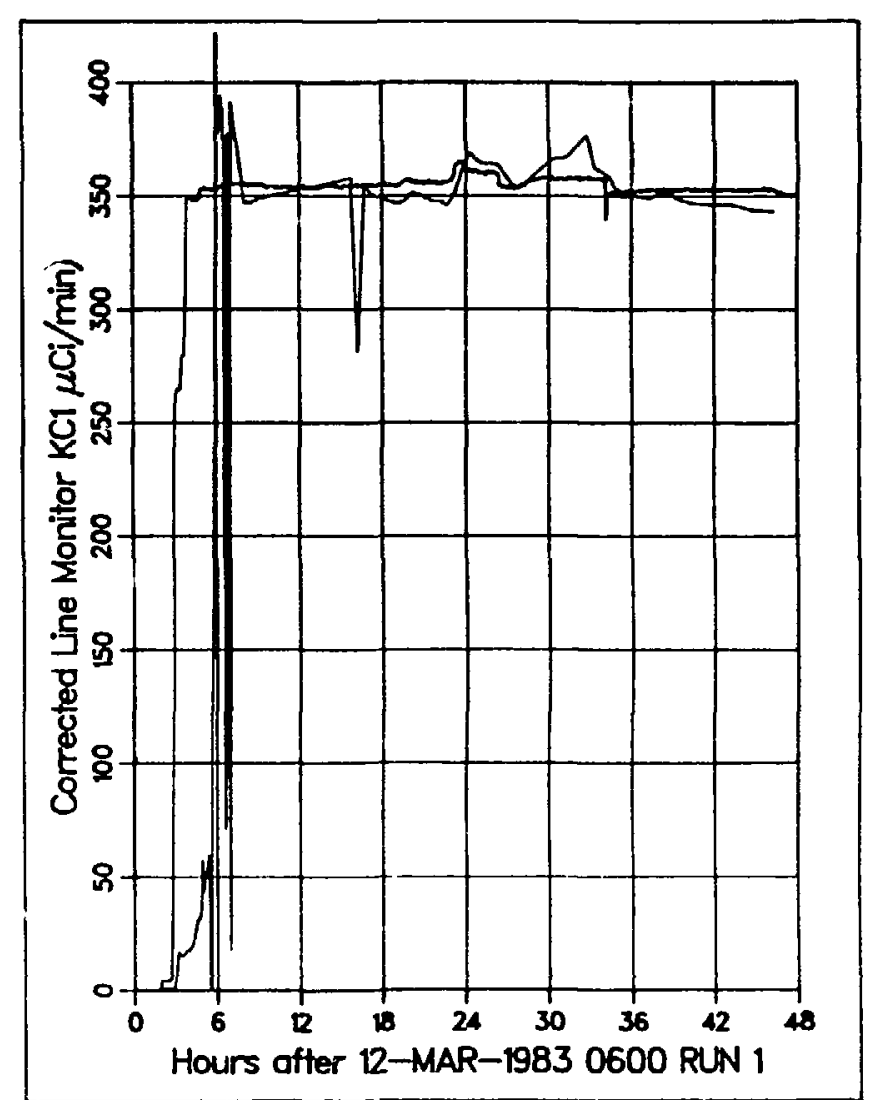

Fig. VI-9. Tritium release rate and generation rate for Run 1 . 
state was achieved very rapidly in the first run. The negative spike in the release curve at hour 17 is due to changing samples, which briefly altered flow. In addition, the temperature excursions at hours 21 and 31 cause significant perturbations in the tritium release curve.

Run 2 was at a nominal temperature of $700^{\circ} \mathrm{C}$ with a sweep gas of helium $+0.1 \% \mathrm{H}_{2}$ flowing at a rate of $100 \mathrm{~cm}^{3} / \mathrm{min}$. The temperature and tritium release data are shown in Figs. VI-10 and VI-11, respectively.

The tritium release curve shows a positive spike, followed by a return to steady-state within approximately three hours. The positive spike indicates that the tritium inventory in the solid is less at higher temperatures. There is a temperature spike at 1600 on March 14, which translateds to a sma.11 peak in the tritium release curve at 1800 on March 14. There was also a reactor setback at 1000 on March 15, which caused brief temporary fluctuations in temperature and tritium release.

Run 3 was at a nominal temperature of $700^{\circ} \mathrm{C}$ with a sweep gas flow rate of $100 \mathrm{~cm}^{3} / \mathrm{min}$, as in Run 2. The concentration of hydrogen in the sweep gas was increased from 0.1 to $1.0 \%$. The temperature and tritium release data are shown in Figs. VI-12 and -13 , respectively. The temperatures were nearly constant throughout the run except for two small transients which occurred at 1500 on March 16 and at 0800 on March 17. The $150^{\circ} \mathrm{C}$ decrease at 1400 on March 17 is the start of Run 4. At the beginning of the run, the tritium release curve shows a very sharp spike, which returns to steady state in less than one hour. The positive spike indicates that the increase in hydrogen level in the sweep gas enhances tritium release.

Run 4 was at a nominal temperature of $550^{\circ} \mathrm{C}$ with a flow rate of 100 $\mathrm{cm}^{3} /$ min for the sweep gas of helium $+0.1 \% \mathrm{H}_{2}$. These sweep gas conditions were used for most of the runs. The temperature and tritium release data are shown in Figs. VI-14 and -15 , respectively. The temperature data are generally rather smooth except for a reactor shutdown at 0900 on March 21, a sma1l transient at 1000 on March 19, and a series of oscillations on March 21 and March 22. A11 these transients are the result of changes in the neutron flux: levels, as can be noted by examination of the tritium production curve in Fig. VI-15. The tritium release curve shows a negative peak at the beginning of Run 4, followed by a slow approach to steady state. The transient in tritium release at hour 20 is from changes in flow caused by changing samples. The tritium release curve does not reach steady state durfing Run 4. 


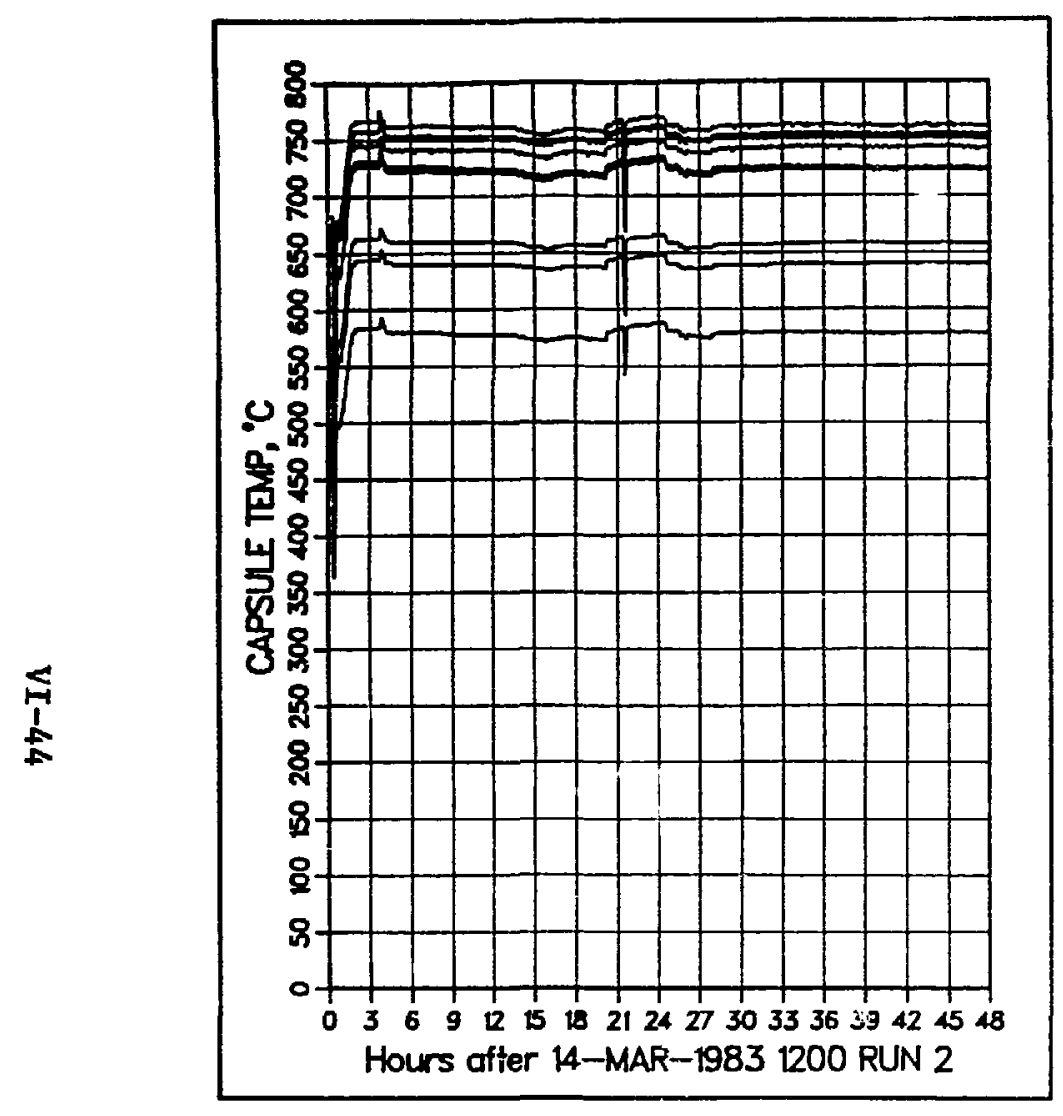

Fig. VI-10. Recorded temperatures for Run 2.

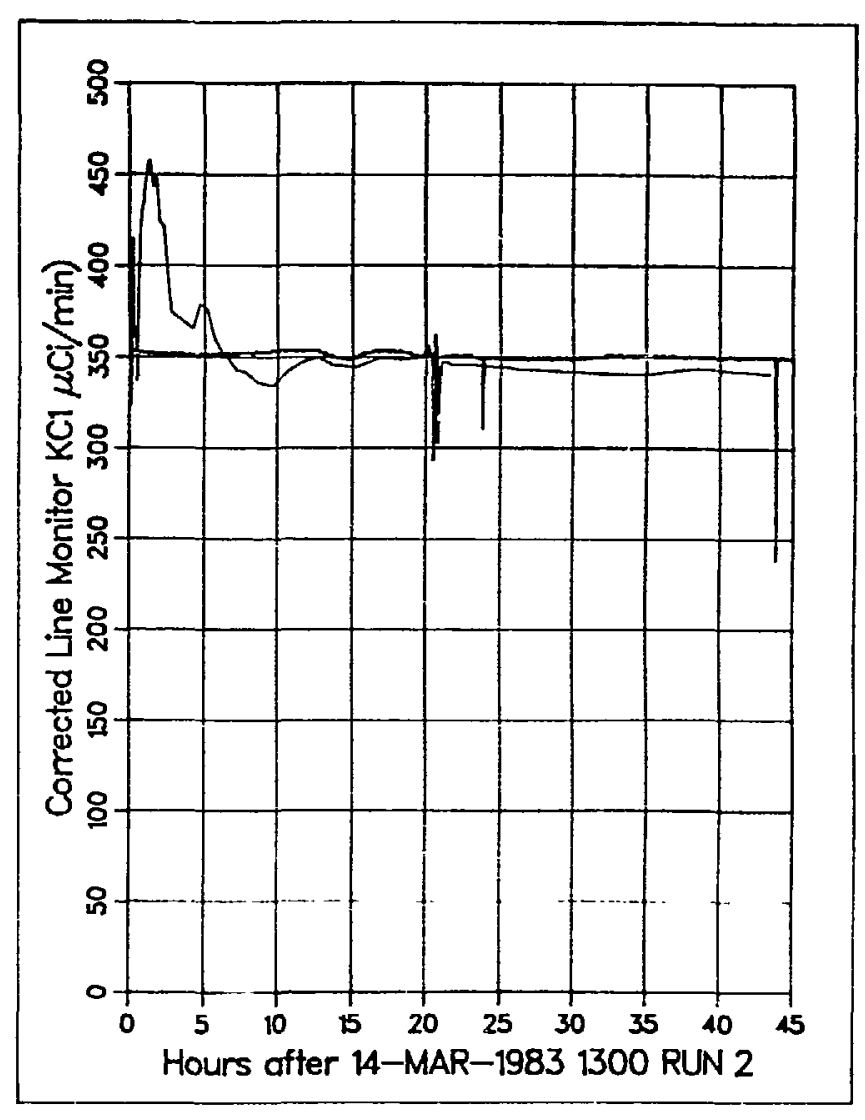

Fig. VI-11. Tritium release rate for Run 2. 


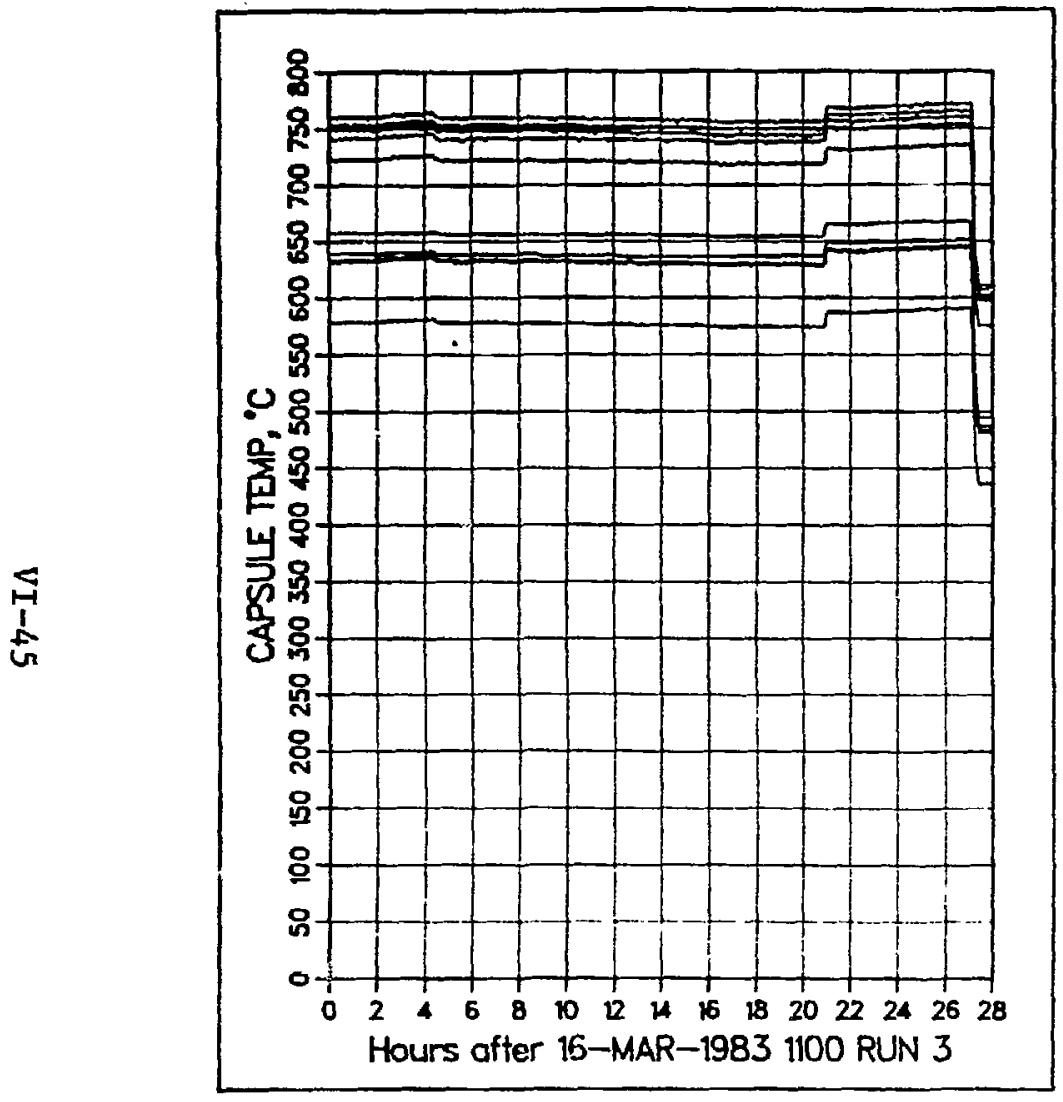

Fig. VI-12. Recorded temperatures for Run 3.

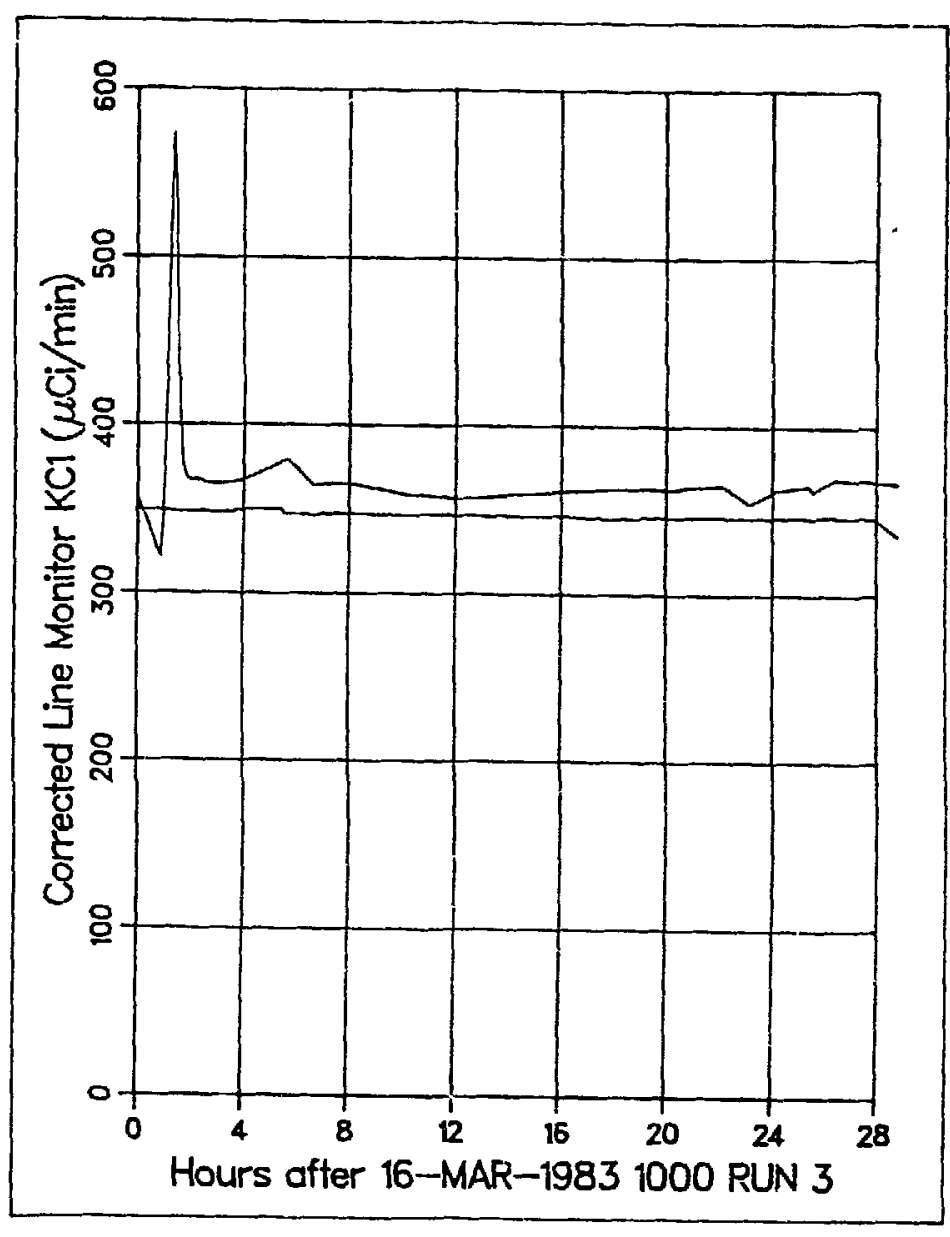

Fig. VI-13. Tritium release rate for Run 3. 


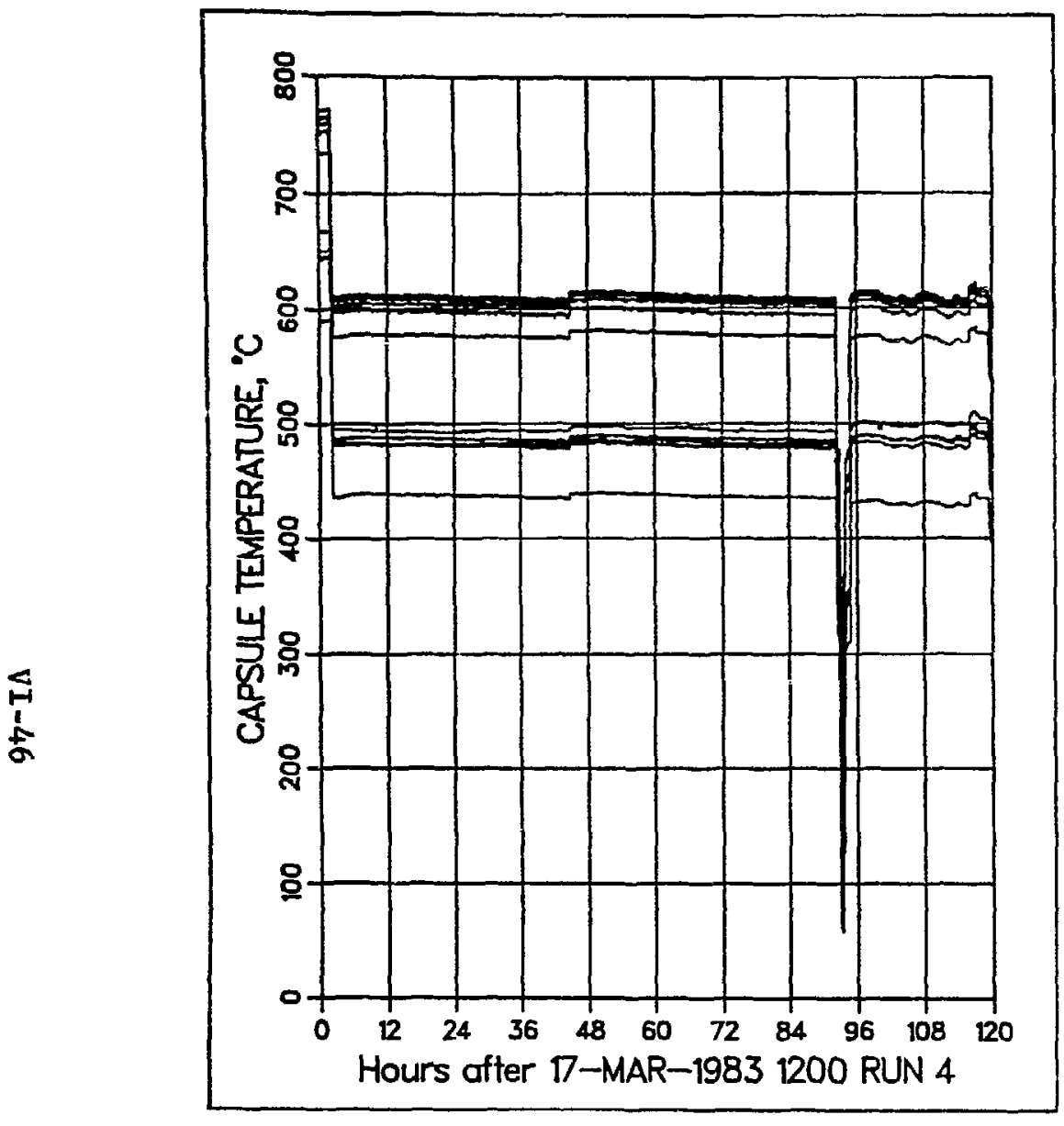

Fig. VI-14. Recorded temperatures for Run 4.

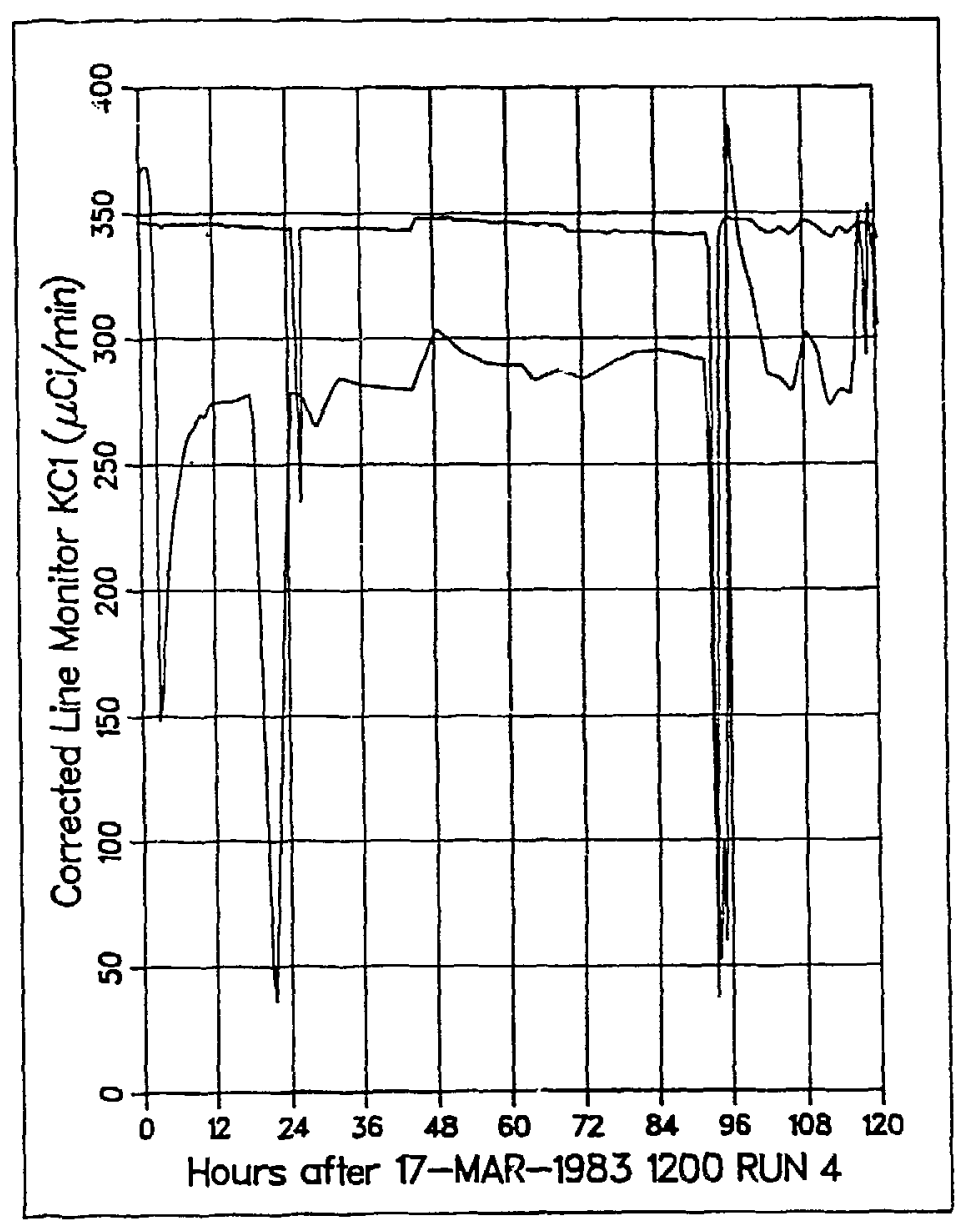

Fig. VI-15. Tritium release rate for Run 4 . 
Run 5 wis at a nominal temperature of $500^{\circ} \mathrm{C}$ with a flow rate of 100 $\mathrm{cc} / \mathrm{min}$ and with a sweep gas composition of helium $+0.1 \% \mathrm{H}_{2}$. The temperature and tritium release data are shown in Figs. VI-16 and -17 , respectively. The tritium release curve shows a negative peak, followed by a very slow approach to steady state. Steady state is not achieved in Run 5 .

Run 6 was at a nominal temperature of $550^{\circ} \mathrm{C}$ with a flow rate of 100 $\mathrm{cm}^{3} / \mathrm{min}$ and for the sweep gas of hellum $+0.1 \% \mathrm{H}_{2}$. The iemperature and tritium release data are shown in Figs. VI-18 and -19 , respectively. Neutron flux and temperature varied by $1 \%$ for the entire run. The tritium release curve shows a strong positive peak, followed by a smooth return to steady state at about $20 \mathrm{~h}$.

Run 7 was at a nominal temperature of $600^{\circ} \mathrm{C}$ with a flow rate of 100 $\mathrm{cm}^{3} / \mathrm{min}$ for the sweep gas of helium $+0.1 \% \mathrm{H}_{2}$. The temperature and tritium release data are shown in Figs. VI-20 and -21 . The temperatures and the neutron flux are again essentially constant for this run. The apparent temperature excursions for two thermocouples (TR801 and TR901) are due to problems with the electronic recording system. The tritium release curve has a drop in the middle of the peak caused by the scale change. Whenever the signal rises above $1000 \mu \mathrm{Ci} / \mathrm{min}$, the scale changes automatically and this causes an offset problem. The tritium release curve shows a large positive spike followed by a return to steady state within $20 \mathrm{~h}$.

Run 8 was at a nominal temperature of $650^{\circ} \mathrm{C}$ with a flow rate of 100 $\mathrm{cc} / \mathrm{min}$ and with a sweep gas composition of helium $+0.1 \% \mathrm{H}_{2}$. The temperature and tritium release data are shown in Figs. VI-22 and -23 , respectively. The temperatures and the neutron $f 1 u x$ levels were essentially constant throughout Run 8, except for a slight drop in flux and temperature at 0300 on March 29. The tritium release curve shows a positive spike followed by a return to equilibrium in about $12 \mathrm{~h}$.

Run 9 was at a nominal temperature of $650^{\circ} \mathrm{C}$ with a sweep gas flow rate of $100 \mathrm{~cm}^{3} / \mathrm{min}$. The sweep gas composition was changed, going from $0.1 \%$ hydrogen added to pure helium with no hydrogen added. The temperature and tritium release data are shown in Figs. VI-24 and -25 , respectively. The temperatures are essentially constant, and all conditions are the same as Run 8 except for the addition of hydrogen to the sweep gas. Removal of the hydrogen additive 


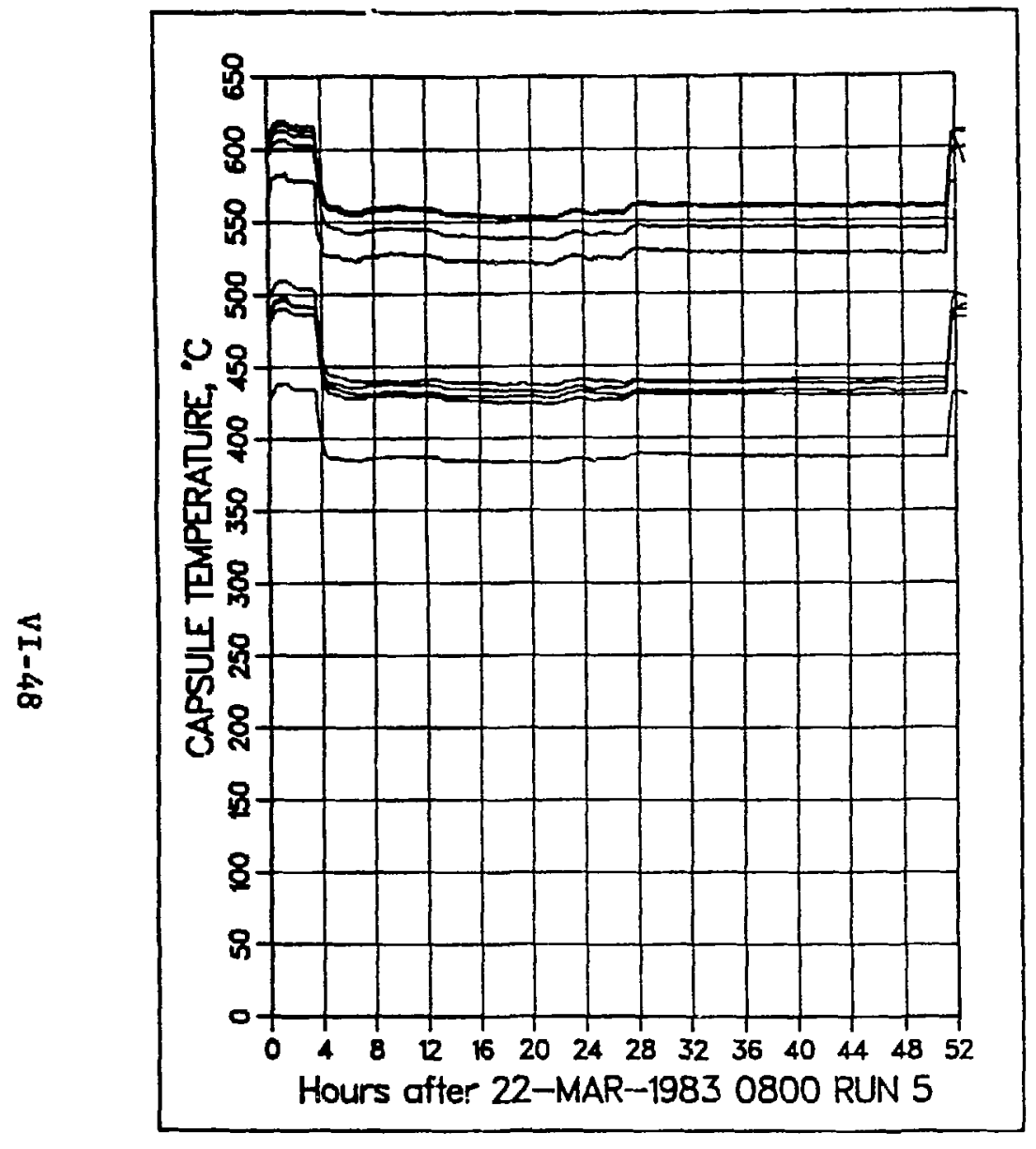

Fig. VI-16. Recorded temperatures for Run 5.

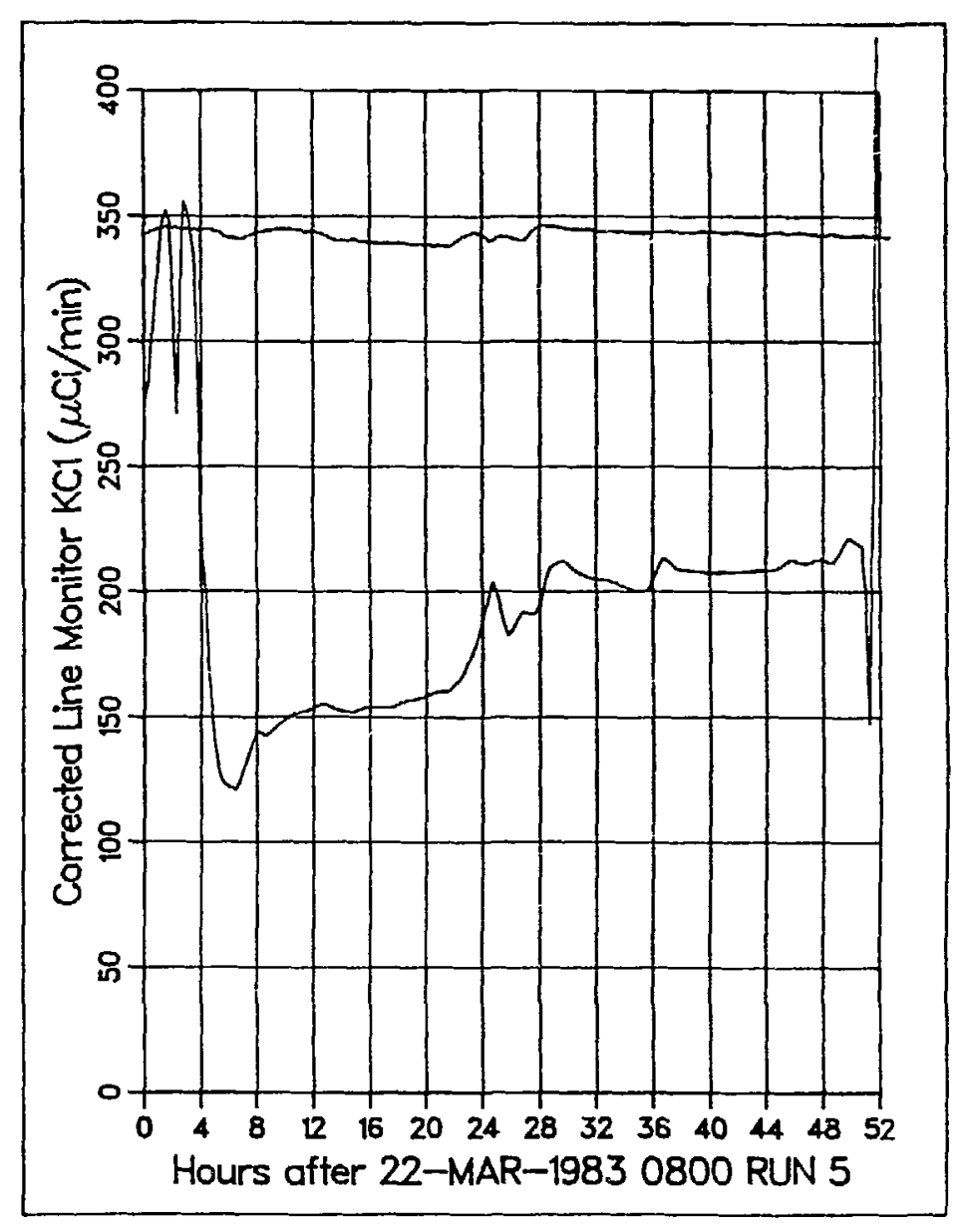

Fig. VI-17. Tritium release rate for Run 5. 


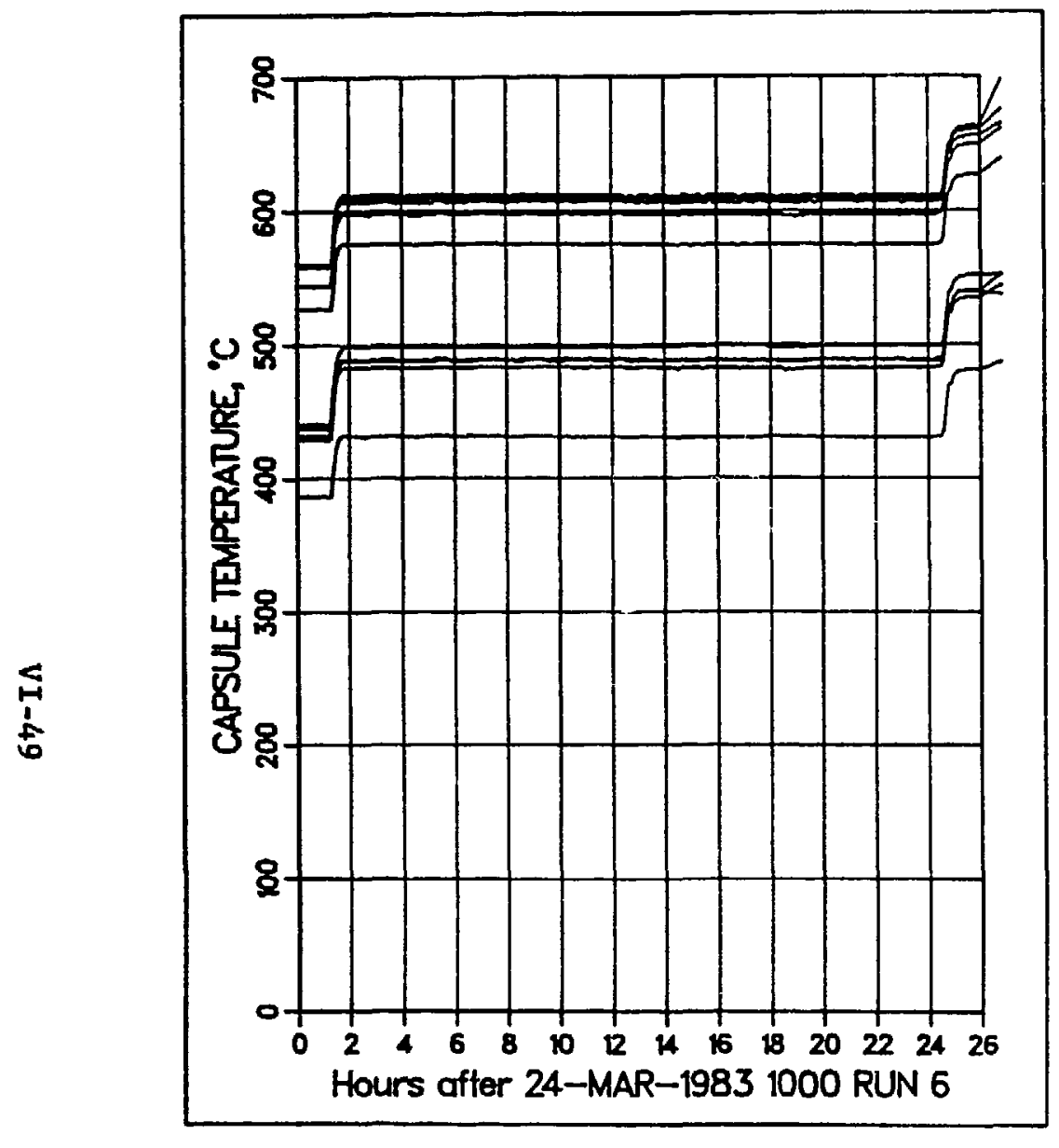

FIg. VI-13. Recorded temperatures for Run 6 .

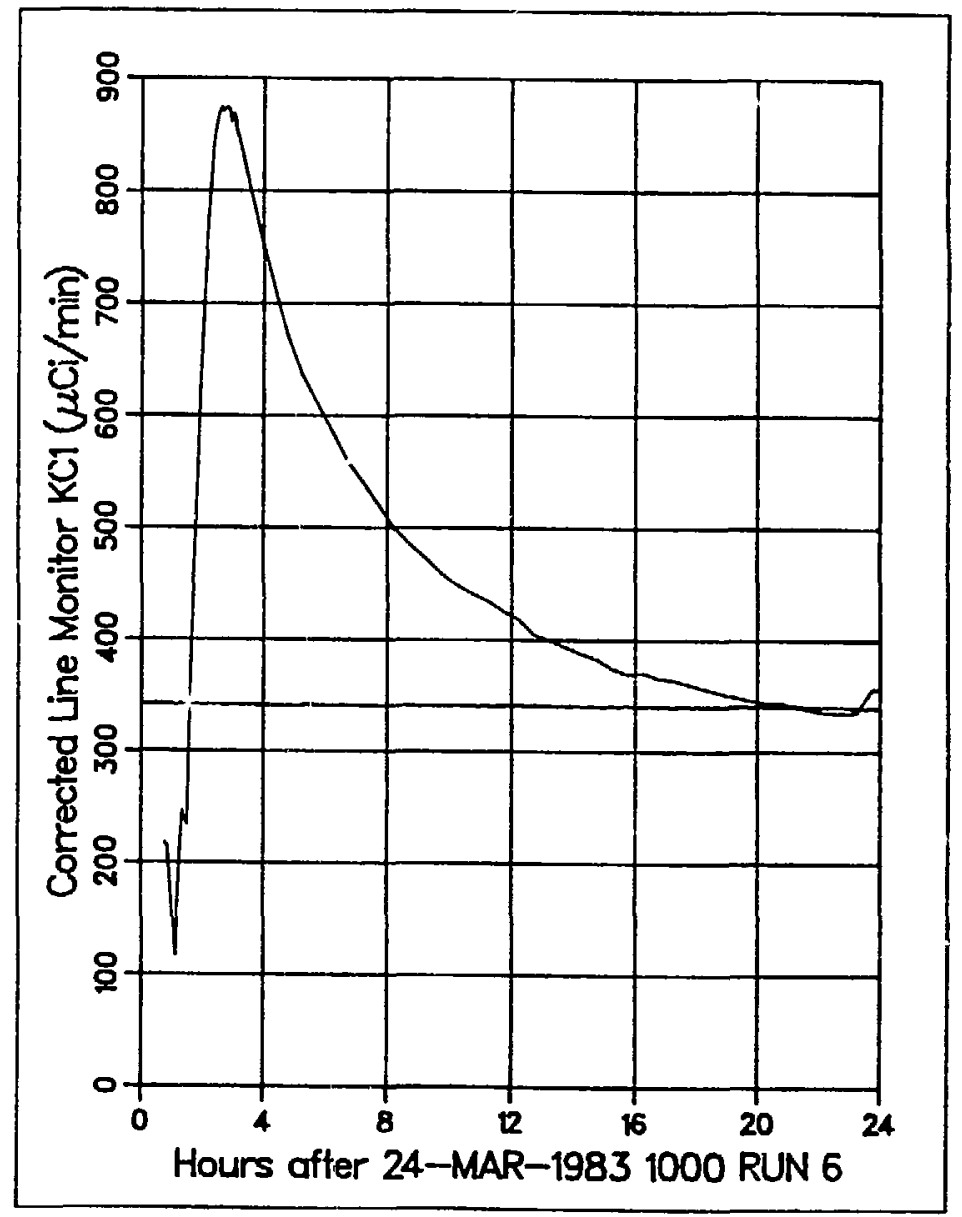

Fig. VI-19. Tritium release rate for Run 6 . 


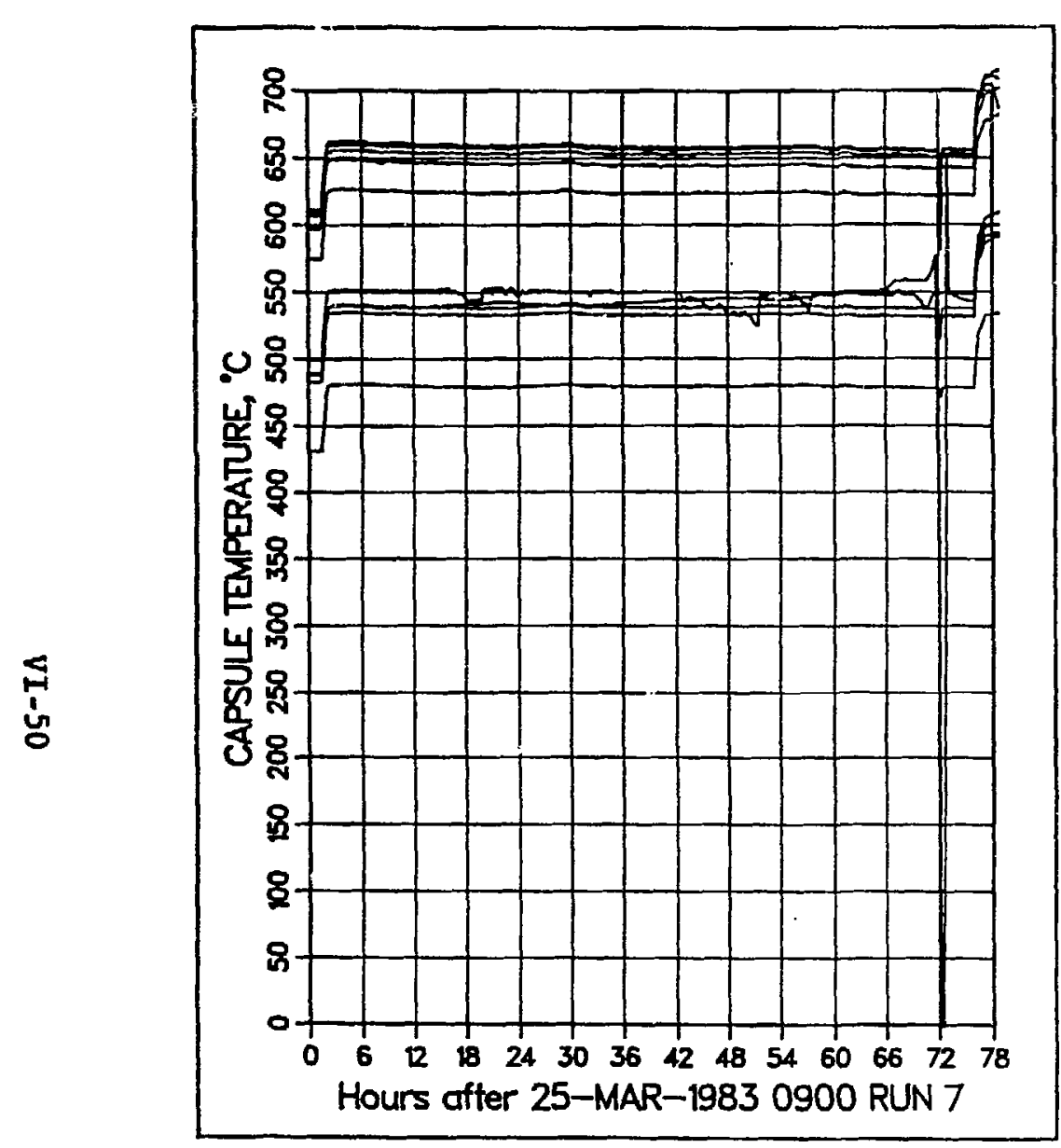

Fig. VI-20. Recorded temperatures for Run 7.

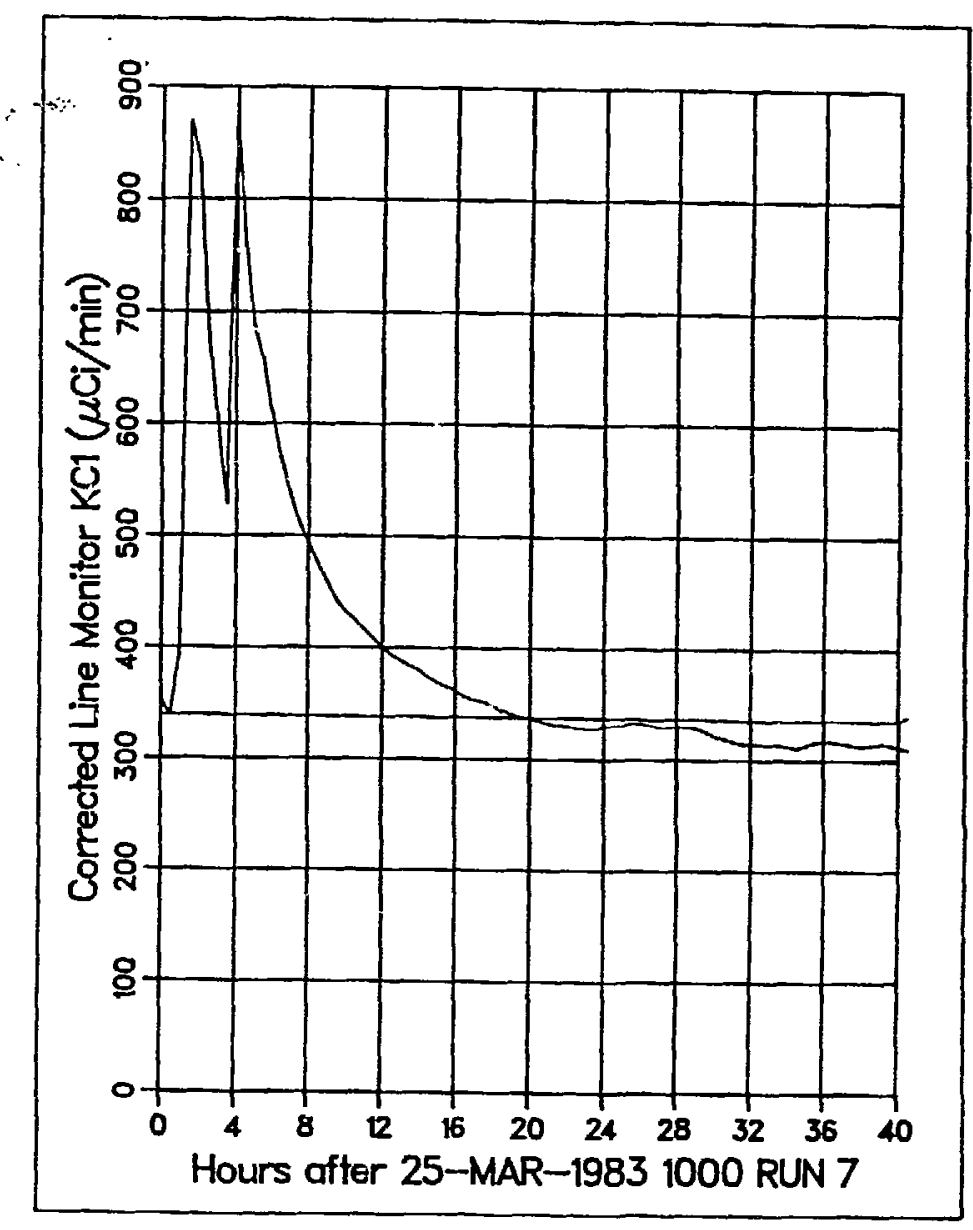

Fig. VI-21. Tritium release rate for Run 7 . 


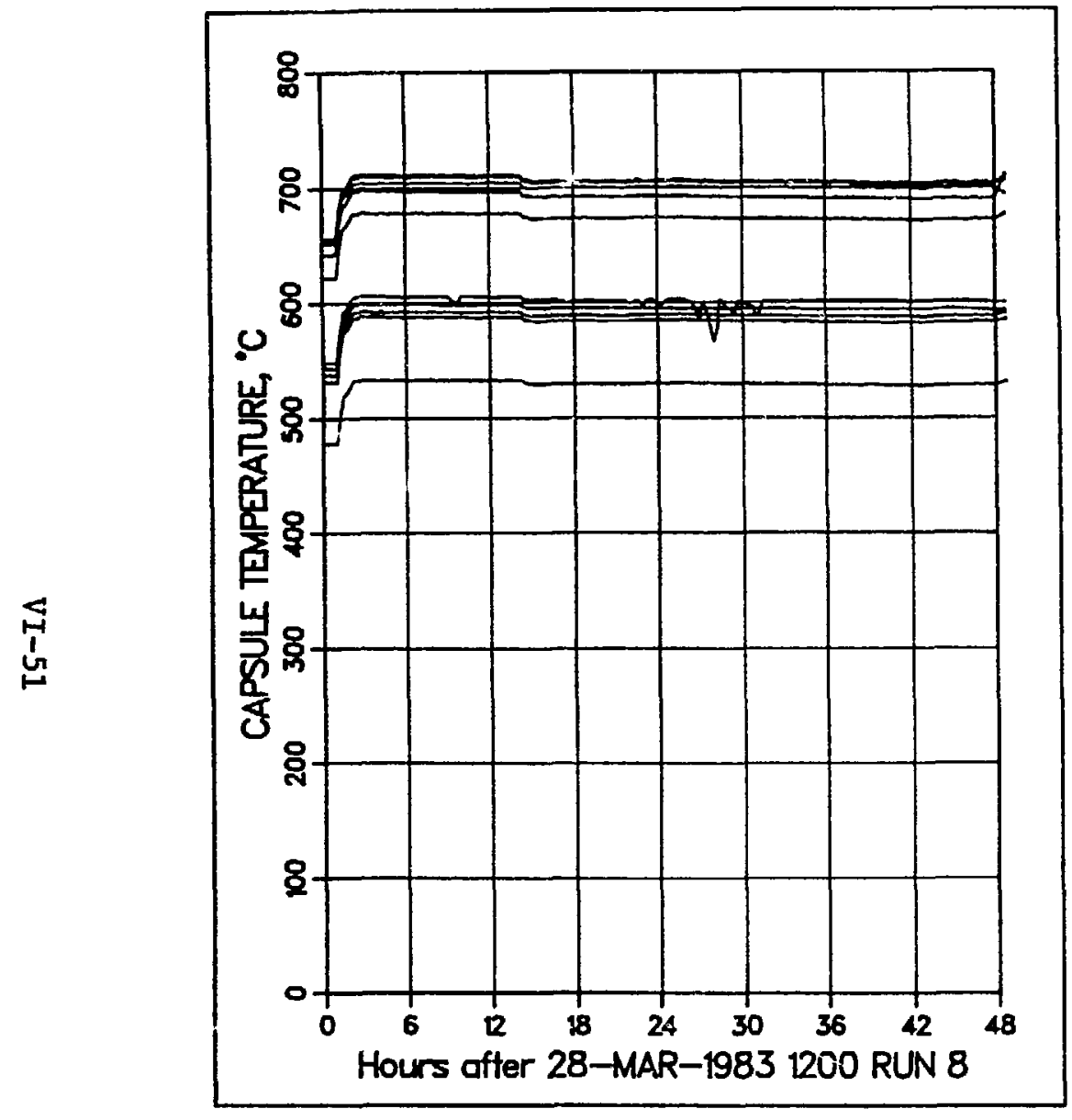

Fig. VI-22. Recorded temperatures for Run 8.

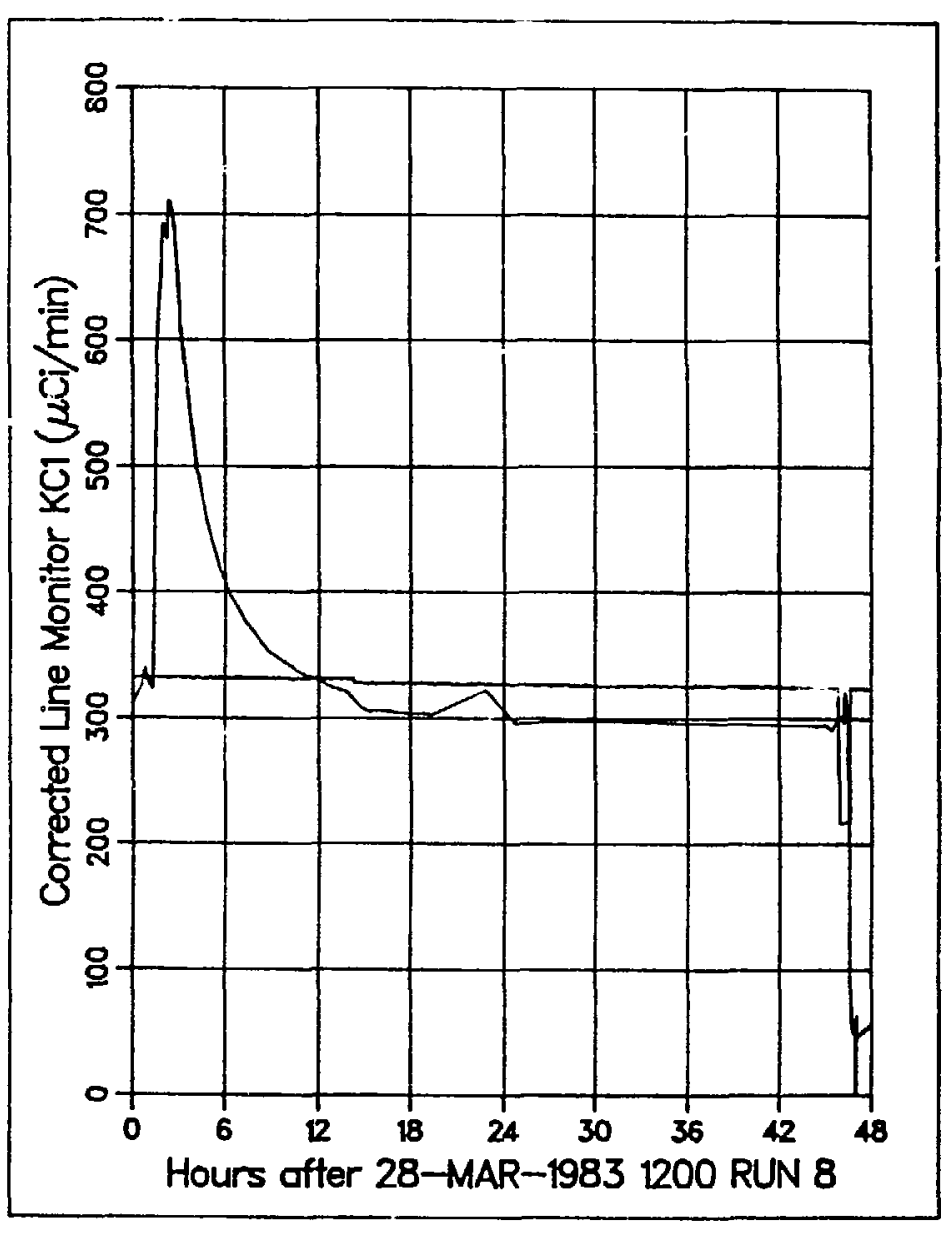

Fig. VI-23. Tritium release rate for Run 8 . 


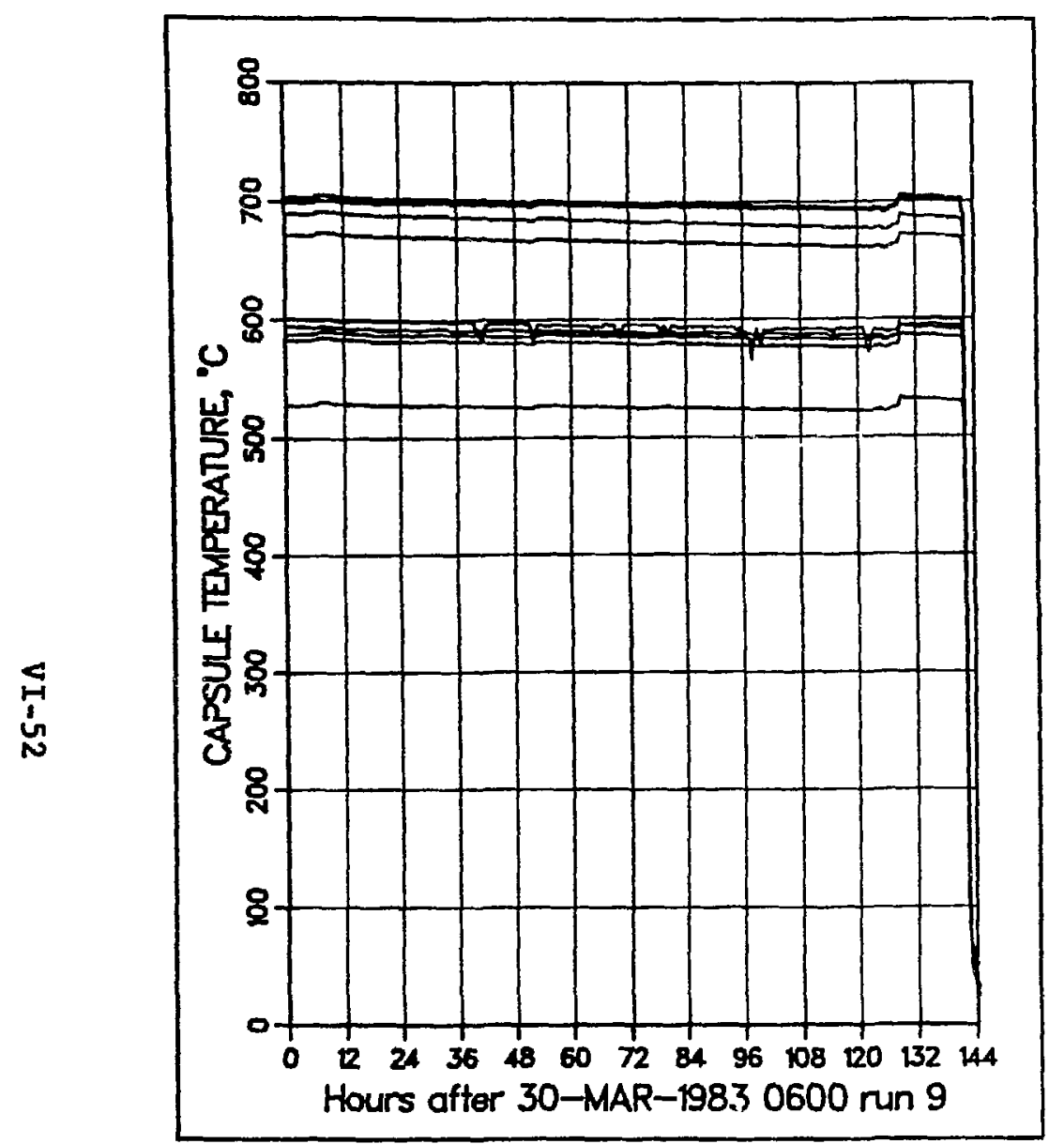

Fig. VI-24. Recorded temperatures for Run 9.

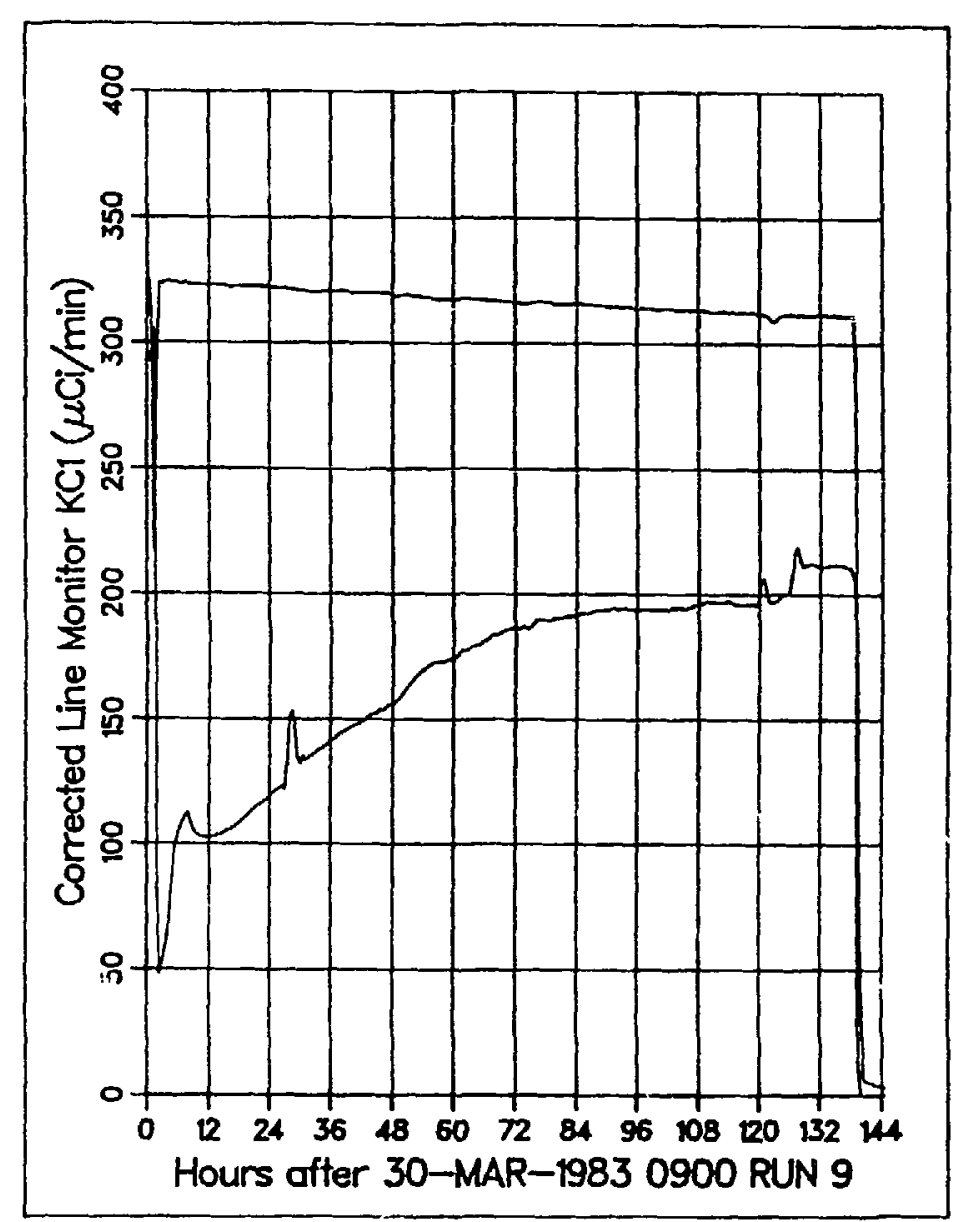

Fig. VI-25. Tritium release rate for Run 9. 
has a profoundly nega:ive impact upon tritium release rate, as is evidenced by the large negative peak and the very slow return to equilibrium. Steady state is not achieved in Run 9.

Runs 10 and 11 were performed to investigate the transport rates of radionuclides at lower temperatures. Run 10 was at a nominal temperature of $400^{\circ} \mathrm{C}$, and Run 11 was at a nominal temperature of $500^{\circ} \mathrm{C}$. The sweep gas was pure helium flowing at $100 \mathrm{~cm}^{3} / \mathrm{min}$. The temperature data for the two runs are shown in Figs. VI-26 and -27 . The tritium release data are given in Fig. VI-28. Tritium release was minimal for these runs and steady state was not achieved.

Run 12 was at a nominal temperature of $550^{\circ} \mathrm{C}$ with a flow rate of 100 $\mathrm{cm}^{3} / \mathrm{min}$ for the sweep gas composition of pure helium. The temperature and tritium release data are shown in Figs. VI-29 and -30 , respectively. Steadystate tritium was not reached in four days.

Run 13 was at a nominal temperature of $600^{\circ} \mathrm{C}$ with a flow rate of 100 $\mathrm{cm}^{3} / \mathrm{min}$ and for the sweep gas of pure helium. The temperature and tritium release data are shown in Figs. VI-31 and -32 , respectively.

There was a reactor shutdown on April 13, as indicated by the low temperatures in Fig. VI-31. The positive spikes in the tritium release curve indicate a net release of tritium after the run had begun, when the reactor was restarted on April 14. The spike is followed by a return to steady state $\sim 100 \mathrm{~h}$ after the reactor restart. At 2200 on Apr 11 16, a slight decrease in flux caused a drop in temperature of $\sim 5 \mathrm{deg}$. This temperature drop causes a noticeable dip in the tritium release curve.

Run 14 was at a nominal temperature of $700^{\circ} \mathrm{C}$ with a flow rate of 100 $\mathrm{cm}^{3} / \mathrm{min}$ for the sweep gas of pure hellum. The temperature and tritium release data are shown in Figs. VI-33 and -34, respectively. The neutron flux and the temperatures are rather constant throughout the run. The tritium release curve shows a positive spike followed by a return to steady-state conditions.

Run 15 was at a nominal temperature of $700^{\circ} \mathrm{C}$ with a flow rate of 100 $\mathrm{cm}^{3} / \mathrm{min}$ for the sweep gas of helium $+0.1 \% \mathrm{H}_{2}$. The temperature and tritium releaze data are shown in Figs. VI-35 and -36 , respectively. The only change from the previous run is the addition of $0.1 \% \mathrm{H}_{2}$ to the sweep gas. The addition of $\mathrm{H}_{2}$ has a dramatic effect: the very large positive spike indicates a 


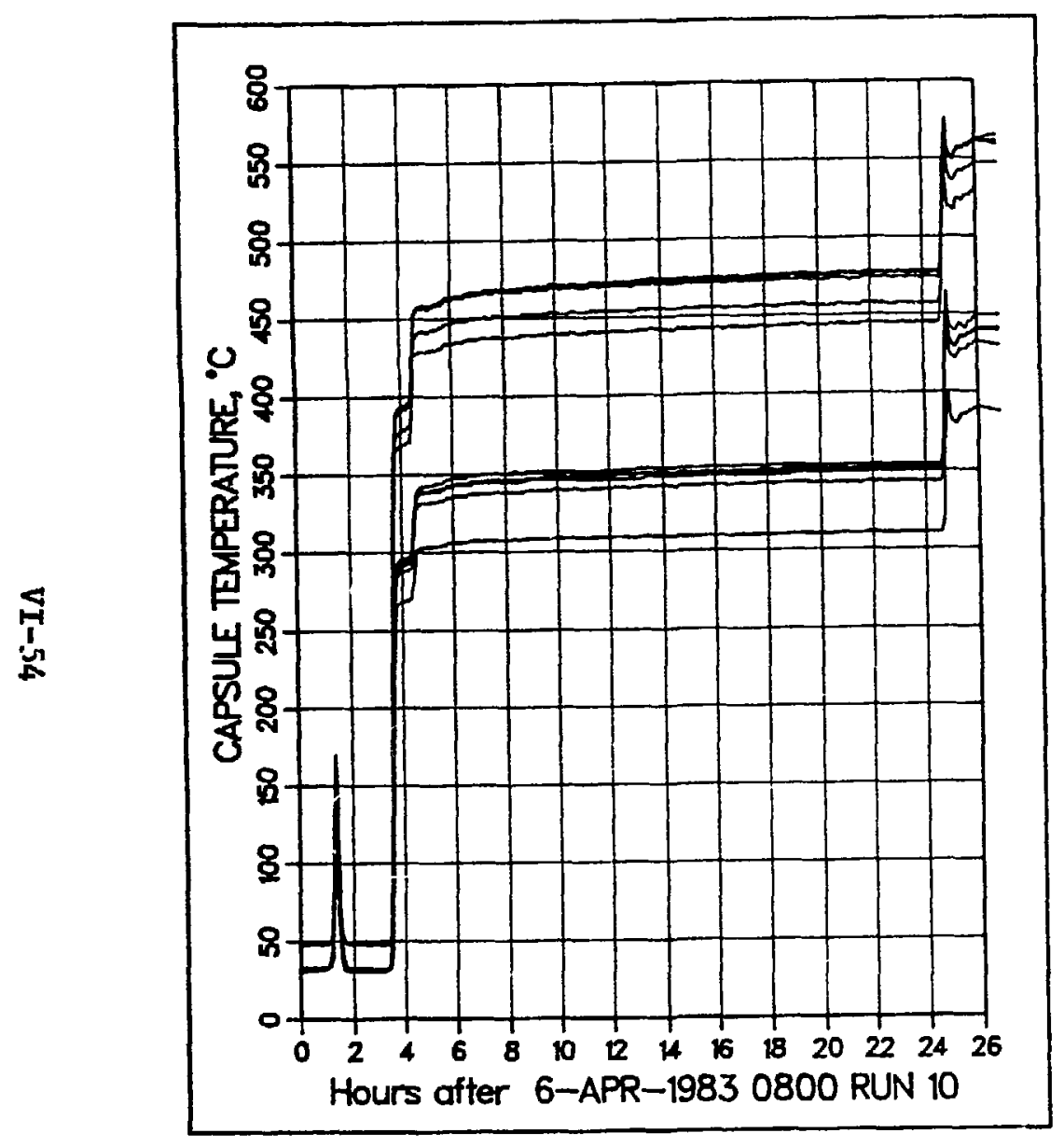

Fig. VI-26. Recorded temperature for Run 10.

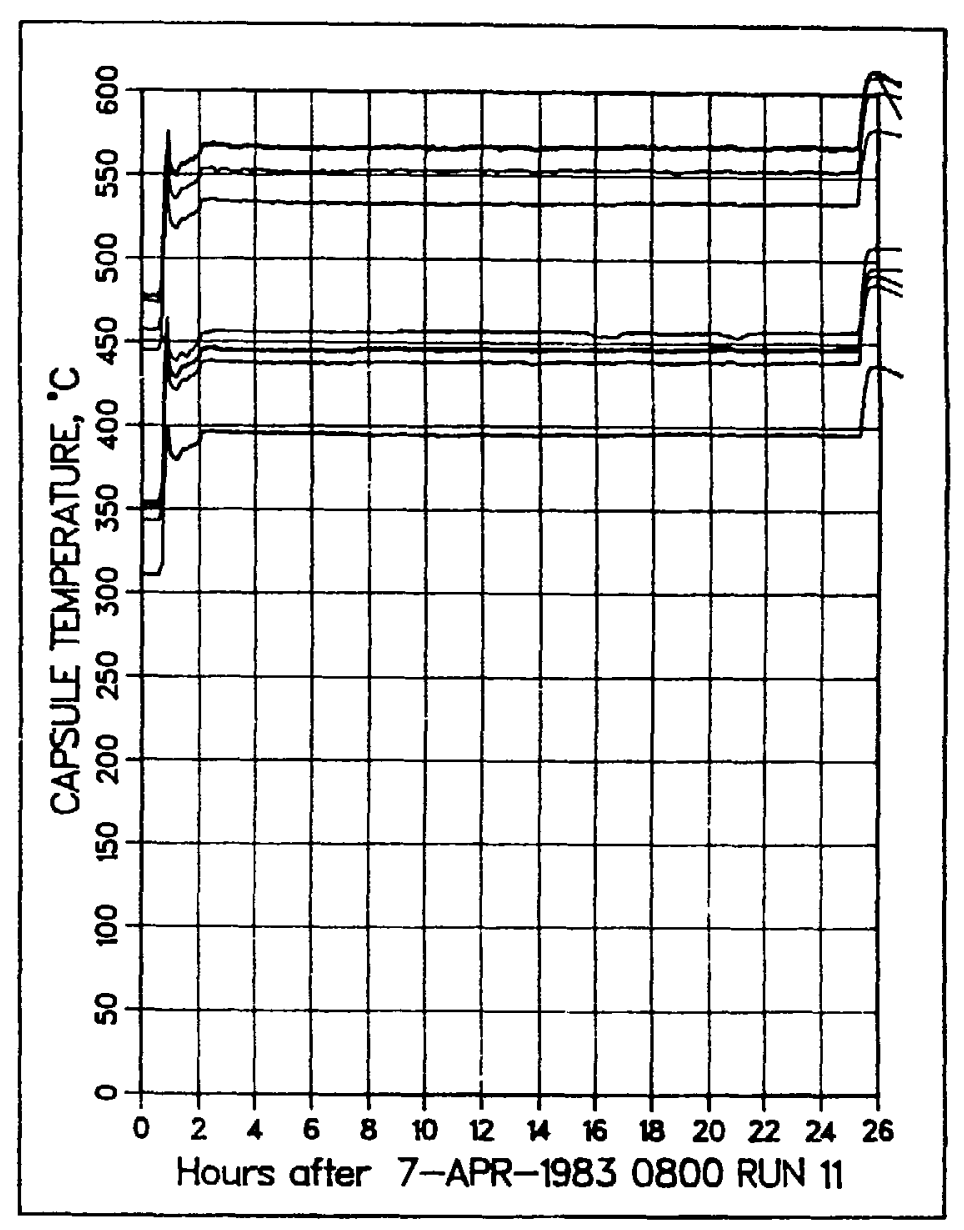

Fig. VI-27. Recorded temperatures for Run 11. 

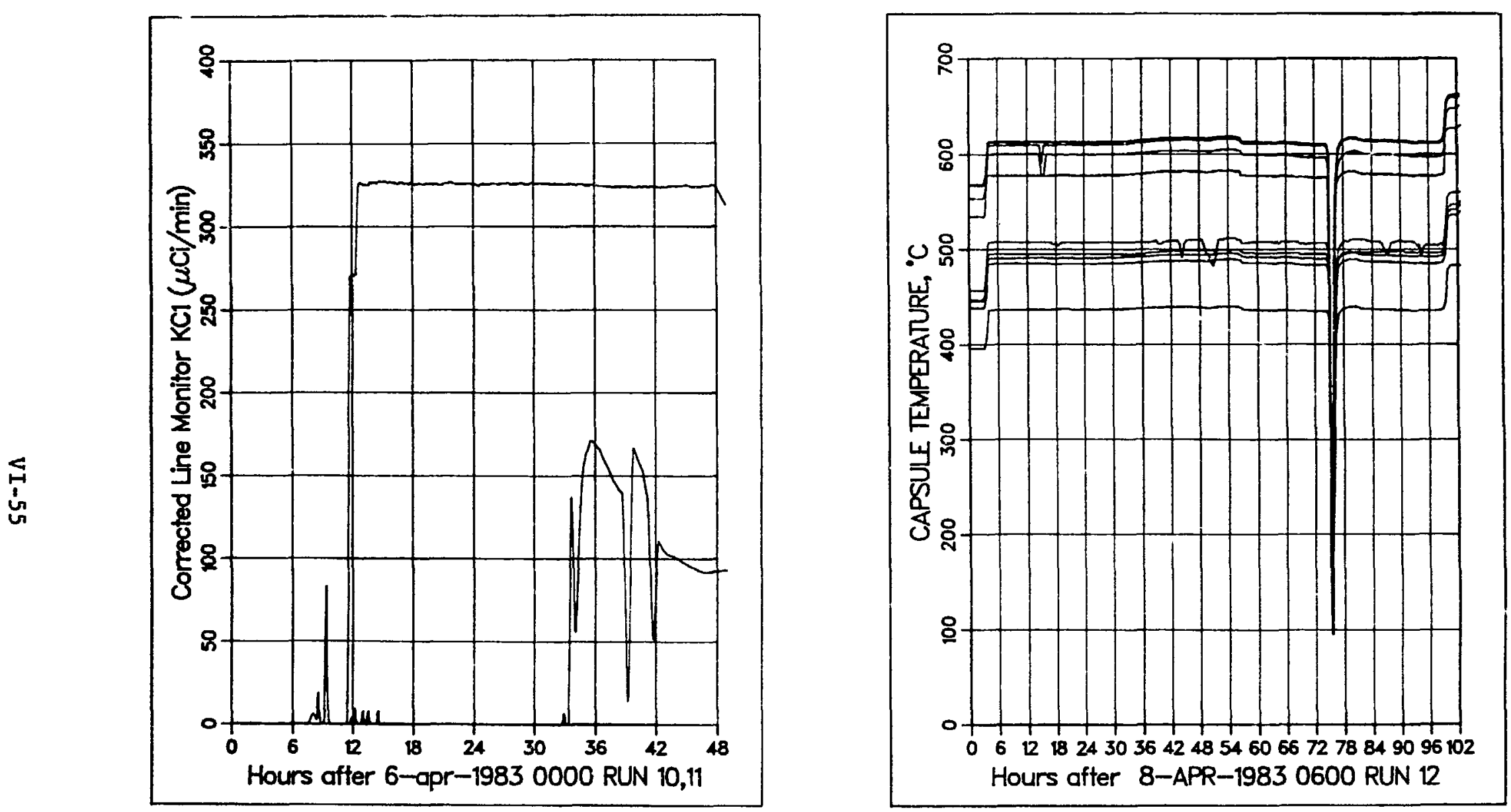

Fig. VI-28. Tritium release rates for Runs 10 and 11 . Fig. VI-29. Recorded temperatures for Run 12. 


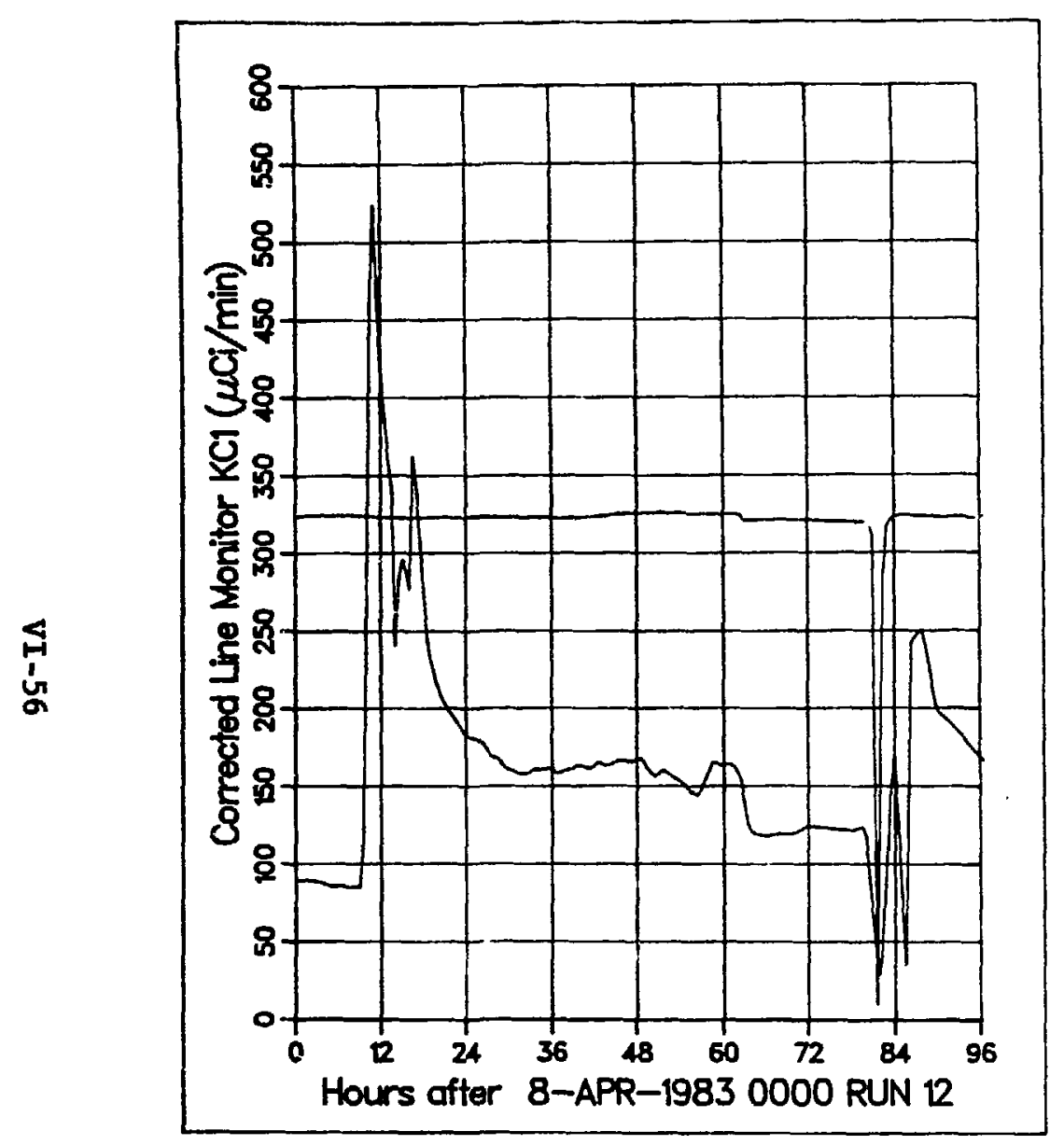

Fig. VI-30. Tritium release rate for Run 12.

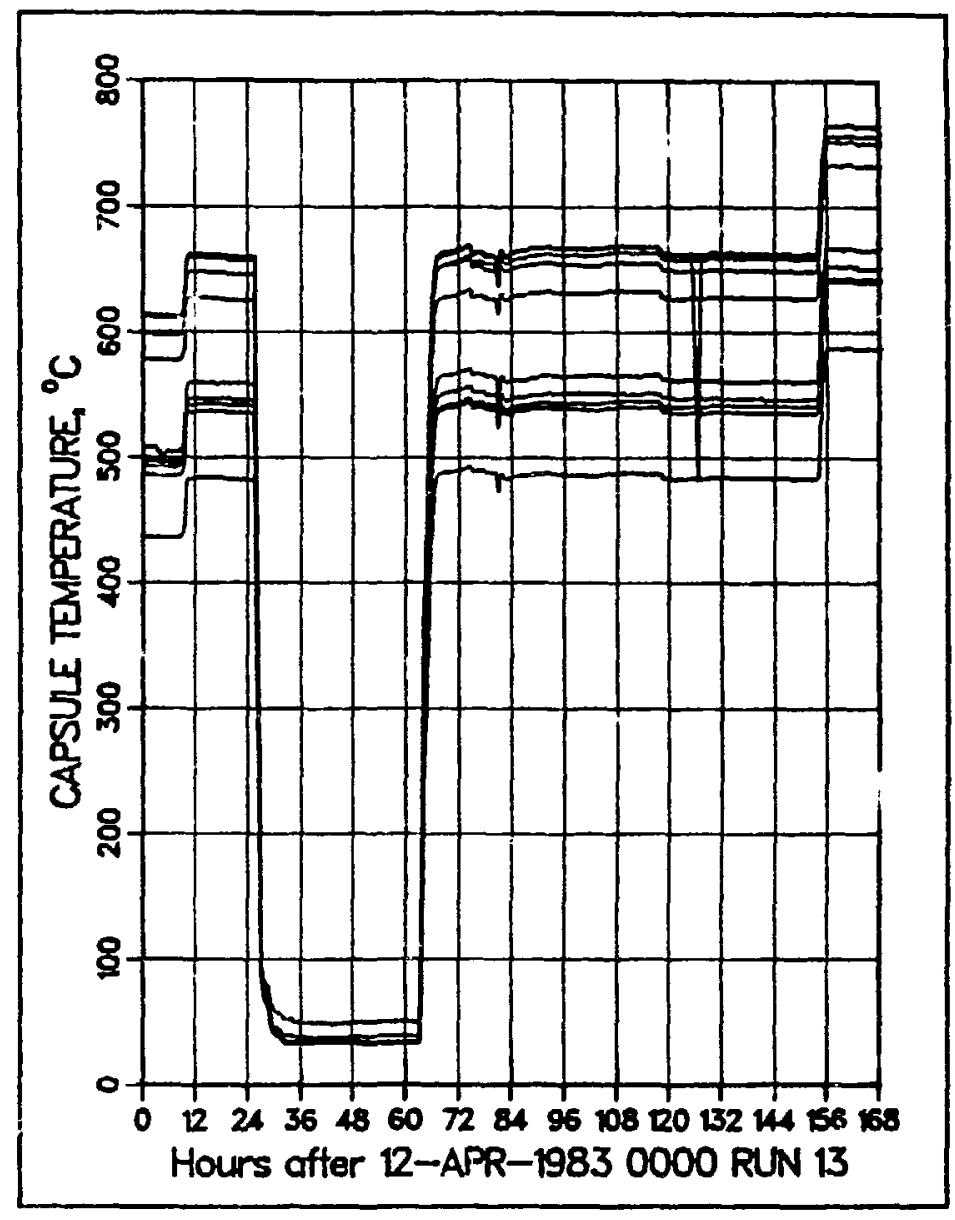

Fig. VI-31. Recorded temperatures for Run 13. 


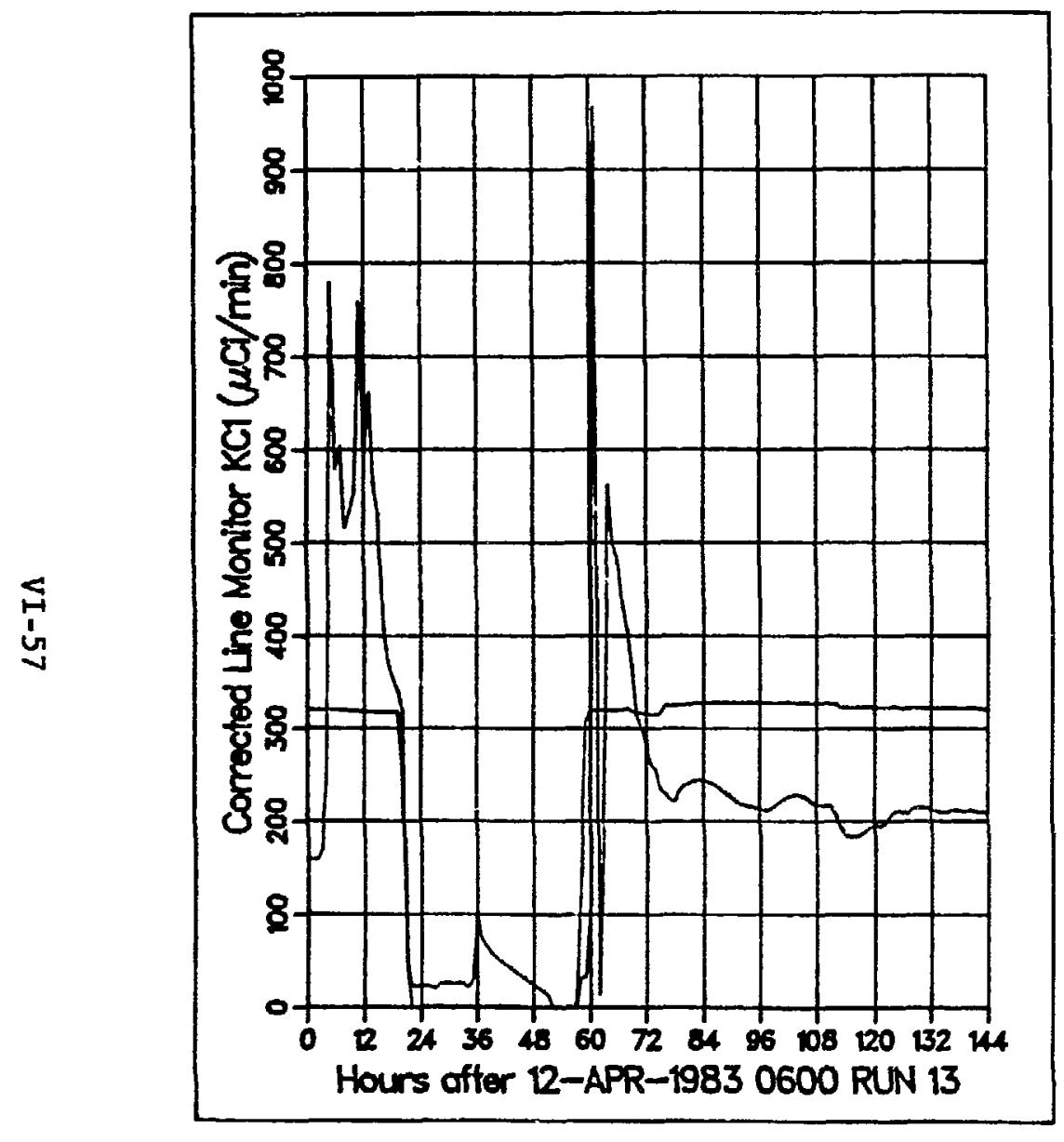

Fig. VI-32. Tritium release rate for Run 13.

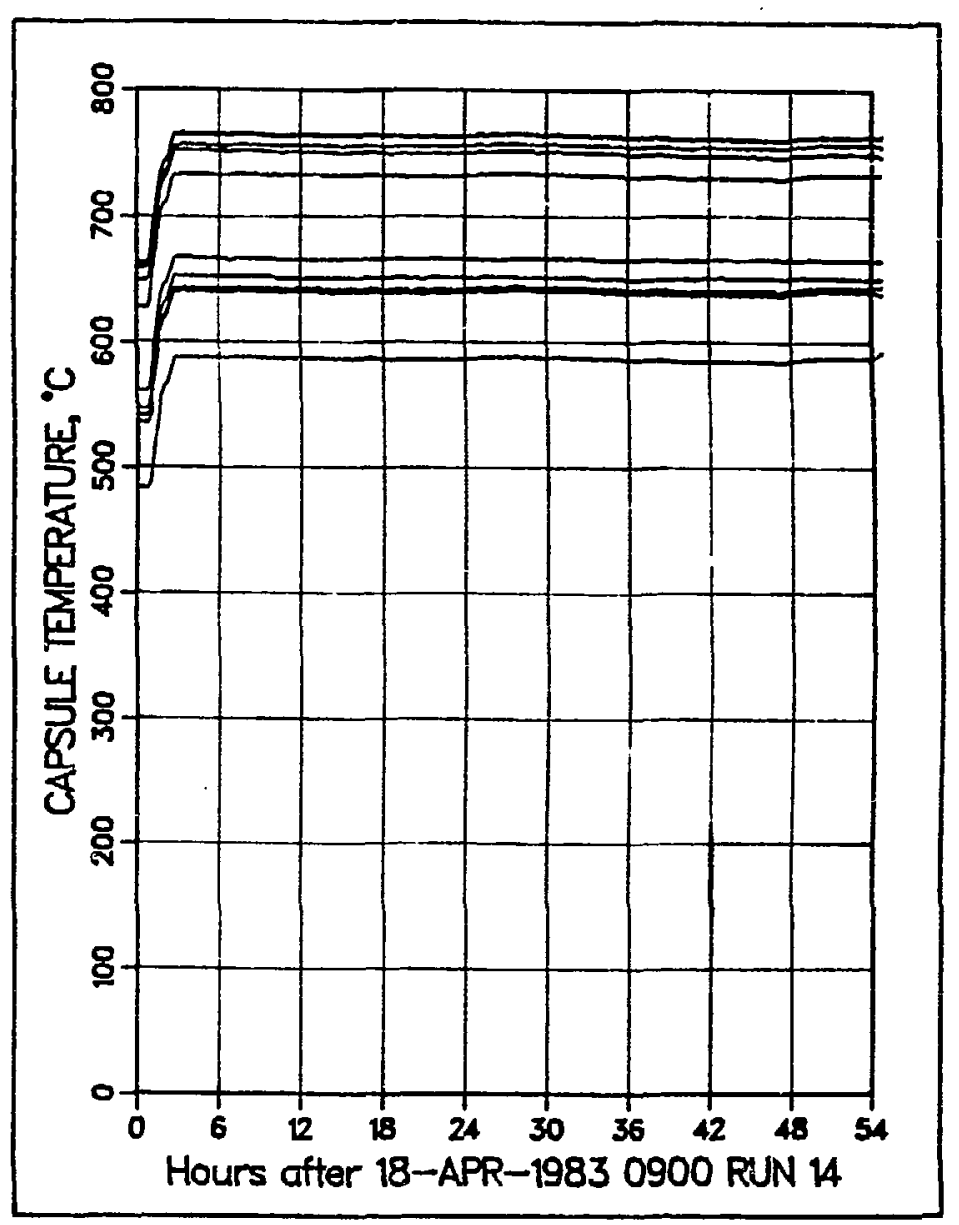

Fig. VI-33. Recorded temperatures for Run 14. 


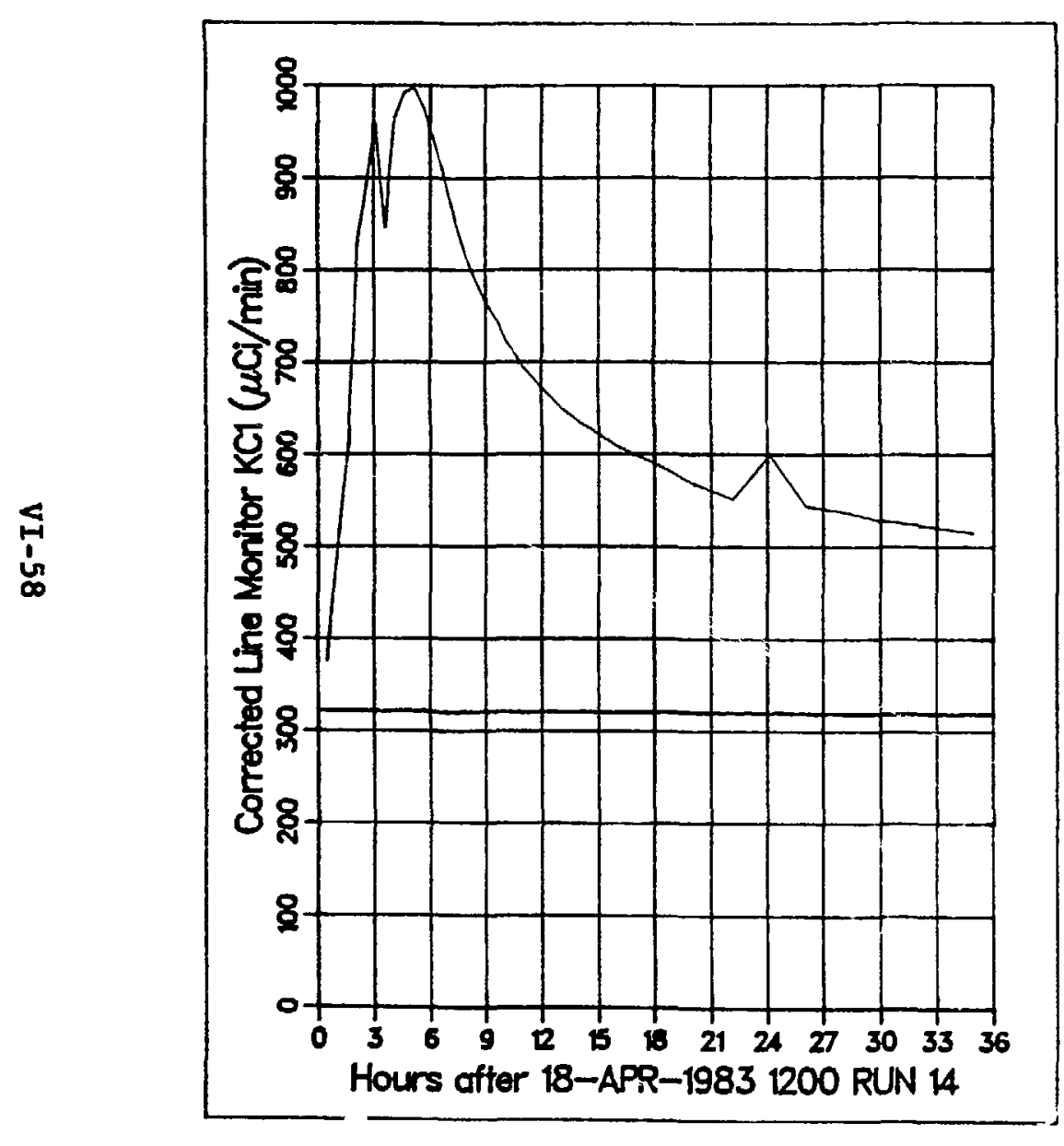

Fig. VI-34. Tritium release rate for Run 14 .

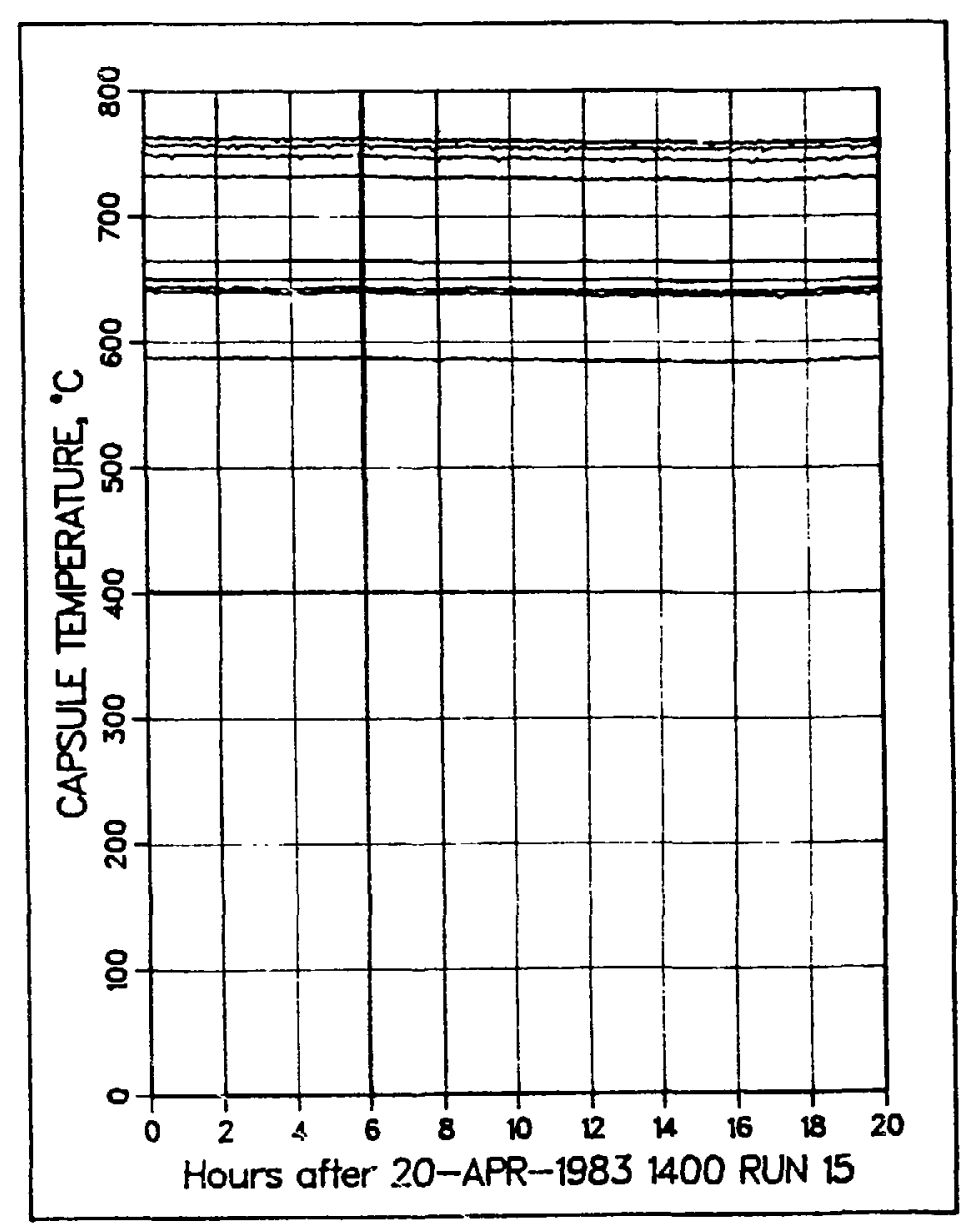

Fig. VI-35. Recorded temperatures for Run 15. 


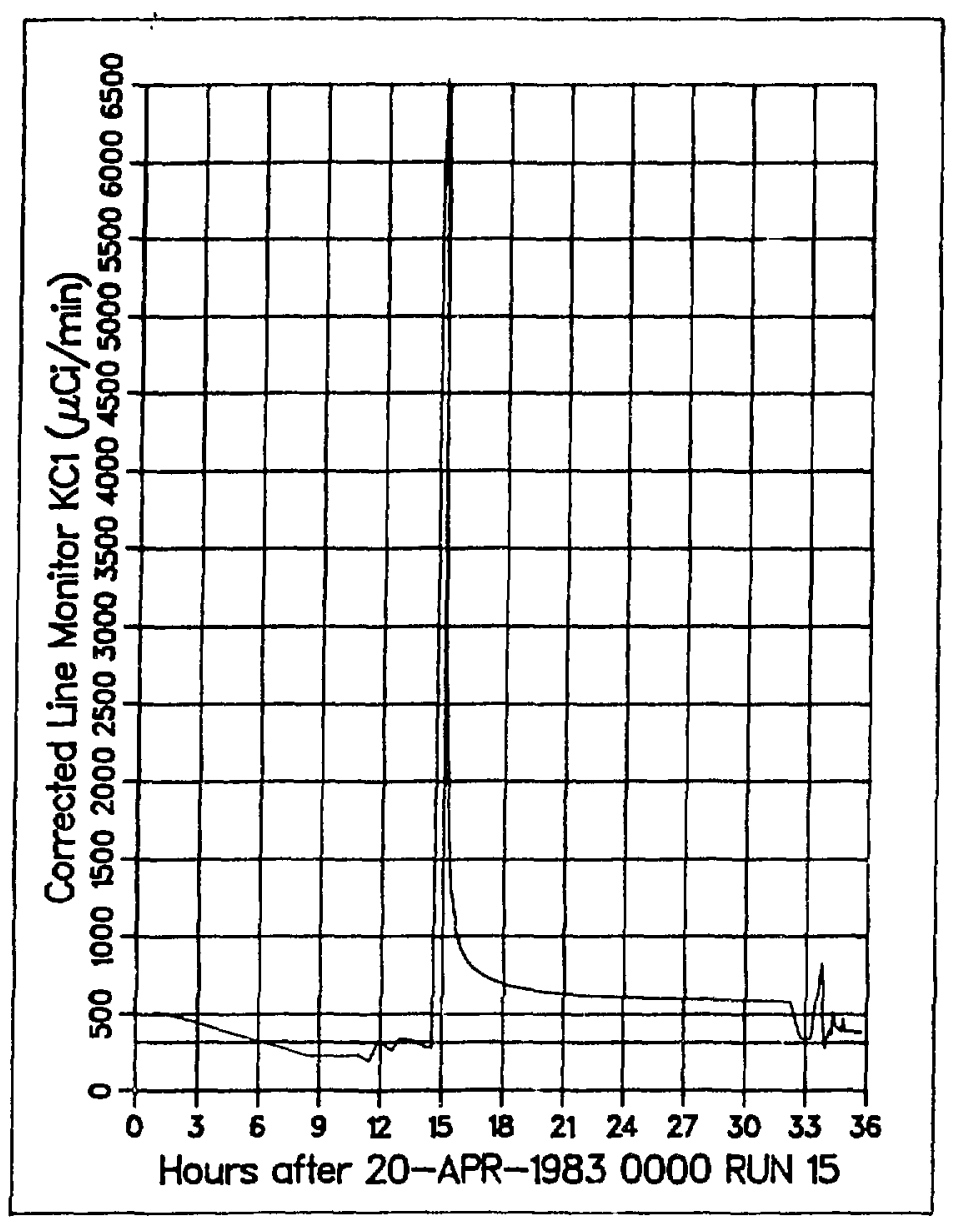

Fig. VI-36. Tritium release rate for Run 15.

large net release of tritium. The adaition of hydrogen appears to significantly enhance tritium release. The spike is followed by a return to steady state.

Run 16 was at a nominal temperature of $700^{\circ} \mathrm{C}$ with a sweep gas flow rate of $300 \mathrm{cc} / \mathrm{min}$ and with a sweep gas composition of helium $+0.1 \% \mathrm{H}_{2}$. The temperature and tritium release data are shown in Figs. VI-37 and -38 . Pressure and flow conditions in the sweep gas are given in Figs. VI-39 through -42 . The only change in conditions from the prior run is an increase in flow rate from 100 to $300 \mathrm{~cm}^{3} / \mathrm{min}$. The increase in flow results in a positive spike at the beginning of the run, followed by a very rapid return to steady state. The second spike seems tc be correlated to a temporary drop in pressure (Fig. VI-39) and a brief Increase in sweep gas inlet flow. For Run 16 overall, the increase in flow seemis to result in an improvement in release. 


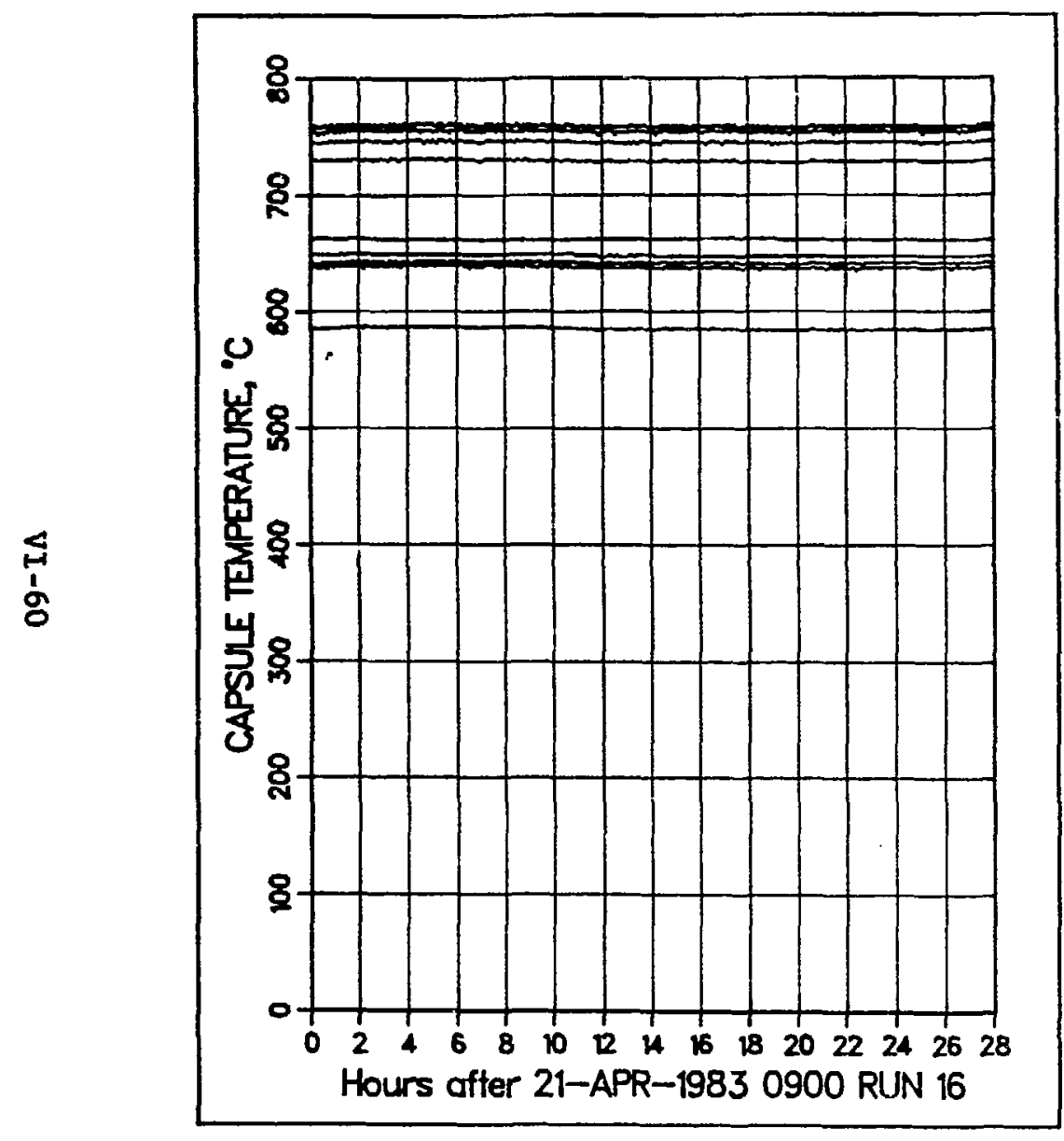

Fig. VI-37. Recorded temperatures for Run 16.

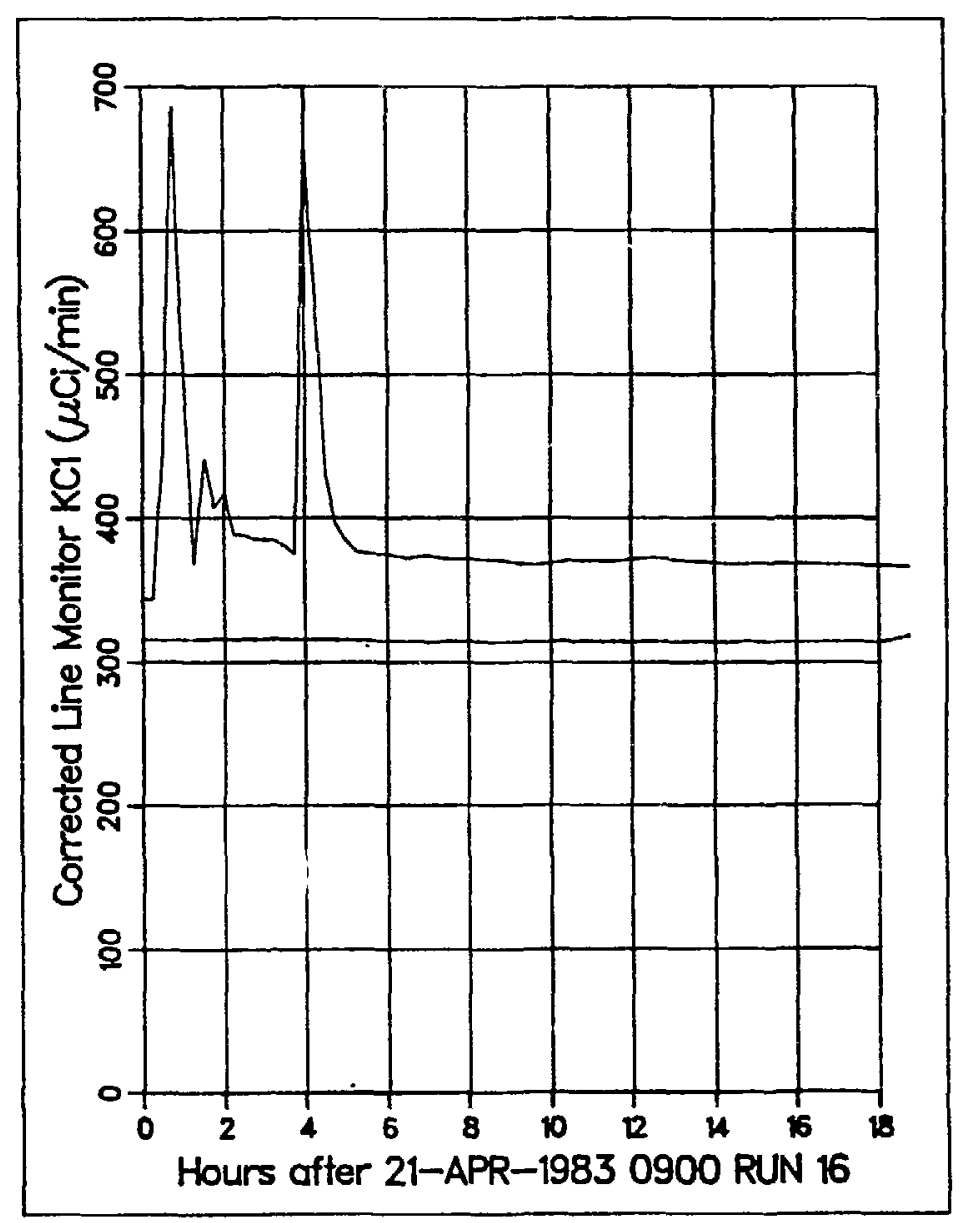

Fig. VI-38. Tritium release rate for Run 16. 


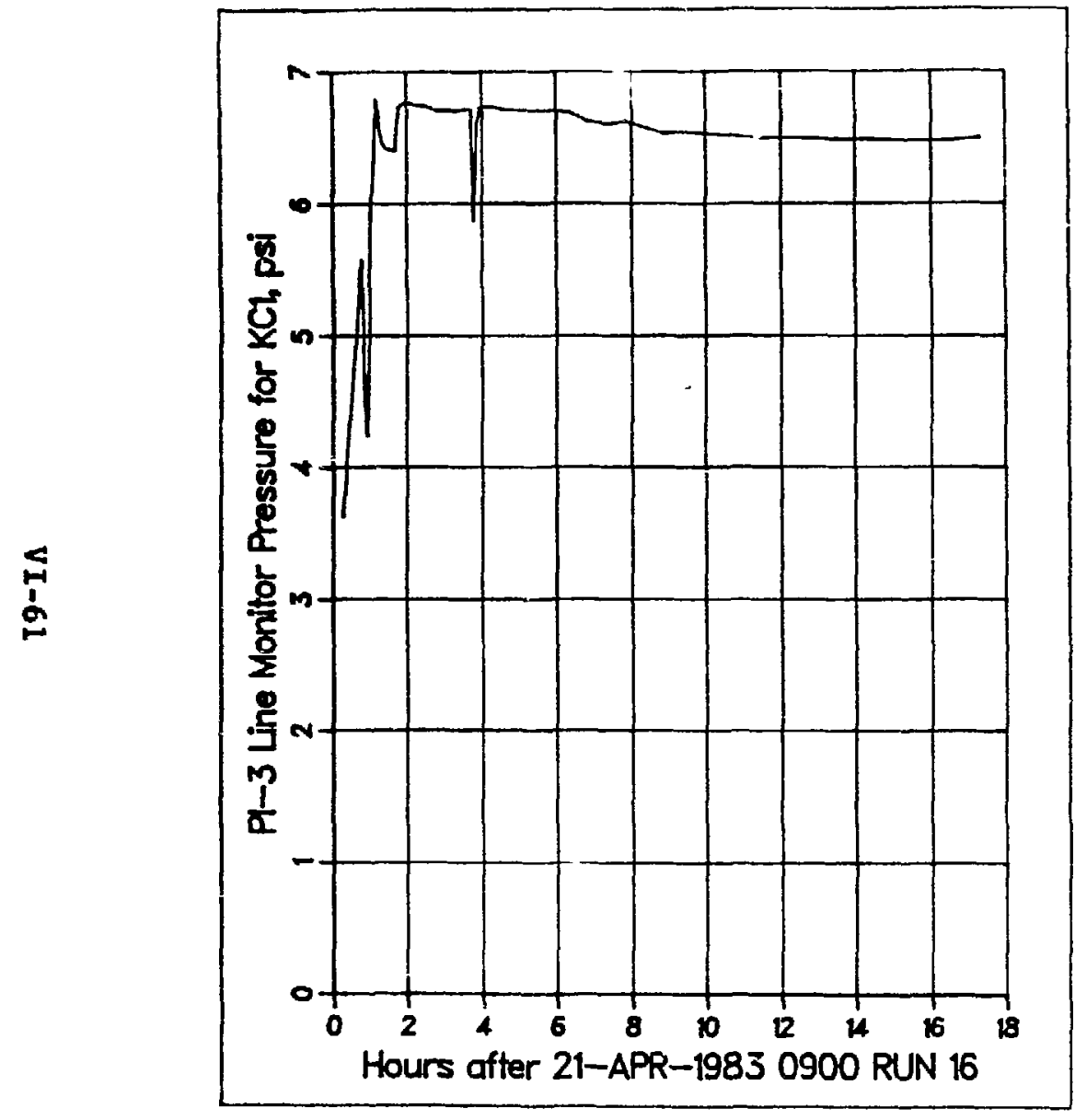

Fig. VI-39. Sweep gas pressure (psig) for Run 16.

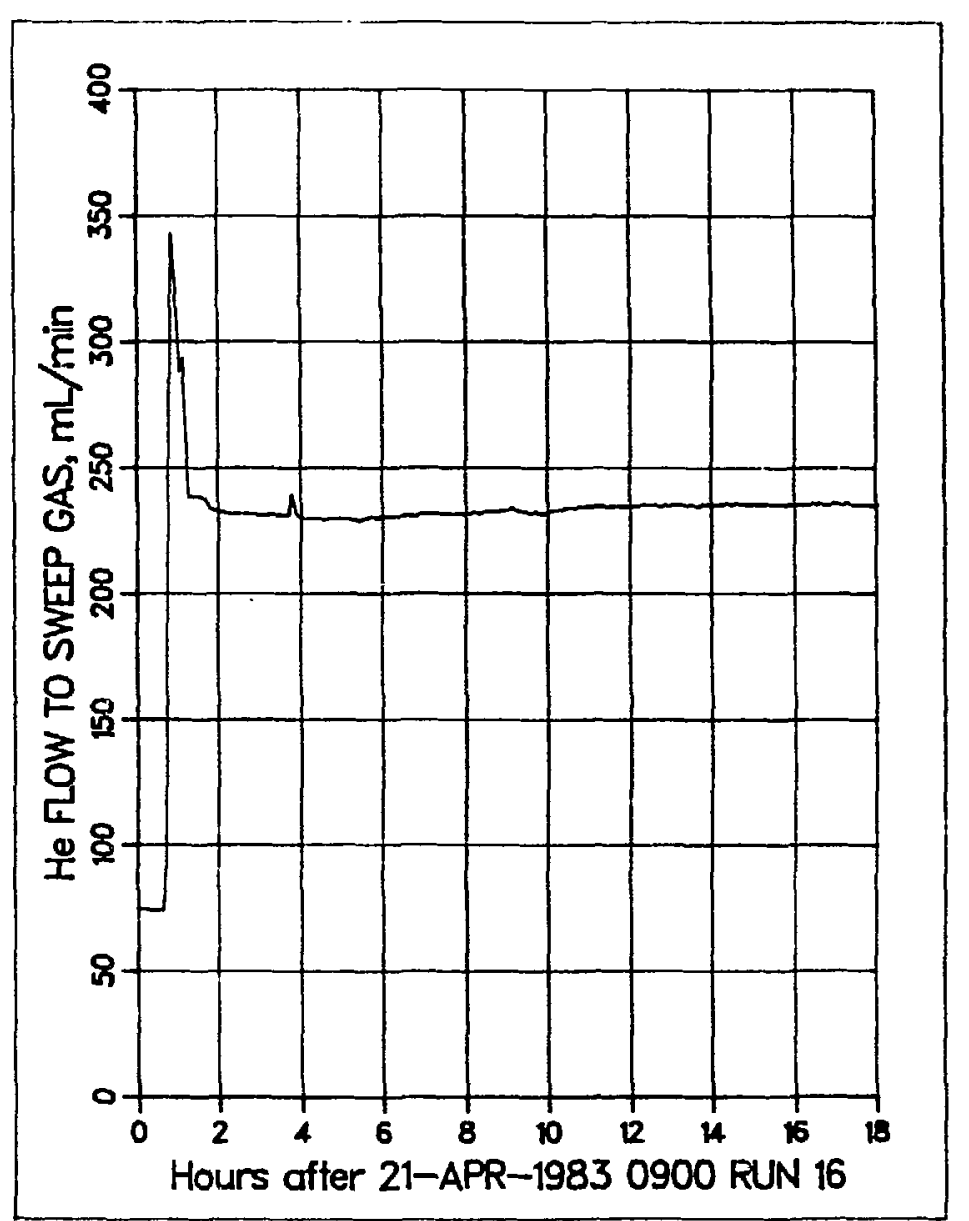

Fig. VI-40. Helium flow to sweep gas for Run 16. 


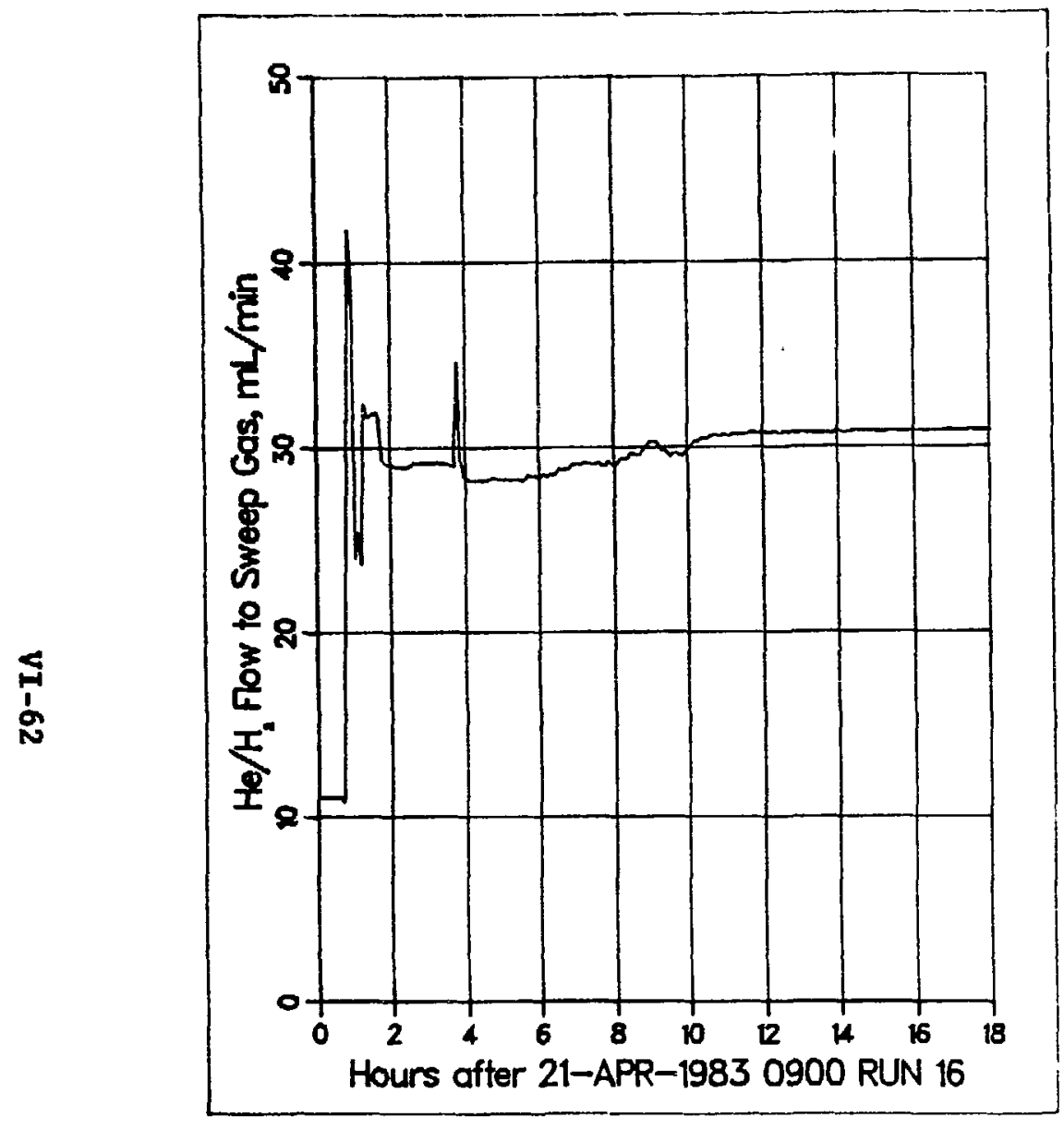

Fig. VI-41. Flow of helium $+4 \% \mathrm{H}_{2}$ to sweep gas for Run 16 .

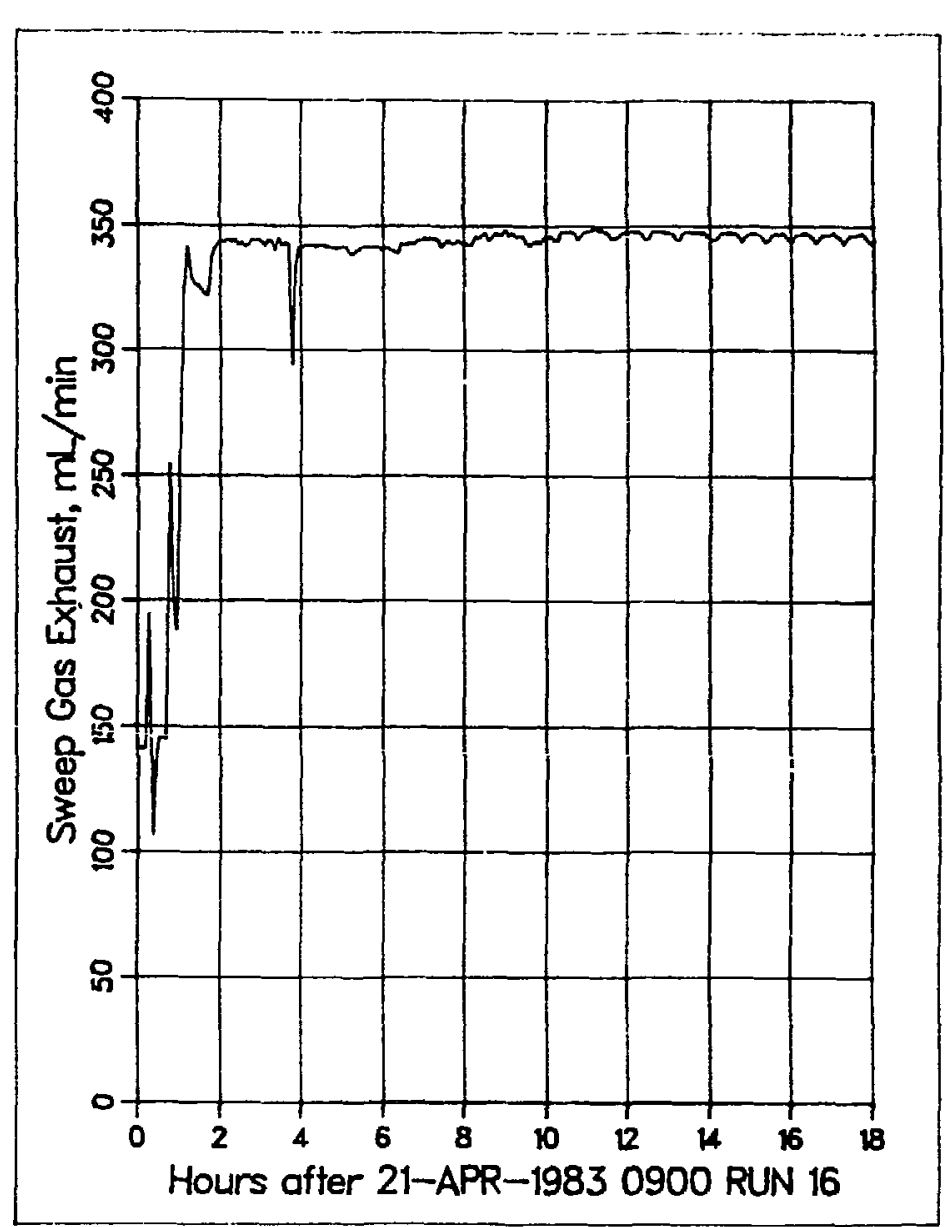

Fig. VI-42. Sweep gas exhaust $\left(\mathrm{cm}^{2} / \mathrm{min}\right)$ for Run 16. 
Run 17 was at a nominal tenperacure of $700^{\circ} \mathrm{C}$ with a flow rate of 30 $\mathrm{cm}^{3} / \mathrm{min}$ for the sweep gas of helium $+0.1 \% \mathrm{H}_{2}$. The run is the same as the prior run except that the sweep gas flow rate is decreased from $300=\mathrm{sw} / \mathrm{min}$ to $30 \mathrm{~cm}^{3} / \mathrm{min}$. The temperature and tritium release data are shown in Figs. VI-43 and 44 , respectively. A fluctuation in release rate occurs at the beginning of the run, followed by a rapid return to steady state. It is not clear what the effect of decreased flow is. This decreased flow does not seem to have a strong effect upon tritium release.

Run 18 was at a nominal temperature of $700^{\circ} \mathrm{C}$ with a flow rate of 100 $\mathrm{cm}^{3} / \mathrm{min}$ for the sweep gas of helium $+0.1 \% \mathrm{H}_{2}$. The temperature and tritiun release data are shown in Figs. VI-45 and -46, respectively. The run has the same conditions as Run 17 except that the flow rate is increased irom 30 $\mathrm{cm}^{3} / \mathrm{min}$ to $100 \mathrm{~cm}^{3} / \mathrm{min}$. An observable peak in the tritiun release curve, followed by a return to steady state within $8 \mathrm{~h}$.

Run 19 was at a nominal temperature of $650^{\circ} \mathrm{C}$ with a flow rate of 100 $\mathrm{cm}^{3} / \mathrm{min}$ for the sweep gas of helium $+0.1 \% \mathrm{H}_{2}$. The temperature and tritium release data are shown in Figs. VI-47 and -48 , respectively. The tritium release curve shows oscillations followed by a negative peak and a return to steady state. The oscillations are brought about by temporary pressure and flow changes resulting from changing samples. The rapid fluctuations in the temperature plots were caused by electronic problems with thermocouple T 2901 .

Run 20 was at a nominal temperature of $650^{\circ} \mathrm{C}$ with gas flow rate of 100 $\mathrm{cm}^{3} / \mathrm{min}$ for the sweep gas of helium $+0.2 \%$ oxygen. The temperature and tritium release data are shown in Figs. VI -49 and -50 . The temperature profiles show a startup period of about $24 \mathrm{~h}$ at low temperature $\left(300-400^{\circ} \mathrm{C}\right)$ preceding operation at full temperature. Again, there were problems with thermocouple TR901. The tritium release shows no significant release of tritium in the HT form. As reported previously, no significant release of tritium in the condensable form occurred during the TRIO experiment. The addition of oxygen did not appear to be beneficial to tritium release.

Run 21 was at a nominal temperature of $650^{\circ} \mathrm{C}$ with a flow rate of 100 $\mathrm{cm}^{3} / \mathrm{mIn}$ for the sweep gas of helium $+0.1 \% \mathrm{H}_{2}$. The temperature and tritium release data are shown in Figs. VI-51 and -52, respectively. The test represents a return to standard conditions following the run with oxygen. The 


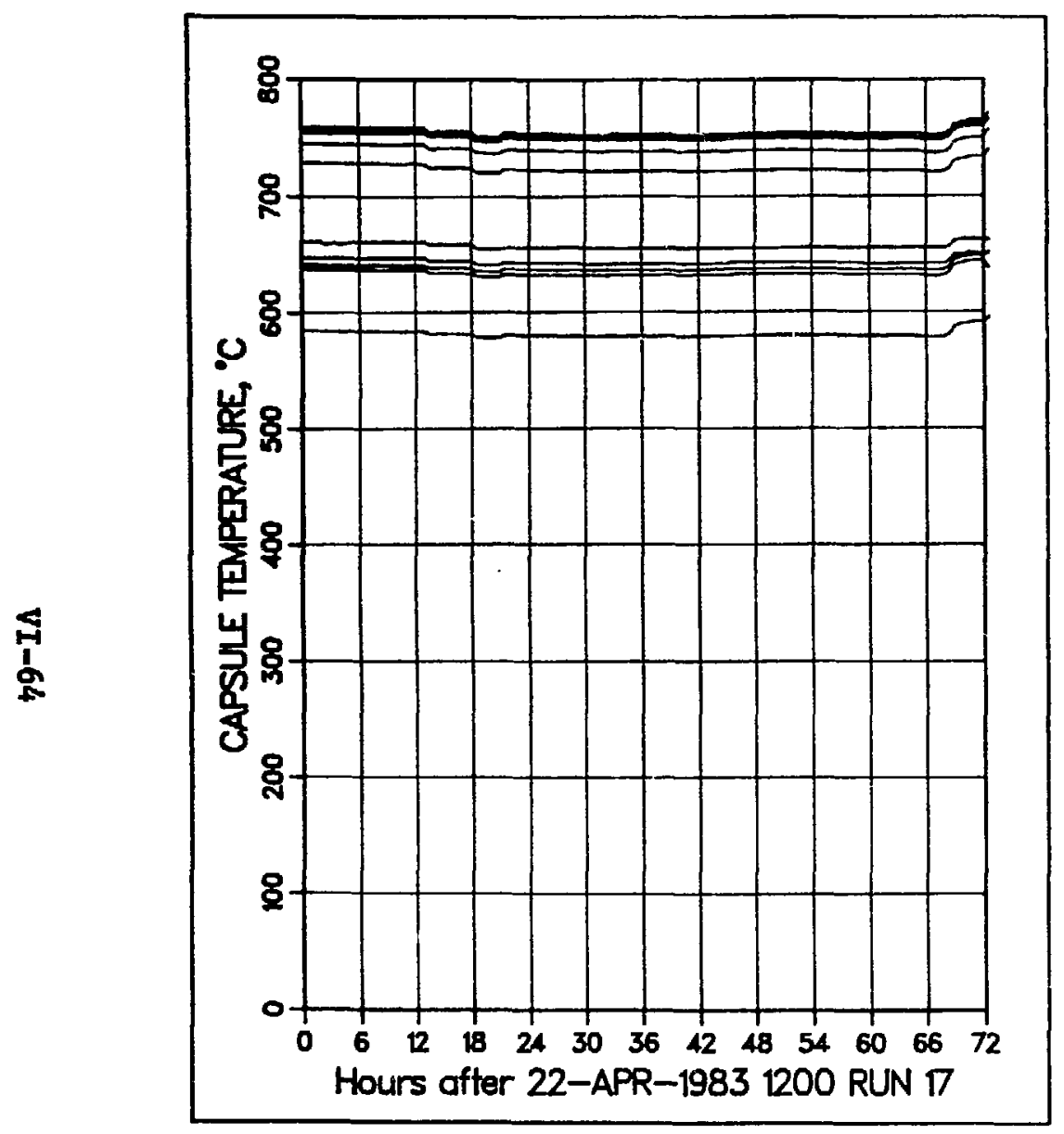

Fig. VI-43. Recnrdad temperatures for Run 17.

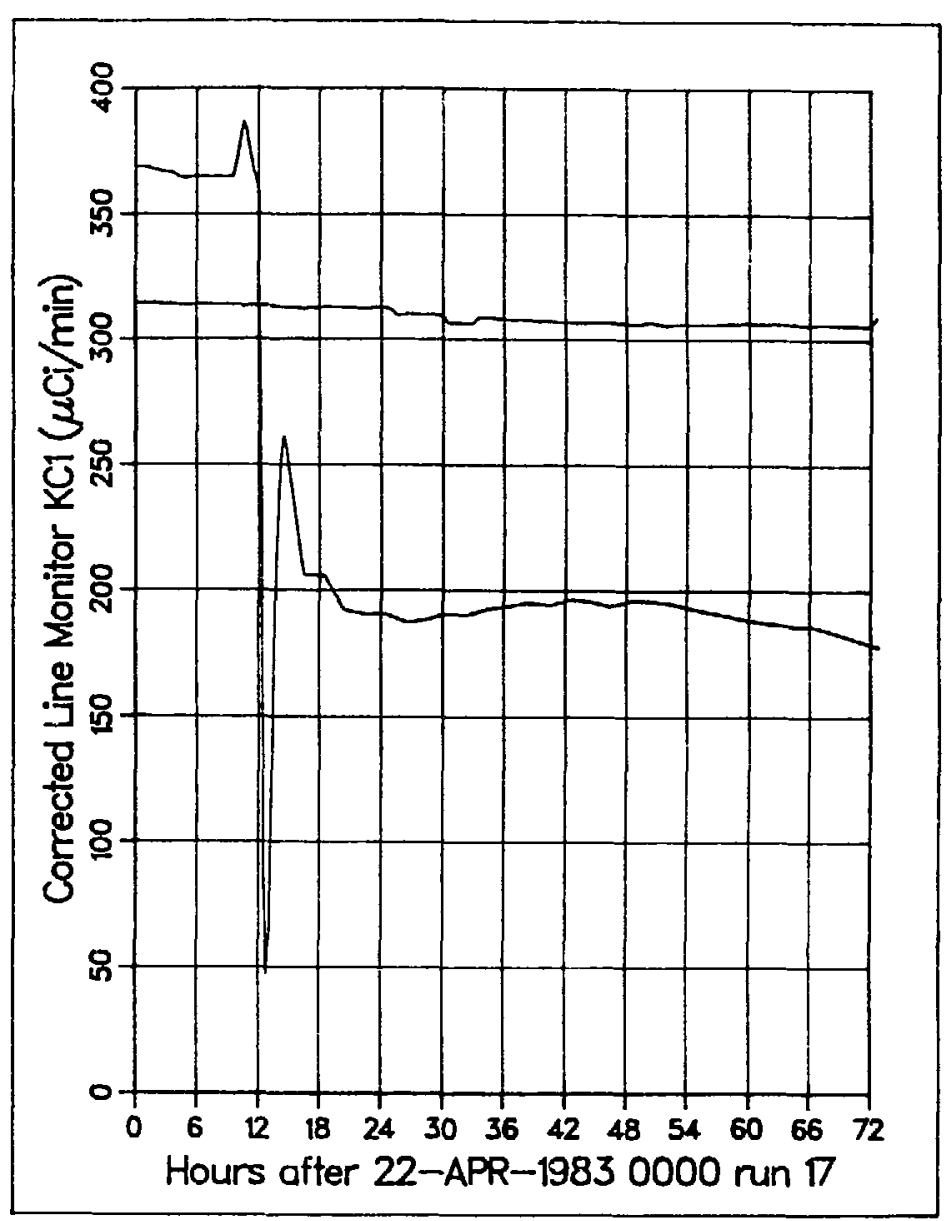

Fig. VI-44. Tritium release rate for Run 17. 


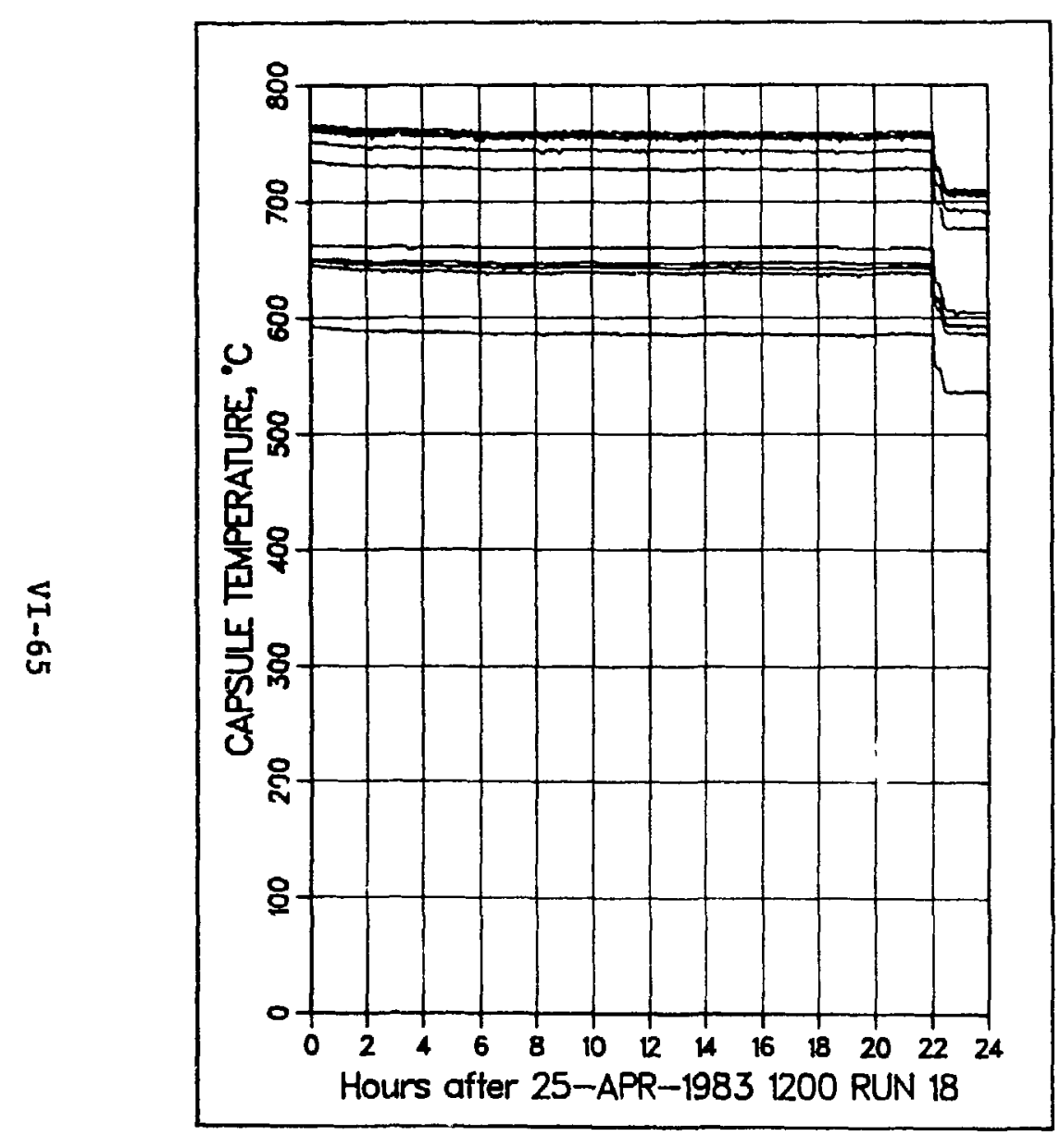

Fig. VI-45. Recorded temperatures for Run 18.

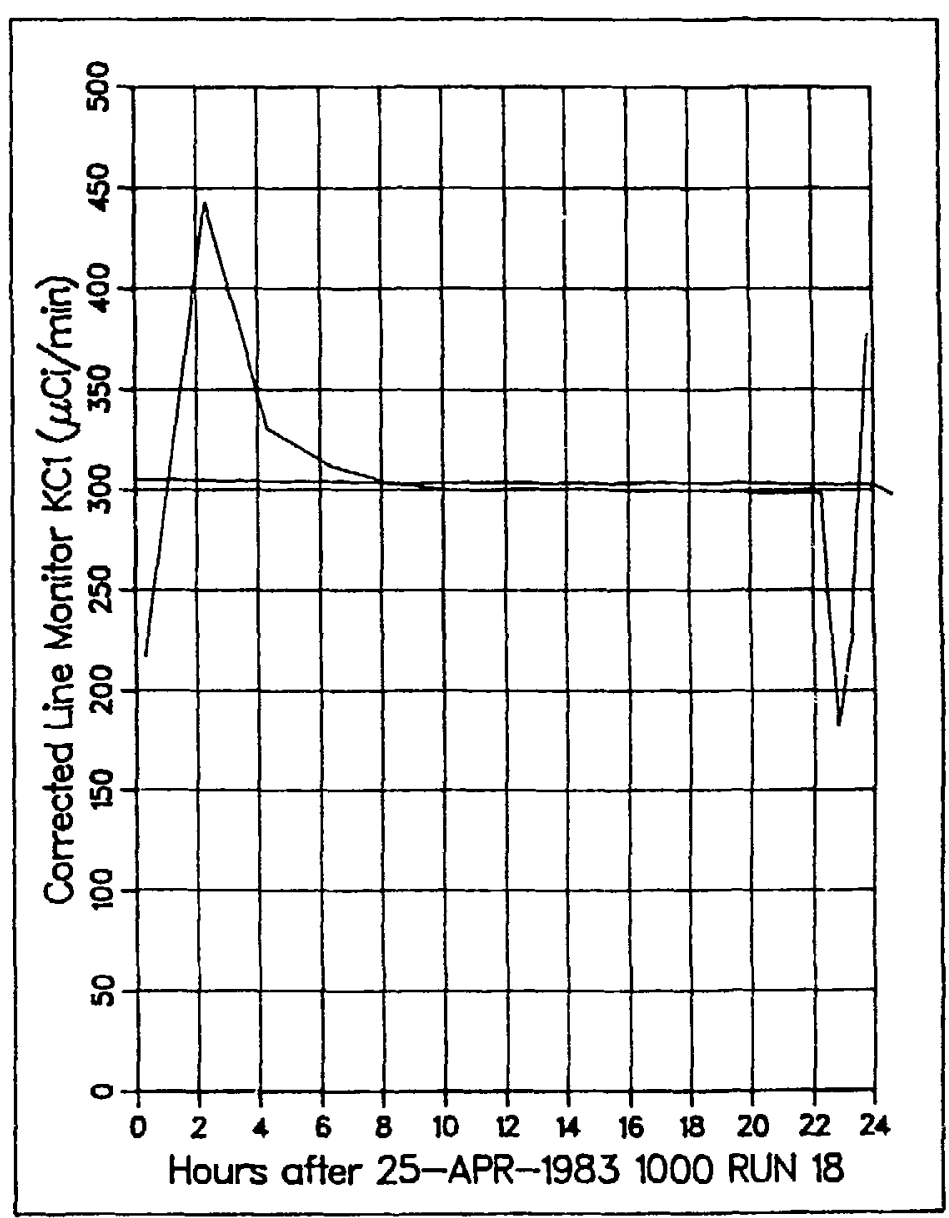

Fig. VI-46. Tritium release rate for Run 18 . 


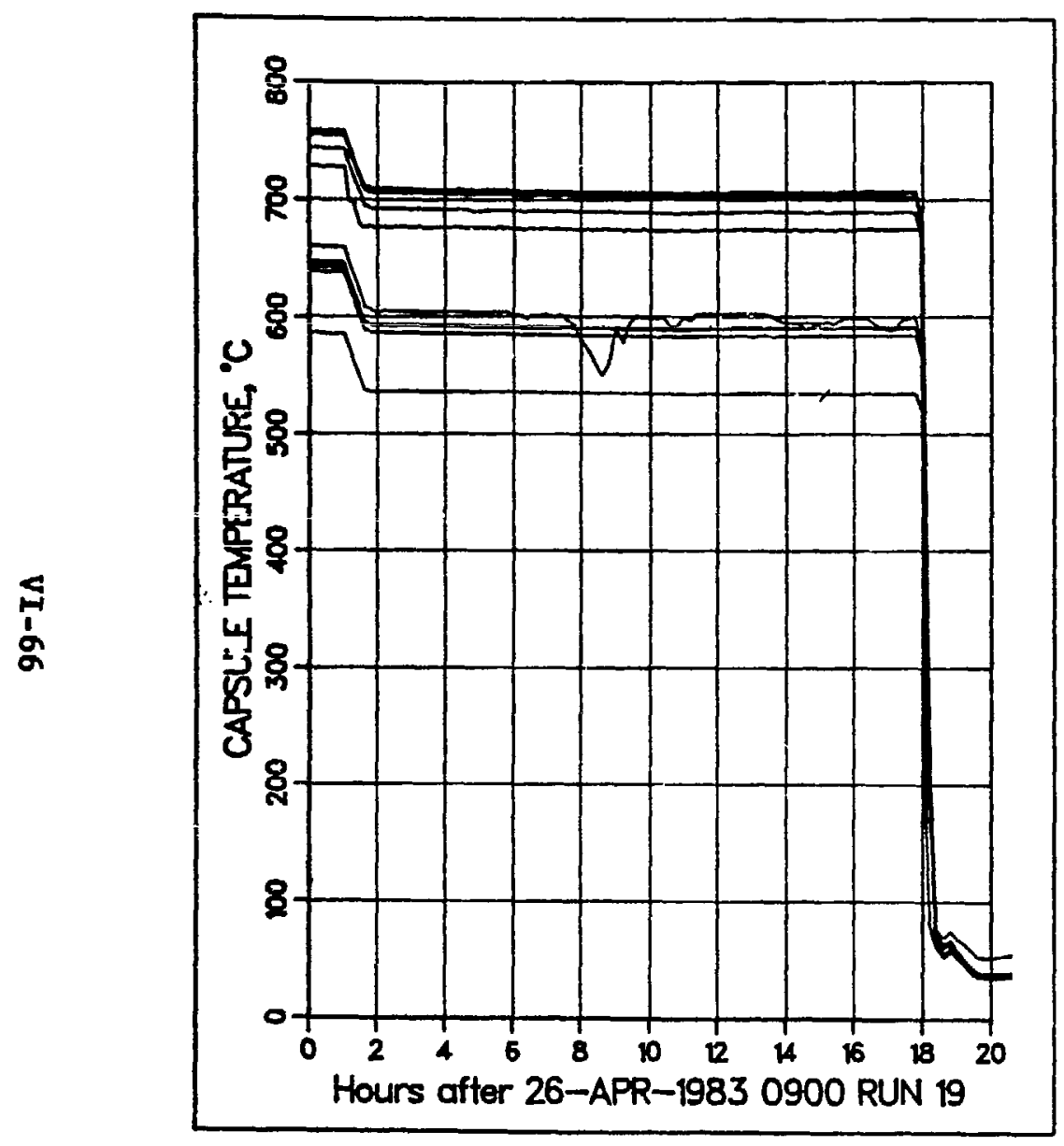

F1g. VI-47. Recorded temperatures for Run 19.

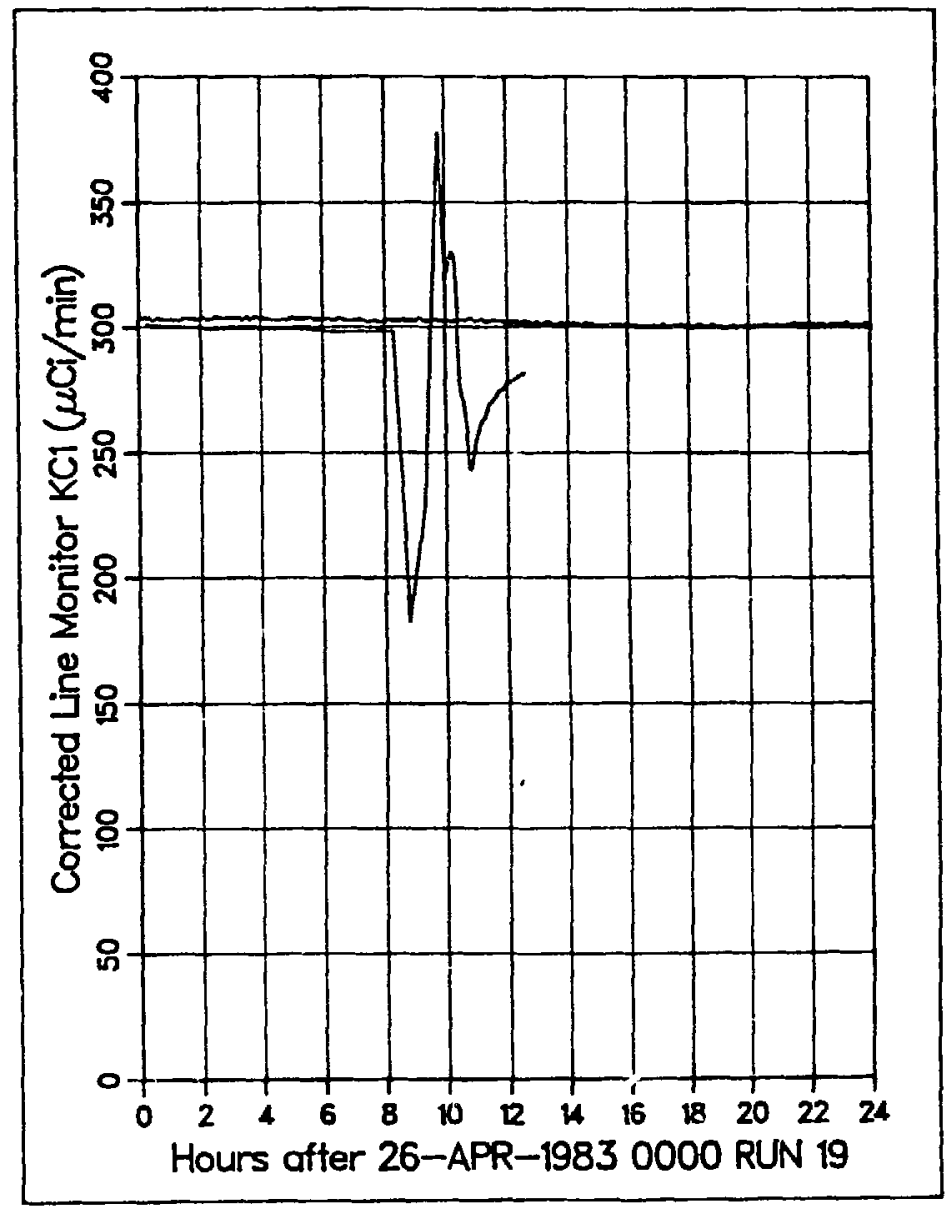

Fig. VI-48. Tritium release rate for Run 19. 


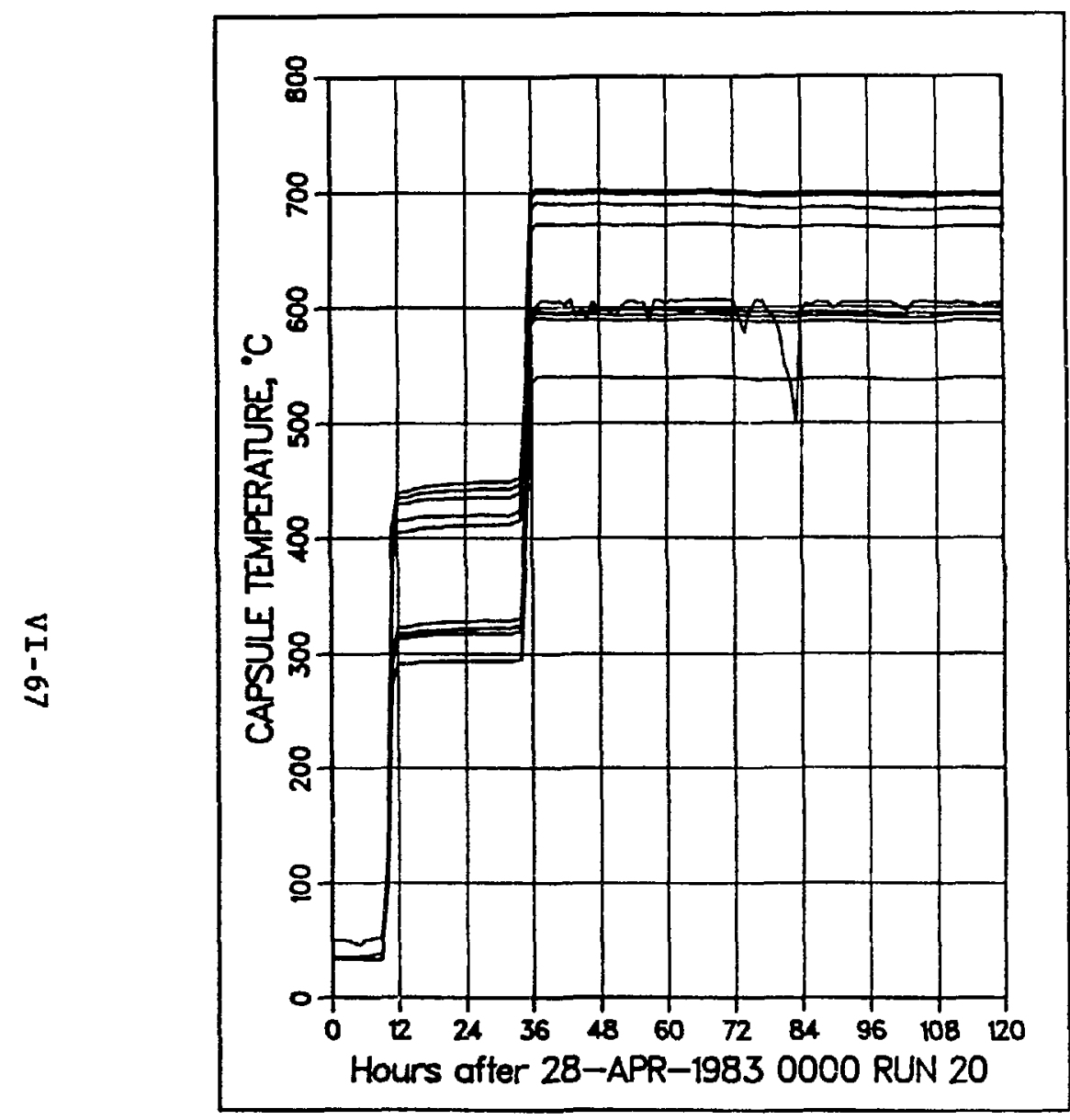

Fig. VI-49. Recorded temperatures for Run 20.

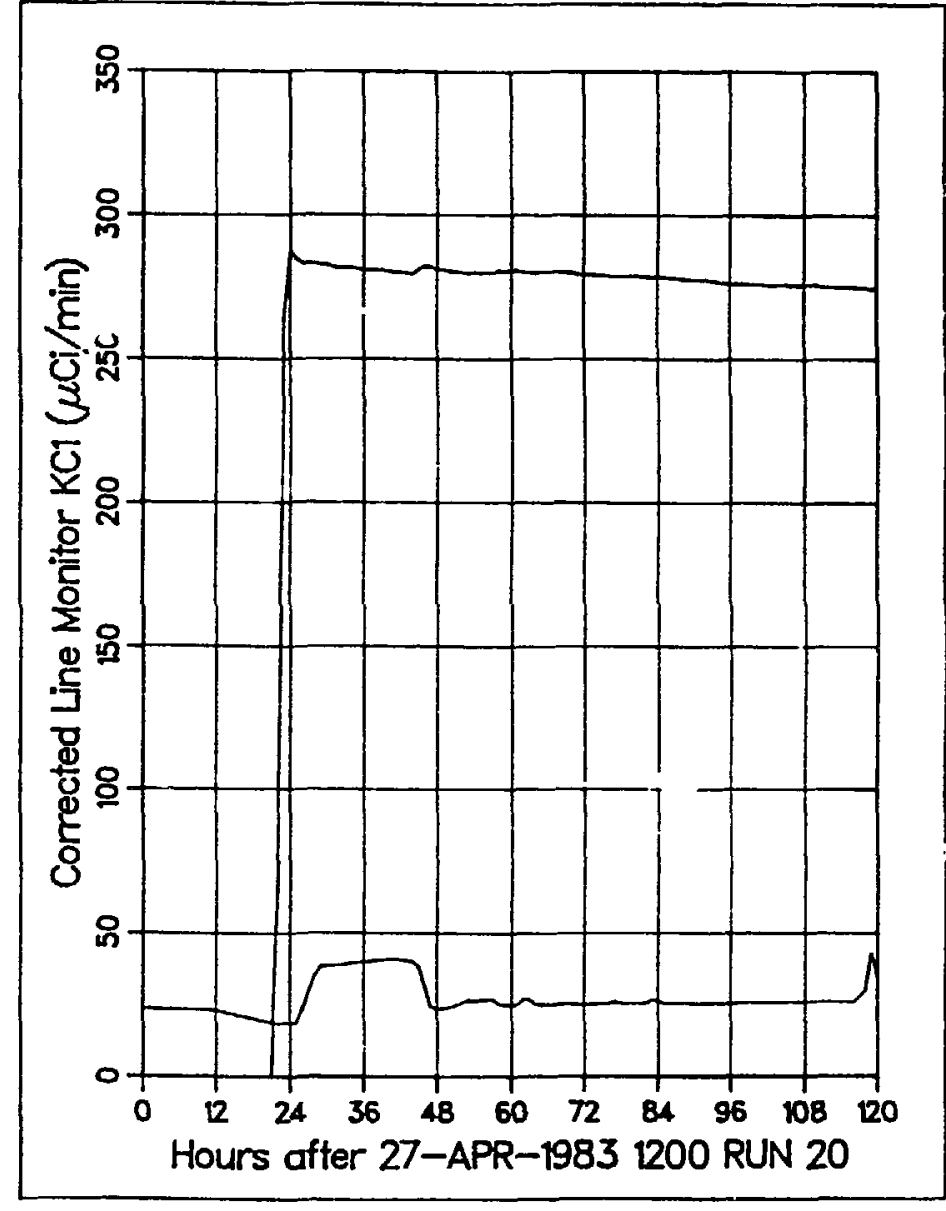

Fig. VI-50. Tritium release rate for Run 20 . 


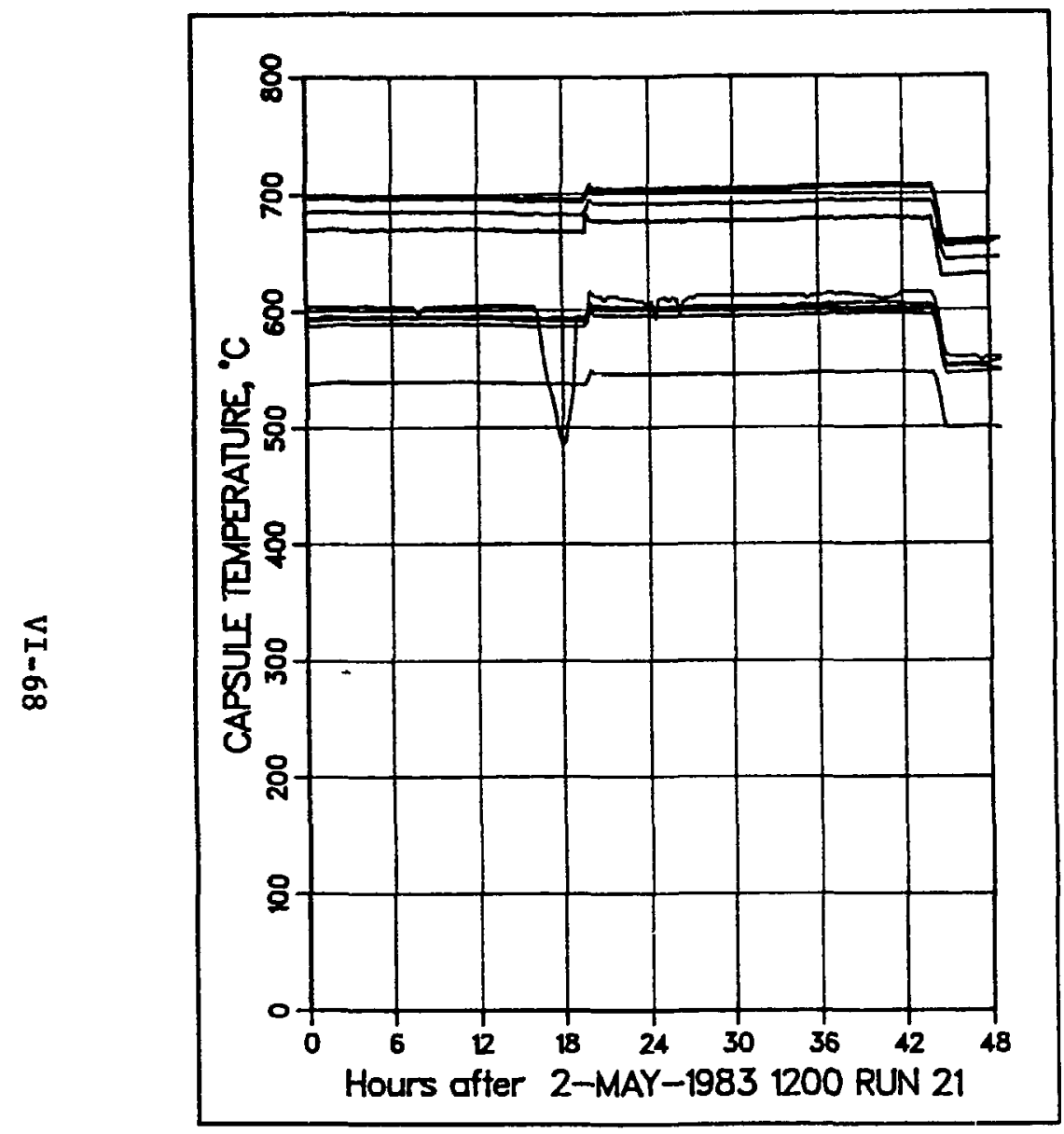

Fig. VI-51. Recorded temperatures for Run 21.

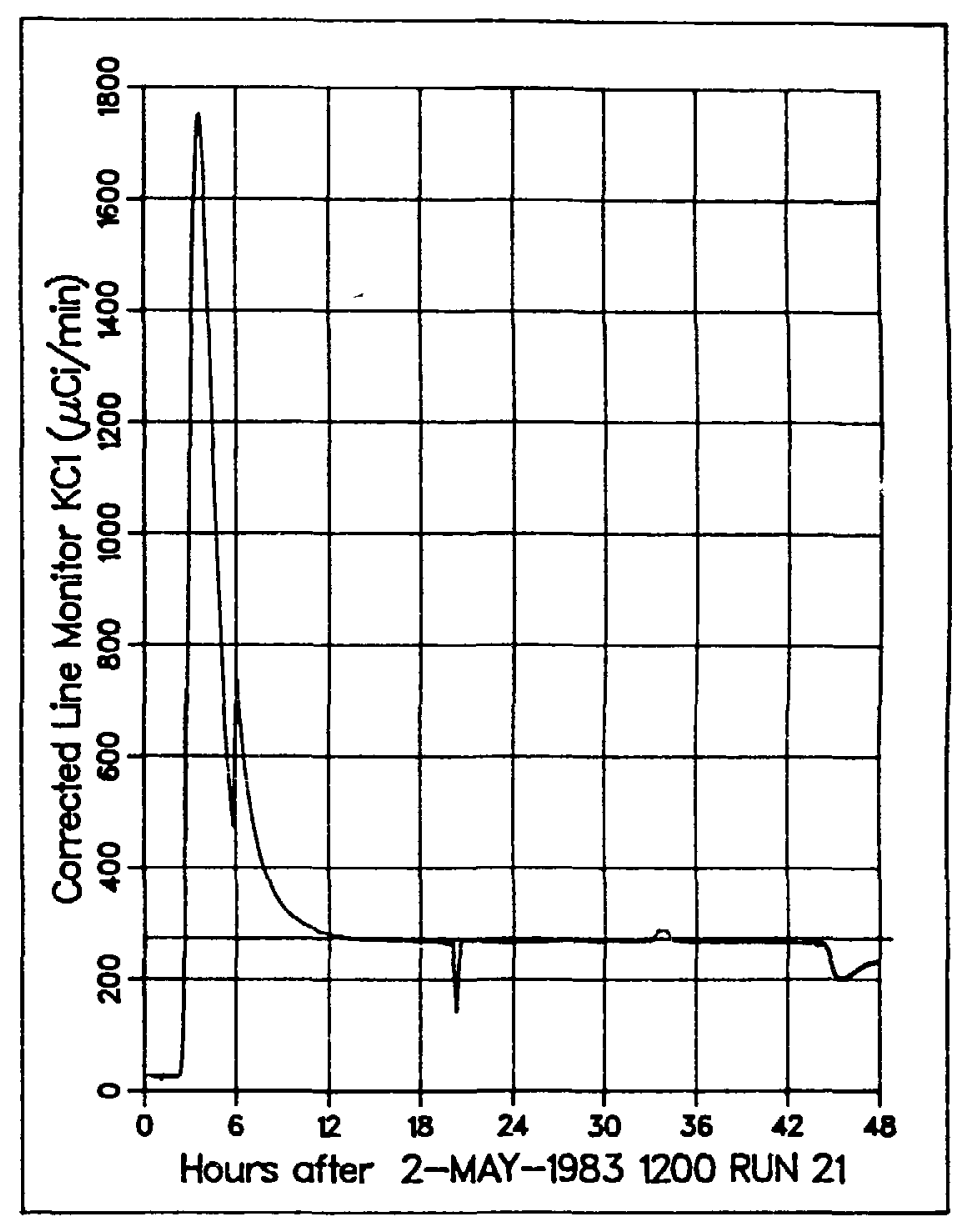

Fig. VI-52. Tritium release rate for Run 21. 
tritium release curve shows a very strong positive peak followed by a return to steady state. The temperature perturbation at 0500 on May 3 did not have a noticeable effect upon tritium release.

Run 22 was at a nominal temperature of $600^{\circ} \mathrm{C}$ with a flow rate of 100 $\mathrm{cm}^{3} / \mathrm{min}$ for the sweep gas of helium $+0.1 \% \mathrm{H}_{2}$. The temperature and tritium release data are shown in Figs. VI-53 and -54 , respectively. The run represents a decrease of $50^{\circ} \mathrm{C}$ from Run 21 . The temperatures were essentially unifors throughout the course of the run. The tritium release curve has a negative peak, followed by a return to equilibrium conditions. There is a fluctuation at 1500 resulting from temporary pressure and flow changes caused by a sample change.

Run 23 was at a nominal temperature of $550^{\circ} \mathrm{C}$ with a flow rate of 100 $\mathrm{cm}^{3} / \mathrm{min}$ for the sweep gas of hellum $+0.1 \% \mathrm{H}_{2}$. The temperature and tritium release data are shown in Figs. VI-55 and -56, respectively. The run represents a decrease in temperature from the prior run. The temperature profiles show the initial temperature drop, followed by an ORR shutdown at 1700 on May 5 , an ORR restart at 1900 on May 6, two reactor setbacks, and a number of sma11 step changes in temperature. Owing to the many changes in temperature and neutron flux, the tritium release curve is very complex. It can be said that the temperature decrease at the start of the run caused a negative peak. The release curve at the end of the run appears to approach steady-state conditions.

Run 24 was at a nominal temperature of $600^{\circ} \mathrm{C}$ with a flow rate of 100 $\mathrm{cm}^{3} /$ min for the sweep gas of helium $+0.1 \% \mathrm{H}_{2}$. The temperature and tritium release data are shown in Figs. VI-57 and -58 , respectively. The run was the same as the previous one except that the temperature was higher by $50^{\circ} \mathrm{C}$. The temperatures and neutron flux were essentially constant for the duration of the run. The increase in temperature caused a large positive spike in the tritium release curve followed by a return to steady-state conditions within $20 \mathrm{~h}$. The drop in the release curve at the peak maximum is due to a scale change in the tritium monitor.

Run 25 was at a nominal temperature of $560^{\circ} \mathrm{C}$ with a flow rate of 100 $\mathrm{cm}^{3} / \mathrm{min}$ for the sweep gas of helium $+0.1 \% \mathrm{H}_{2}$. The test conditions represent a decrease of $40^{\circ} \mathrm{C}$ from Run 24. The temperature and tritium release data are shown In Figs. VI-59 and -60 , respectively. The temperature data show very 


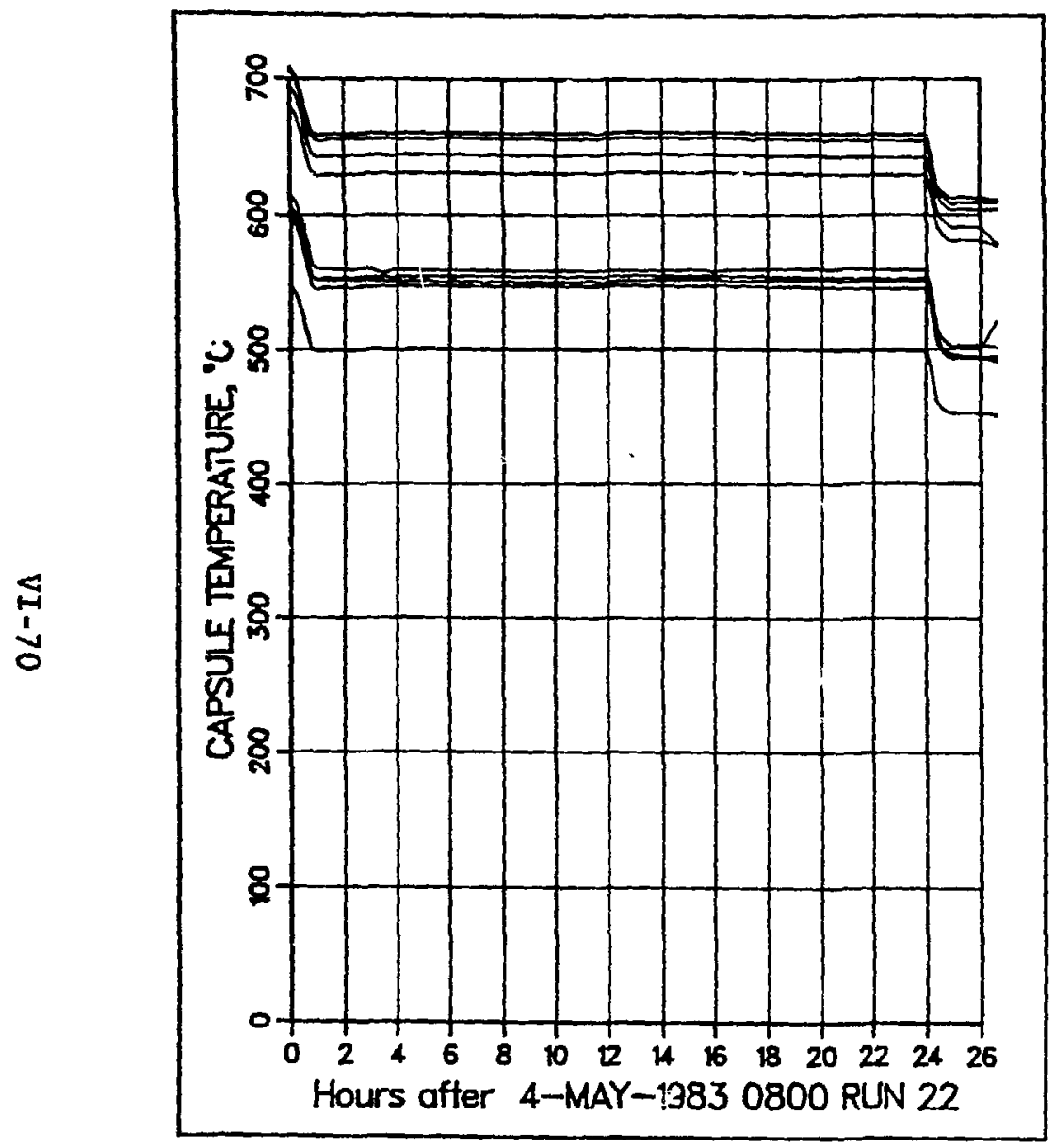

Fig. VI-53. Recorded temperatures for Run 22.

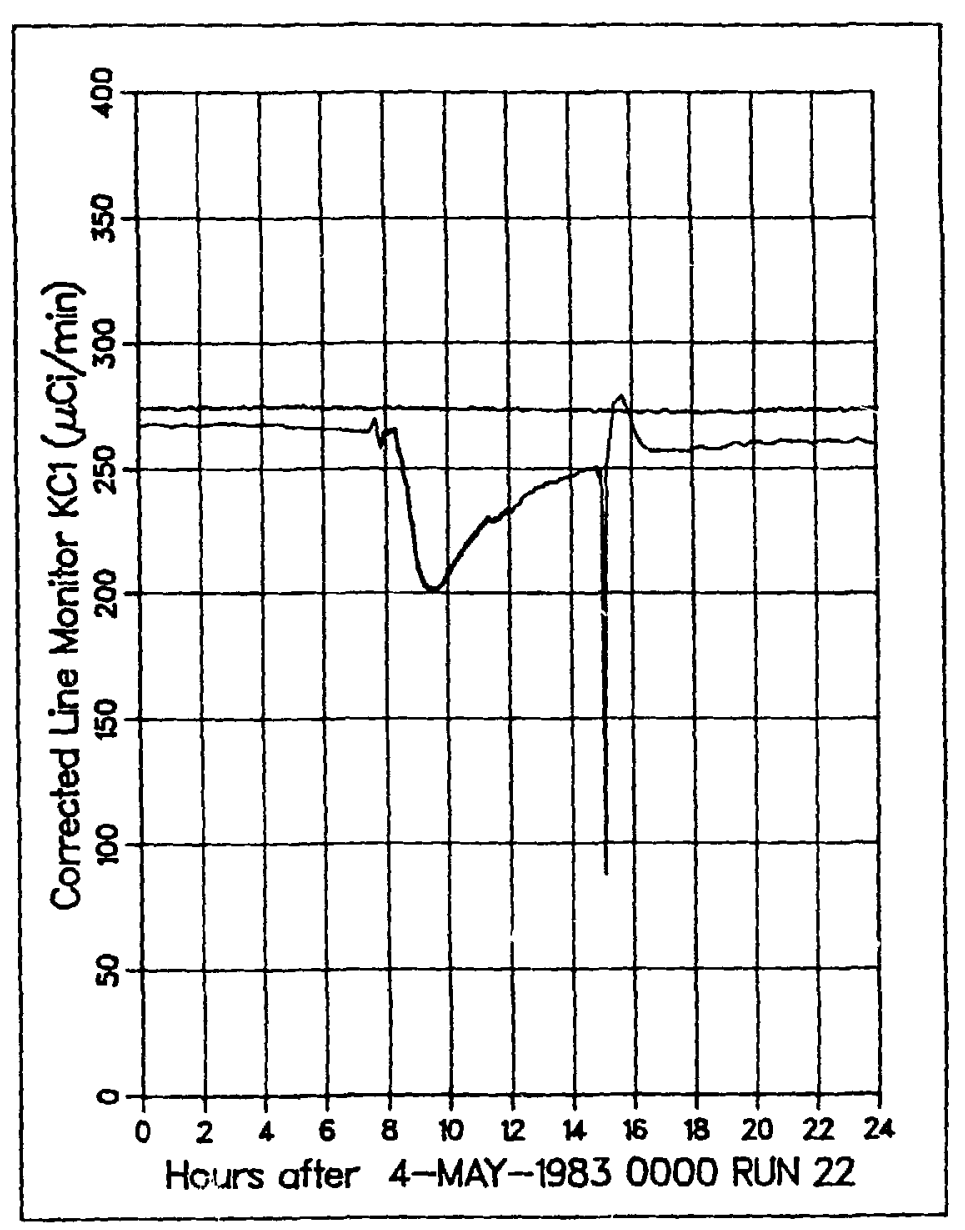

Fig. VI-54. Tritium release rate for Run 22 . 


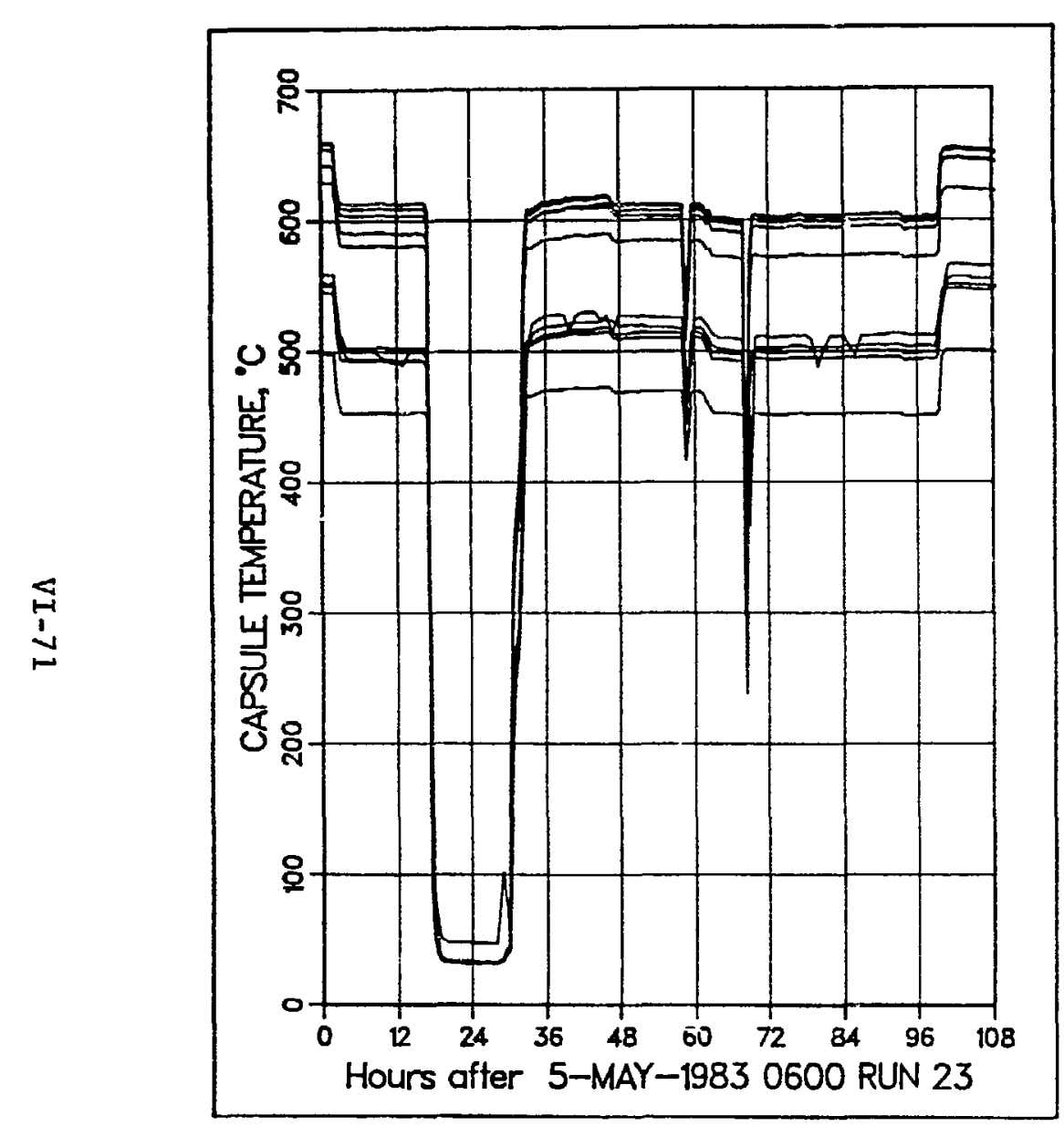

Fig. VI-55. Recorded temperatures for Run 23.

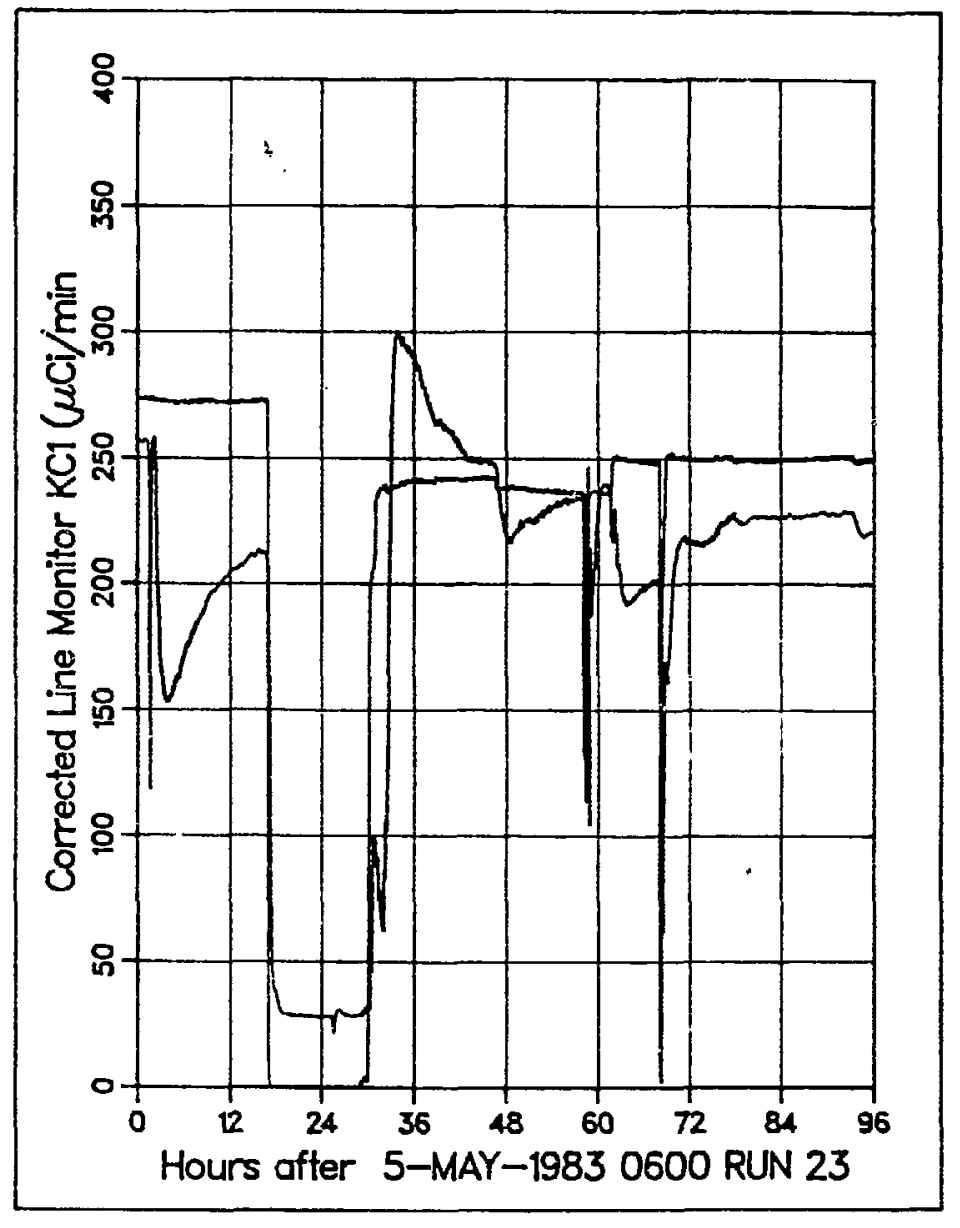

Fig. VI-56. Tritium release rate for Run 23. 


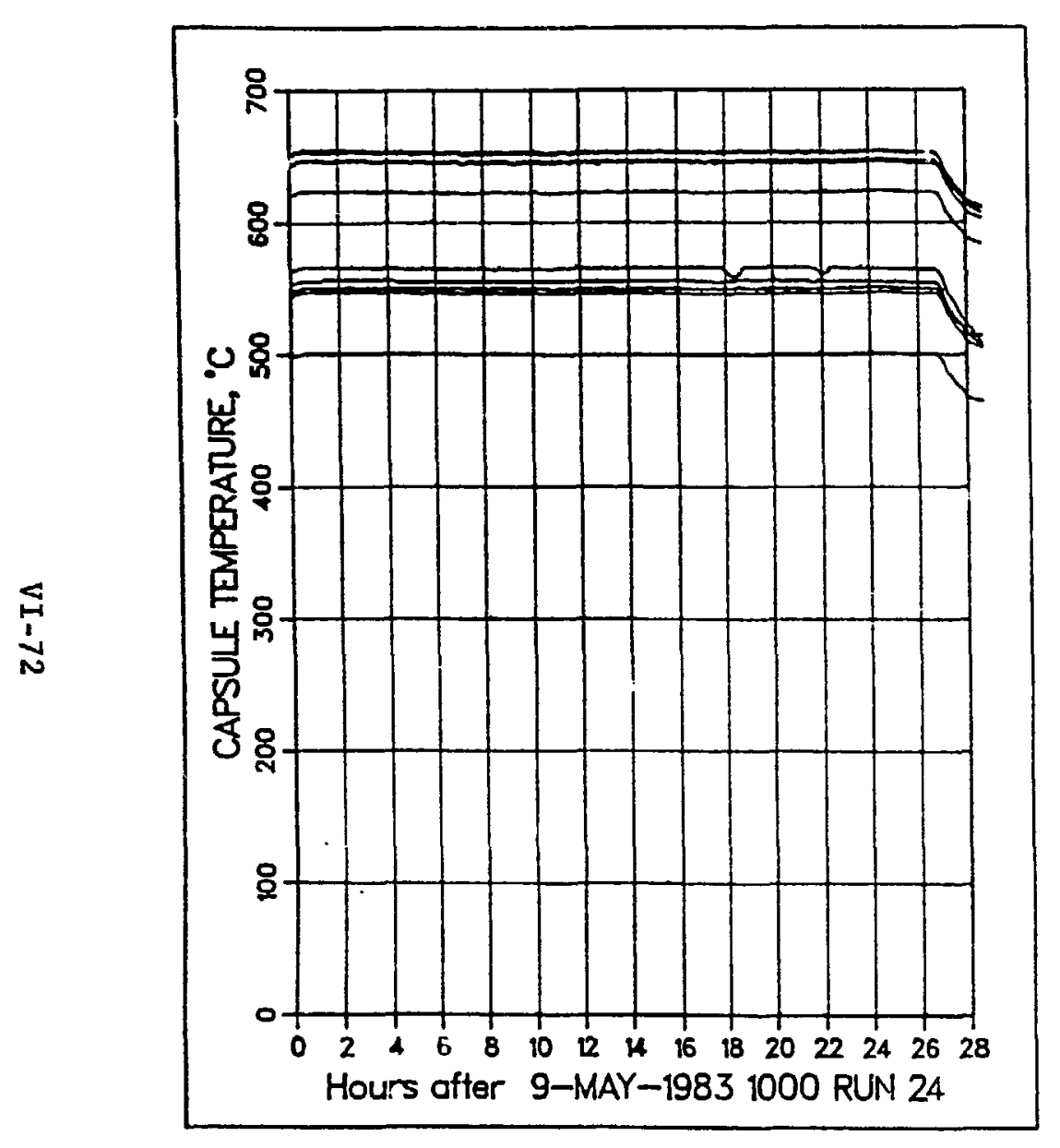

Fig. VI-57. Recorded temperatures for Run 24.

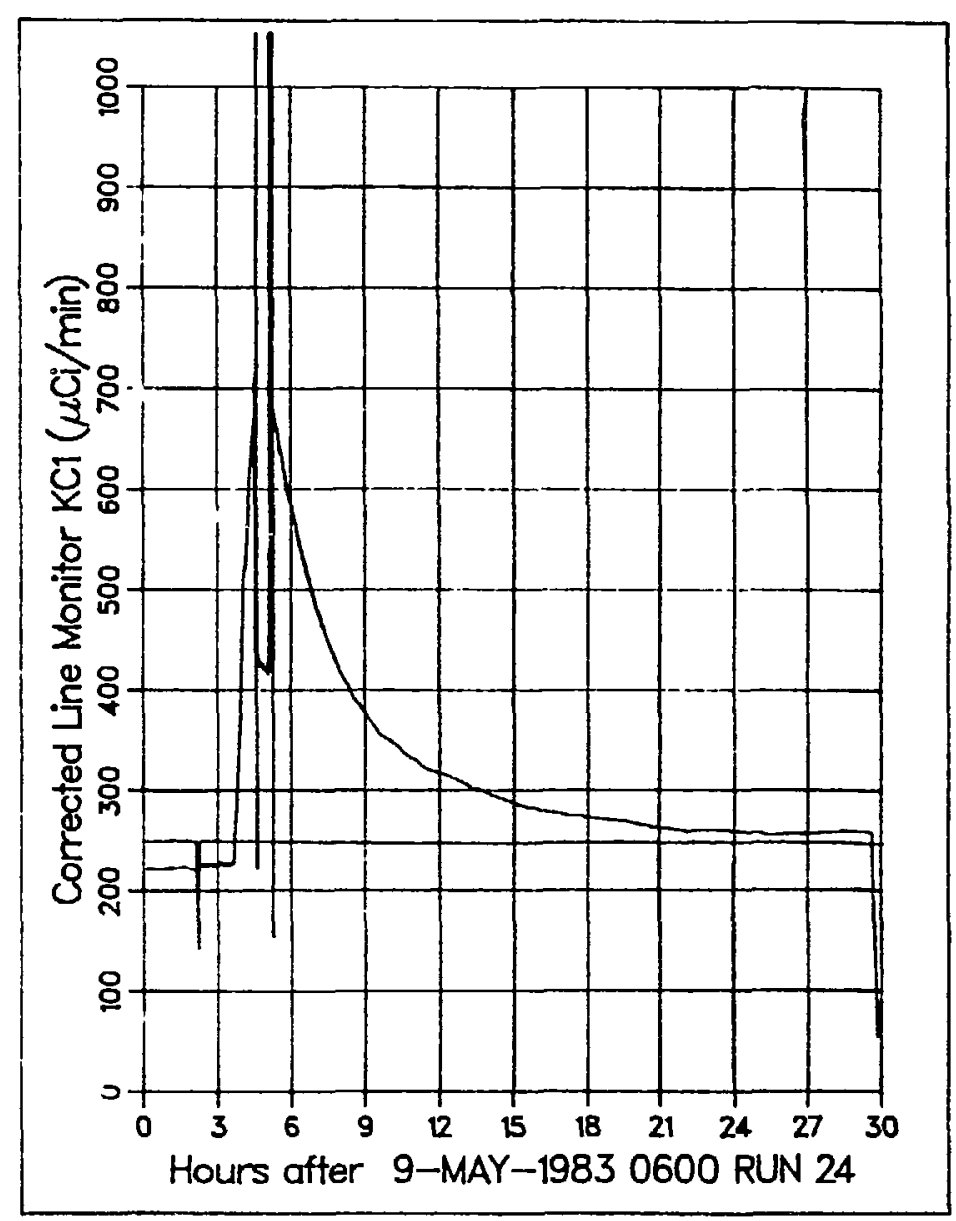

Fig. VI-58. Tritium release rate for Run 24 . 


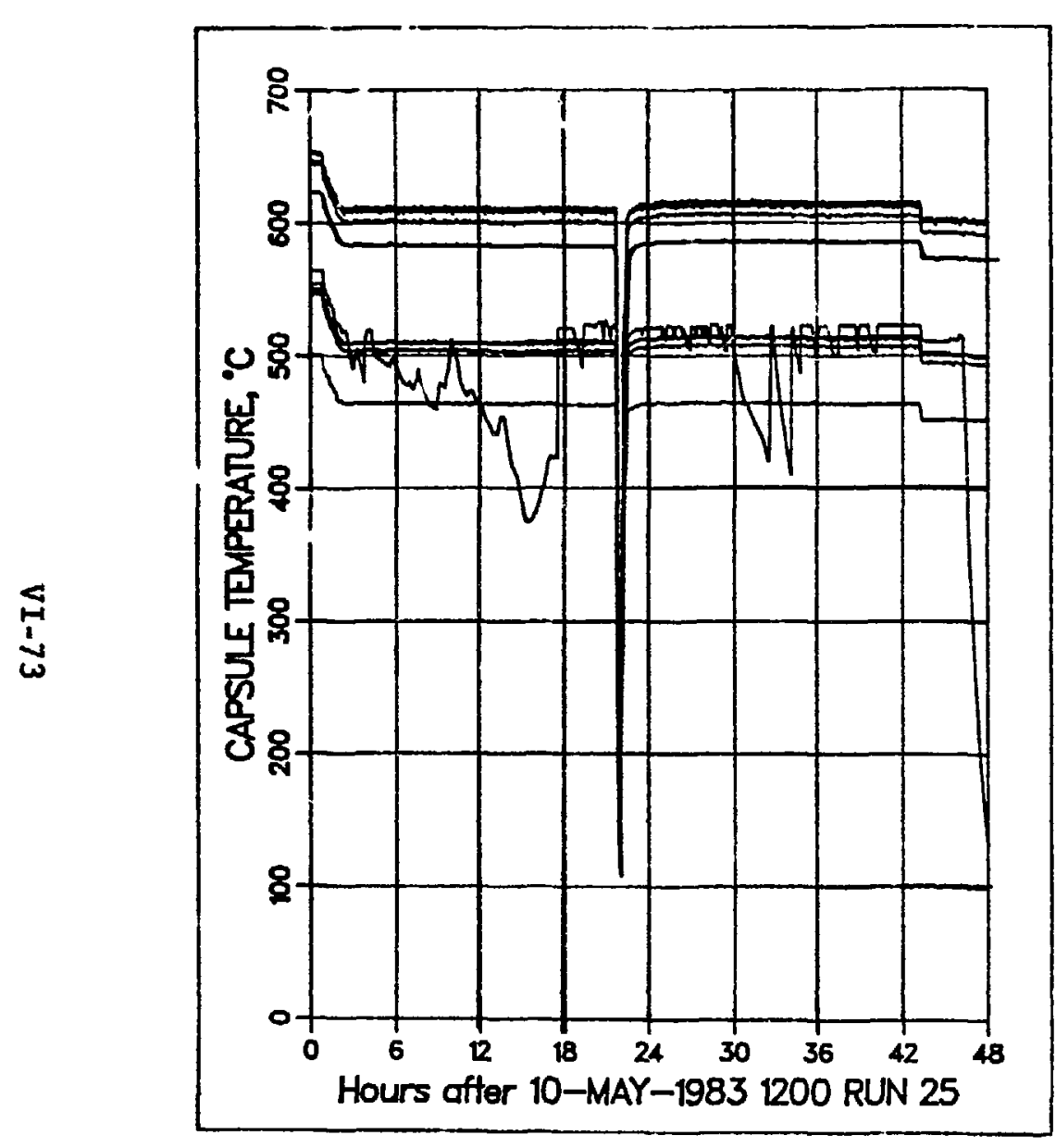

Fig. VI-59. Recorded temperatures for Run 25.

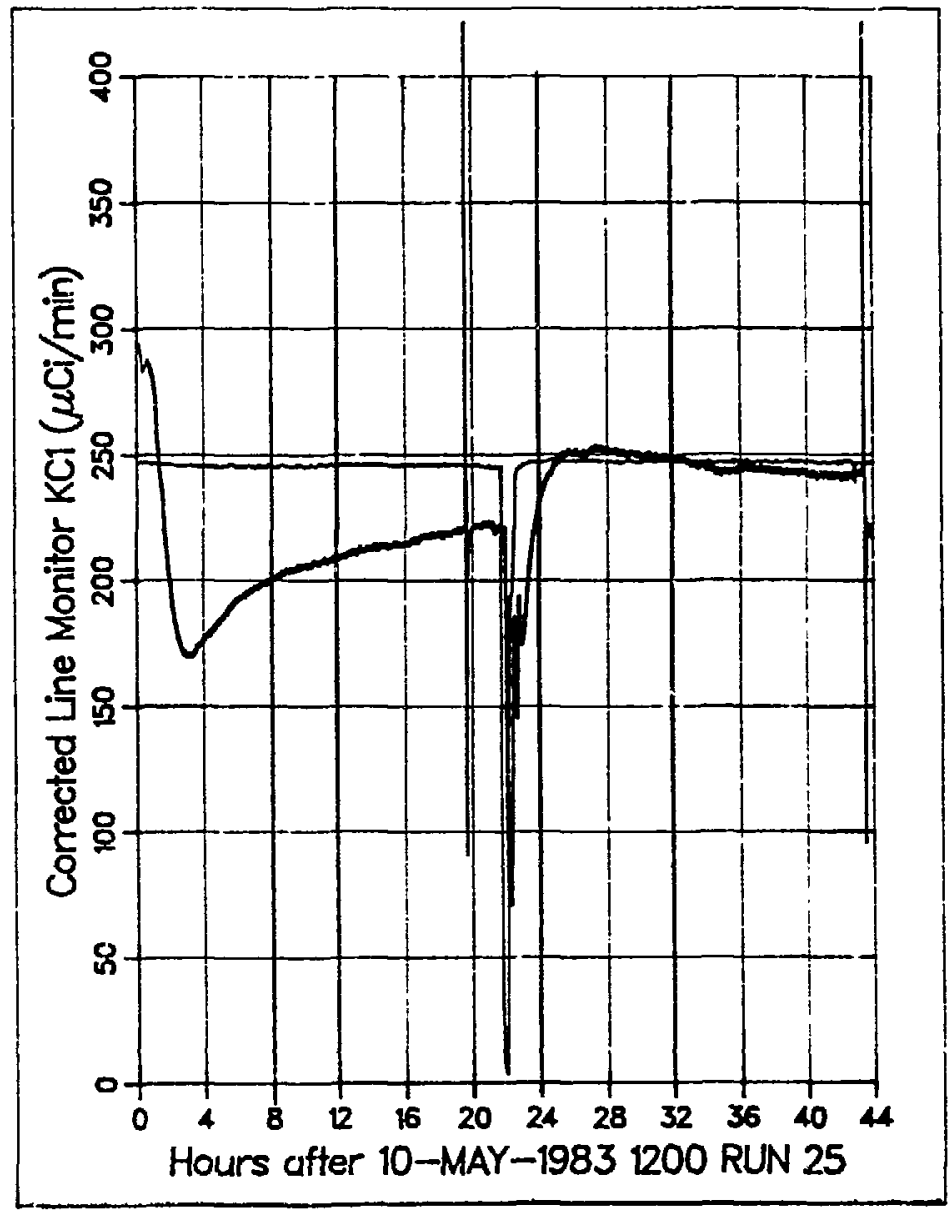

Fig. VI-60. Tritium release rate for Run 25. 
constant temperatures except for a reactor setback which occurred at 1045 on May 11. Thermocouple TR90l continued to be erratic. The tritium release curve shows a negative peak followed by a return to equilibrium. The reactor setback resulted in a significant perturbation in the release curve, owing to the drop in temperatures.

Run 26 was at a nominal temperature of $550^{\circ} \mathrm{C}$ with a flow rate of 100 $\mathrm{cm}^{3} / \mathrm{min}$ for the sweep gas of helium $+0.1 \% \mathrm{H}_{2}$. The temperature and tritium release data are shown in Figs. VI-61 and -62 , respectively. The test conditions for the run represent a temperature decrease of $10^{\circ} \mathrm{C}$ from Run 25 . In Fig. VI-62 there is a small negative peak followed by a return to steady state in $\sim 70 \mathrm{~h}$. A perturbation resulting from a sample change is observable in this figure at 0700 on May 13.

Run 27 was at a nominal temperature of $525^{\circ} \mathrm{C}$ with a flow rate of 100 $\mathrm{cm}^{3} / \mathrm{min}$ for the sweep gas of helium $+0.1 \% \mathrm{H}_{2}$. The temperature and tritium release data are shown in Figs. VI -63 and -64 , respectively. The test conditions of the run represent a temperature decrease of $25^{\circ} \mathrm{C}$ from Run 26 . There was a reactor shutdown followed by a restart at 1400 on May 16 . The temperature profiles show that the nominal temperature was dropped from 550 to $525^{\circ} \mathrm{C}$ at 1200 on May 17. After that time, the reactor power was at $28.5 \mathrm{MH}$ (95\% of full power) for the rest of the run. The temperature drop of $25^{\circ} \mathrm{C}$ results in a negative peak followed by a return to steady state in about $150 \mathrm{~h}$. On the night of May 20, essentially all the sweep gas leaked into a glovebox; the amount lost was calculated to be $0.16 \mathrm{Cl}$. No measurable tritium was detected in the room. The levels of tritium in the glovebox (FIg. VI-65) rose to about $15,000 \mu \mathrm{Ci} / \mathrm{m}^{3}$.

Run 28 was at a nominal temperature of $500^{\circ} \mathrm{C}$ with a flow rate of 100 $\mathrm{cm}^{3} / \mathrm{min}$ for the sweep gas of hellum $+0.1 \% \mathrm{H}_{2}$. The temperature and tritium release data are shown in F1gs. VI-66 and -67 , respectively. The run represents a decrease in temperature of $25^{\circ} \mathrm{C}$ from Run 27 . The temperature readout from TR901 was still giving problems; thus the negative spikes on May 26 are due to recording problems and not temperature variations. There is a fivedegree increase in temperature at 1700 on May 29, owing to a slight increase In neutron flux. The tritium release curve shows a negative peak followed by a slow return to equilibrium after about $170 \mathrm{~h}$ ( 7 days). There are a number of oscillations in the tritium release curve, which were caused by small changes in temperature and in flow conditions. 


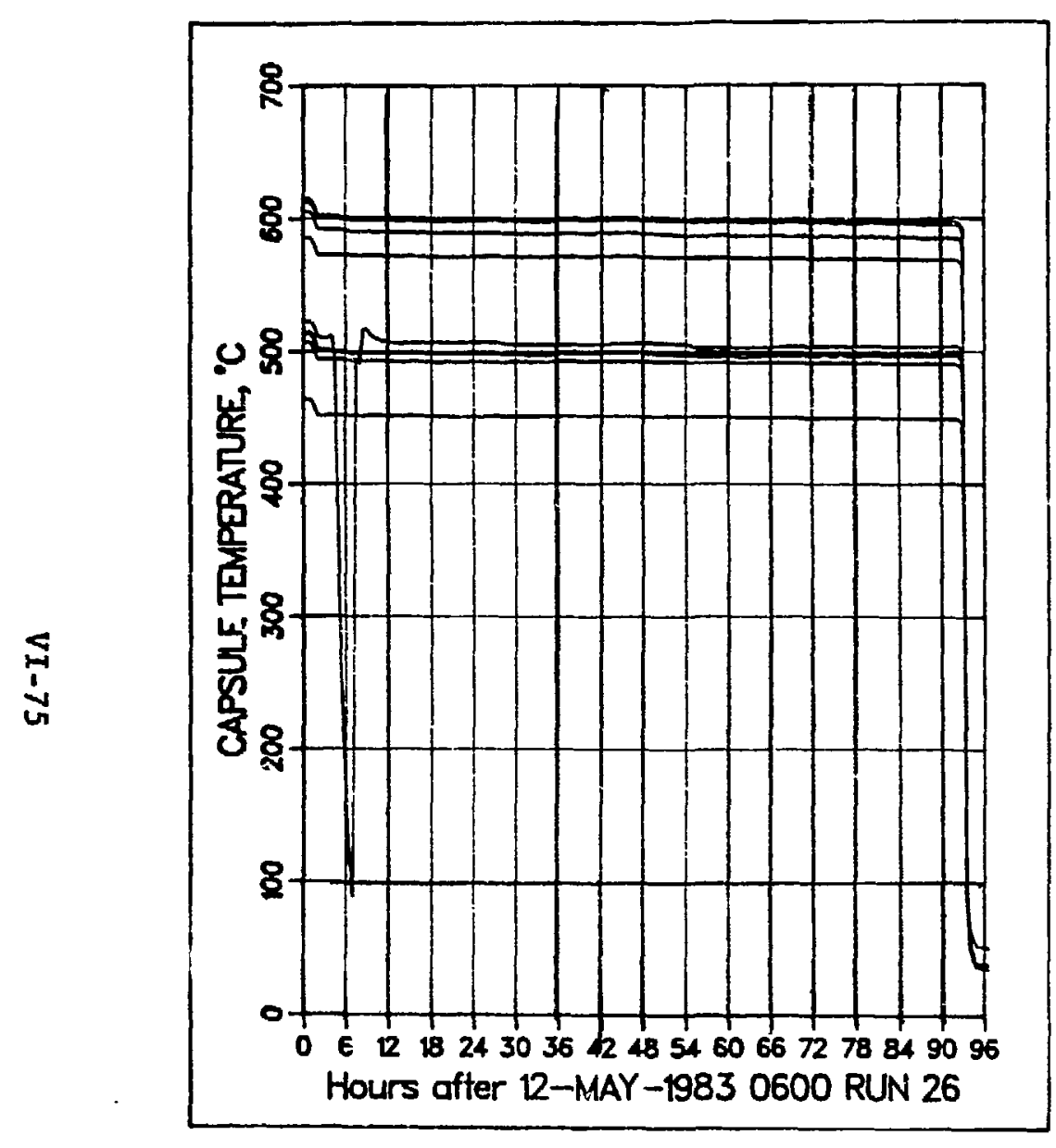

Fig. VI-61. Recorded temperatures for Run 26.

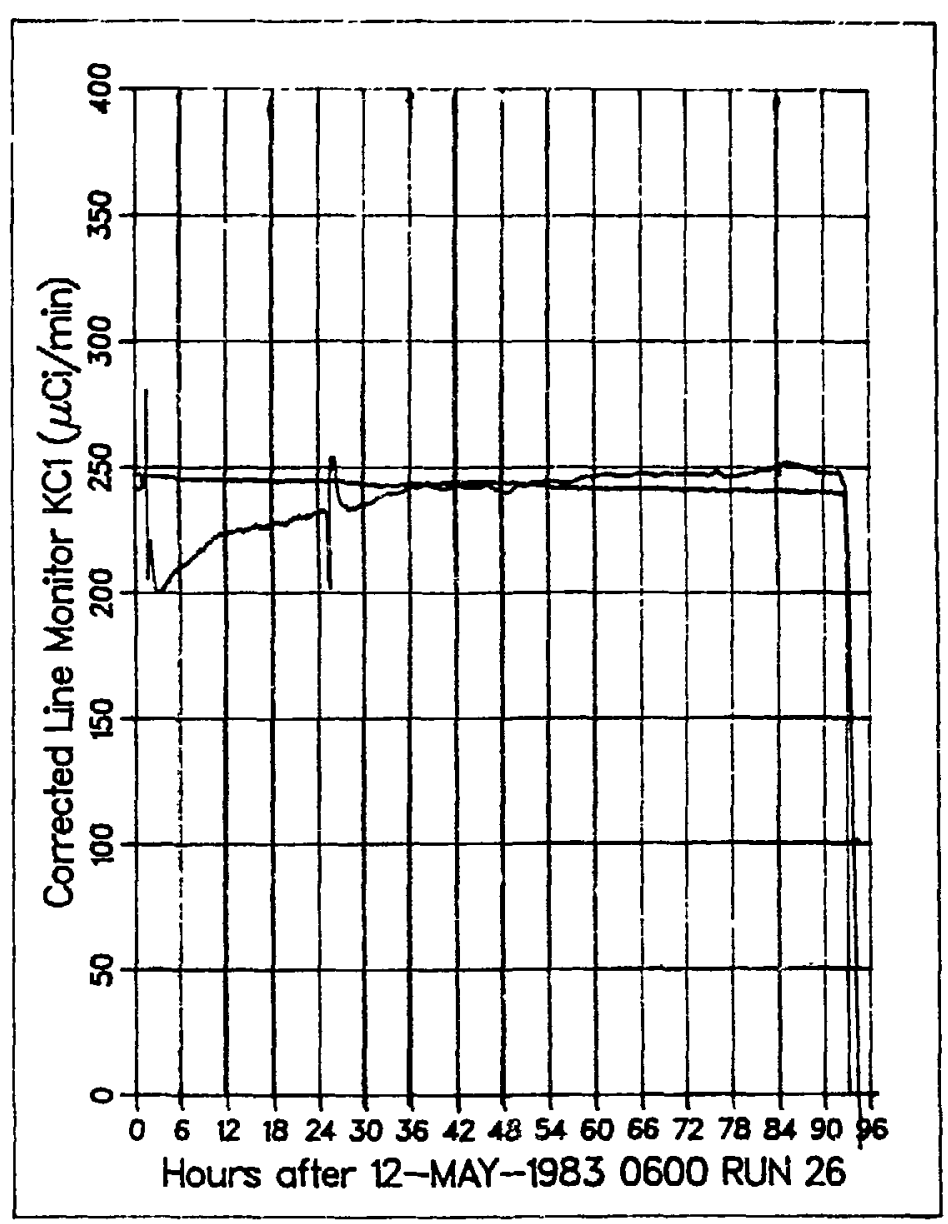

Fig. VI-62. Tritium release rate for Run 26 . 


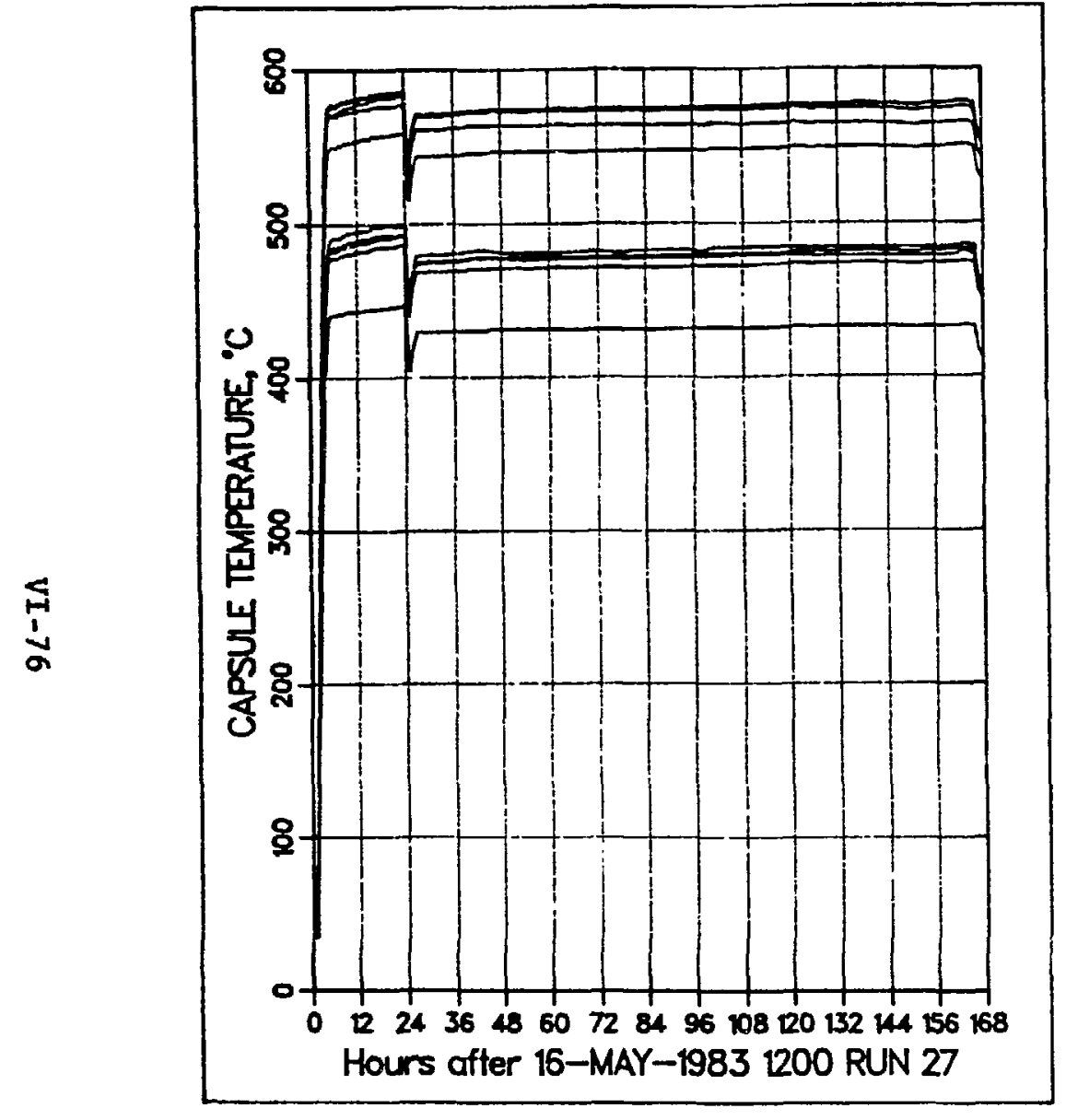

Fig. VI-63. Recorded temperatures for Run 27.

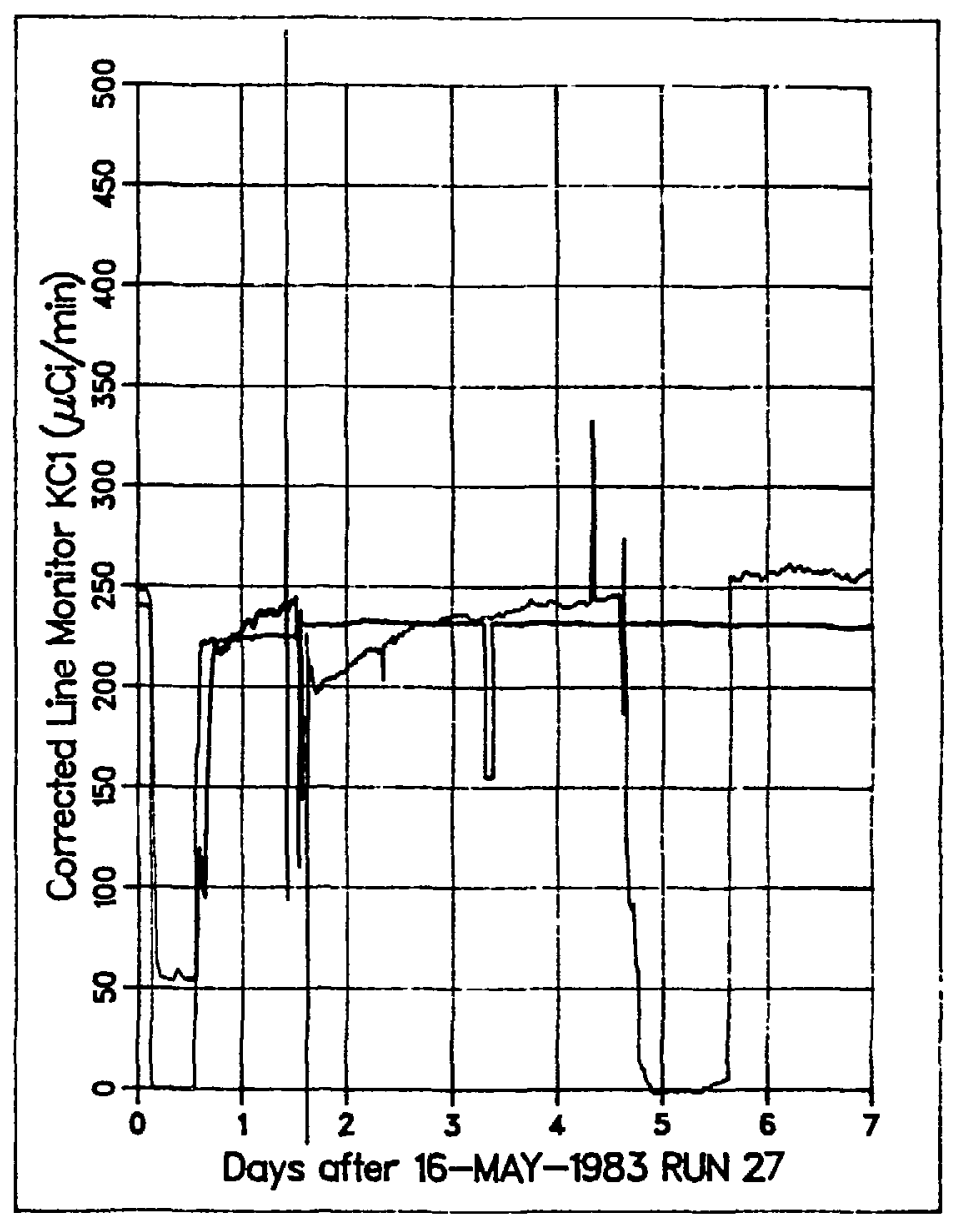

Fig. VI-64. Tritium release rate for Run 27. 


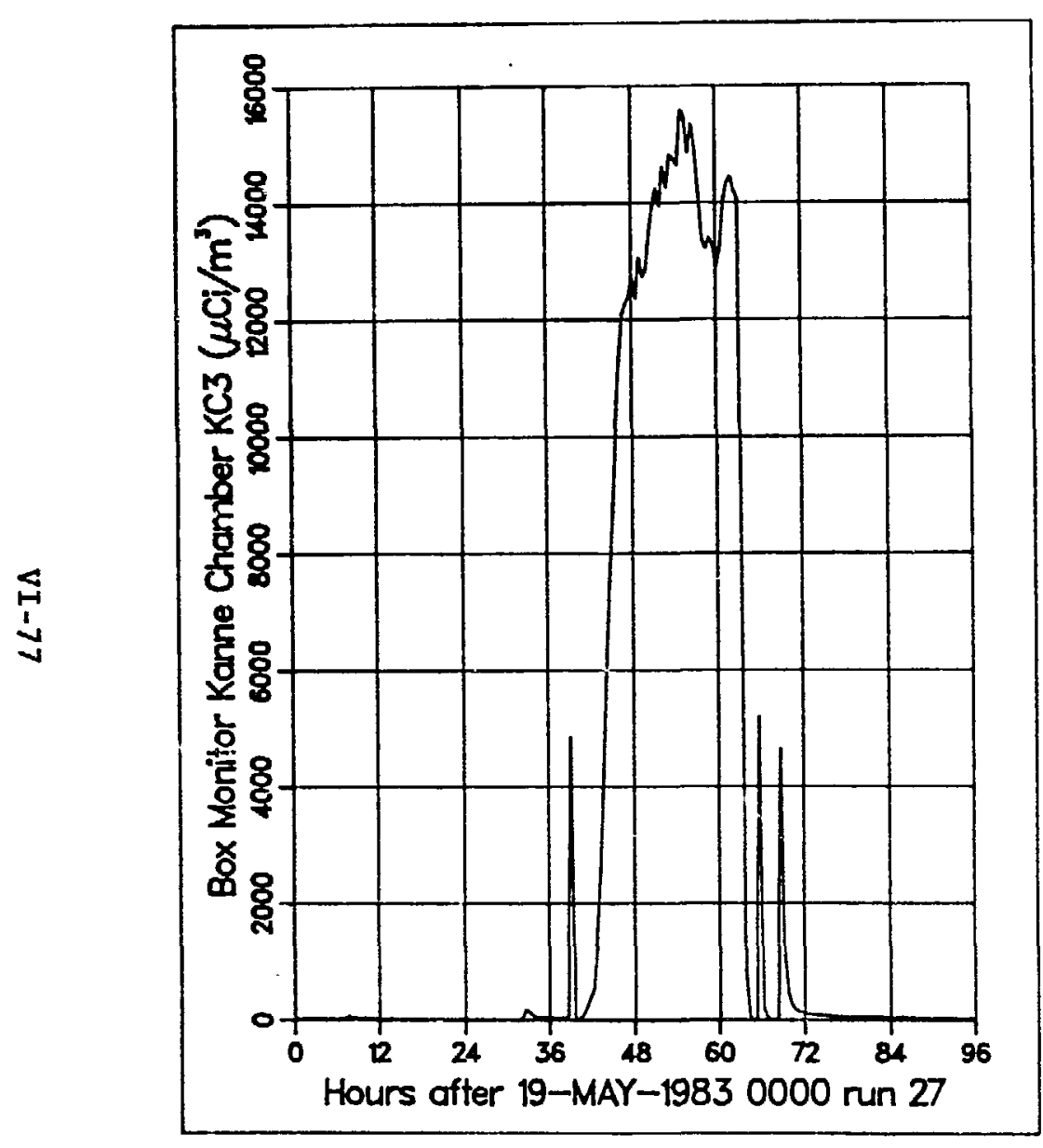

Fig. VI-65. Tritium leaked into glovebox af $t \in r$ May 20.

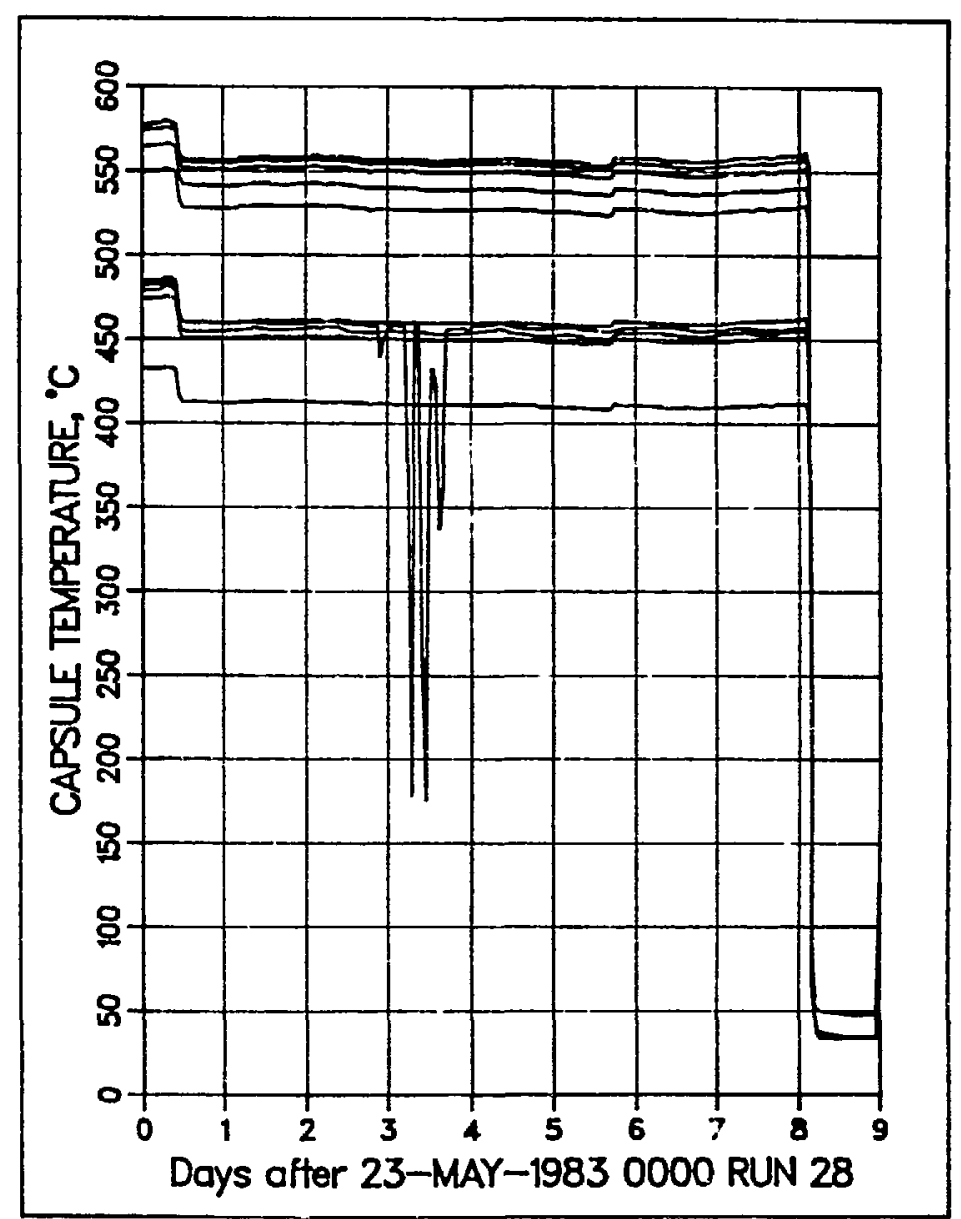

Fig. VI-66. Recorded temperatures for Run 28 . 


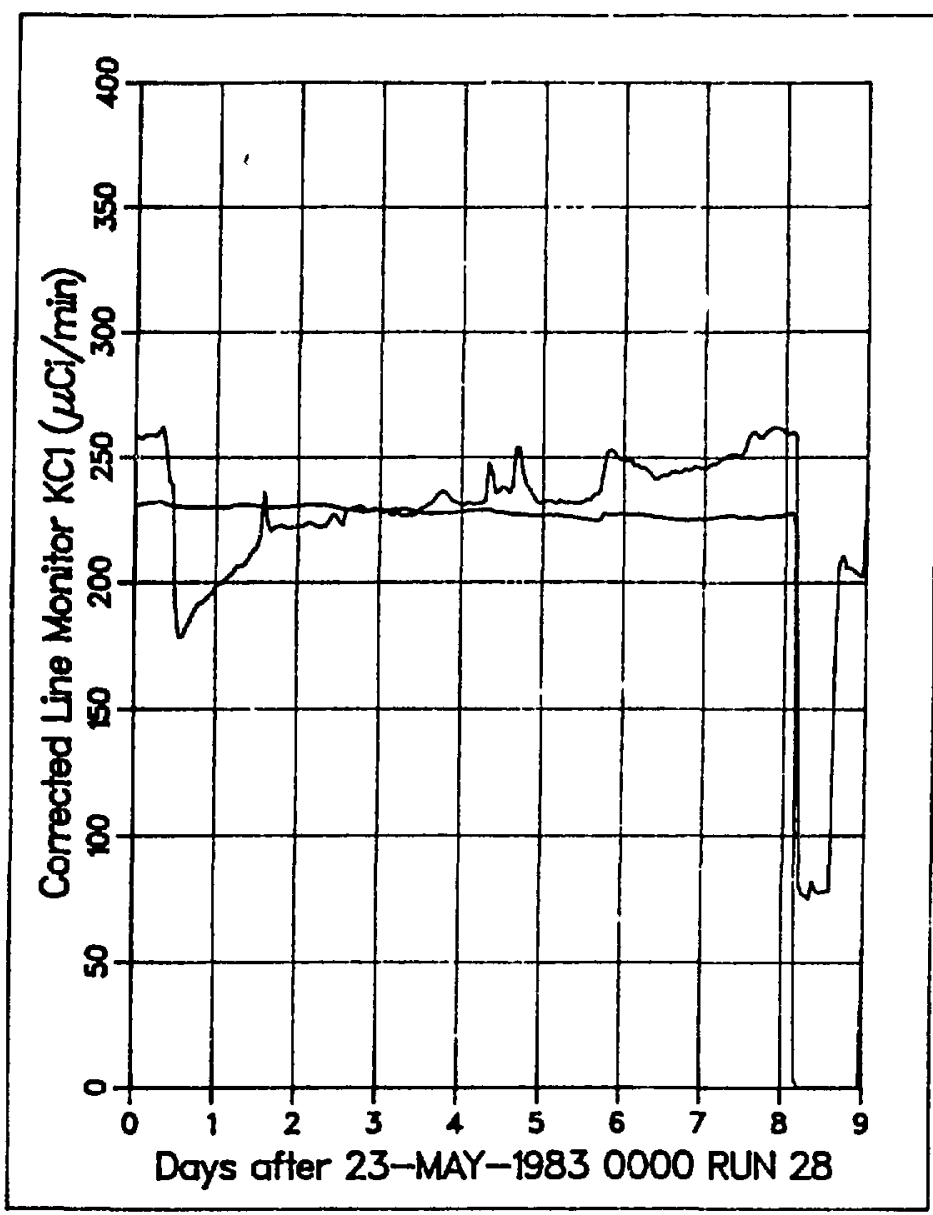

Fig. VI-67. Tritium release rate for Run 28.

Run 29 was at a nominal temperature of $480^{\circ} \mathrm{C}$ with a flow rate of 300 $\mathrm{cm}^{3} / \mathrm{min}$ for the sweep gas of helium $+0.1 \% \mathrm{H}_{2}$. The temperature and tritium release data are shown in Figs. VI-68 and -69 , respectively. The test conditions of the run represent a decrease in temperature of $20^{\circ} \mathrm{C}$ and a threefold increase in flow from Run 28. The temperature profile reflects a reactor shutdown on June 1 and some temperature oscillations on June 2. The tritium release curve shows overall a negative peak followed by a slow approach to equilibrium. The run does not appear to reach steady-state conditions.

Run 30 was at a nominal temperature of $480^{\circ} \mathrm{C}$ with a flow rate of 100 $\mathrm{cm}^{3} / \mathrm{min}$ for the sweep gas of hellum $+0.1 \% \mathrm{H}_{2}$. The temperature and tritium release data are shown in Figs. VI-70 and -71 , respectively. Figure VI-70 shows that the temperatures were essentially constant for the run. The tritium release curve shows a slow approach to equilibrium; steady-state 


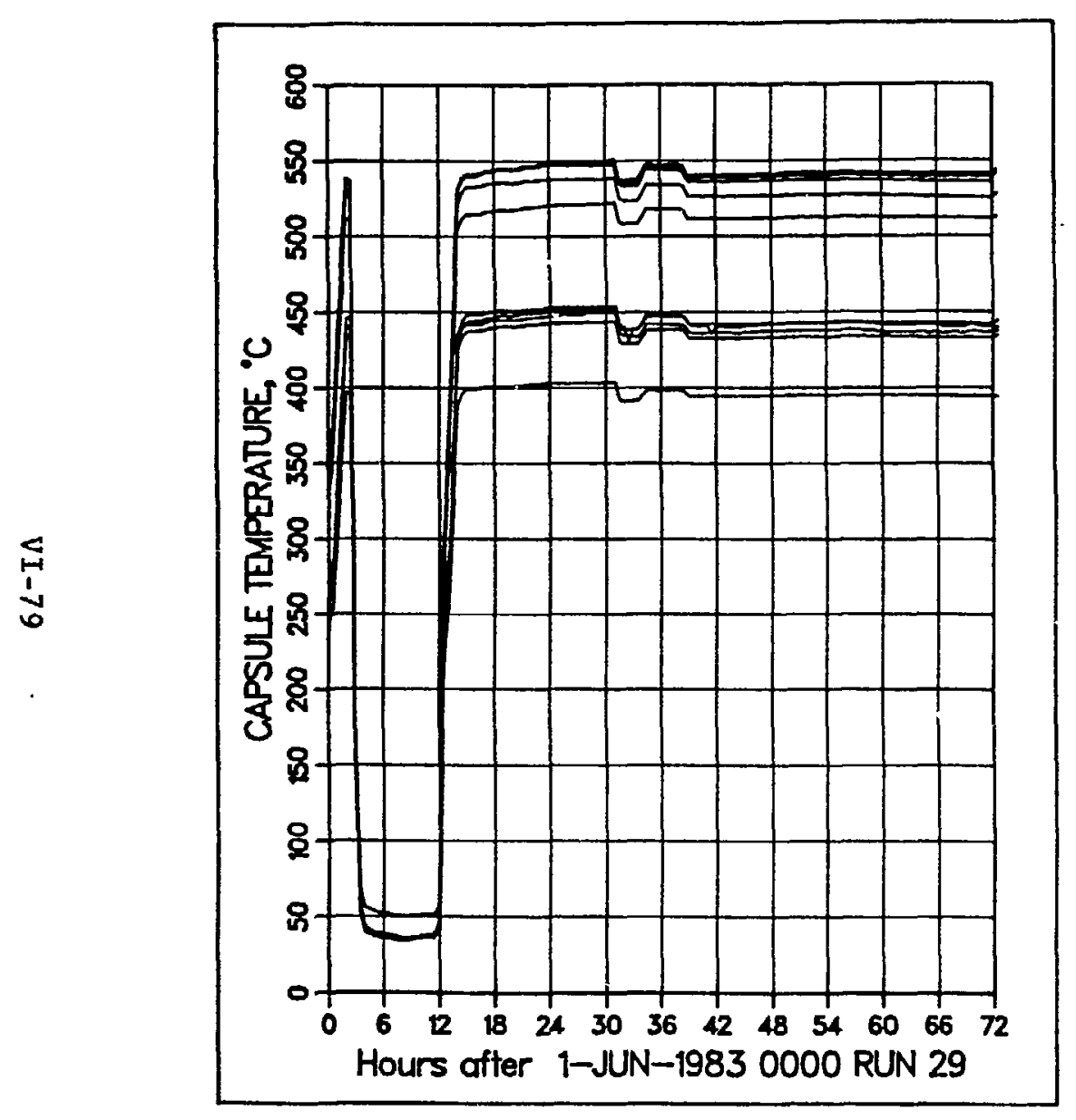

Fig. VI-6ع. Recorded temperatures for Run 29.

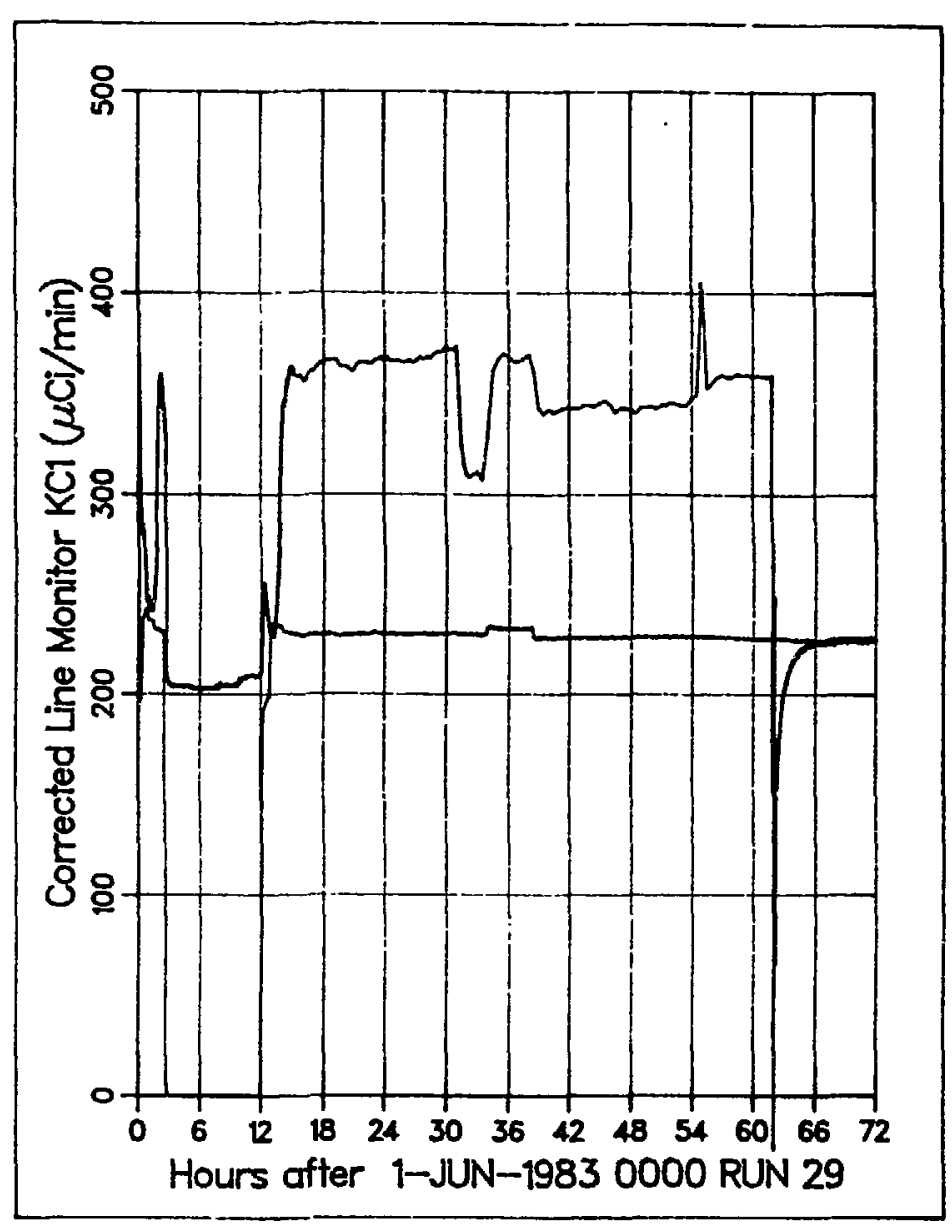

Fig. VI-69. Tritium release rate for Run 29. 


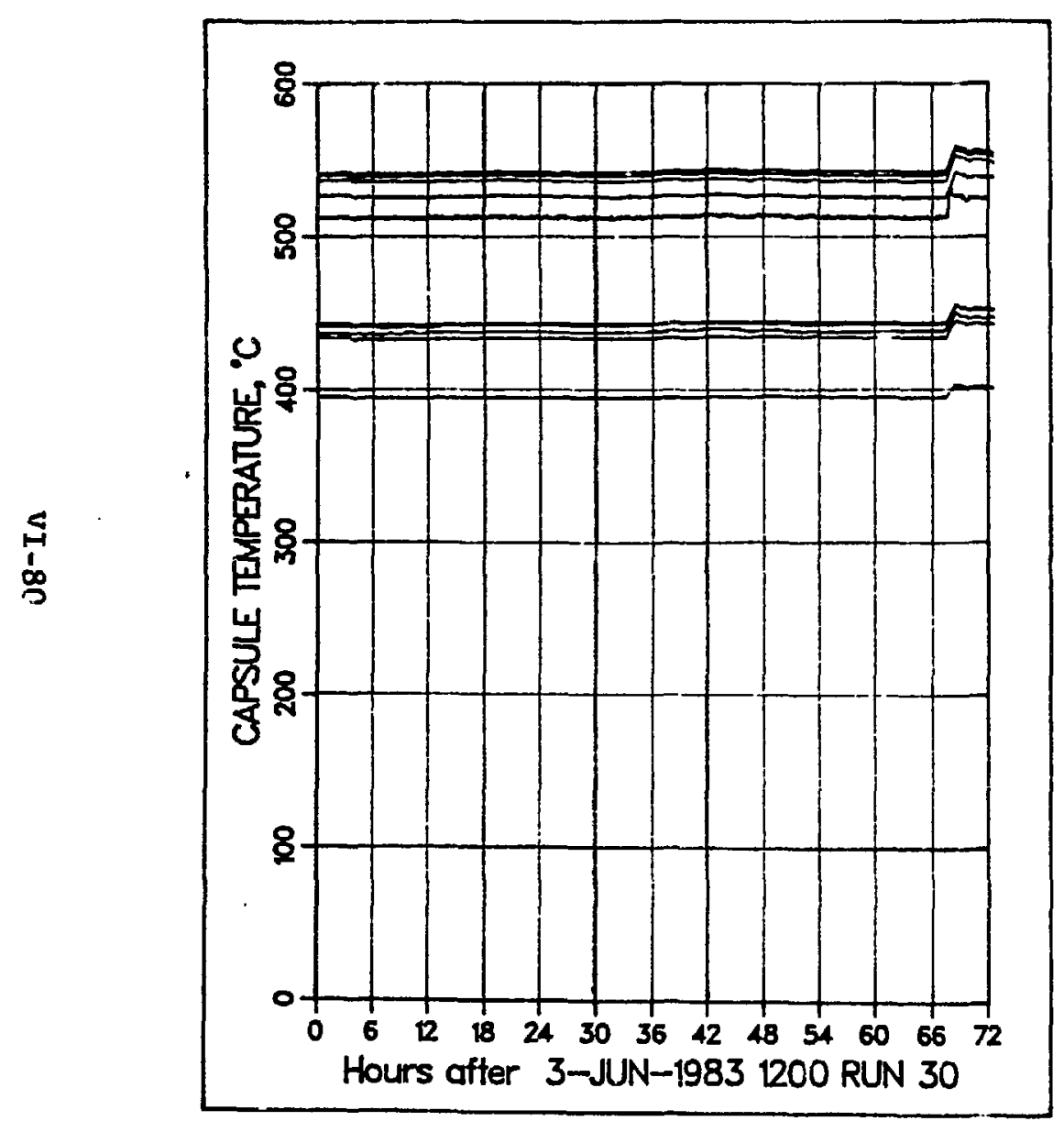

Fig. VI-70. Recorded temperatures for Run 30.

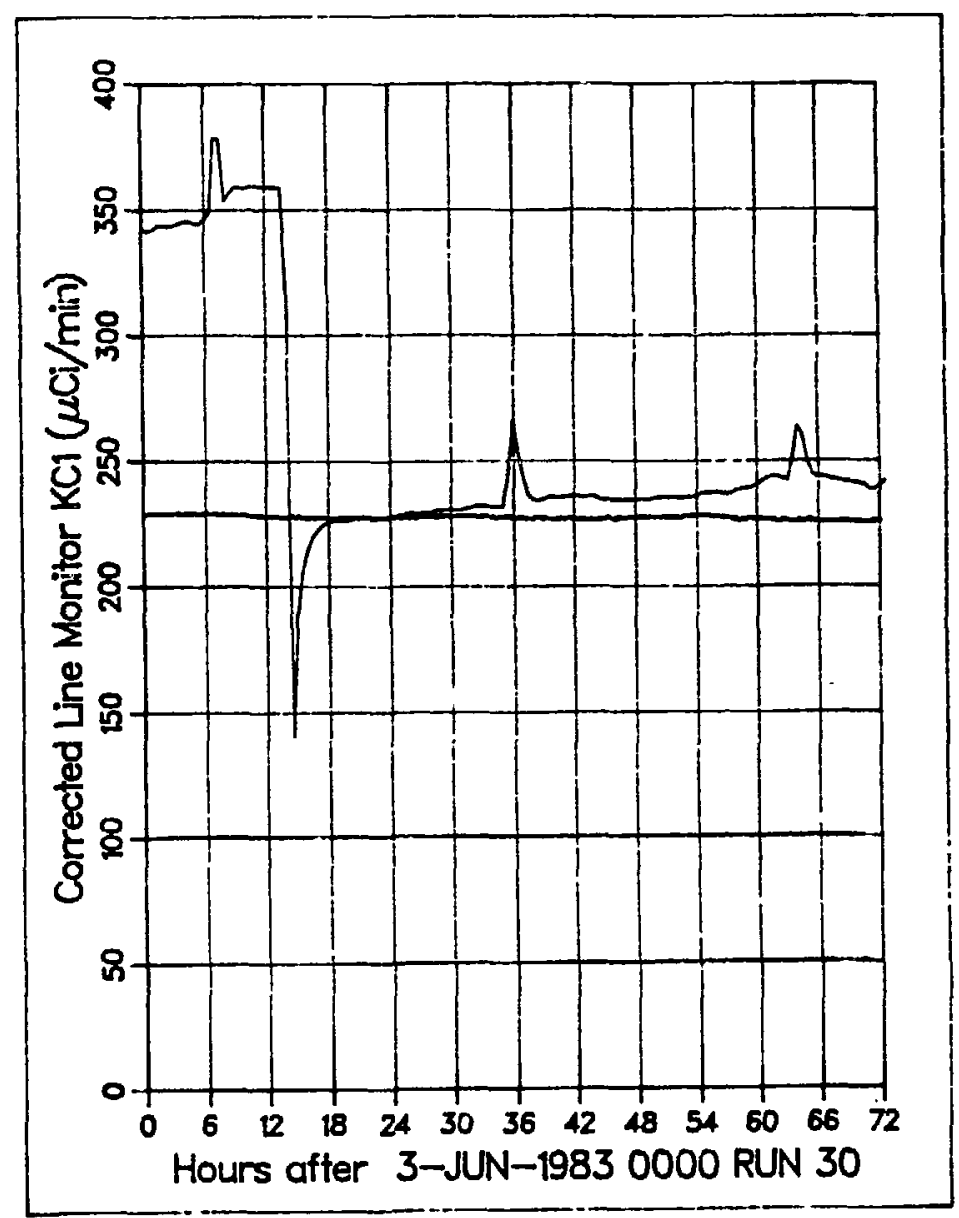

Fig. VI-71. Tritium release rate for Run 30 . 
conditions are needed after $\sim 6$ days at $480^{\circ} \mathrm{C}$, including the time at this temperature from Run 29.

Run 31 was at a nominal temperature of $500^{\circ} \mathrm{C}$ with a flow rate of 100 $\mathrm{cm}^{3} / \mathrm{min}$ for the sweep gas of helium $+0.1 \% \mathrm{H}_{2}$. The temperature and tritium release data are shown in Figs. VI-72 and -73 , respectively. The run represents a $20^{\circ} \mathrm{C}$ temperature increase from Run 30 . The tritium release curve shows a small positive peak followed by a return to steady state In about $40 \mathrm{~h}$.

Run 32 was at a nominal temperature of $550^{\circ} \mathrm{C}$ with a flow rate of 100 $\mathrm{cm}^{3} / \mathrm{min}$ for the sweep gas of helium $+0.1 \% \mathrm{H}_{2}$. The cemperature and tritium release data are shown in Figs. VI-74 and -75 , respectively. The run represents a $50^{\circ} \mathrm{C}$ temperature increase from Run 31. The tritium release curve shows a large positive spike followed by a return to equilibrium conditions. The run was terminated before the system reached steady state. There are again oscillations in the tritium signal owing to changes in scale.

Run 33 was at a nominal temperature of $650^{\circ} \mathrm{C}$ with a flow rate of 100 $\mathrm{cm}^{3} / \mathrm{min}$ for the sweep gas of helium $+0.1 \% \mathrm{H}_{2}$. The temperature and tritium release data are shown in Figs. VI-76 and -77 , respectively. The run represents a return to reference conditions. Since Run 33 was the last one, time was allowed to permit equilibration. The tritium release curve shows a very strong peak followed by a return to steady state within $20 \mathrm{~h}$.

\section{G. Release Rates of HT0, Condensable Forn}

The release rate of the HTO, or the condensable form, was not continually monitored in the way that HT was. Rather, samples were taken over various time intervals. These data wern presented in tabular form in Sec. VI.E. It is of some interest to determine the actual release rate of HTO. Therefore, the data presented in Sec. VI.E were used to calculate HTO release rates. These data are not instantaneous but average release rates over the time the sample was collected. Rather than a smooth curve, the release rate appears as a series of bar graphs. 


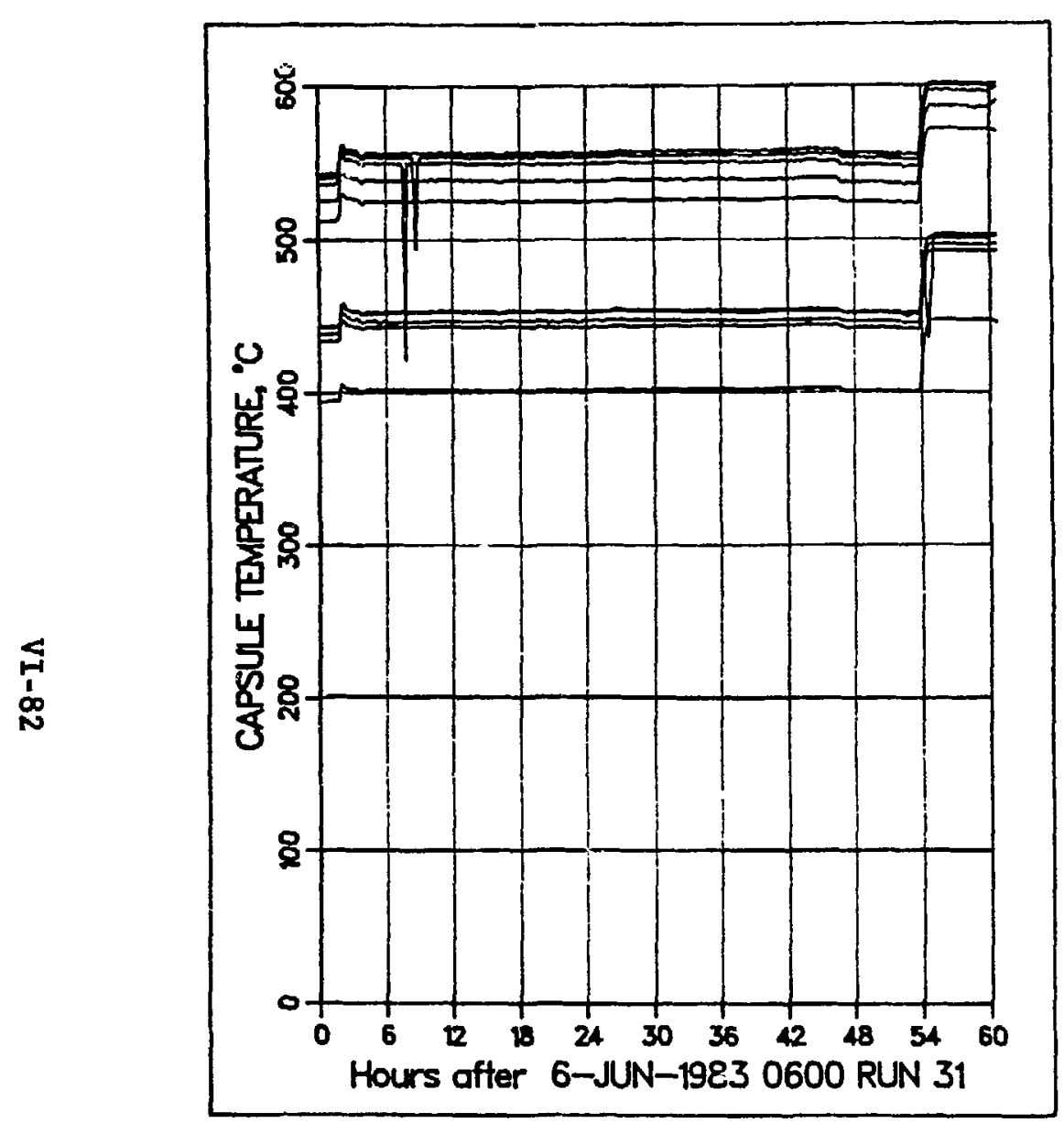

Fig. VI-72. Recorded temperatures for Run 31.

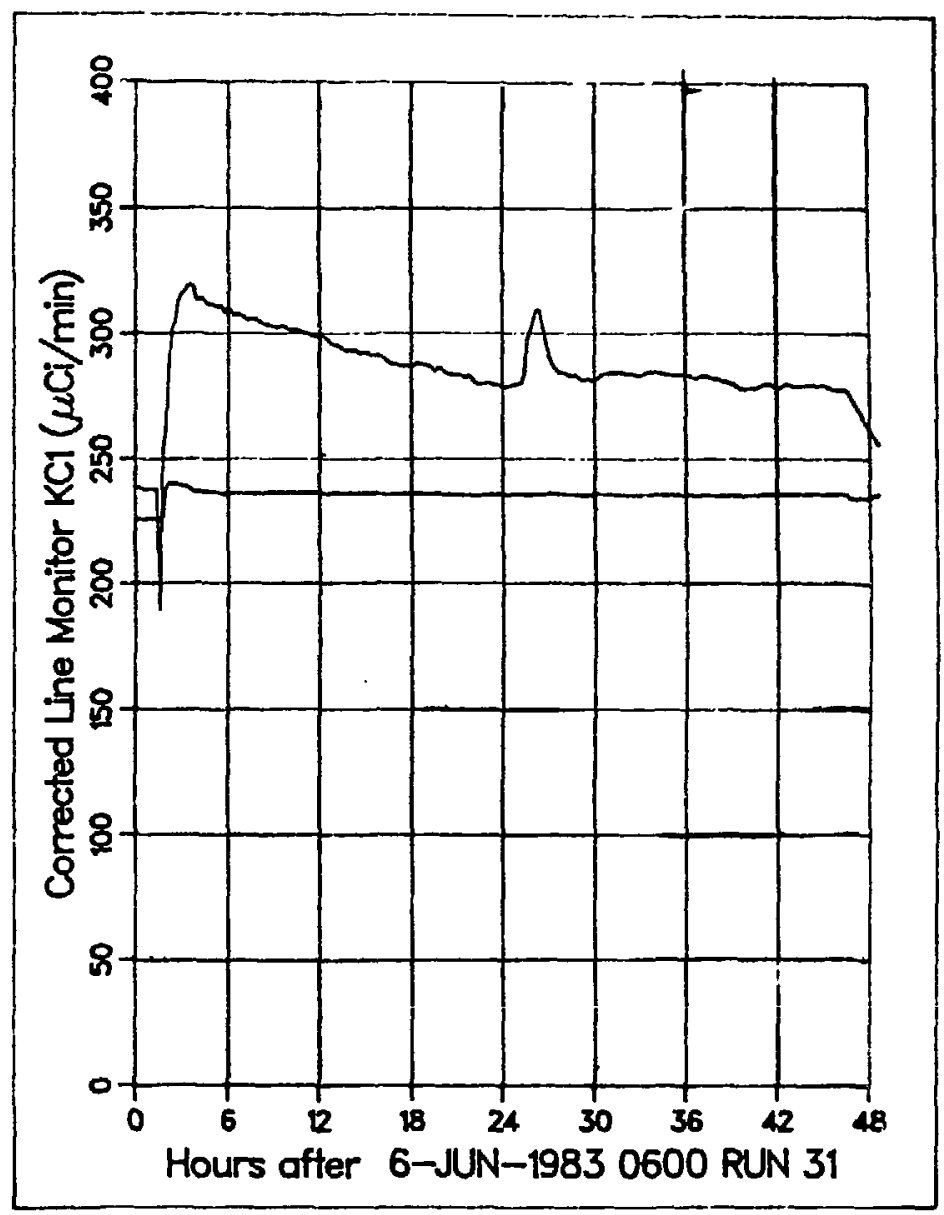

Fig. VI-73. Tritium release rate for Run 31 . 


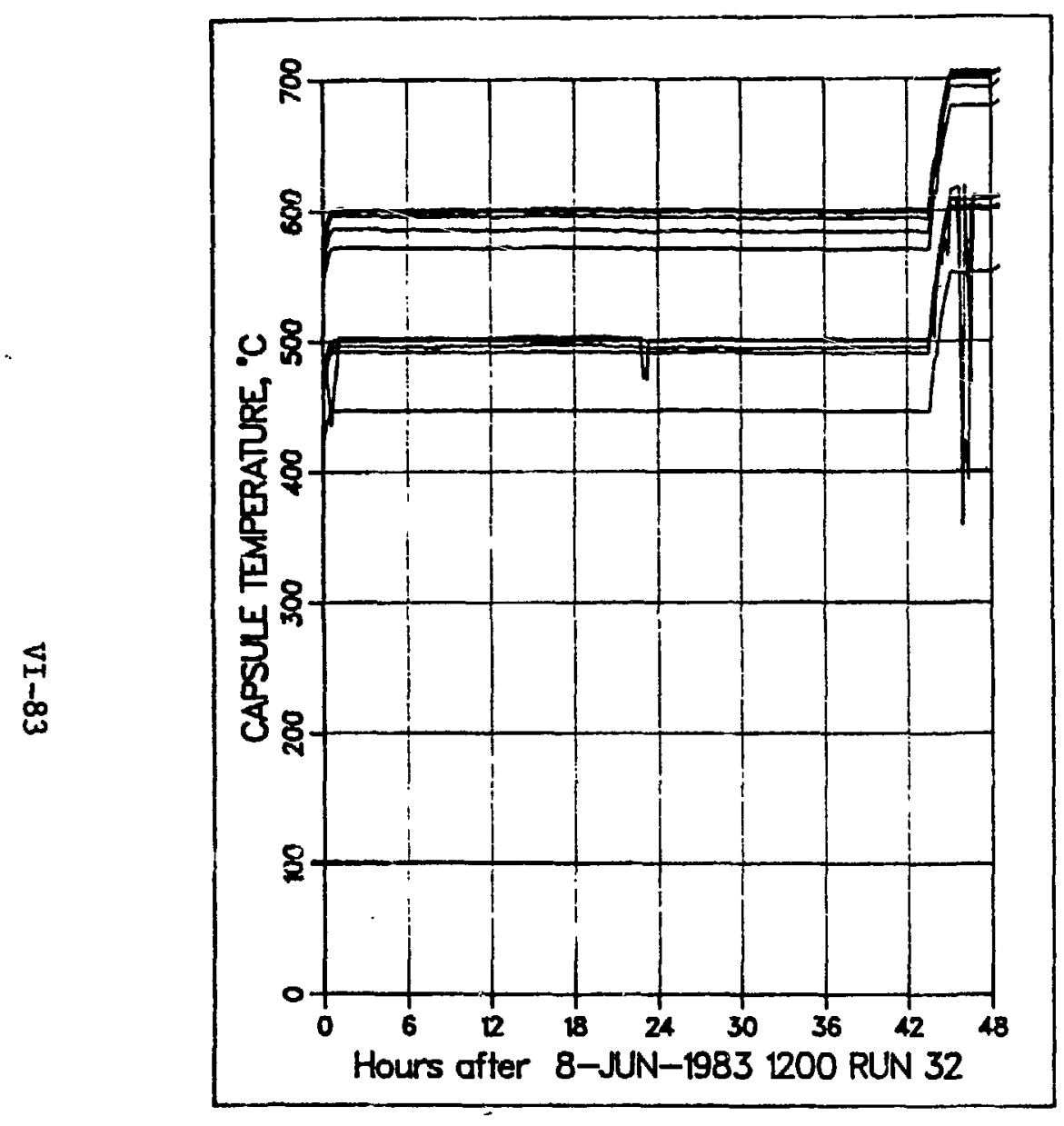

Fig. VI-74. Recorded temperatures for Run 32.

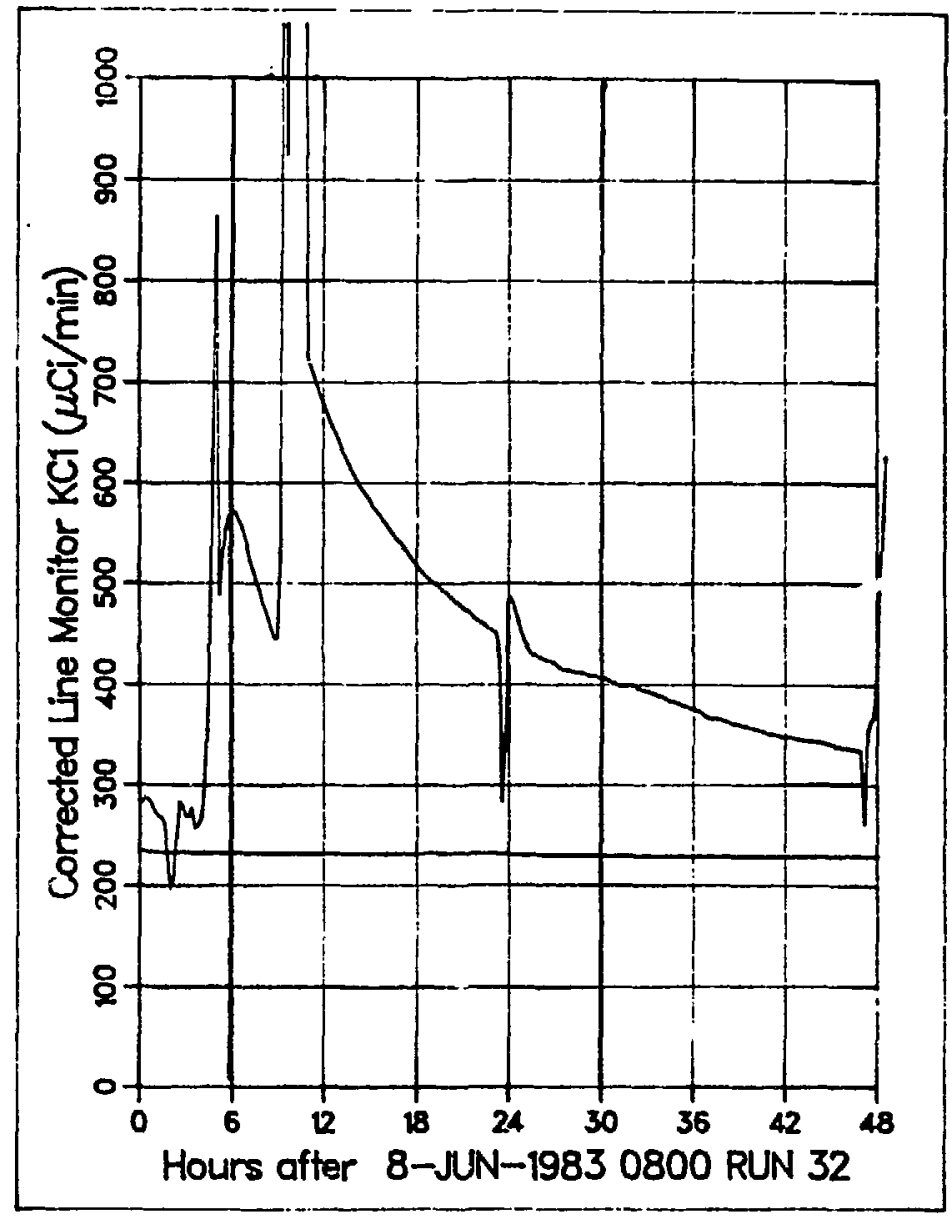

Fig. VI-75. Tritium release rate for Run 32. 


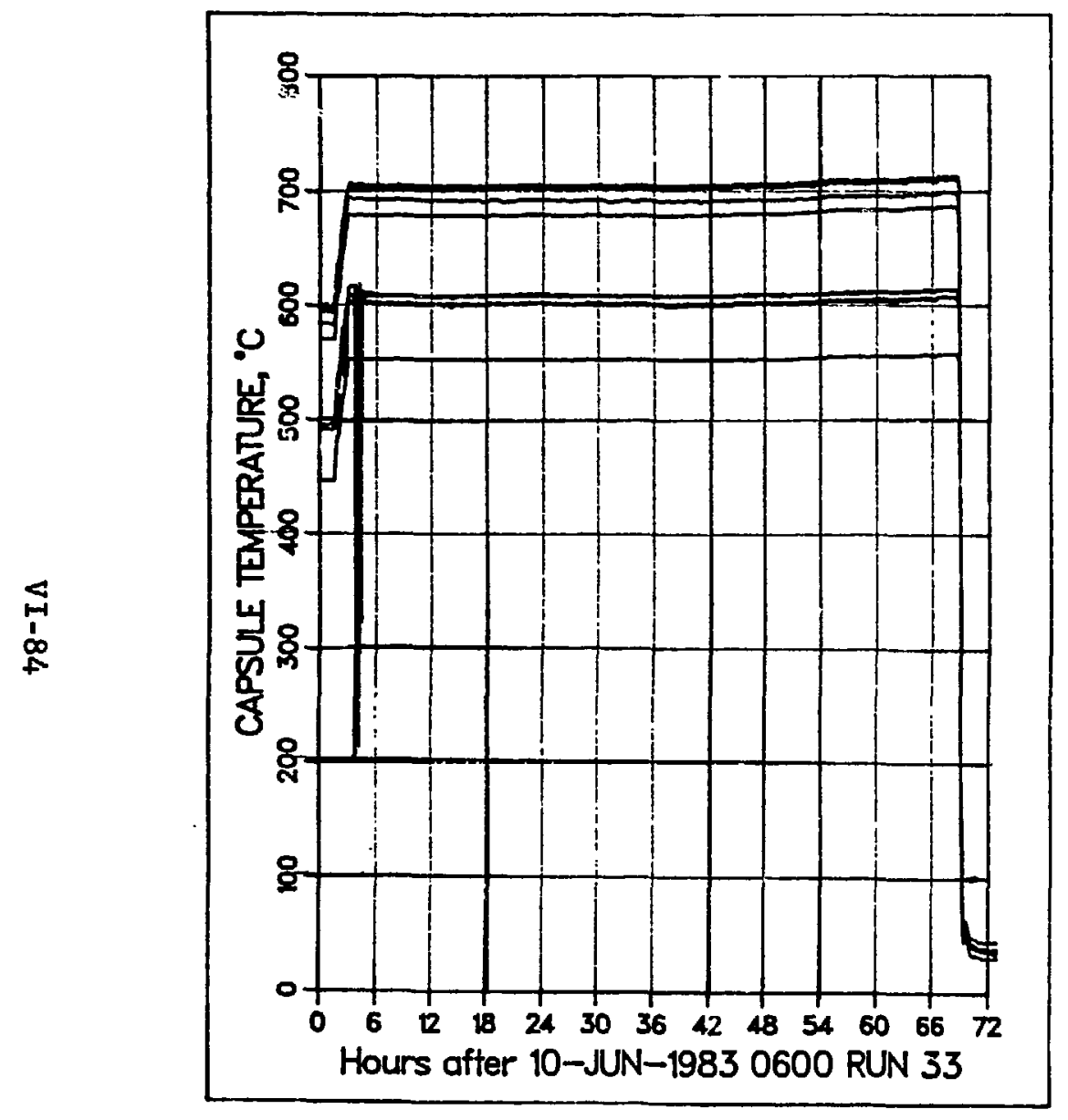

Fig. VI-76. Recorded temperatures for Run 33.

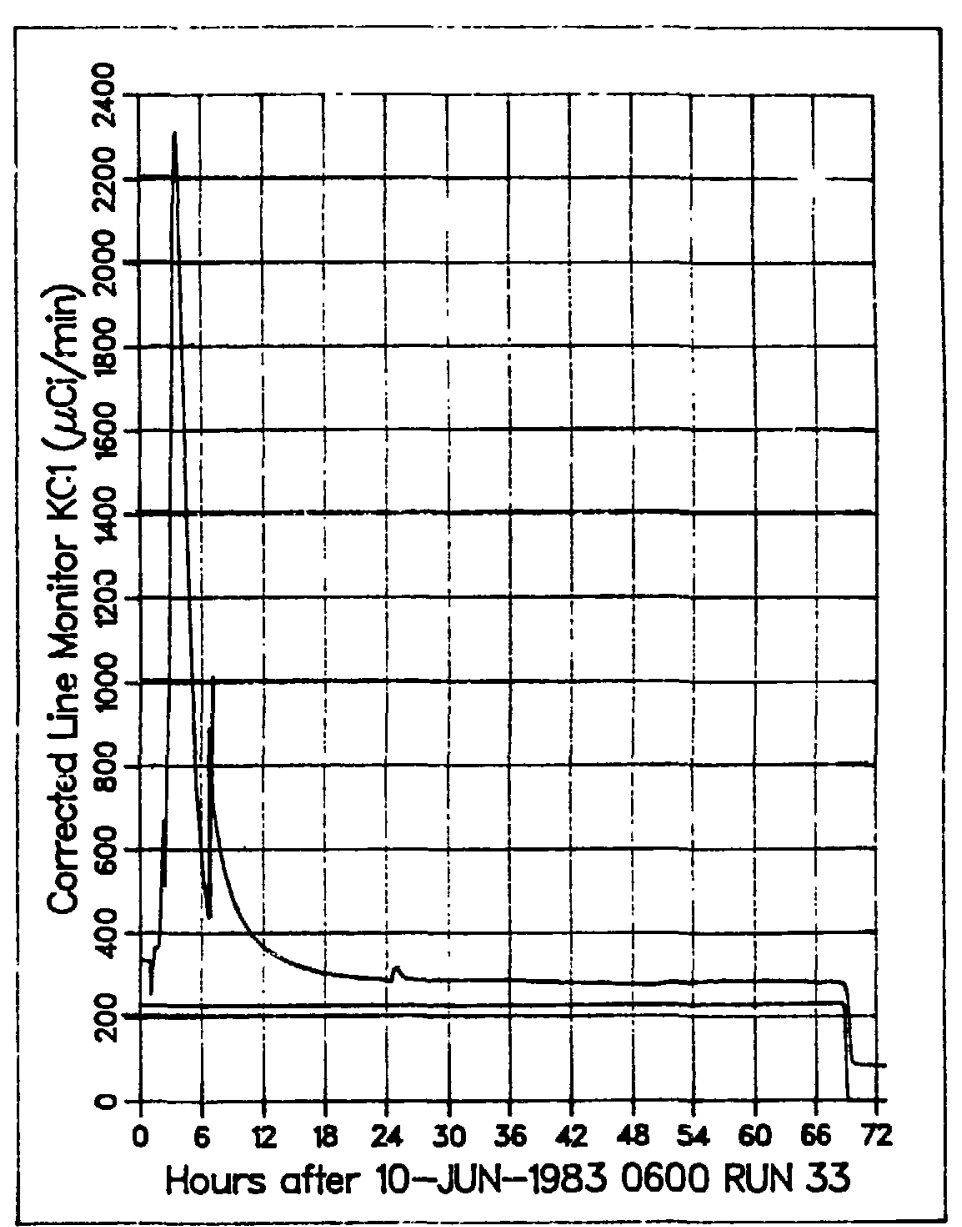

Fig. VI-77. Tritium rele-i_ rate for Run 33 . 


\section{Runs $0-4$}

The HTO release rates for the first four runs are given in Fig. VI-78. One can see a small peak for Run 0 , which coresponds to a small but finite release of $\mathrm{HTO}$ at $\sim 200^{\circ} \mathrm{C}$. Run 1 has a rather large peak of $\sim 50 \mu \mathrm{Ci} / \mathrm{min}$ followed by rapid decline. This peak is $\sim 15 \%$ of the HT release rate (see Fig. VI-9). For an extended period of time, the HTO release rate declines to below $10 \mu \mathrm{Ci} / \mathrm{min}$, then below $1 \mu \mathrm{Cl} / \mathrm{min}$. The initial peak for HTO released appears to be associated with an initial experimental. condition, such as outgassing the final traces of molsture from the $\mathrm{LiAlO}_{2}$ ceramic. The HTO release rate for Run 1 is shown in more detail in Fig. VI-79.

A small peak is observed at hour 180 in Fig. VI-78. Thi peak occurs 24 $h$ after the sharp spike in the HT release curve, at the beginning of Run 3 . Run 3 was at $700^{\circ} \mathrm{C}$ with $1 \% \mathrm{H}_{2}$ added to the sweep gas.

\section{Runs $4-20$}

The HTO release rates for Runs $4-20$ are given in Figs. VI-80 through -83 . During this period the HTO release rate was small but finite. The release rate curves may have some structure, but the peaks do not instantaneously correlate to HT peaks. It appears that delays of about a day may occur between the onse: of large HT peaks and that of HTO peaks. The HTO release curves for Runs 4-19 are discussed below.

The HT curves in Runs 4 and 5, for which the temperature was decreased to $500-550^{\circ} \mathrm{C}$ (Figs. VI-15 ard -17 ) show strong negative peaks. These runs are represented by the first $100 \mathrm{~h}$ in Fig. VI-80. The HTO release curve shows a continual decrease during this period. Run 6 (temperature increased from 500 to $550^{\circ} \mathrm{C}$ ) begins at hour 108 in $\mathrm{Fig}$. VI-80. 'There is a strong positive peak in the HT curve (Fig. VI-19); an observable peak occurs in the HTO curve $12 \mathrm{~h}$ later. Run 7 begins at hour 130 in Fig. VI-80, but because only one sample was taken, it is not possible to resolve any HTO peak. Run 8 begins at hour 12 in Fig. VI-81. There may be an HTO peak at the start of the run and one about $36 \mathrm{~h}$ after the beginning of the run.

Runs $9\left(650^{\circ} \mathrm{C}\right), 10\left(400^{\circ} \mathrm{C}\right)$, and $11\left(500^{\circ} \mathrm{C}\right)$ are represented in Fig. VI-82. All three runs had no hydrogen added to the helium sweep gas. Reactor shutdowns took place at hours 144 and 264; no HTO release is indicated when the 


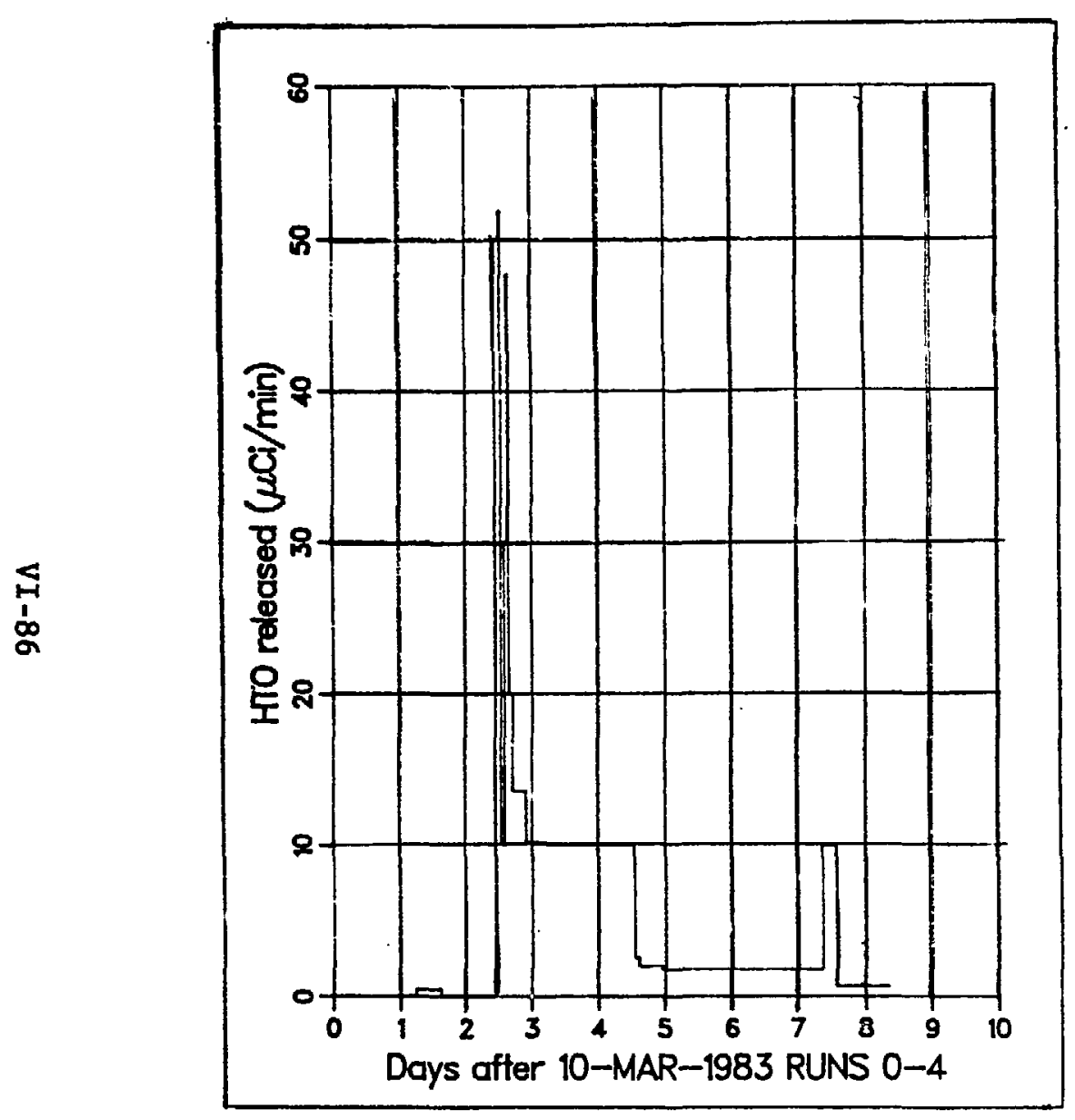

Fig. VI-78, HTO release rates for Runs 1-4.

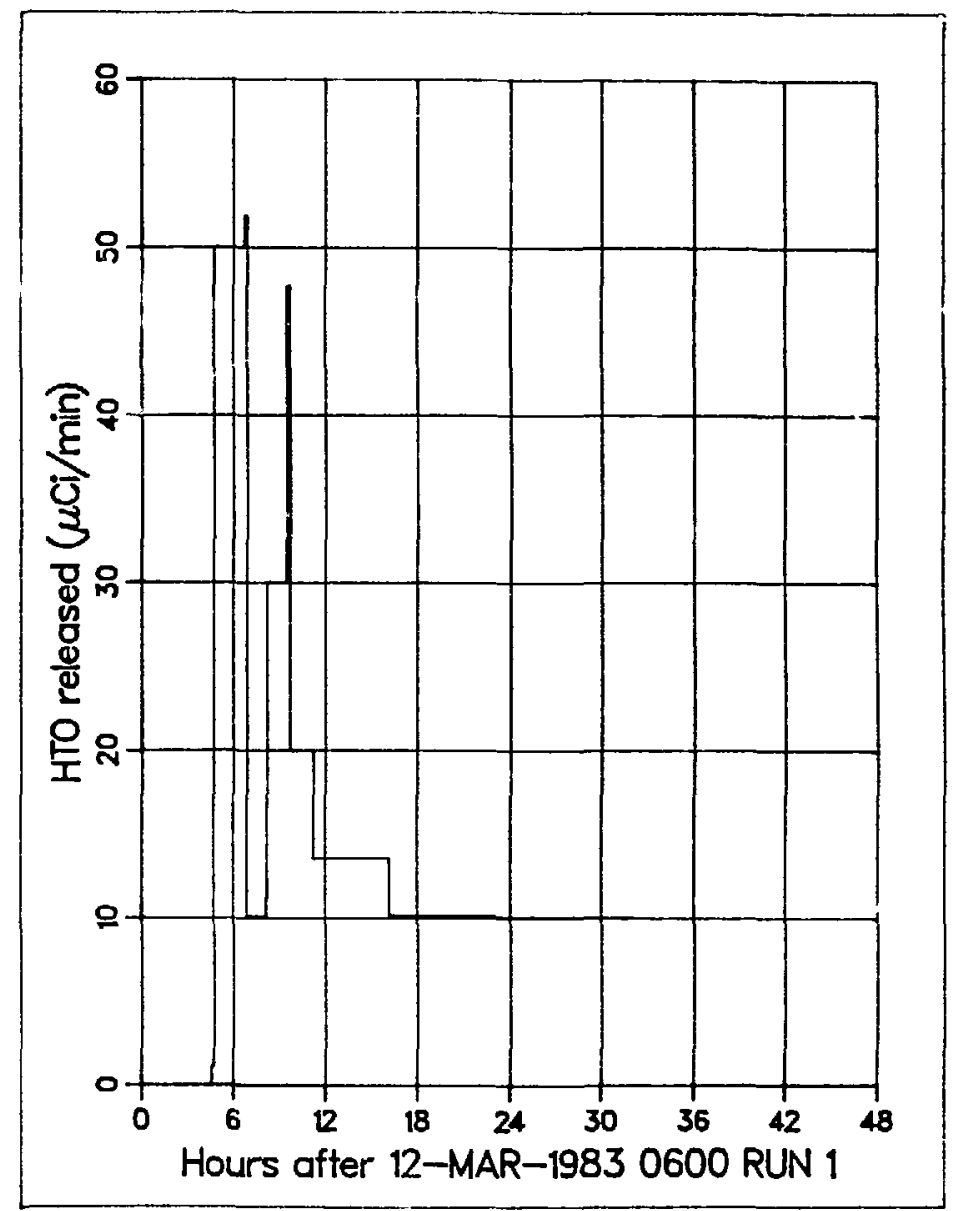

Fig. VI-79. HTO release rate for Run 1. 


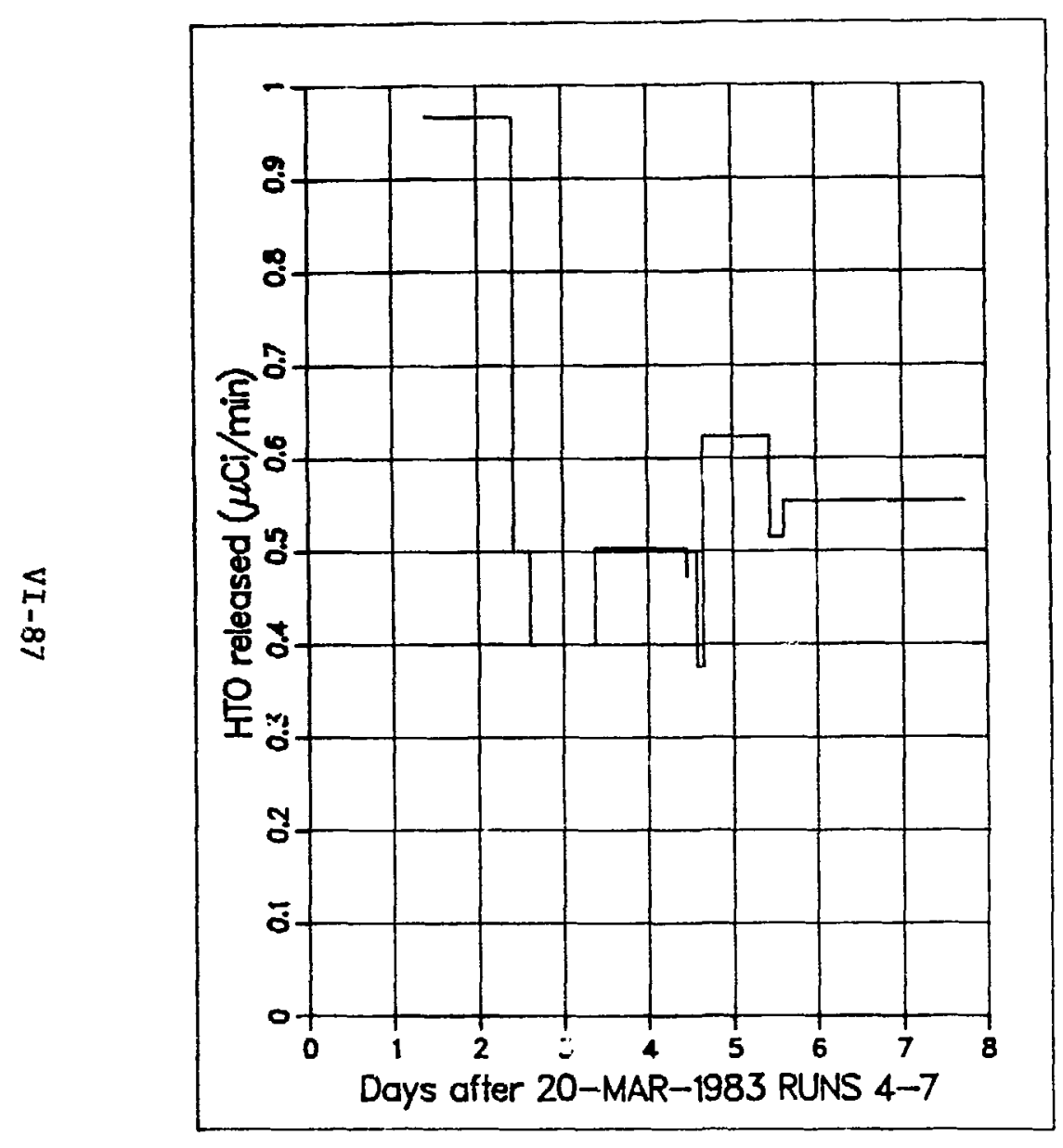

Fig. VI-80. HTO release rate for Runs 4-7.

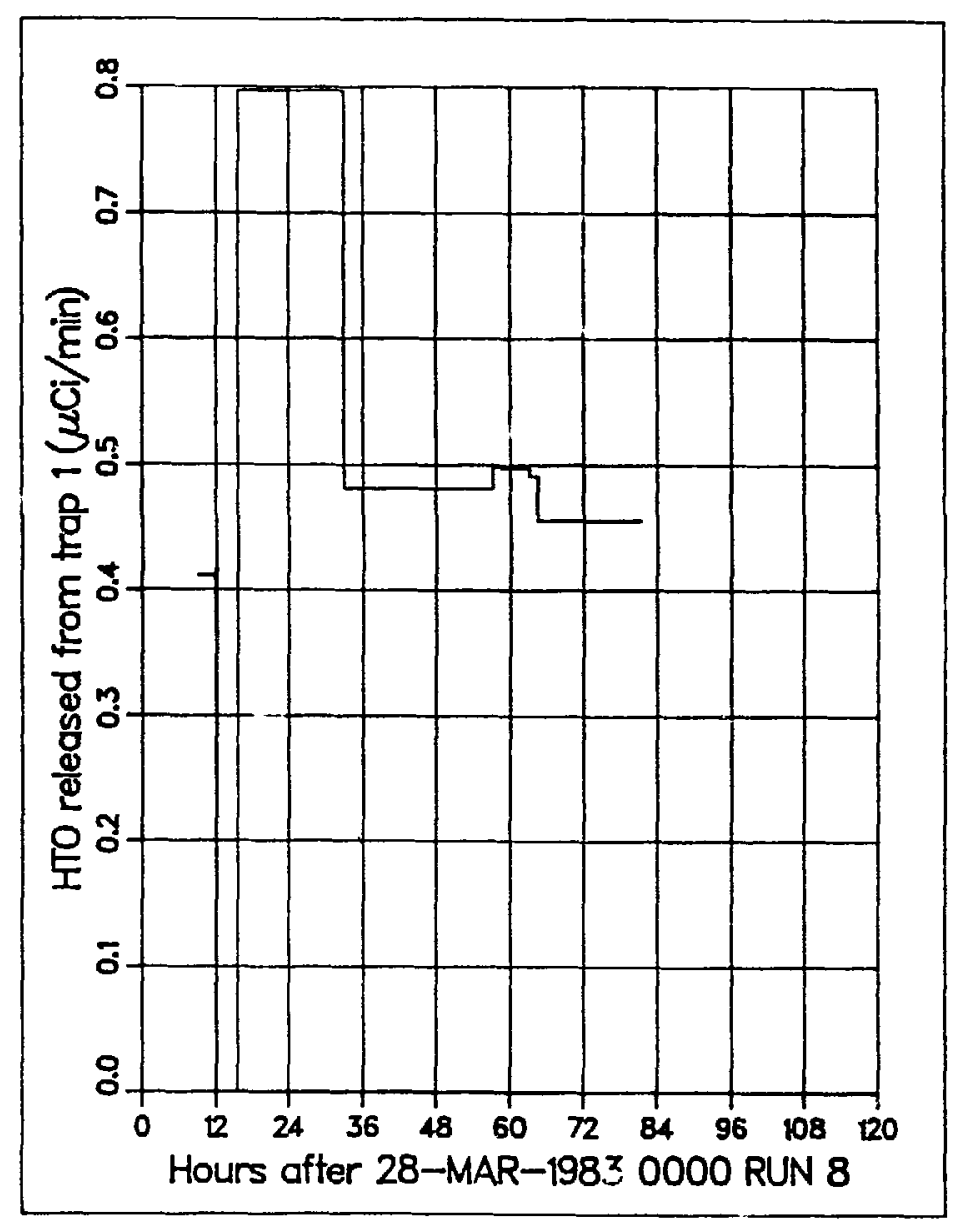

Fig. VI-81. HTO release rate for Run 8. 


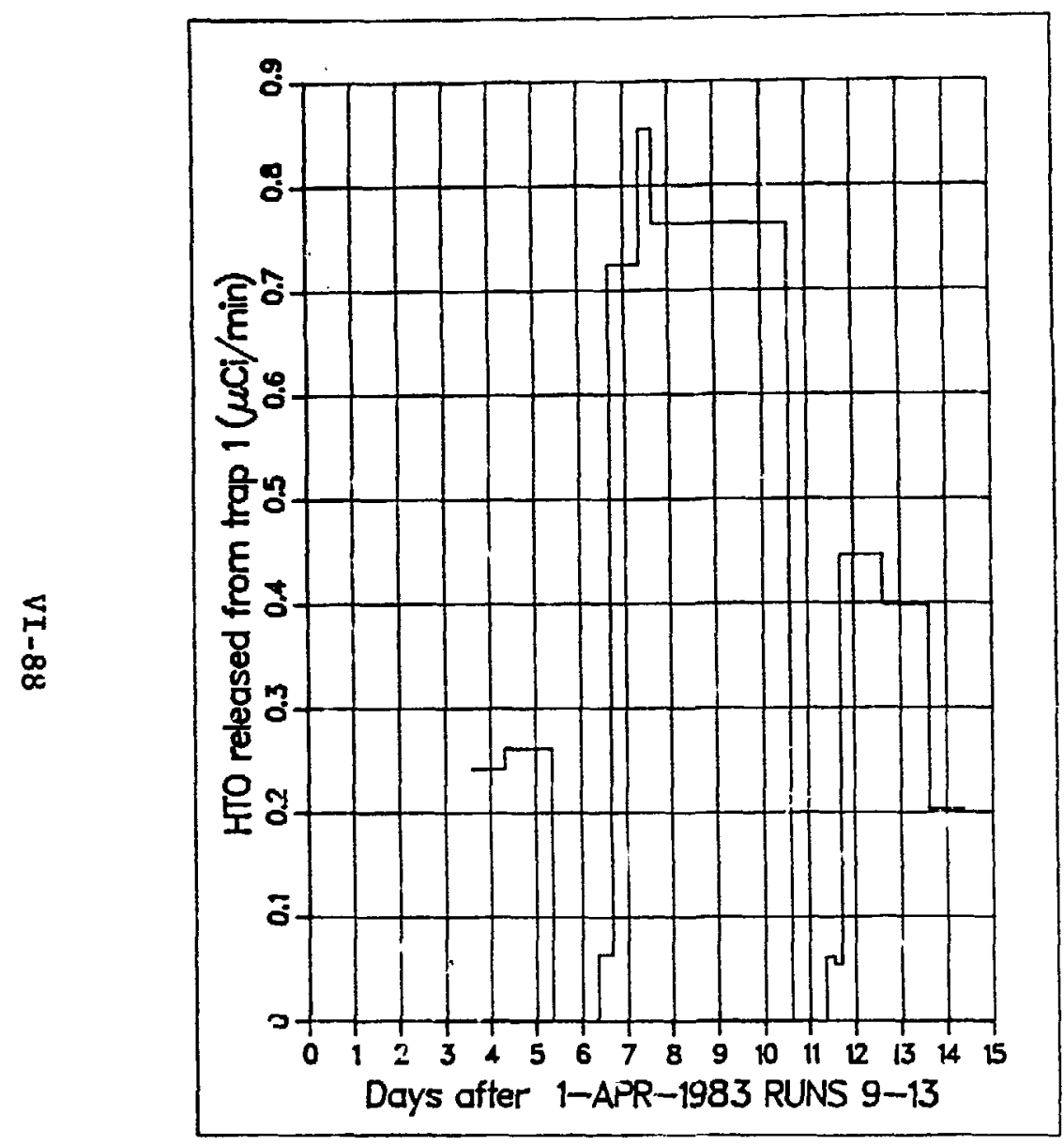

Fig. VI-82. HTO release rate for Runs 9-13.

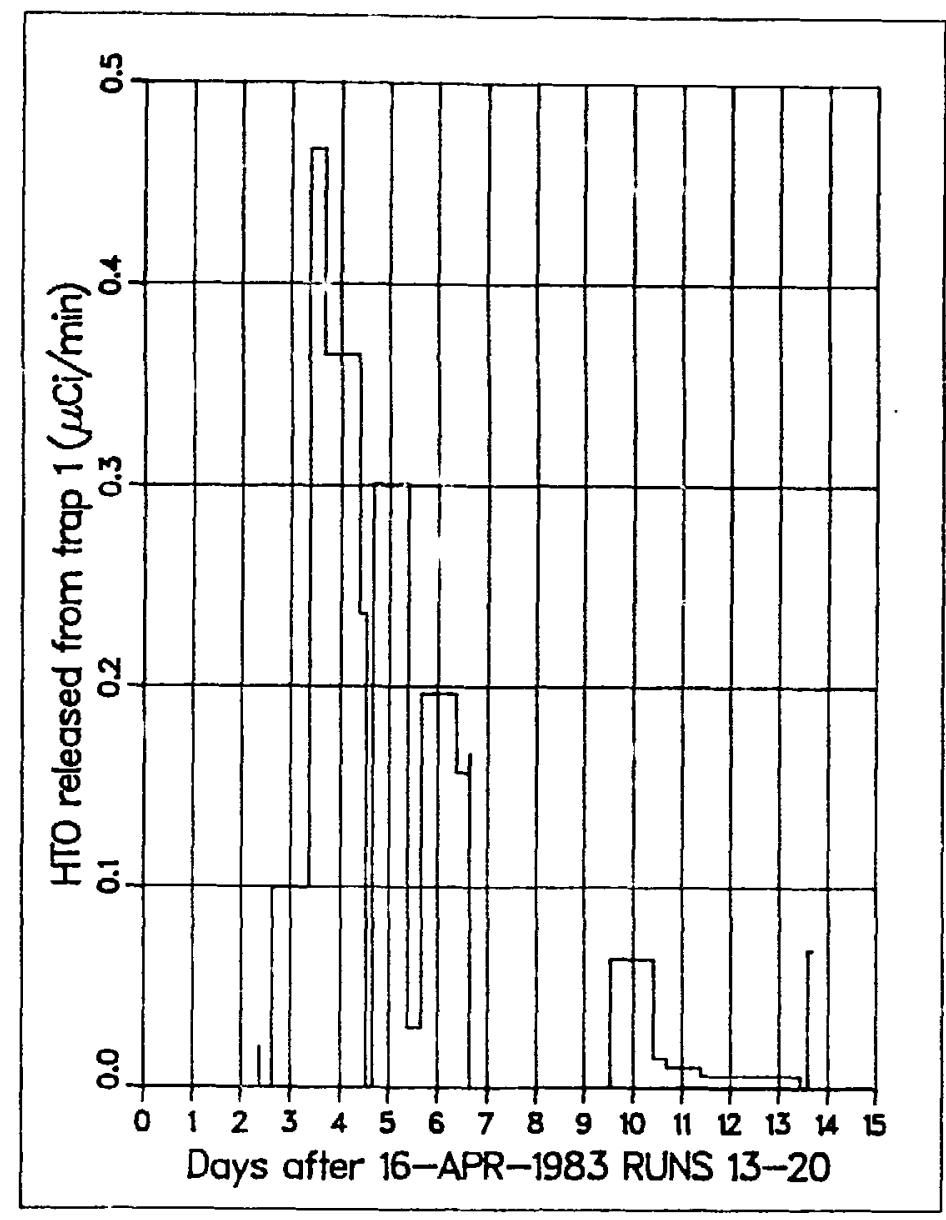

Fig. VI-83. HTO release rate for Runs 13-20. 
reactor is down. In each case, a small spike appears in the HTO release curve $\sim 24 \mathrm{~h}$ after reactor startup.

Figure VI-83 shows the HTO release rate for Runs $13-20$. Run $14\left(700^{\circ} \mathrm{C}-\right.$ no $\mathrm{H}_{2}$ added) begins at hour 60. The HT release curve (Fig. VI-34) shows a strong positive peak at 1700 un April 18 (hou 65 in Fig. VI-83). The HTO release curve shows a peak $\sim 24 \mathrm{~h}$ later. There is not sufficient resolution to tell if there are HTO peaks associated with Rurs 15-19.

\section{Runs 20-30}

Run 20 was at a nominal temperature of $650^{\circ} \mathrm{C}$, with $0.1 \%$ oxygen added to the sweep gas for April 29 until May 2. The HTO release rate for Runs 20-30 are shown in Figs. VI-84 through -87 . During Run 20, essentially no tritium was released, either as HT or HTO. However, for the next several weeks after Run 20, the HTO release curve rose dramatically, to a peak of $150 \mu \mathrm{Ci} / \mathrm{min}$ at the beginning of Run 29 (see Figs. VI-86 and -87). This peak occured 30 days after Run 20 had ended. This large peak also does not seem to correlate with the test conditions of Runs $21-30$; 1 it is therefore attributed to the effects of Run 20. As noted above, HTO peaks often occur $24 \mathrm{~h}$ after the HT peaks. It is inferred that HTO exits the system $\sim 24 \mathrm{~h}$ after it has been released from the breeder in the capsule. The fact that some 30 days elapsed before the HTO created in Run 20 was released suggests that the HTO from Run 20 was trapped on the surface of the breeder material. Over a period of 30 days, the HTO on the breeder surface was gradually released.

In addition to the large HTO peak, there is some evidence of structure in the HTO release curves for Runs 21-30. Run 21 begins at hour 132 in Fig. VI-84. About $12 \mathrm{~h}$ later an HTO peak appears to form. A reactor shutdown occurred at hour 216, followed by a restart at hour 224. An observable HTO spike occured $\sim 20 \mathrm{~h}$ after startup. Run $24\left(600^{\circ} \mathrm{C}\right)$ has a large HT spike at 1100 on May 9 (see FIg. VI-58), which corresponds to hour 300 in Fig. VI-84. Some $30 \mathrm{~h}$ later the HTO curve showed a peak; which may be associated with the phenomena of Run 20. An HTO peak appears $\sim 24 \mathrm{~h}$ after reactor startup (Fig. VI-86), which occurred at hour 108 .

Run 31 is depicted 1n Fig. VI-88. The HT release curve ws given in Fig. VI-73. There appears to be a flow transient at 0800 on June 6 , which generated a sharp, narrow spike in both the HT and HTO release curves. The HT peak 


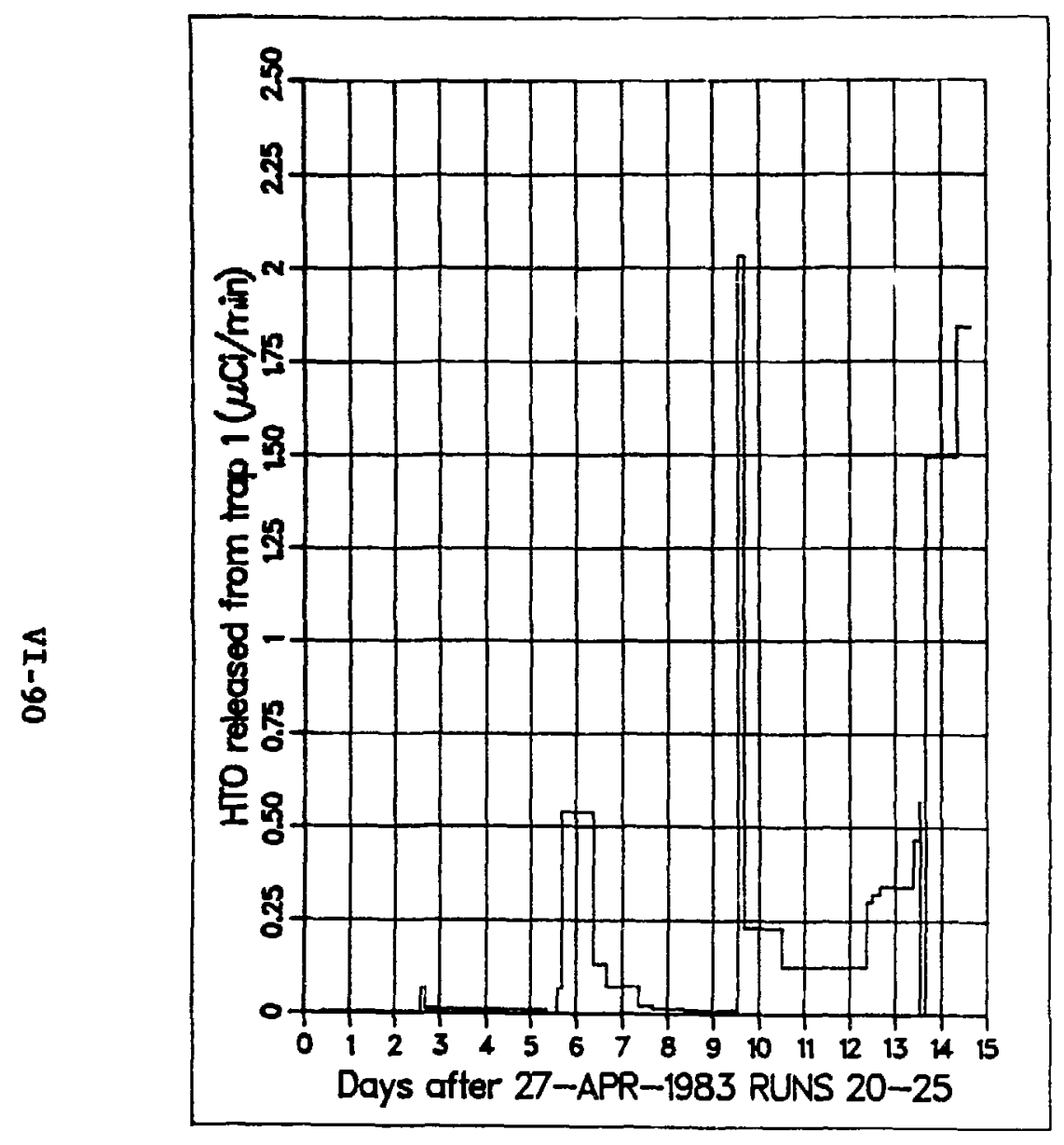

Fig. VI-84. HTO release rate for Runs 20-25.

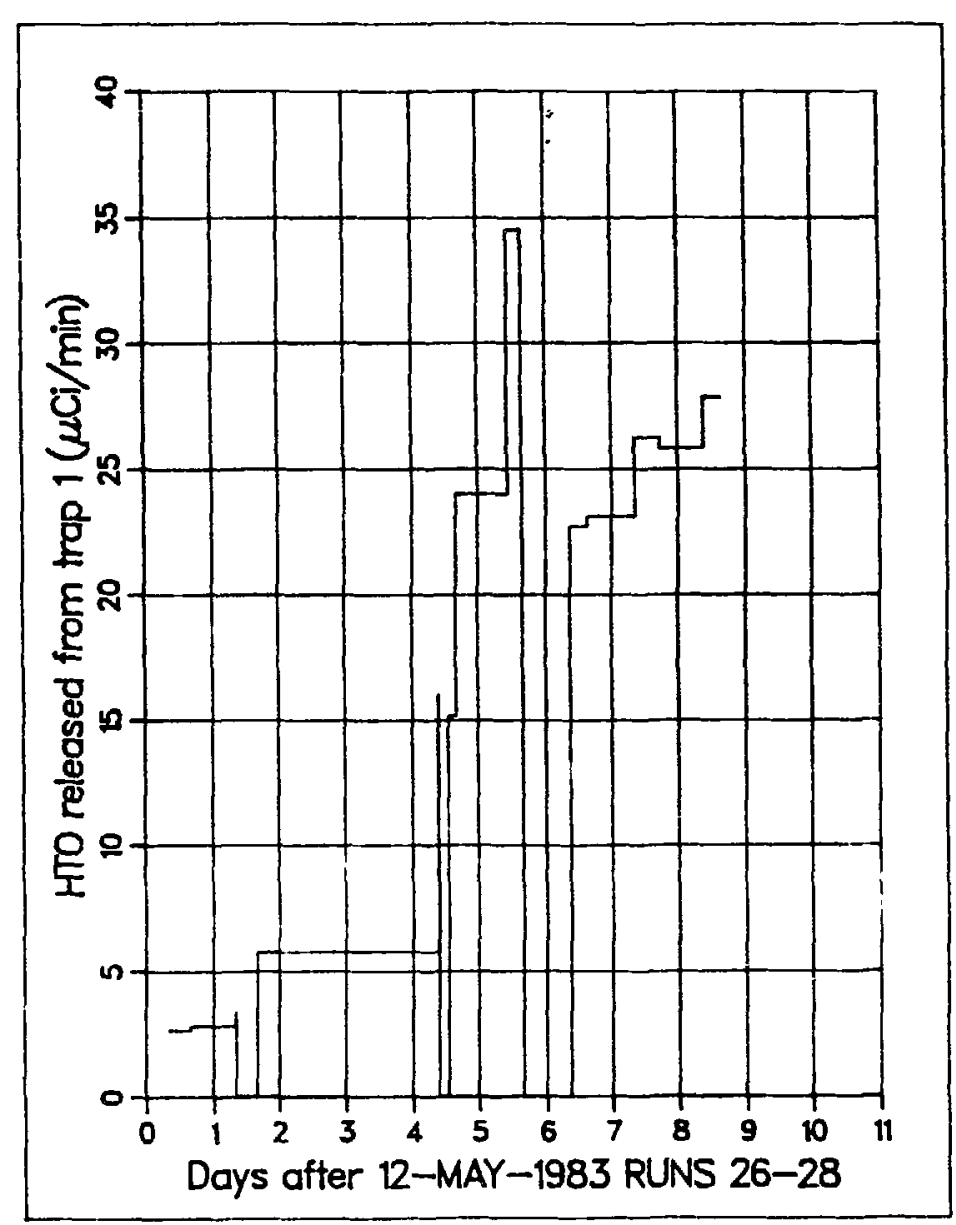

Fig. VI-85. HTO release rate for Runs 26-28. 


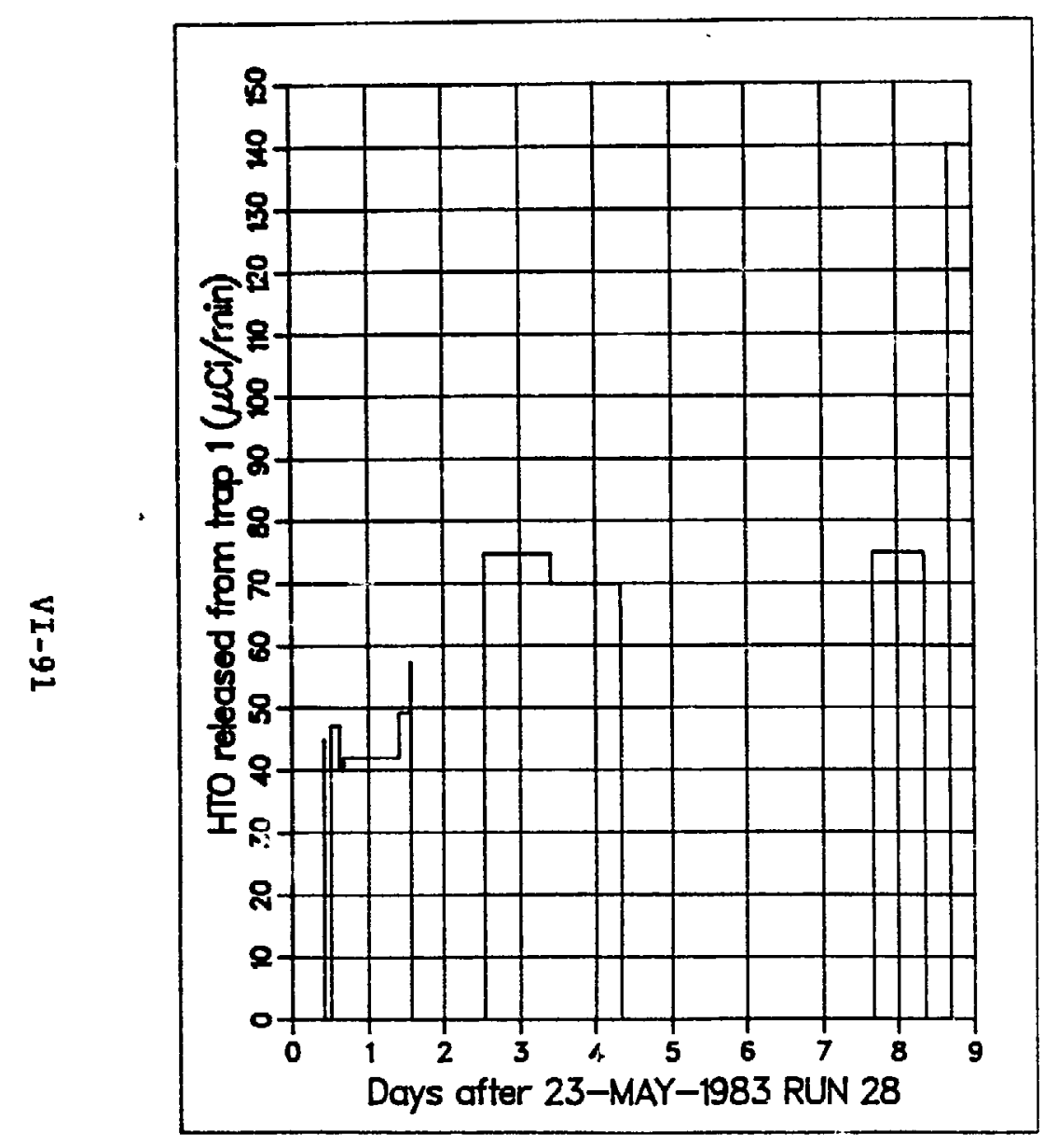

Fig. VI-86. HTO release for Run 28.

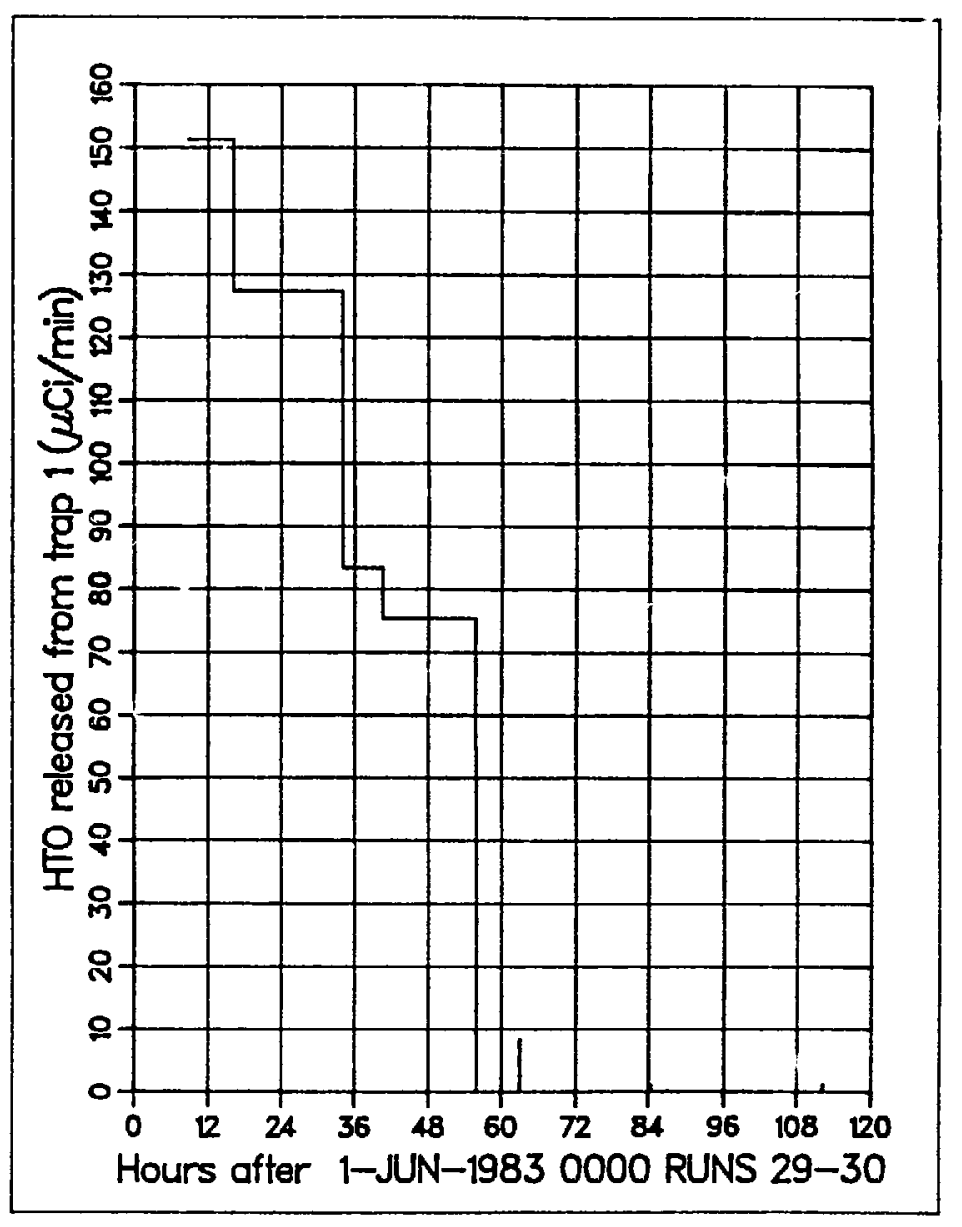

Fig. VI-87. HTO release for Runs 29-30. 


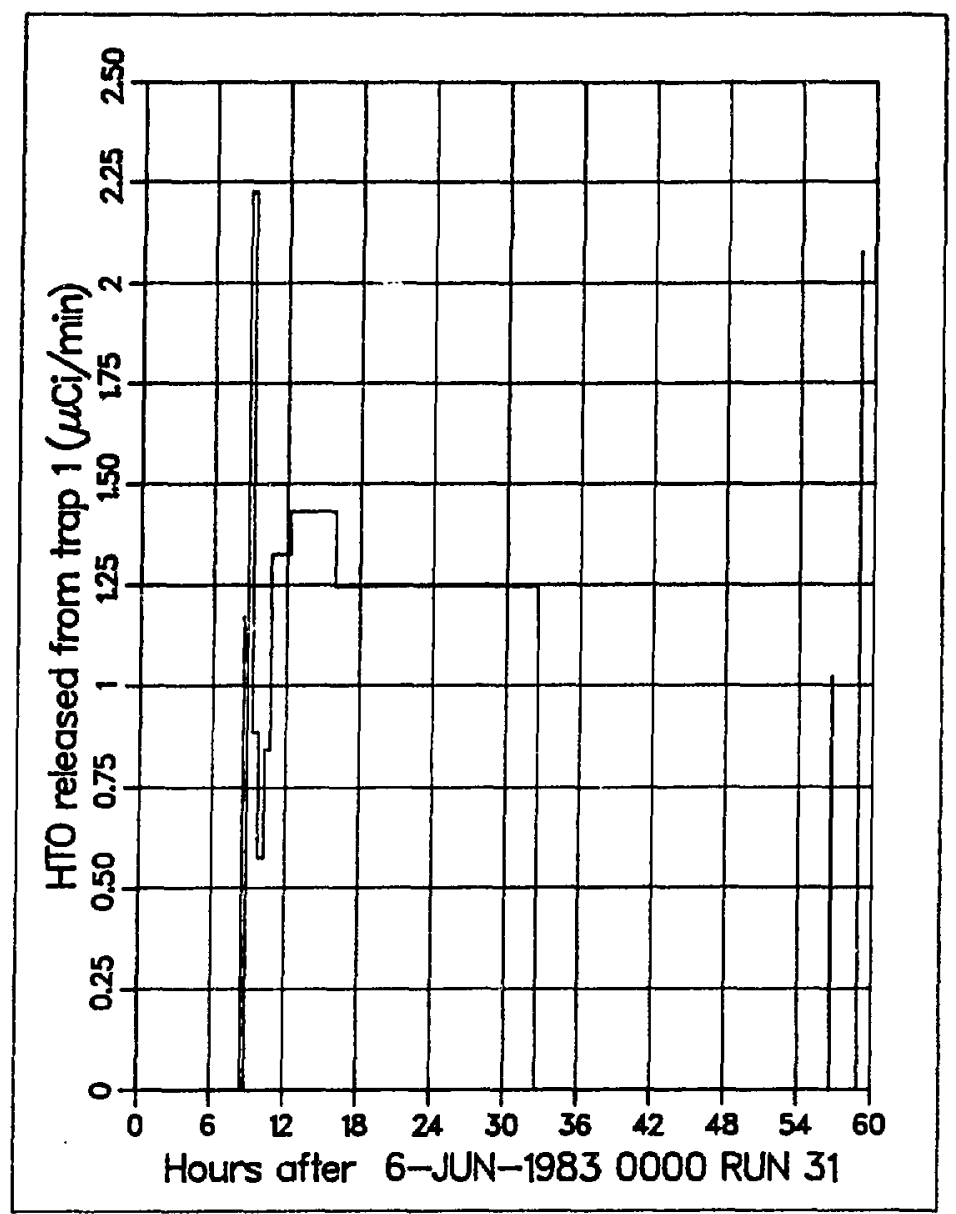

Fig. 88. HTo release for Run 31.

occurred at 1000 on June 6 and is small. The HTO peak, at about 1400 on June 6 , is also small.

Run 32 is depicted in Fig. VI-89. The peak in the HT curve (Fig. VI-75) occurred at 1400 on June 8 . The peak in the HTO curve at hour 56, at the beginning of the next run, may be associated with Run 32 ,

Run 33 is shown in Fig. VI-90. The HT curve (Fig. VI-77) showed a very strong peak at 1000 on June 10 . There is a very strong peak in the HTO curve 10 to 20 h later.

\section{B. Radionuclides}

During operation of the TRIO experiment, a number of radionuclides were observed In the sweep gas stream (Table VI-8). These species were detected by 


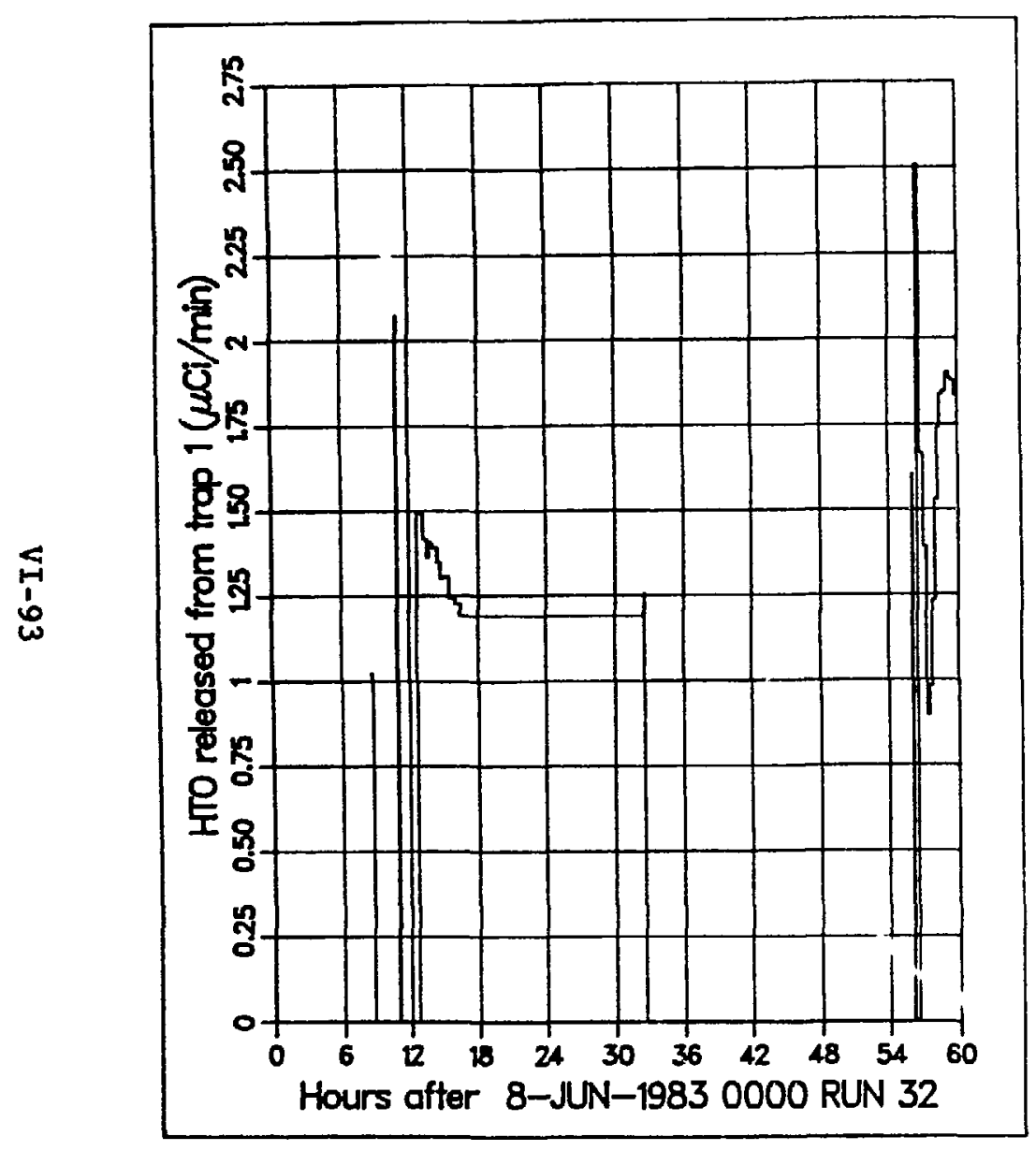

Fig. VI-89, HTO release tate for Run 32 .

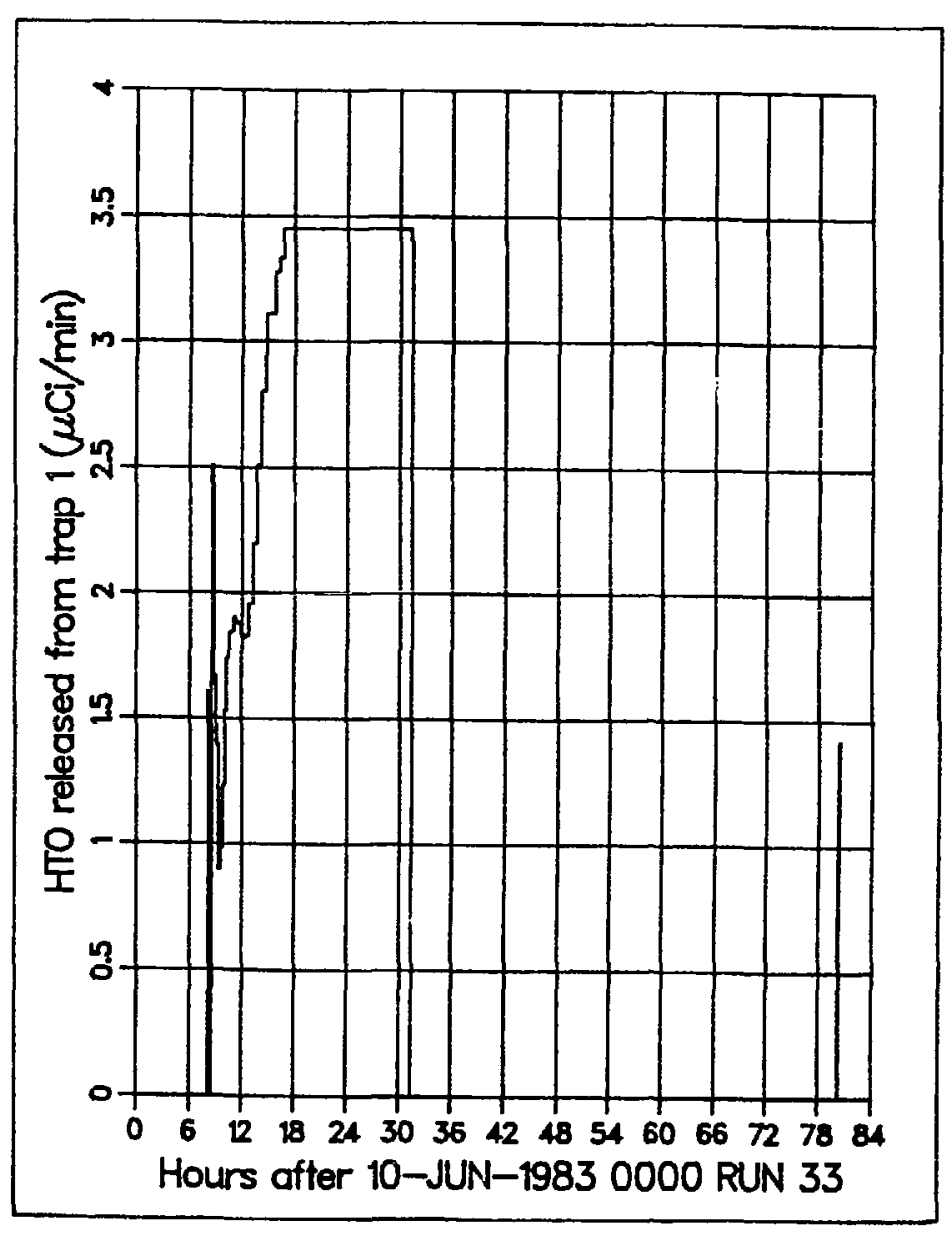

Fig. VI-90. HTO release rate for Run 33. 
TABLE VI-8

Radionuclides Observed

\begin{tabular}{|c|c|c|}
\hline Nuclide & Half-Life & Source \\
\hline$\angle O_{F}$ & $11.0 \mathrm{~s}$ & $\mathrm{Ne}$ \\
\hline $23 \mathrm{Ne}$ & $37.5 \mathrm{~s}$ & $\mathrm{Na}, \mathrm{Ne}$ \\
\hline${ }^{38} \mathrm{C} 18$ & $37 . ? \mathrm{mo}$ & $\mathrm{Cl}$ \\
\hline $41_{\mathrm{Ar}}$ & $1.8 \mathrm{~h}$ & $\mathrm{Ar}, \mathrm{K}$ \\
\hline $85^{\mathrm{m}} \mathrm{Kr}$ & 4. 48 & $\mathrm{U}$ \\
\hline${ }^{87} \mathrm{Kr}$ & $76.0 \mathrm{mo}$ & $\mathrm{U}$ \\
\hline${ }^{89} \mathrm{Kr}$ & $3.15 \mathrm{~m}$ & $\mathrm{~J}$ \\
\hline${ }^{90} \mathrm{Kr}$ & $32.3 \mathrm{~s}$ & $\mathrm{U}$ \\
\hline${ }^{88} \mathrm{Rb} 8$ & $17.7 \mathrm{mo}$ & $\mathbf{U}$ \\
\hline${ }^{89} \mathrm{Rb} 9$ & $15.4 \mathrm{mo}$ & $\mathbf{U}$ \\
\hline $133 \mathrm{Xe}$ & $5.25 \mathrm{~d}$ & $\mathrm{U}$ \\
\hline $135 \mathrm{~m}_{\mathrm{Xe}}$ & $15.3 \mathrm{mo}$ & $\mathrm{U}$ \\
\hline $135 \mathrm{Xe}$ & $9.09 \mathrm{~h}$ & $\mathrm{U}$ \\
\hline $137 \mathrm{Xe}$ & $3.85 \mathrm{mo}$ & $\mathbf{U}$ \\
\hline $138 \mathrm{Xe}$ & $14.2 \mathrm{mo}$ & $U$ \\
\hline $139 \mathrm{Xe}$ & 40.06 & $\mathrm{U}$ \\
\hline $138 \mathrm{Cs}$ & $32.2 \mathrm{mo}$ & $\mathrm{U}$ \\
\hline $139 \mathrm{Cs}$ & 9.4 mo & \\
\hline
\end{tabular}

ganma spectrometry as previously described (Section IV.C). The levels of these specles were not more than $1 \%$ of the observed tritium signal, as measured by the Kanne chamber used to monitor the HT level. As shown, these radionuclides arlse from the presence of trace levels of certain contaminants present in the breeder material. The presence of these radionuclides is $\mathrm{sig}-$ nificant because it provides information on radioactivity levels that have to be handled in a fusion reactor. In addition the data on the transport of neon, argon, krypton, and xenon may provide some insight Into the transport behavior of a very important specles, helium. 
SECTION VII

POST-IRRADIATION EXAMIMATIONS (PIE) 


\section{POST-IRRADLATION EXAMINATIONS (PIE)}

The TRIO experiment is comprised of three phases: pre-irradiation, irradiation, and post-irradiation. Data from each phase provide an essential contribution to the overall experiment. The pre-iruadiation phase (see Sec. IV) includes: appropriate characterizations of the $\mathrm{LiAlO}_{2}$ breeder material, mockup testing of critical experimental components (e.g., gas analysis system), and characterization of the nuclear environment with core mockup tests. The irradiation phase (see Sec. VI) includes the collection of extensive data on tritium release in its various chemical forms and monitoring of neutron flux and temperature profiles. The post-irradiation examinations (PIE) include the following steps: (1) disassembly of the capsule, (2) measurement of tritium retention in the breeder pellets, (3) determination of lithium burnup by measurement of lithium isotopy, (4) determination of thermal flux with dosimetry wires, (5) microstructural characterization of the pellets by scanning electron microscopy, (6) analysis of radioactivity in the pellets, and (7) characterization of the phase stability of the irradiated $\gamma^{-\mathrm{L}_{\mathrm{AlO}} \mathrm{O}_{2}}$ by $\mathrm{X}$-ray diffraction.

\section{A. Capsule Disassenbly}

The large capsule assembly, nearly $3 \mathrm{~m}$ (12 ft) in length (shown as it was fabricated in Fig. IV-8), was removed from the reactor after unbolting the flange at the top and then crimping, disconnectling, and sealing the sweep gas lines with tubing fittings. A $10-m(30-f t)$ length of the sweep gas exit line was also crimped, then disconnected and sealed at both ends and retained for tritium assay. This line led from the top of the reactor through the pool to the gas analysis system. The large assembly was then brought to the $0_{i N R}$ hot cell under water, and the lower portion (45 cm, $16 \mathrm{in.}$ ) was sawed off with a motorized hacksaw. This portion of the assembly contained the test capsule and $\mathrm{LiAlO}_{2}$ breeder specimens. The cut part of the lower assembly was then resealed by immersing it into a pot of epoxy, which is expected to be capable of maint aining its integrity to a dose of $10^{8}$ rads. This assembly was loaded into a 2-R container, and a Garden-2 cask, and delivered to ANL. 
The capsule disassembly was performed in the ANL Alph_-Gamma Hot Cell facility (AGHC); the $\log$ number for the task is AG266A. In this facility, al1 cuts were made using high-speed grinding wheels with brittle blades. No cutting lubricants were used, and the capsule was rotated during cutting to prevent excessive heating. The temperature of the pieces was not measured, but cutting conditions used were the same as operations that did not melt sodium in similar capsules It is therefore estimated that the temperatures did not exceed $60^{\circ} \mathrm{C}$. Photographs were taken after each cut was made and retained in permanent records of the AGHC. Care was taken to retain orientation of all parts with respect to "up" and geographic direction. North was the zerodegree reference angle.

Owing to the large amount of neutron-irradiated steel, the capsule assembly had an activity in excess of $10^{6} \mathrm{R}$. All steel components in the capsule required remote handing. The activity of the lithium aluminate specimens was in the milliroentgens range, and these samples could be handled manually, with appropriate care.

The first cut was through the capsule assembly at a location below the lowest extent of the inner cladding, as shown in Fig. VII-1. The lower portion of the assembly was then discarded. A photograph of the remaining portion of the assembly is shown in Fig. VII-2. The orientation of the photograph is such that the view is looking upward toward the bottom of the inner capsule. The outer cladding and the four fins on the outside of the assembly having been cut through; the cogwheel-shaped centering device at the bottom of the inner capsule is visible. The tube on the outside of the east fin contains dosimetry wires, and the tube on the inside of the east $f$ in is the inlet line for the control gas. Burrs on the inside of the outer cladding resulting from the cut were removed, causing the scratches on the west side of the cogshaped centering plate. As shown, the centering plate and the inner capsule had been shifted directly to the east side, causing the gap to be smaller and the temperatures to be lower on that side. The nominal gap was $0.051 \mathrm{~cm}$ $(0.020 \mathrm{in.})$, and the actual gap dimensions were measured by inserting piano wires of various diameters between the slots in the centering plate into the gap. As can be determined from Table VII-1, the largest gap was on the west side, and the smallest gap was on the east. side. The inner capsule (when at ambient temperature) was displaced from center by $0.023 \mathrm{~cm}(0.009 \mathrm{in}$.$) to$ 

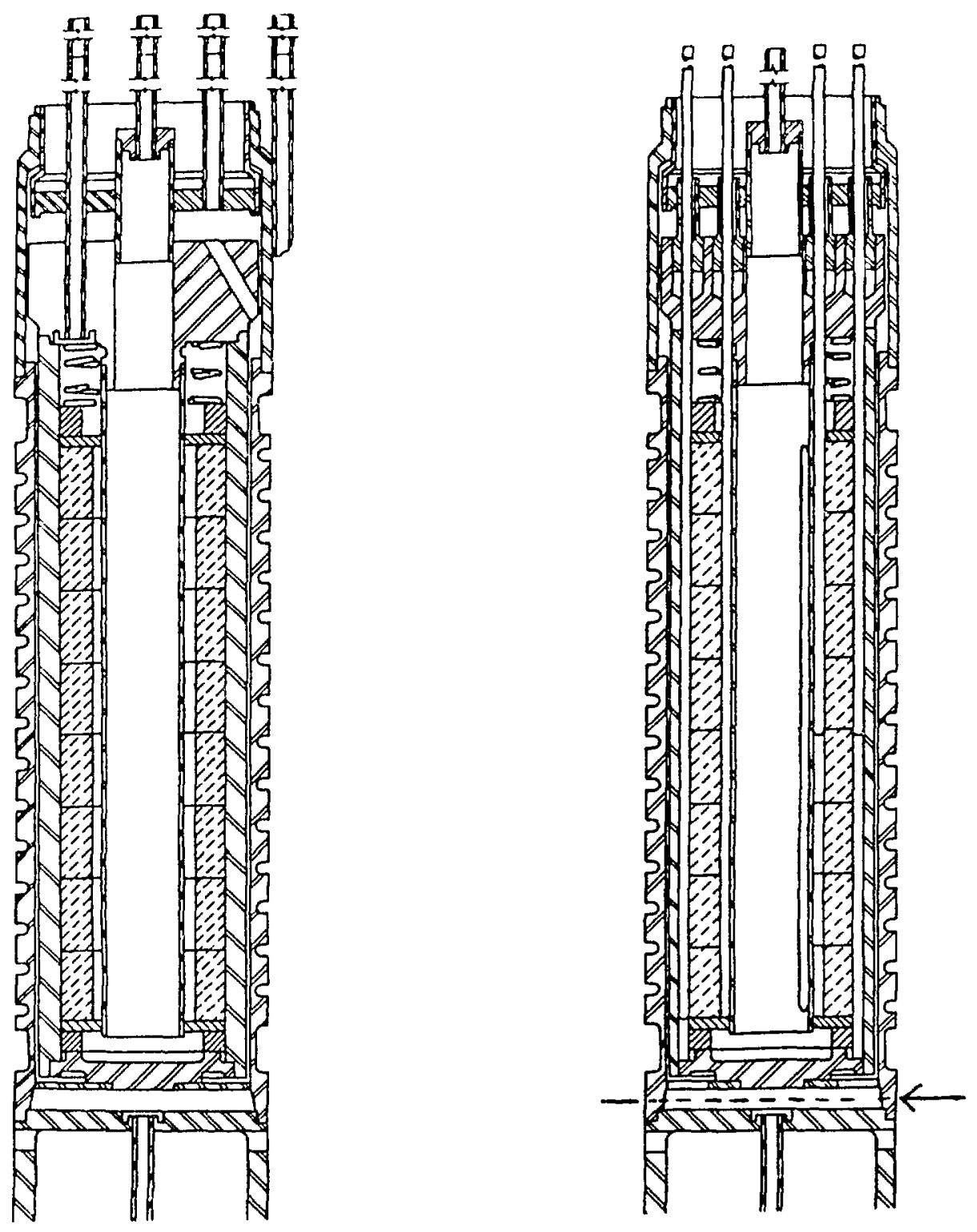

Fi3. VII-1. Drawing of TRIO capsule showing location of first cut for the disassembly procedure. 


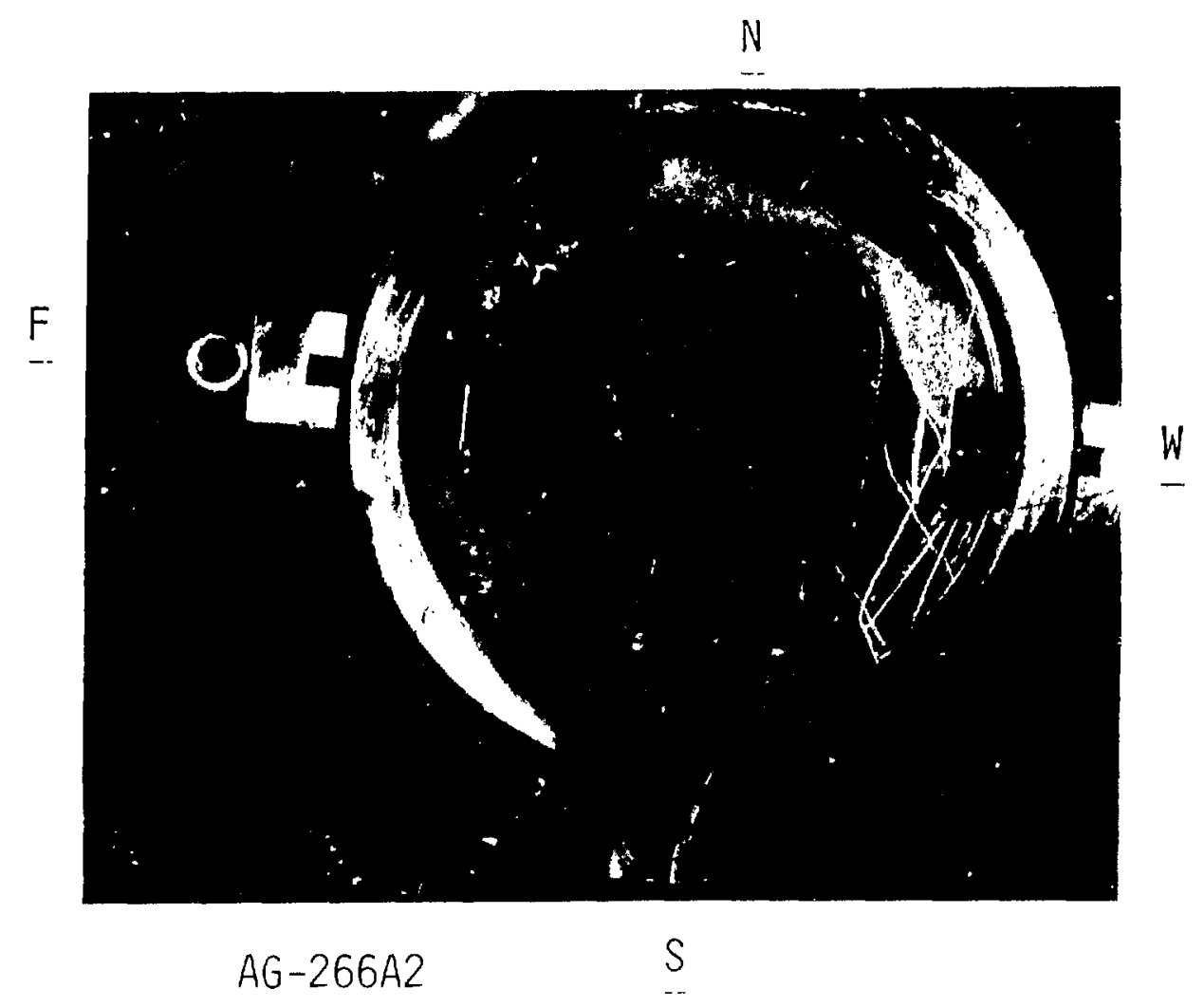

Fig. VII-2. Photograph of TRIo capsule after first cut made. (The view is upward from the bottom.)

TABLE VII- 1

Measured Capsule Asymmetry

\begin{tabular}{c|l|l|l|l|l|c}
\hline \multirow{2}{*}{$\begin{array}{c}\text { Angle } \\
\text { (deg) }\end{array}$} & \multicolumn{3}{|c|}{ Capsulc Asymmetry $^{\mathrm{a}}$ (in.) } & \multicolumn{2}{c}{ Gap (in.) } \\
\cline { 2 - 7 } & 0.012 & 0.014 & 0.020 & 0.024 & Measured & Estimated \\
\hline 30 & + & - & - & - & $>0.012$ & 0.014 \\
90 & - & - & - & - & $<0.012$ & 0.011 \\
150 & + & - (close) & - & - & 0.012 & 0.014 \\
210 & + & + & + & - & $>0.020$ & 0.022 \\
270 & + & + & + & + & $>0.024$ & $0.029^{\mathrm{b}}$ \\
330 & + & + & + & + & $>0.020$ & 0.022 \\
\hline
\end{tabular}

a"+" means wire fit in gap; "-" means that the wire did not fit.

$b_{0.029 \text { fits. }}$ 
the east. The measured temperatures showed that the east side was consistently colder by about $50^{\circ} \mathrm{C}$. This eifect was due to the gap asymmetry. It is likely that the thermocouples located on the east and west sides directly measured the maximum and the minimum temperatures. It is fortunate that the capsule was not off-center to the north or to the south, because no direct way would have been available to determine the entire temperature range.

The outer cladding was then removed and discarded, and the inner capsule was sectioned to recover the breeder pellets. The breeder pellets appeared to be intact, but broke into four to six pieces when picked up. The lithiumaluminate pellets were numbered 1 to 7 from top to bottom. The orientation of each fragment was marked, and each pellet was placed in a separate container (plastic having a push-fit top) under the ambient cell atmosphere of dry nitrogen. Each plastic container was then placed in a metal "paint-can" type container. The hot cell is generally used to examine fission fuels, and the pellets did become contaminated with fission products. Also, the grinding procedures produced substantial quantities of steel dust, and the pellets probably became contaminated with bits of cladding.

\section{B. Tritium Retention in the Lithium Aluminate Pellets}

Pellets 3 and 4 were selected for tritium analysis. Pellet 3 was removed from the AGHC, bagged, placed in a container, and delivered to the Analytical Chemistry Laboratory of ANL. The pellet was in six pieces. The pellet was removed from its containers and reassembled (Fig. VII-3). The activity was quite high, probab'y orring to contamination as discussed above. The total activity was $1.5 \mathrm{R}$ at a distance of $4 \mathrm{in.}$, with a gamma level of $50 \mathrm{mR}$. $A$ imear on one of the pellet fragments (the south sector) showed a gamma level of 1.5 million counts per minute (cpm) gamma and 0.15 million cpm alpha, indicating a substantial amount of loose activity. Owing to the amount of loose activity present, an attempt was made to clean the pellet with an ethanol wash. This procedure lowered the total activity to $200 \mathrm{mR}$, but the gamma level was unchanged. Each pellet fragment was then given an ethanol wash. It was observed that the ethanol rapidly soaked into the sample, because of its porous structure. The tritium content of the ethanol was determined to be $0.22 \mu \mathrm{Ci}$, less than $0.2 \%$ of the amount of tritium in a sample. 


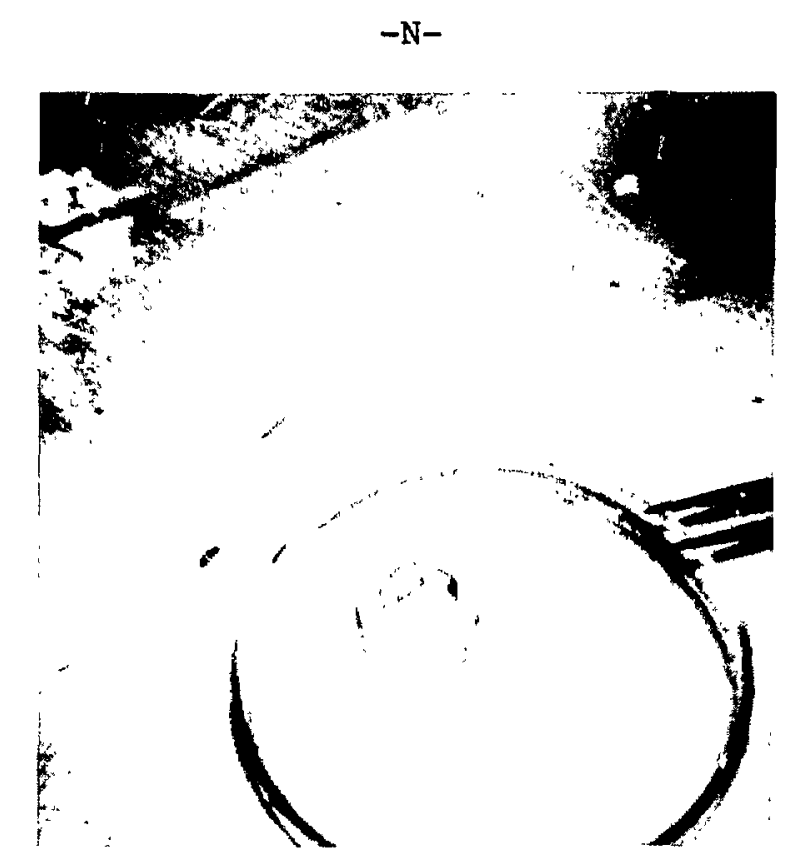

Fig. VII-3. Photograph of irradiated $\mathrm{LiAlO}_{2}$ pellet.

Each pellet fragment (weight about $1 \mathrm{~g}$ ) was dissolved in $150 \mathrm{~mL}$ of $6 \mathrm{~N}$ HCl and then neutralized with NaOH. Aliquots of the solution were taken for measurement of lithium isotopy. To measure tritium content, two 15-mL samples were distilled, and two aliquots of each distillate were analyzed for tritium by liquid scintillation counting. Each aliquot was counted twice. The standard deviation of counts for each distillation aliquot was less than $0.7 \%$, and the results for each distillation aliquot agreed within $1.5 \%$.

A sample of distillate was dried and counted to determine if any carryover of nontritium activity had occurred by distillation. The activity counts were $3 \mathrm{cpm}$ alpha and $30 \mathrm{cpm}$ beta; neither of which was above background. The samples all had at least 40,000 accumulated counts so that the error in counting statistics was less than $0.5 \%$. The background counts were less tha:l $3 \%$ of the sample counts. Counting efficiencies were typically about 40\%, as determined by using NBS standards. The error of a single tritium determination was less than 5\%, fincluding errors in the NBS standard $(<2 \%)$, volumetrics $(2 \%)$, and distillation ( $2 \%)$.

Fragments, roughly in the shape of 60-deg sectors of the pellet, were taken from the east (coldest side) and the west side of Pellets 3 and 4 for tritium assay. Upon dissolution of the samples of Pellet 3 , sniffing for 
tritium detected a measurable amount - the order of $10 \%$ of the content later found to be in the samples. The amount of tritium in the gas phase evolved during dissolution of the samples from Pellet 4 was then determined quantitatively by circulating the cover gas in a closed loop through a calibrated Kanne chamber (tritium monitor). This procedure has an estimated accuracy of $\$ 10 \%$ in the amount of tritium so determined. Once the sealed contafners holding the pellet fragments had been opened, a slight amount of tritium was detected, $<10 \mu \mathrm{Ci} / \mathrm{m}^{3}$ or less than $0.1 \mu \mathrm{Ci} / \mathrm{m}^{3}$, this amount is less than 0.02 $\mu \mathrm{Ci} / \mathrm{g}$ of sample.

The data on measured tritium activities are presented in Table VII-2. The amounts of tritium in the solid range from about 100 to about $600 \mu \mathrm{Ci} / \mathrm{g}$, or 0.01 to 0.06 wppm. The east side, which was at the lowest temperature, had a much higher tritium content than the west side. The amount of tritium evolved from the samples was about $100 \mu \mathrm{Hi} / \mathrm{g}$, representing a content of 0.01 wppm. The average concentration is $303 \mu \mathrm{Ci} / \mathrm{g}$ in Pellet 3 and $391.5 \mu \mathrm{Ci} / \mathrm{g}$ in Pellet 4, a difference of $23 \%$. This difference is attributed to two factors: the retention is highly temperature dependent, and the temperature distributions within pellet fragments is different. The overall average amount of tritium in the solid is $350 \pm 50 \mu \mathrm{Ci} / \mathrm{g}$. Since the amount of tritium which evolved upon dissolution is about $100 \mu \mathrm{Ci} / \mathrm{g}$, the total content is then estimated as $450 \pm 50 \mu \mathrm{Ci} / \mathrm{g}$ an average concentration of $0.047 \pm 0.005 \mathrm{wppm}$. The

\section{TABLE VII-2}

Measured Tritium Activities Retained in Lithium Aluminate Breeder ${ }^{a}$

\begin{tabular}{l|c|c}
\hline $\begin{array}{c}\text { Sample } \\
\text { I.D. }\end{array}$ & $\begin{array}{c}\text { Tritium in Solid } \\
(\mu \mathrm{Ci} / \mathrm{g} \text { of solid) }\end{array}$ & $\begin{array}{c}\text { Tritium Evolved } \\
(\mu \mathrm{Ci} / \mathrm{g} \text { of solid) }\end{array}$ \\
\hline 3, east & $459 \pm 23.0(0.048)$ & - \\
3 , west & $147 \pm 7.0(0.015)$ & - \\
4 , east & $623 \pm 31.0(0.065)$ & $137 \pm 14.0(0.014)$ \\
4, west & $160 \pm 8.0(0.016)$ & $77 \pm 8.0(0.008)$ \\
\hline
\end{tabular}

a The numbers in brackets are tritium concentrations in wppm. 
amount of tritium retained in the total amount of breeder $(42.9 \mathrm{~g})$ is estimated to be $0.019 \pm 0.002 \mathrm{Ci}$. Of the total amount of tritium recovered ( 35.1 Ci), more than $99.94 \%$ was evolved from the breeder and successfully recovered during operation of the experiment. The value of tritium retention, 0.05 wppm, demonstrates that in-situ tritium recovery can work well for solid breeders, and that tritium inventorles in solid breeder blankets can be 1 ow.

The length of stainless steel tubing that led from the top of the reactor tank to the glovebox had been crimped and sealed at each end. In an effort to determine if significant quantities of tritium were absorbed on the walls of the tubing, the tubing was opened and connected to tygon tubing, and $50.0 \mathrm{~mL}$ of water was recirculated through the tubing for $48 \mathrm{~h}$. The amount of tritium leached intc the water was $0.57 \mathrm{mCl}$. Additional leachings produced a total of $0.11 \mathrm{mCi}$. The amount of tritium on the tubing is, therefore, estimated as less than $1 \mathrm{mCi}$.

\section{Lithiu Isotopy}

The ${ }^{6} \mathrm{Li}$ burnup was determined by using 1sotope dilution mass spectrometry. The initial content had been previously measured as $0.55 \%$. The results for the irradiated pellets are given in Table VII-3. The ${ }^{6} \mathrm{Li}$ conter. is $0.38 \%$, representing a burnup of $0.17 \%$.

\section{Dosinetry}

The TRIO assembly contained iron, nickel, and titanium dosimetry wires located in separate tubes on the west and east sides, and in the center of the capsule. The tubes on the west and east sides were on the outside of the capsule assembly and located on the outside surface of the fins (see Fig. IV10). The 0.25-mm (10-mi1) diameter wires were cut into six pieces, each about $1.4 \mathrm{~cm}$ long, and counted by gamma spectrometry, using a Ge(Li) detector. The reaction rates were corrected for decay during irradiation using the flux histories measured by the self-powered neutron detectors (see Sec.VI.B). As already noted, the flux histories showed significant variations not observed in the power history, owing to fuel changes. The effect upon interpretation of the dosimetry data is minimal, since the activations used are long lived. 
TABLE VII-3

${ }^{6} \mathrm{Li}$ Isotope Content of Irradiated TRIO Pellets

\begin{tabular}{l|c}
\hline $\begin{array}{l}\text { Sample } \\
\text { I.D. }\end{array}$ & at.\% $6 \mathrm{LH}^{\mathrm{a}}$ \\
\hline 3, east (1) & $0.379 \pm 0.004$ \\
3, east (2) & $0.379 \pm 0.004$ \\
3, west (1) & $0.378 \pm 0.004$ \\
3, west (2) & $0.378 \pm 0.004$ \\
4, east & $0.383 \pm 0.004$ \\
4, west & $0.385 \pm 0.004$ \\
Average & $0.38 \% \mathrm{~b}$ \\
\hline
\end{tabular}

\footnotetext{
"Values after " \pm " are precision estimated by the analyst.

$\mathrm{b}_{\text {Birnup }}=0.55 \%-0.38 \%=0.17 \%$.
}

The measured activation rates are listed in Täble VII-4. The results represent the average of measurements on the six samples. No gradient was measurable over the length of the wires. As can be seen in Table VII-4, the thermal flux is about the same on the two outside (east, west) positions, but drops $32 \%$ in the center due to neutron absorption from the stainless steel cladding (total thickness, $1 / 4 \mathrm{in.)}$ and from the breeder material. The shielding factor (0.68) was calculated to be 0.74 for the stainless steel and 0.95 for the lithium aluminate breeder. The former number corresponds well to the results for the core mockup test $(0.77$ shielding factor for the steel, see Sec. IV.D). The fast flux gradients are rather steep, decreasing a factor of two from west to east. This decrease is due to the location of the experiment in the A2 position, which is at the edge of the core. This gradient in fast flux has a negligible effect upon tritium production rates and has only a minor effect upon the damage rates. The activation rates are 30 to $40 \% 1$ ower than those in the core mockup test, indicating a correspondingly lower flux in the experiment compared to the core mockup test. 
TABLE VII-4

Measured Activation Rates for TRIO

\begin{tabular}{l|c|c|c}
\hline \multirow{2}{*}{ Position } & \multicolumn{3}{|c}{ Activation Rate, ${ }^{a}$ atom/atom-s } \\
\cline { 2 - 4 } & $58 \mathrm{Fe}(\mathrm{n}, \mathrm{\gamma})^{59} \mathrm{Fe}$ & $54 \mathrm{Fe}(\mathrm{n}, \mathrm{p})^{54} \mathrm{Mn}$ & $46 \mathrm{TI}(\mathrm{n}, \mathrm{p})^{46} \mathrm{Sc}$ \\
\hline \multirow{2}{*}{ West } & $1.24 \times 10^{-10}$ & $5.34 \times 10^{-12}$ & $7.47 \times 10^{-13}$ \\
Center & $8.31 \times 10^{-11}$ & $3.42 \times 10^{-12}$ & $5.26 \times 10^{-13}$ \\
East & $1.22 \times 10^{-10}$ & $2.67 \times 10^{-12}$ & $3.82 \times 10^{-13}$ \\
\hline
\end{tabular}

$a_{\text {Results are the average of measurements on }}$ six samples and normalized to $30 \mathrm{MW}$.

The activities in Table VII-4 were used to adjust the neutron spectrum measured in the core mockup test (Sec. IV.D). The resultant neutron fluences are listed in Table VII-5.

Table VII-5

Adjusted Neutron F1uences in TRIO

\begin{tabular}{l|c|c|c}
\hline \multirow{2}{*}{\multicolumn{1}{c|}{ Energy Range }} & \multicolumn{3}{c}{ Neutron Fluence $\left(10^{20} \mathrm{nv}\right)$} \\
\cline { 2 - 4 } & Center & West & East \\
\hline Total & 19.1 & 28.7 & 19.7 \\
Thermal $(<0.5 \mathrm{eV})$ & 6.93 & 10.3 & 9.52 \\
Thermal $(2200 \mathrm{~m} / \mathrm{s})$ & 5.32 & 9.13 & 8.43 \\
Ep1thermal $(0.5 \mathrm{eV}-0.1 \mathrm{MeV})$ & 6.01 & 8.98 & 5.13 \\
Fast $(>0.1 \mathrm{MeV})$ & 6.15 & 9.05 & 5.0 \\
\hline
\end{tabular}

\section{E. Microstructural Characterization of Irradiated Lithiu} Aluinate Breeder Materla1

The correlation of microstructural parameters (grain size, surface area, porosity, etc.) of the breeder material to tritium release is essential to understanding the nature of tritium transport. Therefore, the miciostructure of the breeder had to be characterized both before and after irradiation. In 
the TRIO experiment, scanning electron microscopy (SEM) photographs were taken of the breeder material before irradiation (see Fig. IV-4). In addition, SEM was used to examine the irradiated lithium aluminate breeder material. Moreover, a small section of thermocouple cladding was examined to investigate interactions between the lithium aluminate and stainless steel cladding. The results of these studies are presented below.

A. fragment of Pellet 3 (from the northwest sector) was chosen for this investigation. The specimen was fractured and SEM photographs (Figs. VII-4 and -5 ) were taken. The microstructure appears to be the same as before irradiation. The grain radius is still $0.1 \mathrm{um}$, and the agglomerates are still about $50 \mu$ in diameter.

A second sample, Pellet 6, was put back together, mounted in epoxy, then ground and polished. The results of the investigation showed that no significant changes in microstructure had occurred as a result of the irradiation in the experiment.

Owing to the radioactivity of the steel components, only a very small meţal sperimen could be examined with the techniques used, which involved some hands-on operations. A small section of thermocouple from the west inside, $\sim$ $\mathrm{cm}$ in length, was studied to look for breeder-cladding interactions. There was some evidence of corrosion, viz., a light-colored material that was deposited on the surface (probably an oxide) was depleted in iron and enriched in chromium.

\section{F. Radioactivity}

A sample of lithium aluminate (the southwest sector of Pellet 3 ) was analyzed by gamma spectrometry and treated as an activation sample. The measured activities and calculated source concentrations are summarized in Table VII-6. Comparison of these results with pre-irradiation elemental and activation analysis (Table IV-3) that the observed impurities as determined before and after irradiation are generally in agreement. However, the PIE sample has more fission products (Table VII-7), probably owing to contamination from the hot cell. In an effort to remove surface contamination, the sample was washed with ethanol, and both the sample and the wash solution were counted. It was found that only the gamma peaks from long-lived fission products namely $1{ }^{34} \mathrm{Cs}$, 

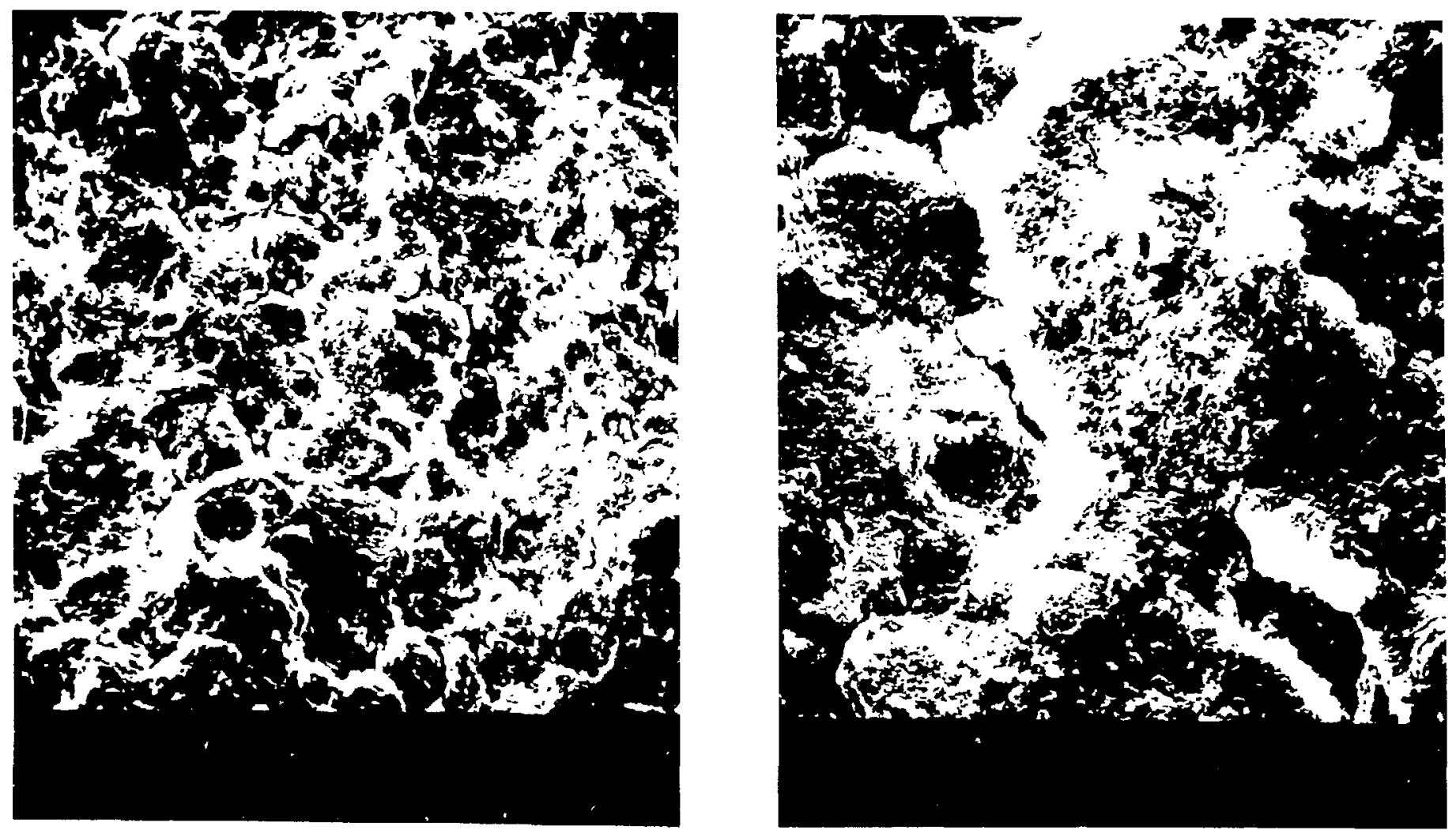

Fig. VII-4. Scanning electron micrographs of irradiated $\mathrm{LiAlO}_{2}$ from TRIO. 

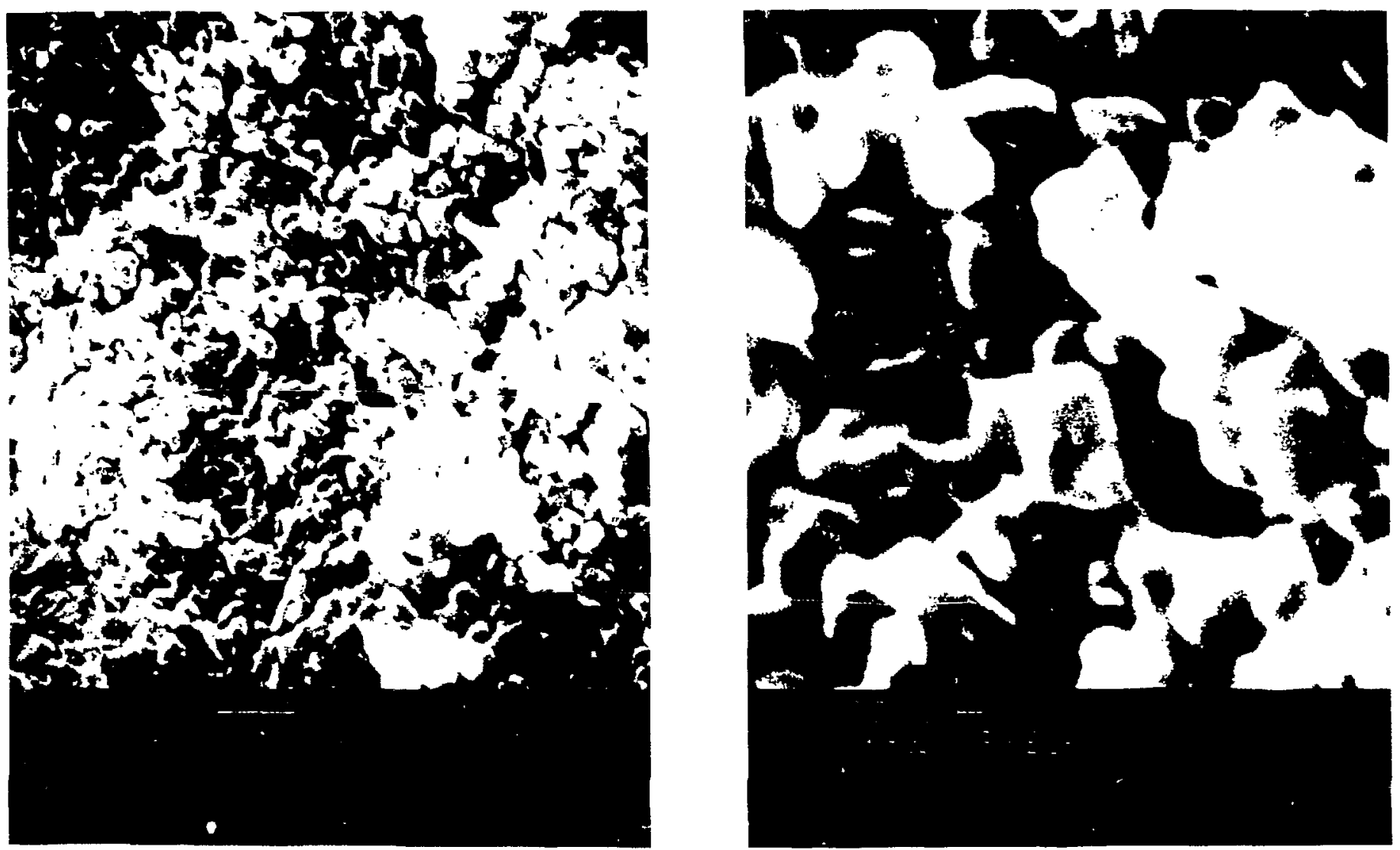

Fig. VII-5. Scanning electron micrographs of irradiated $\mathrm{LiAlO}_{2}$ from TRIO. 
TABLE VII- 6

Gamna Analysis of Irradiated TRIO Lithium Aluminate Samples

\begin{tabular}{|c|c|c|c|c|c|c|c|c|}
\hline \multirow[b]{2}{*}{ Isotope } & \multicolumn{4}{|c|}{ Before Washing } & \multicolumn{4}{|c|}{ After Washing with Ethanol } \\
\hline & $\mu \mathrm{Ci} / \mathrm{g}$ & $\begin{array}{l}\text { Error } \\
( \pm \%)\end{array}$ & Element & wppm & $\mu \mathrm{Ci} / \mathrm{g}$ & $\begin{array}{l}\text { Error } \\
( \pm \%)\end{array}$ & Element & wppm \\
\hline $22 \mathrm{Na}$ & 0.093 & 3.5 & $\mathrm{Na} ?$ & & & & & \\
\hline${ }^{46} \mathrm{Sc}$ & 28.9 & & Sc & 0.088 & & & & \\
\hline${ }^{51} \mathrm{Cr}$ & 489.0 & & $\mathrm{Cr}$ & 40.9 & & & & \\
\hline $54 \mathrm{Mn}$ & 1.65 & & $\mathrm{Fe}$ & 164.0 & & & & \\
\hline${ }^{59} \mathrm{Fe}$ & 6.87 & & $\mathrm{Fe}$ & 138.0 & & & & \\
\hline $57 \mathrm{Co}$ & 0.16 & & Ni? & & & & & \\
\hline${ }^{58} \mathrm{Co}$ & 13.1 & & $\mathrm{Ni}$ & 40.9 & & & & \\
\hline${ }^{60} \mathrm{Co}$ & 8.80 & & Co & 0.38 & & & & \\
\hline $65 \mathrm{Zn}$ & 42.8 & & $\mathrm{Zn}$ & $27 \cdot 0$ & & & & \\
\hline $95 \mathbf{Z r}$ & 1.09 & 4.0 & $\mathrm{Zr}^{\mathrm{a}}$ & 9.5 & & & & \\
\hline $103 \mathbf{R u}$ & 0.49 & 7.4 & $\mathrm{Ru}^{\mathrm{a}}$ & 0.11 & & & & \\
\hline $110^{\mathrm{m}} \mathrm{Ag}$ & 0.49 & 1.9 & $\mathrm{Ag}$ & 0.036 & & & & \\
\hline $12{ }^{4} \mathrm{Sb}$ & 2.31 & 2.9 & $\mathrm{Sb}$ & 0.051 & & & & \\
\hline $134 \mathrm{Cs}$ & 18.0 & & $\mathrm{Cs}$ & 0.341 & 11.0 & & Cs & 0.208 \\
\hline $137 \mathrm{Cs}$ & 0.24 & & $\mathrm{U}$ & & 0.11 & & $\mathrm{U}$ & \\
\hline $141 \mathrm{Ce}$ & 0.23 & 5.0 & $C e^{a}$ & 0.075 & & & & \\
\hline $144 \mathrm{Ce}$ & 9.28 & 10.0 & $\mathrm{U}$ & & 5.66 & 10.0 & $\mathrm{U}$ & \\
\hline $18 l_{\mathrm{Hf}}$ & 8.66 & 5.1 & Hf & 0.33 & & & & \\
\hline $182 \mathrm{Ta}$ & 0.846 & 3.0 & $\mathrm{Ta}$ & 0.011 & & & & \\
\hline
\end{tabular}

${ }^{a}$ Products which can be made either by direct activation or by Fission of uranium. 
TABLE VII-7

Possible Fission Products

(Uralum/Plutonium) of

Irradiation Aluminate Samples

\begin{tabular}{c|c|c}
\hline Isotope & $\begin{array}{c}\text { Before } \\
\text { Washing } \\
\text { (wppm U) }\end{array}$ & $\begin{array}{c}\text { After } \\
\text { Washing } \\
\text { (wppm U) }\end{array}$ \\
\hline $95 \mathrm{Zr}$ & 0.59 & 0.59 \\
$103_{\mathrm{Ru}}$ & 0.19 & 0.19 \\
$1{ }^{37} \mathrm{Cs}$ & 10.9 & 4.9 \\
$141 \mathrm{Ce}$ & 0.84 & 0.84 \\
$144 \mathrm{Ce}$ & 17.4 & 10.6 \\
\hline
\end{tabular}

${ }^{137} \mathrm{Cs}$, and ${ }^{144} \mathrm{Ce}$ were decreased by washing. Thus, there had been some contamination from fission products.

\section{G. X-Ray Diffraction}

Samples of Pellet 3 were taken, powdered, and analyzed by $X$-ray diffraction. The principal phase in all samples was gamma lithium aluminate. lo evidence of alpha or beta phases was observed. The sample from the west inside, which had the highest temperature, showed a minor phase of $\mathrm{LiAl}_{5} \mathrm{O}_{8}$. Both west samples showed a very minor peak of lithium aluminate hydrate, as did the east outside sample. The sample from the east inside had no extra lines. The results show that the material was still lithlum aluminate in the same phase. High temperature and lithium burnup may have been starting to produce the lithium-depleted phase. The trace amounts of hydrolysis product could have been caused by handling of the sample after irradiation. 
SECTION VIII

DATA ANALYSIS 


\section{DATA ANALYSIS}

The raw data accumulated in the course of the experiment, presented in Sec. VII, includes neutron flux, temperatures, and release rates of tritium in the various forms. The translation of the raw data into parameters used for design of fusion reactor blankets is discussed in this section. For example, the raw data on neutron flux and dose are used to determine damage, heating rates, and critium production rates in the $\mathrm{LiAlO}_{2}$ breeder material. The temperature data, in conjunction with the calculated heating rates, are used to determine heat transfer coefficfents and thermal conductivity of the breeder material. The tritium release data, both integral and dynamic, are used to calculate tritium inventory and to quantify the release in terms of specific nechanisms, e.g., Intragranular diffusion. A tritium mass transport coefficient, diffusivity, is thus derived.

\section{A. Dosinetry and Danage Analysis}

\section{Tritium Production Rates}

By use of the determined neutron spectrum (Sec. VII.D), the 1ithium burnup rates were computed. The outer rate was found to be $9.7 \times 10^{-8}$ atom/atom. $\mathrm{s}$ while the inner rate was $6.5 \times 10^{-8}$ atoms/atom.s. These two values were then used to determine the total burnup; an iterative procedure to calculate the time-dependent burnup and resultant decrease in self-shielding. The stainless steel sleeve was about $0.5-\mathrm{cm}$ thick resulting in a neutron absorption loss factor of about 0.69 (calculated with an analytical approximation for selfshielding). This effect was estimated to be about 0.74 from the previous flux measurements in the mockup experiment (Sec. IV.D). The initial lithium selfshielding was about 0.952 , decreasing to about 0.968 by the end of the irradiation. These two factors yield an average lithium burnup rate of $5.87 \times 10^{-8}$ atom/atom.s. Over the 97 days of the run, this would result in a net burnup of $37.7 \%$ of the initial ${ }^{6} \mathrm{Li}$ atoms and a net tritium activity of $39.2 \mathrm{Ci}$. Due to the uncertainties in fluence measurements and self-shielding approximations, the tritium values have an estimated accuracy of 10-15\%. If the measured 1ithium-to-iron activity ratios from the mockup experiment are used, the calculated tritium production is $37.5 \mathrm{C}$. 


\section{Damage Analysis}

The SPECTER computer code was used to calculate damage parameters for the TRIO irradiation in ORR. The dose (rads), gas production, and damage rates are listed in Tables VIII-1 through VIII-3. The dose for the 1ithium aluminate is calculated to be $5.36 \times 10^{12}$ rads, resulting in $0.81 \mathrm{dpa}$. Using the measured value of $4.8 \mathrm{~W} / \mathrm{g}$ for gamma heating (Sec. IV.D), the gamma dose was calculated to be $3.54 \times 10^{12}$ rads. The displacement damage includes $0.30 \mathrm{dpa}$ from the ${ }^{6} \mathrm{Li}$ reactions, plus the damage $(0.51 \mathrm{dpa})$ from the $11 \mathrm{thi} u \mathrm{~m}$, aluminum, and oxygen recoils. The total damage is approximately equal to $0.6 \mathrm{MW} \cdot \mathrm{yr}$, or $\sim 2$-mo operation in STARFIRE. 1

\section{Nuclear Heating Rates}

The data given above on dose rates was translated into nuclear heating rates. The gamma heating rate and the fast neutron heating rate were assumed to be directly proportional to the neutron flux, as continuously measured by the self-powerf neutron detectors. The uncertainty of the gamma and fast neutron heating rates so derived was estimated to be $<20 \%$. The heating rates in the lithium aluminate from tritium production are in direct proportion to the tritium production rate. The uncertainty in the tritium production rate is less than $10 \%$. The uncertainty in the total heating rate is estimated to be less than 15\%. The data for nuclear heating rates are given in Table VIII-4.

\section{B. Analysis of Heat Transfer Data}

The TRIO capsule is illustrated schematically in Figure IV-3. As shown, the thermocouples were located on the inside and the outside surfaces of the breeder pellets, both on the east and the west side. The locations of the thermocouple junctions are also illustrated, there are a total of ten, designated thus: $\mathrm{T} 1$ (east, inside), T2 (east, outside), T3-T6 (west, inside, top to bottom), and T7-T10 (west, outside, top to bottom). The recorded temperatures for the entire experiment were presented in Secs. VI.C and VI.F. To minimize uncertainties, temperature readings were averaged over those time intervals when the readings were nearly constant. Such data wcre taken for the 33 runs, and the results are given in Table VIII-5, which 1ists the run number, the time interval, the number of data points $(N)$, the ten temperatures ( $T 1$ through 
TABLE VIII-1

Speciral Averaged Kerma (MACKLIB) Factors and Damage per Component Nuclide in the $\mathrm{LiAlO}_{2}$.

\begin{tabular}{|c|c|c|}
\hline & \multicolumn{2}{|c|}{ Damage } \\
\hline & $(\mathrm{keV}-\mathrm{b})$ & (rads) \\
\hline Hydrogen & $7.0039 \times 10^{2}$ & $1.2911 \times 10^{13}$ \\
\hline Deuterium & $4.7055 \times 10^{2}$ & $4.3370 \times 10^{12}$ \\
\hline He 11 um & $3.7827 \times 10^{2}$ & $1.7421 \times 10^{12}$ \\
\hline Lithi um-6 & $1.3664 \times 10^{6}$ & $3.3340 \times 10^{15}$ \\
\hline Lithium-7 & $2.7662 \times 10^{2}$ & $7.2845 \times 10^{11}$ \\
\hline Beryllium & $1.8587 \times 10^{2}$ & $3.8018 \times 10^{11}$ \\
\hline Boron-10 & $2.7271 \times 10^{6}$ & $2.1950 \times 10^{15}$ \\
\hline Boron- 11 & $1.3459 \times 10^{2}$ & $2.2555 \times 10^{11}$ \\
\hline Carbon-12 & $1.2014 \times 10^{2}$ & $1.8455 \times 10^{11}$ \\
\hline Nitrogen & $4.9696 \times 10^{2}$ & $6.5403 \times 10^{11}$ \\
\hline Oxygen & $1.0731 \times 10^{2}$ & $1.2364 \times 10^{11}$ \\
\hline Fluorine & $1.2503 \times 10^{2}$ & $1.2131 \times 10^{11}$ \\
\hline Scdium & $1.6840 \times 10^{2}$ & $1.3503 \times 10^{11}$ \\
\hline Magnesium & $7.2434 \times 10^{1}$ & $5.4920 \times 10^{10}$ \\
\hline Al umi num & $1.4903 \times 10^{2}$ & $1.0182 \times 10^{11}$ \\
\hline Silicon & $6.5279 \times 10^{1}$ & $4.2845 \times 10^{10}$ \\
\hline Chlorine & $3.7211 \times 10^{3}$ & $1.9348 \times 10^{12}$ \\
\hline Potassium & $1.8804 \times 10^{2}$ & $8.8648 \times 10^{10}$ \\
\hline Calcium & $1.4646 \times 10^{2}$ & $6.7361 \times 10^{10}$ \\
\hline Titanium & $1.2790 \times 10^{2}$ & $4.9219 \times 10^{10}$ \\
\hline Vanadium & $1.6889 \times 10^{2}$ & $6.1113 \times 10^{11}$ \\
\hline Chromi um & $6.2772 \times 10^{1}$ & $2.2254 \times 10^{10}$ \\
\hline Manganese & $3.1790 \times 10^{3}$ & $1.0667 \times 10^{12}$ \\
\hline Iron & $3.8452 \times 10^{1}$ & $1.2692 \times 10^{10}$ \\
\hline Coba1t & $4.0877 \times 10^{1}$ & $1.2786 \times 10^{10}$ \\
\hline Nickel & $1.0907 \times 10^{2}$ & $3.4247 \times 10^{10}$ \\
\hline Copper & $6.0413 \times 10^{2}$ & $1.7527 \times 10^{11}$ \\
\hline Zirconium & $2.7606 \times 10^{1}$ & $5.5787 \times 10^{9}$ \\
\hline
\end{tabular}


TABLE VIII-1 (Contd.)

\begin{tabular}{|c|c|c|}
\hline & \multicolumn{2}{|c|}{ Damage } \\
\hline & $(k e V-b)$ & (rads) \\
\hline Niobium & $2.9384 \times 10^{1}$ & $5.8301 \times 10^{9}$ \\
\hline Molybdenum & $3.3340 \times 10^{2}$ & $6.4059 \times 10^{10}$ \\
\hline Tin & $2.1604 \times 10^{1}$ & $3.3554 \times 10^{9}$ \\
\hline Tantalum & $2.0110 \times 10^{1}$ & $2.0487 \times 10^{9}$ \\
\hline Tungsten- 182 & $1.9420 \times 10^{1}$ & $1.9670 \times 10^{9}$ \\
\hline Tungsten-183 & $1.7868 \times 10^{1}$ & $1.7998 \times 10^{9}$ \\
\hline Tungsten- 184 & $1.1409 \times 10^{2}$ & $1.1429 \times 10^{10}$ \\
\hline Tungsten-186 & $1.1629 \times 10^{4}$ & $1.1525 \times 10^{12}$ \\
\hline Lead & $2.3588 \times 10^{1}$ & $2.0986 \times 10^{9}$ \\
\hline Thorium-232 & $4.7882 \times 10^{3}$ & $3.8045 \times 10^{11}$ \\
\hline Protractinium & $1.4893 \times 10^{4}$ & $1.1884 \times 1012$ \\
\hline Uranium-233 & $3.0625 \times 10^{7}$ & $2.4229 \times 10^{15}$ \\
\hline Ur anium-234 & $4.7042 \times 10^{4}$ & $3.7058 \times 10^{12}$ \\
\hline Uranium-235 & $2.9552 \times 10^{7}$ & $2.3181 \times 10^{15}$ \\
\hline Ur anium-236 & $2.0044 \times 10^{4}$ & $1.5656 \times 10^{12}$ \\
\hline Uranium-238 & $1.0036 \times 10^{4}$ & $7.7733 \times 10^{11}$ \\
\hline Neptunium & $5.6373 \times 10^{4}$ & $4.3846 \times 10^{12}$ \\
\hline Plutonium-238 & $9.7670 \times 10^{5}$ & $7.5648 \times 10^{13}$ \\
\hline P1 utonium-239 & $5.8538 \times 10^{7}$ & $4.5149 \times 10^{15}$ \\
\hline P1utonium-240 & $6.7214 \times 10^{4}$ & $5.1625 \times 10^{12}$ \\
\hline Plutonium-24l & $6.4246 \times 10^{7}$ & $4.9141 \times 1015$ \\
\hline Plutonium-242 & $4.9176 \times 10^{4}$ & $3.7459 \times 10^{12}$ \\
\hline Americium-241 & $3.565 \mathrm{~s} \times 10^{5}$ & $2.7272 \times 10^{13}$ \\
\hline Americium-243 & $3.8848 \times 10^{4}$ & $2.9470 \times 10^{13}$ \\
\hline
\end{tabular}


TABLE VIII-2

Spectral Averaged Gas Production File (ENDF 533)

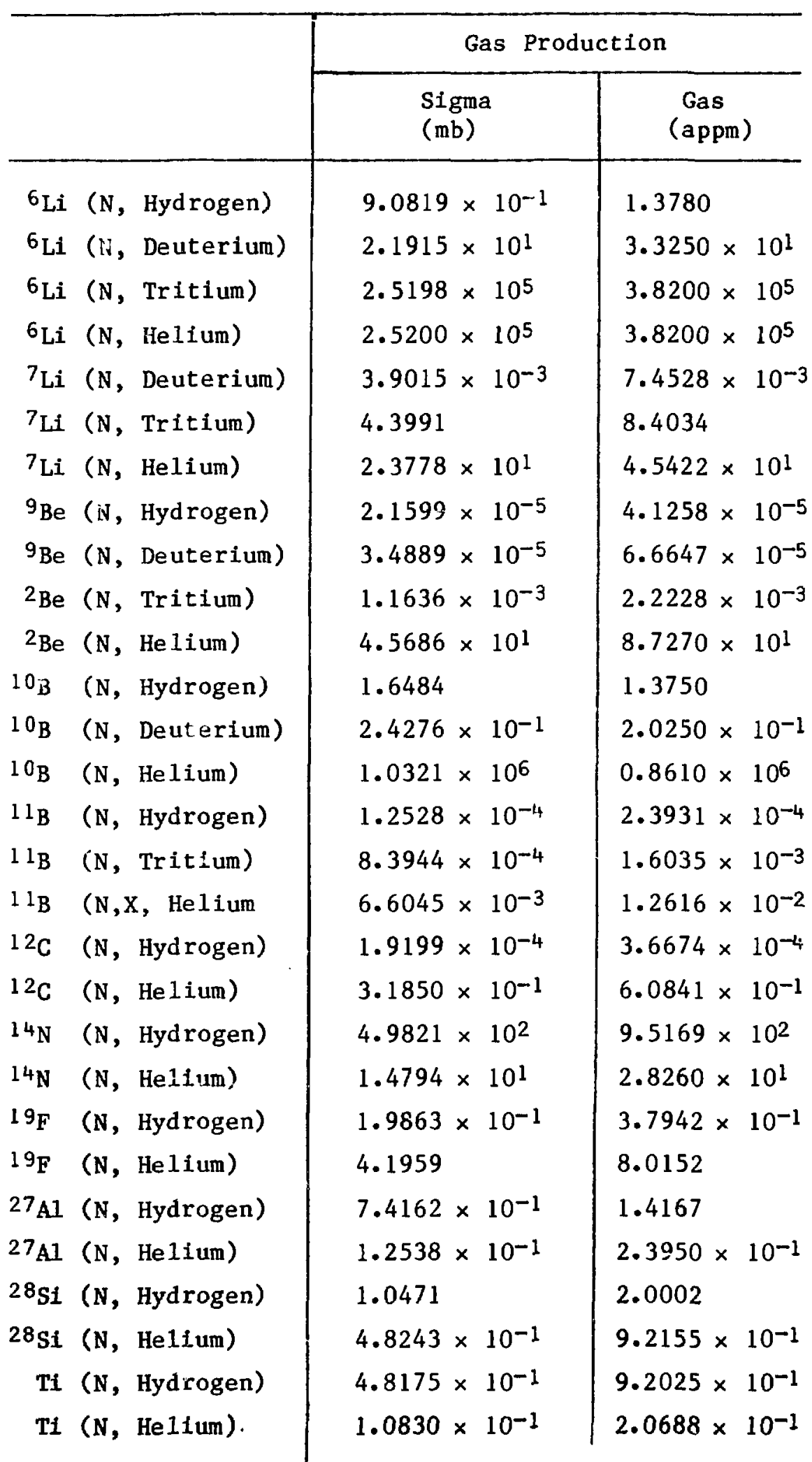


TABLE VIIT-2 (Contd.)

\begin{tabular}{|c|c|c|}
\hline & \multicolumn{2}{|c|}{ Gas Production } \\
\hline & $\begin{array}{l}\text { Sigma } \\
(\mathrm{mb})\end{array}$ & $\begin{array}{c}\text { Gas } \\
\text { (appm) }\end{array}$ \\
\hline V (N, Hydrogen) & $8.6311 \times 10^{-2}$ & $1.6487 \times 10^{-1}$ \\
\hline$V \quad(N$, Helium $)$ & $4.6181 \times 10^{-3}$ & $8.8216 \times 10^{-3}$ \\
\hline Cr (N, Hydrogen) & $7.1945 \times 10^{-1}$ & 1.3743 \\
\hline $\mathrm{Cr}$ (N, Deuterium) & $2.3418 \times 10^{-5}$ & $4.4734 \times 10^{-5}$ \\
\hline $\operatorname{Cr}(\mathbb{N}$, Tritium $)$ & $1.2795 \times 10^{-4}$ & $2.4441 \times 10^{-4}$ \\
\hline Cr $(N$, Helium-3) & $5.6717 \times 10^{-6}$ & $1.0834 \times 10^{-5}$ \\
\hline $\mathrm{Cr}(\mathrm{N}, \mathrm{Helium}-4)$ & $3.2794 \times 10^{-2}$ & $6.2644 \times 10^{-2}$ \\
\hline $55 \mathrm{Mn}$ (N, Hydrogen) & $1.2793 \times 10^{-1}$ & $2.4438 \times 10^{-2}$ \\
\hline $55 \mathrm{MN}$ (N, Helium) & $2.6592 \times 1002$ & $5.0797 \times 10^{-2}$ \\
\hline Fe (N, Hydrogen) & $9.9205 \times 10^{-1}$ & 1.8950 \\
\hline Fe $(N$, Helium) & $5.7466 \times 10^{-2}$ & $1.0977 \times 10^{-1}$ \\
\hline${ }^{59} \mathrm{Co}(\mathrm{N}$, Hydrogen $)$ & $2.6719 \times 10^{-1}$ & $5.1040 \times 10^{-1}$ \\
\hline${ }^{59}$ Co $(N$, Helium) & $2.6745 \times 10^{-2}$ & $5.1090 \times 10^{-2}$ \\
\hline $\mathrm{Ni}$ (N, Hydrogen) & $1.2307 \times 10^{1}$ & $2.3510 \times 10^{1}$ \\
\hline $\mathrm{Ni}(\mathrm{N}$, Deuterium) & $2.2761 \times 10^{-3}$ & $4.3479 \times 10^{-3}$ \\
\hline $\mathrm{Ni}(\mathrm{N}$, Helium) & $8.5119 \times 10^{-1}$ & 1.6260 \\
\hline $\mathrm{Cu}(\mathrm{N}$, Hydrogen $)$ & 2.0937 & 3.9994 \\
\hline $\mathrm{Cu}(\mathrm{N}, \mathrm{Helium})$ & $4.8190 \times 10^{-2}$ & $9.2054 \times 10^{-2}$ \\
\hline
\end{tabular}

\section{TABLE VIII-3}

Damage from Various Sources of TRIO

\begin{tabular}{|c|c|c|}
\hline Source & dpa & rads \\
\hline${ }^{6} \mathrm{Li}(\mathrm{n}, \alpha) \mathrm{t}$ & 0.30 & $1.67 \times 10^{12}$ \\
\hline Fast reactions & 0.51 & $0.15 \times 10^{12}$ \\
\hline Gamna heating & $=$ & $3.54 \times 10^{12}$ \\
\hline Total & 0.81 & $5.36 \times 10^{12}$ \\
\hline
\end{tabular}


TABLE VIII-4

Nuclear Heating Rates

\begin{tabular}{|c|c|c|c|c|c|c|}
\hline \multirow{2}{*}{$\begin{array}{l}\text { Days } \\
\text { after } \\
3 / 10 / 83\end{array}$} & \multirow{2}{*}{$\begin{array}{l}\text { Dafly } \\
\text { Average } \\
\text { Flux }\end{array}$} & \multirow{2}{*}{$\begin{array}{l}\text { Tritium } \\
\text { (Ci) }\end{array}$} & \multicolumn{4}{|c|}{ Nuclear Heating $(\mathrm{W} / \mathrm{g})$} \\
\hline & & & ${ }^{6} \mathrm{Li}(\mathrm{N}, \alpha) \mathrm{t}$ & Fast & Gamma & Total \\
\hline 1 & 0.0000 & 0.0000 & 0.0000 & 0.0000 & 0.0000 & 0.0000 \\
\hline 2 & 0.0000 & 0.0000 & 0.0000 & 0.0000 & 0.0000 & 0.0000 \\
\hline 3 & 0.0469 & 0.0215 & 0.1183 & 0.0086 & 0.2073 & 0.3342 \\
\hline 4 & 0.6887 & 0.3155 & 1.7353 & 0.1269 & 3.0448 & 4.9069 \\
\hline 5 & 1.1146 & 0.5087 & 2.7977 & 0.2053 & 4.9276 & 7.9306 \\
\hline 6 & 1.0975 & 0.4986 & 2.7421 & 0.2022 & 4.8522 & 7.7965 \\
\hline 7 & 1.1076 & 0.5008 & 2.7545 & 0.2040 & 4.8970 & 7.8556 \\
\hline 8 & 1.1080 & 0.4986 & 2.7425 & 0.2041 & 4.8985 & 7.8450 \\
\hline 9 & 1.1052 & 0.4951 & 2.7228 & 0.2036 & 4.8861 & 7.8125 \\
\hline 10 & 1.0939 & 0.4878 & 2.6827 & 0.2015 & 4.8364 & 7.7205 \\
\hline 11 & 1.1157 & 0.4951 & 2.7232 & 0.2055 & 4.9324 & 7.8611 \\
\hline 12 & 1.1136 & 0.4919 & 2.7055 & 0.2051 & 4.9235 & 7.8341 \\
\hline 13 & 1.0858 & 0.4774 & 2.6257 & 0.2000 & 4.8005 & 7.6261 \\
\hline 14 & 1.1225 & 0.4912 & 2.7017 & 0.2068 & 4.9626 & 7.8711 \\
\hline 15 & 1.1251 & 0.4900 & 2.6952 & 0.2072 & 4.9743 & 7.8768 \\
\hline 16 & 1.1300 & 0.4898 & 2.6940 & 0.2081 & 4.9959 & 7.8980 \\
\hline 17 & 1.1276 & 0.4865 & 2.6755 & 0.2077 & 4.9853 & 7.8685 \\
\hline 18 & 1.1245 & 0.4828 & 2.6553 & 0.2071 & 4.9714 & 7.8338 \\
\hline 19 & 1.1216 & 0.4793 & 2.6361 & 0.2066 & 4.9589 & 7.8016 \\
\hline 20 & 1.1173 & 0.4752 & 2.6135 & 0.2058 & 4.9397 & 7.7590 \\
\hline 21 & 1.1066 & 0.4684 & 2.5763 & 0.2038 & 4.8925 & 7.6727 \\
\hline 22 & 1.0902 & 0.4593 & 2.5264 & 0.2008 & 4.8200 & 7.5472 \\
\hline 23 & 1.0976 & 0.4603 & 2.5317 & 0.2022 & 4.8527 & 7.5866 \\
\hline 24 & 1.0937 & 0.4565 & 2.5110 & 0.2015 & 4.8355 & 7.5479 \\
\hline 25 & 1.0901 & 0.4529 & 2.4911 & 0.2008 & 4.8195 & 7.5114 \\
\hline 26 & 1.0870 & 0.4495 & 2.4724 & 0.2002 & 4.8055 & 7.4782 \\
\hline 27 & 1.0843 & 0.4464 & 2.4550 & 0.1997 & 4.7937 & 7.4484 \\
\hline 28 & 0.1809 & 0.0743 & 0.4085 & 0.0333 & 0.7997 & 1.2414 \\
\hline 29 & 0.5851 & 0.2399 & 1.3192 & 0.1078 & 2.5870 & 4.0140 \\
\hline 30 & 1.1384 & 0.4649 & 2.5569 & 0.2097 & 5.0328 & 7.7994 \\
\hline 31 & 1.1403 & 0.4634 & 2.5489 & 0.2100 & 5.0414 & 7.8004 \\
\hline 32 & 1.1457 & 0.4634 & 2.5485 & 0.2110 & 5.0652 & 7.8247 \\
\hline 33 & 1.1515 & 0.4635 & 2.5490 & 0.2121 & 5.0910 & 7.8521 \\
\hline 34 & 1.1111 & 0.4451 & 2.4478 & 0.2047 & 4.9125 & 7.5649 \\
\hline 35 & 1.1502 & 0.4585 & 2.5216 & 0.2119 & 5.0850 & 7.8185 \\
\hline 36 & 0.0920 & 0.0366 & 0.2011 & 0.0169 & 0.4067 & 0.6248 \\
\hline 37 & 0.4045 & 0.1606 & 0.8836 & 0.0745 & 1.7883 & 2.7464 \\
\hline 38 & 1.1710 & 0.4635 & 2.5491 & 0.2157 & 5.1769 & 7.9417 \\
\hline 39 & 1.1905 & 0.4688 & 2.5787 & 0.2193 & 5.2635 & 8.0615 \\
\hline 40 & 1.1794 & 0.4621 & 2.5417 & 0.2172 & 5.2143 & 7.9732 \\
\hline 41 & 1.1800 & 0.4600 & 2.5302 & 0.2174 & 5.2170 & 7.9646 \\
\hline 42 & 1.1801 & 0.4578 & 2.5177 & 0.2174 & 5.2175 & 7.9526 \\
\hline 43 & 1.1787 & 0.4549 & 2.5020 & 0.2171 & 5.2111 & 7.9303 \\
\hline 44 & 1.1762 & 0.4517 & 2.4843 & 0.2167 & 5.2003 & 7.9012 \\
\hline 45 & 1.1741 & 0.4486 & 2.4673 & 0.2163 & 5.1908 & 7.8743 \\
\hline 46 & 1.1601 & 0.4410 & 2.4257 & 0.2137 & 5.1289 & 7.7683 \\
\hline 47 & 1.1596 & 0.4387 & 2.4128 & 0.2136 & 5.1269 & 7.7532 \\
\hline 48 & 1.1587 & 0.4362 & 2.3990 & 0.2134 & 5.1229 & 7.7354 \\
\hline
\end{tabular}

VIII-7 
TABLE VIII-4 (Contd.)

\begin{tabular}{|c|c|c|c|c|c|c|}
\hline \multirow{2}{*}{$\begin{array}{l}\text { Days } \\
\text { after } \\
3 / 10 / 83\end{array}$} & \multirow{2}{*}{$\begin{array}{l}\text { Daily } \\
\text { Average } \\
\text { Flux }\end{array}$} & \multirow{2}{*}{$\begin{array}{l}\text { Tritium } \\
\text { (Ci) }\end{array}$} & \multicolumn{4}{|c|}{ Nuclear Heating ( $\mathrm{W} / \mathrm{g})$} \\
\hline & & & ${ }^{6} \mathrm{Li}(\mathrm{N}, \alpha) \mathrm{t}$ & Fast & Gamma & Total \\
\hline 49 & 1.1549 & 0.4325 & 2.3793 & 0.2127 & 5.1061 & 7.6981 \\
\hline 50 & 0.1449 & 0.0541 & 0.2977 & 0.0267 & 0.6405 & 0.9649 \\
\hline 51 & 0.6410 & 0.2393 & 1.3147 & 0.1181 & 2.8340 & 4.2669 \\
\hline 52 & 1.11811 & 0.4017 & 2.2092 & 0.1991 & 4.7798 & 7.1881 \\
\hline 53 & 1.0824 & 0.4003 & 2.2014 & 0.1994 & 4.7852 & 7.1860 \\
\hline 54 & 1.0765 & 0.3962 & 2.1794 & 0.1983 & 4.7593 & 7.1369 \\
\hline 55 & 1.0736 & 0.3934 & 2.1636 & 0.1978 & 4.7466 & 7.1079 \\
\hline 56 & 1.0724 & $0.39: 1$ & 2.1512 & 0.1975 & 4.7413 & 7.0900 \\
\hline 57 & 1.0792 & 0.3918 & 2.1548 & 0.1988 & 4.7712 & 7.1248 \\
\hline 58 & 1.0367 & 0.3746 & 2.0605 & 0.1910 & 4.5832 & 6.8347 \\
\hline 59 & 0.4697 & 0.1692 & 0.9305 & $0 . \cup 865$ & 2.0764 & 3.0935 \\
\hline 60 & 0.9516 & 0.3418 & 1.8796 & 0.1753 & 4.2072 & 6.2621 \\
\hline 61 & 0.9845 & 0.3521 & 1.9365 & 0.1813 & 4.3525 & 6.4703 \\
\hline 62 & 0.9993 & 0.3559 & 1.9572 & 0.1841 & 4.4179 & 6.5592 \\
\hline 63 & 0.9980 & 0.3539 & 1.9464 & 0.1838 . & 4.4124 & 6.5426 \\
\hline 64 & 0.9824 & 0.3469 & 1.9078 & 0.1810 & 4.3433 & 6.4321 \\
\hline 65 & 1.0 .016 & 0.3521 & 1.9367 & 0.1845 & 4.4281 & 6.5492 \\
\hline 66 & 0.9980 & 0.3493 & 1.9214 & 0.1838 & 4.4121 & 6.5174 \\
\hline 67 & 0.9969 & 0.3475 & 1.9112 & 0.1836 & 4.4074 & 6.5022 \\
\hline 68 & 0.9937 & 0.3449 & 1.8969 & 0.1830 & 4.3933 & 6.4732 \\
\hline 69 & 0.5295 & 0.1832 & 1.0074 & 0.0975 & 2.3408 & 3.4457 \\
\hline 70 & 0.9465 & 0.3264 & 1.7952 & 0.1744 & 4.1847 & 6.1543 \\
\hline 71 & 0.9681 & 0.3325 & 1.8286 & 0.1783 & 4.2802 & 6.2871 \\
\hline 72 & 0.9473 & 0.3240 & 1.7819 & 0.1745 & 4.1883 & 6.1448 \\
\hline 73 & 0.9747 & 0.3320 & 1.8258 & 0.1795 & 4.3091 & 6.3144 \\
\hline 74 & 0.9790 & 0.3320 & 1.8261 & 0.1803 & 4.3281 & 6.3345 \\
\hline 75 & 0.9799 & 0.3309 & 1.8201 & 0.1805 & 4.3322 & 6.3328 \\
\hline 76 & 0.9826 & 0.3304 & 1.8175 & 0.1810 & 4.3443 & 6.3428 \\
\hline 77 & 0.9855 & 0.3300 & 1.8151 & 0.1815 & 4.3571 & 6.3537 \\
\hline 78 & 0.9866 & 0.3290 & 1.8094 & 0.1817 & 4.3620 & 6.3532 \\
\hline 79 & 0.9844 & 0.3269 & 1.7977 & 0.1813 & 4.3522 & 6.3312 \\
\hline 80 & 0.9865 & 0.3261 & 1.7938 & 0.1817 & 4.3614 & 6.3369 \\
\hline 81 & 0.9845 & 0.3241 & 1.7826 & 0.1813 & 4.3526 & 6.3165 \\
\hline 82 & 0.9871 & 0.3236 & 1.7797 & 0.1818 & 4.3641 & 6.3256 \\
\hline 83 & 0.9932 & 0.3242 & 1.7830 & 0.1829 & 4.3909 & 6.3568 \\
\hline 84 & 0.1349 & 0.0439 & 0.2416 & 0.0248 & 0.5964 & 0.8629 \\
\hline 85 & 0.6087 & 0.1979 & 1.0883 & 0.1121 & 2.6910 & 3.8914 \\
\hline 86 & 1.0176 & 0.3296 & 1.8130 & 0.1874 & 4.4987 & 6.4991 \\
\hline 87 & 1.0139 & 0.3270 & 1.7985 & 0.1868 & 4.4825 & 6.4678 \\
\hline 88 & 1.0150 & 0.3259 & 1.7925 & 0.1870 & 4.4873 & 6.4668 \\
\hline 89 & 1.0165 & 0.3250 & 1.7873 & 0.1872 & 4.4940 & 6.4686 \\
\hline 90 & 1.0498 & 0.3341 & 1.8376 & 0.1934 & 4.6411 & 6.6720 \\
\hline 91 & 1.0663 & 0.3378 & 1.8580 & 0.1964 & 4.7144 & 6.7688 \\
\hline 92 & 1.0601 & 0.3343 & 1.8387 & 0.1953 & 4.6868 & 6.7207 \\
\hline 93 & 1.0533 & 0.3307 & 1.8186 & 0.1940 & 4.6569 & 6.6696 \\
\hline 94 & 1.0514 & 0.3285 & 1.8070 & 0.1937 & 4.6483 & 6.6490 \\
\hline 95 & 1.0559 & 0.3284 & 1.8064 & 0.1945 & 4.6682 & 6.6691 \\
\hline 96 & 1.0666 & 0.3302 & 1.8164 & 0.1965 & 4.7156 & 6.7284 \\
\hline 97 & 0.1344 & 0.0415 & 0.2283 & 0.0248 & 0.5942 & 0.8473 \\
\hline
\end{tabular}


TABLE VIII -5

Heat Transfer Data from TRIO: Temperatures

Time

Temperature $\left({ }^{\circ} \mathrm{C}\right)$

\begin{tabular}{|c|c|c|c|c|c|c|c|c|c|c|c|c|c|c|c|c|c|}
\hline Run & Date & on & of $f$ & $\mathbf{N}$ & $T 1$ & $\mathrm{~T} 2$ & $T 3$ & $\mathrm{~T} 4$ & T5 & T6 & $\mathrm{T} 7$ & $\mathrm{~T} 8$ & $T 9$ & $\mathrm{~T} 10$ & TE & $T W$ & TAV \\
\hline 0.1 & $3 / 11$ & 0346 & 0500 & 16 & 208 & 165 & 222 & 221 & 223 & 216 & 180 & 177 & 181 & 175 & 186 & 200 & 194 \\
\hline 0.2 & $3 / 11$ & 2020 & 2120 & 13 & 205 & 162 & 218 & 217 & 218 & 211 & 177 & 173 & 176 & 171 & 184 & 196 & 190 \\
\hline 1.1 & $3 / 12$ & 0856 & 0920 & 7 & 337 & 248 & 361 & $36 \%$ & 365 & 350 & 278 & 274 & 277 & 279 & 292 & 320 & 306 \\
\hline 1.2 & $3 / 12$ & 0936 & 0950 & 7 & 354 & 257 & 380 & 382 & 384 & 368 & 290 & 286 & 289 & 294 & 306 & 335 & 20 \\
\hline 1.3 & $3 / 12$ & 0950 & 1040 & 13 & 421 & 296 & 451 & 455 & 458 & 441 & 338 & 334 & 340 & 348 & 358 & 397 & 378 \\
\hline 1.4 & $3 / 12$ & 1040 & 1050 & 3 & 453 & 322 & 483 & 487 & 492 & 476 & 368 & 365 & 369 & 380 & 388 & 428 & 408 \\
\hline 1.5 & $3 / 12$ & 1054 & 1124 & 7 & 518 & 384 & 547 & 553 & 560 & 548 & 434 & 432 & 438 & 452 & 451 & 496 & 474 \\
\hline 1.6 & $3 / 12$ & 1200 & 2400 & 145 & 628 & 489 & 651 & 661 & 670 & 660 & 540 & 540 & 547 & 564 & 558 & 604 & 581 \\
\hline 1.7 & $3 / 13$ & 0000 & 1800 & 217 & 642 & 499 & 666 & 675 & 684 & 673 & 553 & 552 & 557 & 574 & 570 & 612 & 591 \\
\hline 1.8 & $3 / 13$ & 1800 & 3900 & 253 & 636 & 496 & 660 & 669 & 668 & 667 & 549 & 548 & 553 & 569 & 566 & 608 & 587 \\
\hline 2.1 & $3 / 14$ & 1600 & 3100 & 181 & 722 & 580 & 741 & 753 & 763 & 753 & 634 & 635 & 640 & 660 & 651 & 696 & 674 \\
\hline 2.2 & $3 / 15$ & 1700 & 3500 & 217 & 722 & 579 & 741 & 753 & 761 & 751 & 633 & 634 & 639 & 657 & 650 & 695 & 672 \\
\hline 3.1 & $3 / 16$ & 1100 & 1500 & 49 & 723 & 579 & 742 & 754 & 761 & 750 & 633 & 634 & 639 & 658 & 651 & 696 & 674 \\
\hline 3.2 & $3 / 16$ & 1600 & 3200 & 193 & 722 & 579 & 741 & 753 & 760 & 749 & 633 & 633 & 638 & 656 & 650 & 695 & 672 \\
\hline 3.3 & $3 / 17$ & 0900 & 1300 & 49 & 731 & 587 & 750 & 762 & 768 & 756 & 641 & 642 & 648 & 665 & 659 & 705 & 682 \\
\hline 4.1 & $3 / 17$ & 1800 & 2400 & 73 & 577 & 438 & 604 & 607 & 612 & 598 & 487 & 482 & 483 & 495 & 508 & 546 & 527 \\
\hline 4.2 & $3 / 18$ & 0000 & 2400 & 289 & 579 & 439 & 606 & 610 & 613 & 598 & 488 & 483 & 482 & 494 & 509 & 547 & 528 \\
\hline 4.3 & $3 / 19$ & 1200 & 2400 & 145 & 580 & 439 & 608 & 611 & 614 & 600 & 488 & 482 & 485 & 497 & 510 & 548 & 529 \\
\hline 4.4 & $3 / 20$ & 1200 & 2400 & 145 & 577 & 436 & 605 & 608 & 610 & 596 & 486 & 480 & 482 & 493 & 507 & 545 & 526 \\
\hline 5.1 & $3 / 23$ & 1200 & 2400 & 145 & 531 & 390 & 560 & 563 & 564 & 547 & 439 & 431 & 433 & 440 & 475 & 498 & 486 \\
\hline 5.2 & $3 / 24$ & 0000 & 0800 & 97 & 529 & 388 & 560 & 561 & 561 & 546 & 439 & 431 & 433 & 441 & 474 & 497 & 486 \\
\hline 6.1 & $3 / 24$ & 1200 & 2400 & 145 & 575 & 431 & 606 & 609 & 611 & 598 & 489 & 483 & 488 & 499 & 518 & 548 & 533 \\
\hline 6.2 & $3 / 25$ & 0000 & 1000 & 121 & 575 & 431 & 607 & 610 & 612 & 598 & 489 & 483 & 488 & 499 & 519 & 548 & 534 \\
\hline 7.1 & $3 / 25$ & 1500 & 2400 & 109 & 626 & 481 & 655 & 660 & 662 & 648 & 540 & 535 & 539 & 551 & 5 & 597 & 576 \\
\hline 7.2 & $3 / 26$ & 0900 & 2100 & 145 & 625 & 479 & 654 & 658 & 660 & 646 & 539 & 534 & 542 & 549 & 552 & 596 & 574 \\
\hline 8.1 & $3 / 28$ & 1500 & 2400 & 109 & 679 & 533 & 70 & 710 & 712 & 699 & 523 & 588 & 600 & 607 & 606 & 650 & 628 \\
\hline 8.2 & $3 / 30$ & 0000 & 1200 & 145 & 673 & 529 & 700 & 704 & 705 & 689 & 588 & 583 & 594 & 602 & 601 & 649 & 625 \\
\hline 9.1 & $3 / 30$ & 1200 & 2400 & 145 & 672 & 529 & 699 & 703 & 704 & 690 & 589 & 583 & 593 & 600 & 600 & 648 & 624 \\
\hline 9.2 & $3 / 31$ & 0000 & 2400 & 289 & 671 & 529 & 699 & 703 & 703 & 689 & 588 & 583 & 593 & 599 & 600 & 648 & 624 \\
\hline 9.3 & $4 / 01$ & 0000 & 2400 & 289 & 668 & 526 & 698 & 701 & 700 & 686 & 586 & 581 & 592 & 597 & 597 & 646 & 622 \\
\hline 9.4 & $4 / 02$ & 0000 & 2400 & 289 & 667 & 526 & 697 & 700 & 699 & 685 & 586 & 580 & 589 & 592 & 596 & 644 & 620 \\
\hline 9.5 & $4 / 03$ & 0000 & 2400 & 289 & 664 & 524 & 695 & 697 & 696 & 681 & 585 & 578 & 589 & 594 & 594 & 640 & 617 \\
\hline 9.6 & $4 / 04$ & 0000 & 1200 & 145 & 662 & 522 & 693 & 695 & 693 & 676 & 583 & 576 & 586 & 592 & 592 & 638 & 615 \\
\hline 9.7 & $4 / 04$ & 1800 & 2400 & 73 & 672 & 532 & 701 & 704 & 702 & 687 & 592 & 586 & 595 & 599 & $\mathrm{C} 32$ & 647 & 624 \\
\hline 10.1 & $4 / 06$ & 2000 & 2400 & 49 & 441 & 309 & 472 & 472 & 470 & 454 & 349 & 341 & 346 & 352 & 375 & 407 & 391 \\
\hline 10.2 & $4 / 07$ & 0000 & 0800 & 97 & 442 & 309 & 475 & 474 & 472 & 455 & 350 & 342 & 348 & 354 & 376 & 409 & 392 \\
\hline 11.1 & $4 / 07$ & 1000 & 2400 & 169 & 53 & 394 & 565 & 566 & 565 & 551 & 443 & 436 & 444 & 453 & 464 & 505 & 484 \\
\hline 11.2 & $4 / 08$ & 0000 & 0900 & 109 & 533 & 395 & 566 & 568 & 567 & 553 & 446 & 438 & 447 & 454 & 464 & 507 & 486 \\
\hline 12.1 & $4 / 08$ & 1100 & 2400 & 157 & 578 & 436 & 610 & 612 & 613 & 601 & 490 & 485 & 495 & 508 & 507 & 551 & 529 \\
\hline 12.2 & $4 / 09$ & 0000 & 1800 & 217 & 578 & 437 & 610 & 612 & 612 & 600 & 491 & 485 & 495 & 507 & 508 & 551 & 530 \\
\hline 12.3 & $4 / 10$ & ธธก0 & 0600 & 73 & 582 & 439 & 615 & 616 & 617 & 604 & 494 & 488 & 499 & 508 & 510 & 555 & 532 \\
\hline 12.4 & $4 / 10$ & 1400 & 2400 & 121 & 582 & 439 & 615 & 618 & 617 & 604 & 494 & 489 & 500 & 510 & 510 & 556 & 533 \\
\hline 12.5 & $4 / 11$ & 1600 & 2400 & 97 & 579 & 438 & 614 & 615 & 614 & 601 & 494 & 488 & 497 & 510 & 508 & 554 & 531 \\
\hline 12.6 & $4 / 12$ & 0000 & 0800 & 97 & 578 & 436 & 613 & 614 & 612 & 599 & 493 & 486 & 498 & 509 & 507 & 552 & 530 \\
\hline 13.1 & $4 / 12$ & 1200 & 2400 & 145 & 628 & 484 & 660 & 663 & 662 & 649 & 543 & 537 & 547 & 560 & 556 & 608 & 582 \\
\hline 13.2 & $4 / 15$ & 1800 & 2400 & 73 & 632 & 87 & 663 & 668 & 668 & 655 & 544 & 540 & 550 & 566 & 560 & 612 & 586 \\
\hline 13.3 & $4 / 16$ & 1200 & 2000 & 97 & 634 & +88 & 664 & 669 & 668 & 656 & 545 & 541 & 552 & 565 & 56 & 613 & 587 \\
\hline 13.4 & $4 / 17$ & 0000 & 2400 & 289 & 627 & 483 & 657 & 662 & 66 & 649 & 540 & 536 & 548 & 561 & 557 & 608 & 582 \\
\hline 13.5 & $4 / 18$ & 0000 & 0800 & 97 & 62 & 483 & 658 & 66 & 66 & 649 & 541 & 536 & 54 & 561 & 557 & 608 & 582 \\
\hline 14.1 & $4 / 18$ & 1200 & 2400 & 145 & 732 & 587 & 755 & 764 & 765 & 752 & 642 & 640 & 652 & 668 & 660 & 705 & 682 \\
\hline 14.2 & $4 / 19$ & 0000 & 2400 & 289 & 732 & 586 & 754 & 763 & 763 & 750 & 641 & 638 & 651 & 666 & 659 & 704 & 682 \\
\hline 14.3 & $4 / 20$ & 0000 & 0800 & 97 & 730 & 585 & 754 & 761 & 760 & 748 & $64 !$ & 638 & 650 & 664 & 658 & 702 & 680 \\
\hline 15.1 & $4 / 20$ & 1400 & 2400 & 121 & 733 & 588 & 757 & 763 & 763 & 750 & 643 & 641 & 650 & 664 & 660 & 704 & 682 \\
\hline 15.2 & $4 / 21$ & 0000 & 1000 & 121 & 731 & 586 & 754 & 761 & 760 & 747 & 642 & 639 & 651 & 664 & 658 & 703 & 680 \\
\hline 16.1 & $4 / 21$ & 0900 & 2400 & 181 & 730 & 586 & 755 & 760 & 759 & 745 & 641 & 638 & 649 & 663 & 658 & 702 & 680 \\
\hline 16.2 & $4 / 22$ & 0000 & 1300 & 157 & 729 & 585 & 754 & 760 & 758 & 745 & 642 & 638 & 647 & 662 & 657 & 700 & 678 \\
\hline 17.1 & $4 / 22$ & 1200 & 2400 & 145 & 729 & 585 & 75 & 759 & 758 & 746 & 642 & 63 & 647 & 661 & 657 & 700 & 678 \\
\hline 17.2 & $4 / 23$ & 0900 & 2400 & 181 & 721 & 579 & 747 & 752 & 750 & 736 & 635 & 63 & 641 & 655 & 650 & 694 & 672 \\
\hline 17. & $4 / 24$ & 0000 & 2400 & 289 & 721 & 579 & 748 & 753 & 751 & 739 & 636 & 63 & $6 \dot{4} 1$ & 655 & 650 & 694 & 672 \\
\hline 17. & $4 / 25$ & 0000 & 0800 & 97 & 722 & 58 & 750 & 754 & 751 & 739 & 637 & 632 & 641 & 655 & 651 & 694 & 672 \\
\hline 18 & $4 / 25$ & 1800 & 2400 & 73 & 72 & 58 & 75 & 760 & 75 & 74 & 644 & 639 & 647 & 661 & 658 & 701 & 680 \\
\hline 18.2 & $4 / 26$ & 0000 & 1000 & 121 & 728 & 586 & 75 & 760 & 75 & 74 & 64 & 63 & 64 & 660 & 658 & 701 & 680 \\
\hline 19.1 & $4 / 26$ & 1100 & 2400 & 157 & 677 & 536 & 708 & 710 & 706 & 59 & 593 & 58 & 59 & 605 & 606 & 649 & 628 \\
\hline 19.2 & $4 / 27$ & 0000 & 0300 & 37 & 674 & 534 & 705 & 707 & 702 & 698 & 590 & 583 & 591 & 595 & 604 & 646 & 625 \\
\hline 20.1 & $4 / 28$ & 1100 & 1200 & 13 & 405 & 291 & 440 & 435 & 430 & 415 & 323 & 313 & 318 & 317 & 348 & 374 & 361 \\
\hline 20.2 & $4 / 28$ & 1200 & 1300 & 13 & 406 & 291 & 440 & 435 & 430 & 416 & 323 & 314 & 318 & 316 & 348 & 374 & 361 \\
\hline 20.3 & $4 / 28$ & 1300 & 1400 & 13 & 406 & 292 & 441 & 436 & 431 & 416 & 323 & 314 & 317 & 318 & 349 & 375 & 3622 \\
\hline 0.4 & $4 / 28$ & 1500 & 1600 & 13 & 40 & 293 & 444 & 43 & 43 & 418 & 326 & 316 & 320 & $320^{\circ}$ & 351 & 377 & \\
\hline 20.5 & 128 & 1600 & 1800 & 25 & 409 & 293 & 444 & 439 & 433 & 419 & 326 & 316 & 319 & 320 & 351 & 377 & \\
\hline
\end{tabular}

VIII -9 


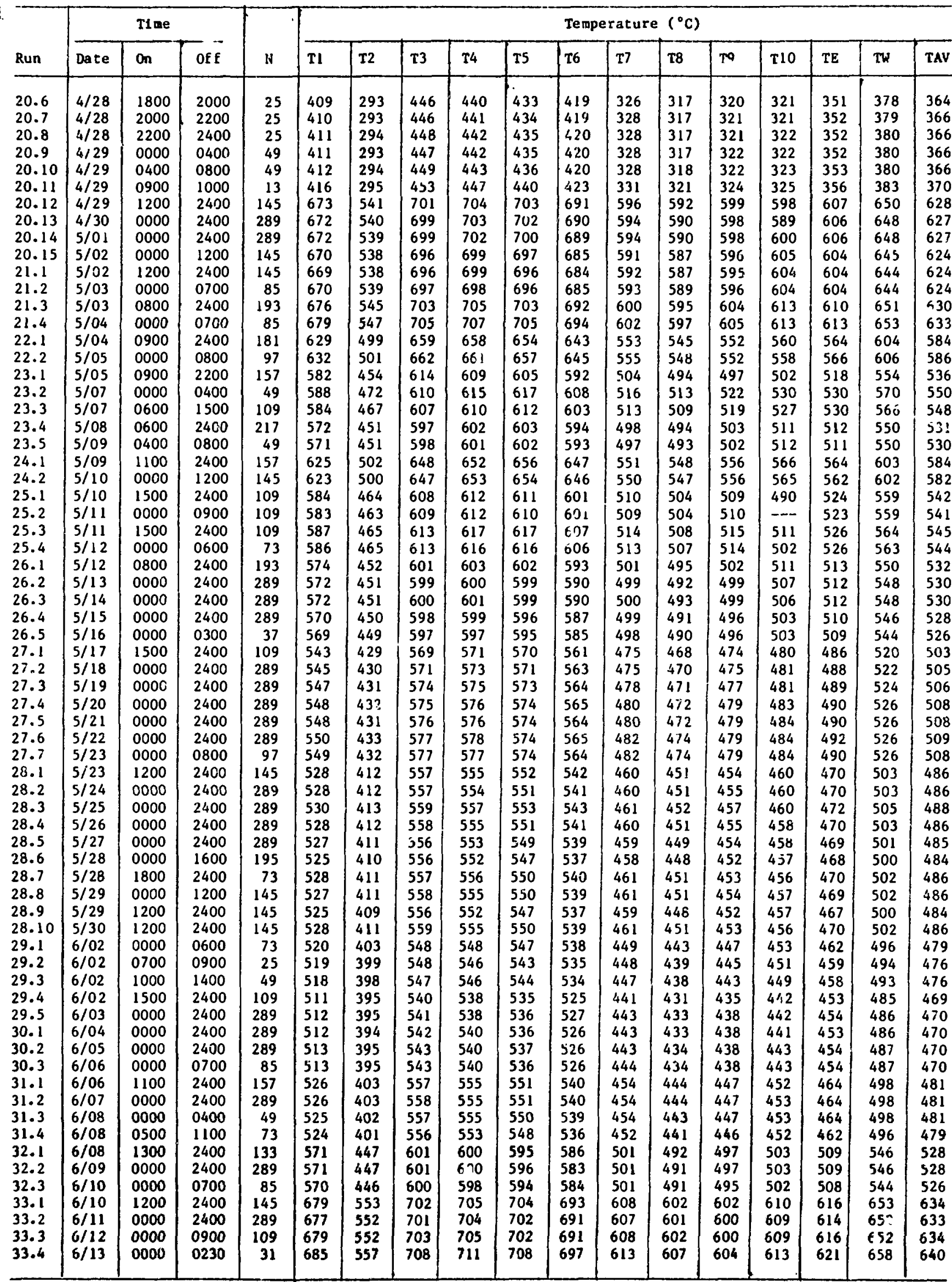


T10), the average temperature in the east and west sides (TE and TW, respectively), and the average overall temperature (TAV). Since thermocouples T3, T7, T9, and T10 were near the ends of the sample, their readings were not used for determining the average temperatures. The measured values have an uncertainty of less than $1 \%$ (see Sec. IV.A). Since the error is likely to be a bias, the averaging of many data points will increase the precision but not the accuracy. Examination of the data in Table VIII-5 revealed that the temperatures followed a very consistent pattern throughout the experiment. This demonstrates that the heat transfer pathways were not changed during the experiment and is a good indication that the structural integrity of the breeder and the capsule was maintained.

The data from Table VIII-5 were used to calculate temperature gradients, which are given in Table VIII-6. Also listed are the thermal neutron flux, the reactor power, and the tritium production rate. These quantities are needed to calculate the heating rates in the capsule.

The error in temperature gradient is calculated as follows. Let us assume the error in a single temperature number is $4^{\circ} \mathrm{C}$ on the west side and $2^{\circ} \mathrm{C}$ on the east side. Since four temperatures are used to calculate the gradient on the west side, the error in the temperature gradient is calculated to be $2^{\circ} \mathrm{C}$. The error for the temperature gradient on the east side (two temperatures) is $1.4^{\circ} \mathrm{C}$. The error for the average temperature gradient is less than $2^{\circ} \mathrm{C}$.

The data from Table VIII-6 allowed calculation of heating rates in the steel (QSS) and the breeder (QLI). The total heat, QTOT, divided by the average temperature gradient, is approximately proportional to $\mathrm{K}$, the thermal conductivity. The calculated values are given in Table VIII-7. The estimated error in the temperature gradient was less than $2^{\circ} \mathrm{C}$, or less than $2 \%$. The uncertainty in heating rates is $15 \%$. The uncertainty in the thermal conductivity values is estlmated to be $15 \%$. The values are nearly all. about 1.2 , except for those at temperatures less than $400^{\circ} \mathrm{C}$, which are higher. The results show that the effects of neutron irradiation have not caused large changes in thermal conductivity. 
TABLE VIII -6

Heat Transfer Data from TRIO: Temperature Gradients

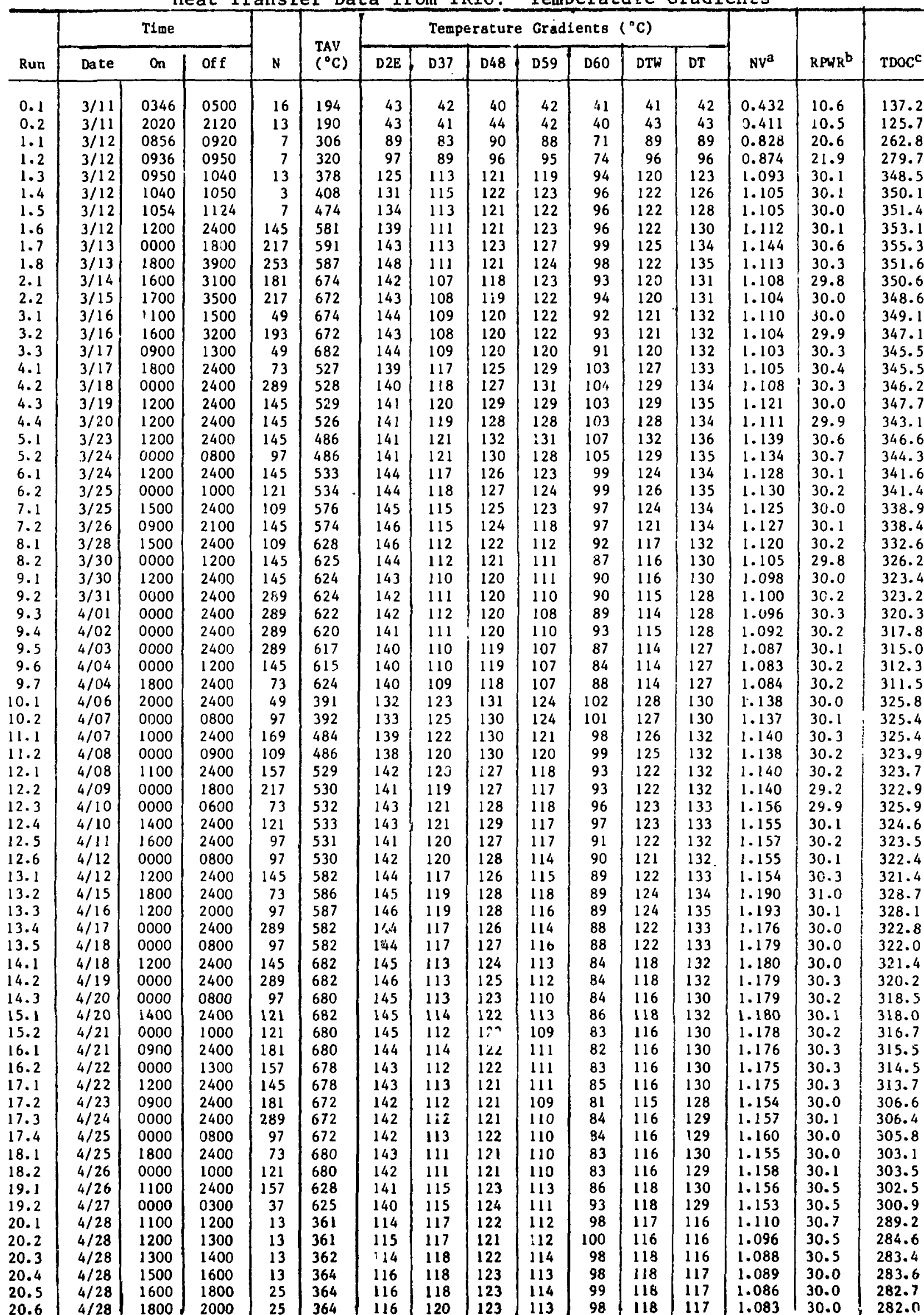




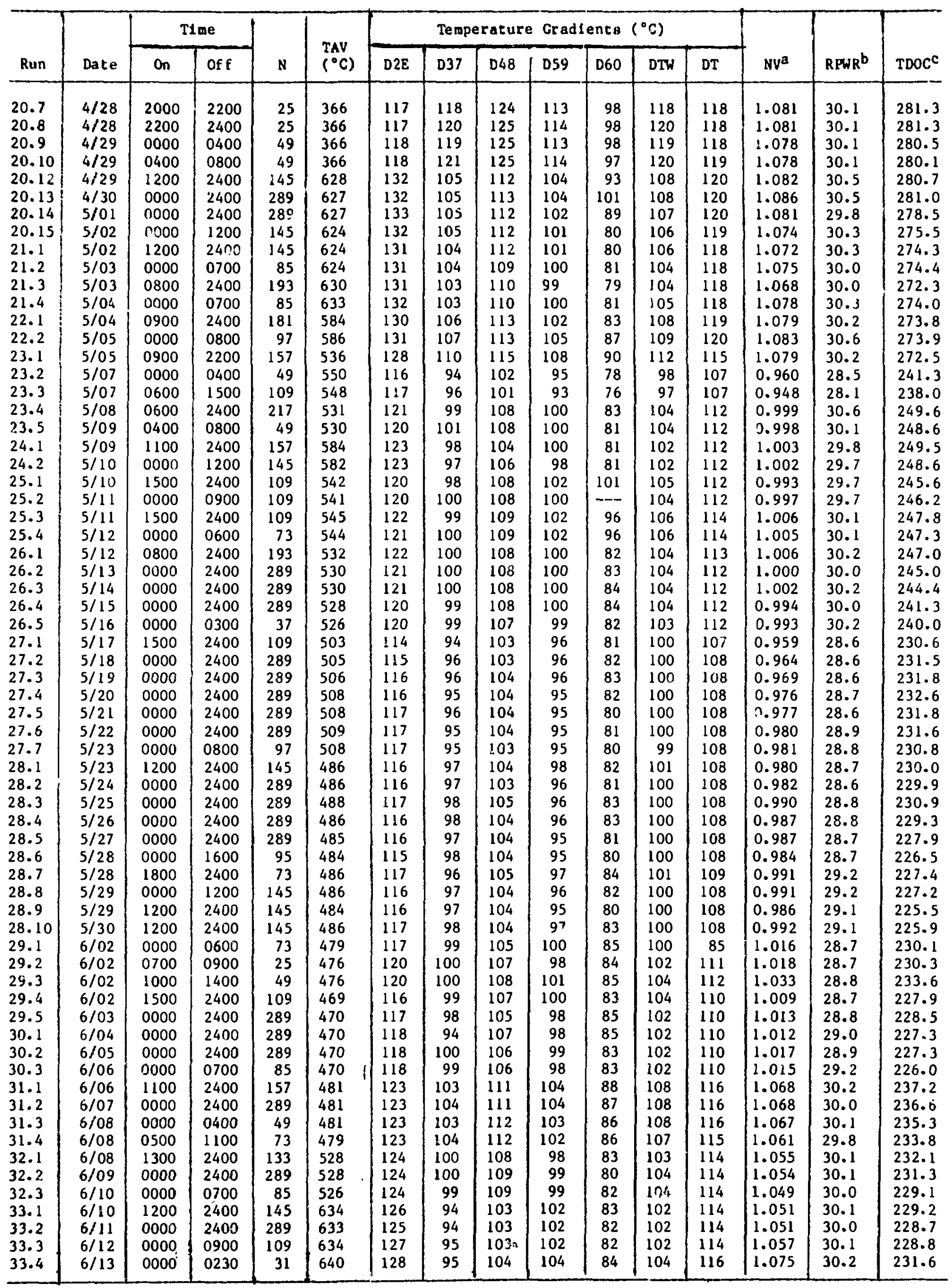

$a_{\text {NV }}$ is the thermal neutron flux, $10^{14} \mathrm{n} / \mathrm{cm}^{2} . \mathrm{s}$.

$b_{\text {RPAR iq }}$ the reactor power, Mw.

$c_{\text {TDOT }}$ is the tritium production rate, $\mu C 1 / m i n$. 
TABLE VIII-7

Heat Transfer Data from TRIO: Thermal Conductivity

\begin{tabular}{|c|c|c|c|c|c|c|c|c|c|c|c|}
\hline Run & Date & TAV & DT & NV & IHTG & GAMM & QSS & QLI & QTOT & $Q / D T$ & $\mathrm{~K}$, est \\
\hline 0.1 & $3 / 11$ & 194 & 42 & 0.432 & 1.076 & 1.986 & 3.09 & 5.12 & 8.21 & 0.195 & 2.43 \\
\hline 0.2 & $3 / 11$ & 190 & 43 & 0.411 & 0.986 & 1.890 & 2.94 & 4.80 & 7.74 & 0.180 & 1.32 \\
\hline 1.1 & $3 / 12$ & 306 & 89 & 0.828 & 2.062 & 3.808 & 5.93 & 9.81 & 15.74 & 0.177 & 1.29 \\
\hline 1.2 & $3 / 12$ & 320 & 96 & 0.874 & 2.194 & 4.019 & 6.26 & 10.38 & 16.64 & 0.173 & 1.27 \\
\hline 1.3 & $3 / 12$ & 378 & $12 ?$ & 1.093 & 2.734 & 5.026 & 7.79 & 12.96 & 20.76 & 0.169 & 1.24 \\
\hline 1.4 & $3 / 12$ & 408 & 126 & 1.105 & 2.747 & 5.081 & 7.91 & 13.07 & 20.99 & 0.167 & 1.22 \\
\hline 1.5 & $3 / 12$ & 474 & 128 & 1.105 & 2.757 & 5.081 & 7.90 & 13.09 & 20.99 & 0.164 & 1.20 \\
\hline 1.6 & $3 / 12$ & 581 & 130 & 1.112 & 2.770 & 5.114 & 7.96 & 13.17 & 21.13 & 0.157 & 1.15 \\
\hline 1.7 & $3 / 13$ & 591 & 134 & 1.144 & 2.787 & 5.261 & 8.19 & 13.44 & 21.63 & 0.161 & 1.18 \\
\hline 1.8 & $3 / 13$ & 587 & 135 & 1.113 & 2.759 & 5.118 & 7.97 & 13.16 & 21.13 & 0.157 & 1.15 \\
\hline 2.1 & $3 / 14$ & 674 & 131 & 1.108 & 2.751 & 5.095 & 7.93 & 13.10 & 21.03 & 0.161 & 1.18 \\
\hline 2.2 & $3 / 15$ & 672 & 131 & 1.104 & 2.735 & 5.076 & 7.90 & 13.05 & 20.95 & 0.160 & 1.17 \\
\hline 3.1 & $3 / 16$ & 674 & 132 & 1.110 & 2.739 & 5.104 & 7.94 & 13.10 & 21.04 & 0.159 & 1.17 \\
\hline 3.2 & $3 / 16$ & 672 & 132 & 1.104 & 2.723 & 5.076 & 7.90 & 13.03 & 20.93 & 0.159 & $1 .: 6$ \\
\hline 3.3 & $3 / 17$ & 682 & 132 & 1.103 & 2.711 & 5.072 & 7.90 & 13.00 & 20.90 & 0.158 & 1.16 \\
\hline 4.1 & $3 / 17$ & 527 & 133 & 1.105 & 2.711 & 5.081 & 7.91 & 13.02 & 20.93 & 0.157 & 1.15 \\
\hline 4.2 & $3 / 18$ & 528 & 134 & 1.108 & 2.716 & 5.095 & 7.93 & 13.05 & 20.98 & 0.157 & 1.15 \\
\hline 4.3 & $3 / 19$ & 529 & 135 & 1.121 & 2.728 & 5.155 & 8.02 & 13.17 & 21.19 & 0.157 & 1.15 \\
\hline 4.4 & $3 / 20$ & 526 & 134 & 1.111 & 2.692 & 5.109 & 7.95 & 13.03 & 20.98 & 0.157 & 1.15 \\
\hline 5.1 & $3 / 23$ & 486 & 136 & 1.139 & 2.719 & 5.238 & 8.15 & 13.29 & 21.44 & 0.158 & 1.15 \\
\hline 5.2 & $3 / 24$ & 486 & 135 & 1.134 & 2.701 & 5.215 & 8.12 & 13.22 & 21.34 & 0.158 & 1.16 \\
\hline 6.1 & $3 / 24$ & 533 & 134 & 1.128 & 2.680 & 5.187 & 8.07 & 13.14 & 21.21 & 0.158 & 1.16 \\
\hline 6.2 & $3 / 25$ & 534 & 135 & 1.130 & 2.678 & 5.196 & 8.09 & 13.15 & 21.24 & 0.157 & 1.15 \\
\hline 7.1 & $3 / 25$ & 576 & 134 & 1.125 & 2.659 & 5.173 & 8.05 & 13.08 & 21.13 & 0.158 & 1.15 \\
\hline 7.2 & $3 / 26$ & 574 & 134 & 1.127 & 2.655 & 5.183 & 8.07 & 13.09 & 21.16 & 0.158 & 1.16 \\
\hline 8.1 & $3 / 28$ & 628 & 132 & 1.120 & 2.609 & 5.150 & 8.02 & 12.96 & 20.98 & 0.159 & 1.16 \\
\hline 8.2 & $3 / 30$ & 625 & 130 & 1.105 & 2.559 & 5.081 & 7.91 & 12.76 & 20.67 & 0.159 & 1.16 \\
\hline 9.1 & $3 / 30$ & 624 & 130 & 1.098 & 2.537 & 5.049 & 7.86 & 12.67 & 20.53 & 0.158 & 1.16 \\
\hline 9.2 & $3 / 31$ & 624 & 128 & 1.100 & 2.535 & 5.058 & 7.87 & 12.68 & 20.55 & 0.161 & 1.18 \\
\hline 9.3 & $4 / 01$ & 622 & 128 & 1.096 & 2.513 & 5.040 & 7.84 & 12.62 & 20.46 & 0.160 & 1.17 \\
\hline 9.4 & $4 / 02$ & 620 & 128 & 1.092 & 2.493 & 5.022 & 7.82 & 12.55 & 20.37 & 0.159 & 1.16 \\
\hline 9.5 & $4 / 03$ & 617 & 127 & 1.0 & 2.4 & 4.999 & 7.78 & 12.48 & 20.26 & 0.160 & 1.17 \\
\hline 9.6 & $4 / 04$ & 615 & 127 & 1.083 & 2.450 & 4.980 & 7.75 & 12.41 & 20.16 & 0.159 & 1.16 \\
\hline 9.7 & $4 / 04$ & 624 & 127 & 1.084 & 2.444 & 4.985 & 7.76 & 12.41 & 20.17 & 0.159 & 1.16 \\
\hline 10.1 & $4 / 06$ & 391 & 130 & 1.138 & 2.556 & 5.233 & 8.15 & 13.01 & 21.16 & 0.163 & 1.19 \\
\hline 10.2 & $4 / 07$ & 392 & 130 & 1.137 & 2.553 & 5.228 & 8.14 & 13.00 & 21.14 & 0.163 & 1.19 \\
\hline 11.1 & $4 / 07$ & 484 & 132 & 1.140 & 2.553 & 5.242 & 8.16 & 13.02 & 21.18 & 0.160 & 1.17 \\
\hline 11.2 & $4 / 08$ & 486 & 132 & 1.138 & 2.541 & 5.233 & 8.15 & 12.99 & 21.14 & 0.160 & 1.17 \\
\hline 12.1 & $4 / 08$ & 529 & 132 & 1.140 & 2.540 & 5.242 & 8.16 & 13.00 & $21 \cdot 16$ & 0.160 & 1.17 \\
\hline 12.2 & $4 / 09$ & 530 & 132 & 1.140 & 2.533 & 5.242 & 8.16 & 12.99 & 21.15 & 0.160 & 1.17 \\
\hline 12.3 & $4 / 10$ & 532 & 133 & 1.156 & 2.557 & 5.316 & 8.27 & 13.15 & 21.42 & 0.161 & 1.18 \\
\hline 12.4 & $4 / 10$ & 533 & 133 & 1.155 & 2.547 & 5.311 & 8.27 & 13.13 & 21.40 & 0.161 & 1.18 \\
\hline 12.5 & $4 / 11$ & 531 & 132 & 1.157 & 2.538 & 5.320 & 8.28 & 13.13 & 21.41 & 0.162 & 1.19 \\
\hline 12.6 & $4 / 12$ & 530 & 132 & 1.155 & 2.529 & 5.311 & 8.27 & 13.10 & 21.37 & 0.162 & 1.19 \\
\hline 13.1 & $4 / 12$ & 582 & 133 & 1.154 & 2.522 & 5.307 & 8.26 & 13.08 & 21.34 & 0.160 & 1.17 \\
\hline 13.2 & $4 / 15$ & 586 & 134 & 1.190 & 2.579 & 5.472 & 8.52 & 13.45 & 21.97 & 0.164 & 1.20 \\
\hline 13.3 & $4 / 16$ & 587 & 135 & 1.193 & 2.574 & 5.486 & 8.54 & 13.47 & 22.01 & 0.163 & 1.19 \\
\hline 13.4 & $4 / 17$ & 582 & 133 & 1.176 & 2.533 & 5.408 & 8.42 & 13.27 & 21.69 & 0.163 & 1.19 \\
\hline 13.5 & $4 / 18$ & 582 & 133 & 1.179 & 2.526 & 5.422 & 8.44 & 13.28 & 21.72 & 0.163 & 1.19 \\
\hline 14.1 & $4 / 18$ & 682 & 132 & 1.180 & 2.522 & 5.426 & 8.45 & 13.28 & 21.73 & 0.165 & 1.21 \\
\hline 14.2 & $4 / 19$ & 682 & 132 & 1.179 & 2.512 & 5.422 & 8.44 & 13.25 & 21.69 & 0.164 & 1.20 \\
\hline 14.3 & $4 / 20$ & 680 & 130 & 1.179 & 2.499 & 5.422 & 8.44 & 13.23 & 21.67 & 0.167 & 1.22 \\
\hline 15.1 & $4 / 20$ & 682 & 132 & 1.180 & 2.495 & 5.426 & 8.45 & 13.23 & 21.68 & 0.164 & 1.20 \\
\hline 15.2 & $4 / 21$ & 680 & 130 & 1.178 & 2.485 & 5.417 & 8.43 & 13.20 & 21.63 & 0.166 & 1.22 \\
\hline 16.1 & $4 / 21$ & 680 & 130 & 1.176 & 2.475 & 5.408 & 8.42 & 13.17 & 21.59 & 0.166 & 1.22 \\
\hline 16.2 & $4 / 22$ & 678 & 130 & 1.175 & 2.467 & 5.403 & 8.41 & 13.15 & 21.56 & 0.166 & 1.21 \\
\hline 17.1 & $4 / 22$ & 678 & 130 & 1.175 & 2.461 & 5.403 & 8.41 & 13.14 & 21.55 & 0.166 & 1.21 \\
\hline & $4 / 23$ & 672 & 128 & 1.154 & 2.405 & 5.307 & 8.26 & 12.88 & 21.14 & 0.165 & 1.21 \\
\hline 17.3 & $4 / 24$ & 672 & 129 & 1.157 & 2.404 & 5.320 & 8.28 & 12.90 & $21 \cdot 18$ & 0.164 & 1.20 \\
\hline 17.4 & $4 / 25$ & 672 & 129 & 1.160 & 2.399 & 5.334 & 8.30 & 12.92 & 21.22 & 0.164 & 1.20 \\
\hline 18.1 & $4 / 25$ & 680 & 130 & 1.155 & 2.378 & 5.311 & 8.27 & 12.85 & 21.12 & 0.162 & 1.19 \\
\hline 18.2 & $4 / 26$ & 680 & 129 & 1.158 & 2.381 & 5.325 & 8.29 & 12.87 & 21.16 & 0.164 & 1.20 \\
\hline 19.1 & $4 / 26$ & 628 & 130 & 1.156 & 2.373 & 5.316 & 8.27 & 12.85 & 21.12 & 0.162 & 1.19 \\
\hline 19.2 & $4 / 27$ & 625 & 129 & 1.153 & 2.361 & 5.302 & 8.25 & 12.80 & 21.05 & 0.163 & 1.19 \\
\hline 20.1 & $4 / 28$ & 361 & 116 & 1.110 & 2.269 & 5.104 & 7.94 & 12.32 & 20.26 & 0.175 & 1.28 \\
\hline 20.2 & $4 / 28$ & 361 & 116 & 1.096 & 2.233 & 5.040 & 7.85 & 12.15 & 20.00 & 0.172 & 1.26 \\
\hline 20.3 & $4 / 28$ & 362 & 116 & 1.088 & 2.223 & 5.003 & 7.79 & 12.07 & 19.86 & 0.171 & 1.25 \\
\hline
\end{tabular}


TABLE VIII-7 (Contd,)

\begin{tabular}{|c|c|c|c|c|c|c|c|c|c|c|c|}
\hline Run & Date & TAY & $\mathrm{DT}$ & NV & THTG & GAMM & QSS & QLI & QTOT & $Q / D T$ & $k$, est. \\
\hline 20.4 & $4 / 28$ & 364 & 117 & 1.089 & 2.225 & 5.008 & 7.80 & 12.08 & 19.88 & 0.170 & 1.24 \\
\hline 20.5 & $4 / 28$ & 364 & 117 & 1.086 & 2.218 & 4.994 & 7.77 & 12.05 & 19.82 & 0.169 & 11.24 \\
\hline 20.6 & $4 / 28$ & 364 & 117 & 1.083 & 2.212 & 4.980 & 7.75 & 12.01 & 19.76 & 0.169 & 1.24 \\
\hline 20.7 & $4 / 28$ & 366 & 118 & 1.081 & 2.207 & 4.971 & 7.74 & 11.99 & 19.73 & 0.167 & 1.22 \\
\hline 20.8 & $4 / 28$ & 366 & 118 & 1.081 & 2.207 & 4.971 & 7.74 & 11.99 & 19.73 & 0.167 & 1.22 \\
\hline 20.9 & $4 / 29$ & 366 & 118 & 1.078 & 2.201 & 4.957 & 7.72 & 11.96 & 19.66 & 0.167 & 1.22 \\
\hline 20.10 & $4 / 29$ & 366 & 119 & 1.078 & 2.198 & 4.957 & 7.72 & 11.95 & 19.65 & 0.165 & 1.21 \\
\hline 20.11 & $4 / 29$ & 370 & 121 & 1.088 & 2.217 & 5.003 & 7.79 & 12.06 & 19.85 & 0.164 & 1.20 \\
\hline 20.12 & $4 / 29$ & 628 & 120 & 1.082 & 2.202 & 4.976 & 7.75 & 11.99 & 19.74 & 0.164 & 1.20 \\
\hline 20.13 & $4 / 30$ & 627 & 120 & 1.086 & 2.205 & 4.994 & 7.77 & 12.03 & 19.80 & 0.165 & 1.21 \\
\hline 20.14 & $5 / 01$ & 627 & 120 & 1.081 & 2.185 & 4.971 & 7.74 & 11.95 & 19.69 & 0.164 & 1.20 \\
\hline 20.15 & $5 / 02$ & 624 & 119 & 1.074 & 2.161 & 4.939 & 7.69 & 11.86 & 19.55 & 0.164 & 1.20 \\
\hline 21.1 & $5 / 02$ & 624 & 118 & 1.072 & 2.152 & 4.930 & 7.67 & 11.83 & 19.50 & 0.165 & 1.21 \\
\hline 21.2 & $5 / 03$ & 624 & 118 & 1.075 & 2.153 & 4.943 & 7.69 & 11.85 & 19.54 & 0.166 & 1.21 \\
\hline 21.3 & $5 / 03$ & 630 & 118 & 1.068 & 2.136 & 4.911 & 7.64 & 11.77 & 19.42 & 0.165 & 1.20 \\
\hline 21.4 & $5 / 04$ & 633 & 118 & 1.078 & 2.150 & 4.957 & 7.72 & 11.87 & 19.59 & 0.166 & 1.22 \\
\hline 22.1 & $5 / 04$ & 584 & 119 & 1.079 & 2.148 & 4.962 & 7.72 & 11.88 & 19.60 & 0.165 & 1.21 \\
\hline 22.2 & $5 / 05$ & 586 & 120 & 1.083 & 2.149 & 4.980 & 7.75 & 11.91 & 19.55 & 0.164 & 1.20 \\
\hline 23.1 & $5 / 05$ & 536 & 115 & 1.079 & 2.138 & 4.962 & 7.72 & 11.86 & 19.58 & 0.170 & $1.25 ?$ \\
\hline 23.2 & $5 / 07$ & 550 & 107 & 0.960 & 1.893 & 4.415 & 6.87 & 10.54 & 17.41 & 0.163 & 1.19 \\
\hline 23.3 & $5 / 07$ & 548 & 107 & 0.948 & 1.867 & 4.359 & 6.78 & 10.40 & 17.18 & 0.161 & 1.18 \\
\hline 23.4 & $5 / 08$ & 531 & 112 & 0.999 & 1.958 & 4.594 & 7.15 & 10.95 & 18.10 & 0.162 & 1.18 \\
\hline 23.5 & $5 / 09$ & 530 & 112 & 0.998 & 1.950 & 4.589 & 7.14 & 10.92 & 18.06 & 0.161 & 1.18 \\
\hline 24.1 & $5 / 09$ & 584 & 112 & 1.003 & 1.957 & 4.612 & 7.18 & 10.97 & 18.15 & 0.162 & 1.19 \\
\hline 24.2 & $5 / 10$ & 582 & 112 & 1.002 & 1.950 & 4.608 & 7.18 & 10.95 & 18.13 & 0.162 & 1.18 \\
\hline 25.1 & $5 / 10$ & 542 & 112 & 0.993 & 1.927 & 4.566 & 7.11 & 10.85 & 17.96 & 0.160 & 1.17 \\
\hline 25.2 & $5 / 11$ & 541 & 112 & 0.997 & 1.932 & 4.585 & 7.14 & 10.89 & 18.03 & 0.161 & 1.18 \\
\hline 25.3 & $5 / 11$ & 545 & 114 & 1.006 & 1.944 & 4.626 & 7.20 & 10.98 & 18.18 & 0.159 & 1.17 \\
\hline 25.4 & $5 / 12$ & 544 & 114 & 1.005 & 1.940 & 4.621 & 7.19 & 10.97 & 18.16 & 0.159 & 1.17 \\
\hline 26.1 & $5 / 12$ & 532 & 113 & 1.006 & 1.938 & 4.626 & 7.20 & 10.98 & 18.18 & 0.161 & 1.18 \\
\hline 26.2 & $5 / 13$ & 530 & 112 & 1.000 & 1.922 & 4.598 & 7.16 & 10.89 & 18.05 & 0.161 & 1.18 \\
\hline 26.3 & $5 / 14$ & 530 & 112 & 1.002 & 1.917 & 4.608 & 7.18 & 10.90 & 18.08 & 0.161 & 1.18 \\
\hline 26.4 & $5 / 15$ & 528 & 112 & 0.994 & 1.893 & 4.571 & 7.12 & 10.80 & 17.92 & 0.160 & 1.17 \\
\hline 26.5 & $5 i s$ & 526 & 112 & 0.993 & 1.883 & 4.566 & 7.11 & 10.77 & 17.88 & 0.160 & 1.17 \\
\hline 27.1 & $5 / 21$ & 503 & 107 & 0.959 & 1.809 & 4.410 & 6.86 & 10.39 & 17.25 & 0.161 & 1.18 \\
\hline 27.2 & $5 / 18$ & 505 & 108 & 0.964 & 1.816 & 4.433 & 6.90 & 10.44 & 17.34 & 0.161 & 1.18 \\
\hline 27.3 & $5 / 1=$ & 506 & 108 & 0.969 & 1.819 & 4.456 & 6.94 & 10.48 & 17.42 & 0.161 & 1.18 \\
\hline 27.4 & $5 / 20$ & 508 & 108 & 0.976 & 1.825 & 4.488 & 6.99 & 10.55 & 17.54 & 0.162 & 1.19 \\
\hline 27.5 & $5 / 21$ & 508 & 108 & 0.977 & 1.819 & 4.493 & 6.99 & 10.55 & 17.54 & 0.162 & 1.19 \\
\hline 27.6 & $5 / 22$ & 509 & 108 & 0.980 & 1.817 & 4.507 & 7.02 & 10.56 & 17.58 & 0.163 & 1.19 \\
\hline 27.7 & $5 / 23$ & 508 & 108 & 0.981 & 1.811 & 4.511 & 7.02 & 10.56 & 17.58 & 0.163 & 1.19 \\
\hline 28.1 & $5 / 23$ & 486 & 108 & 0.980 & 1.804 & 4.507 & 7.02 & 10.55 & 17.57 & 0.163 & 1.19 \\
\hline 28.2 & $5 / 24$ & 486 & 108 & 0.982 & 1.804 & 4.516 & 7.03 & 10.56 & 17.59 & 0.163 & 1.19 \\
\hline 28.3 & $5 / 25$ & 488 & 108 & 0.990 & 1.812 & 4.553 & 7.09 & 10.63 & 17.72 & 0.164 & 1.20 \\
\hline 28.4 & $5 / 26$ & 485 & 108 & 0.987 & 1.799 & 4.539 & 7.07 & 10.59 & 17.66 & 0.164 & 1.20 \\
\hline 28.5 & $5 / 27$ & 485 & $10 n$ & 0.987 & 1.788 & 4.539 & 7.07 & 10.58 & 17.65 & 0.163 & 1.20 \\
\hline 28.6 & $5 / 28$ & 484 & 108 & 0.984 & 1.777 & 4.525 & 7.05 & 10.55 & 17.60 & 0.163 & 1.19 \\
\hline 28.7 & $5 / 28$ & 486 & 109 & 0.991 & 1.784 & 4.557 & 7.09 & 10.60 & 17.69 & 0.162 & 1.19 \\
\hline 28.8 & $5 / 29$ & 486 & 108 & 0.991 & 1.783 & 4.557 & 7.09 & 10.60 & 17.69 & 0.164 & 1.20 \\
\hline 28.9 & $5 / 29$ & 484 & 108 & 0.986 & 1.769 & 4.534 & 7.06 & 10.53 & 17.59 & 0.163 & 1.19 \\
\hline 28.10 & $5 / 30$ & 486 & 108 & 0.992 & 1.772 & 4.562 & 7.10 & 10.58 & 17.68 & 0.164 & 1.20 \\
\hline 29.1 & $6 / 02$ & 479 & $85 ?$ & 1.016 & 1.805 & 4.672 & 7.27 & 10.82 & 18.09 & 0.212 & $1.56 ?$ \\
\hline 29.2 & $6 / 02$ & 476 & 111 & 1.018 & 1.807 & 4.681 & 7.29 & 10.84 & 18.13 & 0.163 & 1.20 \\
\hline 29.3 & $6 / 02$ & 476 & 112 & 1.033 & 1.833 & 4.750 & 7.39 & 11.00 & 18.39 & 0.164 & 1.20 \\
\hline 29.4 & $6 / 02$ & 469 & 110 & 1.009 & 1.788 & 4.640 & 7.22 & 10.74 & 17.96 & 0.163 & 1.20 \\
\hline 29.5 & $6 / 03$ & 470 & 110 & 1.013 & 1.793 & 4.658 & 7.25 & 10.78 & 18.03 & 0.164 & 1.20 \\
\hline 30.1 & $6 / 04$ & 470 & 110 & 1.012 & 1.783 & 4.654 & 7.24 & 10.75 & 17.99 & 0.164 & 1.20 \\
\hline 30.2 & $6 / 05$ & 470 & 110 & 1.017 & 1.783 & 4.677 & 7.28 & 10.79 & 18.07 & 0.164 & 1.20 \\
\hline 30.3 & $6 / 06$ & 470 & 110 & 1.015 & 1.773 & 4.667 & 7.26 & 10.75 & 18.01 & 0.164 & 1.20 \\
\hline 31.1 & $6 / 06$ & 481 & 116 & 1.068 & 1.861 & 4.911 & 7.64 & 11.31 & 18.95 & 0.163 & 1.20 \\
\hline 31.2 & $6 / 07$ & 481 & 116 & 1.068 & 1.856 & 4.911 & 7.64 & 11.30 & 18.94 & 0.163 & 1.20 \\
\hline 31.3 & $6 / 08$ & 481 & 116 & 1.067 & 1.846 & 4.907 & 7.64 & 11.28 & 18.92 & 0.163 & 1.19 \\
\hline & $6 / 08$ & 479 & 115 & 1.061 & 1.834 & 4.879 & 7.59 & 11.21 & 18.80 & 0.163 & 1.20 \\
\hline 32.1 & $6 / 08$ & 528 & 114 & 1.055 & 1.821 & 4.851 & 7.55 & 11.15 & 18.70 & 0.164 & 1.20 \\
\hline 32.2 & $6 / 09$ & 528 & 114 & 1.054 & 1.815 & 4.847 & 7.54 & 11.13 & 18.67 & 0.164 & 1.20 \\
\hline 32.3 & $6 / 10$ & 526 & 114 & 1.049 & 1.798 & 4.824 & 7.51 & 11.06 & 18.57 & 0.163 & 1.19 \\
\hline 33.1 & $6 / 10$ & 634 & 114 & 1.051 & 1.798 & 4.833 & 7.52 & 11.07 & 18.59 & 0.163 & 1.19 \\
\hline 33.2 & $6 / 11$ & 633 & 114 & 1.051 & 1.794 & 4.833 & 7.52 & 11.07 & 18.59 & 0.163 & 1.19 \\
\hline 27 & $6 / 12$ & 634 & 114 & 1.057 & 1.795 & 4.861 & 7.57 & 11.12 & 18.69 & 0.164 & 1.20 \\
\hline & $6 / 13$ & 640 & 116 & 1.075 & 1.817 & 4.943 & 7.69 & 11.29 & 18.98 & 0.164 & 1.20 \\
\hline
\end{tabular}

VIII-15 


\section{Integral Tritiun Release and Tritlun Inventory}

The amounts of tritium accounted for and ${ }^{6} \mathrm{Li}$ and 1 ithium quantities for lithium burnup are given in Table VIII-8. The different methods for determining the various integral quantities are in excellent agreement; all the measurements agree within the estimated error. There is thus confidence that all the tritium is accounted for: $35 \mathrm{Ci}$ was produced and $35 \mathrm{Ci}$ collected. The uncertainty in the total amount produced, the average of the four determinations, is estimated as $3 \%$, or about $1 \mathrm{Ci}$. In addition, as previously discussed (Sec. VII.B), the stainless steel tubing leading from the reactor to the sweep gas analysis system was leached with recirculating water for $18 \mathrm{~h}$, and an insignificant quantity of tritium was recovered.

TABLE VIII -8

Integral Tritium Data

\begin{tabular}{l|c|c|c|c}
\hline Method Used & $\begin{array}{c}\text { Tritium } \\
(C i)\end{array}$ & $\begin{array}{c}\text { 6Li } \\
\text { Burnup } \\
(\%)\end{array}$ & $\begin{array}{c}\text { Lithium } \\
\text { Burnup } \\
(\%)\end{array}$ & $\begin{array}{c}\text { Estimated } \\
\text { Error } \\
(\%)\end{array}$ \\
\hline Tritium collected & 35 & 33 & 0.18 & 5 \\
6Li assay & 32 & 31 & 0.17 & 10 \\
Thermal gradient & 35 & 33 & 0.18 & 10 \\
Dosimetry & $\underline{39}$ & $\underline{38}$ & $\underline{0.21}$ & $\underline{10}$ \\
Average & 35 & 33 & 0.18 & 3.7 \\
\hline
\end{tabular}

The amounts of tritium generated during each run were then calculated, normalized to a total production of $35.1 \mathrm{Ci}$. The tritium residue for each run was calculated, and the tritium inventory at the end of each run from the difference between the total amount generated (CTGEN) and the total amount recovered (CTCOL), as given in Table VIII-9. The tritium retention is known to be zero before irradiation and was measured as $0.019 \pm 0.002 \mathrm{CI}$ at the end of the irradiation. The uncertainty is thus small at the beginning and at the end of the experiment. The uncertainty for the first 15 runs is estimated as $3 \%$ of the amount produced at the end of each run. For the last 18 runs, the uncertainty is estimated as $3 \%$ of the difference between the total amount of 
TABLE VIII-9

Total Tritium

\begin{tabular}{|c|c|c|c|c|c|c|c|}
\hline \multirow[b]{2}{*}{ Run } & \multicolumn{7}{|c|}{ Amount of Tritium (Ci) } \\
\hline & TCOLL & CTCOL & TGEN & CTGEN & DEL T & TINV & \pm \\
\hline 0 & 0.002 & 0.002 & 0.022 & 0.022 & 0.020 & 0.020 & 0.001 \\
\hline 1 & 1.015 & 1.017 & 1.094 & 1.116 & 0.079 & 0.099 & 0.03 \\
\hline 2 & 0.999 & 2.016 & 0.966 & 2.082 & -0.033 & 0.066 & 0.06 \\
\hline 3 & 0.527 & 2.543 & 0.552 & 2.634 & 0.025 & 0.091 & 0.08 \\
\hline 4 & 2.109 & 4.652 & 2.391 & 5.025 & 0.282 & 0.373 & 0.15 \\
\hline 5 & 0.619 & 5.273 & 1.050 & 6.076 & 0.431 & 0.804 & 0.18 \\
\hline 6 & 0.727 & 5.998 & 0.406 & 6.482 & -0.321 & 0.484 & 0.19 \\
\hline 7 & 1.816 & 7.814 & 1.486 & 7.968 & -0.330 & 0.154 & 0.24 \\
\hline 8 & 1.011 & 8.825 & 0.882 & 8.850 & -0.129 & 0.025 & 0.27 \\
\hline 9 & 1.832 & 10.657 & 2.603 & 11.453 & 0.771 & 0.796 & 0.34 \\
\hline 10 & 0.042 & 10.699 & 0.421 & 11.875 & 0.379 & 1.175 & 0.36 \\
\hline 11 & 0.307 & 11.006 & 0.462 & 12.337 & 0.155 & 1.331 & 0.37 \\
\hline 12 & 1.265 & 12.271 & 1.835 & 14.172 & 0.570 & 1.901 & 0.42 \\
\hline 13 & 2.305 & 14.576 & 2.061 & \pm 6.232 & -0.244 & 1.657 & 0.50 \\
\hline 14 & 1.644 & 16.220 & 0.994 & 17.227 & -0.650 & 1.007 & 0.52 \\
\hline 15 & 0.746 & 16.966 & 0.373 & 17.600 & -0.373 & 0.634 & 0.53 \\
\hline 16 & 0.527 & 17.493 & 0.502 & 18.102 & -0.025 & 0.609 & 0.51 \\
\hline 17 & 1.311 & 18.804 & 1.331 & 19.433 & 0.020 & 0.629 & 0.47 \\
\hline 18 & 0.412 & 19.216 & 0.380 & 19.813 & -0.032 & 0.597 & 0.46 \\
\hline 19 & 0.315 & 19.531 & 0.306 & 20.119 & -0.009 & 0.588 & 0.45 \\
\hline 20 & 0.035 & 19.566 & 1.585 & 21.705 & 1.550 & 2.139 & 0.40 \\
\hline 21 & 1.095 & 20.661 & 0.783 & 22.488 & -0.312 & 1.827 & 0.38 \\
\hline 22 & 0.352 & 21.013 & 0.385 & 22.873 & 0.033 & 1.860 & 0.37 \\
\hline 23 & 1.082 & 22.095 & 1.260 & 24.134 & 0.178 & 2.038 & 0.33 \\
\hline 24 & 0.579 & 22.674 & 0.397 & 24.530 & -0.182 & 1.856 & 0.32 \\
\hline 25 & 0.546 & 23.220 & 0.618 & $25.1<8$ & 0.072 & 1.928 & 0.30 \\
\hline 26 & 1.265 & 24.485 & 1.414 & 26.562 & 0.149 & 2.077 & 0.26 \\
\hline 27 & 2.471 & 26.956 & 2.169 & 28.731 & -0.302 & 1.775 & 0.19 \\
\hline 28 & 2.972 & 29.928 & 2.509 & 31.240 & -0.463 & 1.312 & 0.12 \\
\hline 29 & 0.755 & 30.683 & 0.732 & 31.972 & -0.023 & 1.289 & 0.09 \\
\hline 30 & 0.727 & 31.410 & 0.899 & 32.871 & 0.172 & 1.461 & 0.067 \\
\hline 31 & 0.744 & 32.154 & 0.723 & 33.594 & -0.021 & 1.440 & 0.045 \\
\hline 32 & 1.277 & 33.431 & 0.605 & 34.199 & -0.672 & 0.768 & 0.027 \\
\hline 33 & 1.679 & 35.110 & 0.912 & 35.111 & -0.767 & 0.019 & $0.002^{a}$ \\
\hline Total & 35.110 & & 35.111 & & & & \\
\hline
\end{tabular}

${ }^{a_{\text {Measured. }}}$ 
tritium produced ( $35.1 \mathrm{Cl}$ ) and the cumulative total produced at the end of each run.

The experimental conditions, the amounts of the different forms of tritium collected, and the calculated tritium inventories for the 33 runs are summarized in Table VIII-10. Those runs which did not reach equilibrium are indicated by "<" or "〉" as appropriate. There were no experimental tritium permeation data for the first three runs, and the estimated tritium inventory values (in pararenthesis) could be in error by the order of $0.01 \mathrm{Ci}$. This possible error is small in comparison to the estimated uncertainty, however.

\section{Analgsis of Integrai Tritiue Inventory Using Steady-State Diffusion}

A calculational method was devised to explore the degree to which intragranular diffusion is the rate limiting step in determining the tritium retention and release characteristics. For the ideal case of a spherical grain under isothermal conditions with a constant tritium generation rate and the assumption that intragranular diffusion is the dominant mechanism, the tritium inventory (tritium retention) in the grain is given by the relationship:

$$
I_{g}=\left(r_{g}^{2} / 15 D\right) \dot{g}
$$

where $I_{g}=$ the =ritium inventory, $g ; r_{g}=$ the grain radius, $c m ; D=$ the diffusivity, $\mathrm{cm}^{2} / \mathrm{s} ;$ and $\dot{\mathrm{g}}=$ the generation rate, $\mathrm{g} / \mathrm{s}$. The diffusivity has a temperature dependance as:

$$
D=D_{0} \exp (-Q / R T)
$$

where $D_{0}$ is a pre-exponential factor, $Q$ is the activation energy, $R$ is the clas constant, and $T$ is the absolute temperature. In the ideal case (for isothermal conditions), if the logarithm of the tritium retention is plotted versus $1 / \mathrm{T}$, a Iinear relationship is expected.

However, the TRIO experiment had a significant temperature variation; therefore, Eq. (1) has to be integrated over the volumetric temperature distribution in the sample. In this case, if a uniform tritium generation rate $(\dot{G})$ is assumed, the expression for tritium diffusive inventory, $I_{d}$, is:

$$
I_{d}=\left(r_{g}^{2} / 15\right) f \dot{G} /[D(\bar{T})] \text {, }
$$


TABLE VIII-10

Calculated Tritium Inventory for each TRIO Run.

\begin{tabular}{|c|c|c|c|c|c|c|c|c|}
\hline $\begin{array}{l}\text { Run } \\
\text { No. }\end{array}$ & $\begin{array}{l}\text { Conditions } \\
\left({ }^{\circ} \mathrm{C}\right)\end{array}$ & $\mathrm{HT}$ & HTO & PERM & TCOLL & TGEN & TINV & \pm \\
\hline 0 & Tests to $300^{\circ} \mathrm{C}$ & 0.00186 & 0.00006 & $5 \times 10^{-7}$ & 0.002 & 0.022 & 0.020 & 0.001 \\
\hline 1 & $600 /$ STD & 0.985 & 0.026 & $(0.004)$ & 1.015 & 1.094 & 0.099 & 0.03 \\
\hline 2 & 700/STD & 0.985 & 0.009 & $(0.005)$ & 0.999 & 0.966 & 0.066 & 0.06 \\
\hline 3 & $700 / 1 \% \mathrm{H}_{2}$ & 0.513 & 0.011 & $(0.003)$ & 0.527 & 0.552 & 0.091 & 0.08 \\
\hline 4 & $550 / \mathrm{STD}$ & 2.099 & 0.006 & 0.0038 & 2.109 & 2.391 & $>0.373$ & 0.15 \\
\hline 5 & $500 /$ STD & 0.617 & 0.004 & 0.0012 & 0.619 & 1.050 & $>0.804$ & 0.18 \\
\hline 6 & 550/STD & 0.725 & 0.001 & 0.0008 & 0.727 & 0.406 & 0.484 & 0.19 \\
\hline 7 & $600 /$ STD & 1.811 & 0.002 & 0.0031 & 1.816 & 1.486 & 0.154 & 0.24 \\
\hline 8 & $650 / \mathrm{STL}$ & 1.008 & 0.001 & 0.0023 & 1.011 & 0.882 & 0.025 & 0.27 \\
\hline 9 & $650 / \mathrm{No} \mathrm{H}_{2}$ & 1.823 & 0.002 & $0.0068^{a}$ & 1.832 & 2.603 & $>0.796$ & 0.34 \\
\hline 10 & $400 /$ No $\mathrm{H}_{2}$ & 0.040 & 0.000 & 0.0018 & 0.042 & 0.421 & $>1.175$ & 0.36 \\
\hline 11 & $500 /$ No $\mathrm{H}_{2}$ & 0.300 & 0.003 & 0.0040 & 0.307 & 0.462 & $>1.331$ & 0.37 \\
\hline 12 & $550 /$ No $\mathrm{H}_{2}$ & 1.245 & 0.000 & 0.0197 & 1.265 & 1.835 & $>1.901$ & 0.42 \\
\hline 13 & $600 /$ No $\mathrm{H}_{2}^{2}$ & 2.197 & 0.000 & $0.108^{\mathrm{a}}$ & 2.305 & 2.061 & 1.657 & 0.50 \\
\hline 14 & $700 / \mathrm{N} \mathrm{H}_{2}$ & 1.560 & 0.001 & 0.083 & 1.644 & 0.994 & 1.007 & 0.52 \\
\hline 15 & $700 / \mathrm{STD}^{2}$ & 0.715 & 0.000 & 0.031 & 0.746 & 0.373 & 0.634 & 0.53 \\
\hline 16 & $700 / 300 \mathrm{SCCM}$ & 0.503 & 0.001 & 0.023 & 0.527 & 0.502 & 0.609 & 0.51 \\
\hline 17 & $700 / 30 \mathrm{scCM}$ & 1.109 & 0.000 & 0.202 & 1.311 & 1.331 & 0.629 & 0.47 \\
\hline 18 & $700 / \mathrm{STD}$ & 0.380 & 0.000 & 0.032 & 0.412 & 0.380 & 0.597 & 0.46 \\
\hline 19 & 650/STD & 0.294 & 0.000 & $0.021^{a}$ & 0.315 & 0.306 & 0.588 & 0.45 \\
\hline 20 & $650 / 0.2 \% \mathrm{O}_{2}$ & 0.028 & 0.000 & 0.0068 & 0.035 & 1.585 & $>2.139$ & 0.40 \\
\hline 21 & $650 /$ STD & 1.092 & 0.900 & 0.0034 & 1.095 & 0.783 & 1.827 & 0.38 \\
\hline 22 & 600/STD & 0.351 & 0.000 & 0.0013 & 0.352 & 0.385 & 1.860 & 0.37 \\
\hline 23 & $550 / \mathrm{STD}$ & 1.077 & 0.001 & $0.0035^{\mathrm{a}}$ & 1.082 & 1.260 & 2.038 & 0.33 \\
\hline 24 & $600 /$ STD & 0.576 & 0.002 & 0.0014 & 0.579 & 0.397 & 1.856 & 0.32 \\
\hline 25 & $560 /$ STD & 0.537 & 0.007 & 0.0018 & 0.546 & 0.618 & 1.928 & 0.30 \\
\hline 26 & $550 /$ STD & 1.213 & 0.048 & $0.0041^{\mathrm{a}}$ & 1.265 & 1.414 & 2.077 & 0.26 \\
\hline 27 & $525 / \mathrm{STD}$ & 2.128 & 0.333 & 0.0103 & 2.471 & 2.169 & 1.775 & 0.19 \\
\hline 28 & $500 / \mathrm{STD}$ & 2.104 & 0.857 & 0.0107 & 2.972 & 2.509 & 1.312 & 0.12 \\
\hline 29 & $480 / 300 \mathrm{SCCM}$ & 0.543 & 0.211 & 0.0007 & 0.755 & 0.732 & $>1.289$ & 0.09 \\
\hline 30 & $480 / \mathrm{STD}$ & 0.721 & 0.004 & 0.0016 & 0.727 & 0.899 & 1.461 & 0.067 \\
\hline 31 & $500 / \mathrm{STD}$ & 0.740 & 0.003 & 0.0014 & 0.744 & 0.723 & 1.440 & 0.045 \\
\hline 32 & $550 /$ STD & 1.271 & 0.004 & 0.0015 & 1.277 & 0.605 & $<0.768$ & 0.027 \\
\hline \multirow[t]{2}{*}{33} & $650 / \mathrm{STD}$ & 1.666 & 0.009 & 0.0038 & 1.679 & $\underline{0.912}$ & 0.019 & 0.002 \\
\hline & Tota1 & 32.958 & 1.543 & 0.608 & 35.110 & 35.111 & & \\
\hline
\end{tabular}

a"STD" = $100 \mathrm{sCCM}, 0.1 \% \mathrm{H}_{2}$.

$\mathrm{b}_{\text {Permeation sample taken. }}$ 
where $\bar{T}$ is the volume-averaged temperature, and $f$ is a tactor to account for the temperature distribution. The factor $f$ is the ratio between the tritium inventory for the actual distribution and the idealized isothermal inventory at a temperature of $\bar{T}$. For the TRIo experiment, the generation rate is reasonably uniform, having a range of less than $10 \%$ throughout the sample. In addition, although the generation rate does vary considerably throughout the course of the experiment (see Sec. VI), it is reasonably constant during a single run. The grain size did not measurably change over the course of the experiment, as noted in Sec. VII.

For purposes of calculational simplicity, a radial temperature profile is assumed. This temperature profile is characterized by two integrated parameters: $\overline{\mathrm{T}}$ and $\Delta \overline{\mathrm{T}}$ (average radial temperature drop across the pellet thickness). For the capsule geometry (hollow cylinder of outer radius $r_{0}$ and inner radius $r_{i}$ ) and an unknown activation energy $Q$, the $f$ factor is given by

$$
f=f(\bar{T}, \Delta \bar{T}, Q)=(\beta-1)^{-1} \int_{1}^{\beta} \exp [(Q / R \bar{T})(\bar{T} / T-1)] d \eta,
$$

where $\beta=r_{0}^{2} / r_{i}^{2}=2.56 ; \eta=r^{2} / r_{i}^{2} ;$ and $T=10 c a l$ temperature.

Equation (3) can be inverted to solve for the diffusivity as a function of measured and calculated data:

$$
D(\bar{T})=\left(r_{g}^{2} / 15\right) f\left(\dot{G} / I_{d}\right)
$$

The generation rate $(\dot{G})$, the Inventory values $\left(I_{d}\right)$, the volume-averaged temperatures $(\overline{\mathrm{T}})$, and temperature gradients $(\Delta \overline{\mathrm{T}})$ are obtained from data previously presented. The grain radius $r_{g}$ is taken to be $0.1 \mu \mathrm{m}$. If intragranular diffusion is the dominant mechanism, then the calculated diffusivity values should vary linearly with $1 / T$ on a semllog plot. Because $f$ and $D$ depend on the unknown activation energy, $Q$, iteration is required.

The TRIO runs at conditions optimal for tritium release (i.e., hydrogen added to the sweep gas) were postulated to be diffusion controlled. Since the uncertainties in the calculated tritium inventories were smallest at the beginning and at the end of the experiment, the first eight runs and the last six runs were used for the analysis. The data are summarized in Table VIII-11. 
TABLE VIII-11

Calculated Iffusivities from TRIO Data

\begin{tabular}{l|c|c|l|c|c}
\hline $\begin{array}{c}\text { Run } \\
\text { No. }\end{array}$ & $\begin{array}{c}\text { Volume- } \\
\text { Averaged } \\
\text { Temp. } \\
\left({ }^{\circ} \mathrm{C}\right)\end{array}$ & $\begin{array}{c}\text { Temp. } \\
\text { Gradient } \\
\left({ }^{\circ} \mathrm{C}\right)\end{array}$ & $\begin{array}{c}\mathrm{I}_{\mathrm{d}} / \dot{\mathrm{G}} \\
\left(10^{4} \mathrm{~s}\right)\end{array}$ & $\mathrm{f}$ & $\begin{array}{c}\text { Diffusivity } \\
\left(10^{-16} \mathrm{~cm}^{2} / \mathrm{s}\right)\end{array}$ \\
\hline 1 & 592 & 126 & 1.70 & 2.62 & 10.3 \\
2 & 677 & 122 & 1.13 & 2.38 & $14.1^{\mathrm{a}}$ \\
3 & 685 & 122 & 1.56 & 2.36 & $10.1^{\mathrm{a}}$ \\
4 & 627 & 125 & $>6.49$ & 2.99 & $<3.08$ \\
5 & 481 & 125 & $>14.0$ & 3.30 & $<1.58$ \\
6 & 530 & 20 & 8.54 & 2.97 & 2.32 \\
7 & 578 & 130 & 2.73 & 2.73 & 6.65 \\
8 & 627 & 130 & 0.462 & 2.50 & 36.1 \\
28 & 489 & 107 & 34.5 & 2.74 & 0.532 \\
29 & 474 & 107 & $>34.5$ & 2.84 & $<0.551$ \\
30 & 474 & 107 & 37.3 & 2.84 & 0.507 \\
31 & 484 & 112 & 37.4 & 2.88 & 0.513 \\
32 & 531 & 111 & $<20.0$ & 2.64 & 0.883 \\
33 & 642 & 113 & 0.498 & 2.33 & 31.2 \\
\hline
\end{tabular}

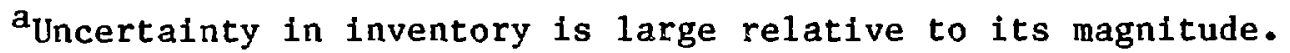

The data were then plotted in Figure VIII-1. The solid circles in the figure represent runs that achieved equilibrium and the open circies represent those that did not. The calculated diffusivity (diffusion coe ificient) shows excellent agreement with the equation

$$
D=1.1 \times 10^{-6} \exp (-35.8 / \mathrm{RT})
$$

where $R=$ the gas constant in $\mathrm{Kcal} / \mathrm{mol}$. The activation energy is 35.8 $\mathrm{Kcal} / \mathrm{mol}$ (150 kJ/mol). The excellent agreement with the 1 inear relationship provides strong evidence that the tritium release for these selected runs is diffusion-controlled. 


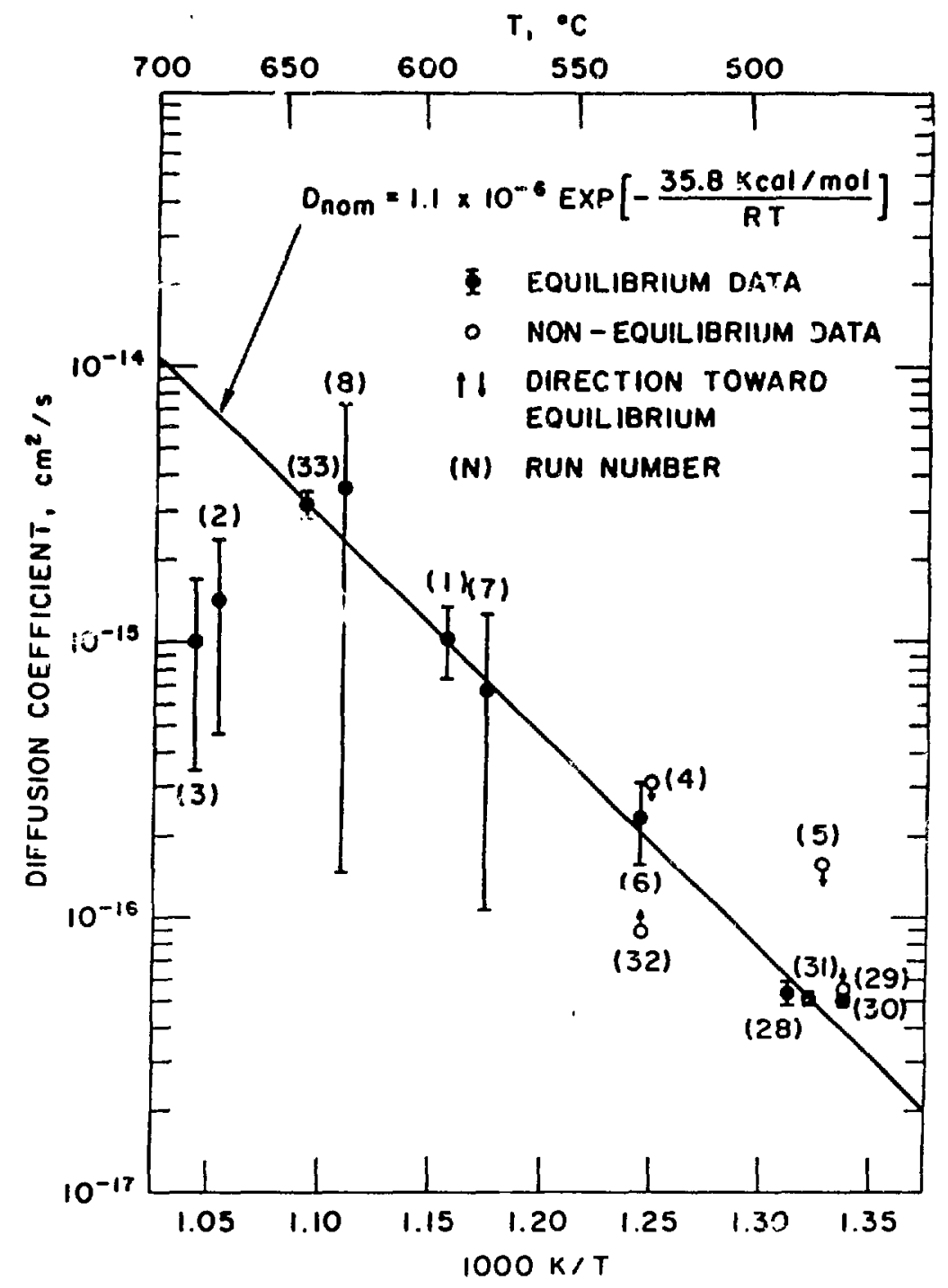

Fig. VIII-1. Diffusion coefficient for tritium in $\mathrm{LiAlO}_{2}$ determined from selected TRIO runs in which intragranular diffusion appeared to be the rate-limiting phenomenon in tritium transport. The one-sigma bands are based on uncertalnties in the experimental generation rate ( $\dot{G}$ ) and inventory (I).

Based on an error generation analysis which includes the uncertainties in generation rate and derived inventory, the diffusion coefficient for LiAJ. $0_{2}$ deduced from the TRIO data can be written as:

$$
\ln \left(\mathrm{D} / \mathrm{cm}^{2} \cdot \mathrm{s}^{-1}\right)=(-13.7 \pm 2.3)-(35.8 \pm 3.9) \mathrm{Kca} 1 / \mathrm{mol} / \mathrm{RT}
$$

If the variability in grain size ( $\pm 30 \%$ ) is also included in the uncertainty analysis, ther the following equation should be used when comparing to single crystal data:

$$
\ln \left(\mathrm{D} / \mathrm{cm}^{2} \cdot \mathrm{g}^{-1}\right)=(-13.7 \pm 1.8)-(35.8 \pm 3.9) \mathrm{Kca} 1 / \mathrm{mol} / \mathrm{RT} \text {. }
$$


No attempts were made to include uncertainties in temperature distribution in the error analysis. While the accuracy of the thermocouples was quite good, the asymmetry of the temperature profile introduces uncertainty into the calculation which could not be easily estimated because of the limited number of data points.

The above relationship was then used to calculate inventories for Runs 9-26. These calculated inventories, given in Table VIII-12, are called the "diffusive inventories". For these runs in general, the diffusion model does not account for all the tritiun retained.

\section{B. Analysis of Dynanic Tritiun Release Data}

The dynamic tritium release curves (see Sec. VI.F) provide additional information on the transport behavior of tritium. Steady-state diffusion analysis was utilized above to investigate runs that appear to be diffusion controlled. An independent way to study these runs is to use transient diffusion kinetics. Described herein is analysis of the dynamic tritium release data using a transient diffusion method.

The dominant mechanism for tritium release is postulated to be intragranular diffusion for selected runs. The grains are assumed to be uniform spheres, $0.1 \mu \mathrm{m}$ in radius. The generation rate is assumed to be uniform and constant for a given run. The steady-state release rate is equal to the generation rate. The time to reach any iraction of the steady-state release rate is sensitive to the diffusivity, and the diffusivity is in turn sensitive to temperature.

First, let us consider an idealized case for a step change in temperature at a constant generation rate. For an isothermal grain with an initial temperature $T_{1}$ and a final temperature $T_{2}$, the time-dependent solutions for the release rate, $\mathrm{k}_{\mathrm{g}}$, and the inventory, $I_{\mathrm{g}}$, are:

$$
\begin{aligned}
\dot{R}_{g} / \dot{g} & =1+\left(D_{2} / D_{1}-1\right)\left(6 / \pi^{2}\right) \sum_{n=1}^{\infty}\left(1 / n^{2}\right) \exp \left(-n^{2} \pi^{2} t / \tau_{2}\right) \\
I_{g} /\left(I_{g}\right)_{S S} & =1+\left(D_{2} / D_{1}-1\right)\left(90 / \pi^{4}\right) \sum_{n=1}^{\infty}\left(1 / n^{4}\right) \exp \left(-n^{2} \pi^{2} t / \tau_{2}\right),
\end{aligned}
$$


TÁRIE VIII-12

Diffusive Jnventories for Runs 9-27

\begin{tabular}{l|c|c|c|c}
\hline Run & $\begin{array}{c}\text { Volume } \\
\text { Averaged } \\
\text { Temp. } \\
\text { No. }\end{array}$ & $\begin{array}{c}\text { Temp. } \\
\text { Gradient } \\
\left({ }^{\circ} \mathrm{C}\right)\end{array}$ & $\mathbf{f})$ & $\begin{array}{c}\text { Diffusive } \\
\text { Tritium } \\
\text { Inventory } \\
\text { (Ci) }\end{array}$ \\
\hline $9^{\mathrm{a}}$ & 630 & 128 & 2.51 & 0.02 \\
$10^{\mathrm{a}}$ & 396 & 127 & 4.10 & $<67.8$ \\
$11^{\mathrm{a}}$ & 488 & 128 & 3.18 & $>2.02$ \\
$12^{\mathrm{a}}$ & 605 & 128 & 2.60 & 0.07 \\
13 & 582 & 128 & 2.08 & 0.12 \\
14 & 684 & 126 & 2.35 & 0.01 \\
15 & 684 & 126 & 2.35 & 0.01 \\
16 & 682 & 126 & 2.36 & 0.01 \\
17 & 677 & 125 & 2.36 & 0.01 \\
18 & 683 & 125 & 2.34 & 0.01 \\
19 & 628 & 126 & 2.50 & 0.04 \\
$20^{\mathrm{a}}$ & 639 & 116 & 2.36 & 0.03 \\
21 & 639 & 116 & 2.36 & 0.03 \\
22 & 588 & 117 & 2.52 & 0.09 \\
23 & 533 & 109 & 2.60 & 0.34 \\
24 & 585 & 109 & 2.43 & 0.08 \\
25 & 546 & 113 & 2.61 & 0.23 \\
26 & 530 & 109 & 2.62 & 0.76 \\
27 & 513 & 106 & 2.64 & 0.56 \\
\hline
\end{tabular}

${ }^{a}$ These runs did not achieve steady state.

where

$$
\begin{aligned}
\left(\mathrm{I}_{\mathrm{g}}\right)_{\mathrm{SS}} & =\left(\tau_{2} / 15\right) \dot{\mathrm{G}} \\
\mathrm{t} & =\text { time from change in temperature } \\
\tau_{2} & =\mathrm{r}_{\mathrm{g}}^{2} / \mathrm{D}_{2} \\
\mathrm{D}_{1} & =\mathrm{D}\left(\mathrm{T}_{1}\right) \\
\mathrm{D}_{2} & =\mathrm{D}\left(\mathrm{T}_{2}\right) .
\end{aligned}
$$

Figure VIII-2 is a plot of Eq. (9) for the case of $D_{2} / D_{1}=19.4$, which corresponds to a step change of $100^{\circ} \mathrm{C}$ from 500 to $600^{\circ} \mathrm{C}$. An observation can be made with regard to Eq. (9). Since the size of the spike in the release rate is given by $\left(\dot{\mathrm{R}}_{\mathrm{g}}\right)_{2} /\left(\dot{\mathrm{R}}_{\mathrm{g}}\right)_{1}=\mathrm{D}_{2} / \mathrm{D}_{1}$, an increase in temperature results in a positive spike and a decrease results in a negative spike with reference to 


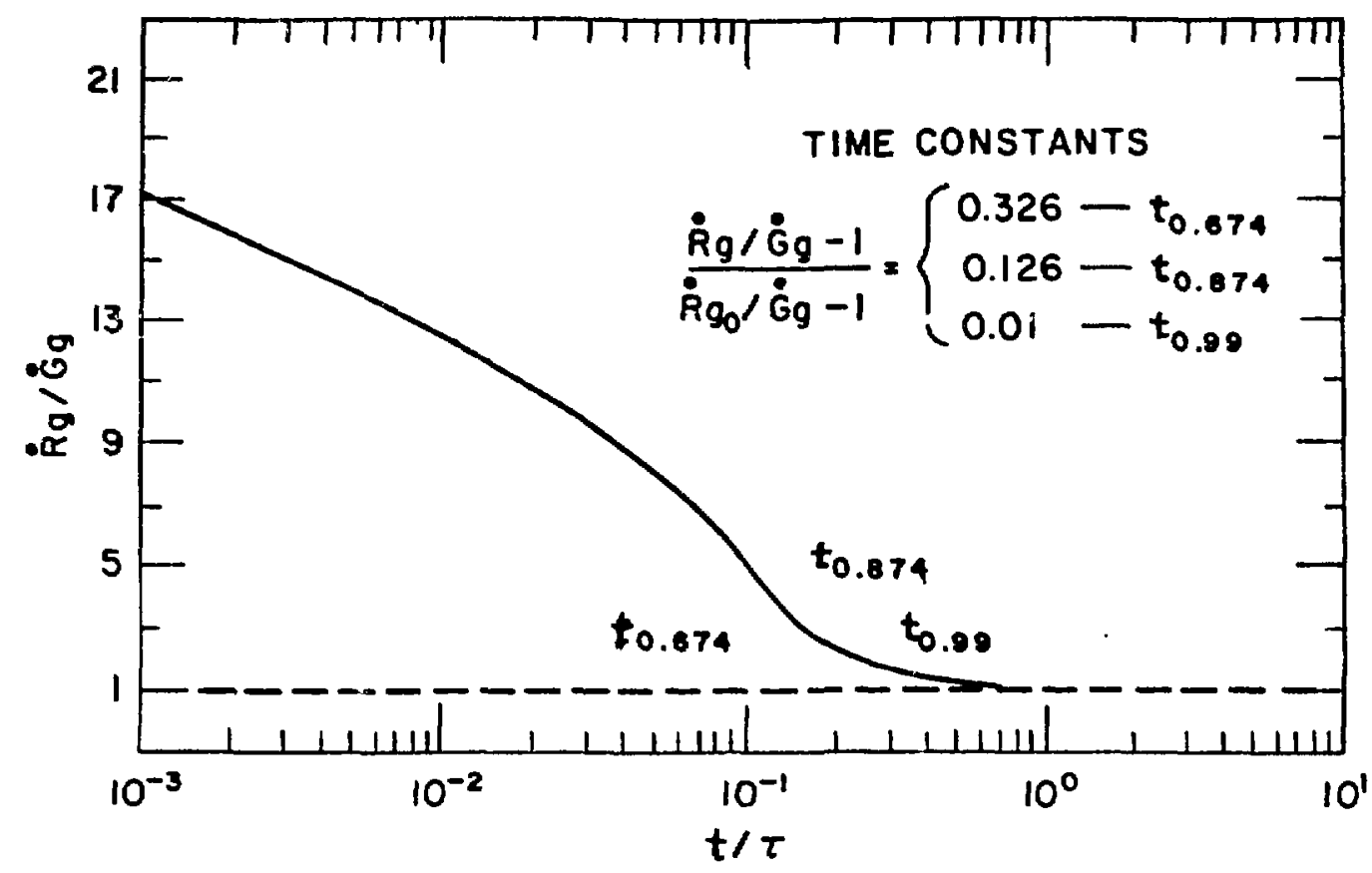

Fig. VIII-2. Fractional release rate for step increase in temperature $\left(\tau_{1} / \tau_{2}=19.4\right)$.

the generation rate line. As previously discussed (Sec. VI.F), this pattern was consistently observed throughout the course of the experiment for nearly a11 33 runs.

The effect of spatial temperature distribution is more complicated than it was for the steady-state case. Let $T_{1}=T_{1}(\eta)$ be the temperature before the temperature jump and $T_{2}=T_{2}(n)$ be the temperature distribution after the jump. Integration of Eqs. (9) and (10) yields

$$
\begin{aligned}
\dot{R} / \dot{G}= & 1+\left(6 / \pi^{2}\right) \sum_{n=1}^{\infty}\left(1 / n^{2}\right)(\beta-1)^{-1} \int_{1}^{\beta}\left(D_{2} / D_{1}-1\right) \\
& \times \exp \left(-n^{2} \pi^{2} t / \tau_{2}\right) d n, \\
I / I_{S S}= & 1+\left(90 / \pi^{4}\right) \sum_{n=1}^{\infty}\left(1 / n^{4}\right) \\
& \times \frac{\int_{1}^{\beta}\left(\tau_{1}-\tau_{2}\right) \exp \left(n^{2} \pi^{2} t / \tau_{2}\right)}{\int^{\beta} \tau_{2} d n} .
\end{aligned}
$$


Figures VIII-3 through -5 show the experimentally determined tritium release rate $\left(\dot{R}_{e}\right)$ versus the analytically determined [(Eq. (11)] release rate $\left(\dot{\mathrm{R}}_{\mathrm{min}}, \dot{\mathrm{R}}_{\text {nom }}, \dot{\mathrm{R}}_{\mathrm{max}}\right)$ for Runs 8,28 , and 31 . In all three cases, the size of the calculated spike is greater than the experimentally determined value. This finding is to be expected because the model assumes an instantaneous temperature change and the monitoring instrumentation lag time is not included in the analysis. The general shapes of the analytical and experimental curves have some similarity, but the quantitative results are somewhat different. Owing to the sensitivity of response to temperature, the differences may be due to the modeling of the temperature profiles.

Equation ( 9 ) can be manipulated to show that the time response of the system depends only on $\mathrm{T}_{2}$ for step changes in isothermal temperatures * Let

$$
F_{\mathrm{e}} \equiv 1-\left(\dot{\mathrm{R}}_{\mathrm{g}} / \dot{\mathrm{g}}-\mathrm{l}\right)\left(\dot{\mathrm{R}}_{\mathrm{g}_{0}} / \dot{\mathrm{g}}-1\right)^{-1} \text {, }
$$

where $F_{e}$ is related to the approach to equilibrium and $\dot{R}_{g_{0}} / \dot{g}=D_{2} / D_{1}$. Substituting $\mathrm{Eq} \cdot(9)$ into $\mathrm{Eq} \cdot(13)$ gives

$$
F_{e}=1-\left(6 / \pi^{2}\right) \sum_{n=1}^{\infty}\left(1 / n^{2}\right) \exp \left(-n^{2} t / \tau_{2}\right)
$$

The variable $\mathrm{F}_{\mathrm{e}}$ is a measure of the extent of approach to equilibrium, being equal to zero at the beginning of the transient and reaching unity at steady state; $F_{e}$ is a function only of $\tau_{2}$, which is equal to $\mathrm{r}_{\mathrm{g}}^{2} / \mathrm{D}_{2}$. Equation (13) can then be used to compare calculated and experimental values of $F_{e}$ for the non-isothermal TRIO runs. This procedure was employed to calculate the time required to reach $67 \%$ of the way to equilibrium. The calculations are compared to the experimental data in Table VIII-13. Once again, the results are encouraging but not conclusive.

While the transient analysis strongly suggests that bulk diffusion is the dominant release mechanism during temperature changes, a more accurate analysis, which models the actual temperature history, is required before this is demonstrated quantitatively. Such efforts, however, would be hampered by the uncertainties in the generation and release rates. 


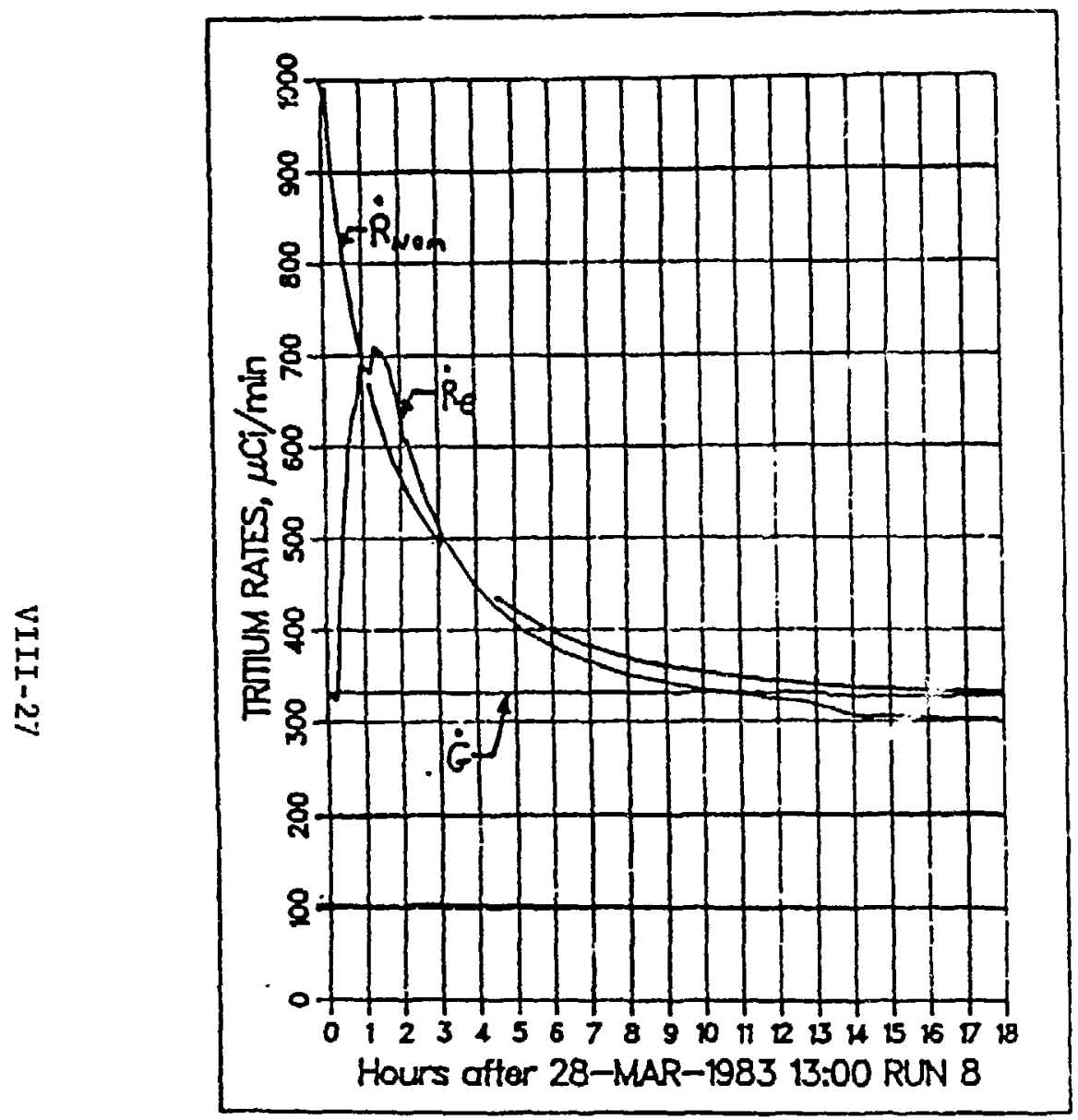

Fig. VIII-3. Comparison between analytical predictions ( $\dot{R}_{\text {nom }}$ ) for Run 8 and TRIO tritium rate $\left(R_{e}\right)$ for a mean temperature increase from $578^{\circ} \mathrm{C}$ to $627^{\circ} \mathrm{C}$. The generation rate $(\dot{G})$ is also shown.

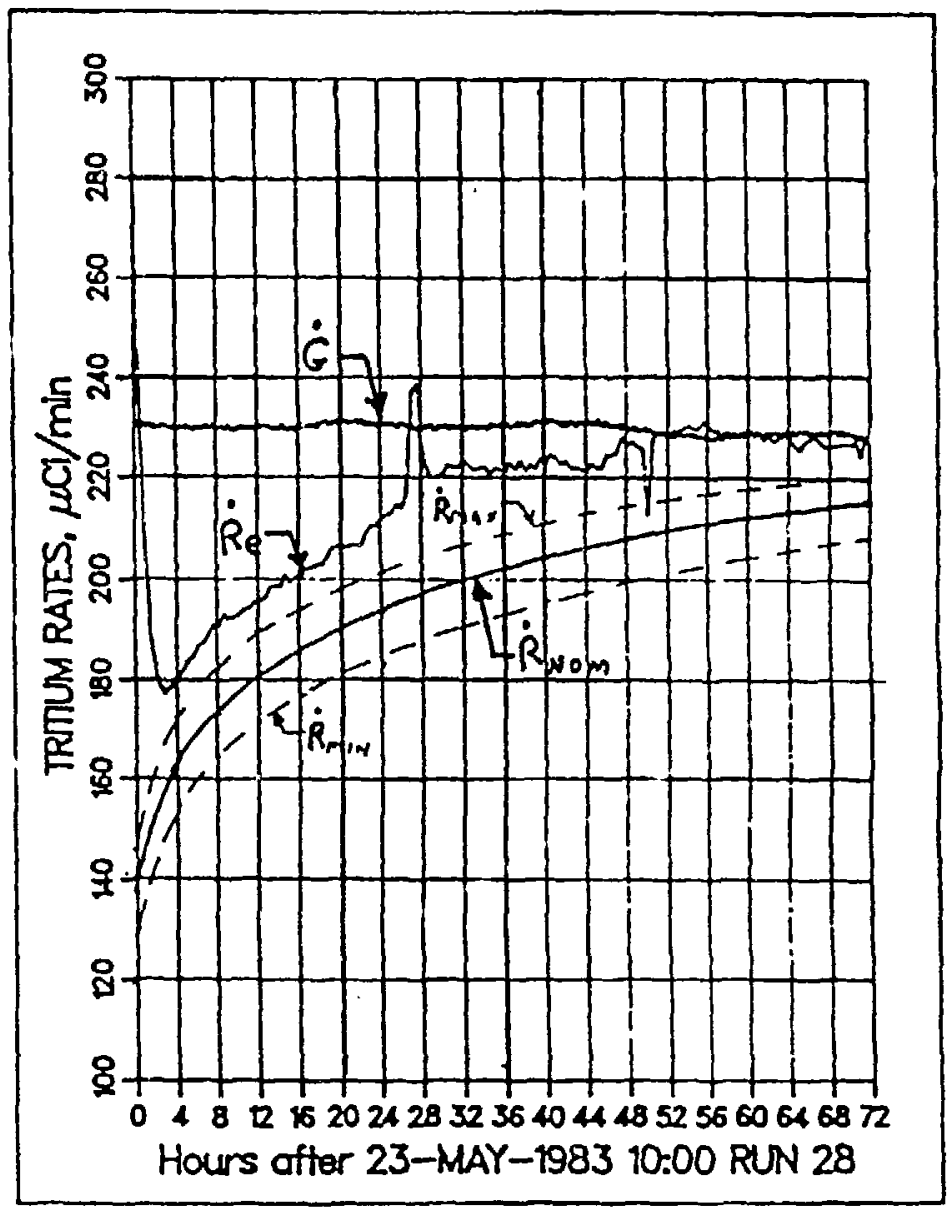

Fig. VIII-4. Comparișon bețween analytical predictions ( $R_{\min }, R_{\text {nom }}, \vec{R}_{\max }$ ) for Run 28 and TRIO tritium release rate $\left(\dot{\mathrm{R}}_{\mathrm{e}}\right)$ for a mean temperature decrease from $513^{\circ} \mathrm{C}$ to $489^{\circ} \mathrm{C}$. The generation rate $(\dot{G})$ is also shown. 


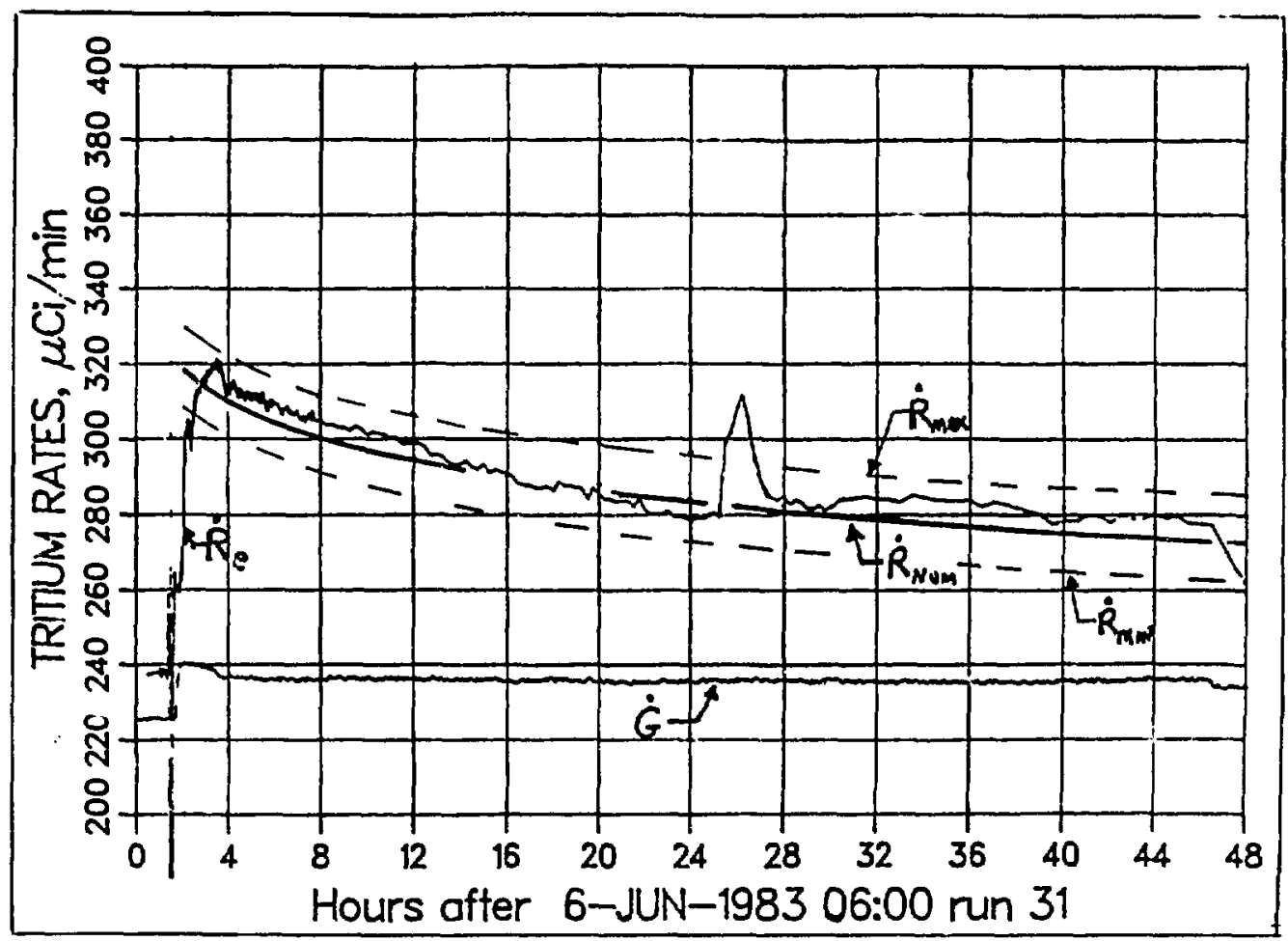

F.g. VIII-5. Comparison between analytical prediction ( $\left.\dot{\mathrm{R}}_{\text {nom }}\right)$ for Run 31 and TRIO tritium release rate ( $\dot{R}_{e}$ ) for a mean temperature increase from $474^{\circ} \mathrm{C}$ to $484^{\circ} \mathrm{C}$. The generation rate $(\dot{G})$ is also shown.

TABLE VIII-13

Comparison Between Predictions and TRIo Results (in Parentheses) for Time to Reach $67 \%$ of SteadyState Release Rate After a Temperature Change

\begin{tabular}{l|c|c|c|c|c}
\hline $\begin{array}{l}\text { Run } \\
\text { No. }\end{array}$ & $\begin{array}{l}\mathrm{T}_{\mathrm{i}} \\
\left({ }^{\circ} \mathrm{C}\right)\end{array}$ & $\begin{array}{c}\mathrm{T}_{i+1} \\
\left({ }^{\circ} \mathrm{C}\right)\end{array}$ & $\begin{array}{l}\Delta \mathrm{T}_{1} \\
\left({ }^{\circ} \mathrm{C}\right)\end{array}$ & $\begin{array}{l}\Delta \mathrm{T}_{i+1} \\
\left({ }^{\circ} \mathrm{C}\right)\end{array}$ & $\begin{array}{c}\mathrm{t}_{0.67}{ }^{\mathrm{a}} \\
(\mathrm{h})\end{array}$ \\
\hline 8 & 530 & 578 & 130 & 126 & $\begin{array}{c}1.8 \\
(2.5)\end{array}$ \\
28 & 513 & 489 & 106 & 107 & $\begin{array}{c}33.0 \\
(21.0) \\
81.0\end{array}$ \\
31 & 474 & 484 & 107 & 112 & $\begin{array}{c}81.0 \\
(38.0)\end{array}$ \\
\hline
\end{tabular}

a

$\left[\dot{R}\left(t_{0.67}\right) / \dot{G}-1\right] /\left[\dot{R}_{0} / \dot{G}-1\right]=0.33 \rightarrow t_{0.67}$ 
SECTION IX

DISCUSSION 


\section{DISCUSSION}

The most Important results from the TRIO experiment are those which contribute to the fundamental understanding of transport of tritium and the transport of heat in a fusion reactor blanket. Since a limited parameter space is tested in an experiment of this kind, models have to be developed so that the results can be extrapolated to anticipated conditions in a fusion reactor blanket. Experimental results were obtained on the amount of tritium retained, or the tritium inventory, as a function of test conditions for the 33 runs. The results also provided quantitative data for intragranular diffusion and information on surface effects and on the chemical species of tritium evolved from the capsule. In addition, the permeation rate of tritium through the inner capsule wall was measured, and an analysis of temperature profiles and heating rates provided data on heat transfer. It was concluded that the irradiation produced measurable amounts of volatile radionuclides and activation products in the lithium aluminate breeder material. These results and their implication for blanket design are discussed.

\section{A. Tritiun Inventory in the Solid Breeder \\ 1. Tritium Inventory Mode1}

A tritium transport model was developed in support of the STARFIRE study. 1 This model is used to predict the steady-state tritium inventory in a solid breeder blanket. Five transport mechanisms were identified: (1) bulk diffusjon, (2) surface desorption, (3) gas-phase transport through interconnected pores in the solid, (4) dissolution -- sometimes called "solubility", and (5) trapping. Each mechanism can contribute to the tritium inventory in the blanket. The total inventory is estimated as the sum of the individual contributions.

For bulk diffusion, probably the best understood of the five transport mechanisms, there is at least some semi-quantitative data for a number of candidate solid breeders. Bulk diffusion is considered to contribute significantly to the tritium inventory in the blanket. Based on diffusion, the tritium inventory is a strong function of grain size and temperature. Activation 
energles for diffusion are typlcally a few tens of kilocalarles per mole. Thus, the tritium inventory rapidly increases as temperature decreases. It is primarily this temperature dependence which establishes the lower temperature limits for solid breeders. 1 Under steady-state conditions, where the tritium generation rate equals the tritium release rate which equals a constant, the following expression has been developed for spherical particles (singlecrystal grains): 38

$$
\mathrm{I}_{\mathrm{d}}=\dot{\mathrm{g}} \mathrm{r}_{\mathrm{g}}^{2} / 15 \mathrm{D}
$$

where $I_{d}=$ the diffusive inventory, i.e., the inventory one would calculate with the assumption that bulk diffusion is the only process contributing significantly to tritium inventory. The other terms are: $\dot{g}=$ the generation rate of tritium; $r_{g}=$ the radius of the spheres; and $D=d i f f u s i v i t y$. For STARFIRE, at $500^{\circ} \mathrm{C}$, if $r_{g}=0.1 \mu \mathrm{m}, \mathrm{D}=10^{16} \mathrm{~cm}^{2} / \mathrm{s}$, and $\tau=6 \times 10^{3} \mathrm{~g} / \mathrm{s}$, the diffusive inventory would be $400 \mathrm{~g}$, assuming uniform temperature throughout the blanket. For the TRIO experiment, assuming the same conditions except that $\dot{\mathrm{g}}=5 \mu \mathrm{Ci} / \mathrm{s}$, the steady-state Inventory is estimated to be $0.33 \mathrm{Ci}$. In practice, the temperatures have a significant range in both the TRIO experiment and in a fusion reactor blanket. Under this circumstance, the volumetric temperature distribution must be integrated to calculate the diffusive inventory.

Surface desorption processes could affect tritium transport, particularly for microstructures having high porosity and small grain size, as is the case for the $\mathrm{LAAlO}_{2}$ material used in TRIO. The tritium inventory associated with surface effects is directly dependent on surface area. Presence of hydrogen gas or water may help tritium release by isotoplcally swamping avallable surface sites. The activation energies for adsorption/desorption are rather low, on the order of a few kilocalories per mole, and the temperature dependance is thus rather weak.

Because of the small grain size of the TRIO pellets, the surface area is large, estimated to be the order of $10 \mathrm{~m}^{2} / \mathrm{g}$. If one assumes a surface site has an area of $10 \mathrm{~A}^{2}$, for the $42 \mathrm{~g}$ of $\mathrm{LAAlO}_{2}$, there are $4.2 \times 10^{21}$ sites, which if completely filled would hold $200 \mathrm{CI}$ of tritium atoms. In the experiment, only a small fraction of the sites is 11kely to be occupled by a hydrogen or a tritium atom. In addition, protium is present at a concentration 
three orders of magnitude higher than tritium for most of the experiment. Under these circumstances, the surface inventory is expected to be orders of magnitude less than $200 \mathrm{Ci}$.

Gas phase transport through the interconnected porosity establishes the tritium partial pressure in contact with the solid breeder. The tritium inventory is estimated by considering the solid to be in equilibrium with the gas phase; the tritium dissolution is then estimated. During the STARFIRE study, considerations of gas phase transport indicated that a reasonable amount of interconnected porosity (20-30\%) was desirable for minimizing tritium inventories.1,18 In addition, larger pore sizes, $>1 \mu \mathrm{m}$, are beneficial. In order to have large pore sizes and, at the same time, small grain sizes, a microstructure having a bimodal pore size distribution was proposed for the breeder material in STARFIRE. ${ }^{1}$ This type of microstructure was selected and fabricated for the TRIO experiment (see Sec. IV.B.)

Tritium dissolution was considered to be a potentially significant problem, particularly for $\mathrm{Li}_{2} \mathrm{O}$, on the basis of some idealized thermodynamic calculations.l The primary assumption in these calculations is that activity coeffients are unity. More recent measurements of $\mathrm{H}_{2} \mathrm{O}-\mathrm{Li}_{2} \mathrm{O}$ phase equilibria have shown that activity coefficlents are very large, approaching 1000 at some temperatures. As a result, tritium dissolution $\mathrm{In}^{\mathrm{Li}} \mathrm{O}^{\mathrm{O}}$ is very small, the order of $1 \mathrm{wppm}$. The tritium solubility in ternary ceramics is expected to be even less. Therefore, tritium dissolution is not expected to make a significant contribution to the tritium inventory.

Trapping could conceivably result in very high tritium inventories in solid breeders. In the STARFIRE study, it was considered a possibility that radiation-induced trapping could lead to unacceptably high tritium inventories. However, the 11 terature contains no applicable data for solid breeders which could have allowed even qualitative limits on this effect to be istablished.

\section{Tritium Inventory Results from the TRIO Experiment}

For the TRIO experiment, the tritium inventory for the 33 runs was given in Table VIII-10. As previously discussed, the data from the first eight runs and the last six runs provided an excellent fit to a steady-state diffusion model. These runs represent optimal conditions for tritium release, namely, 
$0.1 \%$ hydrogen added to the sweep gas stream. It is inferred that, when hydrogen is acided, surface release is enhanced but intragranular diffusion is not appreclably changed. Under these conditions intragranular diffusion is the rate-limiting step for tritium release; the tritium release is diffusion controlled.

For Runs 9-27, the inventory was assumed to be a sum of two components, the diffusive Inventory $\left(I_{d}\right)$ and the surface (Including solubility) Inventory $\left(I_{S}\right)$. The derived diffusion relationship from Runs 1-8 and 28-33 [Eq. (6), Sec. VIII] was used to calculate diffusive inventories for Runs 9-27. Each surface Inventory $\left(I_{S}\right)$ was taken to be the total inventory minus the diffusive inventory $\left(I_{d}\right)$. The results are given in Table IX-1. Certain trends appear upon examination of the data in Tables VIII-10 and IX-1. For Runs $1-8$, the surface inventory term is negligible because the inventories are ascribed to intragranular diffusion. This set of runs had hydrogen added to the sweep gas, generally $0.1 \%$ or a thousand-fold excess with respect to tritium.

Runs 9-14 had no hydrogen added to the sweep gas stream (the actual hydrogen concentration was about $3 \mathrm{ppm}$, or about three times the tritium leve1). For this set of runs the surface inventory increased from a negligible quantity to about $1 \mathrm{Ci}$. As discussed above, the surface site population of tritium could well account for a tritium inventory of $1 \mathrm{Cl}$ for the conditions of Runs 9-14. Addition of $0.1 \%$ hydrogen should decrease the surface inventory by more than an order of magnitude; as a result, the surface inventory for the first eight runs is expected to be less than $0.1 \mathrm{Cl}$. The tritium inventories for the first eight runs appear to be diffusion controlled, and the actual surface Inventories for these runs do Indeed appear to be less than $0.1 \mathrm{Ci}$.

Runs 15-19 represent a return to "standard" conditions; 1.e., hydrogen was again added to the sweep gas. The calculated diffusi inventories for these runs do not account for all the tritium, which is $0.6 \mathrm{Cl}$. However, the uncertainty in tritium inventory for these runs is estimated as $0.5 \mathrm{Ci}$. Therefore, it cannot be concluded that the surface inventory is significantly large for these runs. 
TABLE IX-1

Tritium Inventories for Runs Which Were Not Diffusion Controlled

\begin{tabular}{|c|c|c|c|c|c|c|c|c|}
\hline $\begin{array}{l}\text { Run } \\
\text { No. }\end{array}$ & Conditions ${ }^{a}$ & HT & HTO & PERM & TCOLL & TGEN & $\mathrm{I}_{\mathbf{d}}$ & $I_{s}$ \\
\hline $9^{b}$ & $650 /$ No $\mathrm{H}_{2}$ & 1.823 & 0.002 & 0.0068 & 1.832 & 2.603 & 0.02 & $>0.78$ \\
\hline 10 & $400 /$ No $\mathrm{H}_{2}$ & 0.040 & 0.000 & 0.0018 & 0.042 & 0.421 & $(67.8)$ & -- \\
\hline 11 & $500 / \mathrm{NO} \mathrm{H}_{2}$ & 0.300 & 0.003 & 0.0040 & 0.307 & 0.462 & $(2.02)$ & --- \\
\hline 12 & $550 / \mathrm{No}_{2}$ & 1.245 & 0.000 & 0.0197 & 1.265 & 1.835 & 0.07 & $>1.83$ \\
\hline 13 & $600 /$ No $\mathrm{H}_{2}$ & 2.197 & 0.000 & 0.108 & 2.305 & 2.061 & 0.12 & 1.54 \\
\hline 14 & $700 /$ No $\mathrm{H}_{2}$ & 1.560 & 0.001 & 0.083 & 1.644 & 0.994 & 0.01 & 1.00 \\
\hline $15^{c}$ & 700/STD & 0.715 & 0.000 & 0.031 & 0.746 & 0.373 & 0.01 & 0.62 \\
\hline 16 & $700 / 300$ SCCM & 0.503 & 0.001 & 0.023 & 0.527 & 0.502 & 0.01 & 0.60 \\
\hline 17 & $700 / 30 \mathrm{sCCM}$ & 1.109 & 0.000 & 0.202 & 1.311 & 1.331 & 0.01 & 0.62 \\
\hline 18 & 700/STD & 0.380 & 0.000 & 0.032 & 0.412 & 0.380 & 0.01 & 0.59 \\
\hline 19 & $650 / \mathrm{STD}$ & 0.294 & 0.000 & 0.021 & 0.315 & 0.306 & 0.04 & 0.55 \\
\hline $20^{d}$ & $650 / 0.2 \% \mathrm{O}_{2}$ & 0.028 & 0.000 & 0.0068 & 0.035 & 1.585 & 0.03 & $>2.11$ \\
\hline 21 & 650/STD & 1.092 & 0.000 & 0.0034 & 1.095 & 0.783 & 0.03 & 1.80 \\
\hline 22 & 600/STD & 0.351 & 0.000 & 0.0013 & 0.352 & 0.385 & 0.09 & 1.77 \\
\hline 23 & $550 /$ STD & 1.077 & 0.001 & 0.0035 & 1.082 & 1.260 & 0.34 & 1.70 \\
\hline 24 & $600 / \mathrm{STD}$ & 0.576 & 0.002 & 0.0014 & 0.579 & 0.397 & 0.08 & 1.78 \\
\hline 25 & $560 / \mathrm{STD}$ & 0.537 & 0.007 & 0.0018 & 0.546 & 0.618 & 0.23 & 1.70 \\
\hline 26 & 550/STD & 1.213 & 0.048 & 0.0041 & 1.265 & 1.414 & 0.36 & 1.72 \\
\hline 27 & 525/STDF & 2.128 & 0.333 & 0.0103 & 2.471 & 2.169 & 0.56 & 1.22 \\
\hline
\end{tabular}

NOTE: $" S T D "=100 \mathrm{SCCM}, 0.1 \% \mathrm{H}_{2}$.

${ }^{2}$ Value on the right of slash $(/)$ is temperature $\left({ }^{\circ} \mathrm{C}\right)$; value on left is gas composition.

byydrogen was removed from the sweep gas after Run 8 and up to Run 14. $\mathrm{c}_{0.1 \% \mathrm{H}_{2}}$ was added to the sweep gas after Run 14 and up to Run 19. doxygen was added to the sweep gas in Run 20.

Run 20 had $0.2 \%$ oxygen added to the sweep gas. Very little tritium was evolved during this run, and the retention was more than $2 \mathrm{Ci}$ when the run was ended. Given the temperature $\left(700^{\circ} \mathrm{C}\right)$, the diffusive Inventory was calculated to be negligible in comparison to the total inventory. Furthermore, this excess inventory of about $2 \mathrm{Ci}$ persisted for Runs 21-26 even though hydrogen was added for these runs. In addition, a large amount (about $1.5 \mathrm{CI}_{\mathrm{I}}$ ) of HTO (tritium in the condensable form) was released in Runs 25-29, about a month after Run 20 had ended. About 2 C1 of tritium was retained either in the breeder material or somewhere else in the system for an extended period of time as a result of the oxygen addition. As previously discussed (Sec. VI.G) 
small HTO spikes consistently occurred about one day after large HT spikes. The response time of the system to a release of HTO from the breeder was concluded to be $\sim 1$ day. Therefore, the $2 \mathrm{Ci}$ of excess tritium is associated with either the surface or the bulk of the breeder material.

The last six runs (28-33) appear to be diffusion controlled. The agreement between the calculated diffusivities for Runs 1-8, 28-33, and the derived diffusivity relationship [Eq. (6) in Sec. VIII-D] is quite good, shown in Fig. VIII-2.

There is ancther point regarding Table VIII-10 that is useful to place the data in context. Both the tritium generation rate and the mass of breeder material in a $4000-\mathrm{MW}$ fusion reactor ${ }^{1,9}$ are just about 10 milition times those in the TRIO experiment. Since $1 \mathrm{~kg}$ of tritium is $10 \mathrm{million} \mathrm{Ci}$, the inventories listed in Curies for TRIO would translate to inventories in such fusion reactor blankets in units of kilograms.

\section{B. Intragranular Diffusion}

Diffusivity values have been reported for polycrystalline aiumina, 47-48 single-crystal alumina, 49 and 1ithium aluminate itself.29,50-52 Selected literature data on diffusivity are compared to the TRIO results in Fig. IX-1. Since Wiswall et al. 29 obtained lithium aluminate from the same source used for TRI0, the grain size was assumed to be the same for the two studies. In addition, the diffusivity values were recalculated from their tritium release curves to correct for a small error. Yunker ${ }^{50}$ did not report grain size or diffusivity, but his activation energy agrees with the TRIO results. The grain size for Yunker's experiment was assumed to be $0.3 \mu \mathrm{m}$. The activation energy of $35.8 \mathrm{Kcal} / \mathrm{mol}$ is rather large in comparison to that of other solid breeders, but the value is in agreement with Wiswall et al. ${ }^{29}$ and Yunker. 50 In addition, the activation energy for tritium and hydrogen diffusion in alumina ${ }^{47-49}$ is similar. Both Guggi et al. ${ }^{51}$ and Vasiliev et al. 52 report much lower activation energies and higher diffusivities for tritium in lithium aluminate than derived from TRIO. In both these cases, the particle sizes are reported as two to three orders of magnitude larger than those of TRIO. It is aiso possible that the true grain size in these experiments is much smaller than the grain size which would explain the large difference in magnitude but not activation energy. Another point is that activation energies as high as 


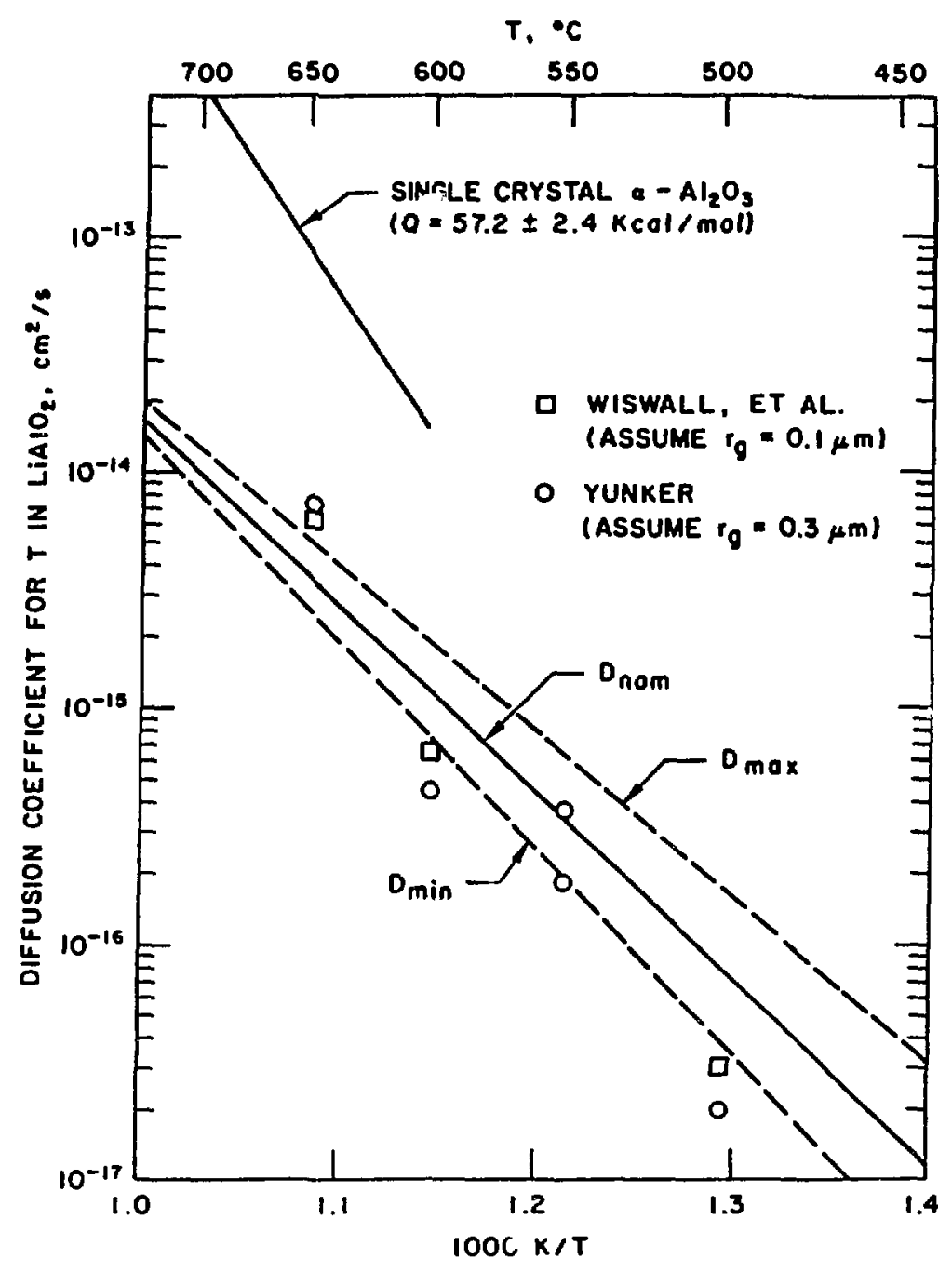

Fig. IX-1. Comparison of selected data for tritium diffusion in $\gamma^{-\mathrm{LAAlO}_{2}}$ with the TRIO correlation (includes uncertainties in grain size, Inventory, and generation rate).

35.8 Kcal/mol are most probably due to intragranular diffusion. other processes such as surface sorption and grain edge diffusion are expected to have much lower activation energies. Based on the high value of the activation energy, the good fit of the experimental data to the diffusivity relationship, and the agreement of the experimental data with results of two other independent studies, it is considered likely that the diffusivity values derived from the TRIO experiment represent intragranular diffusion.

The data for runs at the beginning and end of the experiment indicate that the controlling mechanism for tritium transport, when hydrogen is added to facilitate surface desorption, is intragranula iffusion. Over the limited temperature range, the data fit a single equation. Based on this observation, no evidence exists for trapping, which was earlier considered ${ }^{l}$ to 
possibly be a dominant effect. Thus, for the limited conditions and burnup of the TRIO experiment, trapping is not considered a significant contributor to tritium inventory.

A material such as lithium aluminate could show a wide range of diffusivities owing to impurities and solid-state effects. 53 The diffusivity could be affected by oxide vacancy concentration. Since low oxygen potentials tend to form more vacancies, diffusivity could be enhanced. Conversely, when oxygen potential is high (e.g., Run 20), defect content is reduced and diffusion is correspondingly reduced. This possibility cannot be resolved with the TRIO results.

It is of interest to assess the impact of the derived diffusivity in terms of tritium inventory in a fusion reactor blanket. With the calculational method prevtously described (Sec. VIII.D), the temperature distributions given in the Blanket Comparison and Selection Study (BCSS) ${ }^{9}$ were used to estimate tritium inventories. In that study, the tritium generation rate is $0.006 \mathrm{~g} / \mathrm{s}$ for the entire blanket of the 4000-MW reactor. The maximum temperature is fixed at $950^{\circ} \mathrm{C}$, and the minimum temperature is varied from 300 to $550^{\circ} \mathrm{C}$. As was the case for TRIO, capsule geometry for this study was a hollow cylinder, but the coldest temperatures was on the inside of the cylinder rather than on the outside.

The results of this analysis are presented in Table IX-2, In this table, the temperature distribution is characterized by a volume-averaged temperature (TVAV), over the range from the maximum temperature (TMAX) to the minimum temperature (TMIN). The f factor has the same meaning as before, viz., the ratio between tritium inventory for the actual case and that for the isothermal $(T=$ TVAV) case. The results show that the diffusive tritium inventory rises very rapidly as the minimum temperature is decreased. If the diffusive inventory is not to exceed $1 \mathrm{~kg}$, then the minimum temperature for the blanket must be no lower than $350^{\circ} \mathrm{C}$.

\section{Surface Bffects}

As noted above, the presence of hydrogen appears to enhance the release of tritium. For example, hydrogen addition of $0.1 \%$ (a thousand-fold excess) enhanced tritium release rates and reduced tritium inventories associated with the surface. A simple argument presented above showed that isotope swamping 
TABLE IX-2

Calculated Diffusive Inventories for a 4000-MW Fusion Reactor Blanket

\begin{tabular}{l|l|l|c|c}
\hline $\begin{array}{l}\text { TMAX } \\
\left({ }^{\circ} \mathrm{C}\right)\end{array}$ & $\begin{array}{l}\text { TMIN } \\
\left({ }^{\circ} \mathrm{C}\right)\end{array}$ & $\begin{array}{c}\text { TVAV } \\
\left({ }^{\circ} \mathrm{C}\right)\end{array}$ & $\mathrm{f}$ & $\begin{array}{c}\text { Tritium } \\
\text { Inventory } \\
(\mathrm{kg})\end{array}$ \\
\hline 950 & 550 & 846 & $1.180 \times 10^{1}$ & $7.10 \times 10^{-3}$ \\
950 & 500 & 534 & $3.009 \times 10^{1}$ & $2.16 \times 10^{-3}$ \\
950 & 450 & 823 & $9.384 \times 10^{1}$ & $7.92 \times 10^{-2}$ \\
950 & 400 & 811 & $3.831 \times 10^{2}$ & 0.388 \\
950 & 350 & 800 & $2.080 \times 10^{3}$ & 2.50 \\
950 & 300 & 789 & $3.664 \times 10^{4}$ & 23.5 \\
\hline
\end{tabular}

of surface sites with hydrogen could account for the reduction in tritium inventory when hydrogen was added.

There is further evidence of surface effects. Namely, in Run 3, the hydrogen content was increased from 0.1 to $1 \%$. The HT release curve (see Fig. VI-13) shows a positive spike followed by a very rapid return to equilibrium. The time constant is the order of ten minutes. This rapid response is not characteristic of processes in the bulk of the breeder solid, such as intragranular diffusion, but is characteristic of a surface process, which is much more rapid.

For Run 20, oxygen was added, and very little tritium came out. The tritium inventory increased by about $2 \mathrm{Cl}$ as a result. Run $21\left(0.1 \% \mathrm{H}_{2}\right)$ shows a large positive peak, followed by a return to steady state as shown in the HT release curve (FIg. VI-52). The release curve for Run 21 does not appear to show a different response than other similar runs (e.g., Run 33). Thus, although the tritium retention is higher for Run 2l, the release kinetics do not appear to be affected. The excess tritium, which appears to be in the oxide form, does not seem to inhibit release of the rest of the tritium. If this excess tritium is on the surface, it is probably not affecting surface release rates.

One other issue should be mentioned: The surface could affect the ratio of HT to HTO. This possibility is discussed in the next section. 


\section{Species of Tritiue Bvolved}

The predominant form of tritium evolved during the experiment was the HT, or noncondensable form. Prior to the experiment, the expectation was that the oxide breeder would give rise to the oxide form of tritium. This result has serious implications for blanket design because the permeation rates for HT are much greater than those for HTO, and thus tritium loss rates by permeation could become high. A number of rationales for this observation exist, including kinetic and thermodynamic arguments discussed below.

Perhaps the simplest way to look at the system is first to assume that Iithium aluminate produces $\mathrm{T}_{2} \mathrm{O}$ and the $\mathrm{T}_{2} \mathrm{O}$ reacts with $\mathrm{H}_{2}$ to form $\mathrm{HT}$ and $\mathrm{H}_{2} \mathrm{O}$. For the runs when $0.1 \% \mathrm{H}_{2}$ was added, which is 1000 times the tritium level in the sweep gas, one would expect HT to be the dominant tritium form. However, this rationale does not account for the fact that the noncondensable HT form was dominant even when $\mathrm{H}_{2}$ was not added.

Another possibility is that the structural material, Type 304 stainless steel, is playing a role in the equilibrium process by affecting the oxygen potential of the system. In vapor effusion studies, Guggi et al. 51 noted that the presence of a stainless steel container caused a very significant increase in the vapor pressure of elemental lithium over lithium aluminate. In other words, the oxygen potential was very low in Guggi's test. A series of thermodynamic calculations were performed to assess the effect of the steel cladding on the system. To perform the rather complex set of equilibrium calculations, the SOLGASMIX code was used.

The calculations are for a system that was initially 1 mole of lithium aluminate at $1000 \mathrm{~K}$. It is assumed that 100 mole ppm of the lithium was burned up to form $100 \times 10^{-6} \mathrm{~g}$ atoms tritium, $99 \%$ tritium was released and recovered from the system, and $50 \mathrm{~g}$ atoms oxygen were produced by burnup and remained in the system. The effect of the steel is approximated by assuming the system contains $0.01 \mathrm{~g}$ atom 1ron. The condensed phases are simulated by $\mathrm{LIAlO}_{2}, \mathrm{LIAl}_{5} \mathrm{O}_{8}$, iron, and iron oxide.

In the first calculation, the results of which are shown in Table IX-3, the oxygen activity was fixed at a value derived from the results of Guggi et al $51,2 \times 10^{-35} \mathrm{~atm}$. Based upon these results, the ratio of $\mathrm{T}_{2} \mathrm{O}$ to $\mathrm{T}_{2}$ is very sma11, $5 \times 10^{-6}$. This result is consistent with the TRIO observations. The tritium content of the condensed phase $1 s 5.6 \%$ of the total amount of tritium. 
If the $\mathrm{H}_{2}-\mathrm{H}_{2} \mathrm{O}$ couple sets the oxygen potential (assiming ! ppm water and $0.1 \% \mathrm{H}_{2}$ in the gas phase), the oxygen potential is about $10^{-26}$ atm at $1000 \mathrm{k}$. Under this condition, the HTO:HT ratio is still very small, less than 0.001 .

If the iron-iron oxide couple is dominant, then the oxygen potential is still higher, as shown in Table IX-4. The reduced $T_{2}$ form is only a factor of two higher than the $\mathrm{T}_{2} \mathrm{O}$ form. In this case, over $99 \%$ of the tritium in the system is in solid solution as the tritoxide in the lithium aluminate condensed phase. This represents a considerable increase over the first case (Table IX-3) and indicates that the oxygen potential may affect the tritium retention.

A final calculation, summarized in Table IX-5, corresponds to the oxygen level set at $0.1 \%$ in the gas phase (approximately Run 20). The oxide form does become dominant in the gas phase, but $99.85 \%$ of all the tritium in the system is retained in the solid as the tritoxide.

The above calculations have considerable uncertainty in a number of areas; in particular, the activity coefficient of the tritoxide in solution is unknown. Nonetheless, the trends are meaningful. For example, it was found that the stainless steel cladding miy control the oxygen potential sufficiently to yleld the observed results In the TRIO tests, the cladding temperature was rather high; the tube on the inside was hotter than the breeder material, and the cladding was near the temperature at the colder surface of the breeder. In a fusion reactor with pressurized water coolant, the temperature of the steel structural material will be considerably lower. In this case, the effect of the cladding as a reducing agent will be much less. In addition, if the cladding is oxidized before use with an oxide layer on the steel, the steel may not participate in the equilibrium. Under this circumstance the fraction of the condensable form will be considerably larger.

A second point of interest is that the amount retained in the solid by dissolution rose dramatically as the oxygen potential was increased. The release enhancement observed when hydrogen was added to the sweep gas may be explained by this effect. Conversely, the effect is consistent with the observation that addition of oxygen was detrimental to tritium release. 
TABLE IX-3

Lithium Aluminate Equilibria: Fixed Oxygen Content

\begin{tabular}{|c|c|c|}
\hline Spectes & $\begin{array}{l}\text { Moles at } \\
\text { Equilibrium }\end{array}$ & $\begin{array}{l}\text { Partial } \\
\text { Pressur, } \\
\text { (atm) }\end{array}$ \\
\hline Inert gas & $0.35000 \times 10^{-3}$ & 0.99999 \\
\hline $\mathrm{T}_{2}(\mathrm{G})$ & $0.23414 \times 10^{-8}$ & $0.66895 \times 10^{-5}$ \\
\hline $\mathrm{O}_{2}(\mathrm{G})$ & $0.82058 \times 10^{-25}$ & $0.23445 \times 10^{-21}$ \\
\hline $\mathrm{T}_{2} \mathrm{O}(\mathrm{G})$ & $0.71875 \times 10^{-9}$ & $0.20536 \times 10^{-5}$ \\
\hline LiOT (G) & $0.31388 \times 10^{-14}$ & $0.89680 \times 10^{-11}$ \\
\hline$(\mathrm{LiOT})_{2}(\mathrm{G})$ & $0.73446 \times 10^{-21}$ & $0.20984 \times 10^{-17}$ \\
\hline Lio（G） & $0.72859 \times 10^{-25}$ & $0.20817 \times 10^{-21}$ \\
\hline $\mathrm{Li}_{2} \mathrm{O}(\mathrm{G})$ & $0.64548 \times 10^{-23}$ & $0.18442 \times 10^{-19}$ \\
\hline $\mathrm{LT}$ (G) & $0.86381 \times 10^{-20}$ & $0.24680 \times 10^{-16}$ \\
\hline Li (G) & $0.20134 \times 10^{-16}$ & $0.57524 \times 10^{-13}$ \\
\hline $\mathrm{AlO}_{2} \mathrm{~T}$ (G) & $0.38082 \times 10^{-25}$ & $0.10880 \times 10^{-21}$ \\
\hline A10T (G) & $0.95695 \times 10^{-26}$ & $0.27341 \times 10^{-22}$ \\
\hline Al (G) & $0.21823 \times 10^{-32}$ & $0.62351 \times 10^{-29}$ \\
\hline $\mathrm{LiAlO}_{2}$ (S) & 0.99987 & $\begin{array}{l}\text { Mole Fraction } \\
0.10000 \times 10^{1}\end{array}$ \\
\hline LIOT (L) & $0.99385 \times 10^{-6}$ & $0.99398 \times 10^{-6}$ \\
\hline $\mathrm{LiAl}_{5} \mathrm{O}_{8}(S)$ & $0.25248 \times 10^{-4}$ & $\begin{array}{l}\text { Mole Fraction } \\
0.10000 \times 10^{1}\end{array}$ \\
\hline LIOT (L) & $0.25096 \times 10^{-10}$ & $0.99398 \times 10^{-6}$ \\
\hline
\end{tabular}

Note: Thermodynamic equilibrium condictons in a 1 -mole $\mathrm{LiAlO}_{2}$ system that contains no protium. The oxygen activity is taken as that for the Fe/FeO system. Total system pressure is 1 atm at $1000 \mathrm{~K}$. Assumes that 100-mole ppm burnup of 1ithium has occurred, that $1 \%$ of the produced tritium is st111 present, and that no oxygen has been lost. The system contains, therefore, $0.9999 \mathrm{~g} \cdot$ atom oxygen, $1.0 \mathrm{~g} \cdot$ atom aluminum, and $1 \times 10^{-6} \mathrm{~g} \cdot$ atom tritium. 
TABLE IX-4

Lithfum Aluminate Equilibria: Low Oxygen Activity

\begin{tabular}{|c|c|c|}
\hline Spectes & $\begin{array}{l}\text { Moles at } \\
\text { Equilibrium }\end{array}$ & $\begin{array}{l}\text { Partial } \\
\text { Pressure } \\
\text { (atm) }\end{array}$ \\
\hline Inert gas & $0.35000 \times 10^{-3}$ & 0.99865 \\
\hline $\mathrm{T}_{2}(G)$ & $0.47181 \times 10^{-6}$ & $0.13462 \times 10^{-2}$ \\
\hline $\mathrm{O}_{2}(\mathrm{G})$ & $0.20994 \times 10^{-34}$ & $0.59902 \times 10^{-31}$ \\
\hline $\mathrm{T}_{2} \mathrm{O}(G)$ & $0.23151 \times 10^{-11}$ & $0.66057 \times 10^{-8}$ \\
\hline LiOT $(G)$ & $0.17826 \times 10^{-15}$ & $0.50863 \times 10^{-12}$ \\
\hline$(\mathrm{LiOT})_{2}(\mathrm{G})$ & $0.23657 \times 10^{-23}$ & $0.67501 \times 10^{-20}$ \\
\hline Lio (G) & $0.29168 \times 10^{-27}$ & $0.83226 \times 10^{-24}$ \\
\hline $\mathrm{Li}_{2} \mathrm{O}(\mathrm{G})$ & $0.64635 \times 10^{-23}$ & $0.18442 \times 10^{-19}$ \\
\hline $\operatorname{LiT}(G)$ & $0.30691 \times 10^{-16}$ & $0.87570 \times 10^{-13}$ \\
\hline $\mathrm{Li}(G)$ & $0.50426 \times 10^{-14}$ & $0.14388 \times 10^{-10}$ \\
\hline $\mathrm{AlO}_{2} \mathrm{~T}(\mathrm{G})$ & $0.21628 \times 10^{-26}$ & $0.61710 \times 10^{-23}$ \\
\hline Alot (G) & $0.34000 \times 10^{-22}$ & $0.97013 \times 10^{-19}$ \\
\hline Al (G) & $0.34194 \times 10^{-25}$ & $0.97566 \times 10^{-22}$ \\
\hline $\mathrm{LiAlO}_{2}(\mathrm{~S})$ & $0.10000 \times 10^{:}$ & $\begin{array}{l}\text { Mole Fraction } \\
0.10000 \times 10^{1}\end{array}$ \\
\hline LiOT (L) & $0.56375 \times 10^{-7}$ & $0.56375 \times 10^{-7}$ \\
\hline $\mathrm{LiAl}_{5} \mathrm{O}_{8}(\mathrm{~S})$ & $0.28189 \times 10^{-7}$ & $\begin{array}{l}\text { Mole Fraction } \\
0.10000 \times 10^{1}\end{array}$ \\
\hline LiOT（L) & $0.15891 \times 10^{-14}$ & $0.56375 \times 10^{-7}$ \\
\hline
\end{tabular}

Note: Analogous to Table IX-3 except that the lithium activity is fixed (and the oxygen activity is also thereby fixed) at the value taken from Guggi's study of $\mathrm{LiAlO}_{2}$ in a stainless steel ce11. 
TABLE IX-5

Lithium Aluminate Equilibria: High Oxygen Activity

\begin{tabular}{|c|c|c|}
\hline Spectes & $\begin{array}{l}\text { Moles at } \\
\text { Equilibrium }\end{array}$ & $\begin{array}{l}\text { Partial } \\
\text { Pressure } \\
\text { (atm) }\end{array}$ \\
\hline Inert gas & $0.35000 \times 10^{-3}$ & 0.99900 \\
\hline $\mathrm{T}_{2}(\mathrm{G})$ & $0.11455 \times 10^{-17}$ & $0.32696 \times 10^{-14}$ \\
\hline $\mathrm{O}_{2}(\mathrm{G})$ & $0.35035 \times 10^{-6}$ & $0.10000 \times 10^{-2}$ \\
\hline $\mathrm{T}_{2} \mathrm{O}(\mathrm{G})$ & $0.72624 \times 10^{-9}$ & $0.20729 \times 10^{-5}$ \\
\hline LOT (G) & $0.31567 \times 10^{-14}$ & $0.90101 \times 10^{-11}$ \\
\hline$(\mathrm{LIOT})_{2}(\mathrm{G})$ & $0.74211 \times 10^{-21}$ & $0.21182 \times 10^{-17}$ \\
\hline Lifo (G) & $0.33144 \times 10^{-20}$ & $0.94602 \times 10^{-17}$ \\
\hline $\mathrm{LH}_{2} \mathrm{O}(\mathrm{G})$ & $0.64613 \times 10^{-23}$ & $0.18442 \times 10^{-19}$ \\
\hline LiT (G) & $0.42064 \times 10^{-29}$ & $0.12006 \times 10^{-25}$ \\
\hline Li (G) & $0.44347 \times 10^{-21}$ & $0.12658 \times 10^{-17}$ \\
\hline $\mathrm{AlO}_{2} \mathrm{~T}$ (G) & $0.38299 \times 10^{-25}$ & $0.10932 \times 10^{-21}$ \\
\hline Alot (G) & $0.46599 \times 10^{-35}$ & $0.13301 \times 10^{-31}$ \\
\hline Al (G) & 0.0 & 0.0 \\
\hline $\mathrm{LiAlO}_{2}$ (S) & 0.99987 & $\begin{array}{l}\text { Mole Fraction } \\
0.10000 \times 10^{1}\end{array}$ \\
\hline LIOT (L) & $0.99852 \times 10^{-6}$ & $0.99865 \times 10^{-6}$ \\
\hline $\mathrm{LiAl}_{5} \mathrm{O}_{8}$ (S) & $0.25250 \times 10^{-4}$ & $\begin{array}{l}\text { Mole Fraction } \\
0.10000 \times 10^{1}\end{array}$ \\
\hline LHOT (L) & $0.25216 \times 10^{-10}$ & $0.99865 \times 10^{-6}$ \\
\hline
\end{tabular}

Note: Analogous to Tables $I X-3$ and -4 except that the oxygen activity is set at 0.001 to correspond to a doped sweep gas with $0.1 \%$ oxygen. 
The species evolved could be affected by a number of other factors, such as surface effects and internal kinetic factors. For example, if the diffusion rate of tritium atoms is much faster than that of oxygen atoms, more of the HT form could be released, at least for a short period of time. This process could be affected by surface effects. The experimental results are not sufficient to prove or disprove such effects. It is not considered likely that radiolysis of HTO can account for the experimental results.

\section{B. Tritiun Perneation}

The data on tritium permeation through the primary cladding (see Sec. VI.E) were translated into permeation rates in units of curles per day. The resultant permeation rates are illustrated in Fig. IX-2. The data appear to follow a definite pattern.

During Runs 9-15, no hydrogen was added to the sweep gas, as 1llustrated by the solid squares. For those runs (solid squares in Fig. IX-2), the permeation rate is close to the calculated rate for the tritium pressure and geometry of the capsule, as is shown by the reference lines which used data for austenitic stainless steel.46 When hydrogen gas was added to the sweep gas, permeation rates decreased by an order of magnitude. This effect is attributed to isotope swamping. For the idealized case, a 1000-fold excess of hydrogen would be expected to reduce the permeation by a factor of $\sqrt{1000}$, or about 30. The observed decrease is close to that value.

\section{F. Eeat Transfer Results}

The heat transfer results were presented in Sec. VIII.B. The most significant results were the in-pile measurement of thermal conductivity for the 1ithium aluminate. These data are plotted in Fig. IX-3. The large open circles represent the data for the first few experimental runs; the dots represent the later runs. The estimated precision is $<10 \%$. The data show no significant changes in thermal conductivity as a result of increased irradiation. It is inferred that, for the temperature range of interest, limited radiation damage (equivalent to about two months in a fusion reactor) does not significantly affect thermal conductivity. 

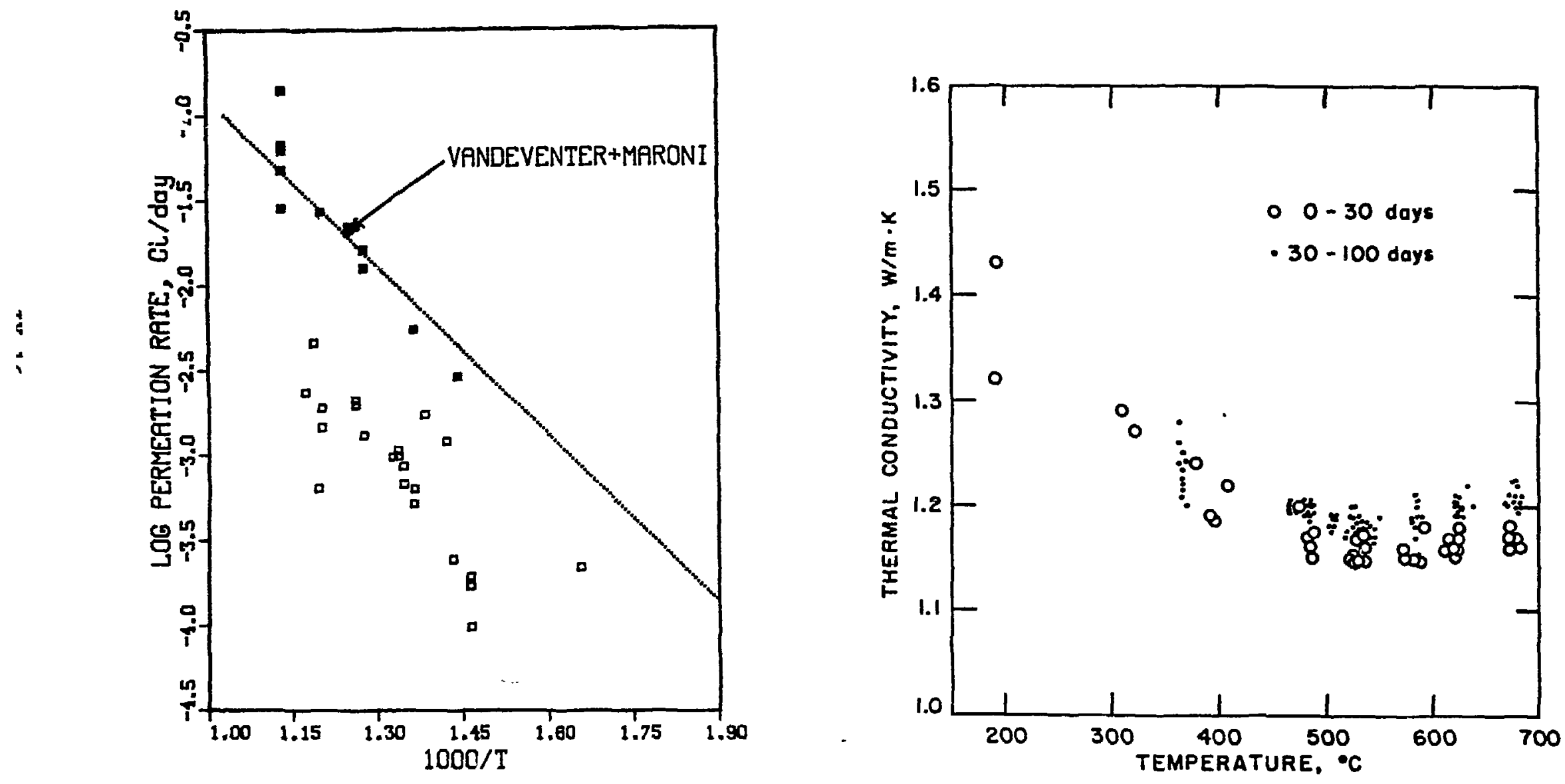

Fig. IX-2. Tritium permeation results for TRIO.

Fig. IX-3. Thermal conductivity of $\gamma-\mathrm{LiAlO}_{2}$ measured in-pile. 


\section{G. Radioactivity}

As previously discussed, impurities in the breeder material resulted in generation of measurable quantities of a number of volatile radionuclides during radiation. The levels measured showed significant temperature dependence, but the data were not adequate to calculate release rates.

Chemical analysis including neutron activation was performed prior to irradiation. In addition, the irradiated material was analysed by gamma spectrometry. The data should be useful in providing guidance to calculations of fusion blanket radioactivity and afterheat. 
SECTION $X$

CONCLUS IONS 


\section{CONClustons}

The purpose of the TRIO experiment was to test in-situ tritium recovery and heat transfer performance of a candidate breeder material, lithium aluminate. Seven objectives vere proposed for the experiment (see Sec. II), and all of them were met. A large amount of data was collected and analyzed. From the results of the experiment, the conclusions are:

1. In-situ tritium recovery can work very well. The inventory levels at the end of the experiment were less than 0.1 wppm. More than $99.9 \%$ of the tritium generated in the lithium aluminate was recovered.

2. Intragranular diffusion was found to be an important tritium transport mechanism. For runs at the beginning and end of the experiment when hydrogen was added to the sweep gas, the experimental data showed excellent agreement with the derived diffusivity given by the relationship:

$$
D=1.1 \times 10^{-6} \exp (-35.8 \mathrm{Kcal} / \mathrm{RT})
$$

The derived diffusivity appears to be consistent with other data in the literature.

3. The tritium inventories and tritium release rates are accounted for by consideration of intragranular diffusion, surface effects, and tritium dissolution ("solubility"). There is no evidence of detrimental or anomalous effects of radiation on tritium transport, such as trapping.

4. Addition of hydrogen to the sweep gas enhanced the release of tritium. This release enhancement is ascribed to improvement in surface release kinetics.

5. The addition of oxygen to the sweep gas was detrimental to release of tritium. This effect is consistent with thermodynamic calculations showing increased retention of tritium as the oxygen potential is increased. In addition, the oxygen might have had a detrimental effect upon the surface of the lithium aluminate. 
6. The predominant form of tritium observed in the experiment was the noncondensable, or HT, form. Thermodynamic calculations correctly predict this form for the experimental conditions. The amount of HT is expected to be less in a fusion reactor blanket, but permeation rates could still be a problem

7. Permeation rates measured in the experiment were extrapolated to rates of about $1000 \mathrm{Ci} /$ day for a fusion reactor blanket, owing to the predominance of the $\mathrm{HT}$ form. Permeation rates of $1000 \mathrm{Ci} /$ day are lower than the rates of tritium currently estimated to transport from the plasma edge through the first wall. Thus, the HT form results in high permeation rates, but such rates may be manageable.

8. The total damage was $0.18 \%$ 1ithium burnup, with 0.81 dpa and a dose of $5.36 \times 10^{12}$ rads. This burnup is equivalent to $\sim 0.6 \mathrm{MW} \cdot \mathrm{yr} / \mathrm{m}^{2}$ or about two months in a fusion reactor 1ike STARFIRE. Under these damage conditions and temperatures ranging nearly up to $800^{\circ} \mathrm{C}$, no evidence was found of significant change in the microstructure of the breeder material.

9. Temperatures were effectively controlled with the gap gas technique.

10. Heat transfer coefficients and thermal conductivity did not significantly change as a result of irradiation.

11. Trace 1mpuritles produced a number of volatile radionuclides, which were observed in the sweep gas. 
SECTION XI

RECOMAENDATIONS FOR FURTHER RESEARCH 


\section{RECOMRNDATIONS FOR FURTHER RESEARCH}

The TRIO experiment produced a considerable amount of useful data on tritium transport and heat transfer in the breeder material of lithium aluminate. More information in these areas is needed, as well as more data on the effects of radiation damage on solid breeder materials in general. The requirements of in-situ tritium recovery establish the operating limits (such as temperature and porosity) for solid breeder blankets. ${ }^{1}$ Tritium recovery from solid breeders is potentially affected by a number of factors, including temperature, temperature gradient, tritium production rate, grain size, porosity, surface area, surface condition (sites), oxygen potential, hydrogen potential, tritium partial pressure, defects/vacancies in the lattice, impurities in the solid and/or sweep gas, and transport of other species (e.g., helium). The role of these factors needs to be quantified in order to stablish meaningful limits for blanket design.

Radiation damage needs to be studied because it can alter some of these factors. For example, radiation damage can change grain size, change porosity, and create defects in the solid.

Temperature is a key factor because it directly affects tritium transport rates and can, particularly in concert with radiation, change the microstructural properties of the ceramic. Owing to the importance of temperature, the control of temperature profiles in a solid breeder blanket is a key issue. Temperature profiles must be controlled both in an experiment of this kind and in a fusion reactor. Therefore, data on heat transfer coefficients and thermal conductivity and the effects of irradiation on these properties is needed.

It is unlikely that a single experiment can effectively solve all the programmatic needs. Nonetheless, TRIo demonstrated that experiments of this type can effectively address many different research needs on breeder blankets. Therefore, it is recommended that more experiments of this type be undertaken. Considered herein is a single experiment which logically follows the successful completion of the TRIO experiment. 
The follow-on experiment should first of all retain the many useful features incorporated in the TRIO experiment. These features include a number of on-line capsule monitors, thermocouples, and flux monitors placed in appropriate locations in the test capsule. In addition, the gas analysis system for TRIO included provision for real-time and integral monitoring of tritium in its various chemical forms, as well as tritium permeation and measurement of impurities. This basic experimental setup should be upgraded to include the following modifications and/or improvements :

1. The temperature gradient should be minimized to simplify the incerpretation of temperature-dependent behavior such as diffusion. It is recommended that the temperature gradient not exceed $50^{\circ} \mathrm{C}$. In addition, it would be beneficial to use a material with a lower activation energy for diffusion. $\mathrm{Li}_{2} \mathrm{O}$ appears to have an activation energy about one-fourth that of $\mathrm{LiAlO}_{2}$.

2. The temperature range should, if possible, span the recommended temperatur limits, viz. $300-1000^{\circ} \mathrm{C}$. Investigation of low temperature allows direct determination of the 1ower temperature limit. Investigation of high temperature allows direct determination of the effects of swelling, sintering, etc., on tritium transport.

3. The experimental system should have improved monitoring capability for oxygen potential becasue of its importance to tritium release. The following items are needed: an oxygen meter, a moisture meter, and a mass analyzer.

4. More quantitative measurements of real-time tritium response are needed. This can be achieved with minor modification of the apparatus and with appropriate provision for extensive manual operations.

5. Experimental runs should go to equilibrium, i.e., each run should last one to two weeks. Longer runs would permit more quantitative measurement of release for volatile radionuclides.

6. In order to reduce uncertainties in measured heat transfer coefficients and thermal conductivity, a more accurate determination of gamma heating rates 15 needed. This could be done by making a number of measurements of gamma heating throughout the course of the experiment. 
7. Because of its high thermal conductivity, $\mathrm{Li}_{2} \mathrm{O}$ is the best cholce of breeder material to study decreases in thermal conductvity owing to radiation damage. In addition, the radial temperature gradient should be at least $50^{\circ} \mathrm{C}$ so that thermal conductivity can be accurately measured.

In addition to consideration of the experimental setup, there are a number of operational choices which should be considered, such as the test matrix, the number of capsules, etc.

The first choice is whether the temperature should be a variable. In the TRIO experiment, the temperature was systematically changed every few days. With this approach, data for many temperatures were obtained from a single test capsule; however, frequent temperature changes complicated the interpretation of the results. A single set temperature has two disadvantages: the amount of data that can be obtained is very Limited, and no information can be derived on temperature-dependent behavior, unless one goes to a large number of capsules. Because irradiation experiments of this kind are expensive to conduct, care should be taken to obtain the maximum amount of data possible. Therefore, each capsule should have the capability of changing the temperature in a controlled way. For purposes of planning, each temperature run should last one to two weeks. For simflar reasons, each capsule should have capabilities for operating with a sweep gas of variable flow and oxygen potential.

The next choice is the number of capsules. The recommended approach is not to do anything that will adversely affect the monitoring capability for each capsule. Thus, each capsule should have appropriate instrumentation. The cost per capsule will be somewhat less for experiments with multiple capsules, but the difference will not be large. The number of capsules will most $1 i k e 1 y$ be set by cost constraints. An option that should be evaluated is running a number of single-capsule tests in series. For the purpose of setting a reference point, it is recommended that the next TRIO-type test include two capsules.

Another choice to be made is the material to be tested. In TRIO, 1ithium aluminate was tested. Lithium aluminate should be similar to a number of the ternary lithium oxides because of its high melting point, low thermal conductivity, and chemical inertness. Lithium oxide, which is rather different in 
the above aspects and is a genarally attractive candidate breeder material, should be tested in a TRIo-type test. Litlifum oxide has been tested in-pile in experiments at JAERI, 27,30 but those experiments were somewhat limited in scope. Lithium oxide remains the most logical choice for the next experiment. The high thermal conductivity and the low activation energy for diffusion are favorable for this material. Therefore, it is recommended that one capsule contain lithium oxide. The selection of material for the second capsule is now an open question. It could be lithium oxide of different morphology, an appropriate ternary oxide, or some advanced material. 
REPERENCES 


\section{REPERENCES}

1. C. C. Baker et a1., "STARFIRE - A Commercial Tokamak Fusion Power Plant Study," Argonne National Laboratory, ANL/FPP-80-1 (1980).

2. B. Badger et al., "UWMAK-II," University of Wisconsin, UWFDM-112 (1975).

3. M. A. Abdou, L. J. Wittenberg, and C. W. Maynard, "A Fusion Design Study of Nonmobile Blankets with Low Lithium and Tritium Inventories," Nucl. Technol. 26, 400 (1975).

4. J. R. Powell et al., "Preliminary Reference Design of a Fusion Reactor Blanket Exhibiting Very Low Residual Radioactivity," Brookhaven National Laboratory, BNL-19565 (1974).

5. K. R. Schultz et al., "Blanket, Shield, and Power Conversion System for a Small Field Reversed Mirror Fusion Center," General Atomic Company, GA-A15533 (1979).

6. R. L. Hagenson et al., "The Reversed-Field Pinch Reactor (RFPR) Concept," Los Alamos National Laboratory, LA-7973-MS (1979).

7. D. W. Kearney et al., "Doublet Demonstration Fusion Power Reactor Study," General Atomic Company, GA-A14742 (1978).

8. D. L. Smith et a1., "Fusion Reactor Blanket/Shield Design Study," Argonne National Laboratory and McDonnell Douglas Astronautics Company, ANL/FPP79-1 (1979).

9. M. Abdou et al., "Blanket Comparison and Selection Study - Interim Report," Argonne National Laboratory, ANL/FPP/TM-177 (1983).

10. M. Abdou et al., "A Demonstration Power Plant Study," Argonne National Laboratory, ANL/FPP/82-1 (1982).

11. D. Steiner et al., "ORNL Fusion Power Demonstration Study," Oak Ridge National Laboratory, ORNL/TM-5813 (1977).

12. K. Sako et al., "Design Study of a Tokamak Reactor," Japan Atomic Ener $3 y$ Research Institute, IAEA-CN-33/G1-2 (1982).

13. U.S. FED-INTOR Activity and U.S. Contribution to the International Tokamak Reactor Phase-2A Workshop, USA FED-INTOR/82-1 (1982).

14. Standard Mirror Fusion Reactor Design Study, Lavrence Livermore National Laboratory, UCID-17644 (1978).

15. ELMO Bumpy Torus Reactor and Power Plant, Los Alamos National Laboratory, LA-8882-MS (1981).

16. E. M. Larsen, R. G. Clemmer, and D. K. Sze, Trans. Am. Nuc1. Soc. 23, 56 (1976), and references therein. 
17. R. G. Clemmer, "The Development of Tritium Breeding Blankets for DTBurning Fusion Reactors," Proc. 4th Top. Mtg. on The Technology of Controlled Nuclear Fusion, USDOE, CONF-801011, p. 526 (1980), and references therein.

18. D. L. Smith et al., "Analysis of In-Situ Tritium Recovery from Solid Breeder Blankets," ibid, p. 560, and references therein.

19. D. Okula and D. K. Sze, "Tritium Recovery from Solid Breeders: Implications of the Existing Data," University of Wisconsin, UWFDM-351 (1980), and references therein.

20. J. W. Davis et al., Proc. Workshop on Tritium Breeding Solids - Research and Development, USDOE, DOE/ET-52039/1 (1981) and references therein.

21. S. Nasu, "Data Base for Breeder Choice," Japan Atomic Energy Research Institute, JAERI-M-8510 (1979) and references therein; also published as Appendix C, Ref. 19 .

22. D. L. Smith et al., "Assessment of Solid Breeding Blanket Options for Commercial Tokamak Reactors," Proc 8th Symp. on Engineering Problems of Fusion Research, San Francisco, IEEE Pub. No. 79CH1441-5, p. 433 (1979).

23. C. E. Johnson, R. G. Clemmer, and G. W. Hollerberg, "Solid Breeder Materials," J. Nucl. Mater. 103, 547 (1981), and references therein.

24. C. E. Johnson and G. W. Hollenberg, "Recent Advances in the Development of Solld Breeder Blankets," Proc. 3rd Top. Mtg. on Fusion Reactor Materials, Albuquerque, New Mexico, September 19-22, 1983.

25. R. G. Clemmer et a1., "The TRIO-01 Experiment: In-Situ Tritium Recovery Results," ibid.

26. G. W. Hollenberg, "Fast Neutron Irradiation Results on $\mathrm{Li}_{2} \mathrm{O}_{2} \mathrm{Li}_{4} \mathrm{SiO}_{4}$, $\mathrm{Li}_{2} \mathrm{ZrO}_{3}$, and $\mathrm{LiAlO}_{2}$," ibid.

27. T. Kurasawa et al., "In-Situ Tritium Recovery Experiment from Lthium Oxide Under High Neutron Fluence," ibid.

28. R. G. Clemmer, R. F. Malecha, and I. T. Dudley, "The TRIO-01 Experiment," Nuc1. Technol./Fusion 4, 83 (1983).

29. R. Wiswal1, E. Wirsing, and K. C. Hong, "The Removal of Tritium from Fusion Reactor Blankets," Brookhaven National Laboratory, BNL-50748 (1977); see also, R. H. Wiswall and E. Wirsing, "Tritium Recovery from Fusion Blankets Using Solid Lithium Compounds-II: Experiments on Tritium Removal and Absorption," Proc. Intern. Conf. on kadiation Effects and Tritium Technology for Fusion Reactors, Gatlinburg, TN, USA, October 1-3, 1975; and R. Wiswall, E. Wirsing, and K. C. Hong, "The Removal of Bred Tritium from Solid Lithium Compounds in Fusion Reactor Systems," Proc. 14th IECEC (1976). 
30. S. Nasu, "A Preliminary In-Pile Test of Tritium Release from $\mathrm{LH}_{2} \mathrm{O}$ Pellets," Japan Atomic Energy Research Institute, JAERI-Memo 9276 (1980).

31. L. Yang et al., "Irradiation Study of Lithium Compound Samples for Tritium Breeding Appilications," GA Technologies, GA-A17407 (1981).

32. D. L. Porter et al., "Irradiation and Compatibility Testing of $\mathrm{LH}_{2} \mathrm{O}$ Materials at EBR-II," Argonne National Laboratory, ANL/FPP/TM-167 (1982).

33. M. S. Ortman and E. M. Larsen, "The Preparation, Characterization, and Melting Point of Lithium Oxide," J. Am. Ceramic Soc., 66(9), 645 (1983).

34. G. W. Hollenberg, in "Fifth Annual Progress Report On Special Purpose Materials for Magnetically Confined Fusion Reactors,". USDOE, DOE/ER$0113 / 2$ (1983).

35. T. Takahashi and T. Kikuchi, J. Nuc1. Mater. 91,93 (1980).

36. R. W. McCulloch, Oak Ridge National Laboratory, personal communication (1983).

37. M. S. Ortman and E. M. Larsen, "Preparation and Melting Point of Octa1ithium Zirconate," Comm. Am. Ceramic Soc. C-142 (August 1983).

38. W. Jost, Diffusion in Solids, Liquids, and Gases, Academic Press, New York, p. 60(1960).

39. R. Treybal, Mass-Transfer Operations, McGraw-Hi11, New York, pp. 79-81 (1968).

40. W. G. Sheehan, M. L. Curtis, and D. C. Carter, "Development of a Low Cost Versatile Method for Measurement of HTO and HT in Air," Mound Laboratory, MLM-2205 (1975).

41. Union Carbide Data Sheet on Linde Molecular Seive Type 4A Pellets (1982).

42. K. R. Thoms, Oak Ridge National Laboratory, personal communication.

43. L. P. Greenwood, "Dosimetry Results for the TRIO test in ORR" in Damage Analysis and Fundamental Studies, Quarterly Progress Report, April-June 1982 , U.S. Department of Energy, DOEE/ER-0046, p. 9 (1982).

44. D. W. Knef, B. M. Oliver, M. M. Nakata, and H. Farrar IV, "Helium Production in Li-6 and B-10 from ORR-TRIO," in Damage Analysis and Fundamental Studies, Quarter1y Progress Report, July-September 1982, U.S. Department of Energy, DOE/ER-0046/10, p. 36 (1982).

45. P. A. Finn et a1., "The Design, Fabrication, and Testing of the Gas Analysis System for the Tritium Recovery Experiment, TRIO-01," 10th Symp. on Fusion Engineering, Philadelphia, PA, December 5-9, 1983. 
46. E. H. Van Deventer and V. A. Maroni, "Hydrogen Permeation Characteristics of Some Austenitic and Ferritic Alloys," J. Nucl. Mater. 92, 103 (1980); see also V. A. Maroni, "Materials Considerations in Tritium Handling Systems," Proc. 1st Top. Mtg. on Fusion Reactor Materials, January 29-31, 1979, Miami, Florida, and referonces therein.

47. E. W. Roberts and J. P. Roberts, Bull. Soc. Franc. Ceram., 77, 3 (1967).

48. V. K. Hauffe and 0. Hoeffgren, Berichte Bunsen-Gese11., Phsyikal. Chem. 74, 537 (1970).

49. T. S. Elleman et al., Proc. 3rd Top. Mtg. on the Technology of Controlled Nuclear Fusion, Santa Fe, NM, Vol. 2, p. 763 (1978).

50. W. Yunker, "Continuous Extraction of Tritium From Irradiated Lithium Aluminate," Hanford Engineering Development Laboratory, TC-1745 (1980).

51. D. Guggi, H. Thle, A. Neubert, and R. Wolfe, "Tritium Release from LiAlO ${ }_{2}$," Intern. Conf. on Radiation Effects and Tritium Technology for Fusion Reactors, Gatlinburg, TN, October 1-3, 1975; see also D. Guggi, H. Ihle, and U. Kurz, "Tritium Release from Neutron-Irradiated LithiumAluminum Oxides," Proc. 9th Symp. on Fusion Technology, EUR-5602, p. 337 (1975).

52. V. G. Vasiliev et al., "Investigation of the Physical-Chemical Properties of Irradiated Inorganic Compounds of Lithium Oxides, Aluminates, and Silicates," US/USSR Workshop on Engineering and Economic Problems of ETF, Moscow and Lenningrad (USSR), September 10-21, 1979.

53. S. W. Tam, Argonne National Laboratory, private communication (1973).

54. F. G. Perry, "Least-Square Spectral Adjustment: The Computer Code STAYSL," Oak Ridge National Laboratory, ORNL/TM-6062 (1979); modified by L. R. Greenwood. 


\section{ACKRONLEDGERNTS}

The authors wish to acknowledge the many people and organizations who contributed to the success of this project. The work was supported by the Office of Fusion Energy, U.S. Department of Energy. Of the many people at Argonne, a few deserve special mention: V. A. Maroni who conceived the idea for the experiment and provided much useful guidance; c. C. Baker who provided guidance, advice, and leadership; C. Hytry who prepared and assembled this document; and the many people from the Chemical Technology Division and the Materials Science Division who made numerous valuable contributions. The authors are indebted to J. R. Conlin, C. D. West, and other people from Oak Ridge National Laboratory. 


\section{DISTRIBUTION FOR ANL-84-55}

\section{Internal:}

$\begin{array}{ll}\text { C. Baker } & \text { C. Johnson } \\ \text { M. Billone } & \text { Y. Liu } \\ \text { D. Bowers } & \text { R. Malecha } \\ \text { Y. Cha } & \text { V. Maroni } \\ \text { R. Clemmer (50) } & \text { R. Mattas } \\ \text { D. Diercks } & \text { B. Misra } \\ \text { A. Fischer } & \text { L. Neimark } \\ \text { F. Fradin } & \text { R. Poeppe1 } \\ \text { L. Greenwood } & \text { D. Porter } \\ \text { D. Gruen } & \text { W. Praeg } \\ \text { P. Finn } & \text { J. Roberts } \\ \text { A. Fischer } & \text { G. T. Reedy }\end{array}$

D. L. Smith

M. Steindler

D. K. Sze

S. W. Tam

H. Thresh

M. Tetenbaum

FPP Files (10)

ANL Contract File

ANL Li braries

ANL Patent Dept. TIS Files (6)

\section{Externa1:}

DOE-TIC, for distribution per UC-20, 20c, 20d, 20e (196) Manager, Chicago Operations office, DOE

University of Chicago Special Committee for the Fusion Program:

S. Baron, Brookhaven National Laboratory

H. Forsen, Bechtel Group, Inc.

J. Maniscalco, TRW, Inc.

G. Miley, University of I11inois-Urbana

P. Reardon, Brookhaven National Laboratory

P. Rutherford, Princeton University

D. Steiner, Rensselaer Polytechnic Institute

K. Symon, University of Wisconsin-Madison

K. Thomassen, Lawrence Livermore National Laboratory

University of Chicago Review Committee for the Chemical Technology Division:

K. H. Keller, U. Minnesota

T. A. Milne, Solar Energy Research Inst.

H. Perry, Resources for the Future, Washington

R. Winston, U. Chicago

W. L. Worre11, U. Pennsylvania

R. Wymer, Oak Ridge National Lab.

E. B. Yeager, Case Western Reserve U.

M. A. Abdou, Untversity of California-Los Angeles

R. Alire, Lawrence Livermore National Laboratory

J. Anderson, Los Alamos National Laboratory

R. Arons, Celenase Resarch Company

A. Bancroft, Chalk River Nuclear Laboratories, Canada

J. Bartift, Los Alamos National Laboratory

S. Berk, U. S. Department of Energy, office of Fusion Energy

S. Buchsbaum, Bel1 Telephone Laboratories, Inc.

G. Casini, Joint Research Centre, Ispra Establishment, Italy

G. Caskey, E. I. DuPont de Nemours, Savannah River Laboratory

G. Catchen, Pennsylvania State University

R. Childs, Oak Ridge National Laboratory

E. Clemmer, Oak Ridge National Laboratory

F. Clinard, Jr., Los Alamos National Laboratory

M. Cohen, U. S. Department of Energy, Office of Fusion Energy

J. Conlin, Oak Ridge National Laboratory 
R. Conn, University of California-Los Angeles

J. Darvas, Commission of the European Communities, Belgium

R. Conrad, JRC Petten, The Netherlands

J. Davis, McDonnel1 Douglas Astronautics Company

S. Dean, Fusion Power Associates, Gaithersburg, MD

D. DeFreece, McDonnell Douglas Astronaitics Company

D. Doran, Hanford Engineering Development Laboratory

I. Dudley, Oak Ridge National Laboratory

F. Dyer, Dak Ridge National Laboratory

K. Ehrlich, Kernforschungszentrum Karlsruhe, Federal Republic of Germany

R. E1chelberger, Rockwell International, Canoga Park, CA

B. Engholm, GA Technologies Inc.

J. Fillo, Brookhaven National Laboratory

P. Fisher, Oak Ridge National Laboratory

C. Flanagan, Westinghouse Electric Corporation, Oak Ridge, TN

T. Galloway, Lawrence Livermore National Laboratory

P. Gildea, Sandia National Laboratories, Livermore, CA

J. Gilligan, North Carolina State University

R. Gold, Westinghouse Electric Corporation, Madison, PA

D. Graumann, GA Technologies Inc.

E. Hager, GA Technologies Inc.

C. Henning, Lawrence Livernore National Laboratory

R. Hickman, Lawrence Li vermore National Laboratory

N. Hoffman, Energy Technology Engineering Center, Canoga Park, CA

3. Hollenberg, Hestinghouse Hanford Company

G. Hopkins, GA Technologies Inc.

P. Hsu, EG\&G Idaho, Inc.

P. Hubberstey, University of Nottingham, United Kingdom

3. Hurley, Los Alamos National Laboratory

J. Hurley, Rockwell International, Golden, Co

). Jassby, Princeton Plasma Physics Laboratory

c. Keller, University of Minnesota

$\therefore$ Kershner, Monsanto Research Corporation

). Kneff, Rockwe11 International, Canoga Park, CA

1. Kinoshita, Japan Atomic Energy Research Institute

3. Krakowski, Los Alamos National Laboratory

3. Kulcinski, University of Wisconsin-Madison

1. Kwast, Netherlands Energy Research Foundation, ECN, The Netherlands

¿. Larsen, University of Wisconsin-Madison

i. Longhurst, EG\&G Idaho, Inc.

?. Lykoudis, Purdue University

.. McGrath, Sandia National Laboratories, Al buquerque, NM

3. Merri11, EG\&G Idaho, Inc.

I. Migge, Hahn-Meitner Institut fur Kernforschung Berlin, FRG

I. Mintz, Sandia National Laboratories, Livermore, CA

i. Moir, Lawrence Livermore National Laboratory

i. Morgan, McDonnel1 Douglas Astronautics Company

$\therefore 0^{\prime} \mathrm{Kula}$, E. I. DuPont de Nemours, Savannah River Laboratory

l. Opdenecker, U. S. Department of Energy, Office of Fusion Energy

l. Overhoff, Overhof $f$ and Associates

i. Pierini, ISPRA, Varese, Italy

$\therefore$ Ruether, U. S. Department of Energy, Of fice of Fusion Energy

1. Rogers, Monsanto Research Corporation

$\therefore$ Roth, CEA, Centre d'Etudes Nucleaires de Saclay, France 

A. Scandora, Science Applications, Inc.
K. Schultz, GA Technologies Inc.
F. Scott, Electric Power Research Institute, Palo Alto
J. Scott, Oak Ridge National Laboratory
T. Shannon, FEDC, Oak Ridge National Laboratory
H. Takeshita, Japan Atomic Energy Research Institute, Japan
A. Tobin, Grumman Aerospace Corporation, Bethpage, NY
P. Tortorelli, Oak Ridge National Laboratory
C. Trachsel, McDonnell Douglas Astronautics Company
V. Vassiliev, International Atomic Energy Agency, Vienna, Austria
R. Verbeek, Comnission of the European Communities, Belgium
L. Waganer, McDonnell Douglas Astronautics Company
H. Watanabe, Japan Atomic Energy Research Institute, Japan
C. D. West, Oak Ridge National Laboratory
P. Wienhold, Institute fur Plasmaphysick, Kernforschungsanlage Jơlich, FRG
H. Willenberg, Mathematical Science N.W.
L. Wittenberg, Univesity of Wisconsin-Madison
W. Wolfer, University of Wisconsin-Madison
C. Wong, GA Technologies Inc.
C. Wu, Institute fur Chemie, Kernsforschungsanlage Jưlich, FRG 